Prehistory of the

Indo-Malaysian Archipelago

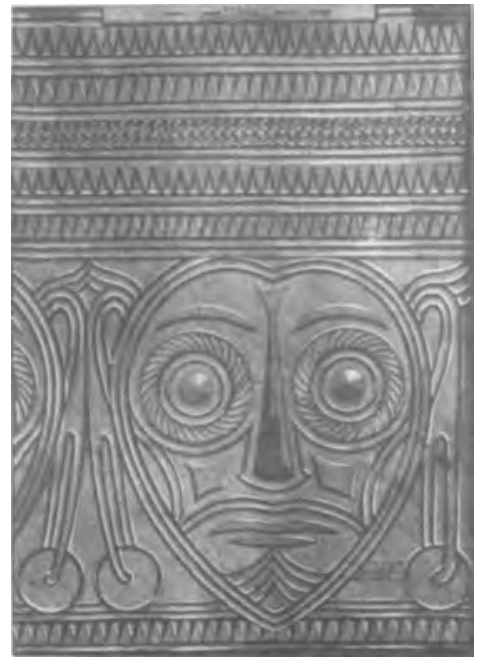




\title{
Prehistory of the
}

\section{Indo-Malaysian Archipelago}

\author{
REVISED EDITION
}

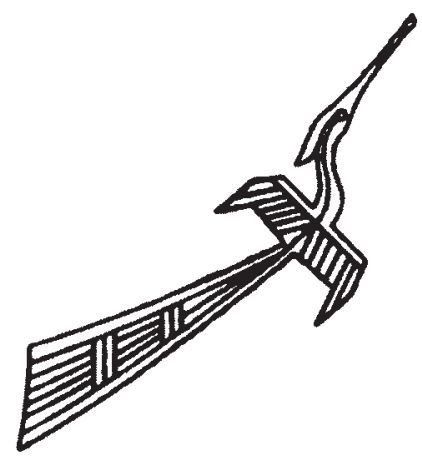

Peter Bellwood

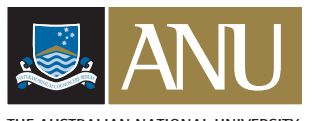

THE AUSTRALIAN NATIONAL UNIVERSITY

E PRESS 


\section{ANU}

E PRESS

Published by ANU E Press

The Australian National University

Canberra ACT 0200, Australia

Email: anuepress@anu.edu.au

This title is also available online at: http://epress.anu.edu.au/pima_citation.html

Previously published by University of Hawai'i Press

National Library of Australia

Cataloguing-in-Publication entry

Bellwood, Peter, 1943- .

Prehistory of the Indo-Malaysian Archipelago.

3rd ed.

Bibliography.

Includes index.

ISBN 9781921313110 (pbk)

ISBN 9781921313127 (online)

1. Prehistoric peoples - Indonesia. 2. Prehistoric peoples

- Malaysia. 3. Indonesia - Antiquities. 4. Malaysia -

Antiquities. I. Title.

959.01

All rights reserved. No part of this publication may be reproduced, stored in a retrieval system or transmitted in any form or by any means, electronic, mechanical, photocopying or otherwise, without the prior permission of the publisher.

Book design by Barbara Pope Book Design

Frontispiece: Man astride a buffalo, with necklace, helmet, and anklets, Pematang, Pasemah. From Hoop 1932. Courtesy: W. J. Thieme

Printed by University Printing Services, ANU

First Edition (C) 1985 Academic Press, Sydney

Revised Edition (C) 1997 University of Hawai'i Press

This edition (C) 2007 ANU E Press 


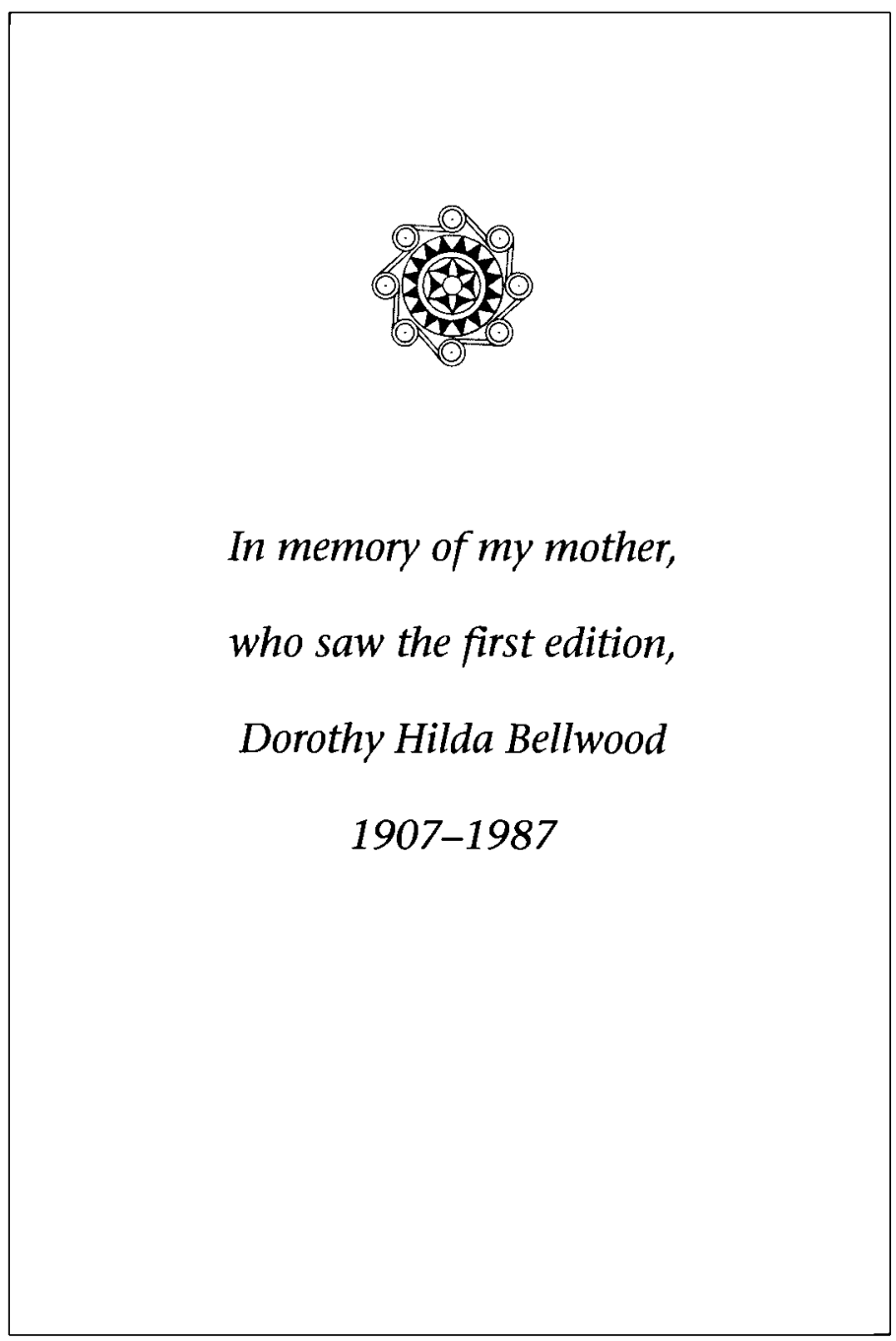





\section{Contents}

Preface to this Edition ix

Preface xv

1 The Environmental Background: Present and Past

I The Indo-Malaysian Archipelago 1

II The Indo-Malaysian Environment 9

III The Pleistocene and Worldwide Changes in Environment 16

IV The Environmental History of the Indo-Malaysian Archipelago during the Pliocene and Pleistocene 22

2 Homo erectus in Sundaland

I The Antecedents 40

II Homo erectus in Java 46

III Homo erectus in Southeast Asia: The Cultural Evidence 55

IV Some Conclusions on "Early" Industries 68

3 Indo-Malaysians of the Last 40,000 Years

1 The Modern Populations of the Indo-Malaysian Region 71

II Genetic and Cranial Data on the Differentiation of Indo-Malaysian Populations 76

III Ancient Populations of Homo sapiens in the Indo-Malaysian Archipelago 82

4 Recent Indo-Malaysian Prehistory: According to the Languages

I Language Families in Southeast Asia and the Western Pacific 97

II Some Linguistic Concepts 100

III The Major Subgroups of Austronesian 104

Iv Dating the Austronesian Family Tree 113

v Indo-Malaysian Linguistic Prehistory: Some Possibilities 117

vı The Papuan Languages and Their Relationships with Indonesia 124

5 The Patterns of History and Ethnography

I The Hunters and Gatherers 131

II The Influences of India and Islam 136 
III The Indo-Malaysian Traditional Agricultural Societies 142

IV Other Ethnographic Features of Austronesian Societies 150

v The Comparative Reconstruction of Early Austronesian Society 152

6 The Hoabinhians and Their Island Contemporaries

I Peninsular Malaysia and Mainland Southeast Asia:

The Hoabinhian and Its Predecessors 158

II Island Southeast Asia: The Later Pebble and Flake Industries, with Variations 171

III The Flake and Blade Technocomplex of the Mid-Holocene 190

7 The Archaeological Record of Early Austronesian Communities

I The Origins of Agriculture 202

II The Beginnings of Austronesian Prehistory 211

III The Neolithic Phase in Island Southeast Asia and Western Oceania 219

IV An Integrated View of Early Austronesian Expansion 241

v The Stages of Austronesian Agricultural Prehistory 249

8 The Archaeological Record of Early Agricultural Communities in Peninsular Malaysia

I The Significance of the Ban Kao Culture and the Malay Peninsular Neolithic 265

9 The Early Metal Phase: A Protohistoric Transition toward Supra-Tribal Societies

I The Dong Son Culture of Northern Vietnam 269

II The Sa Huynh Culture of Southern Vietnam 271

III The Role of India 275

IV Bronze Artifacts of Dong Son and Local Styles from the Sunda Islands and Peninsular Malaysia 277

v The Slab Graves and Iron Industry of Peninsular Malaysia 284

v1 The Early Metal Phase in Sumatra, Java, and Bali 287

VII The Early Metal Phase in East Malaysia and Eastern Indonesia 295

10 A Final Overview

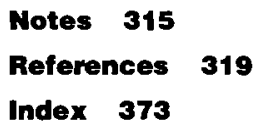

Plates follow page 164. 


\section{Preface to this Edition}

The original edition of this book was published by Academic Press, Sydney, in 1985. The text of this revised edition (henceforth PIMA 2) was completed in 1995, and published early in 1997 by the University of Hawai'i Press. In 2000, a Bahasa Indonesia translation of this revised edition was published as Prasejarah Kepulauan Indo-Malaysia by PT Gramedia Pustaka Utama in Jakarta.

The decision to go ahead with this electronic edition of PIMA 2 was made early in 2007, 10 years after its publication in Honolulu. The book has been out of print for several years, yet no one has written a similar successor, and a readership for its contents still exists; it still scores frequently in citation indices. Furthermore, PIMA 2 presents an overall reconstruction of Indo-Malaysian Prehistory that I am still willing to uphold quite forcefully, despite the need, here and there, for updating and minor modification of opinion. I would therefore like to thank ANU E Press for giving me this opportunity to make this work available again.

Two choices were available to me as this edition approached reality. One would have been to prepare a completely new third edition, a $P I M A 3$, updated throughout. Pressure of other involvements renders this impossible at the moment, and updating a full manuscript of this size would take the best part of a year. I have chosen the easier option, this being to keep the PIMA 2 text in its original form, but to add this short preface in which I refer very briefly to some selected new discoveries and current references.

The first such new discovery must, of course, be the remarkable Homo floresiensis, a dwarfed pre-sapient form of humanity that survived with equallydwarfed stegodons on Flores until the end of the Pleistocene, perhaps as recently as 12,000 years ago; an exciting cave discovery accompanied by plentiful academic intrigue, now described for a general readership by Morwood and van Oosterzee (2007). Although not of central significance for the prehistory of modern humans, the "hobbits" do reflect some interesting potential light on the early movements of extinct hominin species through Asia, possibly as much as 2 million years ago in deep ancestral terms. But how did hobbits and dwarf stegodons manage to survive in Flores for so long, given that modern humans reached Australia long before, by perhaps 50,000 years ago? It is my current (but mutable) opinion that these early modern humans moved through Nusa Tenggara, including Flores, but not Maluku, to their new homes in previously uninhabited Australia and New Guinea. They must have overlapped spatially and chronologically with the hobbits for tens of thousands of years. 
As far as my own research on pre-farming modern human populations is concerned, I now suspect the Tingkayu industry (Fig. 6.7) could be Holocene rather than Pleistocene in date, on the grounds that it has never turned up in cave excavations with well-dated Pleistocene levels (indeed, it has never turned up anywhere else at all). But I am still unsure about this; chronology is always a problem for archaeologists, especially for sites such as Tingkayu that yield no direct dating material. In addition, the shell adzes from Maluku shown in Fig. 25 are likely to be younger than 12,000 to 9000 years in terms of a recently-run series of direct AMS dates on the shell. The Tridacna and Hippopus specimens appear to be only mid-Holocene, in some cases made on ancient shell, and the Cassis specimens could possibly be Neolithic. I am also planning to obtain direct AMS dates for some of the bones of translocated New Guinea marsupials found in Maluku (page 188 below, and see Bellwood et al. 1998; Flannery et al. 1998). Pre-Neolithic translocation is an important issue and dating is still very imprecise.

Some of the post-1995 archaeological findings for the Neolithic and onwards are discussed in various chapters in Southeast Asia: from Prehistory to History (Glover and Bellwood 2004), and in briefer form in my own First Farmers (Bellwood 2005). A number of other recently-edited compilations (especially Sagart et al. 2005; Oxenham and Tayles 2006; Simanjuntak et al. 2006) contain a range of archaeological, archaeolinguistic and biological chapters that fill out many of the issues. The earliest Neolithic culture in Taiwan, the Dabenkeng (spelt, wrongly as it turns out, Ta-p'en-k'eng in the 1997 edition, using the old Wade-Giles spelling), is now confirmed as fully agricultural by at least $3000 \mathrm{BC}$, with rice and foxtail millet cultivation (Tsang 2005). Recent research in Taiwan and the Philippines, especially the Batanes Islands (Bellwood and Dizon 2005) and the Cagayan Valley of northern Luzon (Hung 2005), has strengthened greatly the evidence for Austronesian (Malayo-Polynesian) migration out of Taiwan and into Island Southeast Asia and Oceania after 2000 BC, in part with dentate stamped pottery that occurs in related forms in the Philippines, the Mariana Islands, and in Lapita sites in Melanesia (for my current overall views on Austronesian prehistory, see Bellwood and Hiscock 2005).

Interaction between Austronesian-speaking communities did not stop after colonization of new regions, as indicated already by the movement of New Britain obsidian to Borneo around 3000 years ago (see p 224). Since PIMA 2, new research in Taiwan has established that green jade earrings, either with three projections or with double animal-heads (see Figs 7.7, 9.2 and 9.3), were traded or exchanged between 2500 and 1500 years ago across a vast region that included Taiwan (the source of the some of the jade), the Philippines, Sarawak, southern Vietnam, southern Thailand and eastern Cambodia. One very distinctive type of 
green jade has recently been sourced by electron probe microanalysis to Fengtian in eastern Taiwan (Iizuka and Hung 2005; Hung et al. 2006), and a workshop for the earrings with projections using Fengtian jade has recently been excavated in the Batanes Islands (Bellwood and Dizon 2005). It is possible that these artifacts were made by itinerant craftsmen in workshops spread throughout the Austronesian-speaking world. My suspicion also is that these two artifact forms were reproduced over a millennium later in native nephrite in New Zealand, in the form of the Maori artifacts termed poria kaka and pekapeka, but the question of how the forms survived transmission through a jade-less tropical Polynesia still puzzles me (were they transmitted in perishable materials such as wood?).

While discussing Neolithic issues, I must state clearly that I no longer accept a movement of people from the Malay Peninsula to Sarawak, as suggested in PIMA 2 to explain the appearance around 4000 years ago of rice and cordmarked pottery in Gua Sireh (page 237). Neither the linguistic nor the archaeological evidence support a movement of Austroasiatic-speaking people from Mainland Southeast Asia to Borneo, and Taiwan and the Philippines provide a far more likely origin. It should be noted also that an indigenous development of agriculture in the New Guinea highlands has been supported by new research (Denham 2005, and see related papers in that same volume), whereas evidence for pre-Austronesian agriculture in Island Southeast Asia remains still non-existent. Like New Guinea, research into early agricultural (rice and millet) growing societies in Neolithic China has gone ahead with remarkable intensity (see accounts in Yang 2004), revealing for us a population powerhouse through at least the past 7000 years, indeed a major background player in the Neolithic archaeology of the whole of Southeast Asia.

Populations bring up issues about genetics. In my recent book, First Farmers, I have built up an archaeological, linguistic and biological case for the spreads of early farming populations over very large extents of territory in many parts of the world (see also Bellwood and Renfrew 2002; Diamond and Bellwood 2003). Both PIMA 1 and 2 presented a similar hypothesis for the Austronesian-speaking peoples, with an ultimate origin in southern China and Taiwan and an expansion over half way around the world within the past 4000 years. This expansion led to a fairly clean replacement of earlier languages by Austronesian forms of speech, and a relatively strong spread of a Neolithic technology and economy, albeit with here-and-there survivals of lithic and shell technologies with local pre-Austronesian roots, particularly in eastern Indonesia and Melanesia. Genetically, however, the picture was more complex owing to the human ability for intermarriage and genetic recombination, an ability not available, beyond normal processes of borrowing, to whole languages. As a result, not all speakers of Austronesian languages have identical genotypes. 
I mention this because, when PIMA 2 went to press, a plethora of genetic research into the phylogenies and coalescence times of mitochondrial and Ychromosome DNA lineages had not yet come into being. Nowadays, the international genetics journals are fairly replete with such material. As a non-biochemist who has to keep up to date with all of this and try to work out where it is all going, I can perhaps state that I can believe in the phylogenies and relative ages of these haploid lineages, but not some of the molecular clock absolute dates, which have very large error ranges, a problem exacerbated by very weak methods of calibration to real time (Bellwood 2007:103). There are also many issues concerning the role of natural selection and the ability of $\mathrm{mtDNA}$ and the Y chromosome to plot the histories of whole human populations, as opposed merely to the histories of the lineages themselves. Enough said perhaps at this point, and with some of my colleagues I hope soon to be publishing on this matter (see chapter 12 in Bellwood 2005 for my current views on Southeast Asia).

This brief update has been selective; I have no space to review all the regional archaeology carried out in Malaysia, Indonesia and the Philippines since 1995, from Palaeolithic cave deposits (e.g. Simanjuntak et al. 2001, Simanjuntak 2002 for Java; Barker et al. 2002 for the Niah Caves; O'Connor et al. 2005 for the Aru Islands), through red slipped Neolithic pottery in Taiwan and the Philippines (Hung 2005), to anthropomorphic 2000-year old burial jars from Mindanao (Dizon and Santiago 1996). The major regional journals, such as Asian Perspectives and Bulletin of the Indo-Pacific Prehistory Association, continue to carry many reports every year, as do the in-country national language journals. And then there are all the monographs and $\mathrm{PhD}$ theses, some of the latter supervised here at ANU. However, as stated above, I can still recommend the general framework for Indo-Malaysian prehistory presented in PIMA 2 as a good horse to bet upon. I hope this book still makes good reading in the new millennium.

Peter Bellwood

Canberra

February 2007 


\section{REFERENCES}

Barker, G. et al. 2002. Prehistoric foragers and farmers in South-east Asia: renewed investigations at Niah Cave, Sarawak. Proceedings of the Prehistoric Society 68:147-64.

Bellwood, P. 2005. First Farmers: The Origins of Agricultural Societies. Oxford: Blackwell.

Bellwood, P. 2007. Reply, in Review Feature on First Farmers. Cambridge Archaeological Journal 17:102-6.

Bellwood, P. and E. Dizon. 2005. The Batanes archaeological project and the "Out of Taiwan" hypothesis for Austronesian dispersal. Journal of Austronesian Studies 1 no. 1: 1-33. Taidong, Taiwan.

Bellwood, P. and P. Hiscock. 2005. Australia and the Austronesians. In C. Scarre (ed.), The Human Past, 264-305. London: Thames and Hudson.

Bellwood, P. G. Nitihaminoto, G. Irwin, Gunadi, A. Waluyo and D. Tanudirjo. 1998. 35,000 years of prehistory in the Northern Moluccas. Modern Quaternary Research in Southeast Asia 15:233-75. Rotterdam: Balkema.

Bellwood, P. and C. Renfrew eds. 2002. Examining the Farming/Language Dispersal Hypothesis. Cambridge: McDonald Institute for Archaeological Research.

Denham, T. 2005. Agricultural origins and the emergence of rectilinear ditch networks in the highlands of New Guinea. In A. Pawley, R. Attenborough, J. Golson and R. Hide (eds.), Papuan Pasts, 329-62. Canberra: Pacific Linguistics.

Diamond, J. and P. Bellwood. 2003. Farmers and their languages: the first expansions. Science 300: 597-603.

Dizon, E. and R. Santiago. 1996. Faces from Maitum. Quezon City: Capitol.

Flannery, T., P. Bellwood, J. White, T. Ennis, G. Irwin, K. Schubert and S. Balasubramanian. 1998. Mammals from Holocene archaeological deposits on Gebe and Morotai Islands, Northern Moluccas, Indonesia. Australian Mammalogy 20/3:391-400.

Glover, I. and P. Bellwood eds. 2004. Southeast Asia: from Prehistory to History. London: RoutledgeCurzon.

Hung, H-C. 2005. Neolithic interaction between Taiwan and northern Luzon: the pottery and jade evidences from the Cagayan Valley. Journal of Austronesian Studies 1, no. 1: 109-34.

Hung, H-C., Y. Iizuka and P. Bellwood. 2006. Taiwan jade in the context of Southeast Asian archaeology. In E. Bacus, I. Glover and V. Pigott (eds.), Uncovering Southeast Asia's Past, 203-15. National University of Singapore Press. 
Iizuka, Y. and H-C. Hung. 2005. Archaeomineralogy of Taiwan nephrite: a sourcing study of nephritic artifacts from the Philippines. Journal of Austronesian Studies 1:33-79. Taitung, Taiwan.

Morwood, M. and P. van Oosterzee. 2007. The Discovery of the Hobbit. Sydney: Random House.

O'Connor, S., Spriggs, M. and Veth, P. eds. 2005. The Archaeology of the Aru Islands, Eastern Indonesia. Canberra: Pandanus Books.

Oxenham, M. and N. Tayles eds. 2006. Bioarchaeology of Southeast Asia. Cambridge: Cambridge University Press.

Sagart, L., R. Blench and A. Sanchez-Mazas eds. 2005. The Peopling of East Asia: Putting Together Archaeology, Linguistics and Genetics. London: RoutledgeCurzon.

Simanjuntak, T. 2002. Gunung Sewu in Prehistoric Times. Yogyakarta: Gadjah Mada University Press.

Simanjuntak, T., I. Pojoh and M. Hisyam eds. 2006. Austronesian Disapora and the Ethnogeneses of People in Indonesian Archipelago. Jakarta: LIPI Press.

Simanjuntak, T. , Prasetyo, B. and Handini, R. eds. 2001. Sangiran: Man, Culture and Environment in Prehistoric Times. Jakarta: Yayasan Obor Indonesia.

Tsang, C-H. 2005. Recent discoveries at a Tapenkeng culture site in Taiwan: implications for the problem of Austronesian origins. In L. Sagart, R. Blench and A. Sanchez-Mazas (eds.), The Peopling of East Asia, 63-73. London: RoutledgeCurzon.

Yang Xiaoneng ed. 2004. New Perspectives on China's Past: Chinese Archaeology in the Twentieth Century. 2 volumes. New Haven: Yale University Press. 


\section{Preface}

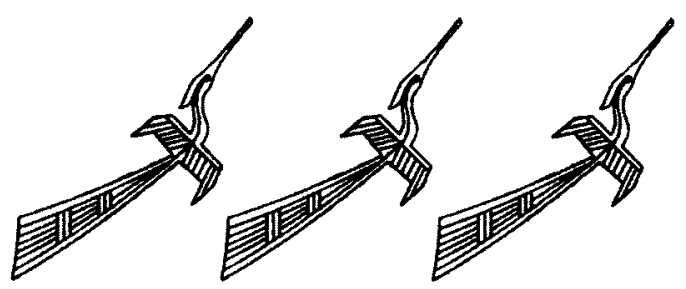

THIS BOOK PRESENTS a multidisciplinary reconstruction of the prehistory of the modern nations of Indonesia and Malaysia as viewed from the perspective of the whole Southeast Asian and Australasian region. Since modern nations' boundaries have little meaning for the student of the remote past, I refer to the region in the following chapters as "the Indo-Malaysian Archipelago." Several interlinked aspects of prehistory are reviewed, mainly from data produced by the disciplines of biological anthropology, linguistics, and archaeology. The overall time span runs from about 2 million years ago to approximately $\mathrm{AD} 1000$. In general, the book ceases with the historical civilizations of the first millennium AD, although it should be realized that prehistory sensu stricto continued in some remote regions to almost the present day.

I would like to acknowledge here the assistance of many of my colleagues who have read parts of the work. These include (for the first edition) Robert Blust of the University of Hawai'i, Roger Green of the University of Auckland, and Australian National University colleagues John Chappell, James Fox, Geoffrey Hope, Robert Kirk, Margot Lyon, Douglas Miles, Cecilia Ng, Alan Thorne, and Darrell Tyron. Helmut Loofs-Wissowa of ANU first suggested that I undertake the project. Colin Groves of ANU and Robert Hall of Royal Holloway, University of London, commented on Chapter 1 in the second edition, and John Chappell provided valuable advice on past sea levels. I wish also to thank Donn Bayard of the University of Otago for valuable editorial comments on the whole second edition manuscript. The Department of Archaeology and Anthropology at the Australian National University has provided invaluable staff assistance, and in this regard I would like to thank Louise Johnson, Jan Lee, and Anthea Bundock for typing and secretarial help. Joan Goodrum, Mandy Mottram, Kevin 
Cowan, and Val Lyon drew many of the maps and charts. Robert Dowhy, Karen Edwards, and the Instructional Resources Unit at ANU produced many of the photographs.

For the second edition I wish also to thank Larry Saha, Dean of the Arts Faculty at $\mathrm{ANU}$, for financial assistance toward the preparation of illustrations.

\section{DATING}

In general, I do not give full radiocarbon determinations with laboratory numbers in the text, but interested archaeologists can locate these through the references (see Bronson and White 1992, and Spriggs 1989 for recent date lists for Southeast Asia). Archaeological sites from about $8000 \mathrm{BC}$ onwards are given approximate $\mathrm{BC}$ or $\mathrm{AD}$ dates according to historical data or the current calibrated radiocarbon chronology (University of Washington, Quaternary Isotope Lab, Radiocarbon Calibration Program Rev. 3.0, 1993). Radiocarbon dated sites older than 10,000 years are generally given a ". . years ago" approximation.

\section{PRONUNCIATION AND PLACE-NAMES}

In Indonesian place-names the "c" is pronounced "ch" as in English "church," "ng" is pronounced as in "singer," and "ngg" as in "finger." Chinese placenames are in Pinyin and Taiwanese in Wade-Giles romanizations. 


\section{Preface}

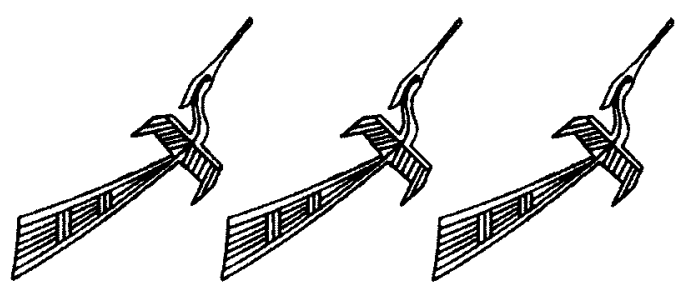

THIS BOOK PRESENTS a multidisciplinary reconstruction of the prehistory of the modern nations of Indonesia and Malaysia as viewed from the perspective of the whole Southeast Asian and Australasian region. Since modern nations' boundaries have little meaning for the student of the remote past, I refer to the region in the following chapters as "the Indo-Malaysian Archipelago." Several interlinked aspects of prehistory are reviewed, mainly from data produced by the disciplines of biological anthropology, linguistics, and archaeology. The overall time span runs from about 2 million years ago to approximately $\mathrm{AD} 1000$. In general, the book ceases with the historical civilizations of the first millennium AD, although it should be realized that prehistory sensu stricto continued in some remote regions to almost the present day.

I would like to acknowledge here the assistance of many of my colleagues who have read parts of the work. These include (for the first edition) Robert Blust of the University of Hawai'i, Roger Green of the University of Auckland, and Australian National University colleagues John Chappell, James Fox, Geoffrey Hope, Robert Kirk, Margot Lyon, Douglas Miles, Cecilia Ng, Alan Thorne, and Darrell Tyron. Helmut Loofs-Wissowa of ANU first suggested that I undertake the project. Colin Groves of ANU and Robert Hall of Royal Holloway, University of London, commented on Chapter 1 in the second edition, and John Chappell provided valuable advice on past sea levels. I wish also to thank Donn Bayard of the University of Otago for valuable editorial comments on the whole second edition manuscript. The Department of Archaeology and Anthropology at the Australian National University has provided invaluable staff assistance, and in this regard I would like to thank Louise Johnson, Jan Lee, and Anthea Bundock for typing and secretarial help. Joan Goodrum, Mandy Mottram, Kevin 
Cowan, and Val Lyon drew many of the maps and charts. Robert Dowhy, Karen Edwards, and the Instructional Resources Unit at ANU produced many of the photographs.

For the second edition I wish also to thank Larry Saha, Dean of the Arts Faculty at $\mathrm{ANU}$, for financial assistance toward the preparation of illustrations.

\section{DATING}

In general, I do not give full radiocarbon determinations with laboratory numbers in the text, but interested archaeologists can locate these through the references (see Bronson and White 1992, and Spriggs 1989 for recent date lists for Southeast Asia). Archaeological sites from about $8000 \mathrm{BC}$ onwards are given approximate $\mathrm{BC}$ or $\mathrm{AD}$ dates according to historical data or the current calibrated radiocarbon chronology (University of Washington, Quaternary Isotope Lab, Radiocarbon Calibration Program Rev. 3.0, 1993). Radiocarbon dated sites older than 10,000 years are generally given a ". . years ago" approximation.

\section{PRONUNCIATION AND PLACE-NAMES}

In Indonesian place-names the "c" is pronounced "ch" as in English "church," "ng" is pronounced as in "singer," and "ngg" as in "finger." Chinese placenames are in Pinyin and Taiwanese in Wade-Giles romanizations. 


\section{Prehistory of the \\ Indo-Malaysian Archipelago}

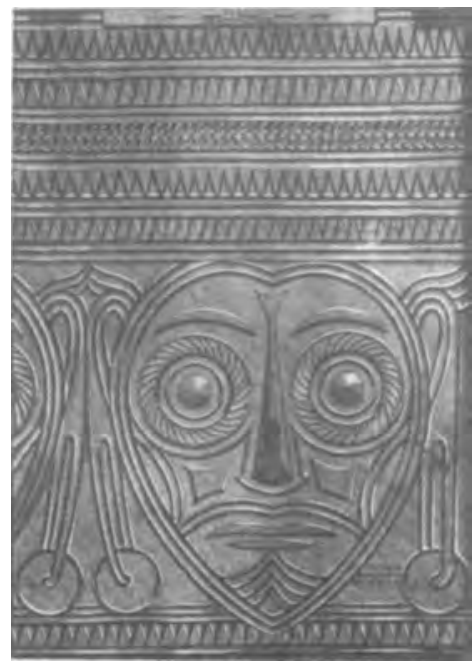





\section{The Environmental Background: Present and Past}

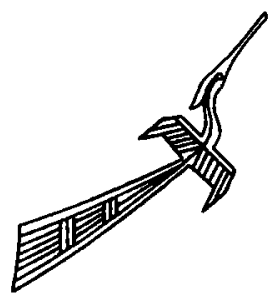

The Indo-Malaysian Archipelago (Fig. 1.1) demonstrates a certain unity in human terms today, in the sense that all its indigenous populations (with restricted exceptions in the Malay Peninsula and the far east of Indonesia) belong to the same major Austronesian-speaking ethnolinguistic group of mankind. The majority belong also to the Southeast Asian branch of the Mongoloid physical stock of mankind, although as I hope to indicate in the course of this book there is no simple one-to-one correlation between biology and language throughout the region. The reality is much more interesting.

However the picture of Indo-Malaysian humanity might appear now, there can be no doubt that it has changed in complex ways in the past. It is first necessary to introduce the archipelago itself from a basically geographical and environmental viewpoint in order to understand some of these changes. The object here is not to duplicate the numerous standard geographical treatises on the region, but rather to emphasize aspects of the environment, past and present, that are likely to have direct interpretative value for the human prehistoric record of the last two million years.

\section{THE INDO-MALAYSIAN ARCHIPELAGO}

The main area of interest includes all the islands of Indonesia and Malaysia (including the Malay Peninsula south of Thailand). The Philippines represent a direct extension northward of the Indo-Malaysian Archipelago, and although they lie outside the main area of concern of this book they will merit extended comment in later sections. Indeed, the prehistory of Indonesia in particular is very closely tied with that of the Philippines, especially in its later stages during 


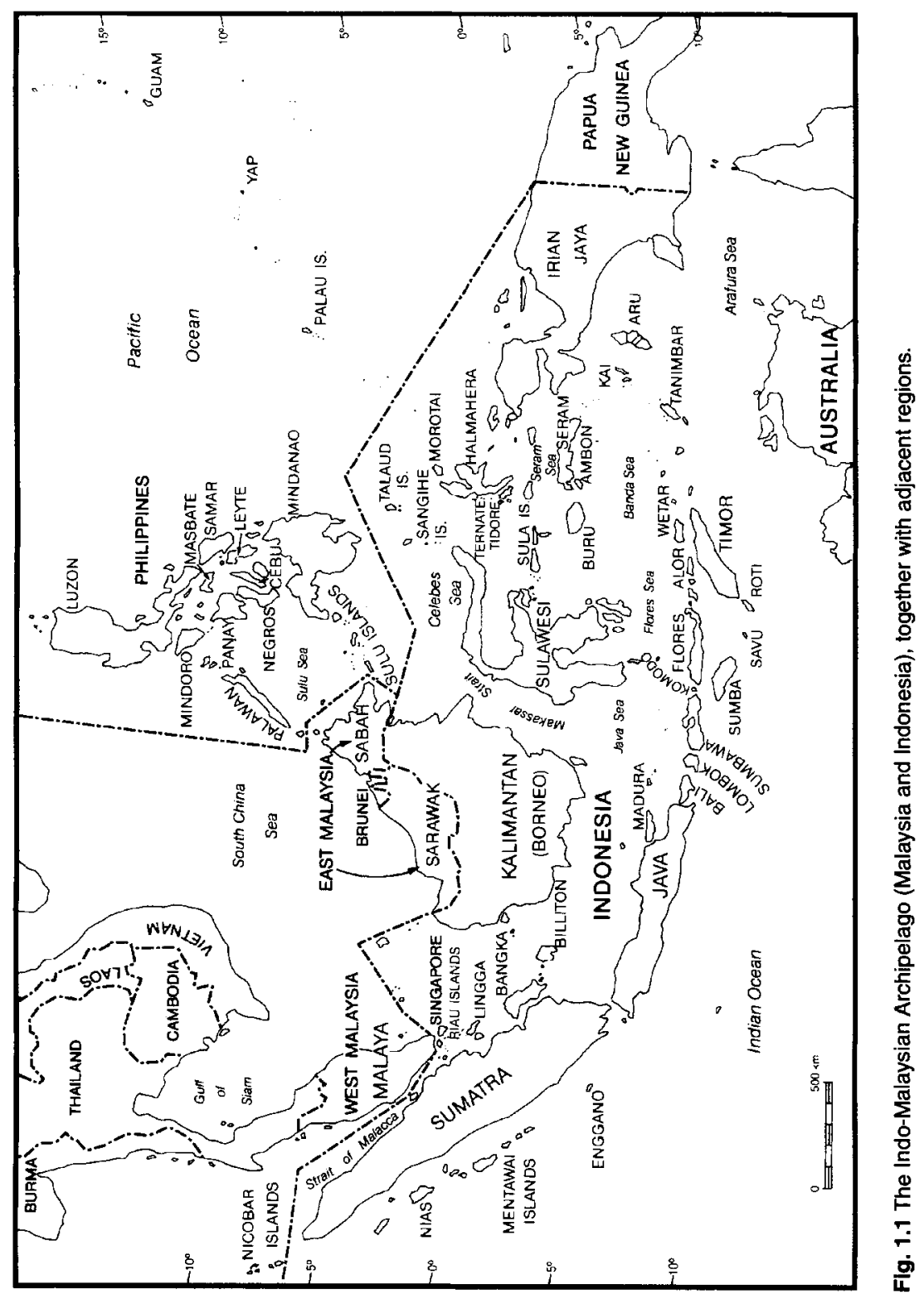


the period of expansion of the Austronesian-speaking peoples. Adjacent regions that will also require extended comment in some following sections include Taiwan, the countries of mainland Southeast Asia (especially Thailand and Vietnam), China (especially the southern half), and the Greater Australasian continent comprising Australia and New Guinea. The latter island, the western half of which is politically now a part of Indonesia, is not included in the IndoMalaysian Archipelago as here defined.

As defined for the purposes of this book, therefore, the "core region" of the Indo-Malaysian Archipelago extends from about $7^{\circ}$ north latitude (northern Peninsular Malaysia and Borneo ${ }^{1}$ ) to $11^{\circ}$ south (Sumba and Timor), and from the western tip of Sumatra to the Moluccas. The region is about 4,200 kilometers long from west to east, 2,000 kilometers from north to south, and supports about 1.8 million square kilometers of dry land, of which about 80 percent lies in Indonesia, with the remainder being in Malaysia (Peninsular Malaysia, plus the states of Sarawak and Sabah on Borneo) and Brunei.

The islands of this region differ greatly in size: Borneo covers 736,000 square kilometers (only slightly smaller than New Guinea), Sumatra comes next with 435,000 square kilometers, then Sulawesi $(172,000)$, the Malay Peninsula $(138,000$ within Malaysia), and Java $(127,000)$. The islands of western Indonesia are in general larger than those of eastern Indonesia (except for Sulawesi), and the reasons for this lie in the structure of the archipelago.

\section{A. The Shelves and Basins}

To understand the human prehistory of Indonesia it is necessary to know something of the geological, climatic, and biotic history of the archipelago. I will commence with some geology, referring to the splendid new color map reconstructions of Southeast Asia covering the past 50 million years produced by the Southeast Asia Research Group in London and now available to Internet users (Hall 1995, 1996).

The Indo-Malaysian islands, "the remarkable festoon of islands that swing around the equator in the East Indies" (Umbgrove 1949), fall into three fundamental structural divisions. The first, forming a direct extension of the Asian mainland in the west and north, comprises the Sunda continental shelf. The second, attached to the Indian Ocean edge of the shelf and extending beyond it east into the Moluccas, comprises the volcanic Sunda-Banda arcuate mountain and trench system. The third, in the northeast, comprises the Sulawesi-Philippine and Halmahera volcanic arc systems (Fig. 1.2, 1.3, 2.10).

The Sunda continental shelf (Tjia 1980), which has the largest area of submerged shelf in the world, has an old and fairly stable tectonic core that has had little recent volcanic activity. Much of it today lies beneath the sediments 


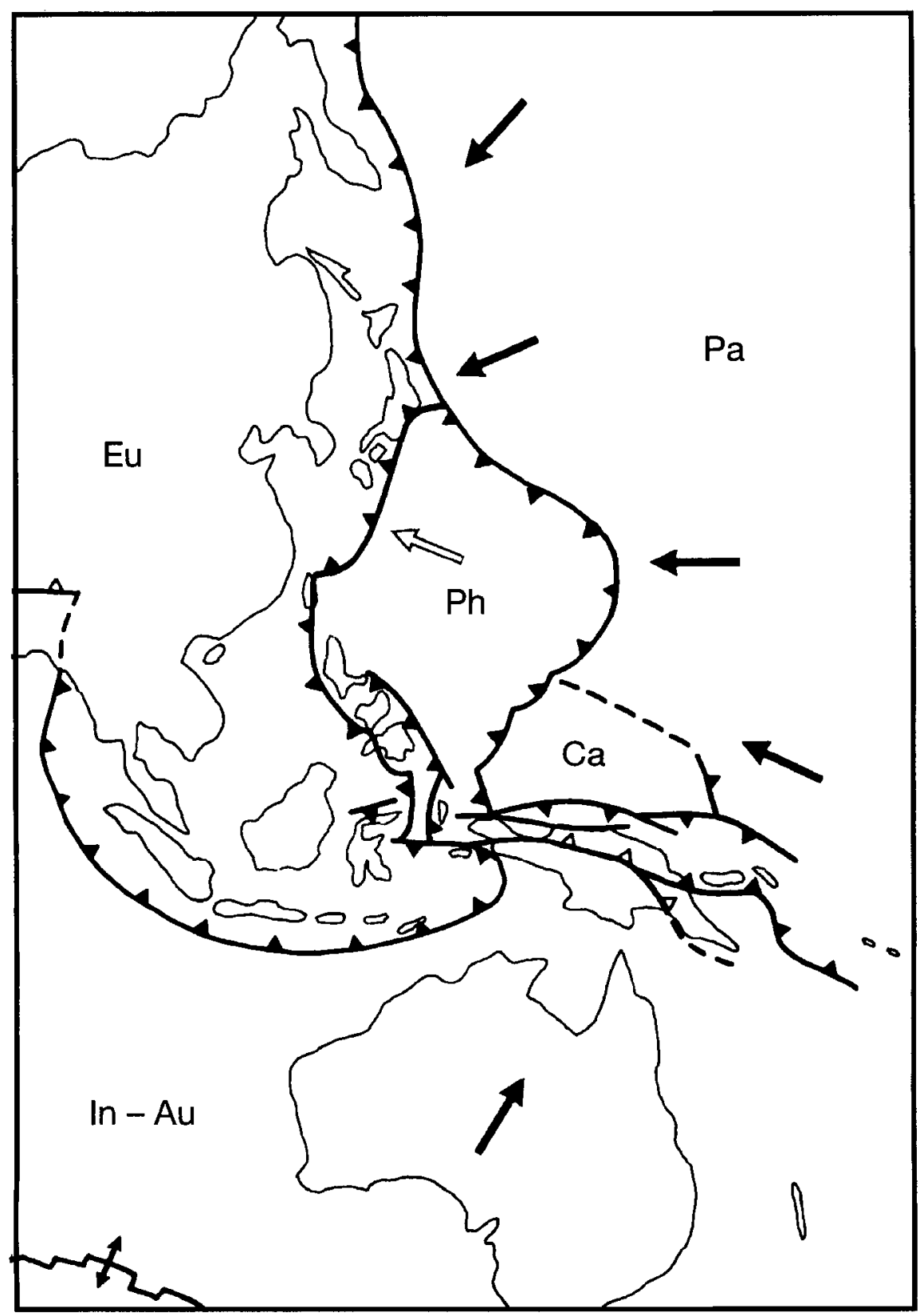

Fig. 1.2 Structural sketch map of eastern Asia, Australasia, and Southeast Asia showing the major plates and subduction zones (thick lines with triangles). Plates are as follows: Eu = Eurasian; $\mathrm{Ph}=$ Philippine; $\mathrm{Pa}=$ Pacific; $\mathrm{Ca}=$ Caroline; In-Au = India-Australia. After Rangin et al. 1990 . 
of the South China and Java Seas as a virtual peneplain worn down by erosion. Present land areas that rise above the old submerged shelf core include the Malay Peninsula, Borneo, and the northern coastal lowlands of Sumatra and Java. The volcanic mountains of Sumatra and Java are actually members of the Sunda-Banda arc system, which has formed along the Indian Ocean rim of the Sunda shelf. It is convenient to refer to the general western Indonesian shelf land mass as Sundaland (see Fig. 1.3), an area which of course contains both the Sunda shelf and a large portion of the present Sunda-Banda arc.

The Sunda-Banda arc includes the highland spines of Sumatra, Java, and the Lesser Sundas and forms one of the most remarkable volcanic mountain arcs in the world. It has been formed by subduction of the Indo-Australian Plate beneath the southern boundary of Indonesia (Rangin et al. 1990) (Fig. 1.2) and continues eastward as far as the southern Moluccas. The process of subduction, described briefly for the Sunda-Banda arc by Ollier (1985), has led to the upwarping of two parallel mountain chains, the inner one volcanic and the outer of uplifted sediments without active volcanoes. The inner volcanic chain includes eighty-two active volcanoes that extend in a curve from Sumatra through Java and into the Lesser Sundas and Moluccas. Outside this arc is a deep marine trench, beyond which the other nonvolcanic outer arc rises to support the small islands off the western coast of Sumatra, as well as Sumba, Timor, and Tanimbar.

So far, therefore, we have two major structural regions in Indonesia: the Sunda shelf and the Sunda-Banda mountain arc system wrought against its edge and beyond it. The latter is still in active construction, as witnessed by numerous volcanic eruptions (such as the famous Krakatoa eruption of 1883) and earthquakes. The third region is not so clearly defined and includes the remaining parts of eastern Indonesia. The Philippines and Sulawesi lie on double arcs similar to the Sunda-Banda arc, and other such arcs continue northward around the western Pacific rim through the Ryukyu Islands, Japan, and the Aleutians. Part of a smaller double arc also appears in Halmahera, farther toward the Pacific.

General accounts of the formation through geological time of the whole Indo-Malaysian region have been given by many geologists and earth scientists and this is a particularly difficult topic to summarize. Those who want current views should consult, as a broad sample of views, Katili $(1974,1975,1991)$, Audley-Charles (1981, 1987), Hamilton (1979), Rangin et al. (1990), McCabe and Cole (1989), Metcalfe (1996), and Hall $(1995,1996)$. The field appears to be one of some disagreement between authorities. For instance, during the 1970s Katili regarded Sundaland as the result of a series of successive volcanic arcs that had been forming since Permian times. According to his thesis, the earliest was represented in the older eroded northerly parts of the shelf, and the latest was 


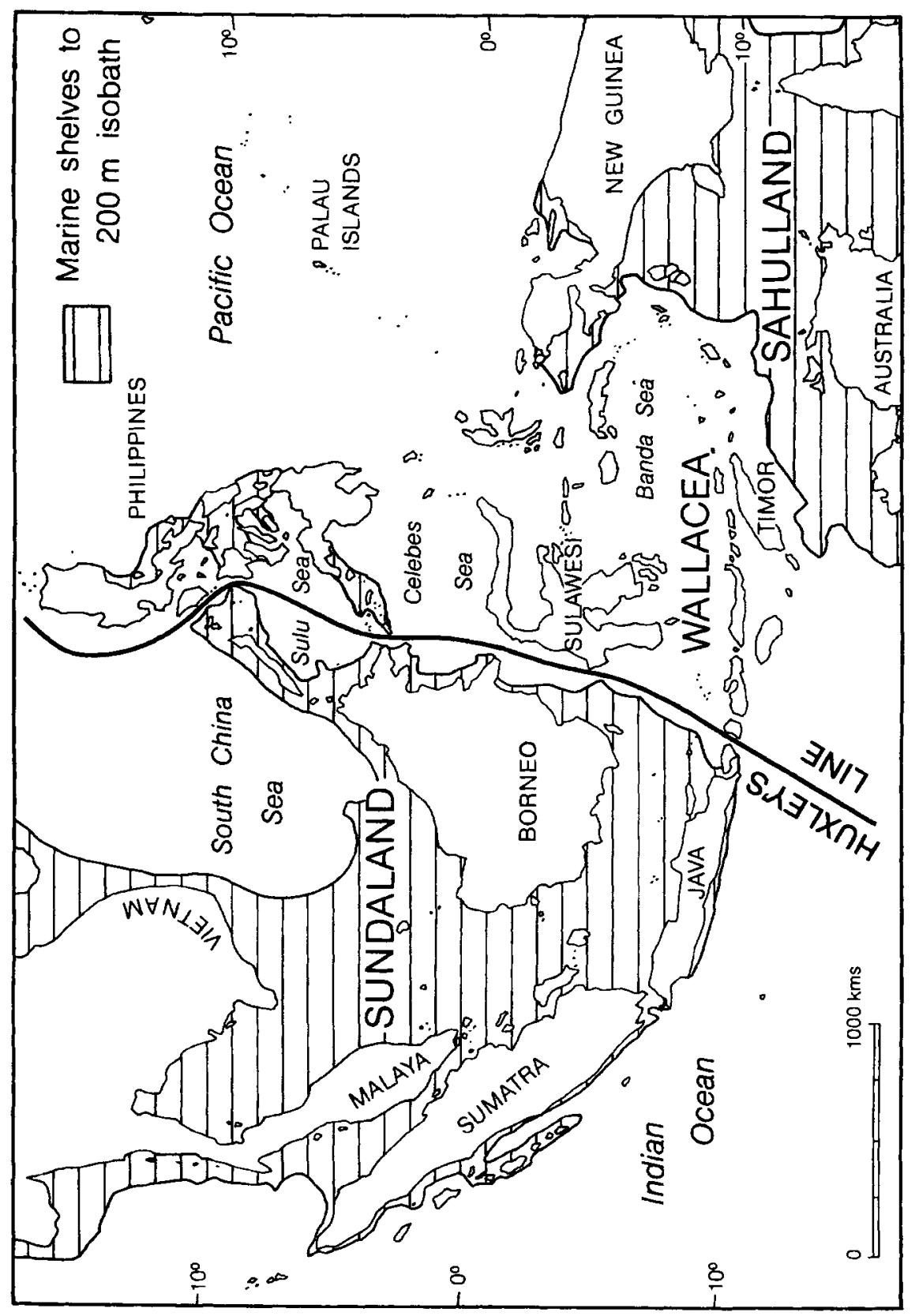

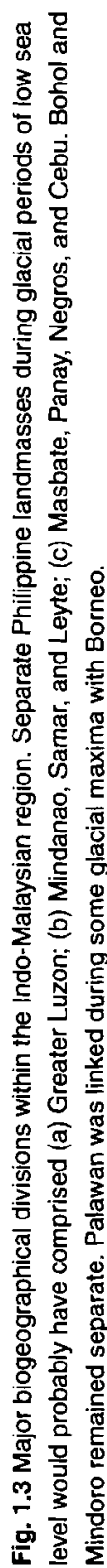


the present Sunda-Banda arc that attained its current configuration during the Pliocene-by which time the emergence of Java was also under way (see also Umbgrove 1949). More recent views, such as those of Metcalfe (1996), consider Sundaland to be a series of fused pre-Cretaceous terranes, some of Gondwanaland origin, rather than purely the creation of a series of volcanic arcs (see also Hutchison 1989). The issue is a complex one and will not be considered in detail; it is not of overall relevance for understanding early human prehistory.

One aspect of continental movement is of relevance, however, because it has allowed some degree of mixing of floras and faunas of very different Asian and Australasian origins. The northward drift of Australia has apparently been continuing at a rate of about $\mathbf{8 0}$ kilometers per million years since this landmass began its northward migration from Gondwanaland early in the Tertiary. The eventual result of this was that the Australian continent, or at least some drifting crustal fragments of it, began to collide with the Banda arc and the eastern part of Sulawesi from possibly 20 million years ago and onward. The geological structure of the eastern region of Indonesia is particularly complex owing to the long history of plate collision and the movement into the region of many small island fragments derived from both the Asian and Australian plate margins (the latter including some portions of the two eastern arms of Sulawesi, plus Timor, Seram, Buru, and the Sula Islands; Metcalfe 1990; Hutchison 1989; Burrett et al. 1991). According to Audley-Charles (1987), the two arms of Sulawesi fused together during the Miocene, about 15 million years ago, although Hall's reconstructions show them as essentially separate until within the past 5 million years.

Whatever the underlying geological sources of the islands of the archipelago, it is clear that it had reached its present basic shape by the time humans first entered it. In terms of human and biotic developments, the major structural divisions can be rearranged to make two basic divisions of more relevance for prehistory. These are Sundaland and Wallacea, with a third area, Sahulland, to the east (Fig. 1.3).

\section{B. Sundaland}

Sundaland comprises the regions on or attached to the present Sunda shelf: the Malay Peninsula, Sumatra, Java, Borneo, Palawan, and other small groups such as the Riau and Lingga Islands. Its eastern edge is marked by Huxley's Line of biogeographers, not to be confused with its better-known antecedent the Wallace Line, which runs south of the Philippines. Much of this area is now covered by shallow sea, but most of it (2.2 million square kilometers) would have been exposed as dry land by low sea levels for long periods during the Pleistocene and especially at the peak of the last glaciation about 20,000 years ago. Drowned 
river channels and sediments in the beds of the South China and Java Seas show this very clearly, although two large freshwater lakes, one north of Java and one in the Gulf of Thailand, would always have existed according to depth contours of the seabeds (Butlin 1993:Maps 8a, 8b). Huxley's Line runs between Bali and Lombok, Borneo and Sulawesi, Borneo and the Sulu Archipelago, then up to include the Calamianes and Palawan, and finally off into the Pacific between Luzon and Taiwan. The eastern edge of Sundaland between Borneo and Sulawesi is partially marked by the remarkable Great Sunda Reef-a partly drowned coral reef extending out from Borneo into the Strait of Makassar to within 44 kilometers of Sulawesi (Bemmelen 1949:Fig. 4) that must originally have grown from the old emergent coastline.

\section{c. Wallacea}

The term Wallacea, after nineteenth-century naturalist Alfred Russel Wallace, was first introduced into the zoogeographical literature by Dickerson in 1928. Dickerson defined the region as that between Huxley's Line and Weber's Linea line in eastern Indonesia that is believed to mark a fifty-fifty balance between the Oriental and Australian faunas. In this book, however, I will adopt a definition more relevant for prehistory: Wallacea includes all those islands lying between the continental shelves of Sundaland and Sahulland, namely the Lesser Sundas from Lombok eastwards, Sulawesi, the Moluccas, and the Philippines (with Sulu, but not Palawan).

Wallacea has evolved as a zone of enormous crustal instability and now exists as a number of islands separated by deep ocean basins (particularly the Sulu, Sulawesi, and Banda Seas), the whole formed by rapid processes of uplift and downfaulting. This area has never formed a continuous land bridge between Asia and Australia and all faunal, floral, and human dispersals through it must have involved water crossings. Some of the enclosed seas have particularly impressive features; for instance, the Sulu Sea is 4,633 meters deep and yet is totally enclosed by high ridges that never sink more than 380 meters below sea level. This means that the temperature of this sea remains fairly even from top to bottom, without the rapid cooling with depth found in the great oceans (Molengraaff 1921). The islands of Wallacea rise from the continuous undersea ridges of the region, and the rate of uplift has been very rapid in places; corals of presumed Pleistocene date have been reported from an altitude of 1,300 meters in Timor, and many islands have series of raised coral coastal terraces. Those at Cape Laundi on the north coast of Sumba have recently been calculated to be rising at a rate of 0.5 meters per 1,000 years (Pirazzoli et al. 1991). On the other hand, corals have been found to depths of 1,633 meters in the bed of the Seram Sea, so downfaulting of great magnitude has also occurred. 


\section{Sahulland}

The Sahul shelf forms a shallow, drowned, and tectonically stable link between the Australian continent and the massive island of New Guinea-it is the Australasian equivalent of the Sunda shelf. The term Sahulland may be used to denote the New Guinea-Australian land masses when both were joined together during periods of low sea level. Environmental changes in northern Sahulland, particularly during the later Pleistocene and Holocene, are of particular significance for an understanding of similar events in Sundaland.

\section{THE INDO-MALAYSIAN ENVIRONMENT}

\section{A. Climate}

As the whole region lies well within the tropics, temperatures are uniformly hot and vary little throughout the day or from season to season. The only major variation in temperature occurs with altitude (average temperature drops $1^{\circ}$ Celsius every 160 meters), but even on the highest peak in Southeast Asia (Mt. Kinabalu in Sabah, 4,104 meters) the temperature never gets colder than an occasional nighttime frost. The only permanent glaciers occur to the east in New Guinea, but only 8 square kilometers of the total 805,000 square kilometers of this island are so covered.

The crucial climatic feature in the region is the rainfall, and for general purposes it is useful to recognize two major zones (Fig. 1.4).

a. The zone within approximately $5^{\circ}$ of the equator, where rain occurs all year round. Most regions do have two slight rainfall peaks, but for practical purposes the rainfall is frequent, heavy, reliable, and the evergreen rain forest grows luxuriantly in constantly damp or wet soils. Peninsular Malaysia, Sumatra, western Java, Borneo, central Sulawesi, the southern and eastern Philippines, and parts of the Moluccas fall generally in this zone, which I will henceforth term equatorial.

b. The zone that extends beyond the equatorial zone both north and south of the equator and is characterized by clearly differentiated wet and dry seasons. Within the Indo-Malaysian Archipelago, this zone forms the warmer equatorward part of a worldwide intermediate tropical zone (Harris 1980) characterized by winter dry seasons of between 2.5 and 7.5 months in length. Within Southeast Asia the intermediate tropical zone includes the mainland north of the Malay Peninsula, the western and northern Philippines, southern Sulawesi, and the Sunda islands from central Java eastwards. The zone ultimately fades into the temperate climates of China and the deserts of central Asia and Australia. Because of the presence of 


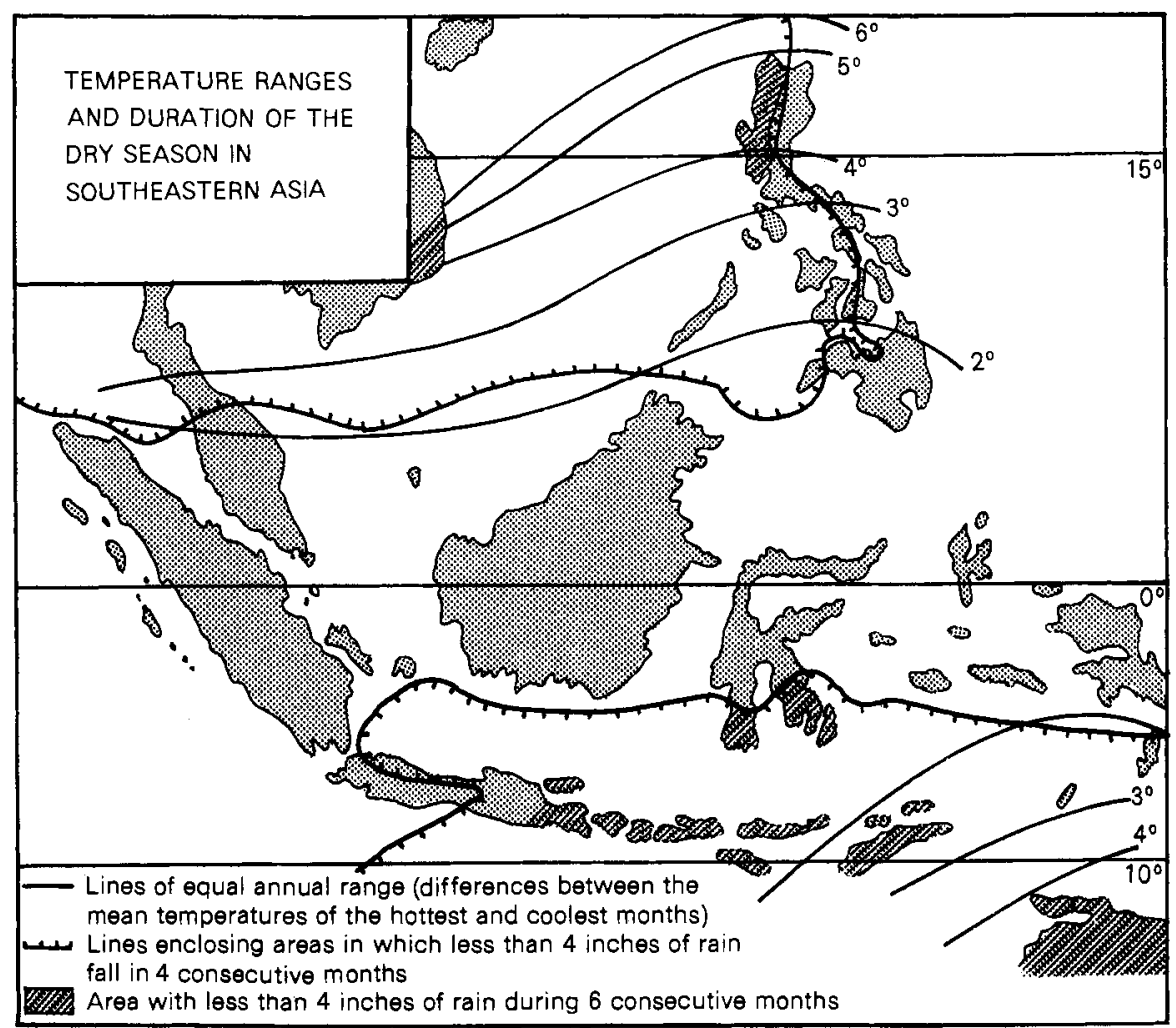

Fig. 1.4 Climatic regimes in the Indo-Malaysian Archipelago. After Robequain 1954. Courtesy: Editions Payot.

the dry season and occasional severe droughts, forests tend to be more open and have a deciduous tendency.

To explain these rainfall variations, I will attempt to summarize this complex topic (see Mizukoshi 1971; Dobby 1976). A major feature of global air circulation concerns the constant exchange of air (which flows as winds) between the equator and the poles. In the tropics, warm air is constantly rising and flowing poleward at intermediate altitudes. It cools, sinks in the latitudes of about 20 to $30^{\circ}$, and flows equatorward again as the trade winds (these blow from the northeast in the northern hemisphere and from the southeast in the southern hemisphere). The tropical zone where the trade winds meet and where air convection is strongest is termed the Intertropical Front, or Intertropical Convergence Zone. This front is not fixed in position, but moves seasonally according to temperatures in the continental interiors of Asia and Australia. 
In January the Asian interior is cold, the Australian interior hot. The resulting pressure gradient outward from Asia deflects the Intertropical Front southward into the southern part of Indonesia and the northern tip of Australia (to about 10 to $12^{\circ}$ south). These areas then receive their rainy season (southern summer) because the front is a constant formation zone of depressions and squalls. In the northern summer (July) the front is pushed far to the north (up to $32^{\circ}$ north) and Mainland Southeast Asia and the northern Philippines then get their wet seasons. The equatorial regions proper tend to have a double wet season because the front passes over them twice in each year.

The Intertropical Front is not the only condition affecting rain in the intermediate tropical regions. When it lies in the northern hemisphere in the northern summer, the southern hemisphere trade winds are sucked across the equator and bring additional moisture from the seas that they cross. The same happens in reverse in January, when the northern trades tend to flow farther south (although they do not get deflected so far because Australia, as a smaller continent, has a much weaker influence than Asia). These extended trade winds become the monsoons, which are usually named after their directions. These directions vary from place to place by deflection across the equator, and their effects can be varied locally by topography and position. For instance, the northeast coast of Peninsular Malaysia, in the northern hemisphere, has its wet season in November-January, actually the southern summer. This is because wet trade winds blow directly onto the coast at this time of the year from the South China Sea.

These climatic variations are of great importance for recent prehistory, and postulated changes in them are also of great importance in the Pleistocene. Typhoons and hurricanes also form in the intermediate tropical zone, but generally occur outside our area of interest. They are common in the northern Philippines, where they blow in from the Pacific Ocean, and likewise in northern Australia and the islands of Melanesia and Polynesia. They are almost unheard of in Indonesia and Malaysia.

\section{B. Landforms and Soils}

Humans, animals, and plants depend not only on climate for their existence, but also on the nature of the ground upon which they live. In the Indo-Malaysian Archipelago there are some very important variations in landforms and soils. These variations lie at the base of the enormous differences in population density seen today between such islands as Java and Borneo. It is my suspicion that they were equally important in prehistoric times.

The main soils of the equatorial ever-wet region are yellow to red leached lateritic formations, often called latosols. These soils are rich in iron and alumin- 
ium, are generally acidic, tend often to be heavy sticky clays, and are generally low in plant nutrients and organic matter. They do, indeed, support dense and luxuriant forests, but these are products of long evolution whereby 50 to 80 percent of the nutrients are accumulated in the biomass and constantly recycled in the upper layers of the soil as vegetation grows, dies, and decays. Once these forests are cleared the cycle is broken, as the nutrients simply leach away through the exposed soil-often with disastrous results.

These lateritic soils are generally characteristic of the equatorial and nonvolcanic lowlands of Sumatra, the Malay Peninsula, Borneo, Sulawesi, and western New Guinea. Today they support low populations because they are fairly infertile, unsuited in traditional cultivation systems to anything but shifting agriculture, and difficult-for reasons of structure and excessive rainfall-to bring under irrigated and terraced rice. Furthermore, the forest itself is ever-wet, hard to clear and burn with simple equipment, and subject to rapid regrowth of weeds and secondary vegetation. In addition, many coastal regions of the Malay Peninsula, eastern Sumatra, and southern and western Borneo have extensive areas of lowland peat soils (Polak 1975), which are very difficult for any simple economy apart from sago management.

The soil patterns change, however, when we move into southeastern Indonesia, from central Java through the Lesser Sundas. Here the soil is constantly enriched by the fertile outpourings of the many volcanoes, particularly where the products are of basic rather than acidic composition, as they are in central and eastern Java, Bali, Lombok, and the Minahasa Peninsula of northern Sulawesi. Most (but not all) of the Sumatran volcanoes are more acidic in this respect and consequently produce soils less favorable for agriculture.

This volcanic replenishment means that the normal tropical trends of leaching and nutrient loss in soils are constantly reversed. The resulting volcanic ashes are often firm and ideally suited for purposes of rice terrace construction, as any visitor to Bali or eastern Java will observe. This lucky combination does not cease here, for these regions have a climate with a definite dry season; this lessens the rate of soil leaching and also promotes a partially deciduous and more open vegetation, which is an easier target for agricultural societies than the ever-wet equatorial rain forest. However, this monsoon vegetation is fragile when subjected to clearance, perhaps more so than the equatorial rain forest, and degraded lands in these regions tend to degenerate to extensive grasslands, particularly where droughts are common.

The present-day results of these differences were very clearly pointed out by Mohr in 1945. From a census taken in 1930 he was able to show that Java and Madura had average densities of over 300 persons per square kilometer, Bali and Lombok about 175, Sulawesi 22, Borneo 4, and Irian Jaya only 0.73 . These figures, even if now outdated (Java had a density of 733 persons per square kilometer in 1983), still tell an important tale. The high Javanese densities are large- 
ly the result of the Dutch introduction of intensive agricultural techniques, including permanent dry-field cultivation after 1830 . Nevertheless, Mohr was able to show very convincingly how high population densities in Indonesia depend on a combination of basic volcanic soils, a nonexcessive rainfall (with a good dry season for cereal ripening and harvest), and a dependence on rice cultivation in permanent irrigated fields. He concluded: "In the Netherlands Indies the population density is a function of the nature of the soil and this is a function of the presence of active volcanoes" (Mohr 1945:262). These differences in soil qualities, when combined with climate, are important for reconstructing patterns of prehistoric agriculture in the archipelago.

One final point of importance concerns the rate of erosion and landform change in the tropics: Archaeological sites are not only hard to find in dense vegetation, but land surfaces can change rapidly in geomorphological terms. Caves and rock shelters are common in Southeast Asia, but few appear to contain deposits much older than 30,000 years; this circumstance may mean that in many cases the caves were buried and thus not available for habitation until geologically recent times, although the possibility that Pleistocene human occupation in equatorial regions was very sparse will also be discussed in Chapter 6. The prevailing high rates of erosion also affect open sites, causing rapid destruction or burial. Some idea of the sheer power of erosional processes in these latitudes can be derived from geomorphic observations: The Solo River of Java carries fifty times more sediment by water volume than does the Rhine (Robequain 1954:26), and Haile (1968:278) has suggested that surfaces exposed to active erosion in the Baram region of Sarawak could have been eroded by as much as 750 meters in the past 2 million years. In the same vein, HanburyTenison (1980) states that limestone surfaces in the Gunung Mulu region of northern Sarawak are eroding at a rate of 0.5 millimeters per year, or about 1,000 meters in 2 million years.

\section{The Flora and Fauna}

The Indo-Malaysian Archipelago forms part of the "Malesia" of botanists; in its ever-wet equatorial regions, the evergreen mixed Dipterocarp rain forest forms

the most complex terrestrial ecosystem in the world. Below about 1000 metres above sea level the forest canopy lies between thirty and fifty metres above the ground and shelters a more or less dense undergrowth of smaller shadetolerant trees, shrubs and saplings criss-crossed by lianes and studded with epiphytes. (Walker 1980:21)

Botanists are always eager to quote impressive statistics about this vegetation: Within Malesia about 10 percent of all the plant species in the world, 25 percent of the genera, and over 50 percent of the families are represented. Over 
25,000 species of flowering plants occur in the region, with 11,000 on Borneo alone. Associated with this variety is a rarity of extensive stands of single tree species. Extreme spatial variation is the rule: No less than 780 species of trees have been recorded from a single 10-hectare plot in northern Sarawak (Hanbury-Tenison 1980).

This equatorial rain forest is characteristic of the lowland regions along the equator that lack dry seasons, but in eastern Java, the Lesser Sunda Islands, and the southern tips of Sulawesi, the longer dry season has favored more open monsoon forests with a deciduous tendency, characterized by stands of casuarina, teak, sandalwood, and eucalypts. In western Java, southern Sumatra, and northern Peninsular Malaysia there is a shorter three- to five-week dry season that also encourages some elements of this type of forest. Local ecological variations also cross-cut the major climatic patterns to create such specialized ecosystems as the coastal mangrove swamps, the limestone forests, and the highmountain moss forests.

From a human prehistoric perspective, it is the broad distinction between the equatorial and the monsoon forests that is likely to be of the greatest significance on a large scale. Modern plant geography also reflects factors concerning the geological history of the Indonesian region that are of importance for human prehistory. The floras of Sundaland are of Asian origin and are rich in species, a reflection partly of the frequency of dryland connections across the subcontinent in the past. The floras of Wallacea, on the other hand, have fewer species, higher proportions of endemic species, and a larger Australian element; some species of eucalypts extend as far as Sulawesi and the southern Philippines, and Agathis species have spread from New Guinea through equatorial Indonesia as far as Peninsular Malaysia. Wallacea may be regarded as a transition zone between two ancient continental areas with quite different floras.

The differences between Sundaland and Wallacea in terms of flora are also reflected in the distribution of animal species-particularly the large mammals that have a fairly prolific fossil record. Basically, Sundaland has an Asian placental mammal fauna that includes many species ranging in size from the elephant downward. Wallacea, on the other hand, has fewer species and a greater proportion of endemic ones, with an increasing Australian marsupial element in the east (Fig. 1.5). (For general surveys see Jacobs 1974, Whitmore 1975, 1981, 1987).

\section{Biogeographical Boundaries}

The sluggishness or absence of faunal dispersal across Huxley's Line into the eastern part of the archipelago is clearly of importance for human dispersal. There have been no Wallacean land bridges of anything more than a very local 


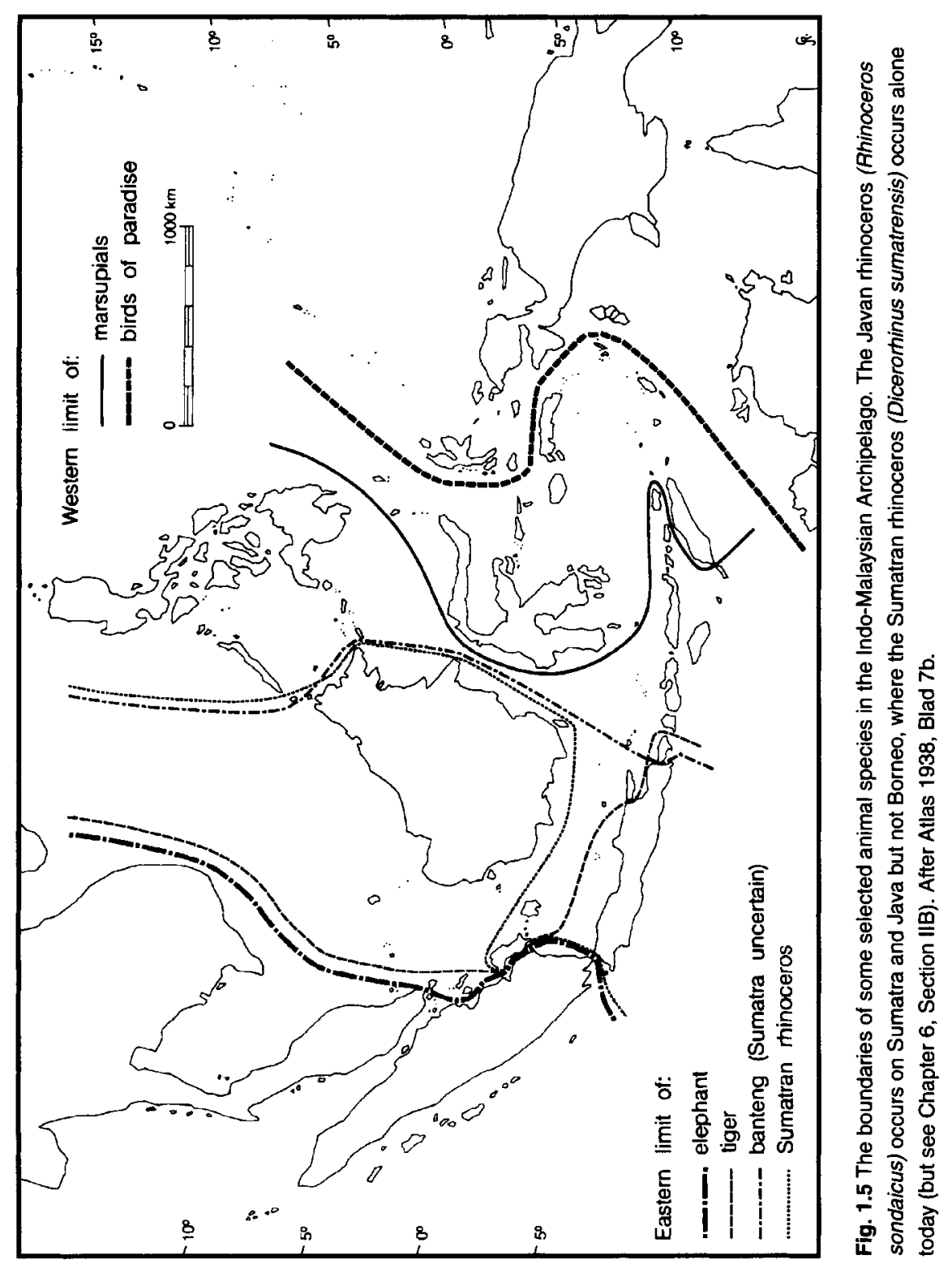


nature within the past 2 million years, an observation underlined by biogeographical as well as geological considerations.

The most important of the biogeographical boundaries is Huxley's Line, the eastern edge of Sundaland (see Fig. 1.3). This is the line beyond which the Sundaland fauna drops off markedly into endemicity and species depletion. Sundaland and Australia have never been joined by continuous dry land, and all faunal dispersals into Wallacea have involved sea crossings with the exception of the few land-bridge cases reviewed below. Of placental mammals, only rats and bats are distributed from Sundaland right through to Sahulland, and of marsupials a number have spread into the Moluccas (Flannery 1995), but only phalangers (cuscuses) ever reached Sulawesi (Groves 1987) and Timor, in the latter case perhaps by human translocation. However, both wallabies and bandicoots were once widely distributed in the northern Moluccas before their extirpation in the Holocene (Flannery et al. 1995) (see Chapter 6, Section IIE). The zone defined as Wallacea (between the Sunda and Sahul shelves) is in fact a zone with a partly endemic fauna of mixed Asian and Australian elements.

Discussions of the significance of Huxley's Line have been numerous and there is still much disagreement about how to subdivide the Wallacean region in zoogeographical terms (see Scrivenor et al. 1942-1943; Mayr 1945; Darlington 1957:462-472; Simpson 1977). The line works quite well for freshwater fish, mammals, and birds (in that order), but is less marked for insects and plants. It also works well between Borneo and Sulawesi, but the Philippine and Lesser Sunda boundaries are hazy. Although Oriental bird faunas drop off sharply down the Lesser Sunda chain from Java, it appears that the reasons are more to do with changing ecology than with the mere presence of sea gaps (Lincoln 1975). Furthermore, there is no sharp break in plant distribution down the Lesser Sundas (Jacobs 1974; Flenley 1979), while the break is sharper from Borneo (with 280 species of Dipterocarps) to Sulawesi (with only 45). In general, it is best to regard Wallacea as a zone of transition rather than as a zone of barriers.

\section{THE PLEISTOCENE AND WORLDWIDE CHANGES IN ENVIRONMEN T}

Having discussed some elements of the natural environment in remote geological time and at the present day, I will now turn to the fundamental question of variations in climate, land-sea distribution, and flora and fauna in the archipelago during the Pliocene and Pleistocene periods. In order to document environmental changes in the Pleistocene, it is necessary to search the literatures of a number of complex and rapidly expanding disciplines in the earth sciences and the natural sciences. There are many viewpoints to explain any given set of phenomena, and rapid obsolescence of any bright new idea 
seems to be an occupational hazard. I hope my scientific colleagues will bear with me while I try to set their data in a perspective relevant for human prehistory.

\section{A. The Pleistocene Period: Definition and Chronology}

Concerning overall chronology, the boundary between the Pliocene and Pleistocene periods has been dated in the past according to three different criteria (Goudie 1983): the onset of mid-latitude glaciation, changes in marine faunas, and changes in terrestrial faunas. The present cycle of mid-latitude glaciation started about 2.5 to 3.2 million years ago (Shackleton and Opdyke 1977), and earlier cycles can be traced back into the Tertiary. But most scholars today accept a Plio-Pleistocene boundary based on the appearance of certain new species of cool-temperature foraminifera in the oceans between about 1.5 and 2 million years ago; this change is marked at 1.6 million years ago by the Calabrian fauna of the Mediterranean (Haq et al. 1977). A similar change also occurs in the Pacific. The Villafranchian land mammal fauna, once considered to appear in the early Pleistocene, is now known to go back well into the Pliocene and is no longer acceptable as a boundary marker.

It is, of course, apparent that both the Pliocene and the Pleistocene are rather artificial divisions of convenience-there are no indications of any major worldwide environmental changes that took place in this particular boundary time span, and hence there is no clear worldwide boundary. This is especially true of Southeast Asia, where it is perhaps not justifiable to separate a Pleistocene from a Pliocene at all. The terminology, however, is too deeply rooted to tamper with at this stage, and I will follow the international chronology adopted for Java by Orchiston and Siesser (1982) and place the Pliocene-Pleistocene boundary at 1.6 million years ago (while noting that a date of 1.8 million years ago is also widely accepted; Howell 1994:254).

The question of subdivisions within the Pleistocene remains. It has been the tradition in the past to place hominid remains, animal faunas, and stone tool assemblages into a framework of early, middle, and late Pleistocene, and there have been a number of strong debates between scholars-particularly with respect to Javanese faunas and stone tools-concerning the division to which a particular fauna or industry belongs. The problem is well illustrated in Bemmelen's geological survey (1949): On page 93, Koenigswald placed the Kali Glagah and Ci Julang faunas of Java in the Pliocene, while on page 99, Bemmelen himself (following Movius) placed these faunas in the Lower (early) Pleistocene. Similarly, Koenigswald (in the above survey) considered the Jetis fauna of Java to be Lower Pleistocene, while Hooijer $(1956,1968)$ placed it in the middle Pleistocene. The situation is becoming even more confused today with the pub- 
lication of conflicting radiometric dates, and it is clear that there are no major pan-Southeast Asian changes in environments, hominids, or faunas that can be recognized as unequivocal boundary markers. I will therefore follow Orchiston and Siesser (1982) and modern African Pleistocene specialists and refer to the period between 1.6 million and 700,000 years ago as early Pleistocene, between 700,000 and 125,000 years ago as middle Pleistocene, and from 125,000 to 10,000 years ago as late Pleistocene. These divisions are for chronological convenience only and it will be noted that they are not of equal duration. The late Pleistocene is the only division with a firm environmental record in Southeast Asia and it equates with the last interglacial and last glacial episodes of the temperate Pleistocene chronologies. Finally, I place the Pleistocene-Holocene boundary at 10,000 years ago following convention; this date falls somewhere toward the end of the worldwide postglacial warming trend.

\section{B. The Consequences of Mid-Latitude Glaciation}

According to present theories (Covey 1984; Broecker and Denton 1990), the mid-latitude glaciations of the past 3 million years have been caused by the interaction of a number of phenomena. Cyclical causes may include variations in the intensity of solar radiation and variations in the earth's trajectory around the sun and the slope of its axis. Other less cyclical causes may include the frequency of ash clouds from volcanic activity and periods of continental uplift in high latitudes. At peaks of glaciation, the icesheets covered three times their present area and extended deep into Europe and North America. During interglacials, conditions returned to something like those of the present, and within the glacials themselves there occurred short warm phases called interstadials, when conditions ameliorated to intermediate levels.

The major worldwide effects of glaciation were to lower sea levels and vegetation zones and reduce temperatures. These changes were all felt quite strongly in tropical latitudes (Rind and Peteet 1985). For instance, in the fairly intensively studied highlands of New Guinea (Flenley 1979; Hope 1980; Haberle 1994), ice sheets covered about 2,000 square kilometers (only 8 square kilometers now) at the last glacial peak 18,000 years ago, the snow line was lowered to 1,100 meters below its present altitude, the tree line was lowered by about 1,500 meters, average highland temperatures dropped by 7 to $7.5^{\circ}$ Celsius, and 57,000 square kilometers of land below the ice were under grassland (as opposed to only 5,000 square kilometers now). ${ }^{2}$ In Southeast Asia the effects of preexisting permanent glaciers are still traceable on the summit of Mt. Kinabalu on Borneo (Flenley and Morley 1978), but any that might have occurred on the high volcanoes of Java and Sumatra will have left no traces owing to subsequent volcanic activity. 
As a result of these changes caused by glaciation, tropical climates on large land masses became drier, rain forests shrank in extent, land bridges were exposed in the Indo-Malaysian Archipelago, and humans, animals, and plants were subjected to alternating phases of island contact and island isolation.

\section{The Cycles of Glacials and Interglacials}

Until the 1960s, Pleistocene climatic cycles were traced mainly from studies of glacial geomorphology in temperate latitudes; the tropics remained rather remote and mysterious. But in the past thirty years, knowledge has been revolutionized by the results derived from deep-sea cores and also from studies on deeply stratified terrestrial gastropod- and pollen-bearing soils. Sediments in the beds of the oceans contain shells of tiny marine microorganisms and these shells contain oxygen in two isotopic forms: ${ }^{16} 0$ and ${ }^{18} 0$. During glaciations, the vast quantities of water trapped in the ice sheets immobilized large amounts of ${ }^{16} 0$ and the cold seas were thus relatively rich in ${ }^{180}$. In interglacials the ratios were reversed. Fluctuations in these ratios have been plotted from deep-sea cores for the duration of the Pleistocene in several areas, and because they are thought to reflect partly the waxing and waning of continental glaciers, they provide excellent evidence of Pleistocene climatic and sea level cycles.

One exceptional core, drilled in the seas of the Solomon Islands, showed that there had been at least seven full glacial maxima with low sea levels since the Matuyama to Brunhes reversal of the earth's magnetic field about 700,000 years ago (Shackleton and Opdyke 1973; Shackleton 1982). It is now known that there have been about twenty full glacials within the past 2 million years, with the same number of intervening true interglacials, plus periodic intermediate interstadials within the glacials themselves. This record has come from deep-sea cores, from deep pollen-bearing soil profiles, from gastropod faunas in loess deposits, and also from the dating of coral reefs as indicators of past sea levels (Kukla 1977, 1981; Chappell 1983; Chappell and Shackleton 1986; Prentice and Denton 1988; Chappell 1994). There is still some disagreement about the duration of the glacial-interglacial cycles, but present views seem to favor long glacials of about 100,000 years separated by much shorter interglacials of perhaps 10,000 years in duration.

Several other general observations emerge: The glacials have become more severe within the past million years, and the glacial waxing and waning cycles themselves are not totally regular. Glacial retreats appear to have been much more rapid than glacial advances (Fig. 1.6, top) and this high rate of environmental change is important for any consideration of the emergence of the present world environmental regime at the end of the last glaciation. It is 

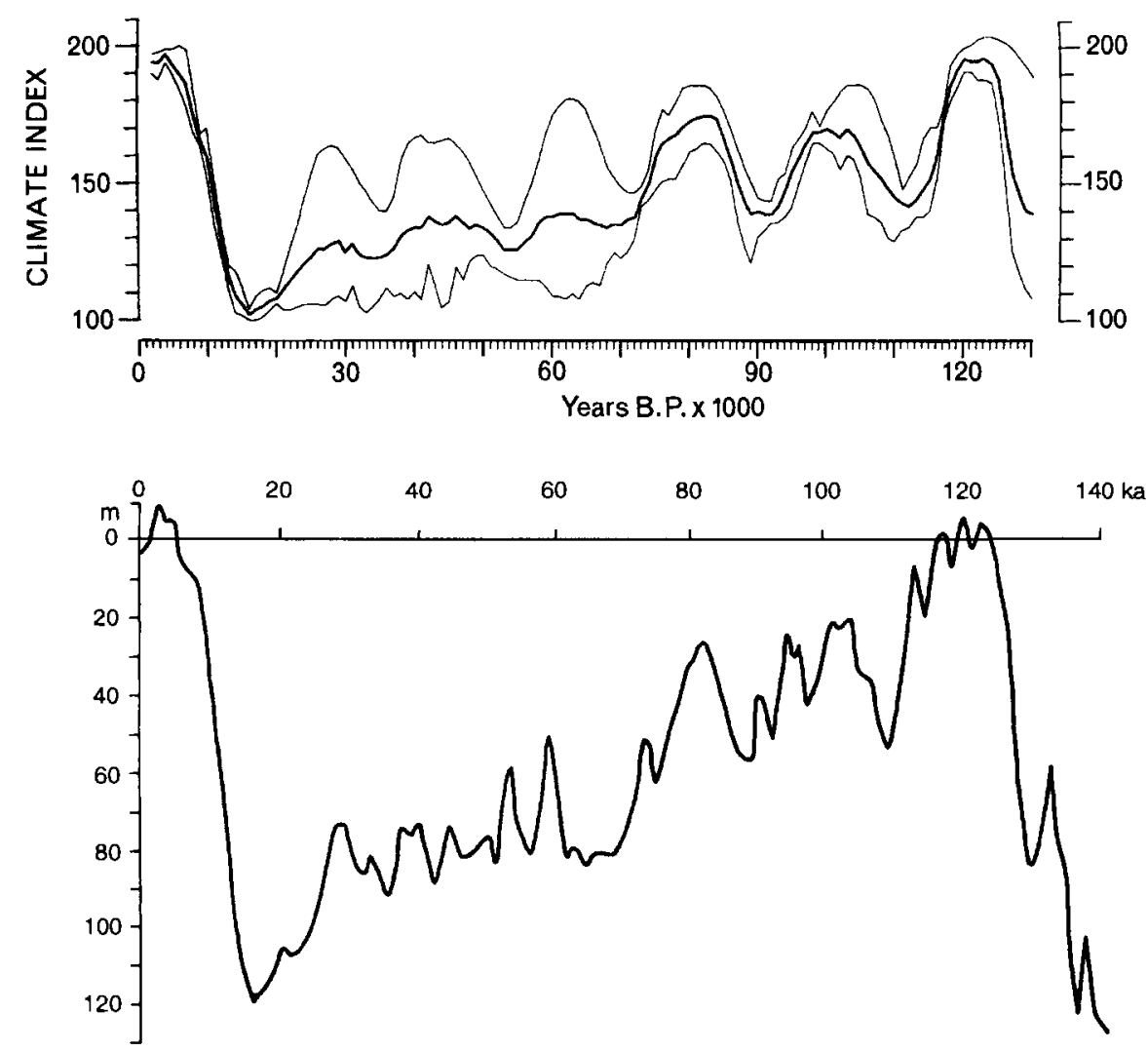

Fig. 1.6 Top: climatic severity index for the past 130,000 years derived from pollen, sea level, and oxygen isotope data. The vertical scale is relative: 100 units correspond to peak glacial conditions, 200 units to the climatic optimum of the mid-Holocene. Uncertainty ranges are also indicated. After Kukla 1981. Courtesy: D. Reidel Publishing Company. Bottom: a sea level curve for the past 140,000 years based on isotopic data from deep-sea cores and supported by data from raised coral reefs on the Huon Peninsula, northeast Papua New Guinea (Shackleton 1987; Chappell et al. 1996). Courtesy: John Chappell. (Note: Although the isotopic curve suggests a level higher than now about 3,000 to 6,000 years ago, evidence from stable regions indicates only minor variation from the present level during this interval.)

also becoming apparent that interglacials are periods of long-term stability of climate, whereas the glacial periods themselves could have witnessed rapid temperature fluctuations owing to changes in ocean circulation patterns (Broecker 1995). This suggests that the close chronological correlation between the relative warmth and climatic stability of the Holocene and the contemporary growth of human cultural complexity may not be entirely coincidental. 


\section{World Sea Level Changes during the Pleistocene}

Large-scale glaciation implies a lowering of world absolute sea level owing to the immobilization of vast quantities of water in the ice sheets. If it is accepted from the oxygen isotope record that there have been twenty glacial-interglacial cycles within the past 2 million years, then world sea levels must have gone through twenty major cycles of glacial falling and interglacial rising-not to mention interstadial fluctuations. Magnitudes of these absolute fluctuations have always been hard to estimate; the most direct indicators come from observations of drowned shelf topography and the dating of old coastline markers such as coral reefs and mangrove timbers.

The calculations are not simple, however, because the earth's surface is not a rigid, unmoving formation washed by fluctuating water levels. It can move in quite a dynamic fashion itself, partly through the mechanism of isostasy, which compensates for the imposition of variable loads such as ice sheets and oceans at changing times and places on its surface. In general, water, ice, or sediment loads promote sinking, while relief from such loads will allow slow upward rebound. These processes are complicated and details need not be of further concern, except to note that isostatic adjustment processes will have operated mainly in the intermittently drowned Sunda and Sahul shelf regions, while many of the Wallacean islands are subject to other kinds of tectonic instability, so that they themselves can rise and fall independently of sea level changes at quite rapid rates. Northern Timor and Sumba, for instance, seem to be rising at the fairly rapid rate of 0.5 meters per thousand years, and northeast New Guinea is rising at up to 4 meters per thousand years (Pirazzoli et al. 1991; Chappell and Thom 1977). As Chappell (1982) has pointed out, each region of the earth must be studied independently with respect to local correlations between the surfaces of land and sea over time.

At present the sea is at a high absolute level in terms of Pleistocene fluctuations, a level previously attained during the last interglacial about 120,000 years ago (Fig. 1.6, bottom). At the last glacial maximum 18,000 years ago, the sea level is widely estimated to have been between 100 and 130 meters below that of the present (Chappell and Thom 1977; Batchelor 1979; Chappell 1994). A high-to-low overall swing of about this magnitude may have occurred approximately every 100,000 years, going back to a million years ago. The swings before a million years ago seem to have been of slightly decreasing vertical magnitude.

When we come to consider more detailed aspects of these fluctuations, we find ourselves confined largely to the last 120,000 years, for which there are obviously more data than for previous cycles. The last interglacial had a fairly short duration-between about 130,000 and 120,000 years ago (Kukla 1981; 
Chappell 1983) - and the seas were at around present absolute levels at this time. Following this, according to the data derived from isotopic analysis of deep-sea cores and from uplifted and dated Pleistocene coral reefs on the Huon Peninsula of New Guinea (Chappell 1982, 1983; 1994; Chappell et al. 1996), sea levels fluctuated many times between relatively high and low points (Fig. 1.6, bottom), although none of these intervening highs appear to have attained the level of the present. The "high" of 28,000 years ago may have reached only 70 meters below present. The implications of these figures are that high stands like that of the present and low stands like that of $18,000 \mathrm{BP}$ were relatively shortlived events during the Pleistocene. Average absolute levels would have been between 30 and 90 meters below present for much longer periods.

These fluctuations in sea level are of great potential importance for prehistory, since low levels make islands larger and also tend to produce land bridges. Shortened sea crossings are particularly important when considering the first settlement of Australia, and this could relate to one of the low points from 35,000 years ago or before (cf. Birdsell 1977). One major problem is that there is still no real agreement on a precise absolute sea level curve; results from other parts of the world are often in conflict (Marcus and Newman 1983), probably owing to local tectonic and isostatic movements. Even within the Indo-Malaysian Archipelago itself, the magnitude of sea level lowering proposed by Linsley (1996) for Sulu is considerably less than that presented in Figure 1.6, and favors a drop to only 40 to 50 meters below present between 58,000 and 23,000 years ago, rather than the 50 to 90 meters suggested here. It would be unwise for archaeologists to demand a universal curve applicable to all regions.

One final matter concerns the disputed question of a world sea level slightly above that of the present during the Holocene. There are raised marine deposits in Sundaland (Tjia 1987; Thommeret and Thommeret 1978), which suggest that sea levels could have been up to 6 meters above present during a warm phase of the Holocene between about 6,000 and 3,000 years ago. These deposits may reflect tectonic or isostatic movement rather than an actual rise in absolute sea level (Chappell 1982, 1994), but they do still indicate that parts of Sundaland may have been relatively more drowned then than they are now. The magnitude of the difference is very small, although the potential here for the destruction of early Holocene archaeological sites on coastlines is clearly of great importance (see Chapter 6).

\section{THE EN VIRONMENTAL HISTORY OF THE INDO-MALAYSIAN ARCHIPELAGO DURING THE PLIOCENE AND PLEISTOCENE}

During the Miocene period (23 million to 5 million years ago) and the Pliocene (5 million to 1.6 million years ago), the archipelago gradually took on its present 
shape as a result of continuing island arc formation and the increasing closeness of the Australian continental plate in the east. By the end of the Pliocene, Sundaland already formed a large emerging continent some 2,000 kilometers from east to west, incorporating much of the present land masses of Peninsular Malaysia, Sumatra, and Borneo (Batchelor 1979), possibly with western Sulawesi still attached (Hall 1996). Some parts of Java had also emerged, although there is current debate on the precise geography of the island at this time (Braches and Shutler 1983-1984).

\section{A. The Pliocene and Early Pleistocene in Java, the Lesser Sundas, and Sulawesi}

In the later Pliocene, about 2 million years ago, a mammal fauna with strong Indian affinities (called "Siva-Malayan" by Koenigswald and related to the Eurasian Villafranchian) gained a footing in the newly emerging western and central Java. It is known from coastal estuarine deposits. Animals present include extinct species of elephant, Stegodon (a proboscidean distantly related to the elephants; Fig. 1.7), hippopotamus, and deer, but apparently no hominids

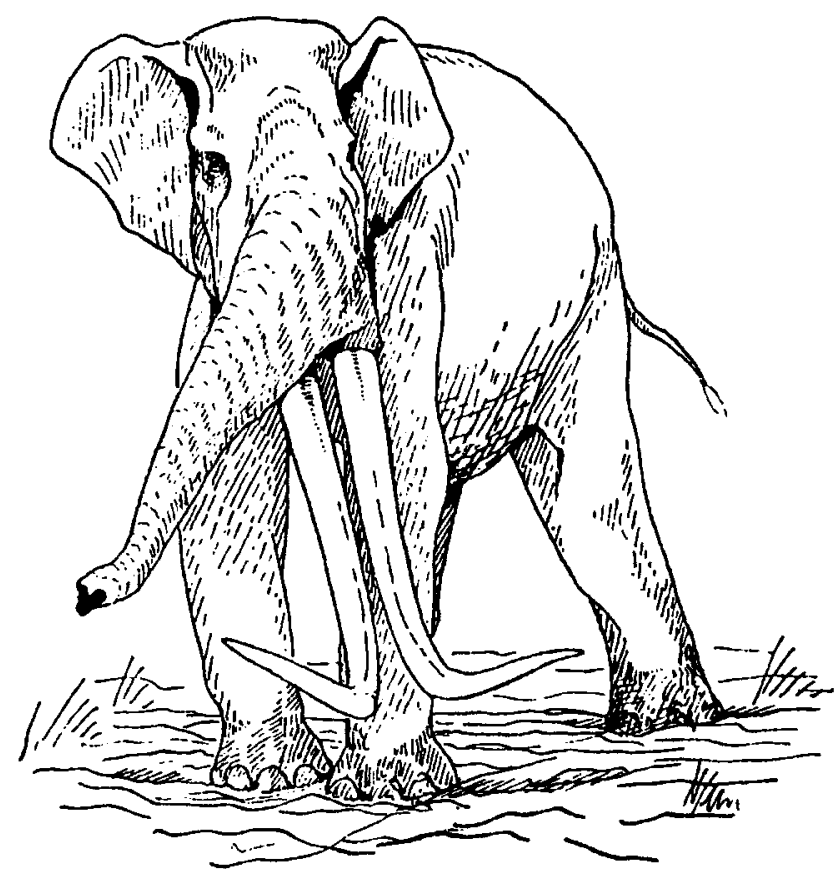

Fig. 1.7 A male Stegodon. In females the tusks were shorter and more widely spaced. From Kurten 1971. Courtesy: Weidenfeld Ltd. 
or forest primates (van den Bergh et al. 1995a). The whole assemblage, with its numerous browsing animals, seems to have been well suited to both forested and more open parklike landscapes.

Although there can be no real certainty with respect to date, it appears that the late Pliocene to early Pleistocene was also a time of three important faunal dispersals eastward, none on present evidence involving early hominids. One of these took place across the Strait of Lombok, from Java and Bali along the Lesser Sundas to Flores and Timor; the other two were from Borneo to Sulawesi and from Borneo to the Philippines (Groves 1985). The Philippines appear never to have had a land bridge to the Asian mainland (Heaney 1985, 1986), but it is possible that western Sulawesi was still attached structurally to Sundaland during the Pliocene (Hall 1996), although not necessarily by continuous dry land.

The link to Sulawesi is documented by the Cabenge fauna from several localities in the Walanae Valley in the southwestern part of the island (Bartstra et al. 1991-1992; Bartstra and Hooijer 1992). Like the oldest fauna on Java, this has Siva-Malayan affinities and contains species of Stegodon and elephant (a small species derived from a larger Pliocene population on Sundaland: Maglio 1973), together with the piglike babirusa and an extinct species of pig. Macaque monkeys and buffalo-like anoas possibly arrived on Sulawesi at the same time, but have not so far been found in the Walanae fauna. A significant chronological marker in this fauna is a giant land tortoise (Geochelone atlas), which appears to have disappeared from Java by about 1.2 million years ago (Bergh et al. 1995a). It was once thought that the Cabenge fauna arrived in Sulawesi via a land bridge through the Sangihe Islands from the Philippines and China, but it is now agreed that it is of Sundaland origin (Groves 1976; Bartstra 1977; Sartono 1979a). The marsupial phalangers presumably rafted from New Guinea to the eastern part of the island during the Miocene, long before the arrival of the Cabenge fauna.

How did the Cabenge placental mammal fauna get to Sulawesi? The biogeographical record suggests that any late Pliocene land bridge would have been short lived, if one ever existed at all (Audley-Charles 1987; Musser 1987). Large mammals can swim, and the absence of a land bridge throughout the following Pleistocene period is agreed upon by all authorities.

The evidence for a land bridge along the Lesser Sunda chain from Java to Timor is even more tenuous. The Wallace Line, which runs east of Bali down the 30-kilometer-wide Strait of Lombok, seems never to have been land-bridged through all of geological time since the Lesser Sundas show no signs of a Sundaland derivation, being instead autochthonous volcanic arc formations (Hall $1995,1996)$. Beyond the Wallace Line the glacial sea level changes would have been insufficient to bridge all the gaps, and even if tectonic movements of the seabed are invoked it seems unlikely that a continuous bridge ever ran all the 
way to Timor. The fossil faunas of the Lesser Sundas are very impoverished and consist only of Geochelone atlas tortoises (Sondaar, 1981, disputes that these belong to the same species as those from Java), large lizards (ancestral Komodo dragons), sporadic rodent species, and species of Stegodon (e.g. Musser 1981; Bergh et al. 1995b for Flores). The latter, however, are of particular importance for the land bridge question because it has been discovered that separate large and dwarfed species, probably derived from Stegodon trigonocephalus of Java, once existed in Mindanao, Sulawesi, Flores, and Timor (Hooijer 1975, 19671968; Sartono 1969, 1973; Bergh et al. 1995b). One species of uncertain size existed on Sumba as well (Sartono 1979b; Hooijer 1981).

To explain these Stegodon distributions, Audley-Charles and Hooijer (1973; see also Hooijer 1975) suggested that Flores and Timor were joined by a land bridge through Alor in the late Pliocene or early Pleistocene, prior to subsidence of the (now 3,000-meter-deep) Timor Sea, and also that Flores was similarly joined to southwestern Sulawesi. Other scholars have been reluctant to accept these postulated land bridges owing to the degree of tectonic movement they demand and the absence of any other faunal similarities. A contrary theory is favored by Sondaar (1981) and Bartstra et al. (1991). As for Sulawesi, they suggest that the Stegodon and tortoise species were able to move from Sundaland into the Lesser Sundas independently, perhaps by swimming. Stegodon thereafter could have undergone independent dwarfing as a result of restriction to relatively small islands and the tortoises could have undergone separate selection for reptilian gigantism. Both these processes for large mammals and reptiles are known from other islands. However, because Bergh et al. (1995b) suggest that the large Stegodon on Flores is actually younger than the pygmy species, it is possible that more than one episode of animal migration was involved.

Interestingly, the large Stegodon on Flores is now claimed to be contemporary with human activity, at about 700,000 years ago by palaeomagnetic reversal dating (Bergh et al. 1995b). This is discussed in more detail in the next chapter.

\section{B. The Pleistocene in Java}

Sometime during the early Pleistocene a new fauna evidently appeared in Java. It was called "Sino-Malayan" by Koenigswald because it shares more species with southern China than did the preceding Siva-Malayan fauna. It is also much richer in species, including many that still exist today. According to many authorities, this fauna developed through three overlapping stages traditionally termed Jetis (the earliest), Trinil, and Ngandong (see Theunissen et al. 1990; and Fig. 1.8 for approximate chronologies). New appearances in the Jetis (Koenigswald 1949; Hooijer 1968; Medway 1972) include rhinoceros, forest primates such as the gibbon and orangutan, and of course hominids. In addition, the 


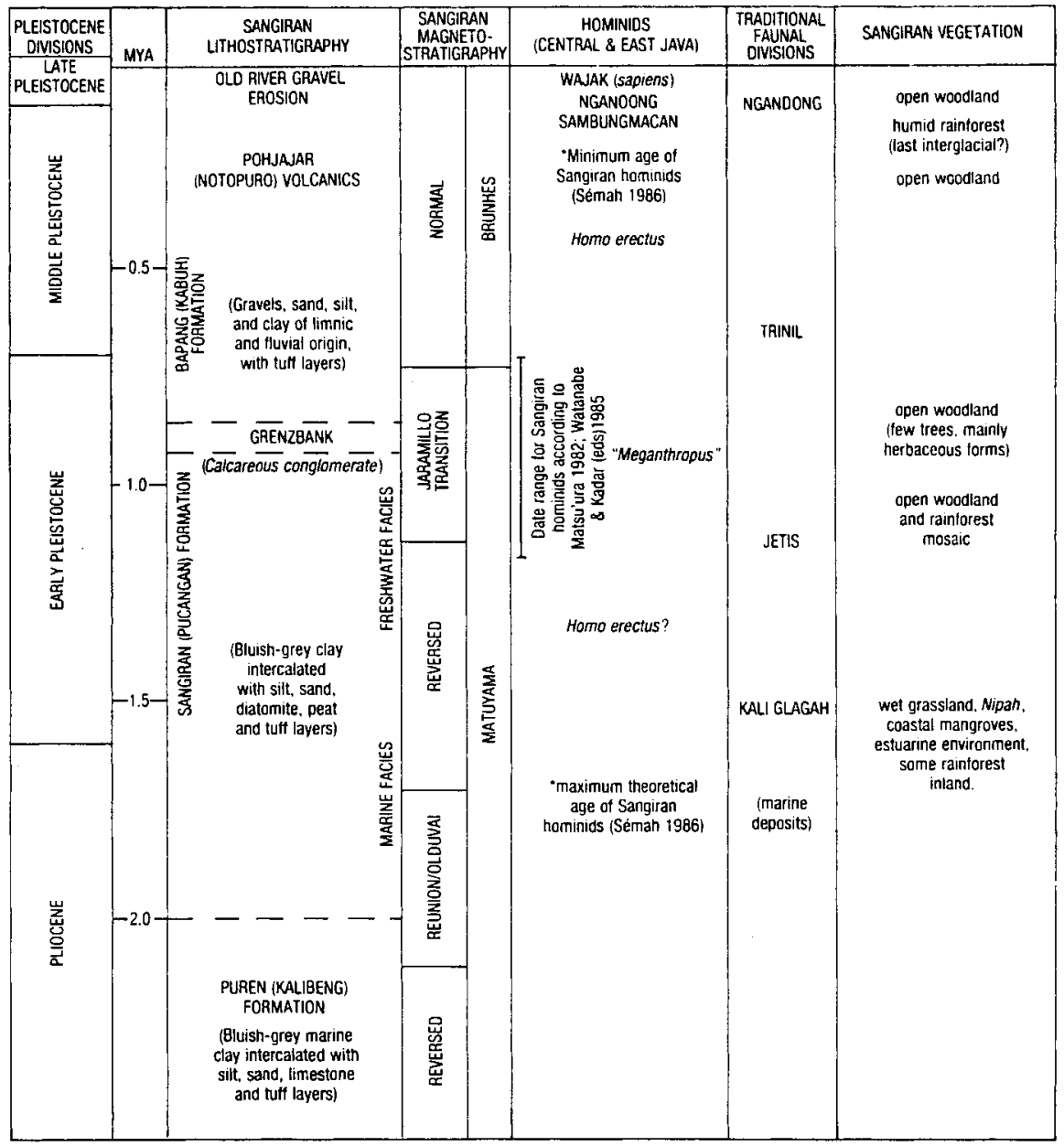

$M Y A=$ millions of years ago

Fig. 1.8 The Sangiran sequence of geological formations, hominids, faunas ("traditional" divisions), and vegetations since the late Pliocene. From Bellwood 1992. Courtesy: Cambridge University Press.

fauna contains a wide range of large herbivores such as elephant, cattle, buffalo, deer, pig, and hippopotamus, and carnivores such as tiger, bear, panther, and dogs of the genus Cuon.

In the past decade or so this faunal scheme has been heavily modified by a new and rather different one, illustrated here in Figure 1.9 (Vos et al. 1982; Sondaar 1984; Leinders et al. 1985; Theunissen et al. 1990; Bergh et al. 1995a). 


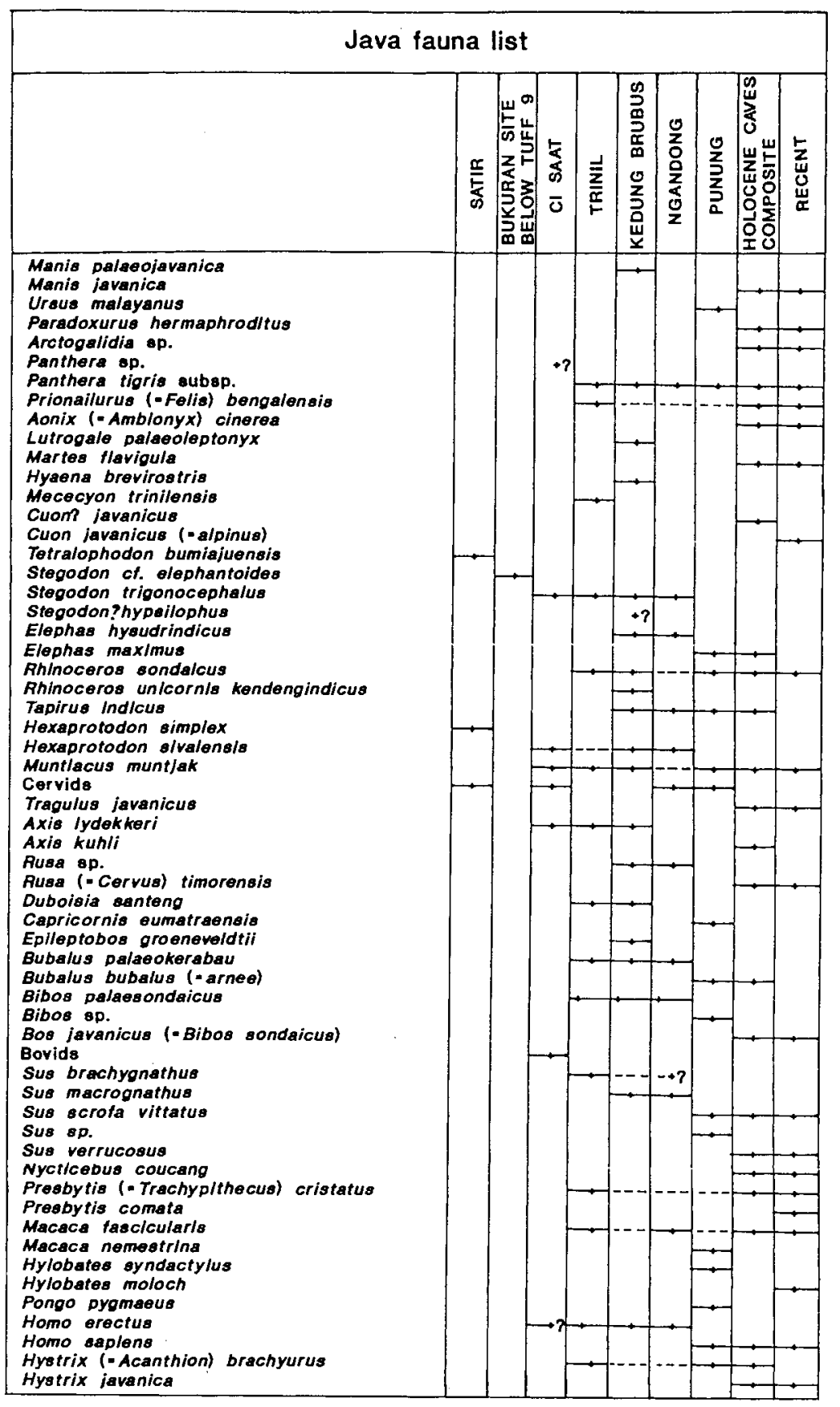

Fig. 1.9 An alternative faunal scheme for Java, as described in Vos et al. 1982 and Bergh et al. 1995a (see text). Dates are approximately as follows: Satir, 2-1.5 mya (million years ago); Ci Saat, 1.2 mya; Trinil, 0.9 mya; Kedung Brubus, 0.8 mya; Ngandong, uncertain; Punung, 110-70 kya. Courtesy: John de Vos. 
This scheme places a number of species later in time, especially the rain forest primates (gibbon and orangutan), that are claimed to have appeared in Java only during the last interglacial period. It also includes a major phase of animal immigration into Java about 800,000 years ago-during the Kedung Brubus faunal stage - at a time when world sea levels were generally low and the island was land-bridged for a long period to the Asian mainland. At present it is not clear if this scheme will completely replace the traditional one; reservations about it have been expressed by Bartstra (1983) and Hooijer (1983). The disagreements have arisen in part because of uncertainties over the exact findplaces of animal bone assemblages-especially those of the Trinil fauna.

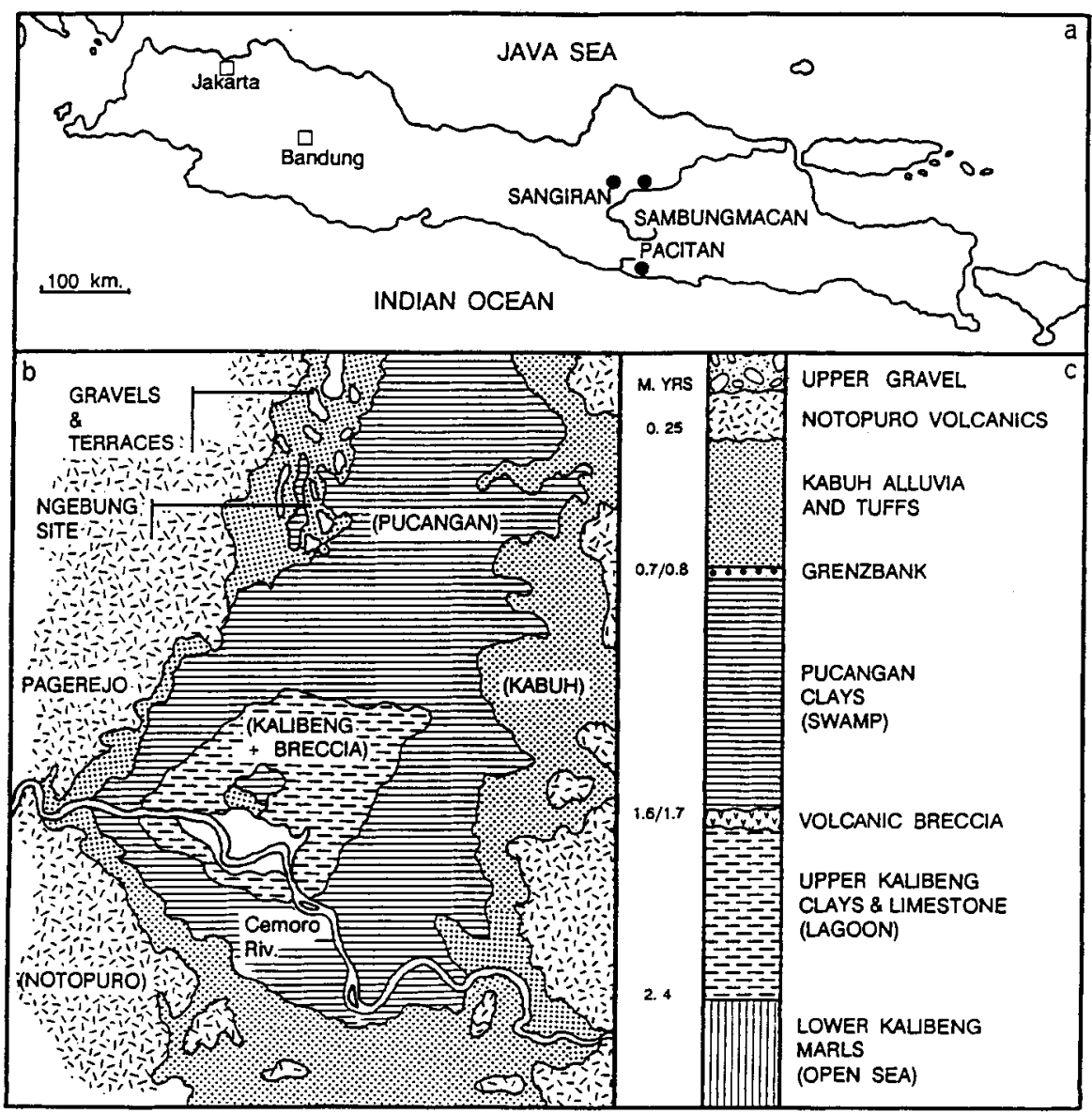

Fig. 1.10 A simplified geological map and section of the Sangiran "dome," showing the location of the Ngebung site. Courtesy: François Sémah. 
The best record of human and faunal evolution in Java comes from a rather dramatic locality known as Sangiran (Plate 1 and Fig. 1.10), where a domed formation of Pliocene and Pleistocene deposits pushed up by volcanic activity has been cut open and exposed by the Cemoro River, a tributary of the much larger Solo. The craterlike exposure here measures approximately 8 by 4 kilometers, and in its rather dissected base are exposed Pliocene estuarine sediments upon which lie patches of terrestrial Pleistocene formations (Matsu'ura 1982; Watanabe and Kadar 1985). During late Pliocene times the region around Sangiran was still partly under the sea, and it appears that a long marine strait occupied much of the area of the present Solo Valley. Sangiran seems to have been quite near the coastline, as recent pollen analyses of the Pliocene estuarine sediments have indicated the presence of mangroves, nipa palms, and pandanus trees (A-M. Sémah 1982a).

Above the Pliocene estuarine deposits in Sangiran come two major terrestrial formations: the Pucangan and the succeeding Kabuh (termed the Sangiran and Bapang formations in Watanabe and Kadar 1985). The Pucangan (Sangiran) formation is exposed through a total thickness of about 160 meters and comprises mainly lacustrine black clays with periods of marine estuarine transgression. The base of the Pucangan has thick estuarine deposits that contain shark teeth and shells of oysters, pearl, and other bivalves. The pollen analyses support the stratigraphy in suggesting a gradual emergence of the land around Sangiran during Pucangan times, and the mangroves were slowly replaced by dryland vegetation with mainly open-country characteristics and patches of rain forest (A-M. Sémah 1982a,b; Watanabe and Kadar 1985). The Pucangan beds contain the earliest hominids.

Above the Pucangan beds at Sangiran lie the Kabuh (or Bapang) alluvial beds, with a calcareous conglomerate and bone-rich marker bed known as the Grenzbank between. The Kabuh beds contain the Trinil fauna; these beds are up to 60 meters thick and have also produced the bulk of the hominid finds. Pollen from the Kabuh layers at Sangiran is predominantly of nonarboreal type (Gramineae, Cyperaceae), but rain forest continued to exist in the general region. It may be that volcanic eruptions promoted some open vegetation at the expense of forest, and the possibility that the climate periodically had a longer dry season than now might also be considered. The middle Pleistocene animal faunas of Java also indicate conditions less forested than now (Medway 1972).

The ages of these deposits are difficult to determine and there is still much disagreement. Ninkovitch and Burckle (1978) dated the base of the Pucangan beds at Sangiran between 1.9 and 2.1 million years ago according to diatom correlations. The locations within the Sangiran sedimentary sequence of dated palaeomagnetic reversals of the earth's magnetic field (Jaramillo Transition and Brunhes-Matayama Boundary; see Fig. 1.8), together with fission track dates on 
zircon grains in volcanic sediments, suggest that the boundary between the Pucangan and Kabuh formations lies somewhere between 0.7 and 0.9 million years ago (F. Sémah et al. 1981; F. Sémah 1982, 1986; Watanabe and Kadar 1985; Hyodo et al. 1993).

The hominid fossils from Sangiran, to be discussed in more detail in the next chapter, come from the upper part of the Pucangan formation, the Grenzbank, and the lower part of the Kabuh formation. Pope (1984) and Bartstra (1983) favor a commencement date for hominids in Java at a little over a million years ago, a date supported by the fission track and fluorine dates from Sangiran listed in Watanabe and Kadar (1985) and by Matsu'ura (1982). In Fig. 1.8, I give a most likely date range for Sangiran Homo erectus of about 1.2 million to 750,000 years ago. This is the date range accepted by most scholars today (e.g., Bergh et al. 1995a).

There are, however, continuing claims by other scholars for much earlier dates. For instance, Siesser and Orchiston (1978) have suggested that foraminifera found in sediment attached to one of the most ancient hominid mandibles from Java (the Pithecanthropus mandible $C$ from the Pucangan formation at Sangiran) are at least 1.6 million years old. The Kabuh formation at Sangiran has produced a surprisingly old potassium-argon date of 1.2 million years (Curtis 1981:16), although the status of this sample has been questioned by Pope (1984). In 1994, the paleontological community received rather a surprise with the publication of an argon-argon date of 1.8 million years for pumice from the Pucangan deposit at Perning, near Mojokerto in eastern Java, that produced (in 1936) a Homo erectus child cranium. Another date of 1.66 million years was given for a pumice layer contemporary with hominid fossils in the Pucangan formation at Sangiran (Swisher et al. 1994). These dates have been challenged by other workers in the field (e.g. Bergh et al. 1996b), and it is only possible to conclude at present that the dating of the Javan Pleistocene and its hominids remains controversial. If the Mojokerto child really is 1.8 million years old, then our whole understanding of the course of human evolution is going to need substantial revision, a possibility discussed in more detail in the next chapter (see also Huang et al. 1995 for claims of similarly early dates from southern China).

Although my tendency is to support the "conventional" chronology for the Javan Pleistocene offered in Fig 1.8, it would perhaps be unwise to be too precise about the overall ages of the Pucangan and Kabuh formations at this stage of research; the boundary between the two may not in fact be synchronous over the whole of central and eastern Java. Given current disagreements, it seems best to regard the Jetis and Trinil faunas as a continuous and intergrading sequence spanning the period from perhaps 2 million to about 500,000 years ago, with periodic new arrivals coming in from the Asian continent, including Homo erectus by at least a million years ago. 
Descendants of the Trinil fauna presumably continued across a nonfossiliferous hiatus in the middle Pleistocene, represented at Sangiran by the volcanic Notopuro deposits, into the late middle and late Pleistocene Ngandong fauna. After the Notopuro tuffs and lahars were deposited in central Java, the area was subject to uplift, causing river rejuvenation and terrace formation. The Ngandong fauna has been found on one of these terraces in the Solo Valley and all its genera apart from Stegodon are still living; it may be regarded as the direct ancestor of the present fauna of Java.

\section{Pleistocene Land-Sea Relationships in the Indo-Malaysian Archipelago}

The question of sea level relative to land surface level during the Pleistocene in Sundaland now arises. The dispersal of animals and hominids might have been affected by the alternate flooding and exposure of this vast area in recurrent cycles of about 120,000 years (Chappell 1994). At present this possibility cannot be easily assessed, and the opinions published to date suggest several lines of disagreement. For instance, Batchelor (1979) has claimed that the Sunda shelf off western Peninsular Malaysia has submarine fan and braided stream deposits, evidence that it was emergent almost continuously until about 500,000 years ago, and even thereafter was not submerged to present levels until the last interglacial, only about 120,000 years ago. On the other hand, Tjia (1980) and Bergh et al. (1996b) are clearly unwilling to accept such a continuously low relative sea level in the early Pleistocene; the latter, following Prentice and Denton (1988), favor a major drop in sea level at about 800,000 years ago, the period of incursion into Java of their Kedung Brubus fauna. There are also some zoological arguments to suggest that Sundaland must have been separated into islands for quite long periods going back into the Pliocene; Chivers (1977) believes that such periodic geographical isolation is required to explain gibbon speciation, and Musser (1982) presents a similar viewpoint for rats.

This apparent difference of opinion about the possibility of a periodic drowning of Sundaland in the early and middle Pleistocene (see also Heaney 1985) cannot easily be resolved at present, mainly because the sea level history of Sundaland cannot simply be read from world absolute sea level curves. Even if isostatic compensation is allowed for, there is always the unknown possibility that localized tectonic movement has occurred. However, if such movement is arbitrarily discounted, then the general shallowness of the present seas over the Sunda shelf would indicate that a drop of only 50 meters with respect to the present land surface would be sufficient to join Peninsular Malaysia, Sumatra, Java, and Borneo to create a sizeable continent. Sundaland could therefore have been exposed as a continental area of varying size for the greater part of the Pleistocene, and its present island configuration may be unusual in terms of geological time. 


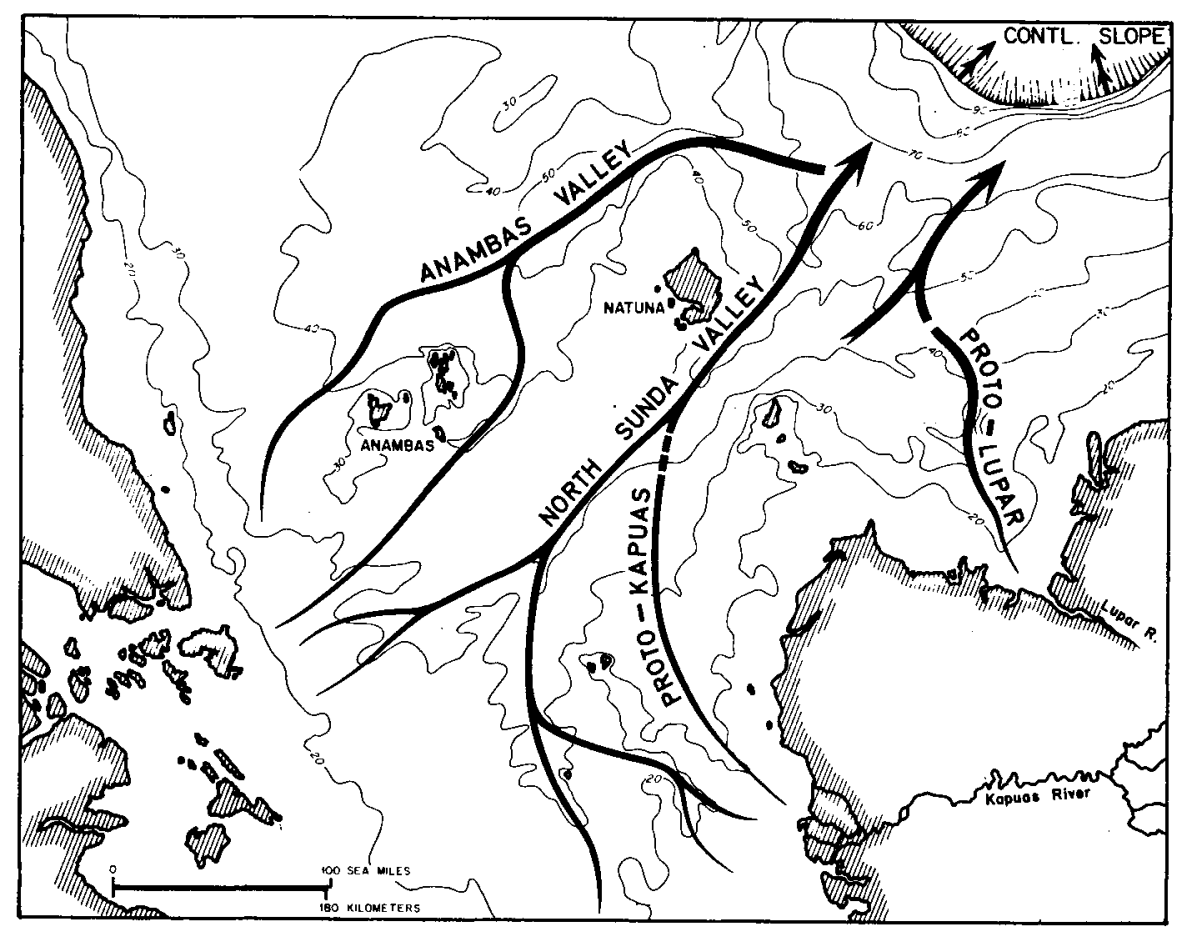

Fig. 1.11 The submarine topography of the northern Sunda Shelf. From Haile 1973. Courtesy: Tsukiji Shokan.

The surface of Sundaland as an emerged continent contains some interesting features. The shallow shelves of the South China and Java Seas are incised by a number of fossil river channels; between Sumatra and western Borneo there are three major ones, termed by Haile (1973) the Anambas, North Sunda (with the Proto-Kapuas as a tributary), and Proto-Lupar valleys. These can be clearly followed to the edge of the Sunda shelf at a depth of about 100 meters (Fig. 1.11). Two large parallel rivers also ran along the bed of the Java Sea between Java and Borneo toward the Strait of Makassar (Umbgrove 1949; Verstappen 1975). Similarities in freshwater fish species between eastern Sumatra and western Borneo indicate that the rivers of these islands were once linked; the Musi of Sumatra and the Kapuas of Borneo in particular were once part of the North Sunda river system. On the other hand, some of these large rivers clearly served as faunal and floral divides of some magnitude; P. Ashton (1972) has pointed out that the Dipterocarp forest trees show some sharp breaks in distribution at the Lupar River in western Borneo, and the presence of the large rivers between Java and Borneo may have slowed down faunal dispersals between these two islands in the Pleistocene. Two large freshwater lakes also occupied the middle of the Gulf 
of Thailand and the area immediately north of western Java, according to seabed contours presented by Butlin (1993:Maps 8a, 8b).

\section{Environmental Conditions of the Last Glaciation in the Indo-Malaysian Archipelago}

The environmental fluctuations of the past 120,000 years-since world climate and sea level were last at around their present configurations-are rapidly becoming quite well plotted. A graph of relative climatic severity over the past 130,000 years has been prepared by Kukla (1981; see Fig. 1.6, top), and this shows quite clearly how relative severity increased gradually and slowly, despite fluctuations, until the last glacial maximum about 18,000 years ago. The amelioration since then has been very rapid, especially after 14,000 years ago (Broecker et al. 1988; COHMAP 1988). This is important for the rate of the last major sea level rise. It has been estimated that parts of the Sahul shelf may have been drowned at rates of 25 to 45 meters horizontal migration per year (Andel et al. 1967), although this movement was interrupted by occasional standstills. Rates of vertical sea level rise probably rose to maxima between 1.0 and 1.5 meters per century (Chappell and Thom 1977:285). On the Sunda shelf, Haile (1973) has postulated a rapid drowning of the course of the Proto-Lupar River, although this could have involved local tectonic downwarping as well.

If we now focus on the last 40,000 years, for which there is increasingly good archaeological and environmental evidence, there is fairly widespread agreement that temperatures fluctuated at intermediate levels until about 25,000 years ago, when they began to downcurve rapidly to reach a nadir at about 20,000 to 18,000 years ago. Climates and sea levels then returned to approximately present conditions by around 8,000 years ago, following a reversion to glacial conditions between 12,500 and 11,000 years ago known as the Younger Dryas Interval (Hughen et al. 1996). In some parts of the world the midHolocene climate was perhaps a little warmer and wetter than at present (Kutzbach 1981; Lamb 1982). The conditions of the last glacial maximum in the tropics are of most interest here, and it is now becoming clear that some of these areas were considerably drier at this time, with evidence for relative aridity, decline of forest biomass, drying of lakes, and extensions of deserts in Australia, Africa, the Middle East, India, and South America (Rind and Peteet 1985; Hammen and Absy 1994; Yan and Petit-Maire 1994). The Bay of Bengal waters were more saline than now, which has been taken to reflect a decrease in summer monsoon rainfall and a consequent decrease in the quantity of fresh water entering the ocean from rivers (Duplessy 1982).

The evidence for Indo-Malaysian climatic conditions of 18,000 years ago is not as clear as it is for some of the larger tropical continental areas, partly 
because this area is relatively complex in terms of land-sea and altitudinal patterning. For instance, in terms of temperature it is apparent that New Guinea highland averages fell to about 7 to $7.5^{\circ}$ Celsius below present averages (see Section IIIB), and tree and snow lines fell by between 1,000 and $1600 \mathrm{~m}$ (but see Note 2). A similar drop in average temperature of 5 to $9^{\circ}$ Celsius has been suggested by Tsukada $(1966,1967)$ for highland Taiwan, and by Newsome and Flenley (1988) for highland Sumatra (see also Stuijts et al. 1988). Such dramatic plunges in average temperature probably did not occur in the tropical lowlands, however, and last glacial estimates for Sundaland locations near sea level tend to fall between 2 and $5^{\circ}$ Celsius below present (Verstappen 1975; Batchelor 1979; Kaars 1991). It is unfortunate that there are as yet no pollen sequences covering the last glacial in the lowland equatorial regions of Southeast Asia. While the evidence does seem to favor a lowland temperature drop of some extent, it was probably of less magnitude than at high altitudes and perhaps less in the oceanic environments of Wallacea than in the more continental regions of Sundaland.

Turning now to rainfall, we may recall that through much of the Pleistocene both Sundaland and Sahulland would have been above sea level as large continental areas. Periods of drowning like the present probably only occurred for short periods. The period of continentality at the maximum of the last glaciation would certainly have produced drier climates. Lower temperatures would reduce convectional rainfall and winds would tend to be drier because they would cross larger land areas and cooler seas. High-latitude glaciation would cause an increased pressure gradient between the Asian and Australian continents and the equator, and the Intertropical Front may have moved outside the limits of the Indo-Malaysian Archipelago for longer periods than it does now. Dry seasons would become longer and even the equatorial regions could have experienced briefer and smaller rainfall maxima (Verstappen 1975; Batchelor 1979; Morley and Flenley 1987). Verstappen (1975:10) has suggested that average rainfall in Sundaland could have been reduced by as much as 30 percent during the last glaciation.

All this suggests that the Indo-Malaysian Archipelago would probably have had much larger areas of monsoon forest during the last glaciation, even if the inner core regions of equatorial forest in Sundaland and New Guinea were relatively little affected. The rain forest itself has certainly maintained a stable composition in Borneo since the Miocene (Muller 1975), but there possibly were landscapes with longer dry seasons and more open vegetation during the successive Pleistocene glaciations, perhaps around the fringes of the Sunda shelf, along which open forest mammals and hominids could have passed more freely southward across the equator. This possibility was pointed out from a floral viewpoint some years ago by Steenis $(1961,1965$; see also Scrivenor et al. 1942- 


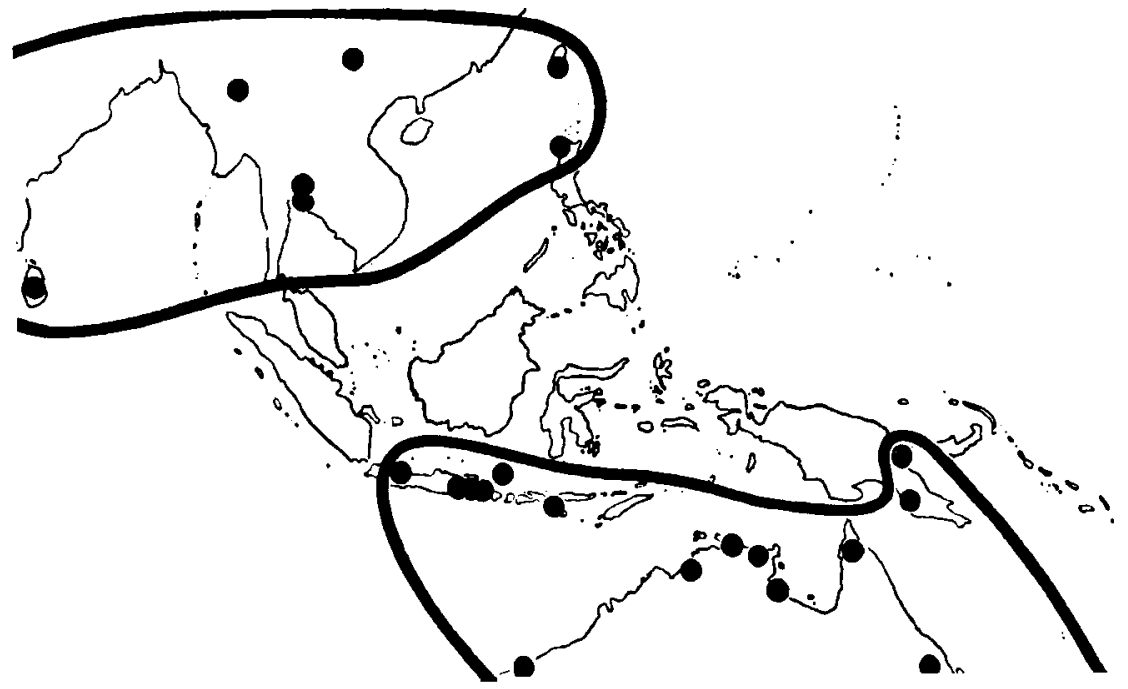

Fig. 1.12 The disjunct distribution of the plant species Rhynchosia minima, which grows under strong dry-season conditions. From Steenis 1961. Courtesy: Herbarium Bogoriense.

1943:148), who showed that several species of Leguminosae and grasses adapted to long dry seasons occur in the northern and southern monsoon areas, but with sharp gaps in distribution in equatorial Indonesia at the present time (Plate 1). To explain these disjunct distributions Steenis suggested that dry season zones or corridors had been more extensive in the past, particularly through Sulawesi and the Philippines. Morley and Flenley (1987:Fig. 5.5) also suggest that a relatively dry "savanna corridor" existed through western and southern Sundaland during glacial maxima.

Other geomorphic observations fill out this picture of late glacial dryness, especially in and around Sundaland. Verstappen (1975) has suggested that the coarse alluvial and colluvial deposits of the last glacial in Peninsular Malaysia indicate tree savanna conditions with considerable erosion and valley filling. Terrestrial deposits beneath the sea on the Sunda shelf include bauxite and laterite pans, kankar nodules, and possibly braided stream deposits, all suggesting past seasonal climates. Confirmatory evidence for a shrinking of rain forests during glacial periods in the tropics has also been claimed by Shackleton (1977) from curves of variation in carbon-13 content in equatorial Pacific foraminifera; he suggests that plant biomass and associated humus, especially in the tropics, increased by a factor of three during the overall warming between 14,000 and 8,000 years ago.

The Sahul shelf has produced similar evidence for glacial dryness. Andel et 
al. (1967) have suggested that it received only between one-third and one-half of its present rainfall in the last glaciation. Nix and Kalma (1972) have reconstructed an open woodland vegetation for the shelf at this time, with a considerable restriction of the southward extent of the New Guinea rain forest. More recent palynological research from seabed cores by Kaars (1989) gives a similar picture, with grassland being widespread on the Sahul shelf between 38,000 and 17,000 years ago.

Some other nonclimatic differences between continental (glacial) and island (interglacial) phases of Sundaland have been outlined by Dunn and Dunn (1977). For instance, at a sea level 100 meters below the present, Sundaland had about twice as much exposed land as it does now, but only about 47 percent of the coastline. This is an important observation, for many ancient economies in this part of the world depended on the sea and periods of high sea level are clearly favorable for increasing coastal resources. On the other hand, interglacial conditions with high rainfall would not favor inland economies based on the hunting of herd animals in open parkland environments. Dunn and Dunn also note another important fact: The Sunda shelf is not flat and the exact extent of the Sunda continent would have varied with sea level. For instance, the island of Palawan, technically speaking a part of Sundaland, is separated from Borneo by a channel 140 meters deep. Because it also has an impoverished mammal fauna, it clearly was not linked to Borneo for long periods, although it certainly was linked at some point during the Pleistocene. Java also might have been an island at times when Sumatra and Borneo were joined to Peninsular Malaysia, as it also has some faunal peculiarities.

We are not really in a position yet to state clearly what impact these Pleistocene climatic and environmental fluctuations had on the floras and faunas (including humans) of the Indo-Malaysian region. The most recent cases of animal extinction at the end of the Pleistocene and in the early Holocene do give some interesting hints, but it is clear that these fluctuations in the Indo-Malaysian latitudes did not have such a massive ecological impact as those recorded for periodically glaciated temperate regions such as Europe and North America.

\section{E. Animal Extinctions and Habitat Shifts in Sundaland in the Late Pleistocene and Holocene}

In 1972, Medway noted that out of two hundred extinctions worldwide during the late Pleistocene, only eleven occurred in Sundaland (this figure is now slightly larger). The 32,000-year-old fauna of Niah in Sarawak has a giant pangolin as its only truly extinct species (Harrisson et al. 1961). This is a very different story from the record of animal extinction in the late Pleistocene in Eurasia, North America, and Australia. Table 1.1 lists some approximately dated exam- 
Table 1.1. Late Pleistocene and Holocene extinctions in Sundaland

\begin{tabular}{|c|c|c|c|}
\hline Species & Site & $\begin{array}{l}\text { Last recorded date } \\
\text { of local or regional } \\
\text { existence }\end{array}$ & Reference \\
\hline $\begin{array}{l}\text { Manis palaeojavanica } \\
\text { (giant pangolin) }\end{array}$ & $\begin{array}{l}\text { Niah Caves, } \\
\text { Sarawak }\end{array}$ & c. $30,000 \mathrm{BC}$ & Medway, 1977a \\
\hline $\begin{array}{l}\text { Tapirus indicus }{ }^{2} \\
\text { (Malayan tapir) }\end{array}$ & $"$ & c. $6000 \mathrm{BC}$ & $"$ \\
\hline $\begin{array}{l}\text { Hylomys suillus }{ }^{1} \\
\text { (lesser gymnure, } \\
\text { an insectivore) }\end{array}$ & $"$ & Late Pleistocene? & $"$ \\
\hline $\begin{array}{l}\text { Melogale orientalis }{ }^{1} \\
\text { (ferret badger) }\end{array}$ & $"$ & early Holocene? & $"$ \\
\hline $\begin{array}{l}\text { Rhinoceros sondaicus } \\
\text { (Javanese rhinoceros) }\end{array}$ & $\begin{array}{l}\text { Madai Caves, } \\
\text { Sabah }\end{array}$ & $6000 \mathrm{BC}$ & Cranbrook, 1988a \\
\hline $\begin{array}{l}\text { Cuon sp. }{ }^{2} \\
\text { (the dhole, a wild canid) }\end{array}$ & $"$ & $6000 \mathrm{BC}$ & $\begin{array}{l}\text { Cranbrook, 1988a } \\
\text { (provisional } \\
\text { identification only) }\end{array}$ \\
\hline $\begin{array}{l}\text { Panthera tigris } \\
\text { (tiger) }\end{array}$ & $"$ & $\begin{array}{l}\text { between } 7000 \text { and } \\
3000 \mathrm{BC}\end{array}$ & $\begin{array}{l}\text { T. Harrison } \\
\text { (pers comm) }\end{array}$ \\
\hline $\begin{array}{l}\text { small deer }^{1} \\
\text { (species unknown) }\end{array}$ & $\begin{array}{l}\text { Guri Cave, } \\
\text { Palawan }\end{array}$ & $3000 \mathrm{BC}$ & Fox, 1970 \\
\hline $\begin{array}{l}\text { Elephas maximus }{ }^{3} \\
\text { (Indian elephant) }\end{array}$ & $\begin{array}{l}\text { Gua Lawa Cave, } \\
\text { central Java }\end{array}$ & early Holocene? & Medway, 1972 \\
\hline $\begin{array}{l}\text { Bubalus sp. } \\
\text { (water buffalo) }\end{array}$ & $"$ & $"$ & $"$ \\
\hline $\begin{array}{l}\text { Neofelis nebulosa } \\
\text { (clouded leopard) }\end{array}$ & $"$ & $"$ & $"$ \\
\hline $\begin{array}{l}\text { Cervus eldi }{ }^{4} \\
\text { (a deer) }\end{array}$ & $"$ & $"$ & $"$ \\
\hline
\end{tabular}

(1. locally extinct; 2 . extinct on Borneo; 3 . extinct on Java; 4. extinct in Indonesia)

ples of late Pleistocene and early to middle Holocene extinctions or habitat shifts in Sundaland. In a review of the whole topic of animal extinction, Medway (1977a) has argued against any major human role in this pattern and has suggested that the rapid environmental fluctuations of the late Pleistocene, especially the final glacial maximum and the subsequent rapid amelioration to warmer, wetter, and more forested conditions, may have been the main causes. However, this cannot account for all species extinctions; the giant pangolin appears to have vanished long before the end of the last glacial, and extinction 
of the Palawan deer seems to have been recent enough for human predation to be considered as a cause. ${ }^{3}$

Of these animals, the giant pangolin is the only totally extinct species; the rest are merely locally extinct. Cervus eldi and wild water buffalo no longer exist in Indonesia today. The gymnure and ferret badger from Niah both survive today on the higher slopes of Mount Kinabalu in Sabah and their presence in the late Pleistocene at Niah supports the evidence for a cooler climate during that period. Medway (1977a) has also suggested that the giant pangolin would have required larger termitaria for its food supplies than exist in equatorial rain forests today, and this again suggests a more open environment in this part of Borneo in the late Pleistocene.

When looking at modern animal distributions (excluding human transportation of such species as deer, monkey, civet, and pig), we find some puzzling patterns (see Fig. 1.5). Elephants occur in Sumatra and Peninsular Malaysia, are extinct in Java, and are not definitely recorded for Borneo prior to recent introductions (Harrisson 1978); the Sumatran rhinoceros occurs in Sumatra, Peninsular Malaysia, and Borneo, but the Javan rhinoceros lives only in Java, Peninsular Malaysia, and Sumatra (the latter only until the 1930s); wild banteng cattle live in Java, Borneo, and northern Peninsular Malaysia, but are not certainly known in Sumatra or southern Peninsular Malaysia; the tiger occurs in Peninsular Malaysia, Sumatra, and Java, but not in Borneo. This list could continue, but I merely wish to point out the existence of these disjunct distributions. The reasons appear to be mainly ecological: ever-wet climates versus seasonal climates and vegetation; the presence of large rivers acting as faunal barriers; the compression of environmental zones against ocean or mountain barriers; and the possibility that some islands were cut off before others by rising postglacial sea levels. Indeed, recent archaeological research is indicating that some of these animals had larger ranges until well into the Holocene. The tiger, Javan rhinoceros and Cuon, for instance, are reported from the Madai excavations in Sabah (Table 1.1), and there is a possible record of elephant from Niah (Terry Harrison, pers. comm.).

Having looked at the histories of the environment, climate, and fauna of the Indo-Malaysian Archipelago from the Pliocene through to the Holocene, it is time to turn to the history of a major mammal species I have so far rather ignored: the human species. 


\section{Homo erectus in Sundaland}

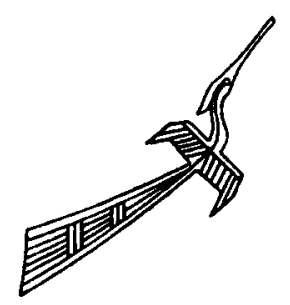

In 1891, a young Dutchman named Eugene Dubois commenced what has now been over a century of human fossil discovery outside Europe: a century that has witnessed some profound changes in scientific views of human origins. Dubois entered-and changed--the history of anthropology in October 1891, near the village of Trinil in the middle Solo Valley of central Java. His discovery, a skullcap (or calotte) of apparent human form, belonged to an archaic human species that he called Pithecanthropus erectus.

Since 1891, many more finds have come to light in Java and the rest of the world. In this section I intend to review the significance of the Javanese erectus populations in their general old World setting. There are now so many articles and sections of textbooks that discuss the finer physical characteristics of these fossils that I will limit myself in this regard to a few central observations. The overall perspective is of most concern here.

During the past thirty-five years, fossil discoveries in the East African nations of Tanzania, Kenya, and Ethiopia have completely overturned previous theories, which quite frequently postulated an Asian center for the earliest phases of human evolution. This view was not surprising considering that most of the major finds during the period from the 1920s to the Second World War had been made in Java and at Zhoukoudian in China, with the exception of the Australopithecines of South Africa, which at that time had yet to achieve full recognition as potential human ancestors.

Scientific understanding of the course of human evolution today is naturally founded on a much fuller fossil record than it was even thirty years ago. The often-confident assertions of paleoanthropologists might lead to a belief that many important problems have been solved, despite their own often funda- 
mental disagreements with each other's interpretations. Common sense, however, would suggest that many surprises may still lie buried in different parts of the Old World. But the advances have certainly been impressive, as one can see by merely comparing recent textbooks with those published about twenty years ago. Paleoanthropology has come a long way indeed since theories of a giant ape ancestry for man were published by Weidenreich as recently as 1945 and 1946. Weidenreich himself was of course a respected and competent scholar who made fundamental contributions to the study of fossil hominids in China and Java, but unfortunately for him he produced his more general theory too soon (an occupational hazard that doubtless is always with us).

\section{THE ANTECEDENTS}

When the original manuscript for this book was prepared in the early 1980s, it was still widely accepted that the oldest primate genus to contain the first recognizable stage of emergent hominid form was a widespread African and Asian Miocene ape called Ramapithecus. Since then, however, the tide of opinion has turned. Ramapithecus has been transferred out of the human lineage and into a possible ancestor for the orangutan.

Today, the quest for human origins is focused most intensively on the Australopithecines of eastern and southern Africa. Even as I prepare this revision, ideas about human evolution are undergoing yet further upheaval as a result of discovering a new genus and species, Ardipithecus ramidus (a presumed forebear of Australopithecus) in deposits dating to about 4.5 million years ago in Ethiopia (Wood 1994; White et al. 1994; Gee 1995). A. ramidus is claimed to be the earliest potential hominid to appear in the African fossil record, and the bones of younger Australopithecine species have been found in large numbers of sites in South Africa, Tanzania, Kenya, and Ethiopia.

These African hominids of the Pliocene undoubtedly contain some of the basic physical and cultural roots of our humanity. For instance, $A$. ramidus is currently placed close to the phylogenetic separation between the human and chimpanzee lines of descent, a separation that might have been encouraged geographically, between 8 and 4 million years ago, with the opening of the African Rift Valley and the development of drier and less forested landscapes in eastern Africa (Tobias 1992; Coppens 1994). Early humans are presumed to have evolved in these areas, whereas the chimpanzees remained closer to the forests in the west. However, as with understanding the tectonic evolution of the Indonesian Archipelago, so too understanding of the course of human evolution becomes more complex and more contested with each new discovery.

When the first edition of this book was being written, biological anthropology was going through a phase of "lumping" in which relatively small numbers 


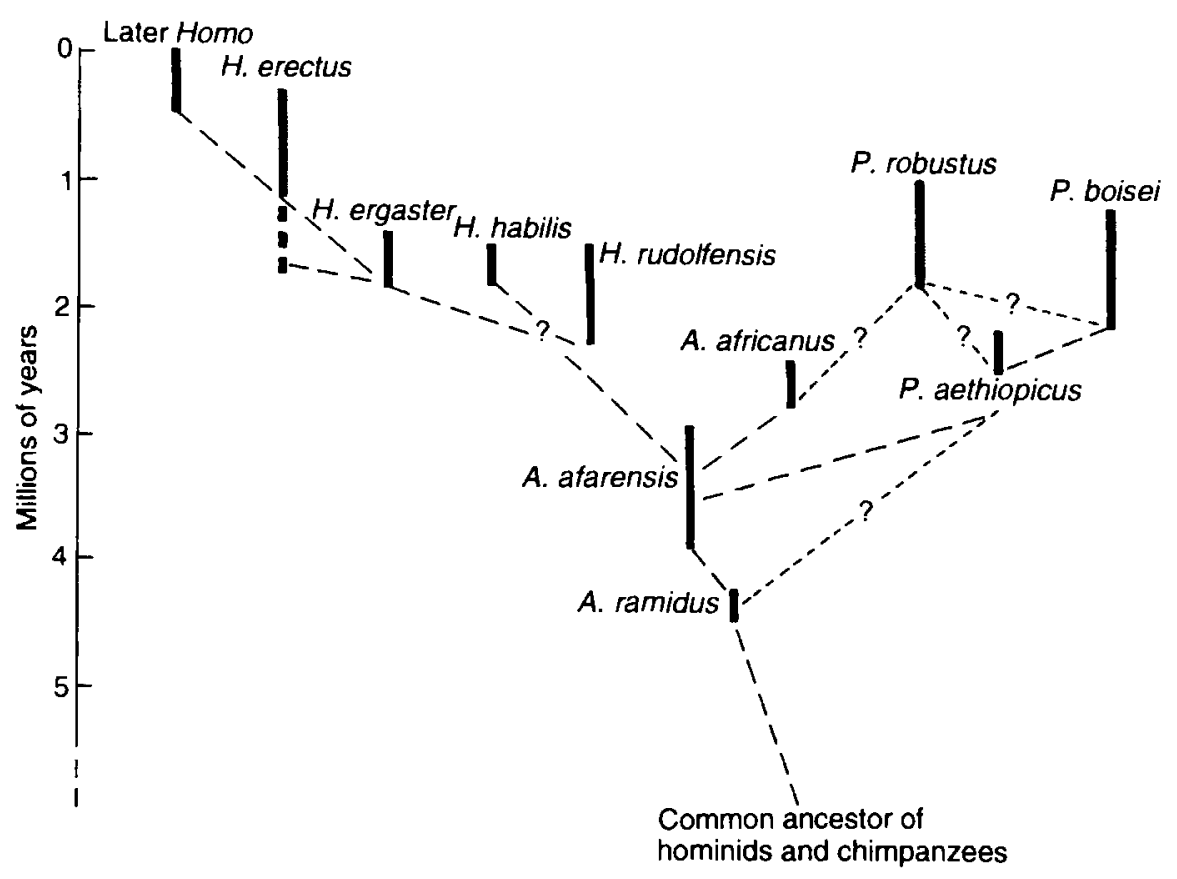

Fig. 2.1 A provisional hominid evolutionary tree. The vertical bars show the known dates of first and last appearances of species. $\mathrm{H} .=$ Homo; $\mathrm{A} .=$ Australopithecus; $\mathrm{P} .=$ Paranthropus (the robust Australopothecines). After Wood 1994. Courtesy: Bernard Wood.

of species were being recognized. In the case of the genus Homo, it was believed that only one species existed at any one time, in the general chronological order Homo habilis $\rightarrow$ H. erectus $\rightarrow H$. sapiens. All of this now seems luxuriously simple; the habilis and erectus grades certainly contained more than one contemporary species if we are to believe many modern commentators (e.g., Groves 1989; Wood 1992; Howell 1994). The original figure 2.1 that accompanied this chapter (reproduced from Johanson and Edey 1981) showed one line of hominid descent from $A$. afarensis through $A$. africanus to $A$. robustus (extinct), and another from $A$. afarensis through $H$. habilis and $H$. erectus to anatomically modern humans. Our understanding of human evolution in 1995 is not so simple. A possible successor evolutionary tree suggested by Wood (1994), far more complicated, now replaces the original (Fig. 2.1).

For this book it is not necessary to prolong the Australopithecine debate as this genus has never been convincingly reported out of Africa (although see Tyler 1992 for suggestions of an Australopithecine presence in Java). The weight of evidence for an emergence of the genus Homo from an Australopithecine ancestor somewhere in Africa now seems to be overwhelming. However, it is 
not clear which species of the generalized habiline (or early Homo) grade-if indeed any of those so far discovered by paleontologists-was/were the ancestor(s) of extra-African Homo erectus and other subsequent human populations. Howell (1994:302) has recently suggested that the ancestral Homo species that dispersed initially out of Africa was the East African species Homo ergaster, which eventually speciated into Homo erectus in eastern Asia (see also Fig. 2.1). Howell, however, like numerous modern anthropologists, regards the Asian Homo erectus as an extinct branch of hominid evolution with no transmission of genes to modern populations. This brings up a major issue of debate to which I shall return.

As far as the line of anatomically modern human descent is concerned, purely behavioral developments in Africa prior to 2 million years ago are likely to have been heavily embedded in a biological developmental matrix involving reinforcement of bipedal posture, increasing hand flexibility, greater cranial capacity (Fig. 2.2), and the development of the human grinding and chewing dentition. The Australopithecines had attained a partially bipedal posture by at least 3.6 million years ago, if not before, according to a series of footprints preserved at Laetoli in Tanzania (Leakey 1981), but the expansion of the brain seems to have occurred mainly later with the genus Homo. Sexual body size dimorphism is well marked among the early hominids, who probably existed on a fairly omnivorous meat and plant diet derived from grassland and savanna territories located close to rivers and lakes (Boaz 1977; Coppens 1994). During the period of transition to the genus Homo, around 2.5 million years ago, there is already direct evidence for stone tool use (Clark 1992; Tobias 1992) and, soon afterwards, inferred but disputed evidence for regular dwelling places or campsites (Binford 1983). Perhaps the record also witnesses the evolution of such concepts as the nuclear family, kinship, the incest taboo, and basic human language (Isaac 1980; Wilson 1980; Lovejoy 1981; Bickerton 1990).

In terms of biological morphology, the earliest populations of erectus grade in Africa and elsewhere can be regarded as evolved habilines. Bernard Wood (1978: 53-54) has described the major skeletal characteristics of the whole erectus group (including the Javanese fossils) as follows: a distinctive cranial shape marked by prominent browridges separated from the rest of the skull by a deep constriction (the postorbital constriction), a low vault with the widest point at the base, extremely thick cranial bones and strongly marked muscle attachments, a broad, large face with large teeth, and an average cranial capacity of about 950 cubic centimeters (Fig. 2.3 and Fig. 2.4). Postcranial remains suggest a body size and posture approaching the sapiens grade, with the important proviso that the erectus grade spans perhaps a million years or more, so some degree of temporal and also regional variation can be expected. Opinions on this matter differ, however, with Wolpoff (1984) and Clausen (1989) claiming signif- 


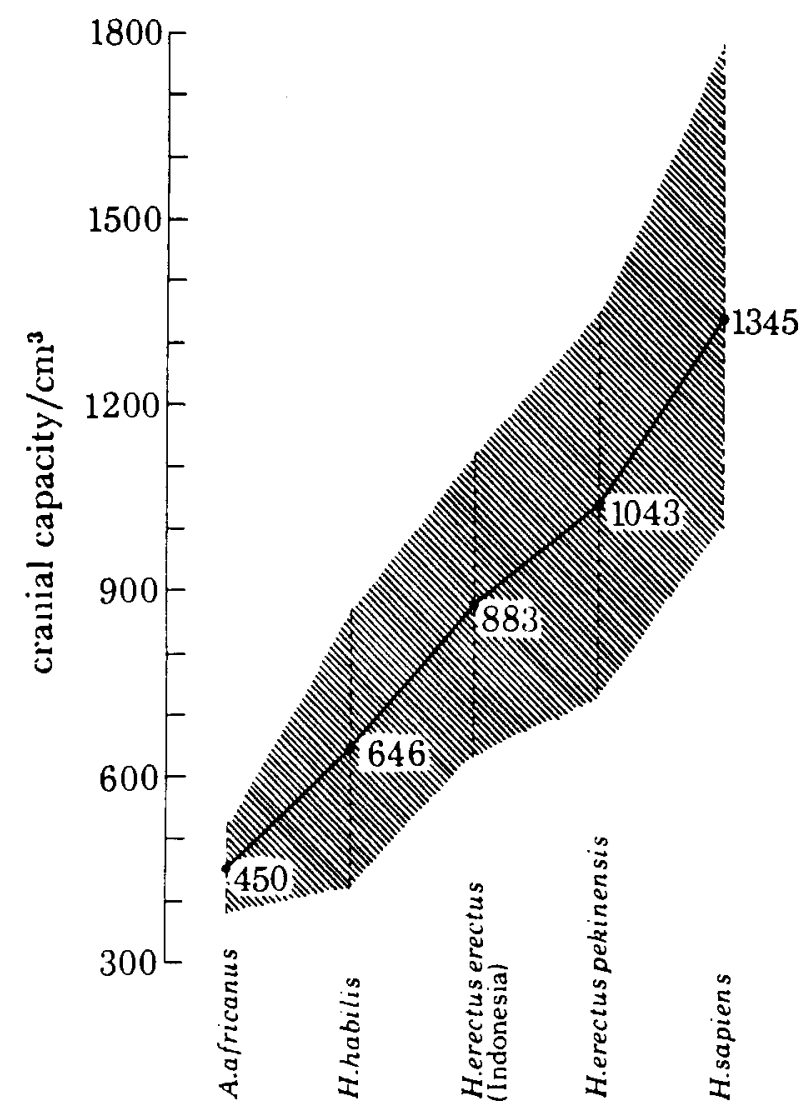

Fig. 2.2 The pattern of cranial capacity increase in human evolution over the past 3 million years (shading indicates 95 percent population limits). From Tobias 1981. Courtesy: The Royal Society, P. V. Tobias.

icant evolutionary change thorough the erectus time span, but Rightmire (1990) claiming virtual stasis.

It is with the early erectus (rather than Australopithecine) evolutionary level that Java takes on a major importance, for this small island contains some of the earliest evidence for human radiation out of Africa through the tropical zones of the Old World. According to different authorities who have claimed absolute dates for the hominids of Java, this radiation might have occurred between 1.8 and 1.0 million years ago (see Chapter 1, Section IVB). I tend to favor the younger age, but freely admit that the whole issue of dating Homo erectus across the Old World is still highly controversial. A date of 1.8 million years for the Perning site in Java (Swisher et al. 1994) would mean that Homo 

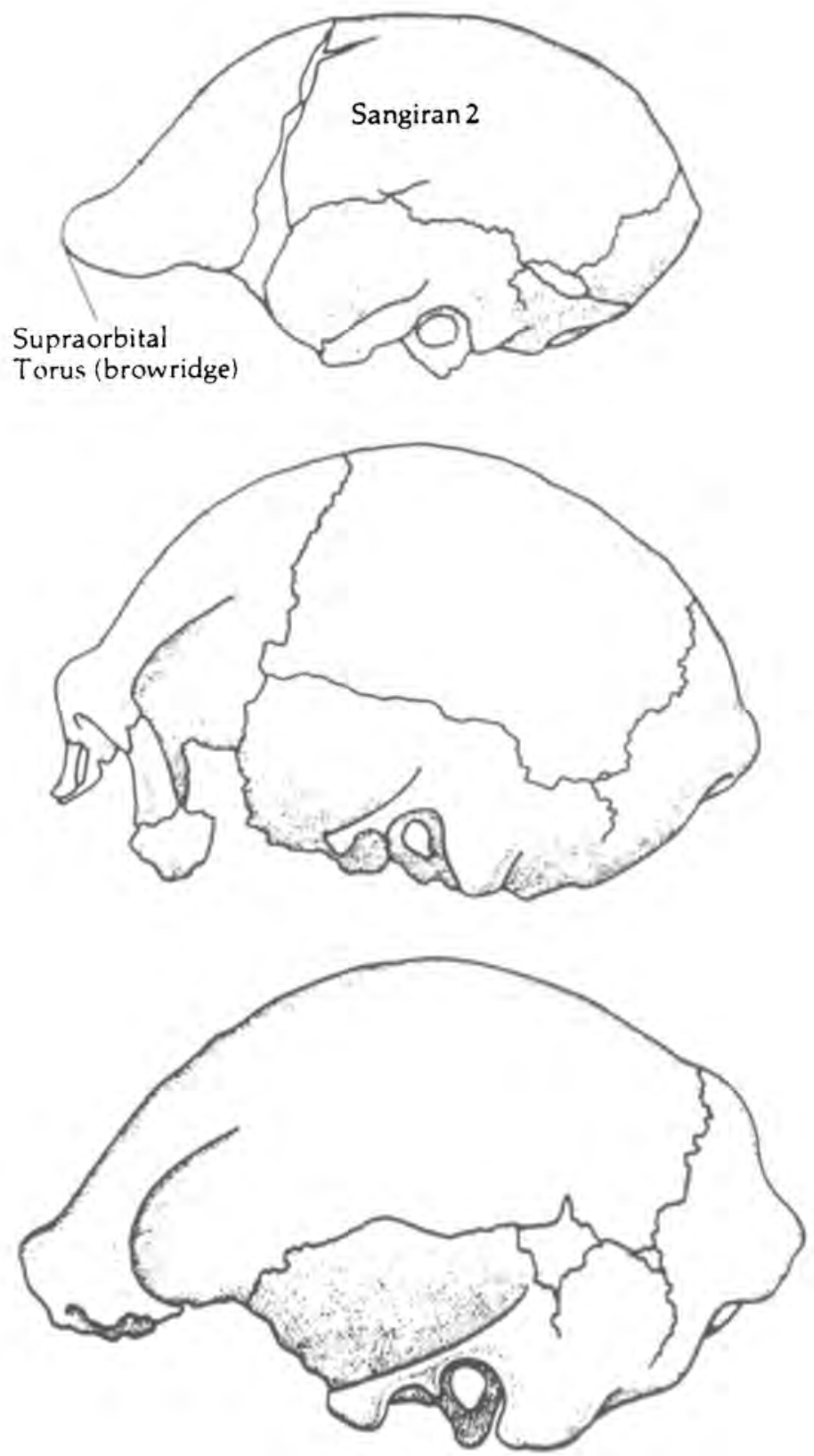

Fig. 2.3 Top to bottom: lateral views of Homo erectus crania from Sangiran (early or middle Pleistocene), Zhoukoudian (middle Pleistocene) and Ngandong (late Pleistocene). To same scale. From Wolpoff 1980. Courtesy: Alfred A. Knopf, Inc. 


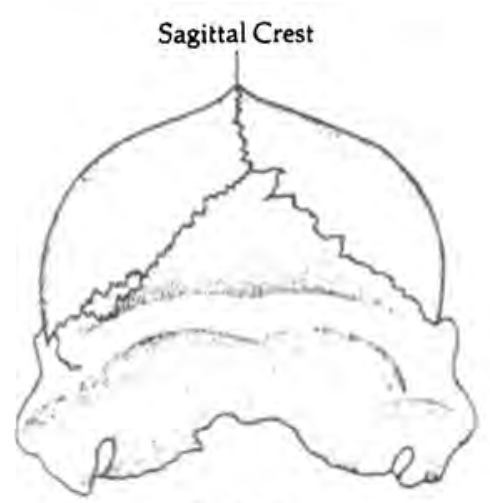

ER 1805

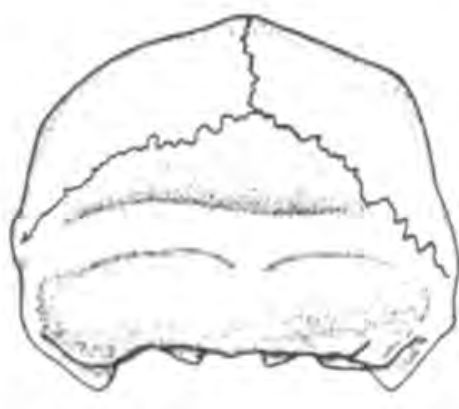

Sangiran 4

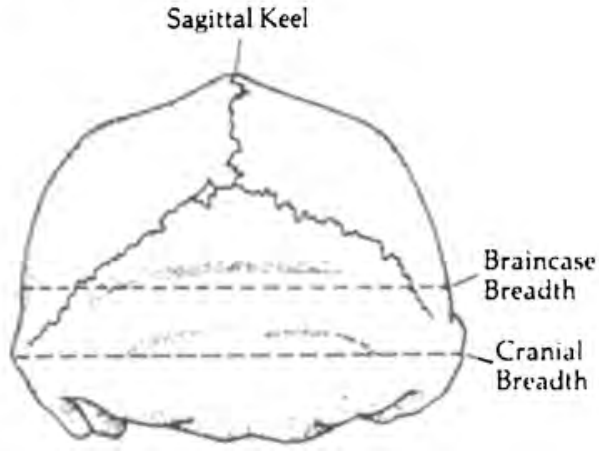

Choukoutien II

Fig. 2.4 Posterior cranial views of ER 1805, a transitional habilis-erectus specimen from Kenya, and later erectus crania from Sangiran and Zhoukoudian (Choukoutien). To same scale. From Wolpoff 1980. Courtesy: Alfred A. Knopf, Inc.

erectus here is older than the erectus-grade population in Africa, which on current evidence dates from about 1.7 million years ago (Rightmire 1990:Fig. 39). The erectus teeth and associated stone tools from Yuanmou in Yunnan (China) have been claimed to date from about 1.7 million years ago (Woo 1980:188; EATQN 15:77), but this date is disputed as being too old by Liu and Ding (1983) who suggest only a middle Pleistocene antiquity (see also Howell 1994:264265). However, there has been a recent claim that a mandible fragment and some possible stone tools from Longgupo Cave in Sichuan date to almost 2 million years ago (Huang et al. 1995). Needless to say, this claim requires fuller verification if it is to be accepted fully. There are big problems of chronology concerning the radiation of Homo out of Africa, and I see no easy resolution at the moment. 


\section{HOMO ERECTUS IN JAVA}

The major find-places for the Javanese fossils have been in the upper Pucangan, Grenzbank, and Kabuh deposits exposed in the anticlinal dome at Sangiran (see Chapter 1, Section IVB and Koenigswald 1956 for descriptive accounts). Additional important fossils have also come from possible Kabuh-equivalent beds at Trinil itself, in central Java, and from Perning, near Mojokerto in eastern Java, but both these sites have major dating problems (see Vos et al. 1982 for Trinil; Swisher et al. 1994 for Perning). At present, all specimens reliably of Pucangan age come from Sangiran (and possibly Perning), and these specimens also appear morphologically to be the oldest in the series. Finally, there is the important late erectus cranial series from Ngandong, Sambungmacan, and Ngawi in the Solo Valley, of late middle or late Pleistocene faunal age.

\section{A. The Pucangan Hominids}

Of the Pucangan sample from Sangiran, the most complete specimen, Sangiran 4 (popularly called "Pithecanthropus IV"; Koenigswald and Weidenreich 1939), comprises the posterior part of a braincase and the lower portion of the maxilla (mid-facial region). Both belonged to a heavily muscled individual with a cranial capacity of about 900 cubic centimeters, with large teeth and particularly large canines (Holloway 1981). A most unusual feature of the dentition is a gap (diastema) between the upper canines and incisors, into which the lower canines would have fitted to allow the jaw to close. Such diastemata are typically an ape phenomenon; the great apes have very large canines, and they do not occur in any other erectus specimen (with the possible exception of the much later Sangiran 17 skull; Thorne and Wolpoff 1981). The Australopithecines from Laetoli and Afar in East Africa do sometimes show this feature (Johanson 1980:48; Wolpoff 1980:134), and it is possible that the diastema in Sangiran 4 implies a relatively early position within the extra-African phylogeny of Homo. Of course, as always there is a counterargument: It has been suggested that the diastema in the Sangiran specimen could be due to "labial displacement of the maxillary incisors because of vertical collapse after loss of the posterior mandibular teeth" (Zingeser 1979). In this case the feature would be simply a result of individual trauma, although the lower jaw of the specimen does not survive to allow direct confirmation.

The Sangiran 4 remains may otherwise be taken as fairly representative of an early Pleistocene, robust Homo erectus population in Java. They come from a stratigraphically high position in the Pucangan beds and there are mandible fragments in older deposits that appear to be of the same basic grade. Most authors until recently have favored attribution of all to a single species-Homo 


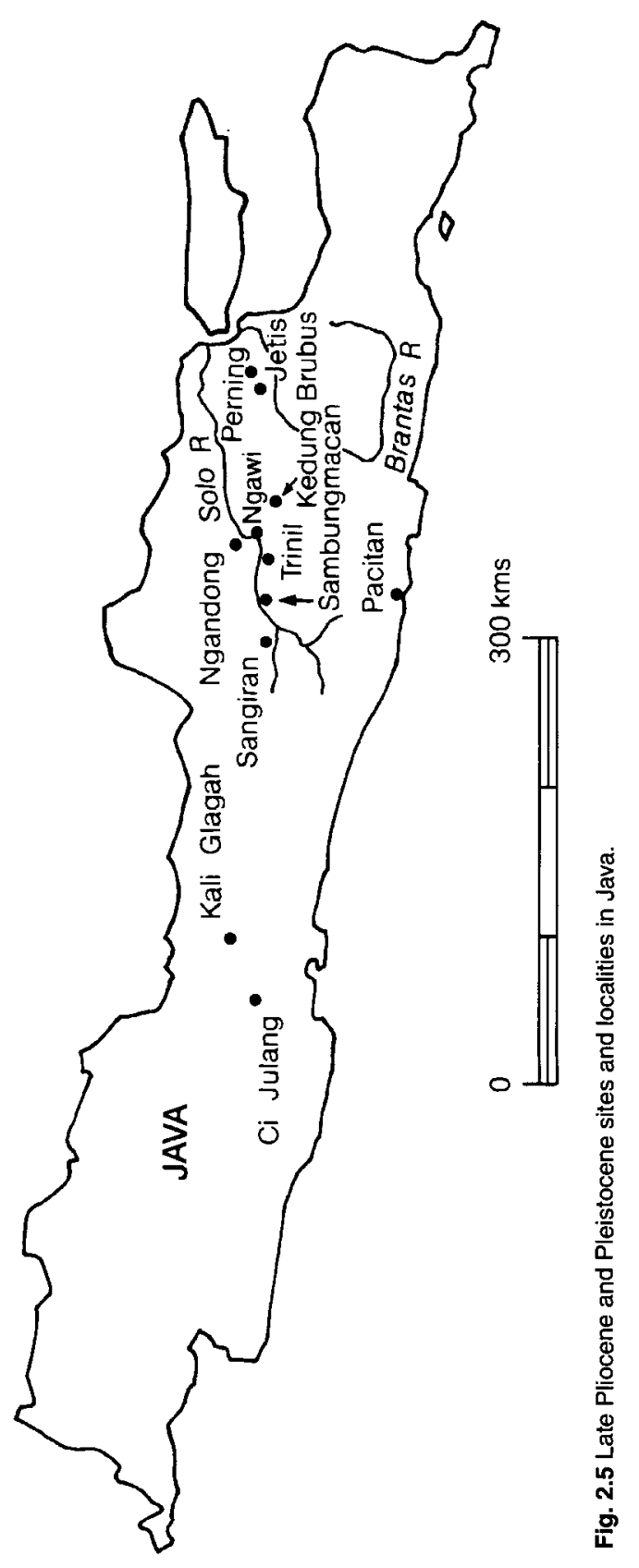


erectus-suggesting that there is no overwhelming reason why the Javanese fossils should not belong to one single species evolving through a long period of time. Rightmire (1990) certainly takes this view, as does Groves (1989).

As with the dating, however, the issue is not simple. The Indonesian paleoanthropologists Jacob (1980) and Sartono (1985) have long favored the existence of more than one contemporary species of pithecanthropine in Java, a view recently supported by Tyler (1992), by Uytterschaut (1992), and by Howell (1994). Part of the debate revolves around the taxonomic status of some massive mandible fragments. The two most important of these fragments were found at giran in 1941 and 1952, one from the upper Pucangan and the other from the lower Kabuh beds. Clearly hominid, but with massive teeth overlapping in size with those of a gorilla, these specimens were named Meganthropus paleojavanicus by Koenigswald and Weidenreich. Weidenreich (1946) adopted the view that Meganthropus was on a direct line of evolution to anatomically modern humans, occupying a position between the more massive-toothed Gigantopithecus and the smaller Pithecanthropus (Homo erectus). This view never became popular and, as the African evidence accumulated, attempts were made to correlate Meganthropus with the robust Australopithecines (or Paranthropus; Robinson 1968) and with Homo habilis (Tobias and Koenigswald 1964). Even today it is still maintained as a genus quite separate from Homo erectus by the Indonesian scholars Jacob (1978a) and Sartono (1975; see also Orban-Segebarth and Procureur 1983). Tyler (1992) also suggests that Meganthropus might be separate from Homo erectus.

So could the Meganthropus jaw fragments represent a situation similar to that in East Africa, where robust Australopithecines continued to exist alongside Homo in the early Pleistocene? Regardless of the fluctuating opinions of paleoanthropologists on this issue, there is still the question of dating. It is hard to be certain whether the hominid remains in question really do overlap in time, although the occurrence of both Homo erectus and Meganthropus in the Grenzbank would suggest that some overlap did occur. In 1970 Lovejoy placed Meganthropus firmly at the larger end of a considerable range of dental size variation in Homo erectus, a view also held by Le Gros Clark (1964), by Bernard Wood (1978:56), and by most contemporary authors since. Although few authorities today seem willing to keep Meganthropus in a separate genus from Homo, I agree with Pope that "the question of the validity and reality of Meganthropus must remain open for the present (1985:69).

\section{B. The Kabuh Hominids}

From the Kabuh Formation, perhaps dating between 1.0 and 0.5 million years ago (see Chapter 1, Section IVB), there is a much fuller set of fossil remains. 
Most specimens again come from Sangiran, and there is also the original find made by Dubois at Trinil: the erectus calotte (skullcap) found in the bed of the Solo River during the dry season of 1891. It has been claimed (Bartstra 1982; Vos et al. 1982; Vos and Sondaar 1982) that the mammal fauna from Trinil is much older than the middle Pleistocene fauna called Trinil from Sangiran, but the Trinil skull itself does fit morphologically with the Sangiran remains attributed to this period. It may be that the fauna and the skull from Trinil are not contemporary, and it must be admitted that there have always been problems with Dubois' records. For instance, he recovered a number of human femora from the general vicinity of the Trinil skull that have often been used to reconstruct a totally modern body posture for Javanese Homo erectus. Fluorine tests (Bergman and Karsten 1952) appeared to support contemporaneity of the skull and femora, even though well-authenticated erectus femora from Africa and Zhoukoudian are considerably more archaic in appearance. However, Day and Molleson (1973) have since thrown doubt on the antiquity of the Trinil femora and certainty is still elusive.

All the other Javanese remains of Kabuh age are cranial or mandibular and represent a population less robust than the earlier Pucangan specimens, but still retaining a high degree of sexual dimorphism. Wolpoff (1980:191) points out that the Javan sample has less projecting browridges than the equivalent African specimens, but more marked facial prognathism, thicker skulls, and stronger muscle attachment areas. Cranial capacities range from 813 to 1,059 cubic centimeters, with an average of 929 (Holloway 1981). Stature probably ranged up to a maximum of around 160 centimeters, and weights may have ranged up to 80 kilograms in the latest populations (Wolpoff 1980:205). It is reasonable to assume that average cranial capacity increased during the time span of erectus evolution, although overall shape varied little until the development of high-vaulted Homo sapiens in the late Pleistocene, and much of the increase in brain size may simply correlate with a slow increase in body size (Bilsborough 1973). It has also been suggested that the thick vault and massive teeth of Homo erectus may relate to continuing selection imposed by the dangers of a hunting life and the frequent use of teeth as tools; the latter would also select for a massive and prognathous face with very powerful muscle attachments.

\section{Farewell to Homo erectus? The Ngandong Remains}

The Javanese remains of Homo erectus considered so far appear to span a long period of time prior to 500,000 years ago, with the majority coming from the later part of the time range. The oldest human remains of anatomically modern type in the region appear to be not more than 40,000 years old, and between 
these two groups there is only one major fossil population-from Ngandongwith related single skull discoveries at Sambungmacan and Ngawi (the latter being on the Solo River, upstream from Ngandong; see Fig. 2.5).

Between 1931 and 1933, Indonesian field assistants employed by the Geological Survey of Indonesia were given the periodic job of excavating a bonebearing terrace about 20 meters above the dry-season level of the Solo River at Ngandong, downstream from Trinil. The whole terrace deposit was about 3 meters thick and the animal bones (about 25,000 were recovered from a 50- by 100-meter excavation) were apparently fairly heavily concentrated in the lower metre of the deposit. From time to time the site was visited by the geologists Ter Haar and Oppenoorth and also by Koenigswald. Over the two-year period the bone collections eventually yielded no less than eleven crania (all lacking faces) and two tibiae of an advanced population of Homo erectus (see Koenigswald 1951, 1956 and Oppenoorth 1932 for eyewitness accounts). It is quite clear that the human skulls were not found together and Koenigswald noted the unusual circumstance that teeth, mandibles, and other bones apart from the two tibiae were entirely lacking; such selectivity was not noted amongst the other animal remains. Furthermore, of the eleven skulls only two had parts of their bases surviving; Koenigswald (1951) postulated that they had been broken open for purposes of brain eating, after which they were used as bowls. The idea of cannibalism has been disputed by Jacob (1967a, 1972), who pointed out that the skull base is a fragile area subject to natural breakage, but the observation still remains that the human bone sample is taphonomically unusual.

The Ngandong crania (Fig. 2.6) were described by Weidenreich just before his death in 1948 (Weidenreich 1951). Like the earlier Javanese remains, they have been classified and reclassified so many times that I will merely say that most authors regard them as large-brained (the average of five skulls is 1,151 cubic centimeters: Holloway 1980) and late members of Homo erectus (Santa Luca 1980). A few, such as Wolpoff (1980:219), regard them as early sapiens on the grounds of a broadening of the upper braincase, but I think most would agree that they represent fairly direct descendants of the Trinil hominids. More hominid fragments have apparently been found recently at Ngandong (Jacob $1978 \mathrm{~b}$ ), and another cranium of Ngandong type (perhaps slightly more archaic) has been found in a river terrace deposit of Ngandong age at Sambungmacan, also on the middle Solo River (Sartono 1979c). In 1987 a new skull, again without facial features, was found at Ngawi, also on the Solo River (Sartono 1991). All are agreed that the "Solo Man" series is post-Trinil, but beyond this are major questions that fall loosely under the headings of context, environment, and date.

Concerning the context of the Ngandong remains, it is clear from Koenigswald's accounts $(1951,1956)$ that the skulls were dispersed amongst other ani- 


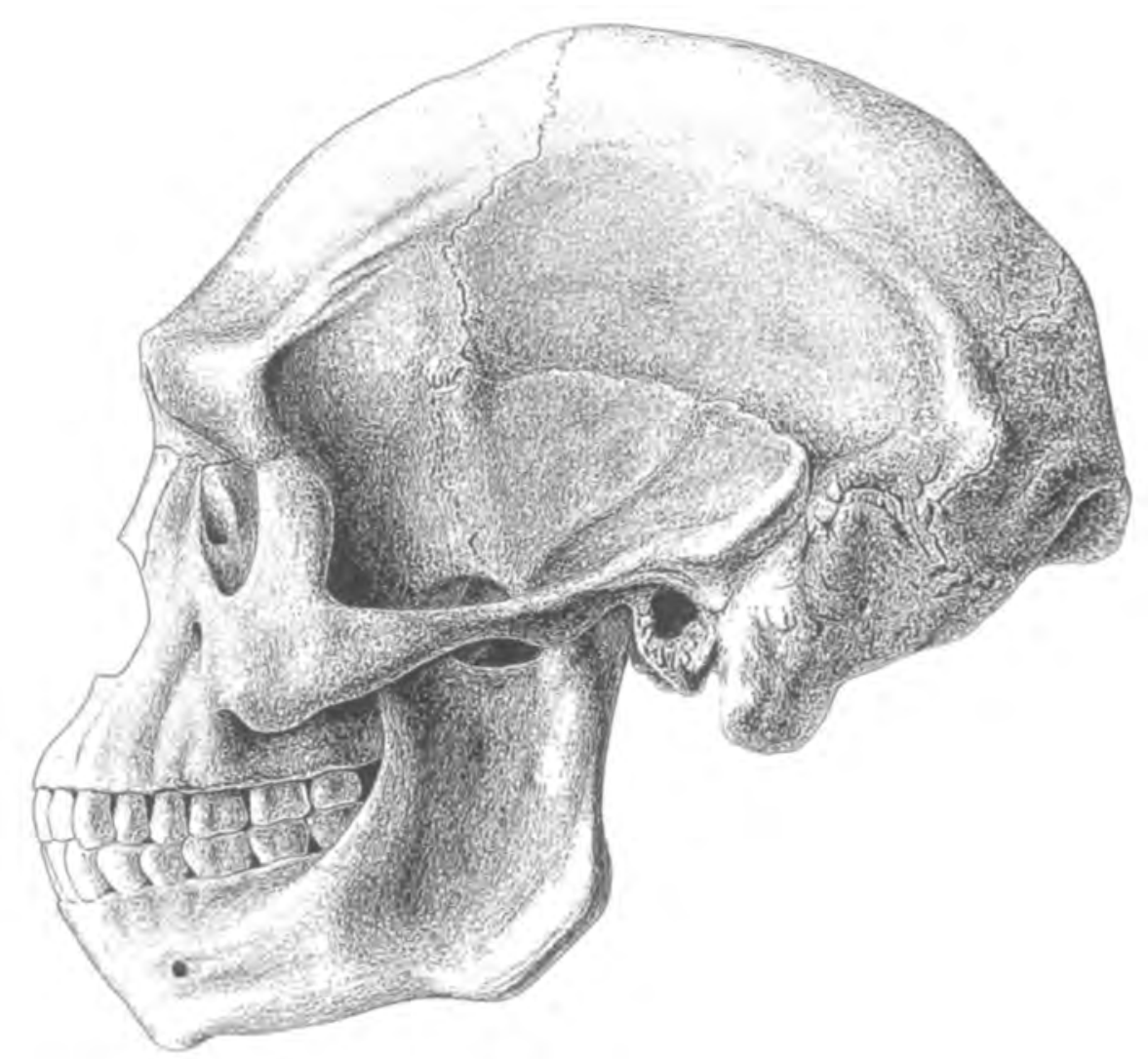

Fig. 2.6 A reconstruction of the Ngandong cranial morphology. From Weidenreich 1951. Courtesy: American Museum of Natural History.

mal bones in what must once have been a quiet bank of sand and gravel, perhaps on the inside of a river bend. Perhaps they were washed there after being cannibalized in a nearby hunting camp; the presence of articulated vertebral columns of cattle could suggest animal butchery in the vicinity. However, if the cannibalism hypothesis is wrong (and there seems to be no very positive way of knowing this), then another idea might explain their predominance. Brain (1978) has noted the predominance of Australopithecine cranial remains over other body parts in the South African site of Swartkrans and describes for comparative purposes his observations of baboon remains left on the ground after cheetah kills. In these situations crania are left complete, together with some of the long bones, but more fragile items such as the vertebral column are completely destroyed. It could be suggested from this that the Ngandong crania and tibiae are the remains of carnivore kills Java had a range of tigers, hyenas, and 
other smaller carnivores in this period). This view would tie in with the total absence of hearths and verified stone tools from the site; these would perhaps be expected if hominids were living close by.

A totally different suggestion to explain the predominance of skulls has been put forward by Santa Luca (1980), who favors the view that the animal bones form a primary deposit, but the skulls were washed out of an older deposit elsewhere and into the site, perhaps by river action. Bartstra and Basoeki (1989), on the other hand, state that the skulls are likely to be in primary position. It is clear that one could go on to ramify hypotheses about the Ngandong remains with no hope of being able to draw any useful conclusions. The answers now will only come from further scientific investigation of sites of the same period.

Concerning the environment of the Ngandong region, we are on firmer ground. The 25,000 animal bones belong to seventeen species (eighteen if one includes dubious Macaca; for lists see Koenigswald 1951; Medway 1972; Sartono 1976). Of these, twelve or thirteen are shared with the Trinil fauna, and the major post-Trinil additions appear to be more modern forms of pig and deer. The only wholly extinct genus is Stegodon. The fauna as a whole hints at a fairly open landscape and, as noted by Koenigswald, some of the buffalo had horns up to 2.25 meters wide, which would argue against the presence of dense forest. The majority of the bones were of deer and cattle (an ancestral banteng), both animals that are more numerous in open landscapes, although they do also occur in small numbers in the dense forests of Sundaland. In addition, one of Oppenoorth's assistants recovered a bone of a crane (Grus grus) from deposits considered to be of Ngandong age at a nearby location called Watualang (Wetmore 1940); this bird winters in southern China today and the bone's presence in Java could suggest a cooler climate then than now.

It is with the date of the Ngandong remains that the most difficulty occurs. The fauna is always classed loosely as late Pleistocene, and the Ngandong terrace deposits certainly postdate the Kabuh beds of central Java (Sartono 1976). However, the fauna is of little help for more precise dating because it is not known when key genera, such as Stegodon, became extinct in Java. Recently the issue of the date of the Ngandong population has been brought into sharper focus with the publication of some provisional uranium/thorium dates on animal bones from the Solo terrace deposits by Bartstra, Soegondho, and Wijk (1988). The dates range from 40,000 to 100,000 years ago with increasing depth, and as the skulls came from the lower layers of the terrace, a date closer to 100,000 years ago seems likely. If the skulls are considerably less than 100,000 years old, then because of their archaic character the undeniable conclusion must be that they are representatives of an extinct sideline of human evolution. This was the stated view of Jacob (1979) and Birdsell (1972:319) and fits current views of a recent African origin for all anatomically modern humans. But on 
this whole issue of continuity versus replacement, the battle lines are still drawn, as I will now indicate. ${ }^{1}$

\section{Broader Perspectives on Javan Homo erectus}

The big issue, of course, is that of whether or not Javan Homo erectus represents an extinct sideline of human evolution with no genetic transmission to living populations, or a partial ancestor for anatomically modern populations, especially the Australoids. Similar questions arise with Homo erectus in China. Two major paradigms currently enliven debate in biological anthropology: the "Out of Africa" (or "Garden of Eden") and the Multiregional (Fig. 2.7). Alan Thorne and Milford Wolpoff (1992) are leading spokespersons for multiregional evolution on a worldwide basis, a paradigm originally formulated by scholars such as Weidenreich and Coon. Multiregional evolution claims that anatomically mod-
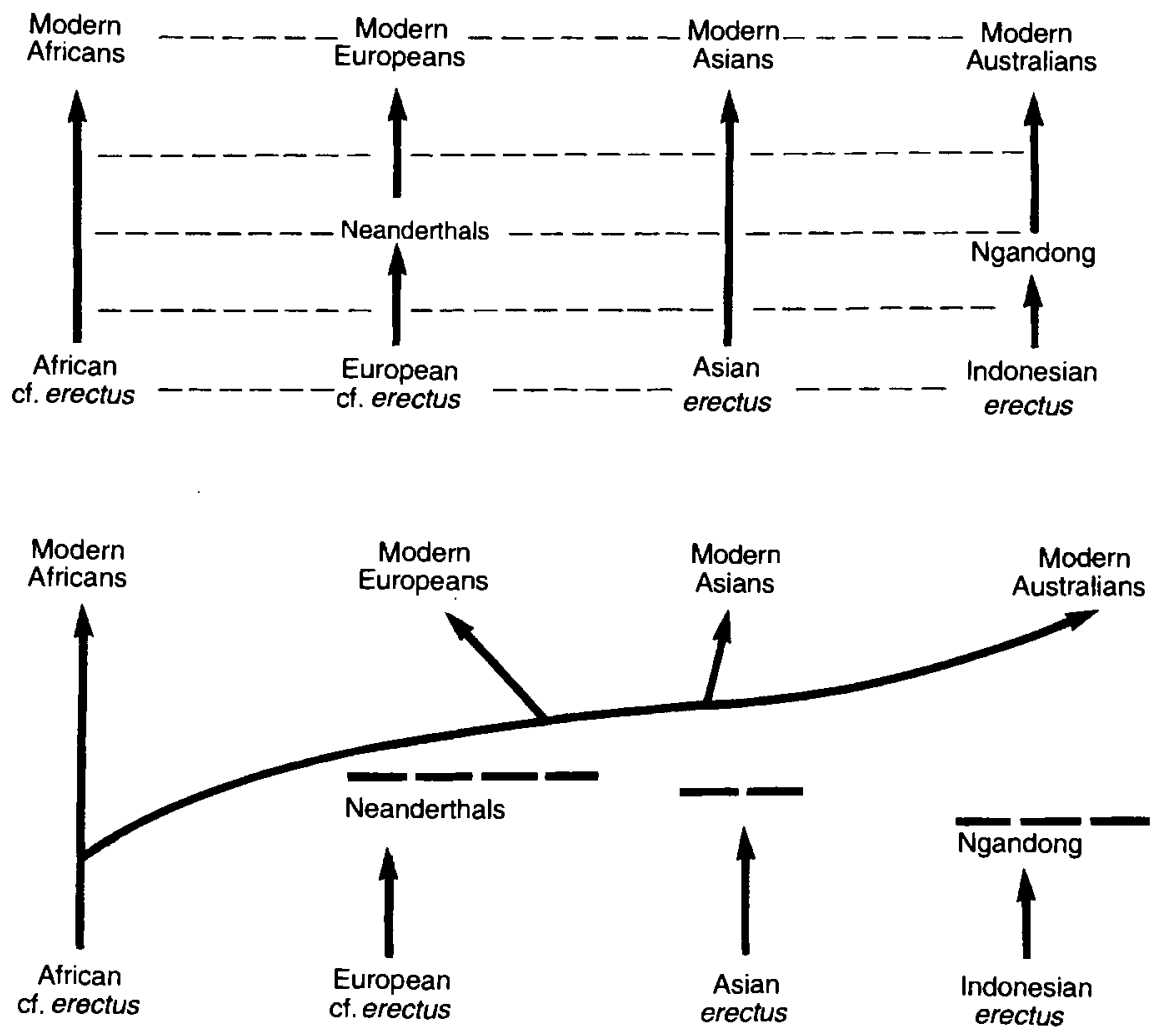

Fig. 2.7 The two major models for the evolution of modern humans. Top: the regional continuity model. Bottom: the out-of-Africa replacement model. After Stringer 1992. 
ern humans across the Old World evolved from local erectus antecedents, with extensive gene flow maintaining species integrity and spreading advantageous mutations across the human range. Thorne and Wolpoff recognize clear morphological continuity, especially in facial prognathism and posterior tooth size, between the Javanese erectus population and certain later Australian sapiens remains of the terminal Pleistocene, particularly those from Kow Swamp in Victoria (Wolpoff et al. 1984; Thorne and Wolpoff 1981, 1992; Wolpoff et al. 1994).

The majority of Chinese paleoanthropologists hold a similar view of erectus to modern continuity for China (e.g., Wu Xinzhi 1996; see also Pope 1992). The major Chinese erectus fossils include the Lantian (Gongwangling) cranium (probably as much as 1.2 million years old; Howell 1994:265), which compares well with the earlier Javan material, and the famous Zhoukoudian population dated between 580,000 and 230,000 years ago (Wu Rukang 1982). The hypothesis of multiregional evolution again claims that the Chinese remains lie morphologically in the line of descent of the modern Mongoloids without major interruption.

Since the first edition of this book was written, the hypothesis of multiregional evolution, which was widely popular in the early 1980s, has come under strong attack-not just by other paleoanthropologists, but also by geneticists. A large number of authorities now regard anatomically modern humans as having spread--perhaps from Africa-between 200,000 and 100,000 years ago, according to the genetic evidence derived from detailed studies of mitochondrial DNA (Cann et al. 1987; Wilson and Cann 1992; Rogers and Jorde 1995). These populations replaced Homo erectus everywhere in the process. The skeletal observation that Javan Homo erectus carries certain unique features (autapomorphies) that are not found in any anatomically modern humans also suggests to many paleoanthropologists that this regional clade cannot be the ancestor of the modern Australoids (Andrews 1984; Groves 1989; Rightmire 1990). Although there are suggestions that a middle-road explanation could be required, favoring radiation out of a restricted homeland combined with some degree of gene flow with preexisting populations (e.g., Bräuer 1992; Smith 1992; and Habgood 1992 for East Asia), the answers are not clear and we are still left with one of the big questions of the 1990s: Is Homo erectus an extinct species, or does it have living descendants?

In view of all this debate, one might ask if the author of this book has an opinion on the ultimate fate of Javan Homo erectus. In my opinion, it cannot be conclusively shown that the Javan hominids are totally extinct, with absolutely no transmission of genes to modern populations. There is still hope for Javan Homo erectus as a partial ancestor for the Australoids, but it is a highly qualified hope-I do not think it is a case of a 100 percent direct ancestry. Humans are migratory animals and it is unreasonable to assume that Java was so isolated 
throughout the Pleistocene that its erectus inhabitants acquired reproductive barrier mechanisms to divide them biologically from the rest of the contemporary hominid population in Eurasia. Periods of mixing with new populations from the Asian mainland must surely have occurred from time to time. Perhaps we can only hope for a really convincing resolution of these issues in future genetic research on ancient bone, especially within the mitochondrial genome. Should anyone believe that these issues are going to be easily resolved, they should look at the great range of opinion presented in recent monographs on the general topic of anatomically modern human origins (e.g., Mellars and Stringer 1989; Bräuer and Smith 1992; Nitecki and Nitecki 1994). Virtually every reputable scholar, and there are many of them, has a different opinion.

\section{HOMO ERECTUS IN SOUTHEAST ASIA: THE CULTURAL EVIDENCE}

It is most unfortunate that all the hominid remains from Java have been found in situations of presumed secondary deposition, devoid of direct cultural context. Stone tools potentially flaked by Homo erectus do occur on the island, but never with human fossils and rarely in securely dated contexts; there are no unambiguous examples of "living floors" of the kind found in Africa and western Eurasia. The possibility arises that Javanese Homo erectus, unlike contemporary Africans and Chinese, did not make tools of stone. Such a situation would be unusual to say the least, and the evidence, which at present is quite vague, deserves careful consideration.

\section{A. Squeezing Blood from Stones}

First I wish to generalize a little about stone tools and to examine the data that can, in theory, be extracted from them. As Isaac (1977a) has pointed out, stone tools can be used in many ways: as markers of human antiquity, as indices of "progress," as symptoms of cultural differentiation in time and place, and as indicators of economic organization. "However," as Isaac has said, "we need to assess the limits to the amount of blood that can realistically be squeezed from these stones" (1977a:5). In practice, many assemblages of stone tools, particularly from earlier time periods, comprise bewildering arrays of overlapping and rather amorphous forms. Prehistoric artisans were rarely turning out models for blueprints, although it has to be assumed that there is some form and patterning in all assemblages. The major difficulty is to separate the meaningful information from the background noise.

Attempts have been made to record processes of manufacture and use of stone tools among peoples who have used them up to recent times, particularly 
in Australia and New Guinea (e.g., Gould 1977, 1980; Hayden 1979). While many of these observations are particularistic, they do serve the essential function of bringing a healthy dose of reality into what was once a field of rather rambling typology. Similar reality is provided by edge-damage analyses based on controlled experiments and high-powered microscopy. The literature in both these fields is growing rapidly, and all basic field research on stone toolbearing sites now has to take account of it. However, the spin-off has so far been restricted and it works best for new material analyzed by aware researchers. New material, in terms of the Southeast Asian subject matter that I am about to consider, is decidedly scarce for any period older than late Pleistocene.

The archaeologist may present data concerning a stone tool assemblage in a manner best outlined in Isaac's useful review (1977a). Technologically defined classes such as cores, flakes, chips, and retouched and shaped forms will first be identified and quantified. At this level the researcher is merely dealing with worked stone from a technological and stylistic viewpoint. The "tool" concept comes in when considering function; this is where the edge-damage research is relevant and is the level at which tools can best be separated from waste material. The moral here is that a piece of apparent waste may turn out to be a tool when the edge is examined (assuming that one can recognize true use wear from other forms of edge damage).

After both manufacturing and edge-damage variables have been quantified, the data can be considered as a whole to suggest the most meaningful overall divisions of the material. In the case of the middle Pleistocene sites at Olorgesailie in Kenya, Isaac (1977b) divided the material into shaped tools, unshaped tools, and unutilized waste. This type of classification, which combines variables of both form and function, cannot be applied easily to any Southeast Asian assemblage because the necessary basic information has rarely been recorded, although I will present some rather generalized observations of my own in due course.

The contents of the above diversion will be familiar to readers who have some background knowledge of archaeology. In my opinion this type of research is of extreme importance and renders much earlier work on stone tools of dubious value. This is sadly so when considering East and Southeast Asia. Earlier researchers here confused form and function by using such terms as chopper, point, scraper, and hand-axe, all of which imply specific activities. In many cases these assumptions may be perfectly right, but the problem is that it is generally impossible to verify whether they are right or wrong. The relevant assemblages were often superficially reported and are now spread in museums, some in quite inaccessible parts of the world. Available illustrations are often of poor quality and selective. 
By now, the reader may grasp that I am tending strongly toward a negative view of the value of stone tools in Pleistocene research in East and Southeast Asia. Perhaps I can reinforce the negativism by pointing out that the above paragraphs have only discussed the stone tool assemblage per se. When considering problems of date and context, the situation becomes even worse; we quickly find ourselves in situations where we are trying to compare one researcher's chopper with the scraper of another, knowing next to nothing of what the tool looks like, how old it is, or the context in which it was found. My own research has told me very clearly that what one researcher calls a middle Pleistocene chopper could well be a discarded waste core less than 10,000 years old.

In any case, the problems exist (e.g., Hutterer 1977), and it is pointless to lay blame. In this review, I am forced to use much of the terminology and the data as they have been published, although I will use my own experience of some of the material to follow a simple line where complexity is not warranted.

\section{B. Pleistocene Stone Tool Industries of East Asia}

The most important Indonesian industries that have been claimed as the handiwork of Homo erectus come from the Ngebung region of Sangiran, from Ngandong, and from riverine locations in south-central Java (the Pacitanian industry). Potential but problematic outliers occur in other parts of Sundaland and Peninsular Malaysia, in Sulawesi, in Flores and Timor, and in the Cagayan Valley of northern Luzon in the Philippines. These industries will be described in this chapter. Late Pleistocene industries of more specialized type that postdate 40,000 years ago are discussed separately in Chapter 6.

The Javanese Pacitanian industry belongs to a widespread group of "chopper/chopping-tool industries" that occur widely in Southeast Asia, China, and India and that have often been contrasted with the "hand-axe" (Acheulian) industries produced in western Eurasia and Africa during the time span of Homo erectus. This distinction has some statistical validity but it is not absolute; the Pacitanian does have hand-axe forms, as indeed do some Chinese and Korean industries (Yi and Clark 1983).

The South and East Asian industries were first described and compared in a comprehensive way by Movius, in a series of long papers (e.g. 1944, 1948, 1955) that have had a rather fundamental influence on all later work. Movius described a number of tool types made on pebbles, tabular chunks, or flakes. These he divided into bifacial and unifacial types: bifacial chopping tools and hand-axes, and unifacial hand-adzes, proto-hand-axes, and choppers and/or scrapers (Fig. 2.8). These definitions were rather intuitive, as Movius was aware, and with the passage of time they have proved too ambiguous for useful com- 


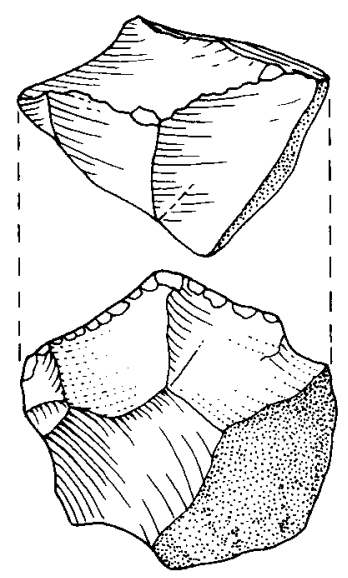

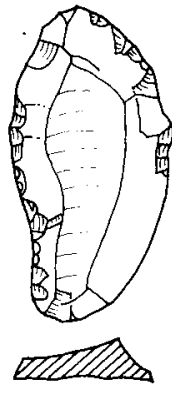

FLAKE

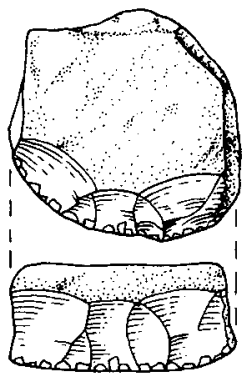

HAND-ADZE

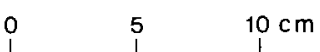

CHOPPING-TOOL
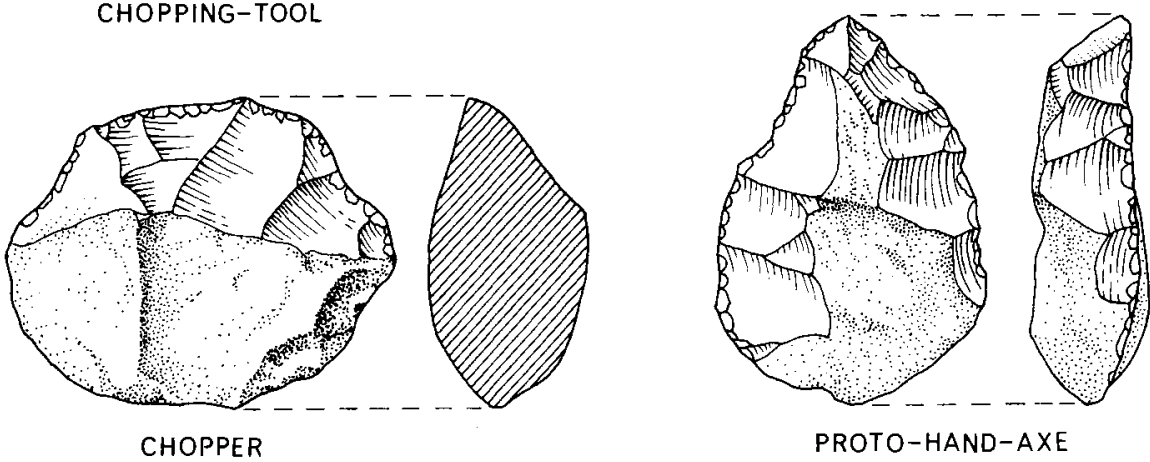

Fig. 2.8 Some major tool forms of the Southeast Asian "chopper/chopping-tool" industries. After Movius 1944; Glover and Glover 1970.

parative purposes. Furthermore, it is clear that many authors have not used these terms as Movius intended, although his definitions were semantically precise (1955:261-262).

My own observations on these classificatory problems stem from my research on later Pleistocene and early Holocene industries, including the Hoabinhian of Peninsular Malaysia and contemporary industries in Borneo, Sulawesi, and the Moluccas. There seems little doubt that these all represent the handiwork of anatomically modern humans of the past 40,000 years. I have elsewhere (Bellwood 1978) referred to all of these industries, which are more fully described in Chapter 6, as "pebble and flake industries." In some remote parts of Southeast Asia they have continued in production into recent historical times, although new technologies did appear in certain regions during the late Pleistocene and Holocene.

Despite local idiosyncrasies that probably depend as much on raw material 
as on varying skills, the Pleistocene pebble and flake industries of Southeast Asia and their Holocene survivals all share in common the production of:

a. Fairly heavy tools made by flaking the edges of riverine pebbles, large flakes, or quarried nuclei-these are normally called "pebble tools" in popular parlance and include most of Movius' categories. Variables such as edge length, edge position, edge angle, extent and position of surface flaking, and unmodified cortex all intergrade. The distinction between unifacial and bifacial working can often be used with profit, especially to isolate an interesting category of bifacial "hand-axes" in the Javanese Pacitanian (Fig. 2.9 a,b). Another useful distinction can perhaps be made with edge angles; the pebble tool forms that Movius defined as choppers, chopping tools and hand-axes tend to have fairly low edge angles, but his hand-adzes and scrapers seem to belong to a differentiable category of steep-edged, flat-based, and thick unifacial tools, usually with edge angles over $70^{\circ}$ (see Fig. 2.8, Fig. 2.9c).

b. Smaller tools made on true flakes struck from cores (Fig. 2.9 e,f), or sometimes on small chunks of stone. These rarely have specific edge or shape characteristics and if they do not reveal clear patterns of retouch, they can only be separated from purely waste material by edge inspection.

c. Manufacturing waste-items not in categories a and b because they show no signs of post-manufacture retouch or usage. They include unutilized flakes, chips (flakelike pieces lacking bulbs of percussion, caused either by core smashing or flake breakage), shattered cores and chunks, and sometimes true discarded cores. The latter are often hard to recognize as true debitage because the removal of flakes causes edge shattering, which can easily be confused with use damage. The term "horsehoof core" (Fig. 2.9d) is used in Australian archaeology to describe one quite characteristic single-platform variant of this class, but there is often confusion as to whether these are to be considered as steep-edged tools or waste items; each example must be examined individually. Other core shapes are less distinctive, but spherical and conical trends in shape can often be observed.

The selection process in these industries is clearly for tool size and edge morphology; overall shape appears to be rather incidental, although shape, edge, and size often correlate to some degree. Common sense would suggest that there are at least two major tool classes: heavy tools with axelike or chopping functions, and tools with scraping and cutting functions. These functional categories do not correspond directly with categories a and $b$ above, and any given stone tool could have served several different functions during its life. 

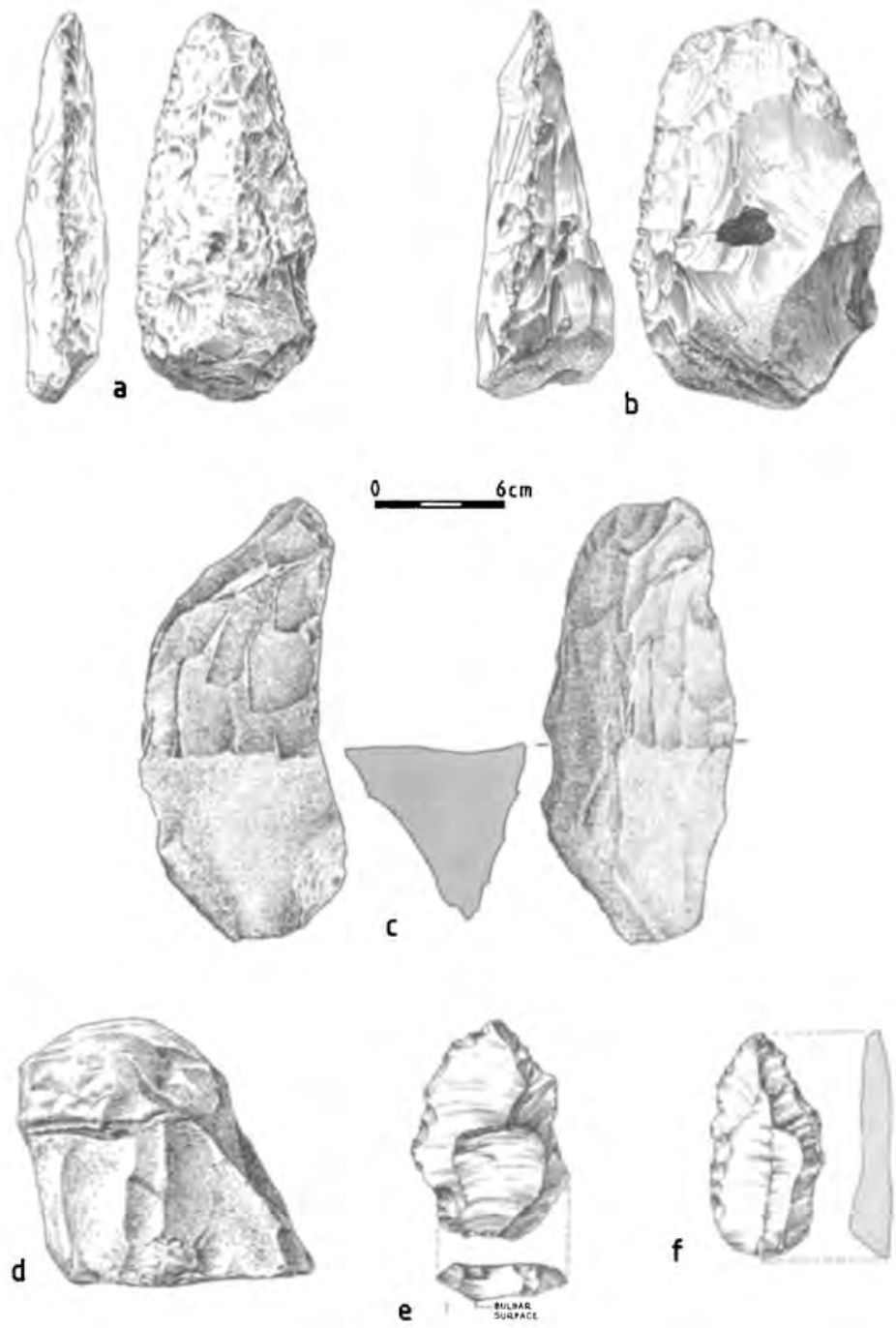

Fig. 2.9 Pacitanian tool forms from Java: (a,b) hand axes; (c) steep-edged and flat-based tool; (d) "horsehoof" core; $(e, f)$ flakes with signs of utilization. From Bartstra 1976; Movius 1944. Courtesy: G-J. Bartstra (a-d); Senckenberg Museum (e-f).

\section{The Pleistocene Industries of China}

Before turning to an examination of the Indo-Malaysian pebble and flake assemblages, I will look at the only area of East Asia where the cultural activities of Homo erectus are clearly documented. The Chinese material is reviewed in a number of selectively illustrated sources (Chang 1986; Aigner 1978a, 1978b, 
1981; Atlas 1980; Jia 1980; Yi and Clark 1983; Chen and Olsen 1990; Olsen and Miller-Antonio 1992), and when these sources are combined it is clear that a lot of potentially detailed evidence is available.

Early Pleistocene sites in China (Fig. 2.10) are surrounded by considerable uncertainty with respect to date and association. The site of Yuanmou, which has produced some rather indeterminate stone tools and a suggestion for the use of fire, is perhaps of early Pleistocene antiquity. Another assemblage claimed to be of early Pleistocene age, again with traces of fire (here in the form of burnt bone), comes from Gehe locality 6053 (Xihoudu) in Shanxi, but Aigner (1978b: 194; see also Woo 1980:196) disputes the presence of true stone tools. Quite clearly, neither of these sites can be considered as unequivocal evidence for the presence of stone tools during the early Pleistocene in China. However, Howell (1994:265-266) accepts the stone tool assemblages from Lantian (Gongwangling) and the Nihewan Basin in northern China as of likely early Pleistocene antiquity. Several other sites, possibly early Pleistocene, are listed by Olsen and Miller-Antonio (1992; see also Huang et al. 1995).

Better material is reported from the middle Pleistocene of central China. Assemblages that may run from the middle into the late Pleistocene have been recovered from Gehe localities 6054 and 6055 and from Dingcun in Shanxi (Fig. 2.10). The major sites of this period occur in the caves at Zhoukoudian near Beijing, where one of the world's most famous populations of Homo erectus has been found in claimed association with evidence for the use of fire, although in this case the assertion has been disputed (Binford and Ho 1985; see Jia 1989 in reply), and the hunting of large mammals such as Pseudaxis, Megaloceros (both forms of deer), and rhinoceros. Artifacts include a range of possible bone and antler tools (Aigner 1978b:182) and by far the best documented sample of middle Pleistocene stone tools from any site in eastern Asia (Fig. 2.11). The industry appears to have a predominance of small retouched flake tools, together with larger pebble and core tools (including some bifacially flaked forms) and flaked stone balls ("bolas stones"; Yi and Clark 1983). Dates for Zhoukoudian range from 230,000 to 580,000 years BP by the uranium/thorium, fission-track, and thermoluminescence methods ( $\mathrm{Wu} \mathrm{Xinzhi}$ 1996).

In southern China the evidence is not as prolific as that from the Yellow River region farther north, but an excellent middle Pleistocene assemblage of retouched flakes and core tools has been excavated at Guanyindong cave in Guizhou. They occur with animal bones from a fauna that includes Stegodon and Ailuropoda, clearly related to the Jetis and Trinil faunas of Java (Olsen and Miller-Antonio 1992:141-143; Howell 1994:269-270). Aigner (1978b:221) has pointed out that the southern industries mostly utilize flakes and that the heavy pebble and core elements tend to be lacking. 


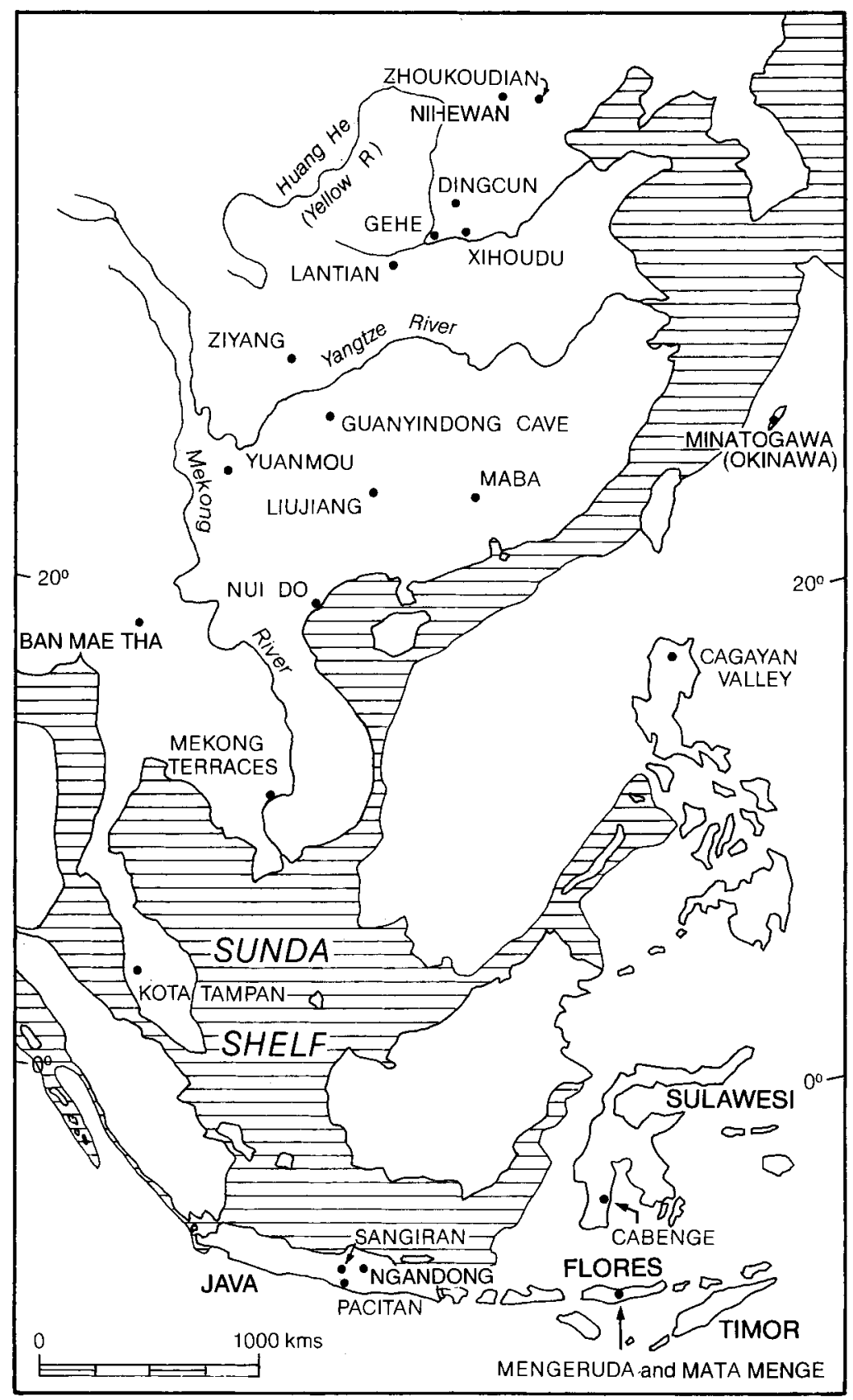

Fig. 2.10 Major fossil and archaeological sites of Pleistocene date in China and Southeast Asia. 

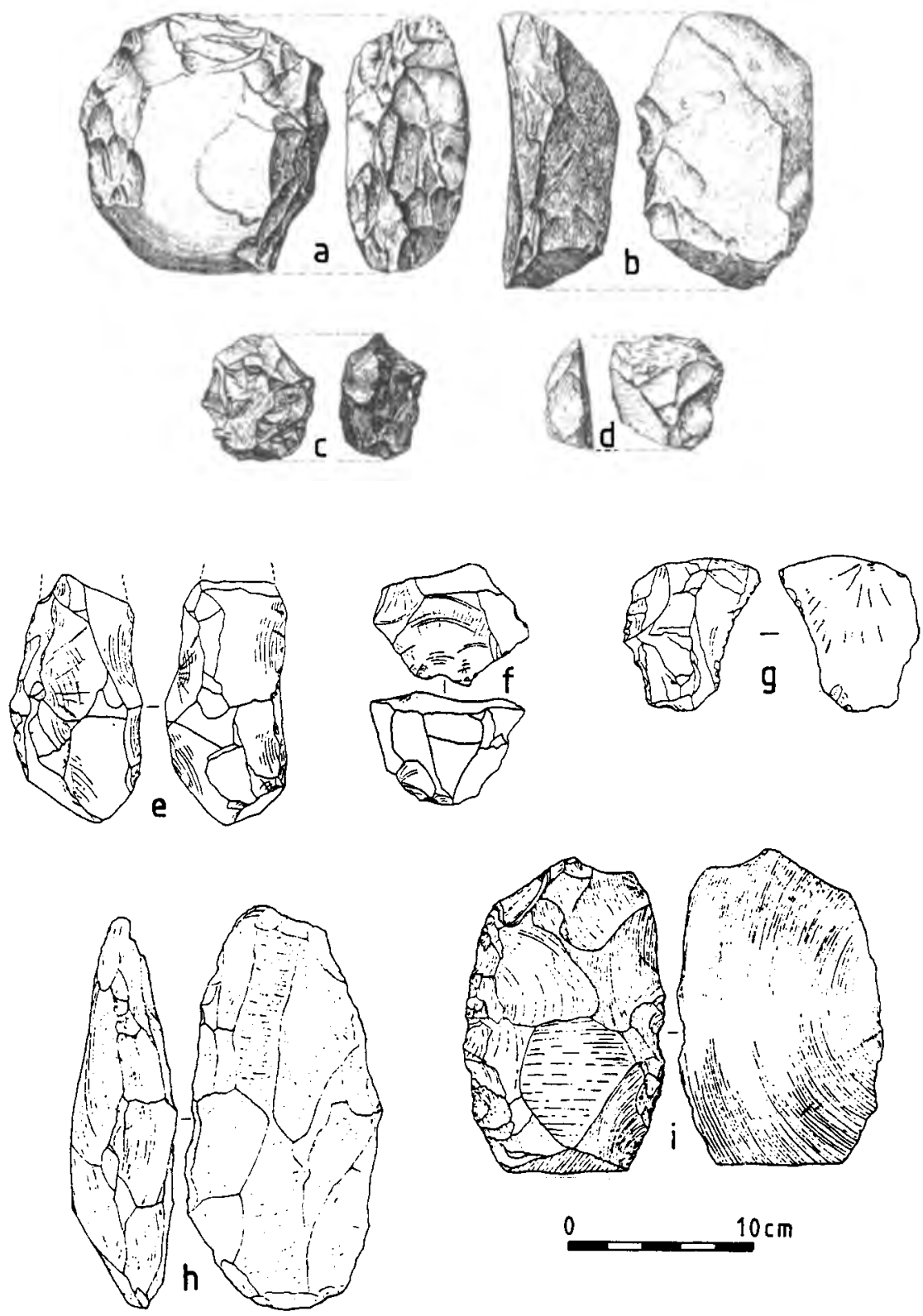

Fig. 2.11 Stone tools from Zhoukoudian (a-d), Gehe (e-g), and Dingcun (h, i): (a) bifacial pebble tool; (b, d) flat-based, steep-edged unifacial tools; (c) core; (e) bifacial core tool; (f) single-platform core; (g) utilized flake; (h) "hand axe"; (i) large retouched flake. From Movius 1944; Yi and Clark 1983. Courtesy: Institute of Vertebrate Paleoanthropology (a-d); University of Chicago Press (e-i). 


\section{Stone Industries Possibly Made by Homo erectus in the Indo-Malaysian}

\section{Archipelago}

(for recent reviews see Allen 1991a; Reynolds 1993)

\section{The Pacitanian}

Tools of this industry (see Fig. 2.9) were first discovered by Koenigswald and Tweedie in 1935 in the bed of the Baksoko River near Pacitan, in south-central Java. Further investigations in 1938 by Terra, Chardin, and Movius led to characterization of the finds as part of the chopper/ chopping-tool complex of Southeast Asia and to an assumption of a middle or late Pleistocene date. Further work was subsequently carried out by Heekeren (1972), who reclassified the material, reported on finds from adjacent valleys, and suggested that the tools were eroding from four implementiferous terraces in the Sunglon and Baksoko Valleys, with the oldest Baksoko material coming from about 15 to 20 meters above the streambed. However, the majority of the tools have been found in secondary positions in the modern streambeds, where they have become rolled and mixed with artifacts of apparent Neolithic provenance.

As described, the industry is made on silicified tuff (the best material), silicified limestone, and fossil wood. It comprises a range of category-a tools (see Section IIIB, above), including bifacial hand-axes and high-backed, steep-angled "scrapers," together with numerous flake tools and waste flakes, some of very large size (Mulvaney 1970). It is not essentially different from the late Pleistocene industries of Borneo, which I will be describing later, but there does appear to be a tendency toward large size. This may be due to collection bias; none of the finds represent contemporary and complete assemblages and it is not clear what the total size range of the material was at any one time.

The most recent work on the Pacitanian has been carried out by Bartstra (1976, 1978a, 1978b; Bartstra and Basoeki 1989). He points out, after exhaustive geomorphological reconnaissance, that alluvial gravels lacking fossils extend up the valley sides to heights of up to 28 meters above the streambeds, but due to slumping and colluvial movement it is not possible to correlate terrace remnants or to recognize individual terraces. Tools are occasionally found in situ up to high gravel levels, and earliest dates according to Bartstra could fall around the middle to late Pleistocene boundary. Bartstra's basic view is that the material could cover quite a long time span indeed, but it is most probably associated with Homo sapiens rather than $H$. erectus.

\section{The Sangiran Flake industry}

In 1934, von Koenigswald found some small rolled and patinated flakes of jasper and chalcedony in the Sangiran dome and started a controversy that still continues today. His claim (stated in Koenigswald and Ghosh 1973) was always 
that the tools originally came from the Notopuro beds-a sequence of volcanic breccias and tuffs that overlie and rest unconformably on the Kabuh layers at Sangiran. These volcanic layers are about 20 meters thick and predate the Ngandong terraces and hominids. They are undoubtedly middle Pleistocene.

The main problem, as Koenigswald admitted, is that the tools were all found on the surface or in superficial layers and the assumption that they originated in the Notopuro beds remains impossible to prove. There has been much disagreement over this in the past. Heekeren (1972:49) claimed that the tools originated from the basal Notopuro, but Bartstra (1974, 1978b; Bartstra and Basoeki 1989) believes the tools are all from recent colluvial and alluvial deposits and cannot be shown to be from the Notopuro beds at all. He suggests an early late Pleistocene antiquity. Furthermore, Bartstra points out that many of the "tools" are no more than nodules of jasper and chalcedony that occur naturally in the area, although there seems little doubt that the rudiments of a genuine assemblage do exist amongst the collections.

The Sangiran industry from these surface sites consists of tools that are very small, well below the average size of the Pacitanian. They comprise small flakes, some with apparent retouch, but little else-the absence of cores and large pebble tools is most unusual. But there are some definite flake tools, including a class of "small bevel-edged flakes" described by Orchiston (1978).

Recently the Sangiran Flake Industry has been brought back into the limelight by the excavation of a few presumed stone artifacts related to this industry stratified in Kabuh deposits between 4.5 and 9 meters above the Grenzbank at Ngebung, in the northwestern part of the Sangiran dome (Fig. 1.10). These deposits are thus of early middle Pleistocene date. The stone items include two small cores, fifteen flakes, and two blades (Sémah et al. 1992; Simanjuntak and Sémah 1996; see also Soejono 1982a for earlier finds at Ngebung), and there are also references to bolas stones and a quartz hammer stone. If the claims are sustained, then the question, "Did Javan Homo erectus make stone tools?" might, after all, be answerable in the affirmative. The evidence is small, but I see no reason to doubt its authenticity.

\section{The Ngandong Industry}

Material associated with the Ngandong fossils, excavated between 1931 and 1933, is sparse and problematical. According to Koenigswald (1951:216), "a few small stone scrapers and some triangular chalcedony flakes were observed, but they have disappeared from our collection." Later commentators, such as Sartono (1976), Bartstra et al. (1976), and Jacob (1978b), seem unwilling to accept that any tools were found in direct association with the fossils at all.

Nevertheless, one of the original investigators, Oppenoorth (1936; see also Stein Callenfels 1936c), was considerably more enthusiastic. He reported workt 1 
bone and antler from the general vicinity of the skulls, together with stone balls of andesite apparently similar to those mentioned above from Zhoukoudian. He also found a spine of a marine stingray close to skull VI. However, according to a geological section presented by Sartono (1976, after Ter Haar), all these items were found in superficial layers of the terrace-above the skulls-except perhaps for the bone tools, which have always remained rather dubious. Oppenoorth did find other tools in terrace deposits in other parts of the Ngandong region: more stone balls at Watualang, a beautiful biserial bone harpoon from Sidorejo, and some chalcedony flakes from the surface at Ngawi (are these the ones mentioned by Koenigswald above?). But none of these items can really be claimed as the handiwork of Ngandong Homo erectus; all may be much more recent, particularly the harpoon (which was originally compared with Magdalenian harpoons from Europe).

This rather sad and confusing story could have a happy ending, given the claim by Jacob et al. (1978) that two unrolled tools of basaltic andesite-a wellmade unifacial pebble "chopper" and a retouched flake--have been found in a late middle or late Pleistocene gravel deposit at Sambungmacan approximately contemporary with the layer that yielded the skull of a late specimen of Homo erectus. The tools appear to be genuine and it may not be unreasonable to claim that the late erectus population of Java also made stone tools, like its possible forebears at Ngebung.

\section{Cabenge, Southwestern Sulawesi}

In Chapter 1, Section IVA, I referred to an important late Pliocene faunaI collection made in the region of Cabenge in the Walanae Valley. From 1947 onward, stone tools have been found in apparent association with these bones, and several authors (e.g. Heekeren 1972:69) have considered them contemporary. Recent geological work has disproved this, however; the tools come from coarse river sediments of presumed but indeterminate Pleistocene age (Sartono 1979a), and the animal bones that occur in these deposits have probably been washed in from older formations (Bartstra 1978c).

Nevertheless, Bartstra (1978c) does note that the patinated tools found in the highest terrace gravels are rather different from the tools of Toalian type found closer to the river; the latter are of undoubted Holocene date (see Chapter 6 , Section IIIB), and the former may be assumed to be older. In a recent paper, Bartstra et al. (1991-1992) suggest that the tools are probably the work of Homo sapiens rather than Homo erectus. Heekeren describes the tools as small thick flakes struck from irregular cores and refers to a range of scrapers and chopping tools. Soejono (1982a) also mentions massive core tools, hand-axes and horsehoof cores. 


\section{Flores, Timor, and Luzon}

In Chapter 1, Section IVA, I discussed the significance of the occurrence of two species of Stegodon, one of normal size and one dwarfed, on a number of eastern Indonesian islands including Flores and Timor. Archaeological interest in this situation was aroused in 1970 when Maringer and Verhoeven (1970a and b) published their results of investigations on Flores. In one region called Mengeruda they claimed to have found stone tools in association with Stegodon bones in scattered exposures in an area about 3 kilometers long. They described a variety of pebble tools, retouched flakes, and one small bifacial hand-axe. Generalized affinities were drawn with the Pacitanian, Sangiran, and Cabenge industries and the suggestion was made that contemporaries of the Ngandong hominids may have been able to venture along the Lesser Sunda chain.

In 1991-1992 an Indonesian-Dutch expedition reexamined the sites visited by Maringer and Verhoeven on Flores and excavated more stone tools from a location near Mata Menge (van den Bergh et al. 1996a). The tools, flakes of chert and basalt, are few but are claimed to be of definite human handiwork and to come from just above a paleomagnetic reversal recorded in a paleosol. This reversal is believed to represent the Matayama-Brunhes boundary at ca. 700,000 years ago. Bones of the large Stegodon trigonocephalus and a giant rat occur in the same layer. Nearby, at Tangi Talo, an older deposit has yielded bones of a pygmy Stegodon, a large tortoise, and Varanus komodoensis-the Komodo dragon-but no stone tools. These finds, newly announced, are exciting indeed and suggest a possible presence of middle Pleistocene Homo erectus within "striking distance" of the Australian continent.

For the other Lesser Sunda Islands and Moluccas there are still no such strong claims for middle Pleistocene tools. Surface finds of "Lower Palaeolithic" type have been reported from many islands, from Lombok in the west (Soejono 1987) through Sumbawa (Soejono 1982a) to Timor in the east (Glover and Glover 1970; Maringer and Verschuuren 1981; Aziz 1981), but stratigraphic contexts are still lacking. The oldest Moluccan industries, to be detailed further in Chapter 6, are contemporary with anatomically modern humans. Similar problems apply to a claimed middle Pleistocene industry from the Cagayan Valley in northern Luzon, Philippines. A genuinely early or middle Pleistocene fauna occurs in deposits exposed in this valley, but as Wasson and Cochrane (1979) note, the stone tools once claimed to be in association with the fauna are probably not; they note also that the industry is quite closely paralleled in terminal Pleistocene and Holocene cave deposits in the area. Pebble tools, horsehoof cores, and retouched flakes are the major forms represented. 


\section{Peninsular Malaysia}

I will close this listing of the Indo-Malaysian stone industries having claimed associations with Homo erectus by referring briefly to the "Tampanian" tools recovered from gravels in the Perak Valley of Peninsular Malaysia. In the principal report published by Walker and Sieveking (1962), the tools-which have certain affinities with the Pacitanian - were linked to high sea level alluvial terrace deposits of early or middle Pleistocene ("First Interglacial") date. However, a number of widely held views on the Tampanian were rudely shaken in 1975, when Verstappen (1975:26-27) pointed out that the "terraces" were better regarded as wash and colluvial deposits formed in a tree-savanna landscape during drier glacial periods. In the same year, Harrisson (1975a) suggested younger affiliations for the tools by attempting to associate them with an overlying late Pleistocene ash shower from the Toba eruption in Sumatra.

Recent work at Kota Tampan by Zuraina Majid (1990) supports Harrisson's view and suggests that the site served as a flaking floor. Majid has related the site to a date of ca. 30,000 years ago based on earlier assessments of the age of the Toba eruption, but new dates for this event suggest an age of perhaps 75,000 years (Chesner et al. 1991). Whatever the final decision on age, the tools appear to be the handiwork of anatomically modern humans; they will be discussed in more detail in Chapter 6 .

\section{SOME CONCLUSIONS ON "EARLY" INDUSTRIES}

When the first edition of this book was published in 1985, none of the IndoMalaysian industries described occurred in dated or even well-stratified contexts. The situation remains similar today, but in my view the newly excavated discoveries at Ngebung in Java and Mata Menge in Flores hold great promise. They may indeed be the handiwork of middle Pleistocene Homo erectus if all claims for these sites are upheld. Having Javan erectus as a toolmaker, however, does not necessarily save this species from the fate of extinction, since other even more evolved hominids such as the Neanderthals also made stone tools and are considered extinct by many modern authorities. Because the sites are few and the gaps in chronology are long, we still have much to learn. 


\section{Indo-Malaysians of the \\ Last 40,000 Years}

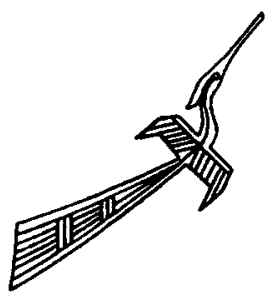

The raw data required for any discussion of origins, distributions, and differentiation amongst recent Indo-Malaysian populations are drawn from two very different and specialized disciplines. These are population genetics, which studies the distributions of the factors that determine heredity, and biological anthropology, which from the point of view of this book is concerned with the analysis of living and skeletal phenotypes (in the latter case, the discipline is better referred to as paleoanthropology). As with the debate over Homo erectus, so too the debate over modern human origins and differentiations is currently quite heated. Answers are not simple, but because modern humans still exist, the answers can perhaps be a little less elusive than those for hominids of half a million years ago.

The present populations of the Indo-Malaysian region are, of course, varied. The variation is expressed as in all human populations through a hierarchy of levels: from individuals through ethnolinguistic groups and geographical zones, eventually to the level of the major races of mankind. The concept of race is clearly quite important when considering prehistoric relationships, but it is a concept that tends to evoke a good deal of argument (e.g., Littlefield et al. 1982), as well as emotion and concern over the dangers of political misuse. From a purely scientific viewpoint, one extreme view states that there are no races-only clines-while at an opposite pole are the "pure race" theories that prevailed in earlier physical anthropology. For an intelligible narrative of prehistory, some concept of race is necessary; it would be unreasonable to claim that there is no geographical patterning to human variation at all. This view would force us to consider every small group independently in terms of its place in a whole-world range of variation-clearly an impossible and unnecessary task. 
Definitions of race are numerous. I will use one given by Buettner-Janusch (1966:184):

A race of Homo sapiens is a Mendelian population, a reproductive community of individuals sharing a common gene pool. All members of our species belong to one Mendelian population and its name is Homo sapiens. This large specieswide Mendelian population may be divided into smaller Mendelian populations, for all practical purposes an indefinitely large number of them.

Buettner-Janusch's last sentence makes it clear that racial classifications are by nature both hierarchical and diffuse; they are ideal subjects for ramified subdivision. If entering the hierarchy at the top, it is traditional to claim that the populations of the Indo-Malaysian Archipelago belong to two of the major geographical races of mankind: the Australo-Melanesians (or Australoids) and the Mongoloids (or, more specifically, Southern Mongoloids). This gross splitting is a heuristic device, for human populations at all times must have shown intergrading or clinal characteristics, just as they do today. But the differentiation is not made lightly; there are strong biological and historical grounds for suggesting that one of these groups (the Southern Mongoloid) has expanded very widely through a prior Australo-Melanesian continuum within the past 10,000 years. Both genetic and skeletal data support this view.

From this perspective the present racial geography of the Indo-Malaysian region is not due entirely to local evolution-without population movementsince the time when the first anatomically modern humans entered the region. It is true that all populations are subject to natural selection, and where breeding groups are small they will be especially subjected to genetic drift for proportions of specific genetic polymorphisms. These types of in situ differentiation amongst relatively or wholly isolated populations have clearly been fundamental to race formation in Homo sapiens, but they are insufficient to explain the large-scale geographical distributions of the races of mankind prior to AD 1500 . Some of these, such as the Southern Mongoloids of the Indo-Malaysian region, have clearly expanded on a very large scale to absorb, replace, or surround preexisting populations.

In already settled areas, major expansions such as this could presumably only have occurred when populations with considerable numerical/demographic and technological advantages impinged on less resistant groups. Prior to the development of agriculture and the attendant and rapid growth in population size and technology, it is unlikely that opportunities for such large-scale expansion into regions already settled would have presented themselves often (Brues 1977:251; Krantz 1980; Bellwood 1994). This, however, is a frequency-dependent and scale-dependent statement, for small groups of hunters and gatherers certainly did move quite large distances during the span of human evolution, 
even into regions previously but lightly settled. But they probably colonized through such previously settled regions only rarely.

If my views on these matters are generally correct, then the major Old World races of mankind, which occur in often widespread and scattered distributions today, would have been more integrated with respect to geographical distribution in the Pleistocene past. In short, the Pleistocene should have witnessed fewer phenotypic boundaries and a more continuous network of gradual clines, broken only by major environmental barriers to human interaction. Organized agriculturalists can quickly dominate sparse groups of tropical forest hunters, but those sparse hunters would be much less likely to have such advantages with respect to other hunters with similar population densities and technology. During the Pleistocene, gentle clines of variation can be expected, while the present pattern-particularly in Southeast Asia-often reveals quite sharp interfaces.

\section{THE MODERN POPULATIONS OF THE INDO-MALAYSIAN REGION}

The vast majority of the inhabitants of this region today are of Southern Mongoloid phenotype. The rising population of over 250 million people is exemplified by such important groups as the Malays, Javanese, Balinese, and Filipinos. It is obvious that the whole archipelago is now a part of the Mongoloid world of East Asia, which also extends into many of the islands of the Pacific and right through aboriginal America. But there are other populations that, although small in number, are of great significance from a historical viewpoint. These comprise the Negritos of Malaysia and the Philippines, and the Melanesians, who extend westward from their own core region around New Guinea into the eastern islands of Indonesia. Following Coon (1966), I define both these populations as being of basically Australoid (perhaps more meaningfully termed Australo-Melanesian) inheritance.

\section{A. The Negritos}

The short-statured Negrito populations (Plate 2) of the region comprise the Peninsular Malaysian Negritos (formerly called "Semang"), who inhabit the mountainous region from Pahang north to the Thai border, and the Negritos of the Philippines who inhabit both coastal and inland localities in pockets of Luzon, northern Palawan, Panay, Negros, and Mindanao (for distributions of the Philippine groups see Chapter 4, Fig. 4.2). The Andamanese, with whom I will not specifically be concerned, are also normally classified with the Negrito group.

The Negritos of Malaysia and the Philippines are traditionally forest and coastline hunters and gatherers and they differ quite sharply from their Mongo- 
loid neighbors, despite occasional intermarriage and the presence of a phenotypically intermediate population in the Senoi of central Peninsular Malaysia (Plate 3). Stature is usually quite small, averaging around 145 to 155 centimeters for males, but there is some overlap here with surrounding populations, many of whom are also of short stature (Polunin 1953; Rambo 1988). In appearance these people are dark, with tightly curled hair (sometimes red or brown), and in facial features they resemble small and gracile Melanesians. The simplest conclusion concerning them is that they are the small-statured representatives of a once widespread Australo-Melanesian population that comprises the extremely varied peoples of Australia and Melanesia today, but that has been absorbed almost entirely into a much more numerous Mongoloid population in Southeast Asia.

Simple conclusions, however, are not always uncontested conclusions. Omoto (1987), on the basis of a multivariate analysis of gene frequencies, has suggested that Philippine Negritos show a relationship to other Mongoloid Filipinos that is not entirely the result of recent intermarriage. Harihara et al. (1992) also point out that Philippine Negritos (Agta) have a high frequency of a 9-base-pair deletion in their mitochondrial DNA genotype, a deletion that is also common in Asian Mongoloid populations and Polynesians. ${ }^{1}$ Hanihara (1990) also presents data from dental measurements that appear to relate the Negritos to the prehistoric Jomonese populations of Japan, together with the ethnographic Ainu of Hokkaido. Teeth, however, are one thing, skulls another. Brace et al. (1991) offer no doubt from craniofacial evidence that the Southeast Asian Negritos are most closely related to Australians and Melanesians (see also Bellwood 1993 for further opinions on Negrito ancestry).

Historical scenarios that might reduce these apparent conflicts will necessarily be reticulate in nature. Both Negritos and Southern Mongoloids must share a degree of common ancestry, as do all modern humans, but with a superimposed history of long subsequent separation followed by recent (about the last 4,000 years) occasional hybridization through intermarriage, especially in the Philippines. The phenotypic differences between Negritos and Southern Mongoloids are clearly sufficient to indicate that both cannot simply be seen as variants within a single intergrading population. Similarly, the Southeast Asian Negritos have also been long separate, perhaps for over 40,000 years, from their distant cousins in Australia and Melanesia.

This still leaves the question of short stature to be explained. It should be noted here that there is no skeletal evidence that would give any support to the idea that the peoples of the Indo-Malaysian region were ever all short-statured Negritos. That they were once all of generalized Australo-Melanesian affinity is, however, a much more supportable proposition. The short stature may be simply a localized independent development. For instance, there are similar 
short peoples in the New Guinea Highlands not otherwise distinct in appearance from their neighbors, who are Melanesians of normal stature. In this case the explanation is probably natural selection within a small part of the range of a generally taller population; small stature may have great adaptive value in mountainous tropical forest environments with limited nutritional resources, where a high ratio of strength to body weight is advantageous (Gajdusek 1970; Howells 1973b:173-174). According to Cavalli-Sforza (1986), small body size in African Pygmies might also help to decrease internal body heat during exercise in a hot, humid climate, thus reducing sweating. Gates (1961) suggested that the New Guinea Pygmies owe their short stature to a local and recent mutation at perhaps a single genetic locus. This view has not been acceptable to many physical anthropologists (e.g., Birdsell 1967:108), but studies of African Pygmies have shown that they are markedly deficient in the production of the insulinlike growth factor IGF-I (Merimee et al. 1981). It is not clear what environmental conditions have promoted this deficiency, but there has presumably been strong selective pressure to favor its survival, and a tendency to short stature in the interiors of other large islands in Melanesia has also been noted.

These views on local adaptation may explain why certain groups have attained short stature, but they still do not explain why all the Malaysian and Philippine peoples of Australo-Melanesian affinity (i.e., the Negritos) are relatively small people. A possible explanation may be that they were already occupying interior or remote environments that selected for short stature when Mongoloid dominance in the archipelago began to develop. Their larger-statured neighbors in regions of high agricultural potential would thus have become absorbed into the present population, while the Negritos, partly through chance and inaccessibility, might have been isolated until recently. Even though Negritos and their neighbors have been in frequent cultural and linguistic contact with agriculturalists (Reid 1987; Headland and Reid 1989), the overall picture suggests that the surviving groups have always maintained a high degree of genetic independence from their neighbors.

It should be noted in this respect that all the Philippine Negritos have adopted Austronesian languages today (Reid 1987), just as all the Malaysian Negritos speak languages in the Mon-Khmer subgroup of Austroasiatic (Benjamin 1976). Prior to the dispersals of the Austronesian and Austroasiatic peoples, the Negritos must have spoken other languages, of which there are indeed some faint traces, especially in the Philippines (Reid 1994a).

\section{B. The Varied Populations of Eastern Indonesia}

The peoples of the eastern Lesser Sunda (Nusa Tenggara) and Moluccas (Maluku) Islands to the west of New Guinea clearly present great variation 
(Plate 4). This is a result of a gradual Southern Mongoloid settlement, much of it historically recent, over the surviving western boundary of Melanesia. The phenotypic racial picture here is markedly clinal and there are few sharp boundaries. The eastern Lesser Sundas were described by Bijlmer (1929; see also Keers 1948), who reported clines in skin color, hair form, and the frequency of the epicanthic (Mongoloid) eye fold in populations from Sumba eastward to Timor. Basically, he regarded the Sumbanese in the west as "Proto-Malays" who are gradually replaced eastward through Flores and Timor by a dominant Melanesian population. Timor and adjacent islands also have interesting juxtapositions of Papuan and Austronesian languages.

The same situation occurs on the island of Halmahera in the Moluccas, although here there is another interesting situation. The Tobelo and Galela of the northern part of the island and of neighboring Morotai, who speak dialects of a West Papuan language, are described by Ishige (1980; see also Wallace 1962:249) as being basically of "Malay stock" with some Melanesian admixture. Because I am currently undertaking fieldwork in this area, I agree entirely with this viewpoint, which is confirmed by recent genetic research (Bhatia et al. 1995). This is clearly another example, like that of the Negritos, where races and languages do not match as might be expected when reasoning from pan-archipelagic patterns of human variation.

\section{The Southern Mongoloid Populations}

The Southern Mongoloid populations (Plates 5 through 9), now numerically dominant in the region, are all speakers of Austronesian languages, with the possible exception of some eastern groups such as the Tobelo and Galela mentioned above. All share considerable physical, cultural, and linguistic homogeneity despite the complex overlays of 2,000 years of Hindu-Buddhist, Chinese, and Islamic civilization. Coon (1966:181) describes them succinctly:

These peoples are mostly short, with a mean stature for males between 157 and 160 centimetres; of medium build; yellowish or brown-skinned; mostly straight haired. ... Among most of them the Mongolian eye fold is rare. Like the Australoids, many of them have large teeth.... They represent a more or less stable mixture between Mongoloid and Australoid elements, with local variations.

A greater degree of Australo-Melanesian inheritance can perhaps be seen amongst those populations that were once called Proto-Malays, as opposed to the Deutero-Malays who are still considered by some authors to represent a second and later migration into the region (e.g., Glinka 1978, 1981). The socalled Proto-Malays included many inland peoples of the larger islands of Indo- 
nesia and the Philippines and of course some of the peoples of the clinal region of eastern Indonesia. Since Mongoloid gene flow has been entering Indonesia throughout historical times, it is clear that the so-called Deutero-Malays, who are basically the populations that inhabit the more accessible areas, have simply had more contact with the Asian Mongoloid world.

If it is possible to state the situation for Indonesia simply, then one can say that a Mongoloid phenotype predominates in the west and north and gradually fades southward and eastward (see also Bellwood 1978:304). In eastern Indonesia, a population that is quite clearly a part of the Melanesian physical and cultural world predominates. A model of Mongoloid expansion into an AustraloMelanesian sphere, allowing for considerable variation within each group, should suffice to explain the picture. The intricate details may always escape us, for the terms Australo-Melanesian and Mongoloid themselves are idealized models, and the Southeast Asian area may have been a clinal zone between these idealized types for many millennia.

\section{Skin Pigmentation and the Southern Mongoloids}

Anthroposcopic traits are poorly understood in terms of genetic inheritance, but it is believed that many of them have complex polygenic bases; hence they are not subject to rapid phenotypic fluctuations caused by genetic drift. Skin pigmentation is one such trait, and the existing variations must have evolved over long periods owing to the action of natural selection in specific environments. It seems reasonable to assume that skin colcr among members of an undisturbed population in a relatively stable environment will remain stable over very long periods of time. From known cases of population expansion, it can be seen that the processes of natural selection that cause skin pigmentation to vary work at very slow rates. For instance, the tropical American Indians are not reported to be noticeably darker than other Native Americans after a settlement period of perhaps 15,000 years (Brues 1977:302), and yet it is clear from Old World observations that tropical latitudes have certainly selected for darker skins over much longer time spans. These observations suggest that human skin color variation is of great antiquity and that the geographical variants seen today commenced development in original homeland environments from the beginning of the Old World radiation of anatomically modern humans.

Skin pigmentation is mainly produced in the deepest layer of the epidermis by melanocytes that produce the black and brown pigment called melanin. The visible color is also affected by the thickness of the outer skin layer, or stratum corneum, which contains keratin. These factors do not vary congruently; African and Melanesian skins are characterized by dark pigmentation but little keratinization, Mongoloid skins have a thick stratum corneum packed with keratin but 
little pigmentation, and European skins lack both pigmentation and keratin. Indeed, human skin colors are formed by the actions of several factors that seem to vary rather independently.

The environmental factors that promote variation in skin pigmentation are still poorly understood. Although many authorities (Krantz 1980; Tasa et al. 1985; Robbins 1991) are willing to accept some latitudinal correlation for skin color as a barrier to the penetration of ulraviolet light (i.e., dark at the equator, lighter toward the poles), there are many explanatory variations on this theme. For instance, Brace (1964) suggested that the fair skins of higher latitudes developed in part because the wearing of clothing circumvented those selective pressures that constantly promoted the production of protective melanin amongst our universally dark-skinned ancestors. Loomis (1967) adopted another explanation, which Brace had rejected: the synthesis of vitamin D by sunlight in the skin. Humans need a balanced quantity of this substance and too much or too little is dangerous, the latter causing skeletal deformation, or rickets. Loomis suggested that human skin is pigmented so that just enough sunlight can penetrate: Dark skins protect from too much, light skins protect from too little in high latitudes where sunshine is weaker.

The conclusion that can be drawn is that skin color is of complex causation, perhaps determined by three to five allelic pairs of genes (Baker 1992:47), and factors involved probably include all those listed above-which of course would vary in relative significance from one environment to another. The reason I have added this discussion, however, is to draw attention to the presence of the relatively light-skinned Southern Mongoloid population in the Indo-Malaysian tropics. It exists in a latitudinal belt that in all other regions of the Old World (Africa, southern India, Melanesia, northern Australia) supports much darker aboriginal populations. Although Southern Mongoloids are darker than Northern Mongoloids, and there is a clear north-south cline in skin color that is even visible within Southeast Asia, I find it hard to escape the conclusion (as also does Brace 1980a) that had the Indo-Malaysian groups evolved entirely within the archipelago, they should be as dark as the latitudinally neighboring Melanesians and Negritos. There is a clear case here of a pattern that does not meet the demands of long-term local natural selection, just as there is in the American tropics.

\section{Genetic AND CRANIAL DATA ON THE DIfFERENTIATION OF INDO-MALAYSIAN POPULATIONS}

When this section was prepared for the first edition of this book, the science of genetics was still making only modest progress in the study of human racial ancestry. In the decade since, there have been such mighty strides in the devel- 
opment of methods for analyzing the sequences of actual nucleotides in nuclear and mitochondrial DNA that we are undoubtedly on the threshold of an outpouring of new data on the remote ancestries of living human populations. To summarize this new material is a daunting task, but so far relatively little of the research has been applied to Southeast Asian populations. For Pacific populations there have been some major advances in recent years, particularly in the identification of population-specific lineages in mitochondrial DNA (Hill and Serjeantson 1989; Chen et al. 1992; Lum et al. 1994; Hagelberg 1994; Melton et al. 1995; Redd et al. 1995). Within Southeast Asia, mitochondrial DNA research has recently shown the importance of southern China as a major region of human radiation and also the apparent survival of Australo-Melanesian mtDNA lineages in small numbers of people in Malaysia and Indonesia (Ballinger et al. 1992). It has also been noted above how a very high proportion of Philippine Negritos have a 9-base-pair deletion in mitochondrial DNA that seems to link them with Mongoloid populations, although new research is showing that this deletion phenotype is associated with many separate lineages in terms of nucleotide substitutions, so the situation might not in reality be very simple.

In the remainder of this section I will retain the basic structure used in the first edition of this book in 1985, with any necessary updating. Therefore, I will test my generalizations about ultimately separate origin zones (despite the possibility of some geographical overlap) for the Australo-Melanesian and Southern Mongoloid populations against data on genetically controlled characteristics. The following discussion is concerned mainly with polymorphic characteristics identifiable in blood, rather than in nucleotide sequences within actual DNA.

At all comparative levels, from two related individuals to whole populations, the human species presents a complex genetic picture of uniqueness, intergradation, and identity, depending upon which genetic characters are under study. The most important ones for charting human prehistory have always been the genetic polymorphisms-systems that can have several states depending on the occurrence of different alleles at specific genetic loci on the chromosomes. The best known of these polymorphisms are perhaps the blood groups, which are of simple inheritance based on variation at only one or a few loci. Some years ago it was commonly believed that pooled blood group frequencies could be used to trace ancestries of specific populations and even major races of mankind. However, it is now known that some blood groups are subject to natural selection over both large and small geographical areas, and among small isolated populations they are particularly susceptible to genetic drift and to a nonrandom sampling process known as the founder effect (Neel 1967). Selection, drift, and the founder effect do of course operate to produce variation in all genetic systems, but some are more resistant to rapid change than others. Those blood groups (such as $\mathrm{ABO}$ and $\mathrm{Rh}$ ) that can change rapidly in frequency in both time and 
space are of little use in tracing ancient connections between the major racial groups of mankind (Simmons 1962, 1976), and their patterns of frequency around the world do not correlate with the distributions of the modern races (Krantz 1980).

In recent years, however, there have been major strides forward in other methods that allow major human populations and races to be "characterized." One important method involves the comparison of populations in terms of their unique alleles and allele combinations (haplotypes), many of which are specific to particular geographical races.

\section{A. Evidence from Population-Specific Genetic Markers}

The genetic systems that are of most use for tracing population origins and ancient connections are those not markedly susceptible to natural selection and resistant to local fluctuation through genetic drift. In other words, single alleles or haplotypes in these systems are considered likely to remain in a population through long periods of time and through long migrations. Where they are distinctive to a particular population and do not occur in others, they can be of great interest for human prehistory. Genetic systems that are strongly subject to disease-associated natural selection, such as the abnormal hemoglobins, do not have those advantages and are not considered here.

There are now known to be a number of fairly stable genetic polymorphisms, apparently unassociated with disease resistance, that do have populationspecific variants. Relevant summaries for all these polymorphisms are given by Kirk (1982; 1986), who demonstrates that Asian and American Mongoloids (including Indo-Malaysian Mongoloids) can be differentiated from Australians and Melanesians on sharp presence-absence occurrences of variants in the Diego red cell antigen system, the transferrin iron-binding serum proteins, the Gm immunoglobulins (Kelly 1990), and the Gc serum protein system. This evidence provides very strong support for the view that the Australians and Melanesians are of reasonably close common origin and are quite sharply separated in many characteristics in blood genetic systems from the Southeast Asians.

\section{B. Evidence from Multivariate Distance Statistics}

This evidence comes from two sources-phenotypic measurements (anthropometric and cranial) and gene frequencies-and studies have tended to be at two levels, one stressing major population affinities and the other being more concerned with microevolutionary patterns of divergence. Anthropometric and cranial distance studies have been confined mainly to the Australian and Oceanic regions (e.g., Howells 1970; 1973a; 1989; Pietrusewsky 1984), and as 
the results do not really contradict those from genetic analyses, I will not consider them here in detail. However, recent analyses of broad geographical coverage by Pietrusewsky (1984; Pietrusewsky et al. 1992), based on a multivariate analysis of thirty-four cranial measurements on skeletal populations, do reveal a sharp level of differentiation between Australo-Melanesian and Mongoloid populations (Fig. 3.1).

Distance diagrams for Southeast Asian and Pacific populations in terms of genetic systems occurring in blood have been presented with increasing frequency since 1973 (e.g., Schanfield in Howells 1973b:76). Most analyses support the evidence given in the section on unique alleles, but this type of analysis has only very recently been extended into Southeast Asia proper. Schanfield (above) reported clear separations between Northern Mongoloids, Southern Mongoloids, and Papuan-speaking Melanesians, but only for five polymorphic loci. Recent studies have used up to twenty-nine loci (Nei and Roychoudhury 1993), and here the results seem to be in basic agreement; they may be summarized as follows:

a. Australians and Melanesians are always more closely related to each other than either is to Southern Mongoloids;

b. Oceanic Mongoloids (Micronesians, Polynesians) group most closely with Southern Mongoloids in Island Southeast Asia (see Hill and Serjeantson 1989);

c. Southern Mongoloids as a whole tend to group closer to Northern Mongoloids (Chinese, Koreans, Japanese) than to American Indians (Nei and Roychoudhury 1993). However, the recent massive worldwide comparison of gene frequencies by Cavalli-Sforza et al. (1994) posits a fairly major separation between Northern and Southern Mongoloids, a separation that is difficult to reconcile with linguistic and cultural data. This is not an issue of direct concern for this book.

Apart from the one last mentioned, these patterns of similarity and difference are clearly unsurprising in the light of the data from the unique genetic markers. It may be asked what further information can come from this kind of research if the results always seem to replicate themselves within broad limits. Perhaps only the future holds the answer to this question, but it is worthy of note that some scholars are quite capable of putting forward new hypotheses that seem to conflict with old ones. For instance, Brace et al. (1991) have suggested, from an analysis of craniofacial evidence, that Polynesians derive from an Ainu-like population in Jomon Japan rather than from Neolithic Southeast Asians, a conclusion that clearly goes against all archaeological and linguistic evidence relevant for this question. So far, this hypothesis has not received much support from other biological analyses. 


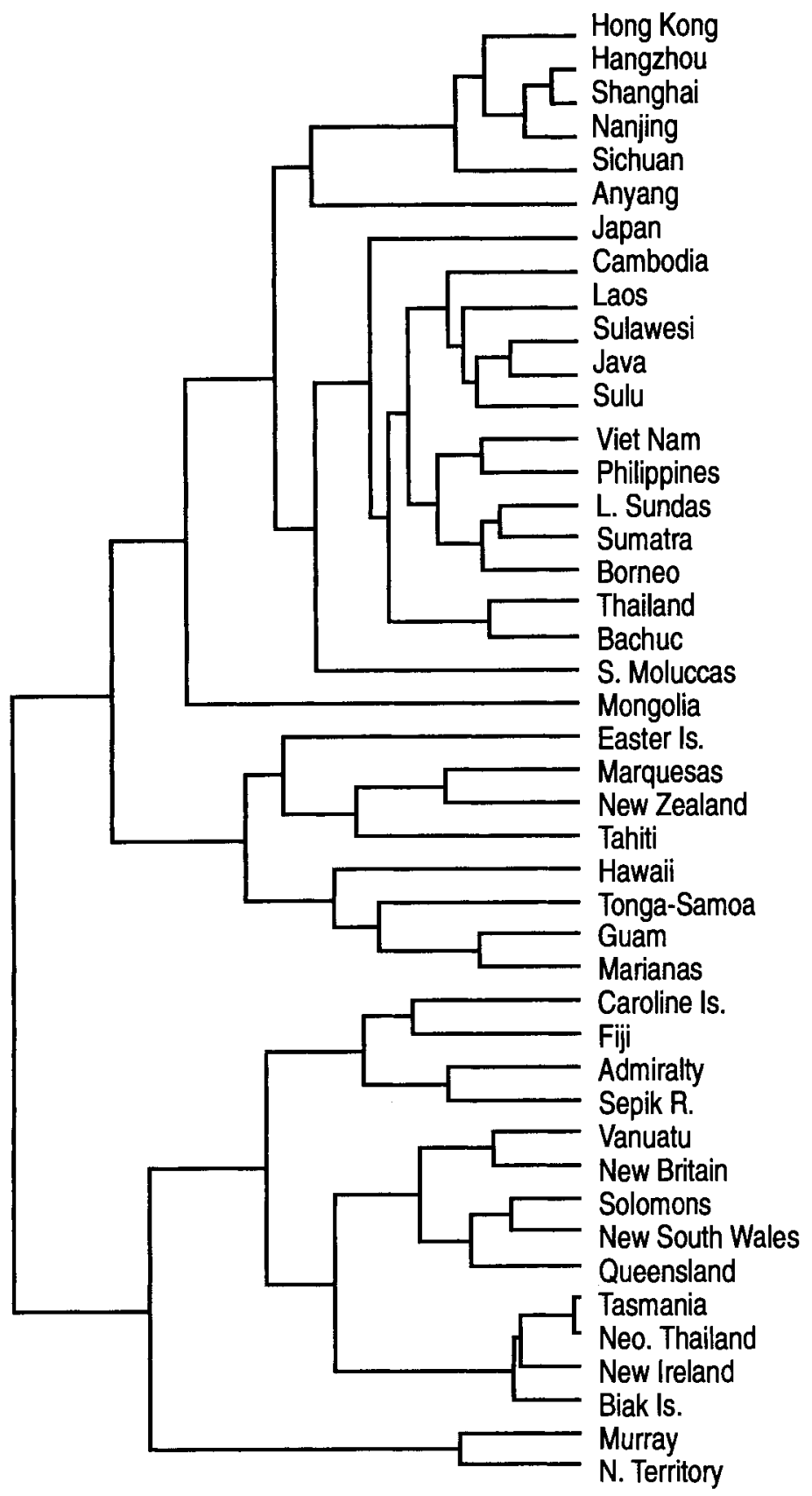

Fig. 3.1 Diagram of relationships for Southeast Asian and Pacific populations based on a muitivariate cluster analysis using thirty-four cranial measurements on samples of male skulls. The upper major branch includes samples in eastern Asia and Polynesia/Micronesia; the lower branch includes samples from Melanesia and Australia. Courtesy: Michael Pietrusewsky. 
Other scholars have been experimenting with information concerning rates of genetic mutation over time to see if specific distance measurements between populations can be correlated with times of separation between them. For instance, Omoto (1981) has suggested from inferred rates of mutation that two groups of Philippine Negritos have been separated from each other for over 10,000 years. The results of such "molecular clocks" are very hard to evaluate, partly because such methods depend on other disciplines for the provision of a basic timescale against which to calibrate the genetic distances.

\section{Smaller-Scale Population Distances}

As well as the attempts to characterize major populations, there have been many attempts in recent years to see how small neighboring populations differ from each other genetically and to see if these differences vary congruently with linguistic differences, rates of intermarriage, geographical distances, and patterns of variation in anthropometric features. Again, almost all of this work has been done in Australia and Melanesia (together with South America), and the results as presented are clearly of more interest to geneticists and ecologists than to prehistorians (e.g., Birdsell 1993:443-446; Friedlaender 1987; J. Wood 1978; Serjeantson, Kirk, and Booth 1983; Stoneking et al. 1990). They are of interest for intensive studies on how genetic variations develop within small isolated populations over time spans of only a few generations, and they can inform about the true genetic significances of language barriers and patterns of intermarriage (for a European example indicating the high genetic significance of local language boundaries, see Barbujani and Sokal 1990).

The genetically varied Melanesian gardening populations are of great interest in this regard, and similar patterns of great local variation characterize some isolated groups of hunters and shifting cultivators in Southeast Asia (see Polunin and Sneath 1953; Lie-Injo 1976; Fix 1984). These are situations where genetic drift can operate at rapid rates, but there always seems to be an encompassing anthroposcopic stability, probably maintained by selection, that not even drift can overcome. I would think that the main interest of these studies for a prehistorian comes from the demonstration that rapid genetic microevolution at identifiable genetic loci, over a period of perhaps 40,000 years across a range of temperate and tropical environments in Australia and New Guinea, has been insufficient to produce major phenotypic differences equal to those we see in the major Australo-Melanesian and Mongoloid divisions today. This brings me back to the observation that the Southeast Asian Mongoloid populations have probably not evolved entirely in the tropical latitudes they now inhabit. 


\section{ANCIENT POPULATIONS OF HOMO SAPIENS IN THE INDO-MALAYSIAN ARCHIPELAGO}

From the genetic information on modern populations presented above, it would seem reasonable to hypothesize-given patterns of trait distribution-that the ancestors of the Indo-Malaysian Mongoloids have moved southward into a region previously settled by Australo-Melanesian populations. The two groups have since hybridized to varying degrees. The diachronic data from ancient skeletal remains should, in theory, allow evaluation of this hypothesis. In practice, however, skeletal remains tend to be fragmentary, often quite poorly dated, and ambiguous in terms of racial correlations. All human populations intergrade, especially in skeletal characteristics, and statements about the affinities of particular crania tend to be probabilistic (older reports often claim more certainty than is now known to be reasonable). While the totality of evidence does suggest that the Southern Mongoloid distribution can only be explained by allowing some importance to expansion, the whole story is complex; we certainly cannot see a clear-cut replacement of populations taking place in the skeletal record. We must allow for intermarriage, local evolution, and also for the important concept that expansion involved more a change in the structure of a Mongoloid-Australoid cline than a migration of uniform and distinct peoples from a remote area such as China.

\section{A. Regional Continuity or Replacement (erectus to sapiens) in Australo- Melanesian and Mongoloid Evolution}

As noted in Chapter 2, some paleoanthropologists today continue to believe that the Homo erectus populations represented by the skeletal series from China and Java have passed on at least some of their locally distinctive morphological characteristics to the present Mongoloids and Australo-Melanesians. This view has a respectable history of support going back to such influential scholars as Weidenreich (1946) and Coon (1962). According to Coon (1962:ix): "A species which is divided into geographic races can evolve into a daughter species while retaining the same geographical races." Coon did come under serious attack from reviewers for his claim that different geographical races crossed the sapiens threshold at different times (Coon 1962:30), but the basic idea that geographical racial differences have been maintained through long periods of human evolution has continued to receive support by Wolpoff and Thorne (e.g., Wolpoff 1985; Thorne and Wolpoff 1992; Wolpoff et al. 1994) for the Australoids and by the same and most recent Chinese authors for the Mongoloids.

On the other hand, as described in detail in Chapter 2, the past decade has seen a considerable growth in the proportion of scholars who favor a relatively 
recent radiation of anatomically modern humans from an African source, perhaps within the past 100,000 years. This view now has a great deal of genetic and craniometric support, and I will not repeat here the previous discussion. When this book was first published in 1985, I accepted the hypothesis of regional continuity. Now, of course, the matter is not so simple. The skeletal evidence from Southeast Asia is too fragmentary and poorly dated to be of central value for resolving this debate, and I see little benefit here in taking a strong stand on the issue. The question of population replacement within the time span of anatomically modern humans is, however, one that must be considered in more detail. It lies at the heart of debate about the origins of current biological patterning within the human populations of Southeast Asia and western Oceania.

\section{B. The Southern Mongoloid Replacement Model for the Indo-Malaysian Archipelago}

The oldest skeletal remains thought to be directly in the ancestry of the Southern Mongoloid populations (together with other related groups such as the ancient Jomon and modern Ainu populations of Japan) include the four partial skeletons dated to ca. 20,000 years ago from a limestone fissure at Minatogawa on the island of Okinawa (Suzuki and Hanihara 1982; Baba and Narasaki 1991) and, from southern mainland China, the late Pleistocene skulls from Liujiang in Guangxi and Ziyang in Sichuan the latter perhaps dated to ca. 35,000 BP; Wu Xinzhi 1996). The Liujiang skull is of great interest: Coon (1962:469) described it as Mongoloid with some Australo-Melanesian features (see also Thorne 1980a:100). This may be telling evidence for the existence of a late Pleistocene clinal zone through Southeast Asia-an area where such a cline may be expected as there are no major latitudinal barriers to gene flow.

For Australoid evolution in the tropical latitudes of Southeast Asia, the record is less complete than that for China because the majority of the relevant specimens date after 30,000 years ago and have been found in Australia; they will be returned to briefly in Section IIID. However, scattered material, mostly postdating 25,000 years ago, is known from various parts of Southeast Asia. Some of the important sites are the caves of Niah and Gua Cha in Malaysia, Tabon Cave in the Philippines, and a scatter of important cave and shell midden sites in Indonesia (for site locations see Fig. 3.2 and Chapter 6, Fig. 6.1).

Niah Cave (the West Mouth) in Sarawak is a major site that will appear frequently in this book; for present purposes, it has by far the best series of dated human remains of any site in Island Southeast Asia, and these include: 


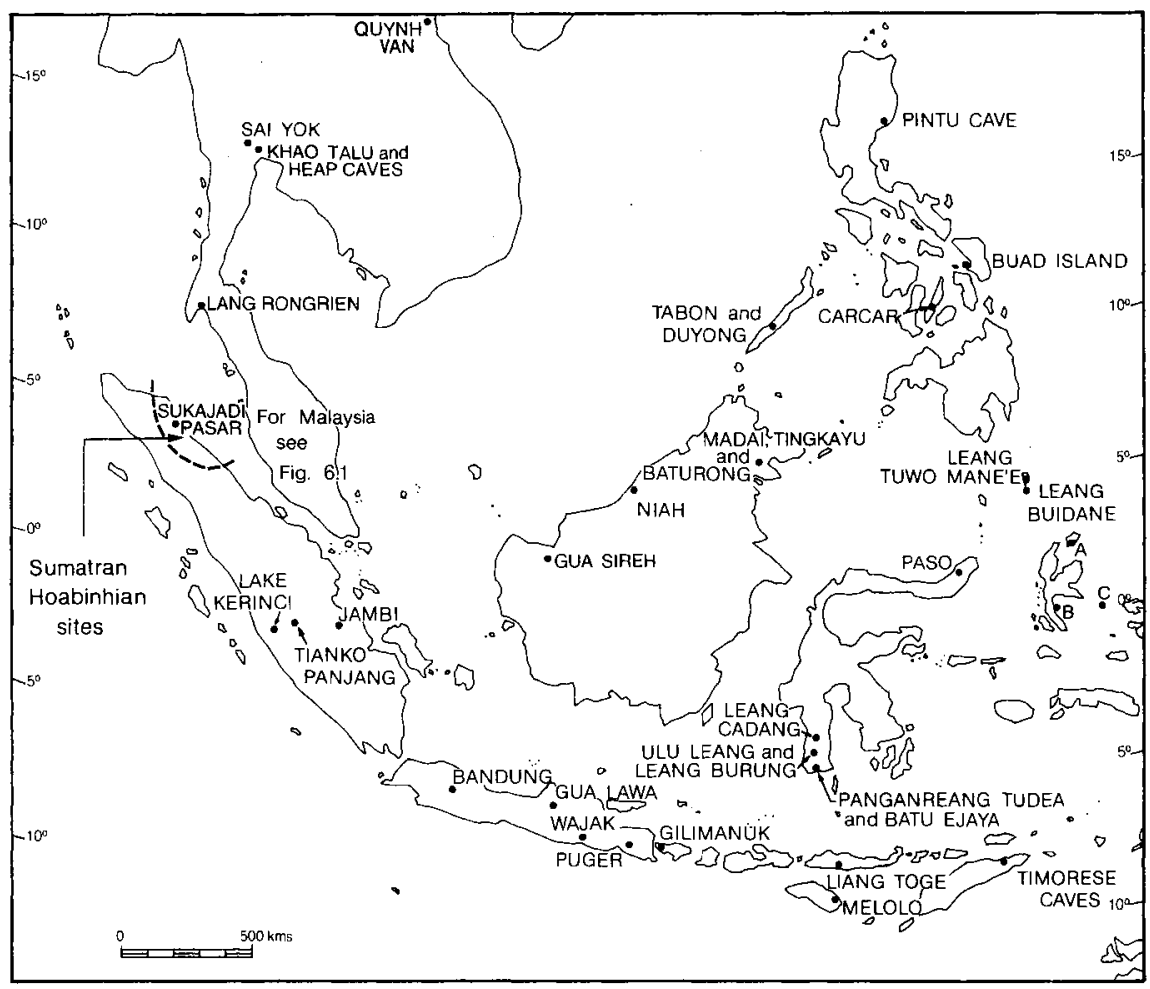

Fig. 3.2 Locations of sites referred to in Chapters 3 and 6. $A=$ Tanjung Pinang and Daeo, $B=$ Gua Siti Nafisah, C = Gua Golo and Gua Wetef.

a. The "deep skull" (Plate 10) (Kennedy 1977), generally associated with a carbon date of ca. $40,000 \mathrm{BP}$, although my inclination is to prefer a younger date on the grounds that the skull must have been buried from a higher level (see Chapter 6, Section IIA).

b. A series of flexed, seated, and fragmentary burials dating between 14,000 and 3,500 BP (Harrisson 1975b; Brooks et al. 1977).

c. Extended burials in coffins or mats dating from possibly $2000 \mathrm{BC}$ to less than 2,000 years ago (Chapter 7, Section IIID).

The situation with respect to the deep skull has been reviewed by Kennedy (1977). The basic analysis of the skull was done by Brothwell (1960), who suggested that it belonged to a young person whose closest morphological affinities were with recent Tasmanians; that is, toward the gracile end of the AustraloMelanesian range of variation. The burial series listed under $b$ has not been 
fully described, but Brooks et al. (1977) provide data on blood groups identified from the bones, and Koenigswald (1958) described the dentitions of the older burials as "Melanesoid." Perhaps all that can be said about this group is that its affinities do not appear to be with the recent Southern Mongoloids, who are more probably represented in group c.

From Tabon and nearby caves on Palawan island in the Philippines, there are two sets of human remains (Fox 1970):

a. A frontal bone and mandible from Tabon Cave dated between 20,000 and 22,000 BP (although the relevant deposits are described as disturbed; Fox 1970:40). The mandible was considered close to the Australian range by Macintosh (1978).

b. Jar burial remains in many caves, all postdating 3,000 years ago and all Mongoloid in terms of incisor shoveling (Winters 1974).

Thus, in both Niah and the Tabon Caves there is some evidence, albeit debatable, for postulating that an Australo-Melanesian population formerly existed in regions occupied by Southern Mongoloids since at least 1000 BC. For Gua Cha the situation is a little different. This site is a rock shelter in Kelantan in central Peninsular Malaysia, a region now inhabited by orang asli (aboriginal) groups of hunter-gatherer Negritos and agriculturalist Senoi; the former being of clear Australo-Melanesian affinity but the latter belonging to a population that has genetic affinities with Mon- and Khmer-speaking populations (Saha et al. 1995). The twenty-seven burials from Gua Cha date from about 10,000 years ago to perhaps 2,000 years ago (Sieveking 1954; Adi 1985; see also Chapter 6, Section IA; Chapter 8) and span both Hoabinhian and Neolithic contexts. Trevor and Brothwell (1962; see also Bulbeck 1982) note that the remains show no change of a racial nature throughout and have general affinities with Melanesians. The Gua Cha remains must be ancestral to some of the present orang asli, especially the Semang Negritos, and possibly form part of the local Senoi ancestry as well. The latter, however, with their Mon-Khmer genetic affinities, surely have a predominant ancestry in Neolithic population movements down the Malay Peninsula from the north (Bellwood 1993). One is obliged to posit some degree of population replacement in the peninsula, even if it is not directly visible in the human remains from Gua Cha itself.

Since the first edition of this book was published, other skeletal remains of claimed Australo-Melanesian affinity have been recovered from Hoabinhian contexts in the caves of Gua Gunung Runtuh in Perak, Peninsular Malaysia, and Moh Khiew in Krabi Province, southern Thailand. The Gunung Runtuh skeleton (Zuraina, ed. 1994) is of a middle-aged male with a deformed left arm and hands, buried in a squatting position with knees drawn up to his chin. Freshwater shells with the skeleton have been Carbon- 14 dated to about 10,000 years 
ago, and the morphology of the individual is within the Australo-Melanesian range. The burials from Moh Khiew Cave appear to be of Australo-Melanesian morphology and to come from Hoabinhian contexts, but only a provisional report is available (Pookajorn 1994). Unlike the series from Gua Cha, however, these samples are small and do not span long time periods.

To fit the information from these sites into a coherent pattern for the IndoMalaysian Archipelago, we must turn to Indonesia. Most of the important material has been reviewed by Jacob (1967a), but more has been recovered in recent years. First I will consider the most problematic site: Wajak in east-central Java, where two crania were found in 1888 and 1890 (the latter by Dubois) in a now-destroyed shelter from which there has survived no direct evidence for date or context (Storm and Nelson 1992). Fortunately, however, it has recently been possible to subject a human femur from the site to bone apatite Carbon-14 dating (Shutler et al. 1994), with a result of about 6,500 вp. Thus the Wajak human and animal bones can probably all be considered early to midHolocene.

But the Wajak crania still raise questions of interest. They have been considered Australo-Melanesian by many authorities and have large brains and faces, but both Coon (1962) and Jacob (1967a) have also noted possible Mongoloid affinities in their flat faces. If the above date is correct, it may be that the Wajak skulls indicate some degree of Mongoloid affinity for Javan populations prior to the period of Austronesian colonization. Unfortunately, the precise morphological affinities of these skulls appear to be obscure owing to problems with reconstruction, but if they are indicative of pre-Austronesian gene flow from mainland Asia into Indonesia, they are of great significance. This latter view was partly espoused by Jacob (1967a:51-52), who considered the Wajak population as possibly ancestral both to Indonesian Mongoloids and to present AustraloMelanesians. I will consider the evidence for this view in the next section, as it has been promoted again recently.

The other Indonesian skeletal remains fall into three rather vague groups:

a. Skeletal remains from several sites on Flores, all presumed to be of Holocene date and clearly belonging to the ancestors of the present mainly Australo-Melanesian population of the island. One adult female of very small stature from a cave called Liang Toge has been dated to ca. $2000 \mathrm{BC}$ (Jacob 1967a:79). Skeletal remains dating from ca. 2,000 years ago recently excavated from the rock shelter of Tanjung Pinang on Morotai Island, north of Halmahera, appear also to have generalized AustraloMelanesian affinities (Bulbeck, pers. comm.). In these easterly regions of Indonesia, still peopled by groups with quite strong Melanesian biological affinities today, such observations are not surprising. 
b. Cranial remains of Australo-Melanesian affinity from regions of northern and western Indonesia that are today inhabited by Southern Mongoloids. The best examples in this group include the large-toothed cranial remains from the cave of Gua Lawa in central Java (Jacob 1967a; Mijsberg 1932); the twelve disturbed skeletons from a destroyed Hoabinhian shell midden at Sukajadi Pasar in northern Sumatra (Budhisampurno 1985); and the skull from the basal levels of Leang Buidane in the Talaud Islands (Bulbeck 1981). None of these samples is directly dated (although the Sukajadi midden has marine shell from a disturbed context dated to ca. 7,500 BP; Bronson and White 1992:508), but all clearly predate the early Metal phase with its associated Southern Mongoloid populations.

c. Skeletal material, mostly postdating 1000 BC (i.e., later Neolithic onwards), that is clearly of Southern Mongoloid affinity, particularly on such criteria as shoveling of the incisor teeth. Material of this type is very widespread; good samples come from Leang Cadang in southern Sulawesi (Jacob 1967a), Gilimanuk in Bali (ca. 2,000 years old, Jacob; 1967b), and Leang Buidane in Talaud (first millennium AD; Bulbeck 1981). In all these cases the populations are clearly ancestral to the present inhabitants of these regions.

Taking this skeletal material at face value, as described in the literature, the most likely hypothesis is that Southern Mongoloid populations have entered the archipelago from the north, mainly via the Philippines as far as Austronesian expansion is concerned but perhaps also via the Malay Peninsula to some extent as well, and have been present since at least $1000 \mathrm{BC}$ in most areas where they are now found; they have clearly never penetrated in any major way into Irian Jaya and adjacent parts of eastern Indonesia. Again accepting old reports at face value, the presumed clinal effects of this expansion in such post-1000 BC populations as those from Melolo in Sumba (Snell 1948) and Puger in eastern Java (Snell 1938) can also be seen.

Let me expand this replacement hypothesis further by describing how it has fared at the hands of authoritative past supporters and how it may relate to historical trends and events. Perhaps the simplest and clearest statement of the replacement view was published by Barth in 1952; he thought that late Neolithic and Bronze-Iron Age populations had been pushed southward out of China by population pressure a little before $500 \mathrm{BC}$. For some reason he believed that there was a sharp ecological barrier between South China and Southeast Asia that Mongoloid populations were unable to cross until they had developed substantial populations with wet-rice agriculture, after which they dramatically burst over the barrier. Such sharp replacement is not supported by the skeletal record, Barth's dates for initial Mongoloid expansion are far too late, and his 
view that North China was the ultimate source for all biological and cultural innovations in Southeast Asia is now known to be wrong. But the basic need for a hypothesis of replacement still remains; indeed, the linguistic evidence for Austronesian dispersal (Chapter 4) makes it unarguable to a certain degree.

As far as the opinions of biological anthropologists are concerned (Barth is a social anthropologist), the basic view of Coon $(1962,1966)$ was likewise that Indonesia and adjacent parts of the Southeast Asian mainland formed the Australoid homeland, overwhelmed by a "great rush" of Mongoloid expansion southward, commencing by at least the Neolithic and culminating in historical times within the past 2,000 years. Coon (1962) also reviewed the early Holocene skeletal material from Mainland Southeast Asian Hoabinhian sites, and there are indications here of a confusing array of Mongoloid and Australo-Melanesian features stretching from northern Vietnam (e.g., Cuong 1986-1987 for Mai Da Nuoc, ca.10,000 BP; Duy and Quyen 1966 for Quynh Van, ca. 6,000 вP) down to Peninsular Malaysia (e.g., Jacob 1967a for Guar Kepah). My own tendency is to regard this pre-Neolithic material as basically clinal between present Australo-Melanesian and Southern Mongoloid foci of variation. For the Asian mainland north of Malaysia, it is not really known when the ancestors of the present Mongoloid populations first began to establish themselves; in the northern part of the region and southern China they may always have been predominantly Mongoloid. For more southerly regions it is probable that Southern Mongoloid expansion was taking place during the Neolithic and early Metal phases, if not before, and linguistic and historical sources suggest that considerable expansion of such groups as the Thais, Vietnamese, and Malays has affected vast areas of the mainland since 3,000 years ago.

A more recent variation of Coon's basic viewpoint has been presented by Howells (1973b, 1976, 1977, 1989), who defined (Howells 1973b) a late Pleistocene province of "Old Melanesia" comprising continental Sundaland, Wallacea, and continental Sahulland. Populations ancestral to modern Australians and Melanesians are stated to have inhabited this region since at least 50,000 years ago (as represented by the remains from Niah, Wajak, and Tabon), and the present Australians and Melanesians now represent the differentiated descendants of Old Melanesian ancestors. For the present day, Howells (1973b:192) defines a province of "New Melanesia," now flanked by the Southern Mongoloid populations who have settled Indonesia, Polynesia, and Micronesia, and regards all these expanding Southern Mongoloids as Austronesian speakers of post-3000 BC southern Chinese origin.

Another recent viewpoint in support of the postulated Southern Mongoloid expansion is that of Brace (1976, 1980a), Brace and Hinton (1981), and Brace et al. $(1984 ; 1991)$. Brace has developed the hypothesis that a reduction of overall tooth size occurred with the development of agriculture. He suggests that the 
Austronesian-speaking Southern Mongoloids (fairly small toothed) had expanded via the Philippines into Indonesia, where they replaced a larger-toothed population still represented by the Australians and Melanesians. Basic gradients in tooth size throughout the region, with the smallest in southern China and the largest in Australia and New Guinea, support this view. Brace and Hinton (1981) went on to stress the importance of soft-food preparation in containers such as pottery as a factor that could relax selection pressures favoring large teeth; pottery is absent in the New Guinea Highlands, although horticulture is of high antiquity there, and tooth sizes have remained large. Again, we are presented with a hypothesis that small-toothed Southern Mongoloids of southern Chinese origin have replaced a more macrodont population in Indonesia and the Philippines. Brace et al. (1991) have expanded this model further in terms of craniofacial evidence and here use the terminology of a "Mainland Asian Cluster" gradually replacing an "Australo-Melanesian Cluster" in population terms.

The views of Coon, Howells, and Brace outlined above are all generally in accord with my own, and I have taken ideas from all three before (Bellwood 1978). Not all authorities, however, present this same viewpoint of recent Mongoloid expansion and replacement. It is now time to turn to another view that may be correct in part and that may necessitate modification of basic replacement theories. This view is that many aspects of the present Southern Mongoloid phenotype have actually evolved within Southeast Asia from the late Pleistocene onward. No one would dispute that intense Mongoloid gene flow into the area has taken place in historic or even latest prehistoric times, but it may be that all the populations of eastern Asia were undergoing similar trends in terms of modernization of skull and facial form throughout the late Pleistocene and into the Holocene. Hence the postulated Southern Mongoloid migrants may have been settling amongst populations who were also evolving in similar ways and who may have contributed more to the present dental and cranial phenotypes of the region than is usually allowed.

\section{The Indo-Malaysian Continuity Model}

This model switches the emphasis strongly away from migration. It has a respectable pedigree, being foreshadowed by Weidenreich in 1945 when he pointed out that brachycephalization (a trend toward an increasing broadness of the skull) could have evolved locally in different populations-an important observation at a time when long skulls and broad skulls were thought to identify different migrating races. Hooijer (1950b, 1952) also pointed out that large teeth alone could not, as others had assumed, be used in the prehistoric Southeast Asian context to identify "Melanesoid" populations. This was not an attempt 
to discount migrations (see Koenigswald 1952 for a spirited rejoinder based on a misunderstanding about this), and Hooijer was merely disputing theories based on teeth in isolation. He was able to show that reduction in tooth size could be a local development and need not necessarily imply a migration from outside by a separate small-toothed population.

In recent years the view of local population evolution within Southeast Asia has become more positive. Turner and Swindler (1978; and see also subsequent Turner references) suggested that late Pleistocene Sundaland was occupied by a population with widely shared dental characteristics that they termed "Sundadont". The present Southern Mongoloids are thought to have retained a Sundadont dentition from this ancestral Proto-Mongoloid population and hence to have developed in situ within Sundaland and adjacent parts of Mainland Southeast Asia. Polynesian and Micronesian dentitions are also within the Sundadont range, thus attesting to their Island Southeast Asian and ProtoMongoloid origins. The "Sinodont" teeth of northeastern Asia and the Americas are also thought to have evolved from an original and more widespread Sundadont-like ancestral form. Melanesian teeth, which are placed in a separate class by Turner and Swindler because of their simplified crown morphologies and low percentages of incisor shoveling, are derived from the same Pleistocene populations that gave rise to the Sundadonts, but have evolved their own local form within Melanesia. Thus, this view suggests that Southern Mongoloids are indigenous to Southeast Asia and share a common late Pleistocene ancestry there with the Melanesians. Craniofacial evidence in support of this view, and contrasting with the above views of Brace, has recently been presented by Hanihara (1993).

Turner's current view (Turner 1987, 1989, 1990, 1992) is that Sundadonty is a basic human pattern and that modern humans have perhaps evolved and dispersed from a Southeast Asian homeland. I find it hard to challenge this view since it is presented without a timescale, although there is virtually no support for a Southeast Asian source for modern humanity in the archaeological record. However, Turner's hypothesis does have support from Kingdon (1993), who suggests that the dark pigmented populations of Africa, Australia, and southern India all originated from coastal hunter gatherers in Pleistocene Southeast Asia. This is an intriguing possibility that merits some consideration, although hard data in support may be difficult to find.

Unfortunately, Turner's analyses do not include any well-dated samples of southern Chinese Neolithic populations. This is important because the possibility arises that the Austronesian dispersal from southern China into Southeast Asia after 5,000 years ago simply represented one group of Sundadonts replacing or absorbing other Sundadonts. If this is correct, then continuity in tooth morphology alone may not be a direct reflection of continuity in actual human 
populations; Southern Mongoloids and Australo-Melanesians alike within the Indo-Malaysian Archipelago might have had similar tooth morphologies throughout.

Can one really argue in such detail-either for or against major population movements-from teeth alone? Perhaps we are back to the objections made by Hooijer (1950b, 1952; see above), but from the other side of the fence. To counter this possibility, Bulbeck $(1981,1982)$ has considered the whole question of local evolution within Southeast Asia in great detail in order to provide stronger support for a continuity hypothesis. He sees the main problem as how to explain the obvious modernization that has taken place within Southeast Asian populations; are these changes due to clade (lineage) changes (i.e., to a Southern Mongoloid migration replacing an Australo-Melanesian population), or are they due to grade changes (i.e., modernization within a single in situ population)? To approach this problem, Bulbeck examined a large amount of Southeast Asian cranial material from the late Pleistocene through to Recent (including many of the remains listed above) and documented what appear to be continuous and unbroken trends throughout: occurrence of the Sundadont dentition; size reductions in teeth, faces, and palates; and a reduction of facial prognathism. On the other hand, there has been a recent increase in the occurrence of upper incisor shoveling, and this is of course a feature most developed in mainland Asian Mongoloid populations.

In his conclusions, Bulbeck stresses that there is nothing in the evolutionary record of recent Southeast Asians that demands a migration of Southern Mongoloids from the north. In the absence of a rigidly defined chronology for the remains, it is clear that the documented changes could be due to changes in clade, in grade, or in both. In terms of teeth, his conclusions parallel those of Turner and suggest that local evolution is at least as good a hypothesis as migration. But it must not be forgotten that these conclusions are drawn only from cranial and dental characteristics; in these areas alone it may be possible to regard Southern Mongoloids as the result of in situ modernization. But I think there is evidence from other sources-those not always considered by physical anthropologists concerned with skeletal remains-that must be considered. I have already mentioned genotype and such phenotypic traits as skin color, but in addition, linguistic evidence indicates that Austronesian speakers with agriculture have expanded throughout the Indo-Malaysian Archipelago and the Pacific Islands within the past 5,000 years; the modern Austronesians must to some degree be descendants of original founder populations that expanded from southern China and Taiwan, even if local genetic input has been considerable.

My own view runs something like the following. The ancient Indo-Malaysian Australo-Melanesians who remained in the archipelago as "cousins" to the descendants of those groups who settled Australia and New Guinea ca. 50,000 
years ago clearly continued to evolve independently on the western side of the Wallacean sea barriers. Many of the changes they underwent were probably shared, to an extent, with more northerly Asian Mongoloid populations. In fact, there may have been continuously intergrading populations from southern China right into continental Sundaland-a clinal Mongoloid-Australoid zone evolving as one rather than two separate populations, but still spanning a sufficiently broad latitudinal zone for natural selection to have quite different results at either end.

This leads directly to the following conclusions:

a. Australia and New Guinea were initially settled from the Indo-Malaysian Archipelago, presumably the Wallacean end of it, by at least 50,000 years ago.

b. Australians and New Guineans have since undergone independent differentiation, but still retain clear traces of their common origin.

c. The Indo-Malaysian populations "left behind" after Australia and New Guinea were initially settled continued to diversify and underwent certain trends in facial and cranial gracilization in common with adjacent Mainland Southeast Asian populations. Some of these changes may have taken place as a result of Pleistocene gene flow from more northerly (ancestral Mongoloid?) populations, but I suspect that changes in local selective pressures, perhaps via undocumented cultural changes, may also have been important. These groups remained phenotypically as Australo-Melanesians, in some cases to the present (the Negritos and some eastern Indonesians).

d. From 3000 вс onward, the Indo-Malaysian region was settled from the north by linguistically related and expanding populations of Southern Mongoloids - the Austronesian-speaking populations. The chronology of this expansion can best be reconstructed from archaeology and linguistics, and it was clearly well underway by about 3000 BC in Taiwan, by $2000 \mathrm{BC}$ in the Philippines and eastern Indonesia generally (with Micronesians and Polynesians hiving off by $1500 \mathrm{BC}$ ), and by perhaps $1500 \mathrm{BC}$ or later in western Indonesia. It should of course be remembered that all the evidence suggests that many of the present Southern Mongoloid populations of Indonesia and Malaysia also have a high degree of AustraloMelanesian genetic heritage.

\section{The Australian Window}

From the Australian region there are further implications that involve the IndoMalaysian Australo-Melanesians. The most simple view of Australian origins 
postulates that only one founder population ever reached the continent and that the patterns of variation in the ethnographic population are due to local selection, plus perhaps some minor later arrivals. This view tells little about human variation in Island Southeast Asia after the period of initial Aboriginal migration over 40,000 years ago, but it does find favor with a number of recent authorities (e.g., Macintosh and Larnach 1976; Howells 1976; Wolpoff 1980; Habgood 1986; Brown 1987).

A quite different view has been espoused for many years by Birdsell (1949, $1967,1972,1977,1993)$. It involves three separate migrations that could each be of great potential significance for the Indonesian region were they to receive support. First, per Birdsell, came Negritos (or Barrineans) from an ultimate African source; this group has had a lasting impact in Melanesia, especially in New Guinea. Second came the Murrayians, from a possible Ainu-like source. Finally the Carpentarians of northern Australia arrived, from a possible southern Indian source. All these populations reached Australia from intermediate locations in Indonesia. In his most recent book, Birdsell (1993) has even ventured to give dates for these expansions: over 40,000 years ago for the Barrineans, ca. 20,000 years ago for the Murrayians, and ca. 15,000 years ago for the Carpentarians. The Barrineans are traced through the Niah and Lake Mungo skulls, the Murrayians through Liujiang, Kow Swamp, Keilor, and Wajak, and the Carpentarians would appear not yet to have ancient representatives (Birdsell 1993:23). Birdsell's belief that Australian variation does not derive from one single founder population alone has recently come back into favor, even if opinions are couched in different terms.

For instance, a view suggested by Brace (1980b) is that Australia was settled first by a large-toothed population, of whom ethnographic descendants survived in the southern part of the continent and in Tasmania. But the populations of the central and northern regions (Birdsell's Carpentarians?) have smaller teeth, and these groups are thought to descend from migrants who entered Australia later. Brace's view has a certain appeal, for it suggests that Australia was reached by successive Australoid groups from Indonesia, who-as noted above -were developing smaller teeth and faces through time.

Alan Thorne is currently the strongest supporter of the view that more than one colonization of Aborigines occurred in Australia. His views come quite close to those of Birdsell, except that he recognizes only two groups on the evidence of ancient skulls. The first is a gracile group best known from Lake Mungo in western New South Wales, where stone tools and associated dates of over 30,000 years ago have been obtained. The second group has a much heavier facial and cranial skeleton, with large teeth and faces that overlap in size with those of later Homo erectus. This second group is known to date between 9,000 and 12,000 years ago at Kow Swamp in Victoria, although the morphology 
might suggest that its ancestors arrived in Australia long before that. Superficially, these two groups could overlap with the Barrinean and Murrayian groups of Birdsell, but Thorne's opinions on the affinities of specific fossils differ considerably from those of Birdsell.

According to Thorne (1980a, b; Thorne and Wolpoff 1981; Sim and Thorne 1990), these two groups represent well-defined skeletal populations of quite different morphology. His suggestions have very clear-cut implications for Australo-Melanesian differentiation within the Indo-Malaysian Archipelago. Thorne's theory is that the Kow Swamp population, plus other "rugged" skulls from various parts of Australia, represent an initial settlement (not a secondary one as suggested by Birdsell) by an Indonesian population derived from a line leading back directly to the Homo erectus population of Ngandong in Java. The other more gracile group, which is represented by the Niah, Wajak, and Tabon remains in Southeast Asia, is thought to represent a second and probably later migration from at least Indonesia, with the possibility of an ultimate Chinese source. As Thorne has stated (1980a:100):

Remains from sites in China, particularly at Liu-Kiang (Liujiang) and Choukoutien (Zhoukoudian), suggest the possibility that the ultimate source of the gracile people of Australia and Indonesia is to be found there.

The implications of both Thorne's and Birdsell's views for the Indo-Malaysian archipelago as well as for Australia are considerable, for if they are correct about a "Chinese connection" during the late Pleistocene, it may be unwarranted to regard the Southern Mongoloids of the Indonesian region as entirely the descendants of a population expansion confined only to the Neolithic and later periods (i.e., confined to the period of Austronesian expansion). Seen in this light, these views may come partly into line with the regional continuity scenarios favored by Turner and Bulbeck. The Indo-Malaysian Archipelago has never been isolated from mainland Asia in the period of modern humans, and population movement, usually on a very small scale but with possibilities for extensive radiation, has always occurred. The same applies to Australia, although here the school of thought that proposes a single origin for all Aborigines seems to be prevailing at the moment.

\section{E. Some Further Observations}

I will finally turn back to the views expressed earlier concerning cultural capacity to support a major population expansion. Small groups of hunters and gatherers might be expected to expand under favorable circumstances, particularly into suitable environments previously uninhabited, but major and rapid migrations into territories already settled by groups with equivalent grades of techno- 
logical and economic organization would be unlikely. The Australian case just described may be a significant and unusual exception if Thorne's interpretation is correct, but in overall support of the generalization it should be noted that studies in the 1950s on resettled Malaysian orang asli groups of forest collectors and recently acculturated shifting cultivators showed fairly clear evidence for decreasing nutritional health and increasing disease (Polunin 1953). Groups such as these, which have been adapting to highly specific local environments for millennia, do not take kindly to upheaval.

On the other hand, it is clear that groups longer adapted to a horticultural lifestyle focused on the partial creation of artificial environments can adapt to movement more easily; the ancestral Polynesians and Micronesians were clearly well adjusted to this strategy, and I suspect that the earliest Austronesian colonists of Indonesia were also. It is amongst these that we can surely find the most significant evidence for any Southern Mongoloid expansion into the Indo-Malaysian Archipelago that might have occurred. I will return to these questions in Chapter 7.

Concerning such pre-Austronesian or pre-Austroasiatic expansions of mainland Asian populations into the Indo-Malaysian Archipelago as might have been involved in an early but secondary colonization of Australia, one can only surmise. Research still has a long way to go in this region and many of the answers will always be elusive. But while it may be unwise to equate entirely the Southern Mongoloid phenotype now present in the archipelago with the linguistic evidence for the past expansion of Austronesian agriculturalists, I am prepared to state my belief that the correlation must be at a very high level. 


\section{Recent Indo-Malaysian Prehistory: According to the Languages}

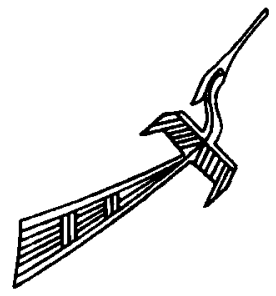

Once they had split up each group forgot the past customs they had enjoyed together and developed different languages because some had short tongues and others long tongues.

Each group found a new name for itself. (From a story related by a Penan headman, Sarawak: Arnold 1958)

The modern traveler who has the good fortune to wander at will through the Indo-Malaysian region will quickly observe that there are many traditional varieties of culture and subsistence economy. This remains true even when one considers the tremendous impacts of modern urbanization and industrialization. Furthermore, there are great variations in language, although these are often not noticed by outsiders owing to the increasing strengths of the national languages, Bahasa Melayu and Bahasa Indonesia (these are actually the same language, originally Malay, with small dialect differences). Linguists in particular have developed precise techniques for drawing inferences about the histories of language families. I consider that any general statements concerning the prehistory of the Indo-Malaysian region within the past 5,000 years must take the linguistic evidence seriously into account. This is especially true for any discussion of recent human population expansion. One cannot work purely from the archaeological data alone. 


\section{Language families IN SOUTHEASt ASIA AND THE WESTERN PACIFIC}

Almost all the peoples of Indo-Malaysia speak languages termed Austronesian (Wurm and Hattori 1983; Pawley and Ross 1993; Bellwood et al. 1995). There are two small exceptions: the Orang Asli of interior Peninsular Malaysia, who speak languages (called Aslian) in the Austroasiatic family; and some peoples of eastern Indonesia who speak Papuan languages, a group of diverse families centered on New Guinea. The Aslian languages are considered in some detail in Chapter 8, while the Papuan languages will be considered at the end of this chapter. When looking at Austronesian linguistic prehistory and the relationships of Austronesian to outside families, research cannot be restricted to Indonesia and Malaysia alone. I will paint a broad picture to begin, and then focus in detail on more localized questions.

Estimates of the number of Austronesian languages now range up to about $1,200,1$ thus making it perhaps the largest in the world in terms of language number and certainly the largest in terms of precolonial extent: over half way around the world, from Madagascar to Easter Island (Fig. 4.1). Over half of these languages are spoken in Oceania, from New Guinea eastward. Present Austronesian speakers are roughly distributed as follows: Indonesia 190 million, Malaysia 18 million, Philippines 66 million, Taiwan 330,000, interior Vietnam 500,000, Madagascar 12 million, and Oceania about 2 million (SBS 1992; Tryon 1995a). Most of the dispersed languages in the vast region of Oceania clearly have very small speech communities.

The Austronesian languages have a geographical distribution that is relatively unbroken except for the outliers in Madagascar and southern Vietnam, the latter having been isolated by recent Vietnamese expansion. There are four language groupings that abut directly on the Austronesian sphere of distribution (Ruhlen 1987) (Fig. 4.2). The first is the Papuan complex, which has about 750 languages in many separate phyla (to use the terminology most favored by linguists working in this region), in New Guinea, western island Melanesia, and a few islands in eastern Indonesia (Wurm 1982; Foley 1986). The second is the Austroasiatic family of Mainland Southeast Asia, a scattered group of about 150 languages (Diffloth 1979; Parkin 1991) that includes Aslian of Peninsular Malaysia, Mon-Khmer, Vietnamese, Nicobarese, some languages in Assam, and the Mundaic languages of northeastern peninsular India. The third comprises the Tai (or Daic) family (Thai, Lao, and related languages) of the central and northern mainland, extending up into southern China (Lebar et al. 1964; Ruhlen 1987). The fourth is the large Sino-Tibetan family (Matisoff 1991), although the main contact between this and Austronesian 


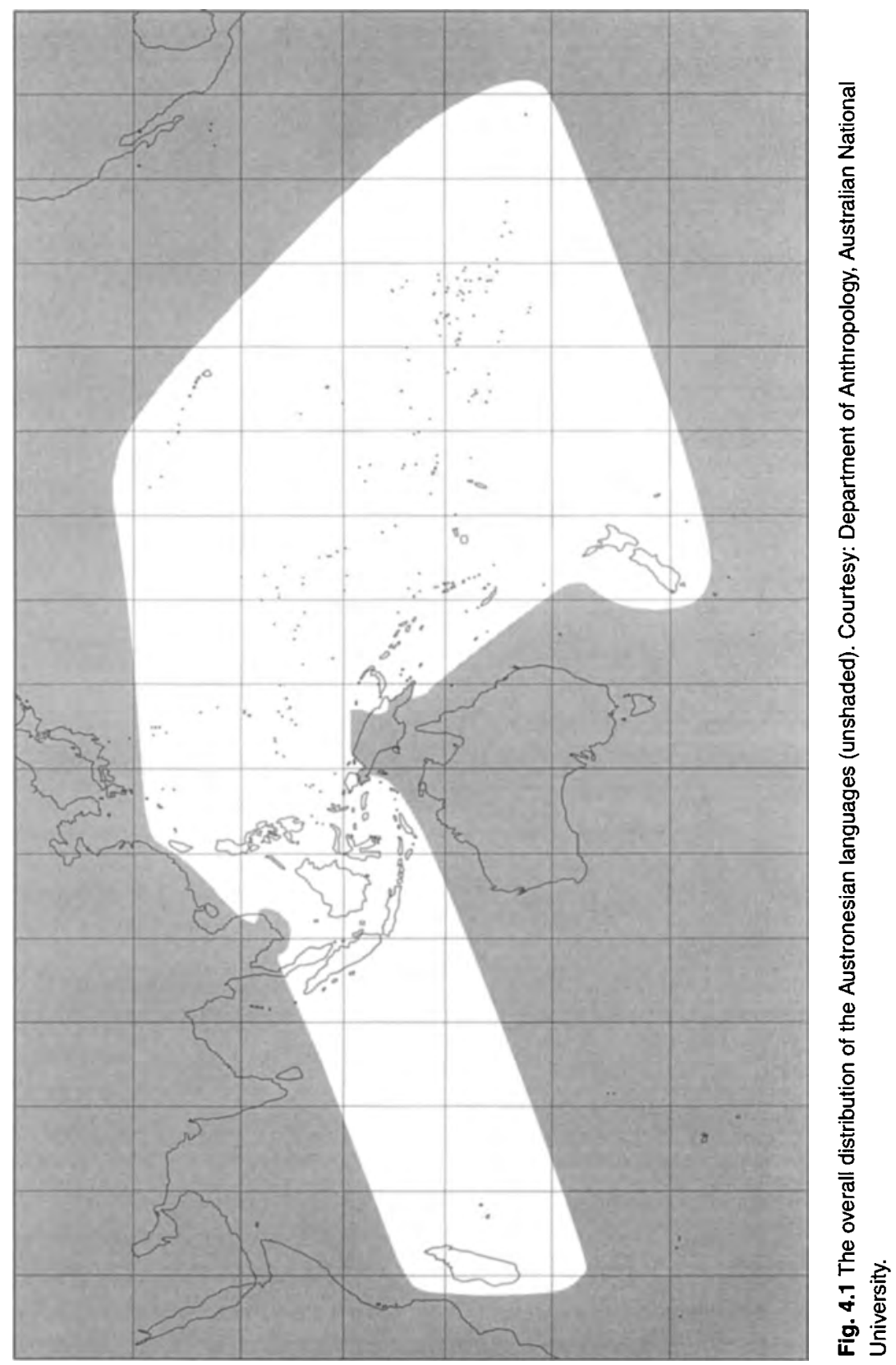




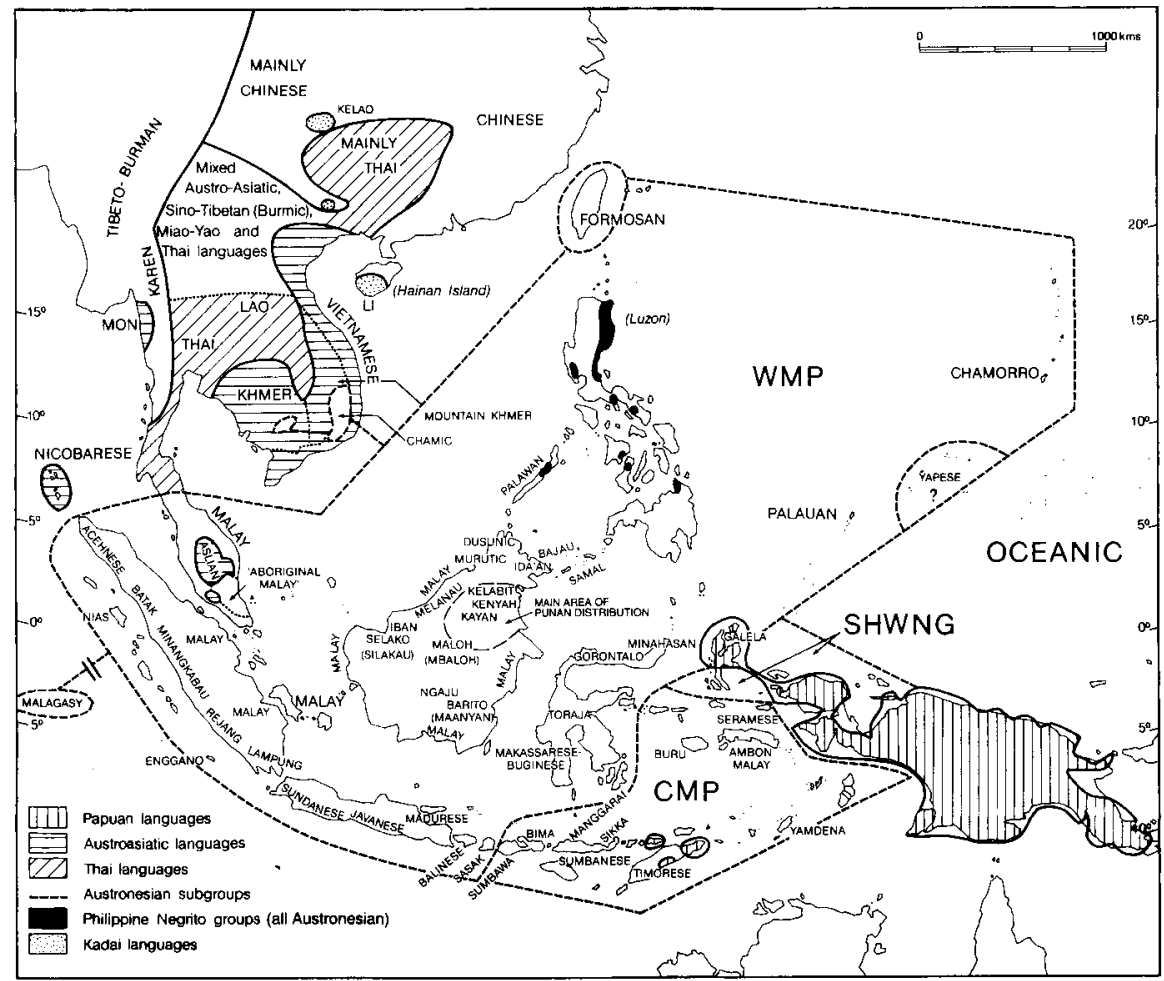

Fig. 4.2 Languages, major Austronesian subgroups, and other language families in Southeast Asia. (Note: "Thai" is nowadays normally spelled "Tai" for the whole family, which includes modern Thai.)

occurs in Taiwan and is of historically recent origin (mainly post-seventeenth century).

When considering geographical distribution alone, the extent of the Austronesian languages is indeed very impressive. It is my view that the expansion of this language family has involved an actual expansion of Austronesian-speaking founder communities through this vast area (Bellwood 1991, 1995a). This may seem self-evident, especially for Oceania, although the possibilities of adoption of Austronesian languages by members of unrelated linguistic groups cannot be entirely disregarded, especially in western Melanesia and amongst the Negritos of the Philippines (Ross 1994; Reid 1994a). If such an expansion has occurred, then it would appear to have reduced the area of prior distribution of the Papuan and Austroasiatic languages, with the exception of Vietnamese. The questions for Austronesian expansion are: When did it occur? How did it occur? and Why did it occur? 


\section{SOME LINGUISTIC CONCEPTS}

According to Swadesh (1964:575), a linguist who has made great contributions to prehistory:

There are three main ways in which linguistics can illuminate prehistory: (a) by establishing facts concerning the common origin and subsequent divergence of languages, implying the earlier unity and subsequent separations of peoples; (b) by discovering diffused features (of phonetics, structure and vocabulary) among languages which bear evidence of prehistoric culture contact; and (c) by reconstructing the vocabulary of old stages of languages so as to bring out suggestions of the physical environment and content of prehistoric cultures.

A number of terms linguists use in their deliberations now require a brief introduction (for a more detailed explanation, see Crowley 1992). Dialects of a language share a common basic vocabulary-usually over 80 percent-sufficient to remain mutually intelligible. If they diverge enough to become no longer mutually intelligible, they become separate languages. This separation is of course gradual as intelligibility does not come or go with one percentage point, and the development of two separate daughter languages from one parent can take a millennium or more, especially if contacts continue (the degree of continuing contact versus separation is essential in this regard). ${ }^{2}$

Languages considered to be related are grouped into families (or phyla in the terminology favored by Papuan linguists), such as Austronesian or Indo-European. All languages are probably related in the final resort, since the roots of language must go back with increasing convergence through all levels of anatomically modern human evolution. But, for comparative purposes, relationship has to be observable in phonology, structure, and vocabulary. When languages have been evolving apart for something over 10,000 years, it seems that such observations can no longer be made with confidence.

Languages in a family can be divided into subgroups with ordered implications for time depth; closely related languages (closely related, that is, in terms of shared innovations) have more recent common ancestors than those more distantly related. Hence, subgroups are hierarchical in terms of geographical space and time depth. A subgroup can be defined as "any group of languages which have passed through a period of common development exclusive of the other languages of the same family and during which period some linguistic change has occurred" (Grace 1959). Uniquely innovated shared cognates of restricted geographical occurrence help to define subgroups, while those that occur within and beyond a particular subgroup are defined as "shared inheritances" (not innovations), and as such refer to a deeper level of subgrouping. A 
cognate is a word deemed through correspondences in sound and meaning to have been inherited by two or more languages from a common ancestor, rather than borrowed from an outside language.

Members of a subgroup also share a common proto-language, an entity reconstructed by identifying cognates (commonly inherited forms) found in daughter languages; the more widespread the cognate, the farther back the protolanguage for which it can be reconstructed. Like subgroups, proto-languages are also hierarchical in space and time, each one corresponding to the base of a subgroup branch in the family tree. Minor proto-languages on the "outer branches" of Austronesian can be shown to have been single languages or dialect chains in many cases, but when moving toward the main trunk and back in time the picture becomes more diffuse and complex. An entity such as ProtoAustronesian (Table 4.1), which existed perhaps 5,000 years ago, may or may not have been a single language as opposed to a chain of related dialects-it is possible that we will never know for certain. However, we can rule out the possibility that it comprised a group of completely unrelated languages; whatever its precise nature, Proto-Austronesian must have been a relatively homogeneous linguistic entity of somewhat restricted geographical extent.

I must also emphasize that the entity reconstructed as Proto-Austronesian is in no real sense a "beginning" for Austronesian; it was not created de novo. What the concept refers to is that point at which the language or chain of languages ancestral to all modern Austronesian languages underwent an initial divergence into two subgroups whose descendant languages have survived essentially apart to the present. Proto-Austronesian is preceded by a phase I will call Initial Austronesian, which in turn goes back to the point at which the ancestral language for the whole Austronesian family split off from its contemporary sister languages. The latter have either developed along different lines to become other modern language families (the Austroasiatic, Tai, and perhaps even Sino-Tibetan families may have this kind of relationship with Austronesian; see below), or some might have become extinct. If they were not written we may never know about this second possibility, but language termination is a common event in linguistic history.

The ancestry of Austronesian thus goes well back beyond the proto-stage, and the family may be visualized as a very large and multibranched limb on the total linguistic tree of mankind. Where the limb departs from another bigger limb (which will in turn join back through others to the ultimate trunk), it is convenient to place the label "Initial Austronesian" (Fig. 4.3). We can only get back beyond the start of this limb by comparing Austronesian languages with those in other families, and many linguists have claimed significant results from such comparisons. Some of these results will be discussed later. 


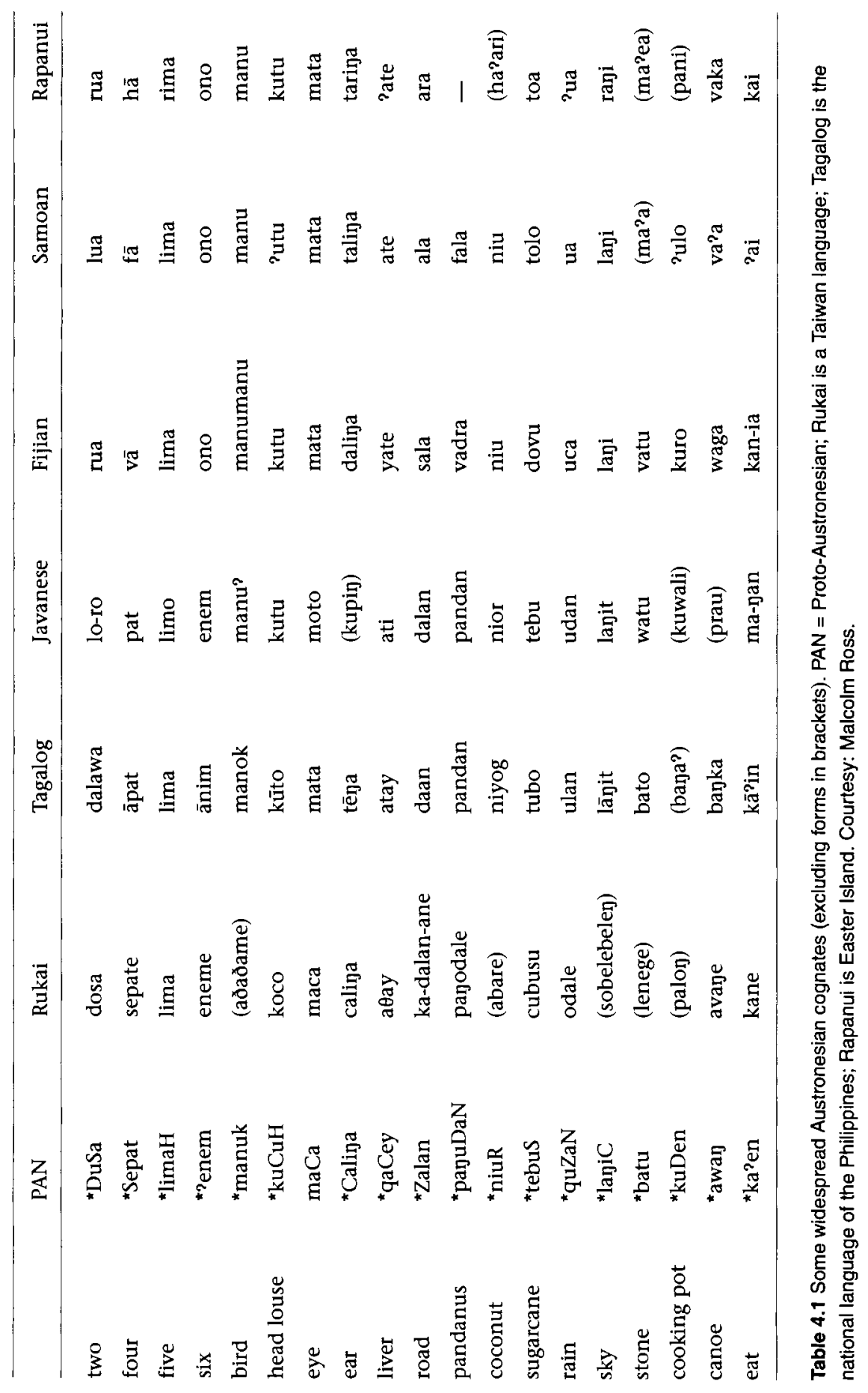




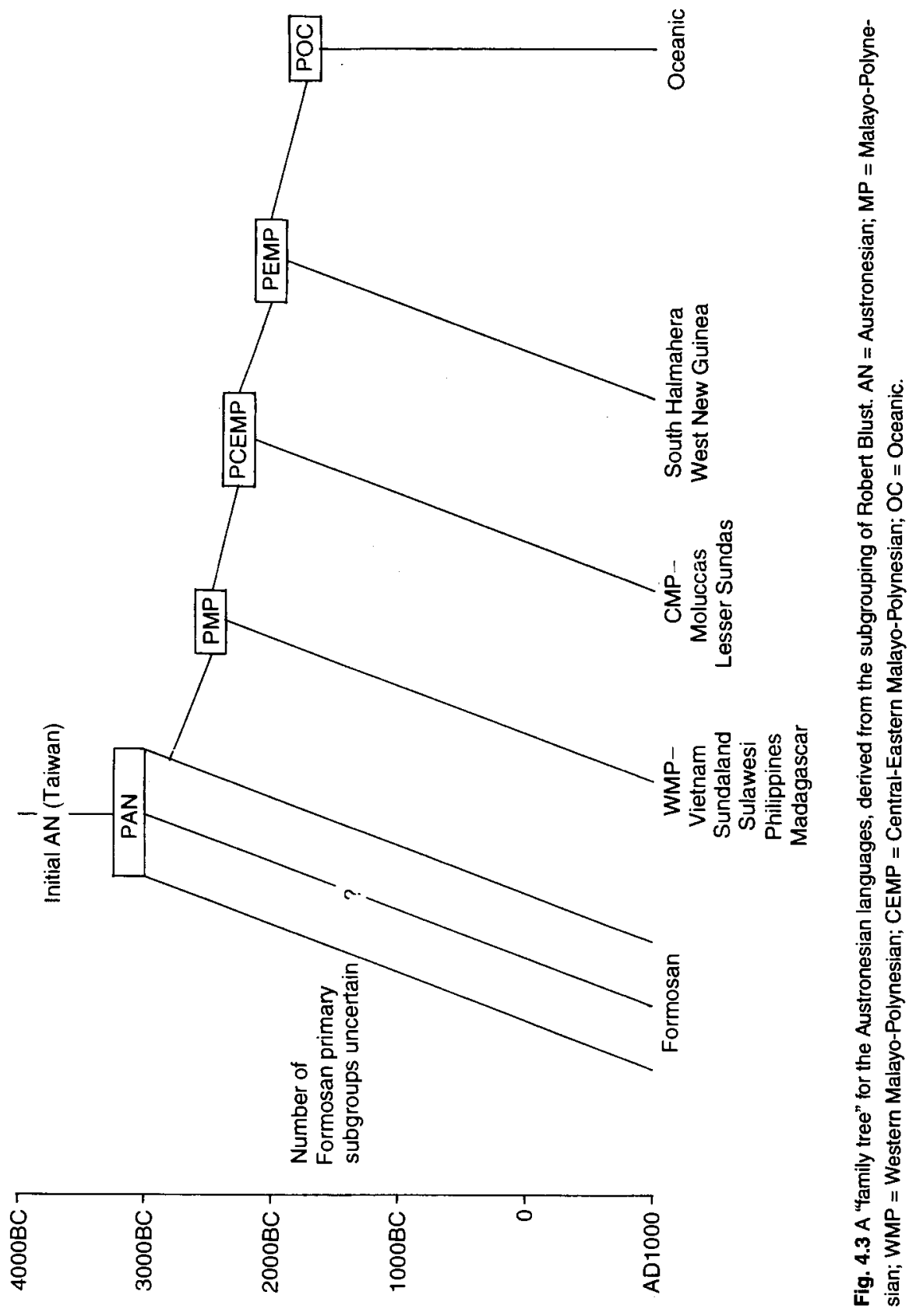


Lexical cognates are also utilized in the subdiscipline of lexicostatistics, which subgroups languages hierarchically in terms of percentages of cognates between all pairs of languages within a group. I will return below to the assumptions of lexicostatistics and its chronological offshoot (glottochronology) when I come to consider the crucial question of the rate of linguistic change. I should, however, make it clear that languages are not subgrouped purely by comparing words; indeed, the technique of lexicostatistics is regarded with suspicion as a classificatory device by many linguists. Our basic knowledge of Austronesian linguistic prehistory comes from much deeper comparisons in the complex fields of grammar (for example, word order in sentences, occurrences of prefixes and suffixes, pronoun forms, verb structures) and phonology (the study of the sound changes through which different languages have progressed). Lexicostatistics alone can in fact lead to a quite erroneous view of Austronesian linguistic prehistory. This is because great lexical diversity alone does not automatically correspond with great time-depth, although there is certainly much truth in the view that great overall linguistic diversity does (Dyen 1975).

\section{THE MAJOR SUBGROUPS OF AUSTRONESIAN}

Comparative linguists are now in general agreement about the basic shape of the Austronesian family tree. Most today use the classification developed by the linguist Robert Blust $(1977,1978,1982,1993,1995 a)$. This classification is based on the occurrences of shared innovations, especially in phonology and in pronoun forms, at the lower levels of the family tree (for details see Ross 1995a; Tryon 1995b). It divides the Austronesian family into at least two major subgroups, of which one, Malayo-Polynesian, includes all Austronesian languages not located in Taiwan (see Fig. 4.3). The Formosan languages themselves include at least one major subgroup, and perhaps between three and six according to recent research (Reid 1982; Li 1985; Blust 1995b). Arguments against the view that the Formosan languages represent one or more primary subgroups have been put forward by Dyen (1995) from a lexicostatistical viewpoint, and by Wolff (1995) from a counterperspective on pronoun forms, but in my view Blust has presented convincing rebuttal (see also Pawley and Ross 1993; Ross 1995b, for support on the Blust tree from verb morphology).

Since the first edition of this book was published, the Blust classification has become almost universally adopted by those linguists who use the comparative method of reconstruction at all levels of language-rather than simply lexicostatistics-and I have decided to delete some of the historical discussion included in that edition. The Blust classification also fits well with the evidence of Island Southeast Asian archaeology (see Chapter 7), although this independent evidence cannot be used directly in its support against other possible sub- 
grouping schemes. But certainly, at the present time, there is no other convincing competitor.

The major subgroups of Austronesian are therefore as follows:

1.1. Formosan (possibly three or more primary subgroups)

1.2. Malayo-Polynesian (all Extra-Formosan languages according to Blust, although Reid [1982] excludes some Northern Philippine languages from Malayo-Polynesian and places them in a separate subgroup intermediate between 1.1 and 1.2; see also Starosta [1995] for an even more complex tree at this level)

2.1. Western Malayo-Polynesian (Philippines, Vietnam, Madagascar, Peninsular Malaysia, Sumatra, Java, Borneo, Sulawesi, Bali, Lombok, western Sumbawa, and two languages-Palauan and Chamorro-of western Micronesia)

2.2. Central-Eastern Malayo-Polynesian (3.1 plus 3.2)

3.1. Central Malayo-Polynesian (Lesser Sundas from eastern Sumbawa eastward, Moluccas except Halmahera)

3.2. Eastern Malayo-Polynesian (South Halmahera and all the Pacific Island Austronesian languages; Melanesia, Micronesia, and Polynesia: 4.1 plus 4.2)

4.1. South Halmahera-West New Guinea

4.2. Oceanic (all Eastern Malayo-Polynesian languages except 4.1)

By translating this family tree into an account of the expansion of the Austronesian speakers, the following founder moves can be listed in chronological order (Blust 1984-1985, 1996):

1. Initial Austronesian and Proto-Austronesian should be located in Taiwan. The founders of the Malayo-Polynesian subgroup then moved into relative separation (maybe even absolute separation) in the Philippines, via Luzon, leaving the other speakers of Formosan languages behind.

2. Founders moved south through the Philippines, then west into Borneo and Sulawesi, and later toward Java, Sumatra, Peninsular Malaysia, and Vietnam. These regions contain the Western Malayo-Polynesian (WMP) languages, which do not comprise a single subgroup (the internal structure of the totality of WMP has not yet been completely defined; some discussion on this follows later).

3. Other founders moved east and south into the Moluccas and Lesser Sundas, giving rise to Central-Eastern Malayo-Polynesian (Blust 1993).

4. Further founder movements gave rise to a separation between Central Malayo-Polynesian and Eastern Malayo-Polynesian, the latter spreading rapidly onward via Halmahera and the Bismarck Archipelago into Oceania. 
It should be noted from the above that the Austronesian languages did not spread into Indonesia via the Malay Peninsula, even though this route was favored at one time (e.g., by Heine Geldern 1932).

\section{A. The Location of Proto-Austronesian}

I have already made it clear that the island of Taiwan is central to the question of the location of Proto-Austronesian. This conclusion has been reached with increasing frequency in recent years, for instance by Dahl (1973), Shutler and Marck (1975), Foley (1980), Harvey (1982), Reid (1984-1985), and the more recent authorities listed above. Many earlier authors, using the results of traditional comparative linguistics, had concluded that the location of Proto-Austronesian would have to lie somewhere in or close to the western region of the Austronesian distribution (excluding Madagascar). For example, Kern (1889/ 1917) favored the Vietnamese coastal region, Haudricourt (1954) favored the coastal region between Hainan and Taiwan, and Benedict (1975) has for the past forty years been strongly in favor of linking Austronesian through Taiwan with the Thai and Kadai languages of southern China. Blust (1982) has shown that words for placental mammal species (pangolin, bovids, monkeys) reconstructed for the Proto-Austronesian vocabulary make a location west of Huxley's Line-in Taiwan or Sundaland-a virtual certainty, although it should be noted this view does depend upon acceptance of Blust's family tree. Indeed, all protolanguage vocabularies can only be reconstructed with certainty if the precise family tree of the language family concerned is also known with certainty, and this is a field where certainty may always be a little elusive.

Nevertheless, taking all these views into account and noting recent trends, there now seems little reason to doubt Taiwan as by far the most likely location for Proto-Austronesian. The most important arguments against Taiwan in recent years have been those by Dyen, whose lexicostatistical classification of the Austronesian languages (Dyen 1962, 1965a) showed that lexical (but not phonological) diversity was higher in western Melanesia than in any other Austronesian region (Fig. 4.4). Dyen's theories on unvarying overall rates of lexical change through time led him to suggest western Melanesia (particularly the Bismarck Archipelago) as the location of Proto-Austronesian-a claim supported by Murdock (1964), but seen today by most authors (including myself) to be impossible, for reasons not connected with linguistics alone (Bellwood 1978: 131-132). I will be returning to Dyen and the question of Melanesia later, but it should be noted that he, as a linguist of broad and respected knowledge, has long been ambivalent about the Formosan languages and has at times admitted their qualifications to represent a primary subgroup of Austronesian (e.g., Dyen 1965b). 


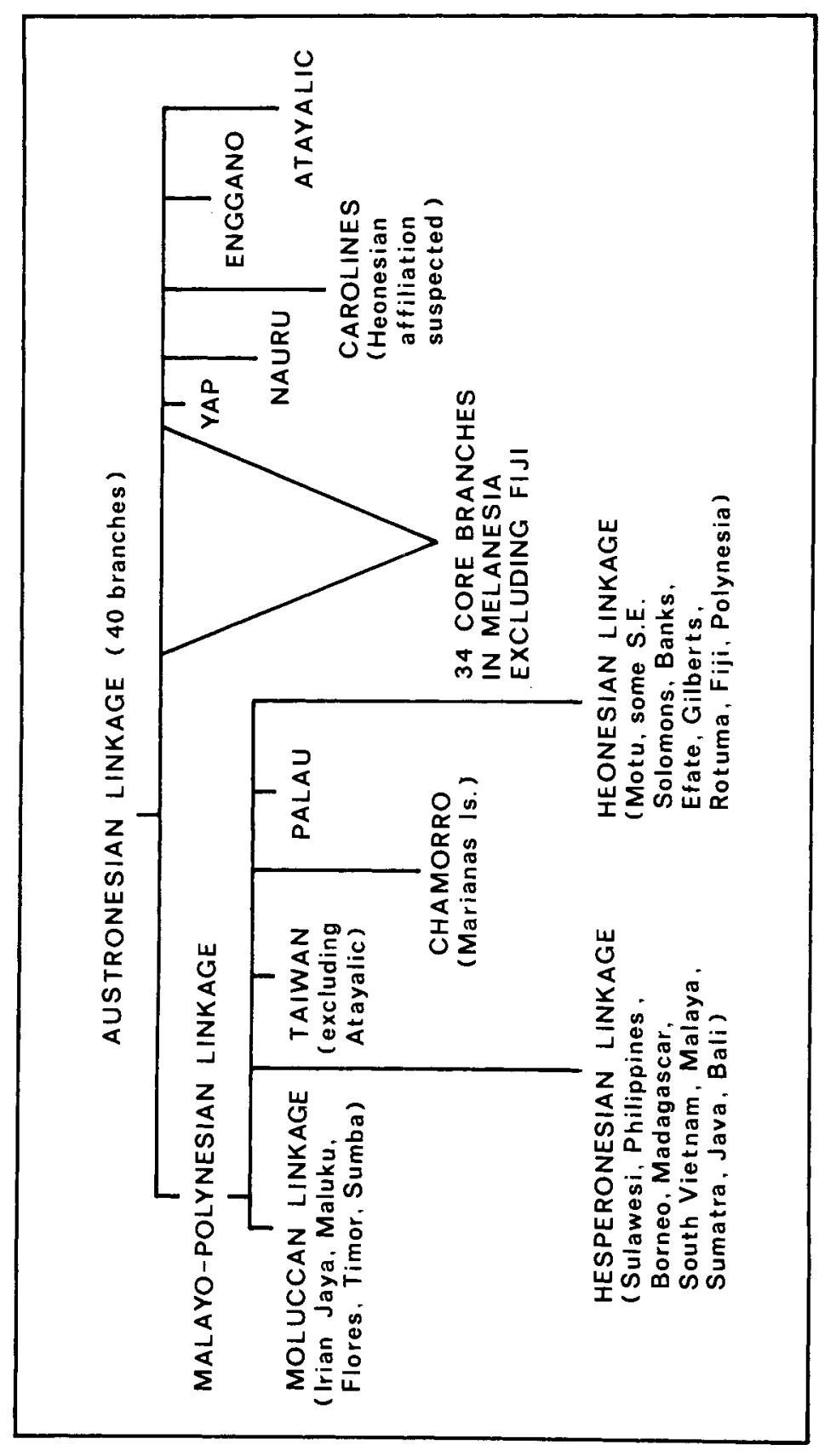

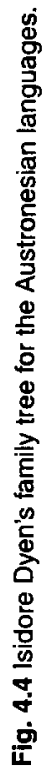




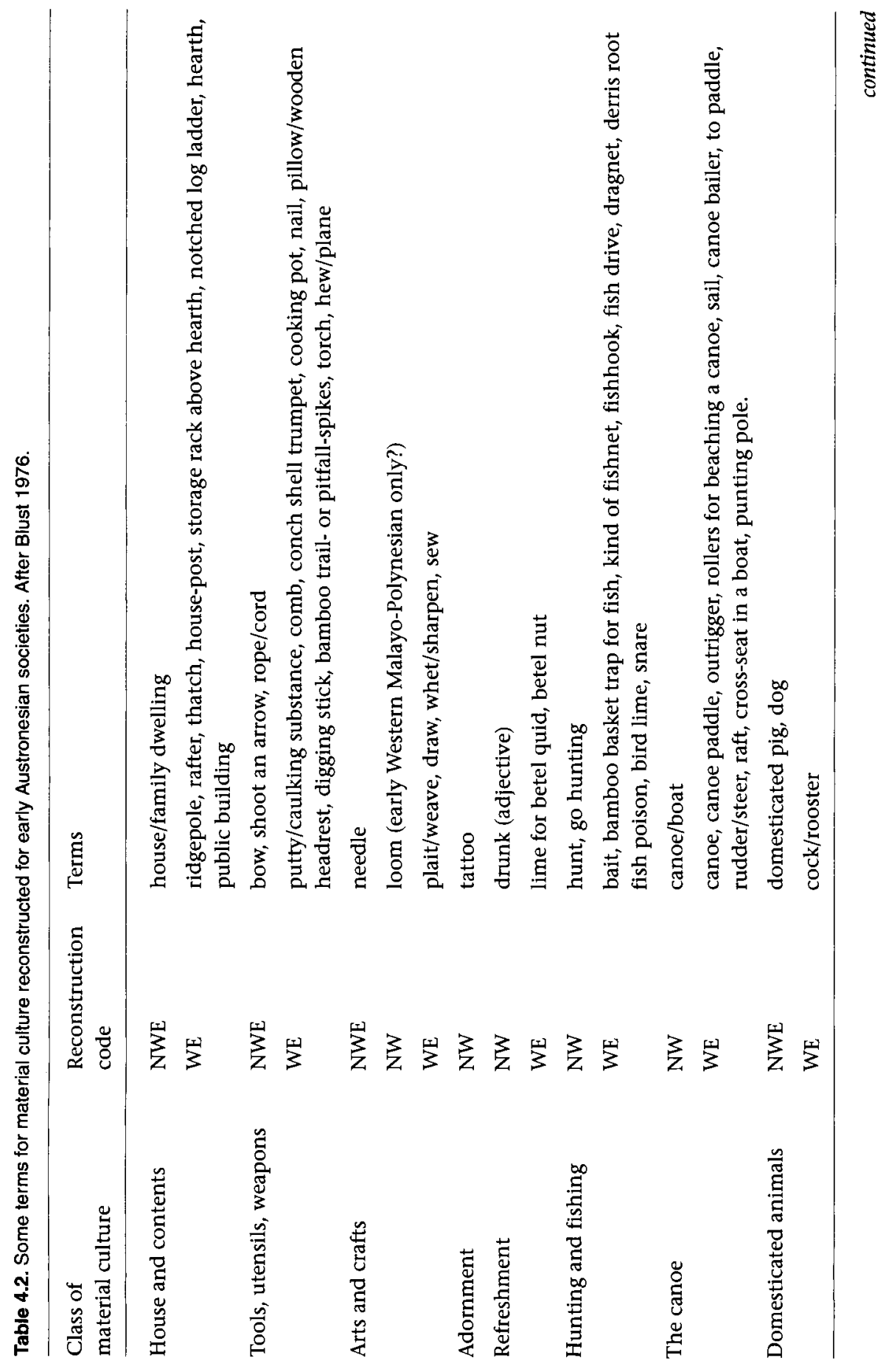




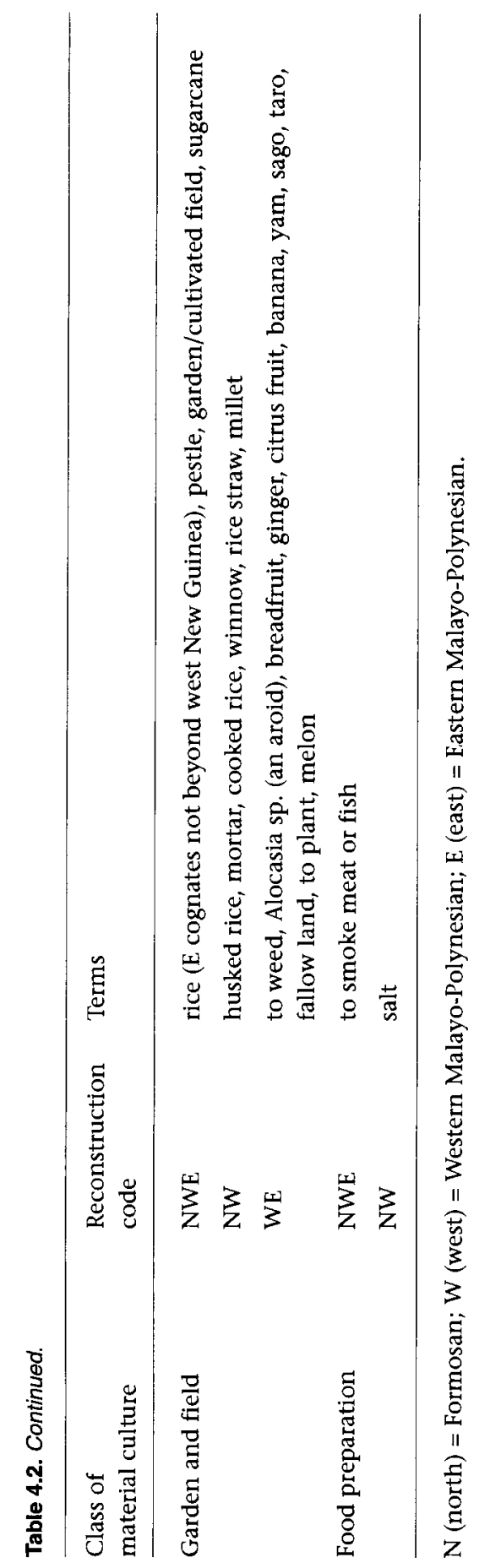


In coming down strongly on the side of Taiwan as the location of ProtoAustronesian, I should make it clear that I do not regard this small island as the absolute "homeland" of the Austronesians. It is simply the place where Initial Austronesian was established and where the first split within reconstructed ProtoAustronesian occurred. The true homeland of the ancestral Austronesians (before the Initial stage) was without any reasonable doubt on the mainland of southern China.

\section{B. The Material Culture of the Proto-Austronesians}

Words and meanings can only be reconstructed for Proto-Austronesian if cognates are found in the languages of two or more of the primary subgroups (Formosan and Malayo-Polynesian) and if borrowing can be positively excluded. The catch here, of course, is that the shape of the family tree must be known with certainty in order to reconstruct a proto-language with total conviction, and as I have indicated above there are still disagreements about this, although I have chosen the tree suggested by Blust as the most convincing one currently available. Nevertheless, despite disagreements, it is generally accepted by all linguists that words having cognate forms in at least one language in the Formosan, Western Malayo-Polynesian, and Eastern Malayo-Polynesian major divisions are almost certain candidates for Proto-Austronesian (see Table 4.1).

A moment's thought will suggest that words reconstructed from Western and Eastern Malayo-Polynesian alone without Formosan cognates cannot automatically be considered as Proto-Austronesian; they can only truly be regarded as Proto-Malayo-Polynesian. Since Proto-Austronesian and Proto-Malayo-Polynesian may be separated by half a millennium in time and a subtropical versus a tropical latitude (i.e., Taiwan versus the Philippines), I believe this distinction to be quite significant. Words for truly tropical plants such as sago and bread-fruit can only be reconstructed for Proto-Malayo-Polynesian, exactly as expected. Words for colder climate species such as rice, millet, and sugarcane go back to Proto-Austronesian, and so these clearly were grown in Taiwan. The fact that there is quite an expanse of latitude here across an important zone of change in world climate clearly makes interpretation a little complex. In Table 4.2, items marked NWE or NW are potentially Proto-Austronesian (NE never occurs), and items marked WE are potentially Proto-Malayo-Polynesian and only possibly Proto-Austronesian. To simplify matters I have not added the Proto-Austronesian reconstructions themselves, and I will leave reconstructions of aspects of society for discussion in the next chapter.

It will be noted from Table 4.2 that most reconstructions are defined as WE, and few have cognates in Formosan. This no doubt reflects the limited information available on some of the Formosan languages (most Formosan aboriginal 
populations now survive inland and have been heavily acculturated to the dominant Chinese population), but another reason may be that Austronesian cultures underwent marked change as they moved southwards into tropical regions, as I will demonstrate later. This becomes rather obvious when one examines the garden and field section, and also canoe terminology (on which see Pawley and Pawley 1994).

I will be making further observations on this list in due course, but it should be noted that the domesticated animals do not include any herbivores (cattle, sheep, or goats). Another point, made by Pawley (1981) and by Blust (1976), is that sound correspondences suggest strongly that material culture traditions (potting, agriculture, fishing, and so forth) were continuous-they were never lost by any widespread groups of Austronesians and later regained through reinnovation or external borrowing. The main point to be noted, however, is that Proto-Austronesians clearly were agriculturalists and had domestic pigs and dogs. In addition, Proto-MaIayo-Polynesians certainly made pottery and sailed between islands in canoes (see also Zorc 1994) ${ }^{3}$ In archaeological terms, these were Neolithic communities, albeit still fishers, gatherers, and hunters wherever these activities were profitable.

\section{The Antecedents of Proto-Austronesian}

The cultural relationships of the Initial Austronesians who crossed from mainland China to Taiwan are universally agreed to be with the mainland, rather than with the island areas to the south, which at this early date were still inhabited by hunters and gatherers who presumably spoke non-Austronesian languages now extinct. Only the Philippine Negritos survive as a distinct biological population from this early phase in the islands directly south of Taiwan, and they have now universally adopted Austronesian languages related to those of their Filipino neighbors (see Reid 1994a, 1994c for a possible non-Austronesian substratum incorporated in Philippine Negrito languages).

One proponent of Austronesian links with mainland languages has been Paul Benedict, who first suggested in 1942 that Austronesian, Thai, and a small group of isolated languages in Hainan and southern China (which he called Kadai) could be classified into one large macrofamily. Since 1942, Benedict has added the scattered Hmong-Mien (formerly called Miao-Yao) languages of southern China and northern Mainland Southeast Asia to the Thai-Kadai-Austronesian family and has coined the term Austro-Thai (now better spelled Austro-Tai) to refer to the whole group (Benedict 1975). He has also reconstructed a large Proto-Austro-Tai vocabulary, which includes terms for field, wet field (for rice or taro), garden, plow, rice, sugarcane, the betel nut complex, cattle, water buffalo, bow and arrow, axe, and canoe. Some of these items are also found in Proto- 
Austronesian, while others, such as cattle and water buffalo, are not, although this may simply mean that these animals were not taken off the mainland during the early period of Austronesian expansion.

If Benedict is right, then these reconstructions apply to Neolithic societies in southern China, whose archaeological remains will be described later. They have always seemed to me to be quite reasonable from a prehistorian's perspective, and Benedict's general views have been supported in part by other linguists such as Dahl (1973) and Reid (1984-1985). However, since the first edition of this book was published, in which I accepted Benedict's views without provisos, the concept of Austro-Tai as a macrofamily of genetically related languages (i.e., languages sharing a common origin) has come under attack. In the past few years there have been several detailed reviews of macrofamily hypotheses involving Austronesian, and one that is now more favored than Austro-Tai is the "Austric" hypothesis, which suggests that Austronesian and Austroasiatic are genetically related at a deep level (Blust 1996; Diffloth 1994; Reid 1994b). This hypothesis was first suggested by Schmidt in 1906 and has had rather a checkered career since that time, but Blust's support is currently quite strong, and Austric has recently received archaeological support from Charles Higham (1996). For Austro-Tai, one current hypothesis (Thurgood 1994) is that the resemblances relate to early borrowing rather than true genetic relationship. Other suggestions exist to the effect that Austronesian may be related to Chinese (Sagart 1994), and even Japanese receives periodic attention as having an-cient Austronesian affiliations, although the evidence here is not widely accepted (Vovin 1994).

From the viewpoint of prehistory, these macrofamily hypotheses are quite important (Bellwood 1994). Whether Austronesian relates to Tai or Austroasiatic and whether the relationship is one of common origin or ancient borrowing, the conclusions are almost the same in terms of historical significance. Ancestral Austronesian languages, prior to the colonization of Taiwan and the period of Proto-Austronesian, were part of a geographical network of languages on the mainland of southern China. These languages were still perhaps undifferentiated into the clear and separate ancestors of existing families, and they formed a network that also included the seeds of early Austroasiatic and early Tai languages, and perhaps also early Sino-Tibetan languages-especially ancestral Chinese according to Sagart, with apparent support from Egerod (1991). The ultimate roots of Austronesian therefore lie in the Neolithic cultures of southern China, a topic to be explored further in Chapter 7. The earliest Austronesians grew rice and probably expanded into the Southeast Asian islands through processes of agricultural demographic growth, as well as through a culturally motivated desire to find new lands. A few archaeologists still refuse to accept this view of a Chinese regional origin for Austronesian, overlooking the details of 
the linguistic evidence (Meacham 1984-1985), but for many years now I have been unable to take such objections very seriously (Bellwood 1984-1985, 1991). Austronesian prehistory is now becoming well founded from a multidisciplinary perspective.

\section{DATING THE AUSTRONESIAN FAMILY TREE}

Before commencing a closer look at linguistic prehistory within Indonesia and Malaysia, I want to digress into the important question of linguistic dating. How can we date reconstructed entities such as Proto-Austronesian? Dating is one of the great scaffolds of prehistory and one that linguists cannot erect easily by themselves. In theory, if the packages of basic vocabulary items (i.e., words for noncultural terms-such as man, woman, and sky-usually compared in lists of 100 or 200 meanings) used for lexicostatistical classifications can be shown to have changed at a known and constant rate through time, then proto-languages should be datable by a mathematical comparison of the shared cognate percentages between selected pairs of their daughter languages. Thus, in theory, all the major splits in the Austronesian family tree can be dated-a feat of no small importance for prehistory-assuming of course that one can know the rate of vocabulary change over a fixed period of time, such as a millennium. This is the basis of the technique of glottochronology, to be discussed further below.

But do such constant rates really exist? Let us start with some simple assumptions about how dialects start to diverge amongst populations who use only verbal communication methods (i.e., without literacy, mass communication media, and so forth). For instance, it may be assumed that people of one ethnic group who live contiguously and interact freely with each other throughout the whole group will maintain a single language over time, regardless of changes that occur within the language itself as a result of the passage of time or borrowing. If a few families move off to a new area and maintain only diminished contact with the main group, they will slowly develop a different dialect, but as long as mutual intelligibility continues to be required, the new group will tend not to drift across the boundary into a separate language (as defined by lack of mutual intelligibility) - unless they become effectively cut off. In a sense, this process of becoming "cut off" may be regarded as optative and as reflecting social structure; in atomizing tribal polities, one may expect it to be more frequent than in large, integrated civilizations.

It may also be assumed that the rate of divergence between two communities speaking an initial common language will be somewhat faster if they are totally separated than if they maintain contact, and much faster if the speakers of one or both languages are in frequent bilingual contact with speakers of other unre- 
lated languages. This introduces an important factor: Linguistic differentiation entirely from within a network of closely related dialects, as might occur on small isolated islands with no external contact or bilingual skills in totally "foreign" languages, will inevitably proceed more slowly than in cases where such isolation is lacking. Such slow differentiation seems to have been the case on large landmasses settled by essentially one language community, such as Madagascar and New Zealand (Pawley and Ross 1993). As for bilingualism, it does not homogenize language or cause individual languages to spread great distances, but it does lubricate diversification; more on this below in the Melanesian context.

In situations where communication over a large region is slowed by distance or difficult terrain but not broken, a sufficiency of time will often produce a dialect chain. In such situations, dialects at distant ends of the chain can often be mutually unintelligible (and thus actually different languages), but the differences will only be very gradual as one moves along the chain, unless it is broken up by expansion of groups within it or by the moving in of unrelated language groups. The existence of such chains does not stop linguistic change, but it may well act to slow it down because linguistic innovations will tend to be widely shared and overlapping in distribution-not localized and concentrated in one community. Many tribal regions of Southeast Asia and Oceania exhibit chains of this kind.

We may conclude from this discussion that the rate of linguistic change following the expansion or splitting of a language community will depend a great deal on rates of subsequent communication, as well as on individual sociolinguistic situations and the presence of other linguistically unrelated populations in the vicinity. Such variables, undocumented for prehistoric communities, can cause problems with chronological calculations. Lexicostatistical studies per se, such as the major Austronesian study by Dyen (1965a) already referred to, do not concern themselves directly with time, but only with classification and subgrouping. However, the time factor is central to an offshoot of lexicostatistics called glottochronology (Gudschinsky 1964). Analyses of languages (mostly Indo-European and west Asian) with long written histories have led to suggestions that basic vocabularies will change at constant rates: 19.5 percent per millennium for a standard 200-word list and 14 percent for a 100-word list. Hence simple glottochronological formulae can, in theory, be derived for calculating how long ago the common ancestor of two or more related languages was in existence.

Two basic assumptions of glottochronology are that the basic vocabularies of all languages change at the same rate and that basic vocabularies in turn are more stable than "cultural" vocabularies and more resistant to borrowing. Certain basic vocabulary items (such as the words for two, five, eye, and louse in the 
Austronesian languages) can be shown to be more resistant to change than others (see Table 4.1), but taken as a whole the rate of overall basic vocabulary change is stated to be constant. The idea of such a constant rate, quite apart from the stability of individual vocabulary items, has of course long been challenged (e.g., Teeter 1963; Bergsland and Vogt 1962). Some languages-such as Icelandic - can be shown to have been extremely conservative, while others-as in Melanesia - can be shown to have changed their vocabularies quite rapidly. Although there exists an insufficient written record for Austronesian languages from which variant rates can be demonstrated directly, ${ }^{4}$ it is possible to throw light on this matter by using another method. Theoretically, all daughter languages of a proto-language should be equally different in basic vocabulary from the proto-language if the overall rate of change of the basic vocabulary is constant through time. A detailed study using this reasoning has been carried out by Blust (1981a), who has compared sixty-two Austronesian languages outside Taiwan with a reconstructed 200-word list for Proto-Malayo-Polynesian.

The results are quite dramatic: Western Malayo-Polynesian languages retain an average of 41 percent of cognates with Proto-Malayo-Polynesian (for example, Malay 59 percent, Minangkabau 50 percent, Tagalog 46 percent, Makassarese 38 percent, Sundanese 35 percent), while Oceanic languages retain only 25 percent on average (for example, Motu 37 percent, but most other New Guinea languages retain only 16 to 30 percent). These differences are significant, although there is much overlap, with some Western languages having low percentages (for example, Yogyakarta Javanese 30 percent) and some Oceanic languages having moderately high percentages (for example, Fijian and Motu 37 percent). Nevertheless, one can hardly escape the conclusion that Western MalayoPolynesian languages have changed, on the average, much more slowly than Oceanic.

Are there any observations that can be made to throw light on this situation? It could be significant that certain languages, such as Atayal of Taiwan and Enggano of western Sumatra, have possibly been subjected to rapid changes due to "word taboos" (i.e., certain sounds, such as those occurring in the name of a dead chief, may acquire a taboo against further utterance; Dyen 1965a:53; Kahler 1978). Other languages, especially in the more stratified Austronesian societies, have different vocabularies for use by or when addressing persons of high status (Grimes and Maryott 1994). Others, such as Atayal again, have slightly different vocabularies for male and female speakers (P. J-K. Li 1983). But all these customs are restricted in occurrence and can hardly explain the overall picture. They are individual idiosyncrasies.

Clearly, there are other more fundamental sociolinguistic factors that are likely to affect rates of linguistic change in preliterate societies. Such variables as the size of the speech community, its internal integrity (the quantity of dialect 
variation), and-most importantly-the external relationships between speakers of a given language and other communities with varying degrees of linguistic relationship, are all sure to be important. The latter factor has already been highlighted above. Different rates of linguistic diversification or convergence (languages do not always grow farther and farther apart) for large urban communities, mobile hunter-gatherers, and small groups of tribal gardeners may perhaps be expected. There are reasons for suggesting that the latter, particularly when they are atomized into small independent groups and have occasion for interaction with speakers of quite different languages, can have very rapid rates of vocabulary change. This has been shown especially for neighboring societies who speak Papuan and Austronesian languages in western Melanesia, where bilingualism, heavy interlanguage borrowing, and even complete language shift - a process that can often carry over major substratum aspects of an abandoned language-are all attested in great detail (e.g., Ross 1994; Dutton 1995; the significance of bilingualism in speeding language change is highlighted by Grace 1985 for New Caledonia).

We have now reached a point where further generalizations will serve little purpose. I have tried to show that the rate of linguistic change will be affected by the degree of communication maintained between related language communities and by various sociolinguistic factors. Small independent populations of tribal horticulturalists, especially if they inhabit a region of existing linguistic diversity, will tend to develop a fast rate of change. Large, integrated civilizations should change more slowly, especially if they have literacy. Basically, the only way to date nodes in the tree of a language family such as Proto-Austronesian is to reconstruct the words for those items of material culture that are likely to appear in the archaeological record, and then to search for those items in the dated stages of that record. For instance, early Austronesians made pots and kept domestic pigs and dogs. Such items occur widely in the Indo-Malaysian Archipelago after about 4,000 years ago but are completely absent before. When they first appear we have a good case for suggesting an Austronesian presence, although this is obviously not a foolproof way of identifying early Austronesians in space and time-other people made pots and kept pigs too, particularly Austroasiatic speakers. Yet this is the best method that we are ever likely to have. To claim greater certainty in tying together the archaeological and linguistic records is to daydream; we can only refine hypotheses.

The time has now come to look at some of the more detailed relationships of the Indo-Malaysian Austronesian languages and to suggest some archaeological correlations (which will be expanded in later chapters) for time-depth. I will not be able to raise glottochronology to the status of a reliable and useful tool, but I will try to give some understanding of the linguistic history of the two regions and to suggest why different rates of linguistic change have occurred. 


\section{INDO-MALAYSIAN LINGUISTIC PREHISTORY: SOME POSSIBILITIES}

In the first edition of this book I wrote that there was no coherent information about the linguistic situation in Indonesia and Malaysia prior to Austronesian expansion, simply because this expansion was so successful and so complete that no traces of anything older had survived (excluding the Papuan languages in the east). Since then we have two important claims, one by Adelaar that there are traces of an Austroasiatic (specifically Aslian of Peninsular Malaysia) substratum in western Sarawak (Adelaar 1995), and another by Reid (1994a) that some of the Negrito languages of the Philippines carry traces of a non-Austronesian substratum, perhaps reflecting an ancient origin as pidgins but since decreolized by continuing close contact with Austronesian populations.

In the case of Sarawak, there is a possibility that early rice agriculturalists might have occupied some regions before Austronesians arrived, according to radiocarbon dates for rice approaching $2500 \mathrm{BC}$ from the cave of Gua Sireh (Bellwood et al. 1992; Ipoi and Bellwood 1991) (see Chapter 7, Section IIID). In addition, it has long been known that Malay, as spoken on the Malay Peninsula, has many loans from the Aslian subgroup of Austroasiatic. Aslian is presumably a descendant of a major pre-Austronesian language grouping of the region. It can thus be assumed that before the breakup of Proto-Austronesian and the expansion of its daughter subgroups southward, the unknown languages of the IndoMalaysian Archipelago were flanked to the west (on the Southeast Asian mainland and in part of Borneo) by ancestral Austroasiatic languages, and to the east (in eastern Indonesia and New Guinea) by ancestral Papuan languages. The affinities of the Philippine Negrito substratum are unknown.

Turning now to the Austronesian languages themselves, I have already made it clear that the location of Proto-Austronesian is best placed in Taiwan. Internal relationships of Formosan languages need not be of concern here, but the rather limited archaeological evidence from the island suggests a date between 4000 and $3000 \mathrm{BC}$ for Initial Austronesian settlement. My reasoning here is simply that pottery, a known cultural and linguistic marker of at least Proto-MalayoPolynesian communities (although not strictly reconstructable to Proto-Austronesian, perhaps because of cognate attrition), first appears in Taiwan at about this time-perhaps 1,000 years before its appearance in any of the islands to the south.

As I have noted, however, the date of Proto-Austronesian does not correlate with the date of Initial Austronesian settlement in Taiwan. Instead, it correlates with the point at which the Initial Austronesian communities of the island, whether speaking one language or several, split irrevocably in such a way that two subgroups were formed, both of which have survived separately to the 


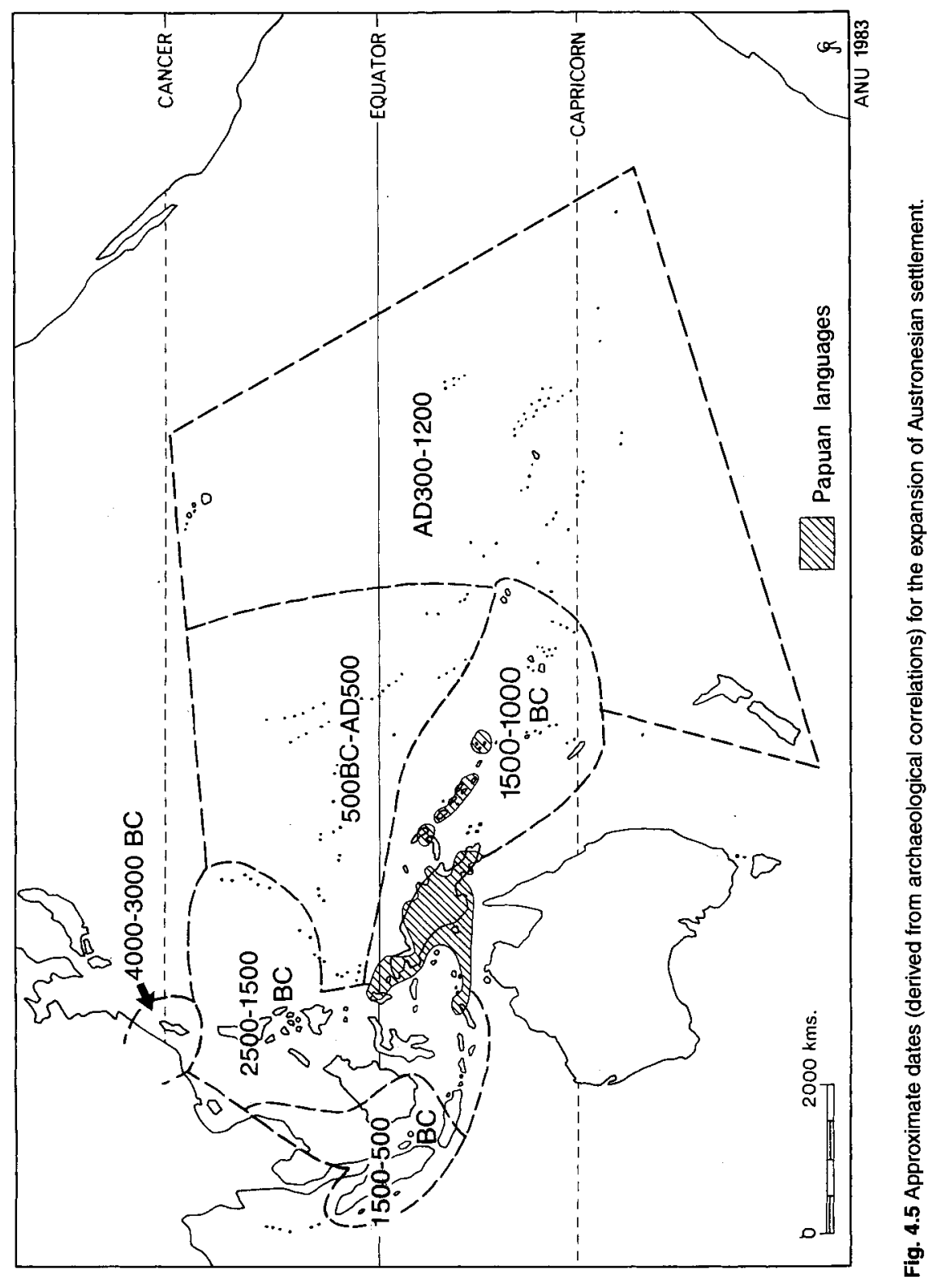


present day. Such separation could have occurred within Taiwan (and must have if Taiwan has more than one primary subgroup of Austronesian), or when people first moved from Taiwan into relative isolation in the northern Philippines. In the same way, Proto-Malayo-Polynesian correlates with a later separation that probably began as people fanned out through the Philippines, before moving south toward Borneo, Sulawesi, and the Moluccas. Proto-Austronesian and Proto-Malayo-Polynesian are not in themselves datable, as discussed above, but taking into account the expansion of relevant forms of material culture in the archaeological record, I would suggest a date of $3000 \mathrm{BC}$ for the breakup of the former and $2500 \mathrm{BC}$ for the breakup of the latter. These dates are a little younger than those preferred by linguists (e.g., Pawley and Ross 1993 suggest 4000-3000 BC for the breakup of Proto-Austronesian), but in my view the archaeologial record is likely to have the last say on this.

At this point, I will return again to the observations made by Blust (1981a) on varying retention rates in the Malayo-Polynesian languages. If the average Oceanic retention figure ( 25 percent) is used to calculate a date for Proto-MalayoPolynesian by using the standard formula of glottochronology ( 80.5 percent retention per language per millennium), then the answer will be around 4000 $5000 \mathrm{BC}$. If the much higher average Western Malayo-Polynesian retention figure (41 percent) is used, then the answer will be about 2000 BC. Such a difference is no small matter, and there can be little doubt from the archaeological record that the Western Malayo-Polynesian rate, in general terms, is the most accurate one for the overall nonwestern-Melanesian prehistory of the Austronesian languages. The Oceanic retention rate is obviously highly depressed and may be considered unrepresentative of the general Austronesian situation. I will look further at the Melanesian languages in due course.

\section{A. After Proto-Malayo-Polynesian}

The dates I am suggesting for the Philippines indicate an Austronesian colonization at about $2500 \mathrm{BC}$, followed by fairly rapid movements to Borneo and Sulawesi by about $2000 \mathrm{BC}$. Whether these movements took place before the Philippines were wholly settled we may never know. The archipelago is highly fragmented, and settlement of remote parts may have continued long after other Austronesian settlers had expanded southward. If this did happen there may be a lesser linguistic diversity and time-depth for some parts of the Philippines than expected, as perhaps documented by Blust (1991) for his Greater Central Philippines subgroup (although Blust relates much of this language expansion to sociocultural dominance patterns after $500 \mathrm{BC}$ ). The Malayic languages have expanded through sociocultural dominance since about 2,000 years ago, as we will see below. 
We now come to the two major divisions within Malayo-Polynesian: Western Malayo-Polynesian and Central-Eastern Malayo-Polynesian. The former has never been established as a true subgroup with rigor, but most of its members share quite high cognate percentages between themselves (over 38 percent according to Dyen 1965a, who termed this subgroup the Hesperonesian linkage). Many languages have large numbers of speakers (approaching 100 million in the case of Javanese) and are spoken over large areas. The overall internal relationships of Western Malayo-Polynesian are unclear, and the lexicostatistical clustering of these languages may be more an artifact of high retention rates from Proto-Malayo-Polynesian than of a true subgrouping relationship. Furthermore, many of the languages of this group have long histories of association with civilizations and have borrowed from each other so intensively that true shared innovations are often very hard to detect. Nevertheless, within Western Malayo-Polynesian a number of localized subgroups can be detected. Most of the Philippine languages form a single subgroup, although there is some disagreement about the details (Reid 1982; Zorc 1986). The languages of northern Sulawesi (Minahasa and Sangihe-Talaud) also clearly relate to those of the Philippines.

Another important subgroup, first proposed as the Javo-Sumatra Hesion by Dyen (1965a), has been examined in detail by Nothofer $(1975,1985,1988)$ under the name Malayo-Javanic. It is stated by Nothofer to include Javanese, Sundanese, Madurese, and the "Malayic" languages (see below), plus Acehnese of northern Sumatra. This subgroup clearly occupies a huge geographical area and as the languages all share quite high cognate percentages, they give the impression of a recent major expansion-perhaps during the first millennium BCalthough archaeological correlations in support are almost nonexistent for much of this region. This impression of recency could be tempered by high retention rates and undetected borrowings, but I think we can hardly escape the working hypothesis that many of the Austronesian languages of western Indonesia, Peninsular Malaysia, and Vietnam were not established prior to 1500-1000 BC, or perhaps even later in some areas.

The internal details of Nothofer's classification have been challenged by Blust (1981b, 1988, 1995b), who doubts the existence of a single subgroup containing Javanese, Madurese, and Malay, and proposes instead a closer subgroup relationship between Malay, Acehnese, and the Chamic languages of southern Vietnam (the Malayo-Chamic hypothesis). Javanese in this scheme is believed to relate most closely to Balinese and the Sasak language of Lombok (Blust 1984-1985). Blust believes the Malayo-Chamic languages spread from a common homeland in western Borneo after about $300 \mathrm{BC}$ and that Austronesian languages were once continuous on the Southeast Asian mainland between Peninsular Malaysia and southern Vietnam, a continuum later broken up by 


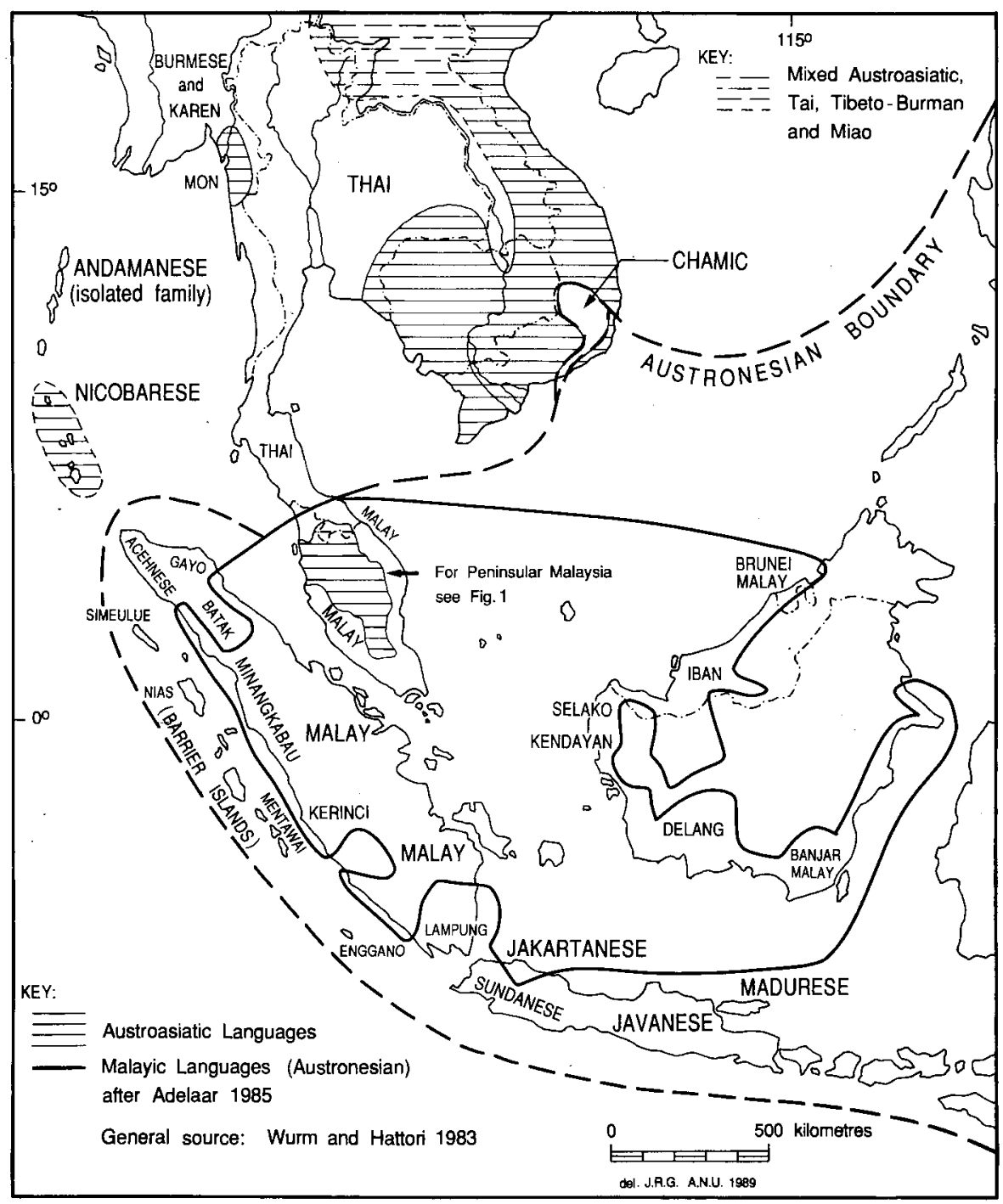

Fig. 4.6 The distribution of the Malayic languages.

historical Mon (Benjamin 1987), Khmer, Thai, and Vietnamese expansion. Acehnese is regarded as a late back-migration from the continent to Sumatra, carrying traces of borrowing from Austroasiatic sources. The close links between Malay and Cham are attested by comparisons of written forms of Old Malay and Old Cham that have survived in inscriptions from the first millennium AD (Marrisson 1975). There seems little reason to doubt that both these mainland groups share a close if not immediate common origin. 
For the Malayic languages (Fig. 4.6), the most recent detailed analyses have been by Adelaar (1992), who proposes a basic division between Malay plus its closest relatives (including Minangkabau and Kerinci of Sumatra and the various Malay dialects spoken in Sumatra, western Java, coastal Borneo, and the Moluccas) and a "Malayic-Dayak" group of related languages on Borneo (Iban, Selako, Kendayan; Hudson 1970). Adelaar and Blust (1988) both agree that the spread of the Malayic languages has taken place from western Borneo since about 2,000 years ago, with much impetus doubtless being given by the growth of the trading empire of Srivijaya focused in southern Sumatra after AD 670. By AD 1500, Malay was a lingua franca over much of the archipelago, especially amongst the Islamic ruling families and their retainers.

Within Western Malayo-Polynesian we therefore see a widespread group of languages-some with many millions of speakers - in continuous contact in historical times and spread over much of Vietnam, Sumatra, Borneo, and all of Java and the Malay Peninsula (Blust 1994b). Much of this language spread has occurred since 1500-1000 BC, according to the linguists-who clearly disagree amongst themselves on details of subgrouping (see Nothofer 1991 for an overall review). There is no clear archaeological evidence from this region that strongly supports this date range or any other (Bellwood 1993), but what is also apparent from the linguistic record is that the other Western Malayo-Polynesian languages in the Philippines and Sulawesi, plus Batak and Gayo in northern Sumatra and the languages of the "Barrier Islands" off western Sumatra (Nias, Enggano, and Simeulue), do not form a part of this recent-spread situation, a circumstance recognized by Nothofer $(1986,1994)$. Nothofer refers to these languages as "Palaeo-Hesperonesian," his implication being that the languages of the north Sumatran and Barrier Island regions in particular have been isolated from the cut and thrust of language expansion in the rest of western Indonesia. The linguistic prehistory of Indonesia is thus "layered," with considerable language replacement happening in centrally placed regions long after initial Austronesian dispersal. We cannot take the view that all the languages of the region have merely evolved in situ since Austronesian dispersal began.

The languages of Madagascar provide us with another interesting example of a late expansion, albeit in this case to a seemingly uninhabited island. The fairly uniform languages here probably originated in a settlement of Austronesians from southern Borneo, where the most closely related languages are Maanyan and Ngaju (Dahl 1951; Dyen 1971). According to Dahl, the presence of certain key Sanskrit loans in the languages of both Madagascar and southern Borneo suggest that the migration postdates $\mathrm{AD} 400$, which is when the first evidence for the influence of Sanskrit on the Western Malayo-Polynesian languages appears. A date during the middle or late first millennium AD seems likely and is supported by Adelaar $(1989,1995)$, who believes, from the study of loan vocabulary, that the southern Borneo people might have been serving as 
crews on ships captained by Malay- or Javanese-speaking overlords. On the issue of late expansion, it should not be forgotten that other Austronesians also reached New Zealand and much of eastern Polynesia, on the opposite side of the world from Madagascar, at about the same time.

On turning to the languages of the Central Malayo-Polynesian subgroup, we move into a very different linguistic situation from that of most of Sundaland. This region has a very large number of languages-about 100 according to Pawley (1974) - and most of them have small numbers of speakers and occupy only small geographical areas. There are linguistic indications that the Central Malayo-Polynesian languages spread very quickly once colonization started (Blust 1993), and the region as a whole has no clear signs of the type of secondary language radiation so visible in Sundaland and in the central Philippines. Blust's analyses indicate that the Central Malayo-Polynesian languages have a higher average retention rate (about 36 percent) from Proto-Malayo-Polynesian than do the Oceanic languages. Thus, while many of these societies are relatively small in scale, the higher retention rate surely reflects a much weaker influence from the pre-Austronesian inhabitants of the region than was the case in western Melanesia (see below).

To date, rather little can be said about internal relationships within Central Malayo-Polynesian, but it is apparent from archaeology that Austronesian settlers had reached Timor by perhaps $2000 \mathrm{BC}$, although the dating here is rather uncertain. The formation of the Eastern Malayo-Polynesian subgroup, which includes the Austronesian languages of southern Halmahera, parts of West New Guinea, and all of the vast Oceanic subgroup, seems to have commenced with the initial Austronesian colonization of Halmahera or the Bird's Head region of West New Guinea. The Austronesian languages of Halmahera have probably been spoken there since $1500 \mathrm{BC}$, and have here undergone much contact with the Papuan languages in the northern part of the island (Voorhoeve 1988, 1994; Bellwood in press). At around $1500 \mathrm{BC}$ the Oceanic languages underwent their very rapid expansion through parts of Melanesia from an immediate homeland in the Bismarck Archipelago. The Oceanic languages form a well-defined subgroup with a common origin in Proto-Oceanic, as established by Dempwolff, Milke, and other more recent authors such as Grace, Pawley, and Ross (see Pawley 1981; Ross 1988, 1989; Pawley and Ross 1995).

In the first edition of this book I felt obliged to provide a fairly long argument in favor of equating Proto-Oceanic with the beginnings of the Lapita archaeological culture of island Melanesia at about $1500 \mathrm{BC}$. Today, this equation is so firmly accepted by linguists and archaeologists alike that it no longer needs lengthy justification. In New Guinea and adjacent parts of western Melanesia, the Oceanic speakers were apparently preceded by Papuan speakers, who may well have had systems of arboricultural food production in coastal regions and quite sizeable populations before Austronesians first arrived. Since 
that time, the Melanesian Austronesian languages have undergone more rapid diversification than their cousins in Indonesia or Polynesia owing to intense contact-induced change and even language shift. Melanesia may be regarded as an area where small, independent, and relatively egalitarian communities, in frequent contact with a large array of different linguistic communities and practicing bilingualism as a major method of communication for trade and social interaction, have become subjected to very high rates of lexical diversification (Dutton 1995).

The same situation does not hold for most of Sundaland and may never have done so, although the relative linguistic homogeneity seen there now may be partly due to the integrating effects of 1,500 years of Hindu-Buddhist and Islamic civilization. Those islands that were little affected by these civilizations, such as Sulawesi, the Philippines, and the islands of eastern Indonesia, do show much more linguistic diversity, both in terms of the large numbers of languages with small numbers of speakers and the degrees of difference between them.

\section{THE PAPUAN LANGUAGES AND THEIR RELATIONSHIPS WITH INDONESIA}

The Papuan languages have been mentioned above in connection with contactinduced change in the Austronesian languages, but they also deserve a brief consideration in themselves because of their reflection of the prehistory of the large island of New Guinea-a prehistory that has been of considerable importance for the eastern part of Indonesia. Within Indonesia (excluding Irian Jaya) they are spoken in central and eastern Timor, Alor, Pantar, and on Morotai and the northern half of Halmahera. Elsewhere in the Moluccas and Lesser Sundas all the languages are Austronesian.

A superficial glance at a map might suggest that these Papuan enclaves are simply remnants of an earlier and larger distribution overrun by Austronesian speakers, but recent research on the Papuan languages as a whole may make such a view rather simplistic. In the case of northern Halmahera, for instance, all the Papuan languages are closely related and clearly record recent radiation, although their first establishment on the island could well be pre-Austronesian (this is simply not clear; see below and Voorhoeve 1988, 1994 for discussion). Here I simply want to take an overall look at the prehistory of the Papuan languages, especially that espoused in a number of papers by Wurm $(1978,1982$, 1983).

The main documented phases of expansion in the prehistory of the Papuan languages, according to Wurm, are as follows (and here I am simplifying what Wurm presents as a rather more complex story): 


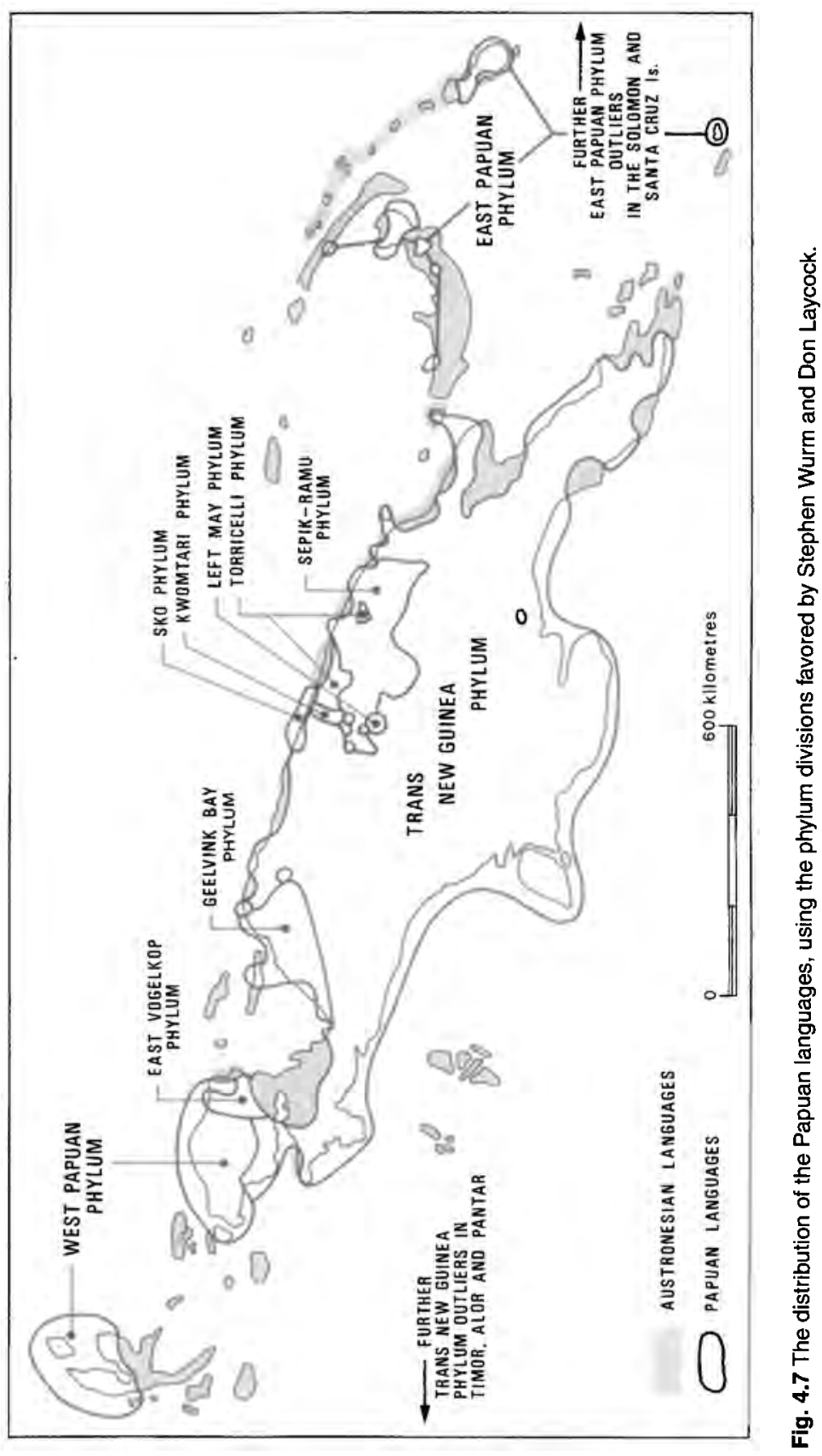


1. New Guinea was first settled about 60,000 years ago (according to inference based partly on Australian archaeological dates), but no present-day Papuan languages descend directly from this early linguistic phase.

2. About 15,000 and 10,000 years ago the first Papuan speakers, possibly in two separate groups according to pronoun forms, settled the New Guinea region, including the eastern Indonesian islands mentioned above. The dates may be regarded as little more than guesses. The languages of Halmahera and Morotai, which are classified in the West Papuan Phylum, descend directly from one of these two periods of linguistic expansion (although Voorhoeve does not record specific agreement with this view; see above).

3. About 3000 вС a major expansion of the Trans New Guinea Phylum of languages took place. This expansion began west of New Guinea, according to Wurm, and occurred after Austronesians had already arrived in the general region, according to the evidence of loan words (a suggestion that perhaps makes the claimed date of $3000 \mathrm{BC}$ a little too old). The Trans New Guinea Phylum languages spread initially along the northern coast of New Guinea and also to Timor, Alor, and Pantar, where they replaced the earlier West Papuan languages.

4. Since 1500 BC, the Trans New Guinea Phylum languages, strongly influenced by Austronesian loan words acquired in the vicinity of the Markham Valley, have expanded in the highlands of New Guinea. The Phylum now contains about 500 languages and covers about four-fifths of the Papuan linguistic area. It is perhaps an excellent example of how a successful linguistic expansion can wipe out traces of earlier diversity.

The basic conclusions relevant for Indonesia that can be drawn from Wurm's accounts are clear. According to him, Halmahera was settled by Papuan speakers long before any Austronesian presence in the area. Timor, Alor, and Pantar might have been settled by two separate groups, one long before and the other contemporary with an Austronesian presence. New Guinea has a far more complex history.

There are aspects of Wurm's reconstruction that I think need some comment. First, in a very detailed review of Papuan linguistics by Foley (1986), the view is taken that the Trans New Guinea Phylum is not a valid construct and that the Papuan languages as a whole are, in reality, highly fragmented into about sixty separate families. On the other hand, Pawley (1995) and Voorhoeve have searched and found widespread lexical cognates across the area of the Trans New Guinea Phylum, thus suggesting that it could have some genetic basis as a true language family. Whatever the ultimate verdict on the Trans New Guinea Phylum, there is still the problem that Wurm derives all his strata of migration from Indonesia at separate times. It seems just as likely that many could represent expansions from within the large pool of population supported 
by the large size and varied resources of New Guinea. For instance, there is now some excellent evidence indicating that New Guinea Highlanders developed their own localized form of horticulture prior to 6,000 years ago, long before Austronesians could have arrived on the New Guinea coasts (Bellwood 1996b). This would be sufficient to support a considerable increase of population over that possible with a hunting and gathering economy, and it would be sufficient to explain the expansionary success of the Trans New Guinea Phylum. It could also explain the remarkable resilience that the Papuan languages have shown in holding their domination of the New Guinea region, despite Austronesian settlement. Foley (1986) also believes that some of the language phyla he recognizes have resulted from agricultural population expansion. Unfortunately, there are no easy answers here, but as with Austronesian, it is important to recognize that not everything in the Papuan linguistic pattern has developed in situ through all time.

So far, most of the linguistic opinions I have presented in this chapter have been what I term "standard"; despite quibbles over details, they are all generally acceptable to the majority of linguists. One exception to this generalization is of course the hypothesis of Dyen that the Austronesian languages developed in and spread from Melanesia. The past twenty years of research in linguistics and archaeology leave little hope for a hypothesis that was originally so clearly presented and yet can now be shown to be incorrect. It was therefore with some surprise that I once read an article by Terrell (1981) that seemed to be harking back to Dyen's viewpoint, albeit from a totally different theoretical perspective. Terrell suggested that the Papuan and the Austronesian language families in Melanesia could have had a common origin and that the divergences between them evolved within Melanesia with the passage of time and a fast rate of linguistic change. As Terrell (1981:251) stated: "In short, in the interests of parsimony, do not invoke migrations beyond necessity."

The point I wish to make to close this chapter is that I believe the linguistic reconstruction I have presented fits the available facts in the most convincing way. Migrations can, in fact, be quite parsimonious explanations for patterns of human variation, although I would agree with Terrell that they are not always the answer for every problem in prehistory. Fortunately, few archaeologists today take the strong antimigrationist stance that dominated the discipline in the early 1980s. Indeed, a debate about the processes of cultural and biological evolution, with a partial focus on the issue of migration, has just erupted in the anthropological literature (Moore 1994; Bellwood 1996d). Terrell clearly adopted a totally different stance from mine; one that was reasoned and logical, but one that (in my view) was heavily dependent on one rather peripheral region of the Austronesian world (western Oceania) for its facts and theories. We may never know which answer, if any, best explains the Austronesian dispersal, but some answers surely have more interdisciplinary support than others. 


\section{The Patterns of History and Ethnography}

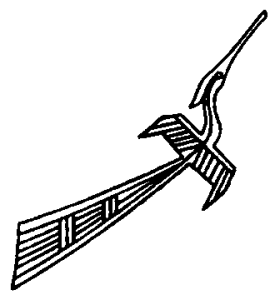

It is not possible to give an exact figure for the number of different ethnic groups in the Indo-Malaysian Archipelago, partly because of problems of definition and boundary recognition very similar to those discussed for languages. Also, the ongoing assimilation of small groups to large majorities and urban lifestyles probably means that the overall number has been continually decreasing this century. The major Human Relations Area Files compilations (Lebar et al. 1964; Lebar 1972) describe about 100 groups for whom there exist good literatures, and Hildred Geertz (1963) has given a total figure of 300 for Indonesia (some of the more important are shown in Chapter 4, Fig. 4.2, and Chapter 8, Fig. 8.7). As one would expect, there is considerable cultural variation, due in part to differences that developed between Austronesian societies in prehistoric times and in part to the varying influences of Indian, Islamic, and European traditions during the past 1,500 years.

I should perhaps explain here my basic stance on the background to cultural variation in the small-scale traditional societies of the archipelago. Many ethnologists in the past adopted a view that observable variations reflected the successive migrations into the region of different cultures and their associated racial groups (e.g., Hose 1926; Loeb 1935; Kennedy 1937; Cole 1945). This is true in part, but sometimes the enthusiasm for cultural "strata" overflows. I am still surprised at how often these "waves" of Veddoids, Proto-Malays, and DeuteroMalays, together with their cultural idiosyncrasies, are repeated without question in modern books on the history and peoples of the region, but this is a matter that I will not pursue. My own view is simple: the Negritos and their traditional hunting and gathering lifestyle must be considered as autochthonous to the Indo-Malaysian Archipelago, whereas the agricultural lifestyles of 
the Austroasiatic Senoi of Peninsular Malaysia and the Austronesian speakers are to a great (but not total) extent the results of cultural and population expansion from more northerly latitudes. The explanations for variation in the Austronesian group require not mixing between clearly differentiable and successive races and cultures, but the slow expansion and adaptation of an originally relatively unified, early Austronesian ethnolinguistic population, combined with intergroup contact and the successive influences of external civilizations. In the terminology favored by Moore (1994), the generation of cultural diversity in the Indo-Malaysian Archipelago has been both rhizotic (via interaction between adjacent groups) and bifurcative (via diversification through population radiation) (see Bellwood 1996d for some theoretical discussion of these concepts).

The ethnolinguistic complexity of the Indo-Malaysian region makes the process of prehistoric reconstruction more difficult than for an area of relative cultural homogeneity such as Polynesia. Furthermore, in Indonesia and Malaysia the boundaries between cultures were perhaps more diffuse in prehistoric times than now. Much of the ethnic consciousness that characterises the region today may have become sharpened in recent centuries as groups have symbolized their different identities in response to degrees of pressure from other migrating groups or incorporation in state and colonial systems (e.g., see Dentan 1975 for the Senoi Semai; King 1982, Rousseau 1990, and King 1993 for Borneo). Nevertheless, I do not hold to the view, proposed most forcefully by Fried (1975), that "ethnicity" is almost entirely a product of state-level outsider pressure. Group identity through common origin, common language, and common territory surely existed in prehistoric societies, even if we have difficulty in identifying nodes of such identity in the archaeological record.

While factors of ethnic identity can serve to differentiate populations, separate identities can also be "pooled" against outside pressures, as Nicolaisen (19771978) has described for small groups in Sarawak in the face of recent Iban and Kayan expansion (see also Sellato 1994 for Borneo nomads). Indeed, on large islands such as Borneo where population densities are low and ethnic groups seek to attract rather than repel outsiders as new members, ethnicity can be decidedly fluid (Rousseau 1990, Tillotson 1994). As Rousseau points out for central Borneo (Rousseau 1990:302):

It is erroneous to expect a priori a specific correspondence between ethnicity, culture, language, common origin (or belief in common origin) and social systems. It is preferable to see these various elements as overlapping plates which move in different directions while influencing each other.

One cannot of course generalize very usefully about ethnic identity on a pan-archipelagic scale because every region has followed a different course of development. Large-scale assimilatory societies such as those of the Javanese 
and the widespread Malays have undoubtedly expanded over a great deal of earlier diversity, while on the other hand the Aslian peoples of Peninsular Malaysia have apparently stressed their ethnic identities in order to resist such assimilation (Benjamin 1986). Each region now presents an ethnic "picture" that reflects its settlement and cultural history and its degree of incorporation into a native state or colonial polity. Each cultural region must therefore be considered on its own merits.

On looking at the societies of the region in broad terms-with respect to descent, political systems, influences from Indian and Islamic civilizations, and so forth-it is apparent that there are three major groupings, defined most clearly by H. Geertz (1963). These are: (1) the partly Indianized wet-rice cultures centered on Java (now Muslim) and Bali; (2) the coastal Islamic societies (especially Malay and Buginese) that have become very widespread through the archipelago as a result of the trade and commerce focused on the Islamic sultanates since the fifteenth century; and (3) the small-scale traditional and mainly interior or remote island populations. In the following pages I will concentrate attention on some of the societies in the third category, as in many ways these have remained closest to their Austronesian cultural ancestries. The cosmopolitan societies of the first two categories, such as present-day Javanese and Malays, will only be mentioned in certain historical contexts. Furthermore, most of the small-scale traditional groups have undergone at least some change as a result of contact with modern civilization. My descriptions will clearly favor traditional customs and behavior as described in historical or ethnographic records.

In terms of descent, the most commonly used terminology divides the smallscale traditional societies of the region into unilineal (patrilineal or matrilineal) and cognatic (including bilateral) categories (Murdock 1960a, 1960b). This terminology is now enshrined in the literature and it does of course refer to differences in descent reckoning that are of social significance for determining marriage and inheritance patterns. But this does not necessarily mean that all Austronesian societies can be placed unequivocally in distinct unilineal and cognatic categories, or that such categories imply totally separate evolutionary trajectories. Furthermore, the dichotomy does not always reflect Indo-Malaysian reality, because in practice many societies follow both unilineal and cognatic principles, depending upon context. Examples of the real-life multitude of cross-cutting contexts include affiliation to a corporate group, location of postmarital residence, inheritance of swidden land or of wet-rice fields, inheritance of status, and membership in burial or irrigation associations.

I will first introduce the surviving hunting and gathering societies, whom I believe do demand separate consideration, and then document the arrival and distribution of Indian and Islamic influences, before turning to the main discussion of the small-scale traditional agricultural populations of Indonesia and Malaysia. 


\section{THE HUNTERS AND GATHERERS}

The equatorial rain forests of the region shelter a number of hunting and collecting societies that have either survived assimilation by, or have adapted out of, the ever-expanding agricultural economies. Some of these groups, such as the Negritos of Peninsular Malaysia and the northern Philippines, are no doubt "pristine" hunters and gatherers who have long resisted total acculturation by surrounding cultivators. Others, such as the Austronesian-speaking hunters and gatherers of interior Sumatra and Borneo, have probably adopted this way of life as a result of change from a partial agricultural ancestry.

The Negrito peoples of the Andaman Islands, Peninsular Malaysia, and the Philippines are of course of great significance in any discussion of the overall prehistory of the archipelago. Although all the Philippine Negritos have now adopted Austronesian languages and some groups have become partly acculturated to a lifestyle of shifting cultivation (for example the Pinatubo and Ayta of western Luzon; Fox 1953; Brosius 1990), there can be no doubt that the Negrito populations as a whole have local ancestries that long predate those of the Southern Mongoloid Indonesians, Filipinos, and Malaysians. Recent linguistic research on Luzon Negritos suggests the existence of a non-Austronesian substratum according to Reid (1994a), and it also seems likely that the Malaysian Negritos have adopted their present Mon-Khmer (Aslian) languages from their agriculturalist Senoi neighbors (see Chapter 8). Some of these groups have long been in contact with agriculturalists (Headland and Reid 1991) and have clearly intermarried with them in some cases (especially in the Philippines), yet only in rare cases have they adopted systematic agriculture (for studies of forager-agriculturalist interactions see Headland 1986; Eder 1987). There is much empirical fodder here for those interested in the origins and spread of agriculture, and I will return to these matters in Chapter 7.

Superficially, the Negrito lifestyle is simple: Small bands of families with rather fluid membership and informal leadership move in a nomadic fashion through the forest, sleeping in camps of lean-tos and shelters. Cultural simplicity is expressed partly through an absence of many features characteristic of the surrounding cultivators. Some of these, such as the absence of headhunting, land tenure, pottery (except in the Andamans), weaving, and alcoholic beverages (Cooper 1941) are no more than one would expect given the nature of Negrito society and economy. Other features, such as the practices of body scarification amongst the Philippine Aeta, face painting, and the wearing of porcupine quills through the nose by the Peninsular Malaysian Negritos (Carey 1976) and the general absence of ear ornaments, tattooing, and dental mutilation, all serve to set these groups apart from surrounding Austronesian cultivators.

While there is not space here to list the many details reported about Negrito hunting and gathering lifestyles, I do wish to question an old view that the 
Negritos represent a direct and totally static window on the Pleistocene past, as implied by some earlier authors (e.g., Cooper 1941; Burkill 1951). It is true that the hunting and collecting economy and the associated band forms of social organization are of great antiquity, but the Negritos have been in contact with outsiders for several millennia and those who survive today, especially in Peninsular Malaysia and the Philippines, have clearly done so by adapting to changing circumstances and pressures (Headland and Reid 1989). Alas, they will not be able to adapt for much longer and most in the Philippines are now facing cultural extinction (Headland 1988; Eder 1987). Peterson (1978) has described how the Aeta of northeastern Luzon have formed trading and labor relationships with Philippine agriculturalists; the Aeta provide hunted meat and labor in return for cultivated produce. But this is clearly a temporary adaptation, similar to that described succinctly by Sellato (1994) for the Punan (non-Negrito) foragers of Borneo. The ultimate fate of the hunters, after centuries and perhaps even millennia of living in sporadic contact with agriculturalists, seems now to be assimilation into low-status membership of the agriculturalist populations.

In central Peninsular Malaysia the pressures have not been as great, and the Negritos here (often called "Semang"; see Endicott 1979, 1984; Rambo 1988 for recent descriptions of their lifestyle) have been able to continue their forest lifestyle through a conscious emphasis on differences between their culture and those of adjacent Malay and Senoi cultivators. This is a conclusion recently drawn by Benjamin (1986), who shows how certain features of Negrito social life, such as avoidance practices and prohibitions that promote marriage outside the group, wide social contact, and freedom of movement, help to ensure the mobility that these people need for survival. ${ }^{1}$ The Negritos have no corporate descent groups and the independent nuclear families are thus allowed to move and make new camp relationships freely. In recent years some of these Negrito groups have become settled and partially converted to agriculture, a process leading to rapid population growth (Gomes 1982), but one that seems to have been far less common in precolonial times amongst the Semang than among the Philippine Negritos. No Semang are known to have made precolonial shifts to agriculture.

The hunters and gatherers of the Indonesian islands are in a different category from the Negritos. Both biologically and linguistically, they are relatively undifferentiated from their Austronesian-speaking agriculturalist neighbors (Indonesia has no Negrito populations). The Indonesian foragers dwell mostly in the inland forests of Borneo and Sumatra, but their general avoidance of cultivation is not a good reason for assuming that they necessarily represent an ancient stratum of Austronesian-speaking hunters and gatherers. The bestknown groups of these Indonesian hunters and gatherers include the Kubu of the lowland swamps of Sumatra (who speak dialects of Malay; Loeb 1935), the 
Punan of interior Borneo, and the rather controversial Tasaday of interior southern Mindanao. In eastern Indonesia there are also the Togutil (Papuan speakers) of northern Halmahera. I will restrict my comments to the Punan and Tasaday because they have both spawned rather voluminous recent literatures.

The Punan (some groups are called Penan, as reviewed by Needham 1954) occupy many forested areas of inland Sarawak and northern interior Kaliman$\tan$ (see Fig. 4.2). They dwell in temporary camps (Plate 11) of a few families, hunting with blowpipes, exploiting stands of a small dryland species of wild sago (Eugeissona utilis) that grows below 1,000 meters, and collecting the fruits of wild rambutan, durian, and mangosteen trees (Hose and McDougall 1912; Sellato 1994; Sather 1995). Linguistically, there is no apparent unity; many groups seem to be related closely to nearby cultivators, an important point stressed by Hoffman (1986). Many groups collect forest items such as beeswax, birds' nests, camphor, and rattan for trade purposes, often leading to close relationships with surrounding agriculturalists such as the Kayan. Such close relationships may have caused acculturation in some Punan societies, as witnessed by their sporadic adoptions of horticulture (Nicolaisen 1976), ironworking, and systems of ranked headmanship (Arnold 1958). It is my own belief, however, that these features need not in totality be the result of recent acculturation, but may simply reflect the fact that the Punan have always straddled the boundary between settled horticulture and forest hunting and gathering, with only some groups shifting entirely toward the latter economic mode.

Since the first edition of this book was published, the Punan have played an important role in two important international debates. The first, which also encompasses the Negritos, concerns the question of whether or not hunters and gatherers could ever have lived in interior equatorial rain forest without regular access to agricultural foodstuffs via trade. According to Headland (1987; for the Philippines), Rambo (1988), and Kuchikura (1993; both for Peninsular Malaysia) they could not. The debate has been given worldwide significance by Bailey et al. (1989), who suggest that interior wet rain forests in Africa, Asia, and America were generally uninhabited before agriculture began. However, the archaeological record for Peninsular Malaysia indicates that foragers did inhabit such regions (as accepted by Bailey et al.), albeit in small numbers and probably not everywhere, and have done so for at least 10,000 years (Endicott and Bellwood 1991; and see Chapter 6). It is quite possible that these foragers practiced clearance activities in order to encourage useful plant growth, especially of fruit trees and tubers, but there are no signs of agriculture in the archaeological record prior to about $2000 \mathrm{BC}$. On the other hand, much of interior Borneo has evidently never supported human populations to judge from modern population distributions, so the hypothesis may work in part for the Punan. The issue is a complex one to which I shall return. 
This brings up the second question. If the present Negritos (who are not represented in Borneo) are descendants of ancient and preagricultural forest foraging groups, then who are the Punan? Are they "genuine" hunter-gatherers like the Negritos, or the products of "devolution" from a partially agricultural ancestry? According to Hoffman (1986), the Punan developed initially as commercial hunter-gatherers linked to and derived from agricultural populations. Sellato (1994) has presented a diametrically opposed view-that the Punan have always been hunter-gatherers and have only recently come into contact with cultivators. There is no archaeological record in support of either view, and neither seems fully convincing. With Sather (1995), I prefer a middle road.

If the linguistic reconstructions of early Austronesian society described in the previous chapter have any merit at all, then clearly there is little scope for any widespread and ancient Austronesian hunting and gathering adaptation in Indonesia without any linkage to agriculture. Unlike the Negritos, the Punan have probably not been foragers since the Pleistocene, and their ancestors could not have entered Borneo as isolated foragers. However, as Sather points out, the initial Austronesian expansions into the archipelago probably combined economies of agriculture, fishing, and foraging. As Austronesians penetrated upriver into the rain forests of Borneo, with their extensive stands of sago and varied animal fauna, some groups-especially those already accustomed to a coastal foraging economy-might have been tempted to turn to upriver foraging nomadism (Brosius 1988). From this viewpoint, Punans have always had some contact with cultivators, but as subsistence foragers rather than as the commercial foragers suggested by Hoffman. If the Punan adaptation was totally independent of the Austronesian agricultural tradition, we would expect to find independent populations of Punans throughout the deep interior rain forests of Borneo. Yet if one looks at the distribution of population on the island, as presented very clearly in sheets 41 and 42 of Wurm and Hattori (1983), it becomes clear that Punans live only where there are relatively close agricultural populations (see also Fig. 4.2). There are extensive areas of quite uninhabited rain forest in interior central and northern Borneo where neither type of population occurs. I see little alternative to regarding the Punan as having penetrated the rain forest right from the start, hand in hand with agriculturalists.

This presents one of the great mysteries of Borneo. Why, unlike Peninsular Malaysia and the Philippines, were there no Negritos in the interior here-no apparently autochthonous foragers? Did the Borneo rain forests really keep them out, as suggested by the above proponents of the empty rain forest theory? I confess to not knowing the answer, partly because no archaeological record exists for the relevant preceramic time periods in the deep interior of Borneo. One day, perhaps we will have such a record.

The conclusion about the part-agricultural ancestry of the Punans can be 
stressed even more forcefully with respect to the Tasaday of Mindanao in the Philippines, a group who achieved media prominence through their "discovery" in 1970 (Fernandez and Lynch 1972; Nance 1975; Yen and Nance 1976). The Tasaday band was living in a cave in the interior rain forest at an altitude of about 1,300 meters above sea level. In 1972 it comprised thirteen adults and fourteen children (twelve boys and two girls). The culture of this group was decribed as being simple in the extreme: a number of widespread Austronesian customs such as tattooing, betel chewing, and tooth filing were practiced, but the people did not hunt, had no baskets or carrying devices, lacked the bow, and used only flaked or edge-ground stone tools. The food supply came mainly from fruits, wild yams (the tops of which were replanted after harvest), grubs, and hand-caught fish and frogs.

Since their discovery the Tasaday have moved in and out of controversy, with many scholars claiming that they were deliberately created "fakes" (see Headland 1992 for a full discussion of different viewpoints on this). I regard them as a genuine but very recent conversion to foraging, perhaps as a result of a feud causing their ancestors to flee and hide in the rain forest. Linguistically, the Tasaday speak a dialect of the Manobo languages of the nearby cultivators, and their separation appears to have occurred after the arrival of the Spanish in the Philippines (L. A. Reid, pers. comm.).

\section{A. The Orang Laut}

Other Austronesian groups who once practiced a rather unusual economy on the cultural fringes of the Indo-Malaysian world are the orang laut (sea people), who are concentrated in two separate regions: along the coasts of the Strait of Malacca and in the Riau Archipelago, and on the northeastern coast of Borneo and in neighboring Sulu (Sopher 1965; Sather 1995). Smaller groups also live down the eastern side of Sulawesi and in pockets in the Lesser Sundas and northern Moluccas. Favored habitats are protected mangrove or coral reef coasts, especially where there are many small islands, as in Riau and Sulu. Traditionally, each family lived permanently at sea in a houseboat complete with a cabin, sleeping facilities, and a cooking place (the latter often a pottery stove). The Bajaus (Sama Bajaus or Sama Dilaut) of Sulu and eastern Borneo still lived wholly in boats until around 1930, but most groups throughout the archipelago have become settled on land in recent decades.

Traditional Bajau houseboat anchorages comprised a number of independent nuclear families with a very fluid bilaterial organization (Nimmo 1972). Large groups of families sometimes combined for major fishing operations, and some groups shared the usage of small islands for burial. But Bajau houseboat families generally lived independent lives fishing, combined with trading with and col- 
lecting for landbased Samal and Tausug communities; during the period of the Sulu Sultanate in the eighteenth and nineteenth centuries they seem to have been used widely as collectors of marine produce for trade (Warren 1981; Sather 1985; Pallesen 1985). The orang laut of the Riau-Lingga Islands were also rather heavily influenced and controlled by the Malay sultanates of the region and maintained a mobile trading lifestyle until the turn of this century. However, in all regions the pressures to settle down on land are now so strong that the true houseboat lifestyle probably will not survive intact for much longer.

The orang laut lifestyle appears to have developed locally in more than one region. The western groups speak dialects of Malay, but the Bajau language is closely related to the Samal language spoken by many settled agricultural people in the Sulu Archipelago. Hence Sopher's view that the Bajau migrated in Islamic times from the Malacca Strait region cannot be entirely correct (Nimmo $1967,1968)$. In the core regions of Sulu and the Malacca Strait, there were certainly orang laut communities reported in the sixteenth century (Sopher 1965), and Pallesen (1985) uses linguistic evidence to suggest that the Sama Bajau people were in Sulu by at least AD 800. Recent excavations at Bukit Tengkorak in Sabah have produced evidence for a strongly maritime-focused economy dating back to $1000 \mathrm{BC}$ (see Chapter 7), but whether sea nomads proper were involved at this early date is uncertain. In the first edition of this book I suggested that the sea nomad lifestyle had developed as a specialized economic adaptation within the exchange and trade networks of the archipelago during the past 1,500 years. Now I am not so certain; perhaps this lifestyle contains a tantalizing record of more ancient Malayo-Polynesian adaptations long past.

\section{THE INFLUENCES OF INDIA AND ISLAM}

I will approach my main discussion of the small-scale traditional and agricultural societies of the archipelago by first outlining the transformations that have affected many of the western and the more accessible eastern regions during the past 1,500 to 2,000 years. The phenomena of "Indianization" and "Islamization" in Southeast Asia have long been major fields of historical study (for recent general surveys see Wheatley 1983; Hall 1985; Kulke 1990; Tarling 1992), and here I will only touch on some of the major points.

Indian trading enterprise in Southeast Asia and complementary Austronesian sailing to India (and later to Madagascar) appear to have commenced during the first few centuries $A D$ with the discovery of monsoon sailing across the Bay of Bengal (Wolters 1967; Christie 1984-1985; Glover 1990a; Ray 1991). This is evidenced by the recent archaeological finds of Indian pottery at Sembiran in Bali (to be discussed in Chapter 9). By the fifth century AD, some trade routes may also have linked the archipelago directly with China, and small numbers 
of Chinese pottery vessels and bronze items dating from the Han Dynasty and onwards are reputed to have been found in southern Sumatra and eastern Java. ${ }^{2}$ The initial Indian trade contact appears to have stimulated the development in some western regions of apparently native trading states from the second century AD onward (see Chapter 9, Fig. 9.4)—states such as Champa in central Vietnam, Funan around the Gulf of Siam (Coèdes 1947), several small states in the narrow isthmus of southern Thailand, and the rather hazy Ko-ying in Sumatra or western Java (Wheatley 1961; Wolters 1967, 1979; Mabbett 1977; Hall 1985; Higham 1989). However, a widespread and early colonization of Indians in the archipelago is most unlikely to have occurred according to the linguistic evidence, for the Indian loan words in Austronesian languages are almost all from Sanskrit - a language that would not have been in everyday use amongst Indian traders at the time. As Gonda (1973) has noted, there are no Prakrit loans and no trade pidgins in evidence, both of which would be expected had heavy colonization or actual conquest taken place.

The major religious and political processes of Indianization at the court level are now agreed by many authorities to have developed some centuries after this initial period of trade. The first indications that the native rulers of these IndoMalaysian trading states were beginning to model themselves on the Pallava kings of Tamil Nadu and their contemporaries appear around the fifth century, when inscriptions in Sanskrit written in Pallava script record kings with the Sanskrit name ending -varman ("protégé of") in eastern Borneo and western Java (Casparis 1975; Meer 1979). By the seventh century, the sources of the Indian influences seem to have shifted toward northern India and the Pala kingdom of Bengal (Bernet Kempers 1959), and for the first time really tangible Indianized kingdoms with divine rulers and magnificent Hindu or Buddhist monuments began to appear in the archipelago.

The Sumatran Buddhist trading state of Srivijaya, founded about 670 (Wolters 1967; Hall 1976, 1985), was probably the focus of an interlocked group of trading towns in eastern Sumatra (especially Palembang) and the Malay Peninsula. Its trade base was partially derived from the collection and export of native forest products such as pine resin, camphor, and benzoin (Wolters 1967; Miksic 1985), bolstered no doubt by tribute from ships passing through the Strait of Malacca. Srivijaya does not have a very coherent archaeological record (although see Adhyatman 1984 and Manguin 1992 for some recent ceramic finds dating between the eighth and thirteenth centuries from Palembang), and the contemporary Javanese agrarian kingdoms are much better known owing to their superb monuments: the great Buddhist stupa termed the Borobudur was constructed by the Sailendras in the eighth or early ninth century, and splendid Hindu temples were constructed slightly later in the region of Prambanan (Plate 12). After AD 930, the political focus of Javanese civilization shifted to eastern 
Java and culminated in the Majapahit kingdom of the fourteenth century, which was eventually to decline under the pressure of Islam.

The Hindu and Buddhist beliefs on which these kingdoms were founded were almost certainly brought into the archipelago by Hindu Brahmans and learned Buddhists. The former may well have been invited by native rulers to bolster their authority with the rituals and architecture of a major world civilization. The role of the Brahmans in the process of Indianization is generally agreed to have been crucial-and far greater than that attributable to traders (Bosch 1961; Leur 1967; Gonda 1973). Brahmans were necessary to consecrate rulers, who in many cases were identified with the Hindu gods Siva or Vishnu. It was perhaps through them that the majority of the Sanskrit loan words, which fall mainly into the intellectual and administrative categories, were introduced into Austronesian languages. As might be expected, the languages with the most Sanskrit loans are those associated with long-lived civilizations, such as Chamic and Javanese. Malay also acquired many loans through the kingdom of Srivijaya, which has bequeathed to posterity a small number of seventh-century inscriptions in Old Malay. Malay has also been the medium for the more recent spread of Sanskrit loans to many non-Indianized parts of Indonesia, and even as far as Irian Jaya (Gonda 1973).

The geographical impact of Indian influence in the archipelago was always focused heavily on the lands around the Strait of Malacca and the Java Sea. Hence it was of maximum strength in eastern Sumatra, the western Malay Peninsula, Java, and Bali, but it rapidly dwindled in parts of highland Sumatra (for example amongst the Batak) and was almost nonexistent in Nias and Mentawai. In eastern Borneo, the Kutei kingdom of the fifth century seems to have left few descendants, and in general this island, plus Sulawesi, the Lesser Sundas, and the Moluccas, were only affected by Indian civilization in a most superficial way. Traditions that the fourteenth-century Javanese kingdom of Majapahit once controlled the whole of the archipelago are hard to evaluate; Naerssen (1977) has suggested that it claimed tribute from the whole of Island Southeast Asia except for northern Sulawesi and the Philippines, but this may be a substantial exaggeration (Rausa-Gomez 1967; Hall 1968:87). In the Philippines, some Sanskrit loans appear to have spread as a result of Malay enterprise after the tenth century (Francisco 1965; Scott 1968; Postma 1991), and this period also saw the commencement of extensive trade with China, through which the Philippines were drawn increasingly into the wider Indo-Malaysian world.

Some caution is clearly necessary in interpreting the real significance of Indian influence. The great strength and tenacity of Austronesian cultural tradition are evident throughout the whole period, whether in ancient inscriptions in Old Malay or Old Javanese, in the terraced design of the Borobudur, or in certain cosmological concepts (Alkire 1972). The everyday life of the Javanese 
peasant was probably little changed by the far-off existence of a Hinduized court. One major feature of Hindu society in India, the caste system, has had only a limited impact in Southeast Asia, surviving today only in Hinduized Bali. A view that the Austronesian societies adapted certain Indian influences by select invitation only is not without attraction.

Today, the only ethnic group in Southeast Asia to have maintained a coherent-even if highly modified-Hindu tradition is the Balinese (Geertz 1980; Hobart et al. 1996). Prior to Dutch government this island was divided into a number of small statelike domains, with rulers and nobles belonging to widespread, high-ranking, and intermarrying patrilineages. Commoner kin groups have always tended to be localized in individual villages, but commoners also belong to corporate organizations such as temple groups and irrigation societies (subaks; Geertz 1972; Meer 1979) that cross-cut lineage and village boundaries. There is also a priesthood of Brahman derivation, but any visitor to Bali will quickly observe that the picturesque and ubiquitous temples owe little to Indian styles of architecture; there are even indications that some of them preserve aspects of a more ancient "megalithic" tradition (Sutaba 1976) that is widespread throughout the Austronesian world and that received one of its most coherent expressions in the shrines (marae) of late prehistoric Polynesia. The importance of ancestor worship has always characterized Balinese society (Sutaba 1996), just as it has all the other far-flung Austronesian societies that have not converted entirely to Islam or Christianity.

Apart from Hinduism and Buddhism, the only other major religion to affect the archipelago in pre-European times was Islam, which has become the national religion of Indonesia and Malaysia. Its spread has been much more recent than that of the Indian religions, and as a result its history is much better known. The major sultanates only preceded the Portuguese by less than a century. By the eighth century, communities of Arab and Persian Muslims were already settled as traders in Guangzhou (Canton) and other southern Chinese cities. The spread of Islam into Indonesia occurred several centuries later, and linguistic evidence suggests that the Arabic and Persian loan words in Austronesian languages came for the most part directly from India (Gonda 1973; Hall 1977). How the religion came to spread so quickly through the archipelago is not clear, but a combination of trading enterprise, missionary conversion, and the acuity of native rulers who sought power through alliances with well-connected outsiders probably suffices as an explanation (Kumar 1979). By the fourteenth century a trade network, mainly in Javanese and Malay hands, had been set up to bring spices such as cloves, nutmeg, and camphor from the Moluccas. This, added to the well-established trade network from China through the Philippines and around Borneo, undoubtedly provided an excellent channel for the propagation and spread of Islam. By the late thirteenth-century Islamic influ- 
ence was well established in northern Sumatra; a Muslim tombstone found on the northwestern coast of this island is believed to date from 1206 (Ambary 1981). During the fourteenth century a number of Islamic sultanates developed in this region (Miksic 1979), and from 1400 through to the growth of Portuguese power in the early sixteenth century the spread of Islam took place with great rapidity. Sultanates and trading ports developed in Malacca (Melaka), along the northern coast of Java, around the Borneo coast (with important states in Brunei and Banjarmasin), on the island of Jolo in Sulu, and on the islands of Ternate and Tidore off Halmahera. In the seventeenth century two more important trading states were developed by the Makassarese and Buginese of southern Sulawesi (Macknight 1973; Bulbeck 1992; Pelras 1996).

The early centuries of the second millennium witnessed some major transformations in terms of outside interest in the islands of Southeast Asia. For example, the sheer volume of ceramics imported from China during the Song and later dynasties contrasts starkly with the absence (at least in any quantity) of such material during the first millennium. The trade in spices and "forest products," which earlier was perhaps a rather sporadic affair, also spread to encompass virtually the whole archipelago. The islands were thus rapidly brought into contact with many groups of outsiders-not just Muslims, but with other groups such as Chinese and Thais. There are even some slight hints that ethnic Chinese may have settled in some places as craftsmen prior to the spread of Islam (e.g., Manning et al. 1980 for Kota Cina in Sumatra; Cheng 1969 for Sarawak, but contested by Christie 1985). Virtually the whole archipelago became connected to the greater Asian world between the tenth and fifteenth centuries to an extent far greater than in the earlier Indianizing period.

The spread of Islam was one major reflection of this and of course helped to speed up the process toward the end of this time span. By 1521, trans-archipelagic trading was established on a frequent and formal basis, as can be seen from the presence of special port officials (shahbandar) in Malacca to handle trade from regions as far apart as the Moluccas, Java, Luzon, Banjarmasin, and Palembang (Pelras 1981; Andaya and Andaya 1982; Cortesão 1944). One of the major effects of all this intensified trade and contact was that certain groups were able to take advantage of newly emerging options in order to expand widely through the archipelago. The Bugis and Makassarese of southern Sulawesi have been the most important of such groups in recent history, but the Malays have had the greatest impact of all, as they had the advantage of a westerly location around the trade highway of the Strait of Malacca, which enabled them to make their moves very early. Indeed, the whole phenomenon of the spread of the Islamic sultanates is closely tied in with the spread of Malay language and culture. This does not mean that all the Malay communities of the archipelago result entirely from migrations out of the Malay Peninsula; the process has been far more complex and assimilatory than this. 
Today, coastal Islamic groups who speak dialects of Malay and who identify themselves as Malays with localized epithets (e.g., Brunei Malays, Banjar Malays) form a homogeneous belt of peoples around most of the coastal regions of Borneo, eastern Sumatra, and the Maiay Peninsula (see Fig. 4.6). Had the Javanese not had such highly developed earlier civilizations, this island might also have supported many such coastal groups, but the great literary and demographic strength of Javanese civilization clearly made it resistant to cultural domination (Supomo 1995). The modern Malay language perhaps descends from a lingua franca that developed along the shores of the Strait of Malacca, especially in the old Srivijayan heartland of southeastern Sumatra, in southern Peninsular Malaysia, and in the Riau and Lingga Islands. This development cannot be dated with precision, but it must have been underway by at least the time of the foundation of the important sultanate of Malacca in 1414. From this period onward, the use of the Malay language spread rapidly through the coastal regions of the western archipelago, and even as far as Ternate and Tidore in the northern Moluccas. Both the language and the culture have taken on some decidedly assimilatory characteristics; at the present time people can "enter" Malay culture (masuk Melayu) by converting to Islam and speaking the Malay language. Hence most of the coastal Malays of Borneo are almost certainly of local origin in a genetic sense, although ruling classes do often have traditions of foundation marriages with Johor or Malaccan noble families.

The structures of the Moslem sultanates present intricate details that I can hardly hope to summarize here, but I will close this section with a pocket view of Brunei, one of the most important of these trading states (Brown 1970, 1978). During the Song dynasty Brunei was apparently known to the Chinese as P'o-ni (although Christie 1985 equates P'o-ni with Santubong in western Sarawak), and long before the arrival of Islam it seems to have been developing in size and renown, partly on the rich pickings from the trade routes linking southern China and the Strait of Malacca (Bellwood and Omar 1980; Omar 1981). By 1515 the ruler had converted to Islam (Nicholl 1975) and the rather elaborate ruling bureaucracy, which dominated northern Borneo (in spite of Spanish hostility) until the eventual reduction of its territory by the Brookes of Sarawak in the nineteenth century, appears to have acquired great wealth from the taxation of riverine districts all the way from Pontianak in western Borneo to the southern Philippines. In 1521 the sultan's court was described by Antonio Pigafetta, a survivor of Magellan's expedition, in terms that give an impression of considerable wealth and splendor. The Brunei nobles and commoners of today belong to an ethnic category that has been called Brunei Malay since at least sometime in the nineteenth century; the native (non-Brunei Malay) populations are of a lower social status, some being Muslim and Malay speaking (the Kedayans), while others are of more varied religious and linguistic affiliation (Bajau, Melanau, Dusun, and Murut). The Brunei Malay commoners themselves, 
who live in and around the riverine town of Bandar Seri Begawan, probably originated through the assimilation of local populations into the high status Malay lifestyle from the early years of the sultanate.

It will be apparent by now that the bulk of the present-day population of the archipelago, excluding the small-scale traditional agricultural groups who are still to be considered, has a way of life that no longer has much connection with the prehistoric Austronesian past. Furthermore, there is one important aspect of the past century that must not be overlooked. The population of Indonesia is now almost 200 million, of whom about 100 million live in crowded and agriculturally intensified Java. But the fifteenth-century population of Java was only about 4 million (Sudjoko 1981:3), and in 1815 the Raffles census reported it as 4.6 million; it then increased to 29 million by 1900 (McDonald 1980). The total population of the archipelago between the sixteenth century and 1820 probably fluctuated around 8 million (C. Geertz 1963; Reid 1980), indicating a level of demographic stability that is certainly not present today.

\section{THE INDO-MALAYSIAN TRADITIONAL AGRICULTURAL SOCIETIES}

I will now consider the small-scale traditional agricultural societies in the third category defined by H. Geertz in 1963 (see beginning of this chapter). In terms of descent ideology, the societies of Sumatra and the Lesser Sundas tend toward unilineal norms (as do the Chams of Vietnam), while those of Peninsular Malaysia, Borneo, Sulawesi, and the Philippines are basically cognatic (mainly bilateral, but occasionally with ambilineal descent reckoning). These distinctions are by no means as clear or necessarily as historically significant as some of the pre-1950s writers suggested, and the unilineal-bilateral "dichotomy" may be simply a reflection of other more fundamental differences in social structure. For instance, unilineal (and also ambilineal) kinship reckoning can only be expressed within a framework of corporate descent groups that have a membership greater than that of the individual nuclear or stem family. Societies lacking such corporate descent groups, such as the hunter-gatherers and some of the horticultural societies of Borneo and the Philippines, are necessarily bilateral.

These observations reflect little more than the logic of descent ideology and could lead into a discussion of correlations between different aspects of social structure, which I am rather unwilling to undertake. Perhaps I can suggest that, amongst the small-scale traditional societies, there are correlations between the existence of corporate landowning descent groups, stability of land use and tenure, and relatively high population densities (e.g., Miles 1972; Rousseau 1987, 1990:303 for Borneo). Conversely, there are apparent correlations between bilateral kinship reckoning and the absence of corporate descent groups, mobility 
in land use, and relatively low population densities. As far as kinship reckoning is concerned, the significant differences-more significant perhaps than issues of matri- or patrilineality - may thus be between those societies (both unilineal and cognatic) that have corporate landholding descent groups and those purely bilateral societies that do not. I will return to this matter later in this chapter, but I should add that social anthropologists have not to date been concerned with the study of such correlations on a pan-Austronesian or even a pan-IndoMalaysian scale, and there may be a great deal of historically significant information in this field still awaiting exposure.

\section{A. The Unillneal Societies}

In Sumatra, societies with strong patrilineal tendencies are found in the northern highlands (Aceh, Gayo, Batak), in the south of the island (Rejang), and in the isolated island of Nias off the western coast (Loeb 1935; Beatty 1990). In eastern Indonesia they are found, intermixed with small matrilineal enclaves, from Flores eastward and in the Moluccas (Loeb and Broek 1947). Examples of this kind of organization have been described for many groups; for instance, the Bataks of northern Sumatra inhabit villages-formerly defended by embankments —of large patrilineage houses with mat partitions for individual families (Loeb 1935). The island of Nias has a similar system. Originally, each new settlement would perhaps have been founded by members of one patrilineage, but as settlements grow they become more complex; Cunningham (1967) describes a village in Timor that has seventy-eight lineages represented in its population (although six of them form a definite majority). Lineages always become dispersed through processes of growth and fission over time, and many groups-such as the Batak-still call the localized lineages and the larger clans by the same name (marga).

Societies with strong matrilineal tendencies include the upland Chams of Vietnam, the Minangkabau of Sumatra, and the Minangkabau-derived population of Negri Sembilan in Peninsular Malaysia, plus a number of groups amongst the mainly patrilineal societies of the Lesser Sundas and Seram, such as the Tana 'Ai of Flores described in detail by Lewis (1988). The best-known group is undoubtedly the Minangkabau of Sumatra, whose village sections are focused on land- and house-owning matrilineages with uxorilocal residence. Traditionally these matrilineages were grouped into four Minangkabau-wide clans and further into two moieties (Loeb 1935). However, status positions are inherited by males, and noble lineages have a very strong tendency toward patrilineal descent.

One interesting feature of matrilineality amongst the Minangkabau of Sumatra and Negri Sembilan has been its survival in the face of Indian and 
Islamic cultural traditions within which patrilineality has always been stressed. The Minangkabau are not a remote and isolated population; in Sumatra they had Indianized rulers by the fourteenth century and they have been under the influence of Islam for the last 300 years (H. Geertz 1963; Kumar 1979). In Negri Sembilan the matrilineal system has survived as an enclave within Islamic Malay society since at least the sixteenth century, and here some aspects of matrilineal ideology appear to have spread into neighboring small-scale traditional societies such as the Temuan and the Semelai (Carey 1976). Hence the Minangkabau represent a situation where matrilineal descent within corporate landholding lineages has obviously been very stable in the recent past. But, as with the unilineal-bilateral distinction, it is difficult to show that patrilineal and matrilineal ideologies are always permanent, opposed, and nonoverlapping.

Most patrilineal societies, especially the stratified ones, have a system whereby the groom's family pays a bride-price to the family of the bride and the bride is then "released" by her family to live virilocally. But poorer families often cannot afford to pay the bride-price, in which case the husband lives uxorilocally, often in a position of low status (e.g., Cunningham 1967 for Timor). Even if a bride-price is paid, initial postmarital residence will normally be uxorilocal for a year or so; this custom also occurs amongst the cognatic societies of Borneo and Sulawesi. In matrilineal societies such as the Minangkabau there is no brideprice, and here the bride's family sometimes pays a dowry to that of the groom, who will live uxorilocally.

Amongst the patrilineal societies it is clear that the ideal of virilocal residence is not always practiced, either because the bride-price is not paid or because females and their lineages hold important rights to land (as in Mentawai, where women own and inherit rice and taro plots). It can be seen, therefore, that in decisions about postmarital residence and child affiliation to one or the other parental lineage, there will often be a strong tendency towards ambilineality in real life (e.g., see Ellen 1978 for the Nuaulu of Seram). So it may come as no surprise to find that the Rejang of Sumatra turned from a patrilineal and virilocal ideology toward matrilineality around 1930, after bride-price payments were eliminated owing to economic circumstances and pressure from Islam (Lebar 1972:32, quoting Jaspan). In western Timor and eastern Flores there are also situations where closely related ethnic groups can have either patrilineal or matrilineal tendencies (Schulte Nordholt 1971; Metzner 1982).

It is apparent from this that fluctuation from one norm toward the other can occur quite rapidly in some circumstances, although I hesitate to theorize about general causes or to postulate whether the role of bride-price is generally one of cause and effect. But it is necessary to warn against a view that the patrilineal and matrilineal ideologies necessarily represent ancient and long-lasting differences between different Austronesian societies. 


\section{B. The Cognatic Societies}

Cognatic societies that practice shifting cultivation with low population densities are found throughout large parts of Borneo (King 1993), Sulawesi, the Philippines, and Peninsular Malaysia. Many of the Borneo societies still inhabit distinctive raised longhouses with adjoined family living quarters linked by a common veranda, although this tradition is rapidly disappearing today. In the egalitarian and truly bilateral societies without descent groups, such as the Dusun of Sabah (Appell 1978) and the Iban of Sarawak (Freeman 1960, 1981), the individual two- or three-generation families form independent corporate groups who can make alliances with other families for decisions concerning residence and land use. Amongst the Iban, the land- and property-owning family is called a bilek; it survives from generation to generation as new members are born or join through marriage. Iban longhouses can hold up to fifty bilek families living in side-by-side dwelling apartments facing on to a shared verandathe whole structure being up to 200 meters long. Families can move from one longhouse to another if they wish; some villages consist of just one such structure, others of two or more.

The Iban do, however, represent one pole of Borneo variation, as cognatic landholding descent groups of greater generational depth are sometimes found in other societies, especially amongst the stratified societies of central Borneo (Rousseau 1990), among the Maanyan of Kalimantan (Hudson and Hudson 1978), and the longhouse-dwelling Selako (Schneider 1978). From the available ethnographies it seems that a similar range of variation in social structurefrom independent family units to societies with larger descent groups-also occurs in Sulawesi (e.g., see Nooy-Palm 1979 for Sa'dan Toraja cognatic lineages), although here there is a tendency for related families to share multifamily houses (quite magnificent ones in the case of the Toraja: Plate 13) rather than to build independent units in longhouses. Basically, the rather transient bilateral social formations without descent groups characteristic of societies such as the Dusun and the Iban seem to be well adapted to situations where land and labor are not in short supply (Frake 1956). It is interesting to note that corporate descent groups can develop in societies of this type in modern situations of cash cropping, especially of rubber, where land rights become more permanent and where a larger pool of labor is required (Miles 1972).

\section{Political Integration and Ranking}

The Indo-Malaysian traditional societies exhibit relatively small-scale systems of political integration and ranking. Many are basically alliances between egalitarian and independent villages, while others are focused on ranked lineage sys- 
tems that encompass one or more villages or a territorial unit such as a river valley. There is no indication that true states with specialized bureaucracies and the powers to maintain allegiance by force developed anywhere in the region before the Indian and Islamic periods. However, prior to such contacts it is possible that Indo-Malaysian societies evolved in some places (perhaps on Java?) into small-scale ranked chiefdoms or "domains" similar to those of parts of ethnographic Micronesia and Polynesia (although perhaps lacking some of the more extreme expressions of chiefly divinity and power recorded in eighteenthcentury Polynesia).

Ranking in Indo-Malysian small-scale traditional societies is based on a number of principles, the main one being that the descendants of the group that founded a settlement and first cleared the land will tend to preserve high status. Ancestors bulk large wherever we look in traditional Austronesian society, whether in art (Feldman 1985) or in mythology and tradition (e.g., see Lewis 1988 for Flores). Leaders often rise to power because of their ability to trace clear descent from a founding ancestor of a lineage or tribe. I have recently discussed the great importance of "foundership" as a factor giving rise to both migration (one needs to migrate, even if only a little, to become a founder!) and the rise of inequality in Austronesian societies as whole (Bellwood 1996c; Slamet-Velsink 1995). So we have a kind of "founder principle" that can be applied to the ranking of lineages, whether unilineal or ambilineal, but this ranking is also normally open to constant rearrangement through individual cleverness and the manipulation of wealth (as well attested for the links between feasting and chiefship in Nias; Marschall 1980; Beatty 1990). Rank can therefore be both inherited and achieved at the same time in a great many societies.

High-ranking founder lineages provide for the incumbents positions of secular and religious power in many societies-such persons have an important say in village affairs, are entitled to occasional presentations of food and labor from their "subjects," and normally control decisions about land use within the group territory. These lineages generally display their status through the ownership of wealth items: Chinese jars, ancient beads, megalithic monuments, fine weapons, drums, and so forth. Another kind of wealth is expressed through success in agriculture and the raising of livestock, especially pigs, the products of which can be used in prestige feasting. A powerful lineage can also reinforce its position through intermarriage with members of high-ranking lineages in other regions; this procedure has the double function of setting nobles apart from commoners and of expanding valuable alliances. Bride-price also often increases in value with rank; this can reinforce tendencies toward high-rank endogamy, and it can enable powerful lineages to increase their manpower by requiring males unable to meet the necessary payments to reside uxorilocally (e.g., see Forth 1981 for eastern Sumba). 
Such processes of rank enhancement might give the impression that a successful lineage, if it wished, could expand its power almost indefinitely. This is most certainly not the case. Lineage affiliations in real life are notoriously complex, oral genealogies can be manipulated, families wax and wane in terms of wealth and size and-more importantly-as soon as one lineage leader shows signs of increasing power in an unpopular way, there will either be fission or a revolt (as discussed in detail by Leach 1954 for societies of a similar level of organization in Burma). A state cannot develop from a small-scale traditional society unless the emerging leaders can monopolize power and convert the network of military and economic alliances between sections into a centripetal flow toward themselves. This never happened anywhere in the Austronesian world until the period of the Indianized states of the middle and late first millennium AD.

Turning now to look at the expressions of rank and class and their supporting ideologies throughout the traditional regions of the archipelago, one finds that societies tend to be fairly egalitarian in Peninsular Malaysia and parts of Borneo (not central), Sulawesi, and the Philippines, where population densities are low and where there is a dependence on shifting cultivation with bilateral organization and considerable family mobility. Villages are normally independent of each other and leadership is frequently by election, rather than by inheritance within a separate class of nobles. As groups come to depend more on permanent landholdings for wet rice or tree crops (as in the Sunda islands), the separation of noble and commoner classes becomes more marked. This is especially true for those societies that have had close associations with the Islamic sultanates and the networks of international trade. The latter, of course, have provided many of the prestigious wealth items that so frequently provide material support for rank. In general (see beginning of this section), it is apparent that the existence of rank and class divisions in the small-scale traditional societies tends to correlate with the existence of corporate descent groups of unilineal or ambilineal type, as it is between such groups that differential statuses are displayed. It is not true, however, to assume that all cognatic societies necessarily have no class structure; aristocracies are present in the cognatic societies of central Borneo, apart from the Punan (Rousseau 1990; King 1993). In this region of relatively low population density, the ruling lineages appear to be able to coerce their followers into settlement-endogamous marriage arrangements, thus ensuring the maintenance of larger supporting populations (e.g., see Alexander 1993 for the Lahanan).

Amongst the relatively egalitarian societies there is considerable local variation. The Austroasiatic-speaking Senoi of Peninsular Malaysia dwell in villages led mainly by councils of influential elders or by elected headmen (Lebar et al. 1964; Dentan 1968). So too do the Malay-speaking Jakun and Temuan (Carey 
1976), although in the latter groups there are now district headmen as a result of Islamic Malay influence. Many groups in Sulawesi and Borneo have elected headmen and other nonheritable leadership positions at the village level; one group in this category is the Iban of Sarawak.

The Iban have attracted much interest owing to their phenomenal rate of expansion from the Kapuas Basin of western Kalimantan through vast areas of Sarawak within the past 400 years. By 1850 they had expanded to settle most of the Rejang Basin (St. John 1974), and during the late nineteenth century they continued onward to encroach upon the borders of Brunei. Their expansion involved the clearance for shifting rice cultivation of enormous areas of virgin equatorial rain forest, and Freeman (1955:25) reports the case of one bilek family that moved over 300 kilometers in one lifetime. King (1976) and McKinley (1978) have suggested that this expansion was not simply due to population pressure or shortage of land. King believes that the values and beliefs connected with the need to acquire human heads to increase health, prosperity, and status may also have been significant (as also suggested by Hose 1926 for Kayan expansion).

Yet despite the Iban successes in colonization and in assimilating other groups, they never had permanent leaders until they came firmly under the control of the Brooke government after 1841 (Brown 1978; Freeman 1981). Their society was basically classless and egalitarian according to Freeman (1981); each longhouse had nonhereditary guardians or leaders for a number of specific spheres of activity, including law (adat), warfare, and the opening up of new lands. Men achieved these statuses through individual prowess and charisma and through success in agriculture and collecting heads. However, recent studies (Jawan 1992; Sather 1990) suggest that status can form around successful Iban founders or wealthy men when settlements have become stable; as one might expect, the migratory phase is the least conducive to the formation of rank.

I turn now to examine some of the more stratified societies of the region. I have already noted the widespread principle that descendants of founder lineages tend to be of high rank and to control many of the important decisionmaking positions. Some groups in Sumatra use titles of Indian origin for highranking persons (for example, the Singa Maharaja of some Batak groups), but such occurrences do not alter the basic observation that systems of rank and class are quite widespread in Austronesian societies (particularly in Oceania), and they must in some form be of great antiquity.

In the unilineal societies of Sumatra and the Lesser Sundas, the district and village leaders are drawn from high-ranking lineages, often descended from founder figures, and they are usually entitled to labor services from commoners as well as food shares (e.g., see Loeb 1935 for the Bataks). High-rank endogamy 
has given rise in many ethnographic societies to noble, commoner, and slave (war captive and debtor) classes (e.g., see Cunningham 1967 for the Atoni of Timor; Forth 1981 for eastern Sumba). Fox (1977a) has described the numerous small states (or "petty feuding domains") of the past few centuries on Roti and Savu; the hierarchies here were based on ranked patrilineages. On Roti twelve of these small states were in existence in 1690; during the nineteenth century the number increased to eighteen. The Savu states were linked by the sharing of islandwide lunar rituals, and their leaders had heavily ritualized functions; on Roti there appears to have been less integration between the units. However, both Fox (1977a) and Forman (1977) have stressed that these localized hierarchies and their supporting tribute-collecting arrangements were probably intensified by Portuguese and Dutch trade and colonial interference.

One society that does reveal an interesting and presumably indigenous system of ranking is that of southern Nias, off the western coast of Sumatra. Village chiefs here held hereditary titles controlled by noble patrilineages, and there was also an important hereditary male priesthood. The massive chiefs' houses and the unique megalithic monuments of Nias have long been famous as material creations of this intensely ranked society (Schnitger 1964), and it appears that Nias chiefs were considered semidivine, like some of their Polynesian counterparts. They became powerful spirits after their deaths and only they were allowed to wear gold ornaments and to hold intervillage feasts. Chiefly status for the living and the dead was the motivation behind the creation of the spectacular stone structures, and chiefs were also able to keep slaves.

The competitive feasting that bolstered chiefly status on Nias involved great presentations of wealth, especially of pigs, of which up to 1,500 were killed in reported cases. Similar periodic and massive pig slaughters are also characteristic of many societies in Melanesia, and they may once have been more common in Indonesia; the Islamic prohibitions against eating pork, which never reached Nias, have of course had a great impact elsewhere in this region. Nias chiefs could also accumulate and loan wealth with similar profit motives (see Suzuki 1959:40-41) as the Big Men of Melanesia, and I suspect that this type of competitive achievement imposed on a basic system of hereditary ranking was once characteristic of many ancient Austronesian societies.

As already noted, some of the cognatic societies of central Borneo also have class systems and aristocracies based on inheritance, family alliances, and the ownership of highly valued objects. Such groups include the Kenyah, the Kayan, and the Maloh (Hose and McDougall 1912; King 1978, 1993). The Kayan in particular-despite their low population density-maintained three or four social strata from nobles to slaves (Rousseau 1978). The chiefs retained much of their status through intermarriage with chiefly families in other villages, while commoners tended to be village-endogamous. SIave sacrifice on the 
death of a chief (as in Nias) is also reported to have occurred amongst the Kayan and the Melanau of Sarawak (St. John 1974).

\section{OTHER ETHNOGRAPHIC FEATURES OF AUSTRONESIAN TRADITIONAL SOCIETIES}

It is not my purpose here to list all the material correlations of the small-scale traditional societies in the archipelago, but some features drawn from the comparative ethnographic record are of obvious interest for prehistory. Settlements, for instance, are normally focused on village-type nucleations for social reasons and (in the past) for defense. Houses (Waterson 1990; Fox 1993; Plates 13-16) are almost universally rectangular, with the great longhouses of Borneo at the upper end of the size range: up to 200 meters long and sometimes raised 10 meters off the ground. The Minangkabau, Batak, and Toraja have particularly fine multifamily houses with some superb artwork, but in most coastal areas where outside influences have been strong, much smaller nuclear or extendedfamily houses are the norm. Circular houses are not common, but they are constructed in Enggano, western Flores, and by the Atoni of Timor.

Most traditional villages in the archipelago also had in the past one or more special houses in which sacred paraphernalia such as hunted heads, ancestor relics, and lineage symbols and valuables were kept. Sacred storage houses of this kind often served also as temples and as foci for meetings; the small godhouses described for the Simalungun Bataks of Sumatra by Bartlett (1934) were inside fenced enclosures that were also used for the growing of sacred plants and for assemblies-functions rather like those of the marae temple structures of Polynesia. Bartlett, incidentally, regarded these Batak sacred houses as preIslamic survivals, and the widespread distribution of such structures in the Indo-Malaysian Archipelago and in Oceania argues for their great antiquity.

Amongst items of portable material culture, it should be noted that most ethnographic communities either made or had trade access to pottery and iron (Marschall 1968). The clothing of early Austronesian societies was of bark cloth, beaten from the inner bark of a number of local trees such as Manila hemp (abaca, Musa textilis), paper mulberry, and breadfruit, but use of the backstrap loom for weaving has spread through most parts of the archipelago and into parts of western Oceania since at least Proto-Western-Malayo-Polynesian times. Bows and arrows and spears are of at least proto-Austronesian antiquity, but the blowpipe is probably more recent. This device, used with poisoned darts or clay pellets, was developed in or around Borneo according to Jett (1970). It was used throughout the archipelago and taken-presumably by the initial Austronesian settlers-to Madagascar (Fig. 5.1). The blowpipe has also been adopted by the Austroasiatic-speaking Negritos and Senoi of Peninsular Malaysia, although the 


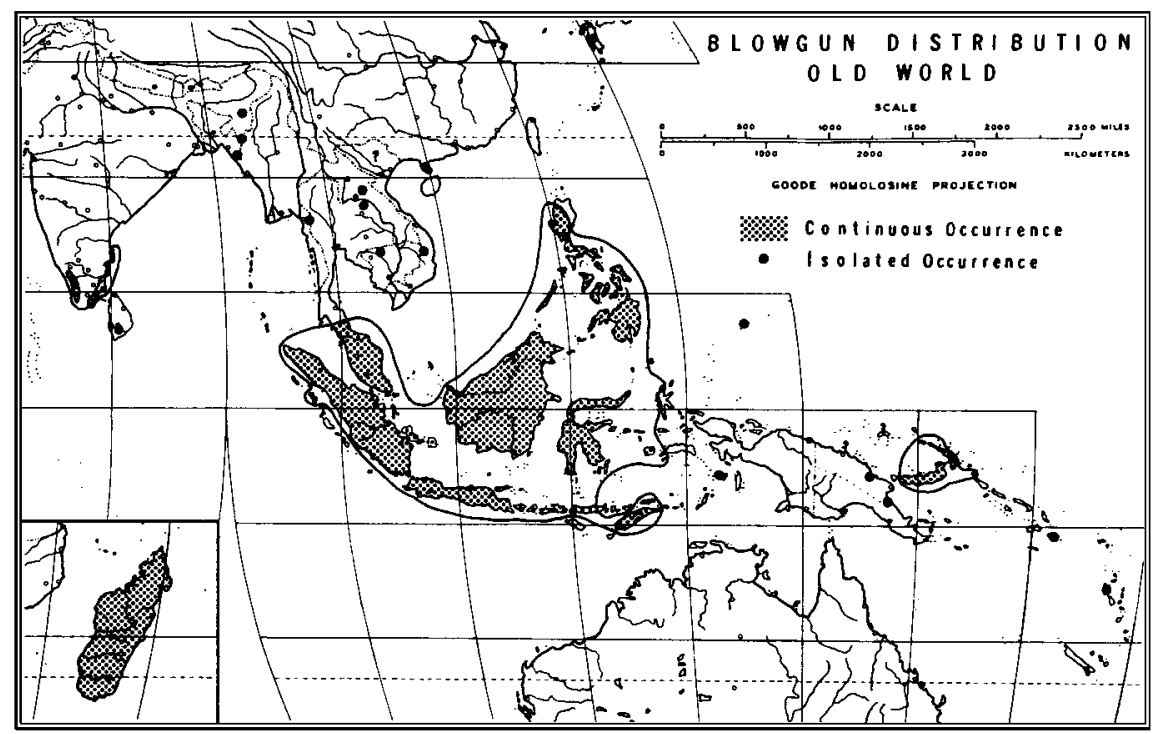

Fig. 5.1 The distribution of the blowgun. From Jett 1970. Courtesy: Association of American Geographers; S. C. Jett.

Philippine Negritos have retained the bow. To my knowledge the pellet bow of India and Mainland Southeast Asia has never been used in the Southeast Asian islands.

Tattooing is a fairly universal trait in the Austronesian world (see Plate 6). For instance, Murut men in northern Borneo traditionally tattooed stars on their shoulders to denote captured heads (Rutter 1929). Deformation of the skulls of infants has not been widely reported, but the Melanau of Sarawak depressed the foreheads of young girls, the people of Minahasa (north Sulawesi) practiced cradleboarding (Hickson 1889:213), and Maceda (1974) reports cases of fairly recent head deformation and trepanation from the Philippines. The practice of headhunting was widespread in the larger islands (it would clearly have been impractical on the smallest ones); I have already mentioned its association with status and expansion amongst the Iban and Kayan of Borneo. Downs (1955) reports that the Bare'e Toraja of Sulawesi used to form raiding parties of ten to twenty men to take heads for the rituals associated with mourning ceremonies, to consecrate sacred houses, and to prove bravery. Mourning rituals were also a major stimulus for headhunting in Borneo (Metcalf 1982).

Most groups in the archipelago who have not been influenced heavily by Indian, Islamic, or Christian traditions practice secondary forms of burial, in which the defleshed bones are eventually stored in a receptacle of some kind. A 
full account of secondary burial rituals for high-ranked Berawan in Sarawak is given by Metcalf (1982); the details are too complex to summarize here, but they make one realize just how much is sure to have been lost in the archaeological record. Interesting ethnographic examples of archaeologically recoverable receptacles for both primary and secondary burials include the stone sarcophagi and stone urns of the Bataks of Sumatra and the Minahasans of northern Sulawesi (Bellwood 1978: Figure 8.24; Dalrymple 1984); the common use in Borneo of large stoneware jars, often cut open to take crouched primary burials (e.g., see Harrisson 1962 for the Kelabits; Massing 1981 for the Benuaq of Kalimantan; Metcalf 1982 for the Berawan); and the common use of megalithic structures, particularly in Borneo and the Lesser Sundas (Schneeberger 1979; Sukendar 1985b; Hoskins 1986; Newton and Barbier 1988). Borneo and Sulawesi in particular have an immense range of wooden burial structures, in the case of Borneo often consisting of wooden mausolea or log coffins that clearly have limited scope for archaeological survival unless placed in caves (as log coffins often were: see Bellwood 1988 for Sabah). Also, in Java there are indications that "charnel houses" raised on posts were used for secondary burial prior to the period of Indianization (Stutterheim 1956).

\section{THE COMPARATIVE RECONSTRUCTION OF EARLY AUSTRONESIAN SOCIETY}

In the final section of this chapter I will present observations from comparative ethnology to supplement the list of features reconstructed linguistically for early Austronesian society in Chapter 4 . The problem can be approached in two ways: either by broad pan-Austronesian comparisons, or by trying to find isolated societies that might have preserved earlier cultural patterns. I will examine the second approach first, if only to reject it totally. I have already shown that isolated hunter-gatherer groups such as the Tasaday and Punan are not valid candidates for ancient reconstructions, and neither are the presumably longisolated societies of Mentawai and Enggano off the western coast of Sumatra. Traditionally, the Mentawaians lacked betel chewing, pottery, metal, rice, and the blowpipe, and the people of Enggano had a similar list of absences that also included weaving and cattle. Both groups used stone tools and depended on taro cultivation until recent times (Loeb 1935; Lebar 1972). Yet one has only to examine the linguistic list of early Austronesian reconstructions (see Chapter 4, Table 4.2) to see that while metals, the blowpipe, cattle, and possibly weaving are relatively recent in the archipelago, three of the items (pottery, betel chewing, and rice) are of at least Proto-Malayo-Polynesian antiquity. So these are cultures that have presumably lost the three items during their ancestry, rather than being fossilized pre-pottery or pre-rice survivals. They clearly reflect local 
adaptation and some loss of cultural items rather than a totally pristine and conservative ancient stratum.

Broader comparative reconstructions are of more value, but there are pitfalls. For instance, simple observations that pile houses, headhunting, and megaliths are widespread are of little assistance in indicating antiquity without linguistic support, and when such entities are studied in isolation they can give peculiar results. I need hardly stress the impracticability of Perry's view (1918) that megalithic monuments in Indonesia were introduced by sun-worshipping, "stoneusing immigrants."

However, when turning to concepts and customs in the religious and social spheres, where simple trait diffusion is perhaps less likely, we can make some headway. In the realm of religion it is clear that beliefs centered on spirit animism and ancestor cults are so widespread and deep seated that they must be of great antiquity (Newton and Barbier 1988). Shamans (i.e., inspirational priests or mediums who are able to converse with spirits through trances) are particularly widespread in Austronesian societies, particularly in Oceania. A dualism of male-sky (e.g., Lowalangi on Nias, Rangi in New Zealand) and female-earth deities, concepts of supernatural and mystical power (mana in Polynesia, semangat in Malay; Winstedt 1953:19), and taboo (tapu in Polynesia, rebu in Batak; Loeb 1935:94-95) are also virtually pan-Austronesian. Blust (1981c) has presented a linguistic reconstruction for a Proto-Austronesian term referring to supernatural punishment for offending ancestors or superior persons (i.e., breaking a taboo), and he has also traced other ritual activities to possible ancient borrowing from Negrito societies.

On the matter of status positions in early Austronesian society, Blust (1980) has reconstructed the Proto-Malayo-Polynesian term datu for a lineage official. Pawley (1981) has suggested that Proto-Oceanic society was stratified with terms for hereditary chief, firstborn son of a chief, and a person of low status. Historical evidence predating AD 800 from Java shows that the term ratu (raka in the Kalasan inscription of $A D$ 778) was applied to a head of a district grouping of several villages (Naerssen 1977; see also Meer 1979; Christie 1986). The implication of this is that central Javanese society in the immediate pre-Sailendra period was probably ordered into a number of small embryonic states or chiefdoms, although this is perhaps no more than would be expected at this period, given subsequent developments in this region.

I will turn finally to the reconstruction of aspects of Proto-Austronesian social organization. Goodenough (1955) has compared societies in the Philippines and Oceania to reconstruct a cognatic type of society for this early phase (see also Loeb 1935), possibly with landholding ambilineal descent groups. More recently, Blust (1980) has presented a totally different reconstruction based on the Lesser Sunda evidence as interpreted by Wouden (1968). The 
details of this are complex, but basically he thinks that early Austronesian societies were organized around a double unilineal descent system, with each person belonging to a separate exogamous matrilineal and patrilineal lineage. Each society would have had four maximal lineages-two matrilineal and two patrilineal-which would have been paired (one of each) into two exogamous moieties.

Both these reconstructions are in obvious opposition and it is not easy to select one or the other as being most plausible (see Blust 1994 and Fox 1994 for further technical debate on the issue). As pointed out by Fox, it is quite possible that the earliest Austronesian societies used both cognatic and unilineal ideologies in different contexts, as do many small-scale traditional Austronesian societies and even more cosmopolitan groups such as the Balinese today. Tendencies toward unilineal and ambilineal descent reckoning may therefore have developed in those societies that evolved ranked corporate groups under situations of high population density and permanency of land use. Since I have already discussed such correlations in this chapter, I will not pursue them further, but I think it should be stressed that Proto-Austronesian society, whatever its precise nature, must have held the seeds of all the traditional and nonoutsider-imposed variations that are evident today (see also Fox 1985).

Another final possibility that may be significant for eastern Indonesia (and also Melanesia) is that the unilineal tendencies amongst Austronesian societies here could reflect very strong influences from the pre-Austronesian populations of the region. These were presumably related to the ancestors of the present Papuan-speaking and predominantly unilineal populations of New Guinea and western Melanesia. Close relationships between some aspects of the societies of eastern Indonesia and New Guinea have also been pointed out by Lebar (1972:124) and Kennedy (1937). However, the issue is complex and may never be fully resolved; I can only present here my own rather intuitive views on the matter. 


\section{The Hoabinhians and Their Island Contemporaries}

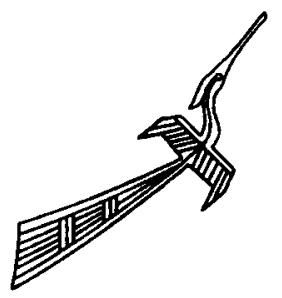

I will now turn to the preceramic archaeological record of the late Pleistocene and early Holocene periods in the Indo-Malaysian Archipelago. In this time span there are a number of long-occupied, radiocarbon-dated, and stratified sites (for locations see Chapter 3, Fig. 3.2 and Fig. 6.1), and all associated human remains are of anatomically modern physical type. Prior to the appearance of pottery, most stone industries in the region consisted of flaked rather than polished stone tools, although edge-ground pebble tools do occur in some sites (such as Niah in Sarawak and Kota Tampan in Peninsular Malaysia). Indeed, well verified edge-grinding of late Pleistocene date is also reported from Australia, New Guinea, Vietnam, and Japan (see below). The rather limited economic evidence at present available for the whole of the Indo-Malaysian Archipelago suggests a universal economy of hunting and gathering prior to about $2500 \mathrm{BC}$. During the Holocene the stone tool industries became more varied, and after $2500 \mathrm{BC}$ new items-pottery and fully ground stone adzes are the most visible-spread through the region.

As I have shown in Chapter 4, the linguistic evidence clearly attests a slow expansion of Austroasiatic and Austronesian-speaking agricultural groups during the last five millennia, but this expansion was not a geographically unified and totalitarian process of replacement. The hunting and gathering lifestyle has been progressively eroded but it has certainly never disappeared entirely, and flaked stone tools continued to be used by both hunting-gathering and agricultural groups until the recent past in some areas. Agriculturalists have also continued to hunt and gather. Hence in recent millennia different technologies and economies could and did occur in neighboring and contemporary sites, creating a mosaic (Hutterer 1976). The archaeological record has to be consid- 


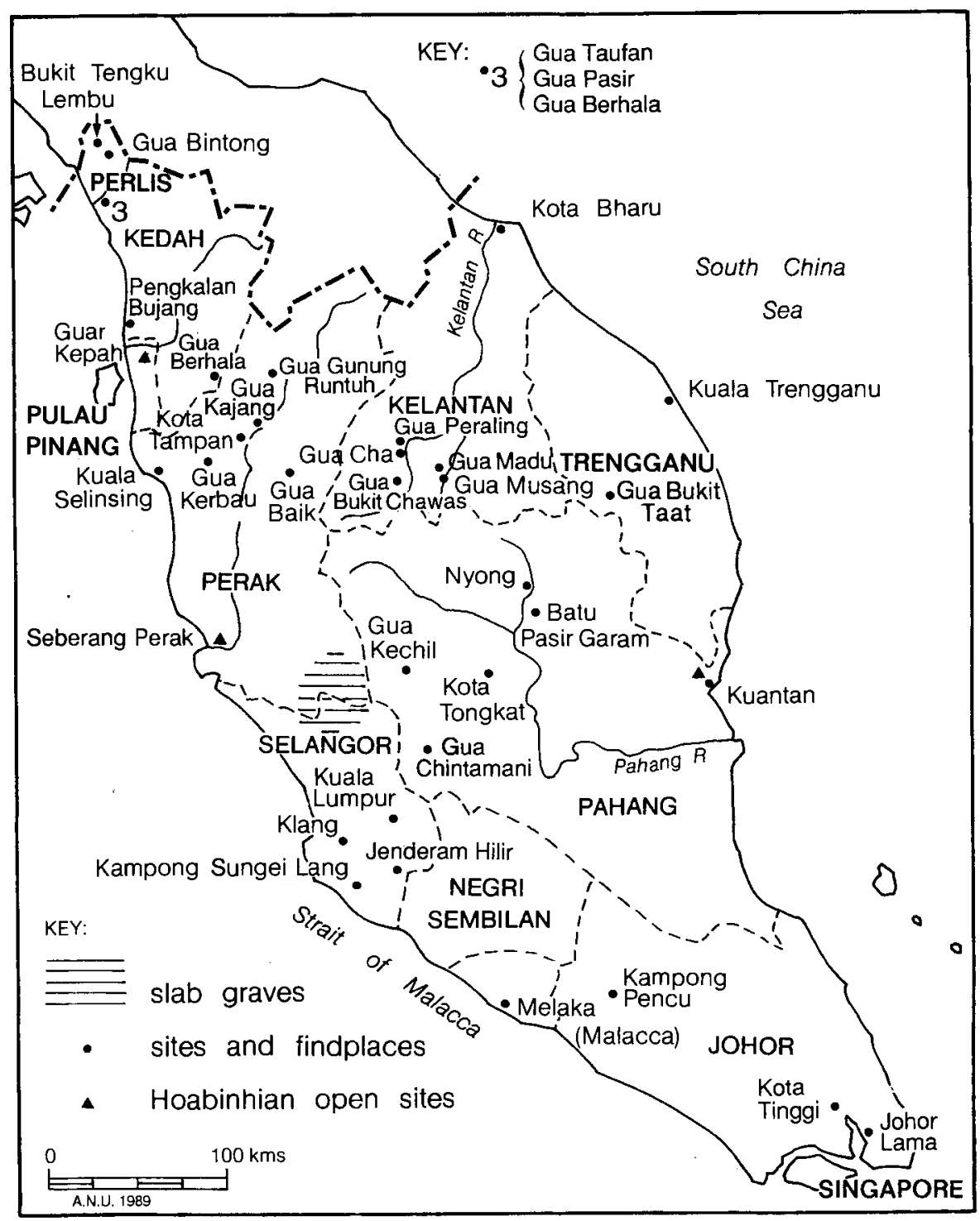

Fig. 6.1 Major archaeological sites in Peninsular Malaysia.

ered partly in terms of synchronic regional variation and not totally in terms of pan-archipelagic technological and economic phases following one after the other.

Before going further, it is necessary to give a brief recapitulation of the paleoenvironmental evidence from the archipelago, particularly pertaining to the 
last 30,000 years, within which the great bulk of the preceramic dated sites lie (see Chapter l, Section IVD). The most dramatic environmental changes would undoubtedly have been caused, particularly in the Sundaland region, by a drop in sea level from about minus 70 to minus 120 meters between 30,000 and 20,000 years ago (see Fig. 1.6), and then a much more rapid rise to the present level between approximately 15,000 and 8,000 years ago. Apart from drowning an unknown number of coastal archaeological sites-to the obvious detriment of modern archaeological studies-the postglacial sea level rise carved the Sundaland continent into the islands that exist today. Economically, the change would have had certain benefits from the increased length and environmental variety of coastline, but there might also have been less favorable changes for human population densities, particularly through the expansion of everwet rain forest, both altitudinally and latitudinally, with the prevailing warmer and wetter climate.

Although the postglacial climatic amelioration perhaps had little effect in the core regions of the equatorial rain forest close to the equator, it would presumably have had more impact on fringing areas of seasonal climate, where monsoon forest or parkland vegetation may have been more extensive during the last glacial period. An increasing density of vegetation in these areas would have affected hunting populations through a diminution in mammal biomass, which decreases dramatically as one moves from optimal savanna conditions, through parkland, toward rain forest. For instance, modern densities of wild banteng cattle range from about ten to fifteen animals per 100 hectares in Javan savanna grasslands down to only one to two animals per 100 hectares in rain forests (Pfeffer 1974). Rain forest faunas present additional problems in that animals rarely herd together; in addition, many species are arboreal, making them more difficult to hunt. This atomistic pattern also characterizes rain forest vegetation, with many species mixed in a mosaic of small numbers of individuals rather than in large stands. Such patterns tend to promote nonspecialized economies and low population densities amongst hunting and gathering populations. Even today, huge regions of interior Borneo rain forest are quite uninhabited by agriculturalists and foragers alike. In view of these points, perhaps it is no coincidence that most of the late Pleistocene flaked stone assemblages in the archipelago come from such seasonally dry regions as central Java, southern Sulawesi, the Lesser Sundas, and parts of the Philippines; human collecting and hunting populations would presumably have been denser here.

Yet there is a puzzle in this. If the archaeological record for the Indo-Malaysian Archipelago is taken at face value it suggests that inland rain forest occupation occurred mainly during the warmer and wetter phases of the late Pleistocene and early Holocene (Endicott and Bellwood 1991; Bellwood 1990a, 1993). So far there is only very limited evidence for occupation a long way inland dur- 
ing the drier last glacial maximum, around 20,000 years ago. Such evidence is represented, for instance, by a few riverine shells at Gua Sireh in Sarawak, a site which then would have been located about 500 kilometers inland (see p. 175). There appears to be no evidence for occupation of the Peninsular Malaysia interior rain forests at this time. This goes against expectations, and the reasons are not clear. Perhaps the very expansion of the equatorial forest itself caused those people living toward its boundaries, especially in regions formerly under monsoon forest, to try new and ultimately more successful methods of trapping and subsistence and hence to increase their population densities. Perhaps food supplies are actually greater in some equatorial rain forests than in the monsoon forests, although if this is true it would go against the body of theory postulating that equatorial rain forests are not good locations for foraging (this issue was discussed at some length in Chapter 5, Section I). Perhaps also, in periods of very low sea level, people occupied mainly the coastal regions, which at that time would have been far away from the present-day equatorial interiors of Peninsular Malaysia and Borneo. At present we simply do not know the answer to this puzzle, but the last glacial maximum "gap" certainly seems to be real.

\section{PENINSUlaR MALAYSIA AND MAINLAND SOUTHEAST ASIA: THE HOABINHIAN AND ITS PREDECESSORS}

Prior to Austronesian settlement, the Malay Peninsula and the adjacent coasts of northeastern Sumatra belonged culturally to the mainland of Southeast Asia rather than to the islands. Between about 18,000 and 10,000 years ago, Hoabinhian assemblages first appeared throughout this region, and there seems little reason to doubt that in Peninsular Malaysia they were made by populations ancestral-either fully or in part-to the present Austroasiatic-speaking orang asli (Negritos and Senoi; Solheim 1980). These groups had ceased to make flaked stone tools long before recorded history, but the Negritos have preserved a hunting and gathering way of life that may be regarded as a modified descendant of the inland Hoabinhian economy.

The term Hoabinhian has been in use since the 1920s to refer to a stone tool industry characterized by distinctive pebble tools flaked over all of one or both surfaces (Fig. 6.2). Hoabinhian sites are found all over the mainland of Southeast Asia, westward to Burma, and northward to the southern provinces of China and perhaps Taiwan. So far, all radiocarbon-dated Hoabinhian assemblages fall between outer limits of 18,000 and 3,000 years ago; it is possible that some Hoabinhian tool manufacture continued into even more recent times in some regions. The greatest "density" of Hoabinhian occupation, particularly in southerly regions such as Thailand and Malaysia, undoubtedly occurred in the early Holocene. 


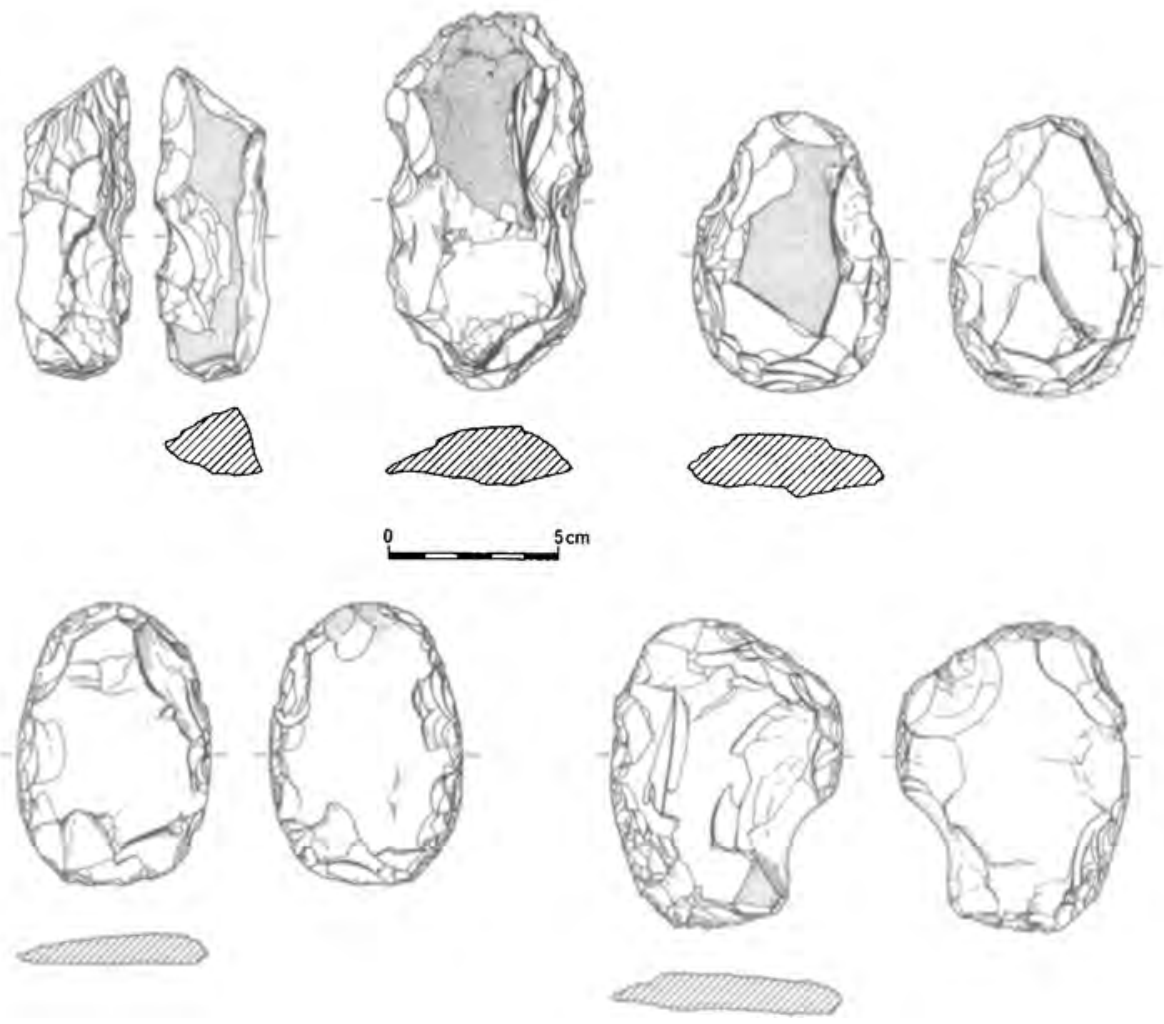

Fig. 6.2 Hoabinhian tools from Gua Cha. Note the incipient waisting on some.

\section{A. The Antecedents of the Hoabinhian}

Late Pleistocene lithic predecessors of the Hoabinhian have been discovered in recent years (Reynolds 1993), particularly in northern Vietnam, southern Thailand, and Peninsular Malaysia. In Vietnam an antecedent pebble tool industry termed the Sonviian has been dated to between 23,000 and 11,000 radiocarbon years ago (Ha Van Tan 1978, 1980, 1985a, 1991; New Researches into Prehistory of Viet Nam 1988). The Sonviian differs from the Hoabinhian in having mainly end- and side-flaked pebbles, rather than pebbles flaked all over one surface, and there is clearly overlap between the two both in technology and in time (Hoabinhian industries first appeared in Vietnam around 18,000 years ago). Whether the differences are culturally significant or simply reflect raw material variations remains to be seen, but it is clear that there is no sharp break in lithic evolution in Vietnam during the late Pleistocene and early Holocene.

The situation of apparent continuity also occurs in Peninsular Malaysia at 
the site of Kota Tampan in Perak-a site already introduced in Chapter 2, Section D6-because of older (mistaken) views about a potential association with Homo erectus. Since 1987 Kota Tampan has been researched by Zuraina Majid, who has shown that it served as a manufacturing locus for pebble and flake tools of quartzite, located close to a former lake about 25 kilometers long possibly dammed up by a landslide in the Perak River valley (Zuraina and Tjia 1988; Zuraina 1990, 1991). The tools are in soil evidently sealed by a layer of volcanic ash from an eruption of the Toba volcano in northern Sumatra, dated by fission tracks in zircons to about 31,000 years ago (Zuraina 1990:89-90). ${ }^{1}$ The tools comprise a few pebble tools, one edge-ground tool, and large numbers of flakes; as illustrated they show some resemblance to the Sonviian of Vietnam and may thus be regarded as a potentially ancestral Hoabinhian industry. However, in Malaysia-unlike Vietnam-there are no occupations dated anywhere between Kota Tampan and the full Hoabinhian, which started there apparently around 13,000 years ago. The last glacial maximum is a void.

The same problem applies to the final site to be described here, a site that adds yet another twist in the form of a technological noncontinuity prior to the Hoabinhian. This site is a rock shelter called Lang Rongrien in Krabi Province, southern Thailand (Anderson 1987, 1990). Lang Rongrien now lies 12 kilometers inland near the head of Phangnga Bay, but during the last glacial maximum it would have been up to 135 kilometers inland. In its upper layers it contains a fairly standard Hoabinhian industry (underlying Neolithic burials) dated to the early Holocene, with a strong bifacial aspect similar to those from the Malaysian sites to be described below. Beneath the Hoabinhian in Lang Rongrien lies an archaeologically sterile layer of rock fall, then beneath this is a basal layer with chert pebble and flake tools dated from four charcoal radiocarbon samples to between 38,000 and 28,000 years ago. Some of the basal tools are of tabular form with bifacial edges, and some of the small debitage looks as if it might derive from bifacial working (see Anderson 1990: Figs. 45-53 for illustrations). This debitage appears from the illustrations to be a little similar to the bifacial flaking debitage from Tingkayu in Sabah (Section IIB), although Anderson does not interpret it in this way. A sequence similar to that from Lang Rongrien is reported from the cave of Moh Khiew in northern Krabi (Pookajorn 1994), but full details of this are not yet published. Moh Khiew also has a basal biface industry dated to $26,000 \mathrm{BP}$, seemingly followed by a Hoabinhian layer dated only to the end of the Pleistocene.

In his conclusions, Anderson points out that the Lang Rongrien basal industry with its nonpebble focus is clearly not ancestral to the pebble-tool Hoabinhian, an opinion with which I concur. But how one explains this apparent disjunction is another matter. If the basal industry of Lang Rongrien really is related to the biface industry from Tingkayu, it could represent a specific radia- 
tion of a population that has not been picked up elsewhere (except possibly at Moh Khiew). Whatever the answer, in the Malay Peninsula it appears that the Hoabinhian represents a later radiation-following a hiatus in last glacial maximum occupation-into the expanding postglacial equatorial rain forests of the early Holocene. It should be remembered, of course, that there is no evidence for such a hiatus in more northerly regions such as China, central/northern Thailand, or Vietnam; the sequence of occupation there seems to be continuous from at least 23,000 years ago right through to the commencement of the Neolithic.

\section{B. Some Hoabinhian Basics}

In Peninsular Malaysia and Sumatra the true pebble-tool Hoabinhian, as defined by all-over flaking of one or both surfaces of river pebbles, does not appear to extend back in time for more than 13,000 years. Hoabinhian sites are found mostly in rock shelters, but there are a few coastal shell middens in Sumatra and Peninsular Malaysia that seem to belong to the present period of sea level after 8,000 в . These middens have never been satisfactorily investigated; most have now been destroyed for lime (McKinnon 1991). In addition, some inland open sites with Hoabinhian stone tools have been reported. However, the excavation record is highly skewed toward the inland rock shelters in the many limestone massifs that dot the jungles of the Malay Peninsula.

The characteristic tool types of the Hoabinhian are unifacially or bifacially flaked flat river pebbles of an approximate fist size, often with cutting edges all around their peripheries (Fig. 6.2). They come in a variety of shapes, from oval through rectangular to triangular, and some occasionally have waisted forms. Bifacially worked tools appear to predominate in most sites in Peninsular Thailand (e.g., Lang Rongrien) and Malaysia, but unifacial forms predominate elsewhere in Thailand, Burma, Laos, Cambodia, and Vietnam. The industry (or technocomplex, after Gorman 1971) has been excavated most prolifically in the limestone massifs of northern Vietnam, where it is associated with flake tools, stone mortars and pounders of various sizes, bone points and spatulae, and flexed burials often dusted with red ochre (hematite).

In Vietnam there is considerable industrial variation within the Hoabinhian time span, and many sites also have edge-ground tools, apparently dating back to 18,000 BP in Xom Trai Cave (Ha Van Tan 1991; Pham Ly Huong 1994). Indeed, overlapping with the late Hoabinhian in time and place is a variant industry that has long been known as the Bacsonian-in reality just an aspect of the Hoabinhian characterized by a high proportion of edge-ground tools. The Bacsonian, which is recognized separately from the Hoabinhian by Vietnamese archaeologists, is stated to date mainly from about $11,000 \mathrm{BP}$ onward; 
this date clearly marks a time when edge-ground stone tools started to become common. Pottery (mostly plain or vine/mat impressed rather than cordmarked) was also widespread in Vietnam by at least 6,500 BP and seems to overlap genuinely with Hoabinhian/Bacsonian tools in the shell mound of Da But in Thanh Hoa Province (Bui Vinh 1991). In this case we may be witnessing a local adoption of pottery making and perhaps also agriculture by indigenous late Hoabinhian populations; more on this in Chapter 7.

One major question, of course, concerns the role of the Hoabinhian itself in any local development of agriculture in Southeast Asia. It should be noted that the Hoabinhian technocomplex covered a vast area, extending virtually from the equator in Sumatra to beyond the Tropic of Cancer in southern China. In the far southern regions, I remain fairly convinced that it had no true agricultural status, but there can be less certainty for northern Vietnam and northern Thailand. On this question there are still only the results of Gorman's excavations in Spirit Cave in northwestern Thailand (Gorman 1970, 1971; Glover 1977b:11-17), where remains of a number of edible fruits and legumes appeared in terminal Pleistocene Hoabinhian levels. None of these remains is from a definitely domesticated species (Yen 1977), and current opinions on the status of the Spirit Cave Hoabinhian economy regard it as part of a foraging lifestyle that might have continued in remote valleys in northern Thailand until 1,000 years ago (Higham 1989:59-61; Bellwood 1992:88). On the other hand, Kuchikura's (1993) studies of wild yam densities in Peninsular Malaysian rain forests lead him to suggest that inland hunter-gatherers there could not have existed in completely undisturbed environments without access to agricultural foods. This need not imply that Hoabinhians were necessarily agriculturalists; perhaps they used their pebble tools to clear and ring-bark vegetation in order to encourage forest floor vines, such as yams, to thrive (a scenario also suggested for the late Pleistocene New Guinea Highlands by Groube 1989). Whatever the real nature of the Hoabinhian economy-and I am certainly unable to accept that it was one of systematic field agriculture-it is now apparent that true agricultural economies based on the cultivation of rice were present in many coastal and lowland valley regions of the Southeast Asian mainland well before 4,000 years ago. There is no clear evidence to suggest that these economies were developed entirely by resident Hoabinhians.

\section{Peninsular Malaysia}

Having given this contextual introduction to the Hoabinhian, I will now turn to its most southerly expressions in Peninsular Malaysia (see Fig. 6.1) and Sumatra (Fig. 3.2). In Peninsular Malaysia a number of inland Hoabinhian caves and shelters have been excavated in the many limestone massifs scattered 
through the northern states of Perlis, Kedah, Perak, Pahang, Terengganu, and Kelantan. In addition, coastal shell middens once existed in the northwestern states of Pinang and Perak. The majority of sites were excavated during the 1920s and 1930s and the reports can only be described as brief. However, the important site of Gua Cha in Kelantan-excavated in 1954 and more recently by a Malaysian National Museum team in 1979-has produced a firm record. During the last fifteen years Malaysian archaeologists have excavated several more sites, including Gua Bukit Ta'at in Terengganu (Nik Hassan Shuhaimi et al. 1990; the basal date here is 9,000 BP), Gua Gunung Runtuh in Perak (Zuraina 1994), Gua Kelawar in Perak (Adi and Zulkifli 1990), and Gua Peraling and Gua Chawas in Kelantan (see below). First, we examine Gua Cha.

This massive limestone rock shelter lies in a remote inland region of equatorial rain forest on the bank of the Nenggiri River, a tributary of the Kelantan River, which flows into the sea at Kota Bharu. In 1954 three large trenches were excavated in the shelter by Sieveking, who published a detailed report on the contents of the Neolithic layers (Sieveking 1954) but gave only stratigraphical observations on the underlying Hoabinhian. In order to throw light on a number of questions concerning the Hoabinhian, the shelter was excavated on a small scale again in 1979 by Adi Taha of the Malaysian National Museum (Adi 1985). I will combine the results of both excavations here.

The Hoabinhian layers at Gua Cha are up to 170 centimeters thick and rest on sterile alluvial deposits. According to sediment analyses the Hoabinhian deposit itself is also of alluvial origin and was clearly formed by occasional flooding of the shelter by the neighboring river (Hughes, in Adi 1985). The industry is a surprisingly homogeneous collection of bifacially flaked flat river pebbles (see Fig. 6.2), with a minority component of cruder pebble tools together with flake debitage and a number of river pebbles that may have served for crushing and pounding-some have red ochre stains. Bone tools were absent, despite their occurrence at other Peninsular Malaysian sites such as Gua Bintong in Perlis (Fig. 6.3). The homogeneity and emphasis on bifacial working of the Gua Cha industry are both quite striking, and radiocarbon dates indicate a commencement soon after 10,000 years ago with a fairly decisive termination a little before $1000 \mathrm{BC}$. A number of primary flexed or secondary burials had been placed in the Hoabinhian deposits; none contained certain grave goods, but one young flexed male excavated in 1979 (Plate 17) had a stone-slab pillow and a body cover of tufa chunks dusted with red ochre. Another unexcavated burial lay beneath two limestone slabs.

The diet and economy of the Gua Cha Hoabinhians were investigated from three angles. First, an examination by Bulbeck (1982) of the occurrence of caries in the teeth of the burials excavated in 1979 suggested considerable consumption of sweet foods such as fruits and honey. Second, flotation of the deposits 

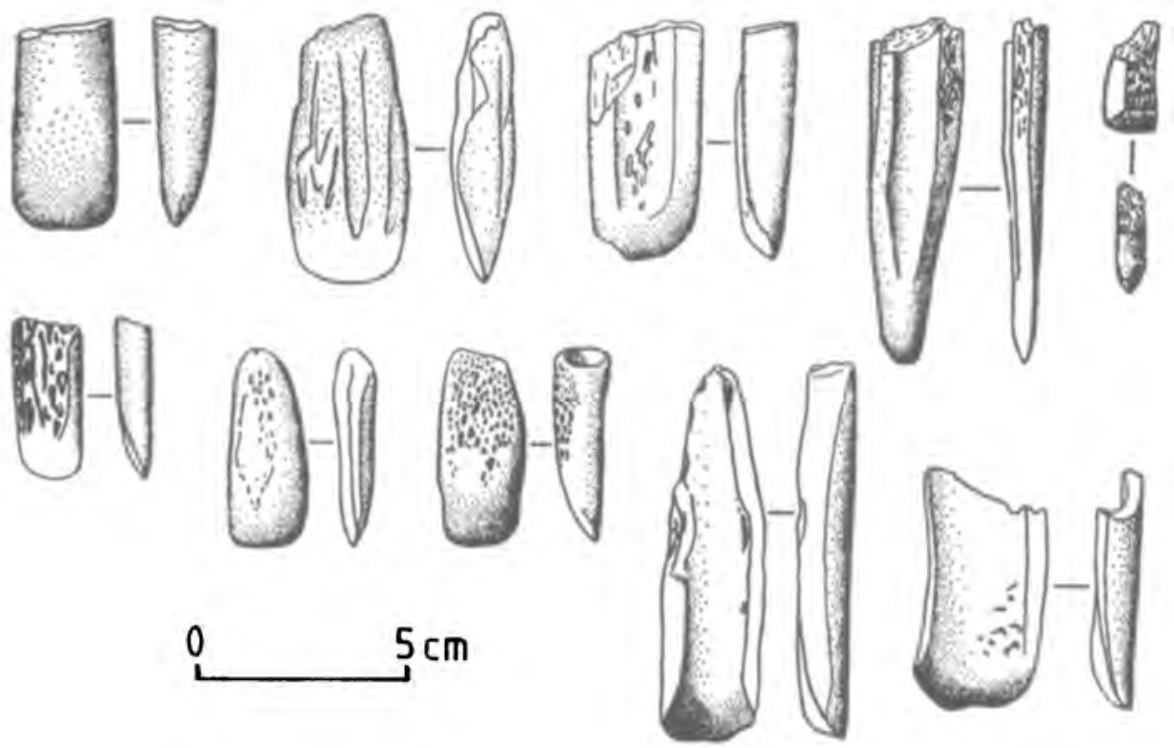

Fig. 6.3 Spatulate bone tools from Gua Bintong, Perlis. From Collings 1937a. Courtesy: National Heritage Board, Singapore.

produced a large quantity of charcoal but unfortunately no recognizable plantfood remains; in this regard it is important to note that a large quantity of carbonized rice was found in an upper layer of the site dated to about 900 years ago, so this cereal would have been detected had it been present earlier in carbonized form (silica phytoliths, since discovered in sediments in nearby Gua Chawas [see below], were not searched for when the site was excavated). Therefore there is no evidence to suggest cereal cultivation at Gua Cha-either Hoabinhian or Neolithic. Finally, large numbers of animal bones were found throughout the Hoabinhian layers; pigs (Sus scrofa and the bearded pig Sus barbatus) were the most commonly killed animals, and it is possible that large numbers of bearded pigs were killed during mass river crossings, as described by Hislop (1954) for Peninsular Malaysia and by St. John (1974, I:138) for northern Borneo. Sieveking (1954) found about twenty-five small heaps of jaws and skull fragments of juvenile pigs in one sector of the Hoabinhian deposits, and young animals seem to have been favored as prey in other species, too. The latter included deer of several species, bear, monkeys, gibbons, rats, squirrels, flying foxes, and (more rarely) rhinoceros and cattle. This species list is very similar to that found in Hoabinhian sites as a whole (Gorman 1971:Table 2). It is also worth noting that small quantities of freshwater shellfish were found in the site, but marine shells were absent as in most inland Hoabinhian sites (Bellwood 1993). 

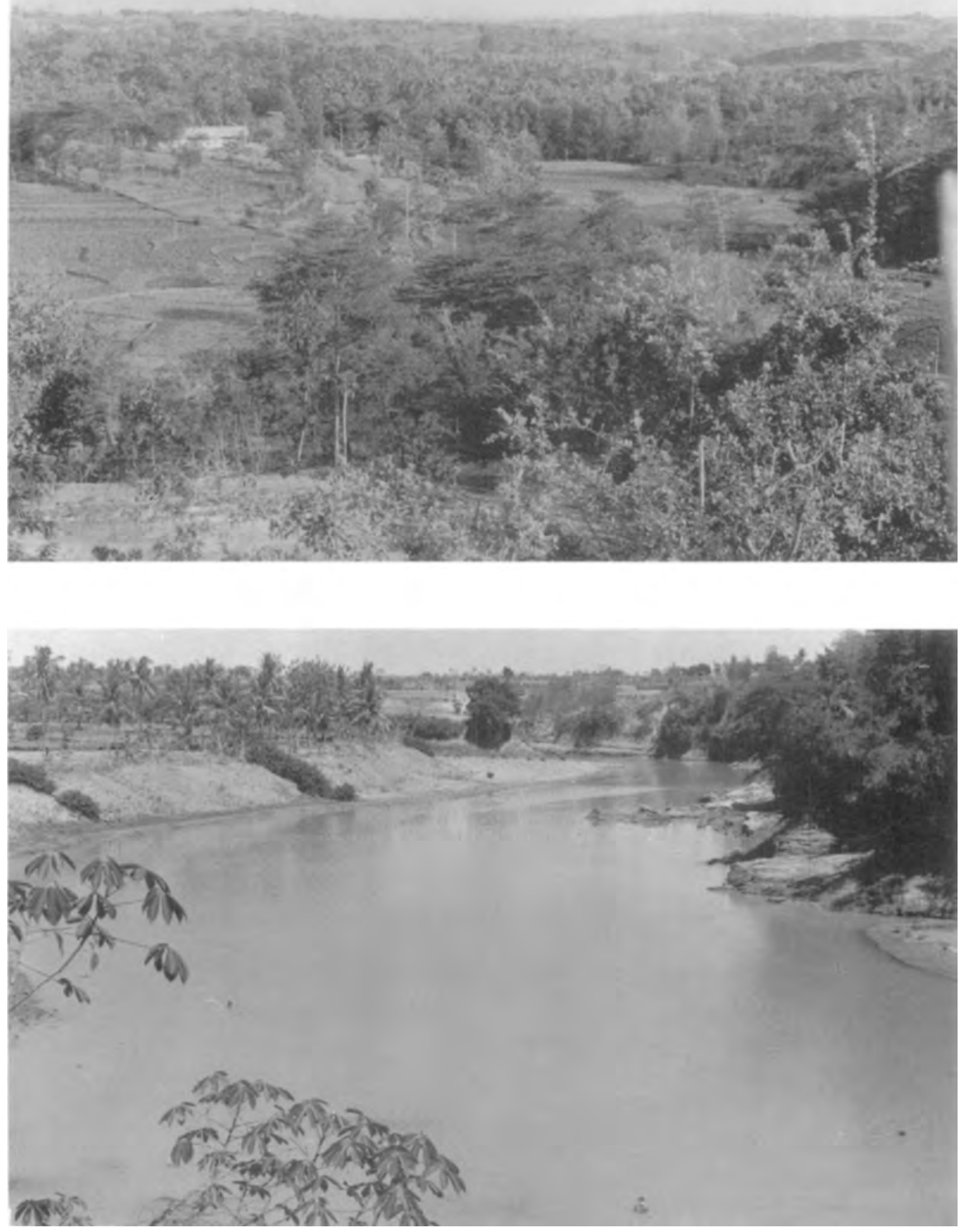

Plate 1. Top: the dissected Sangiran "dome," central Java. Bottom: the Solo River at Trinil, central Java. 


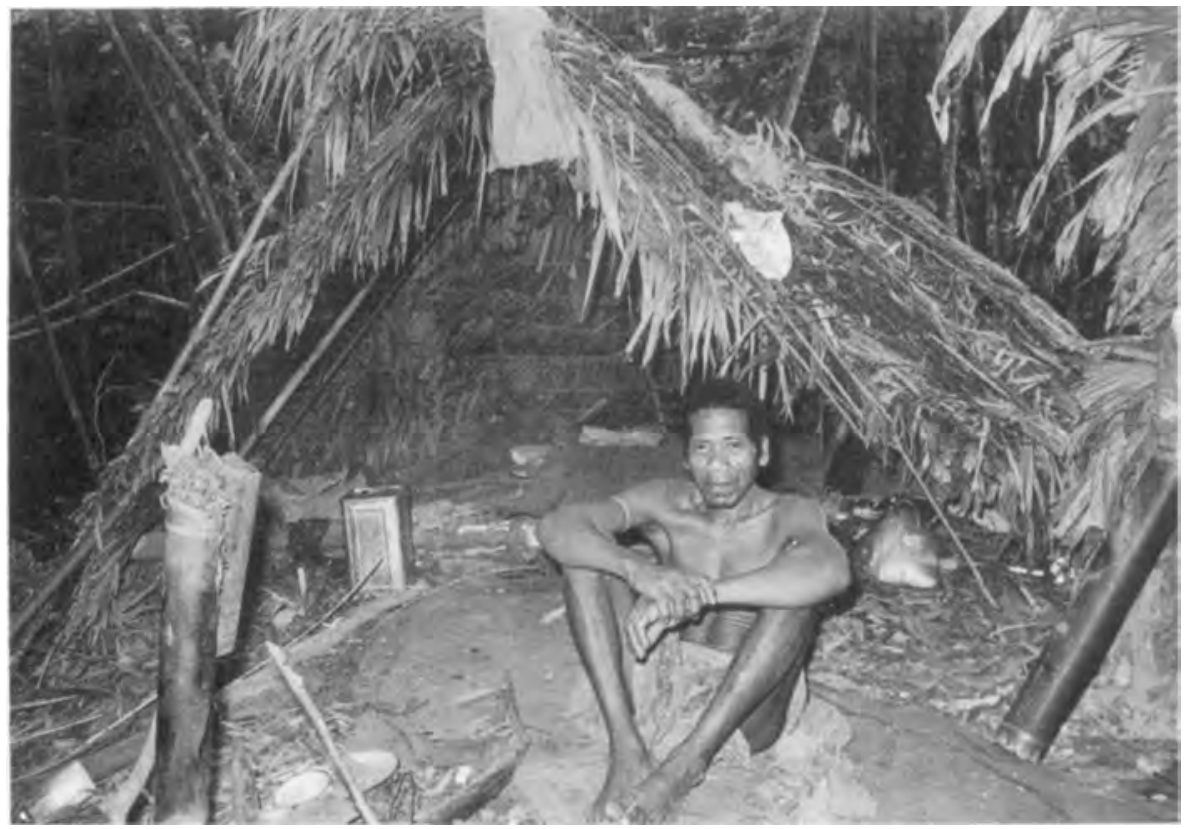

Plate 2. Batek Negrito man and forest camp, Kelantan, Peninsular Malaysia. Photo by Geoffrey Benjamin.

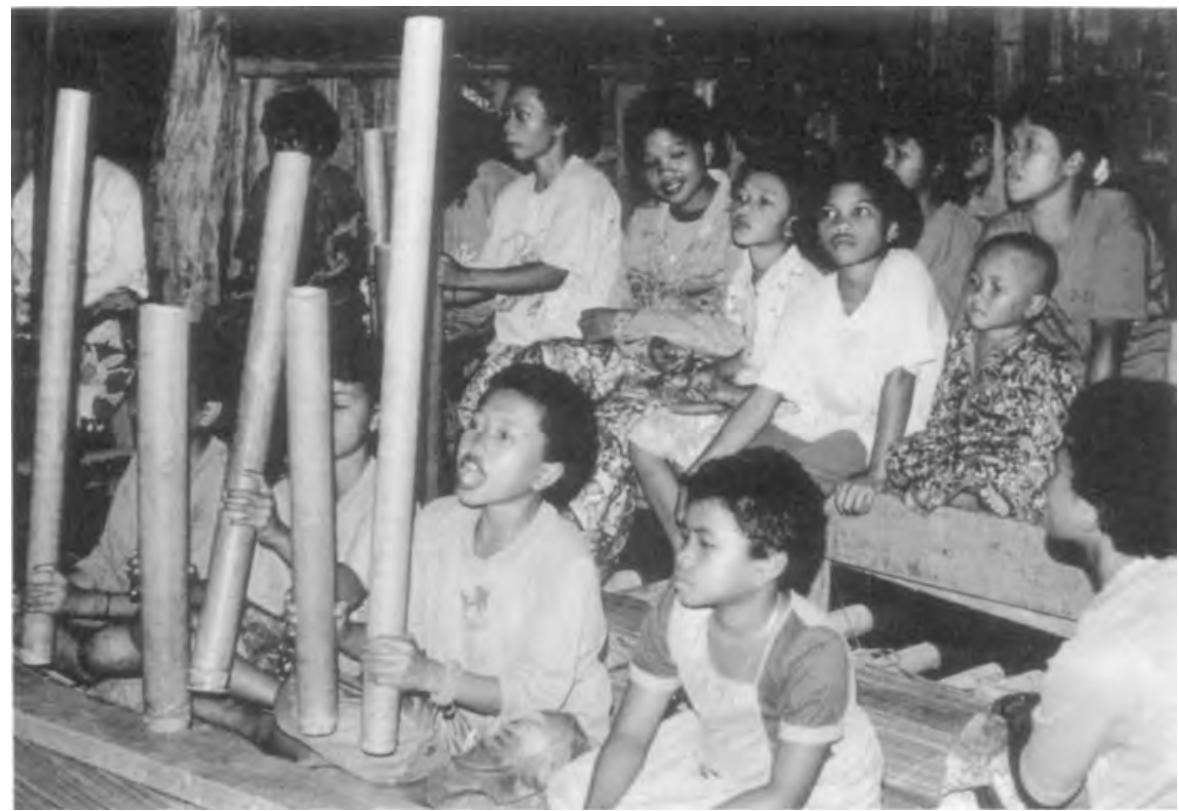

Plate 3. Temiar (Senoi) women and children during a siwang ceremony, Kampung Tohoi, Kelantan, Peninsular Malaysia. 


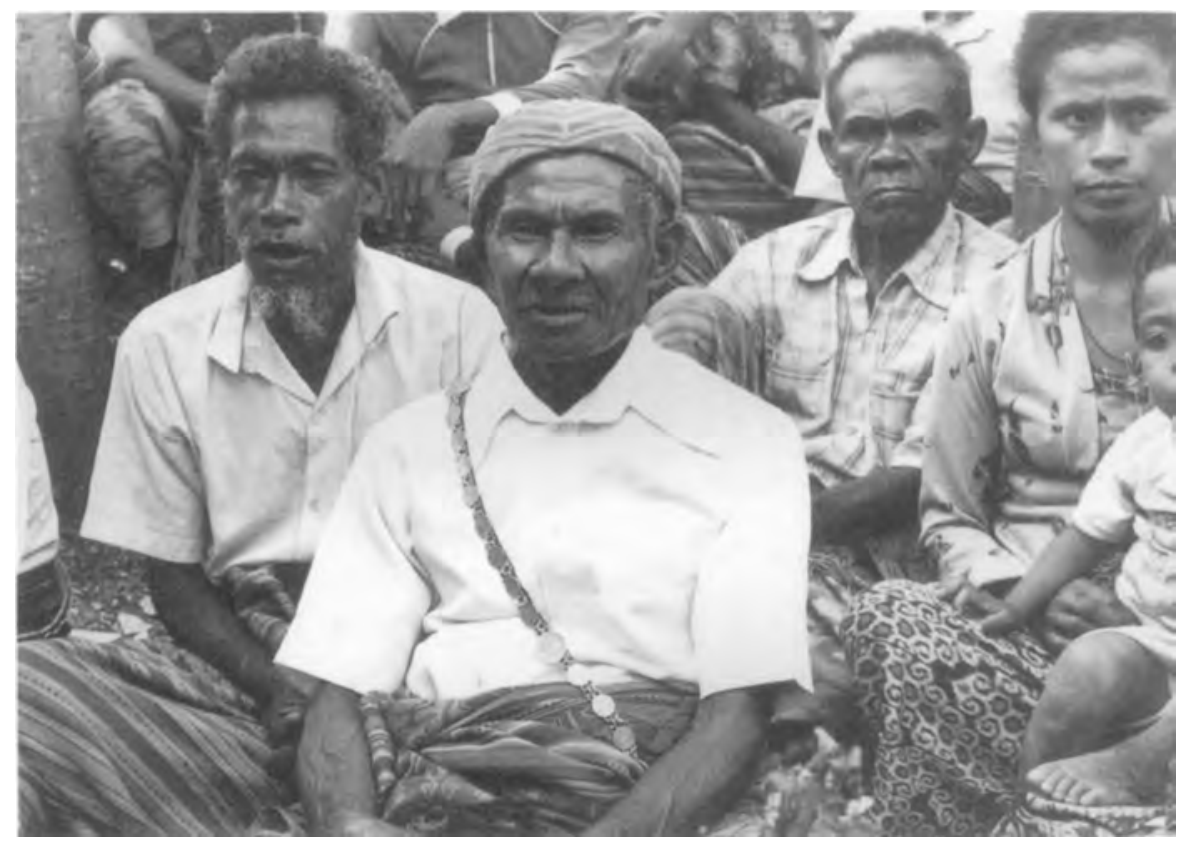

Plate 4. Atoni elders, South Amanuban, western Timor. Photo by James Fox.

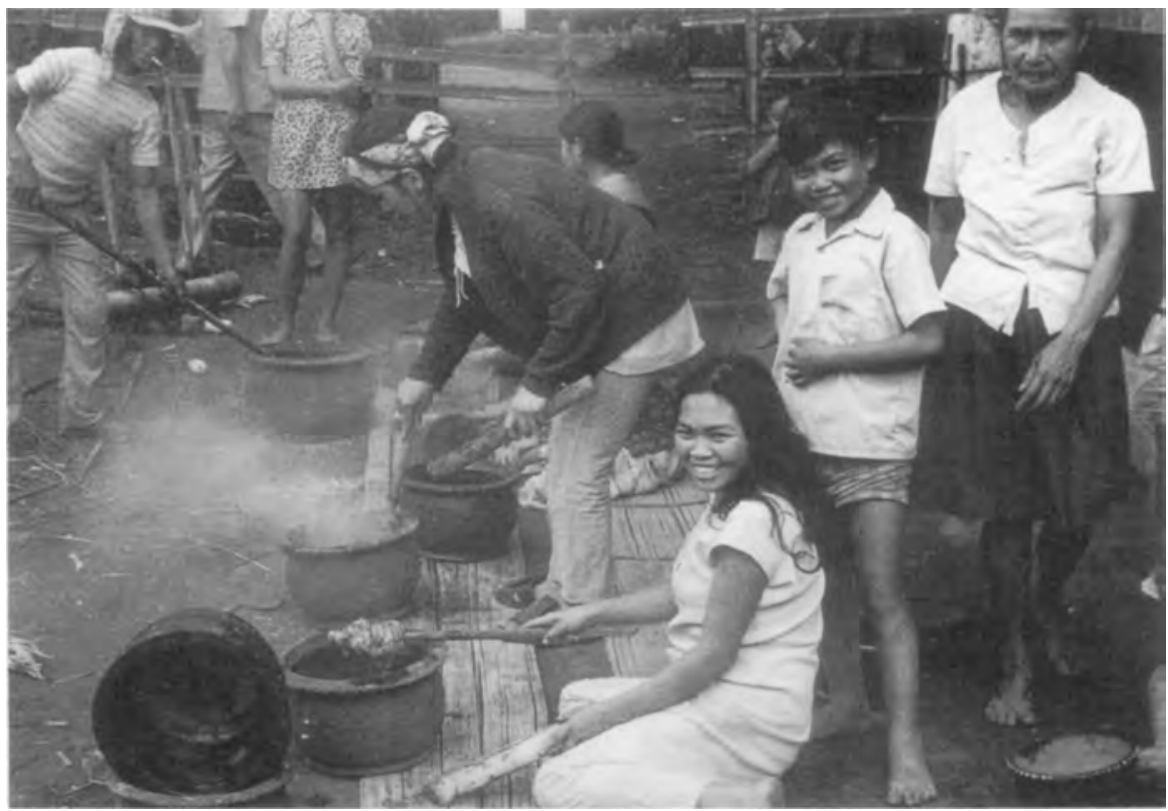

Plate 5. Minahasans of the Lake Tondano region, northern Sulawesi. Pottery still hot from firing is being given a waterproof resin glaze. 


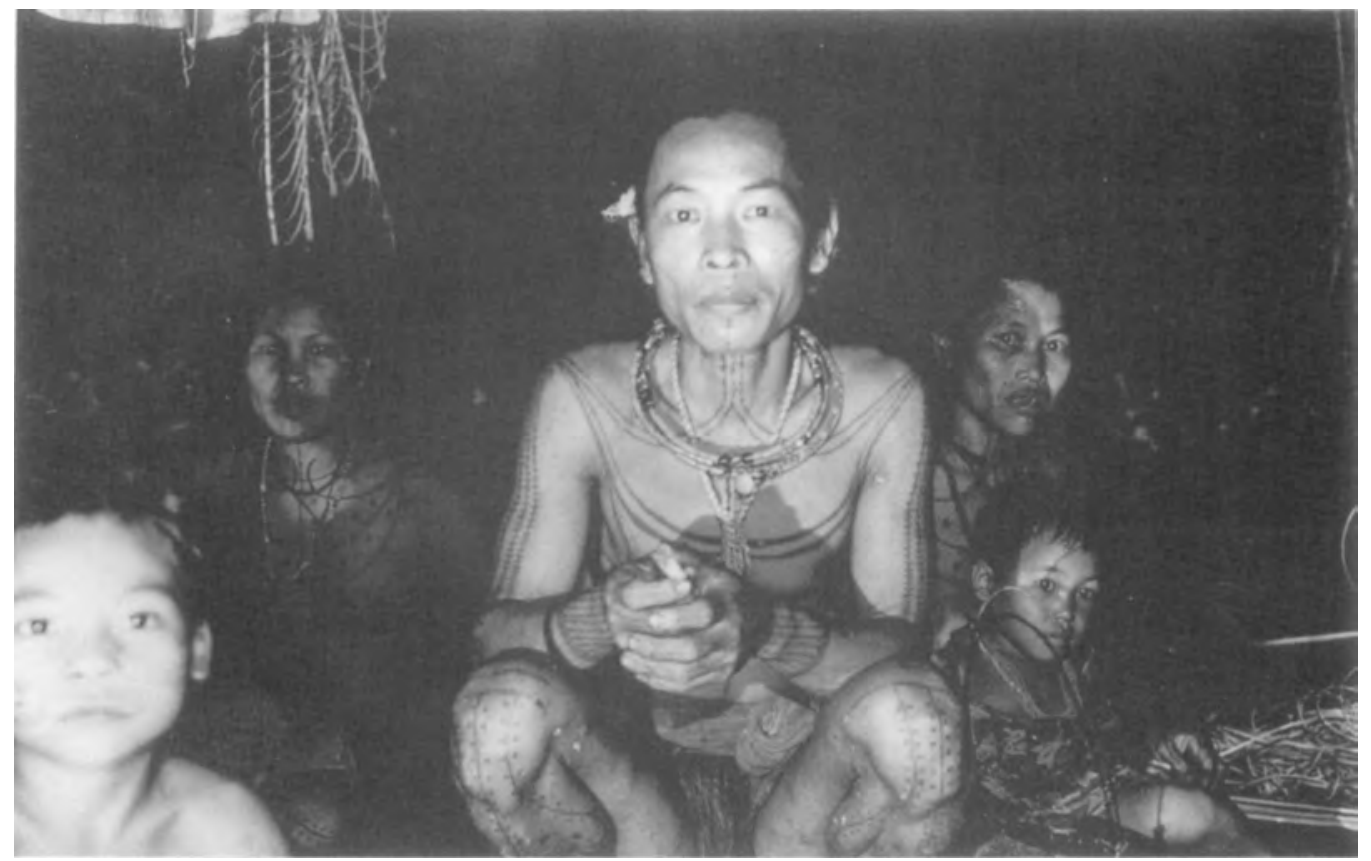

Plate 6. A family on Mentawai, off the western coast of Sumatra. The man is tattooed. Photo by Vernon Weitzell.

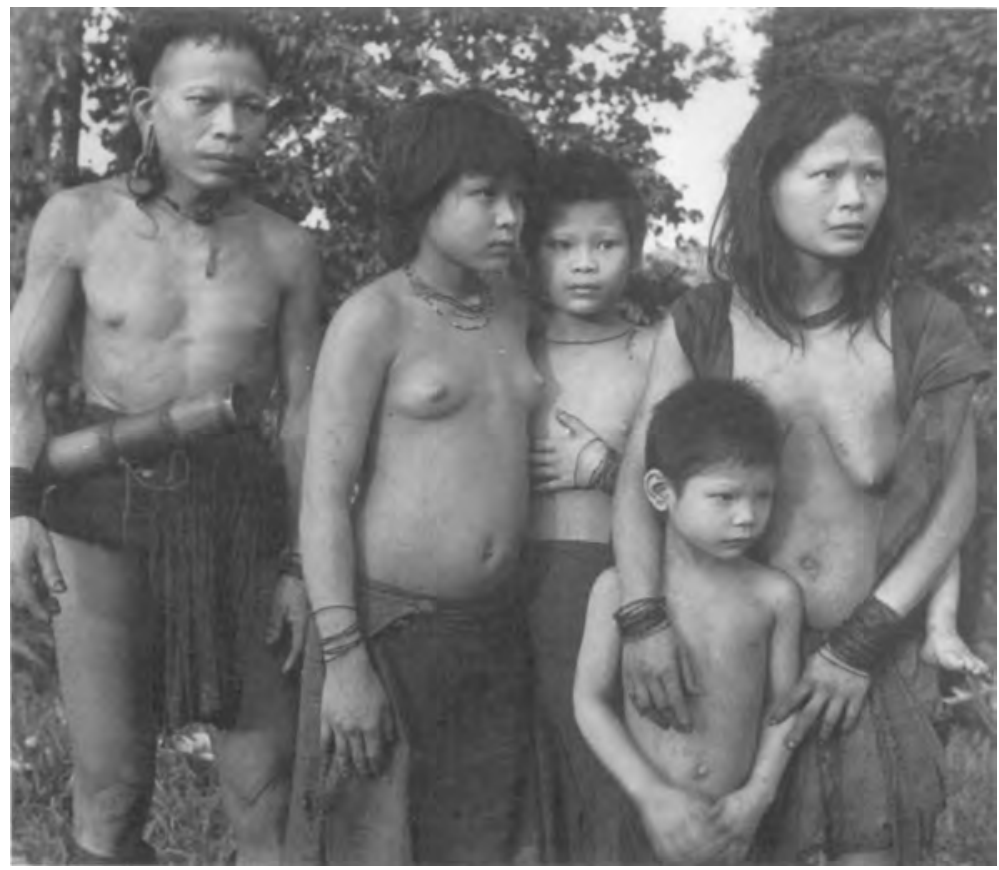

Plate 7. A Punan family at Lio Matu, upper Baram River, Sarawak (1953). Photo by Hedda Morrison. 
Plate 8. Right: A Murut boy, Sabah, photographed in 1910. Courtesy: Sabah Museum (G. C. Woolley collection).

Plate 9. Below: Minangkabau women at Payakumbuh, central Sumatra. Photo by Cecilia Ng.
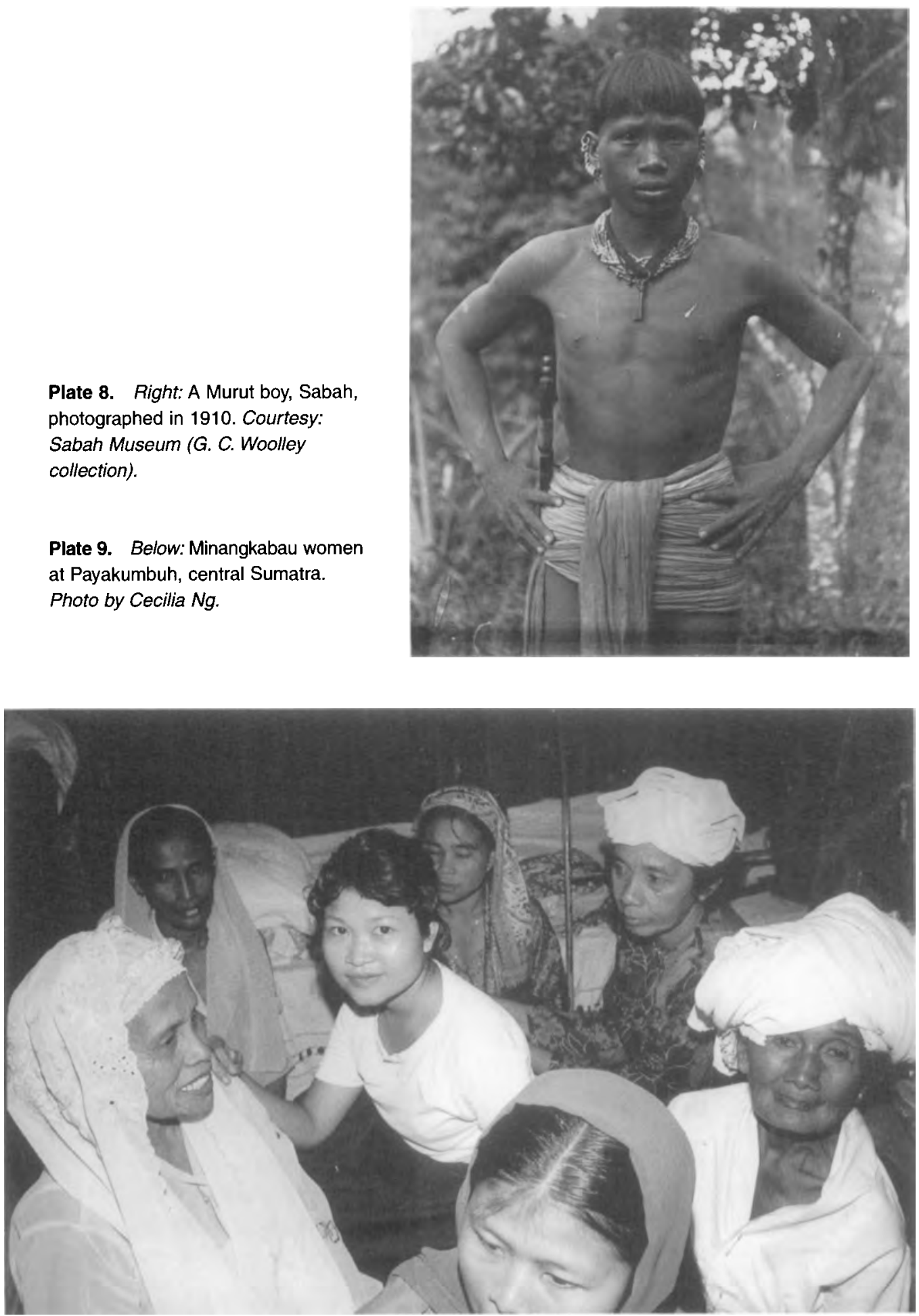


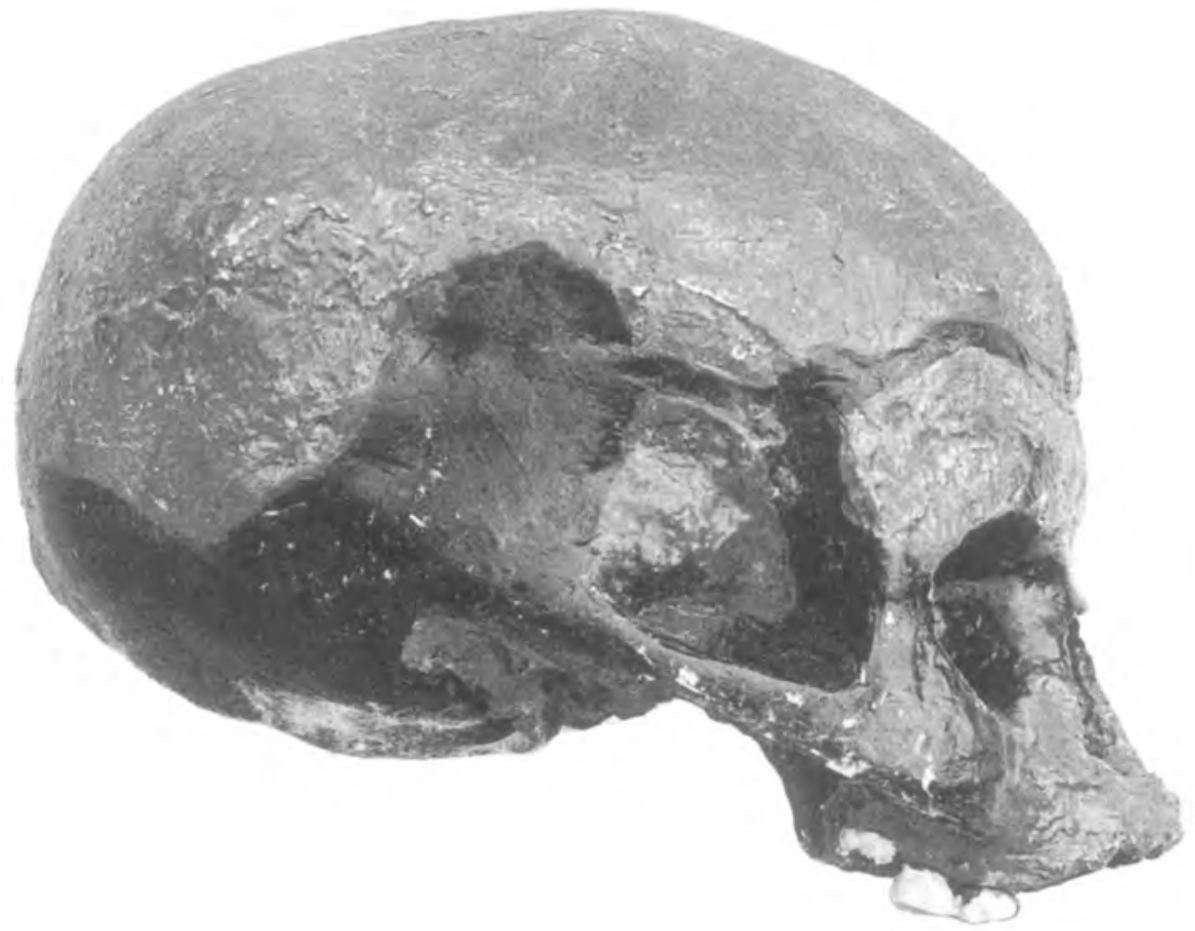

Plate 10. The "deep skull" from the West Mouth at Niah. Courtesy: Sarawak Museum.

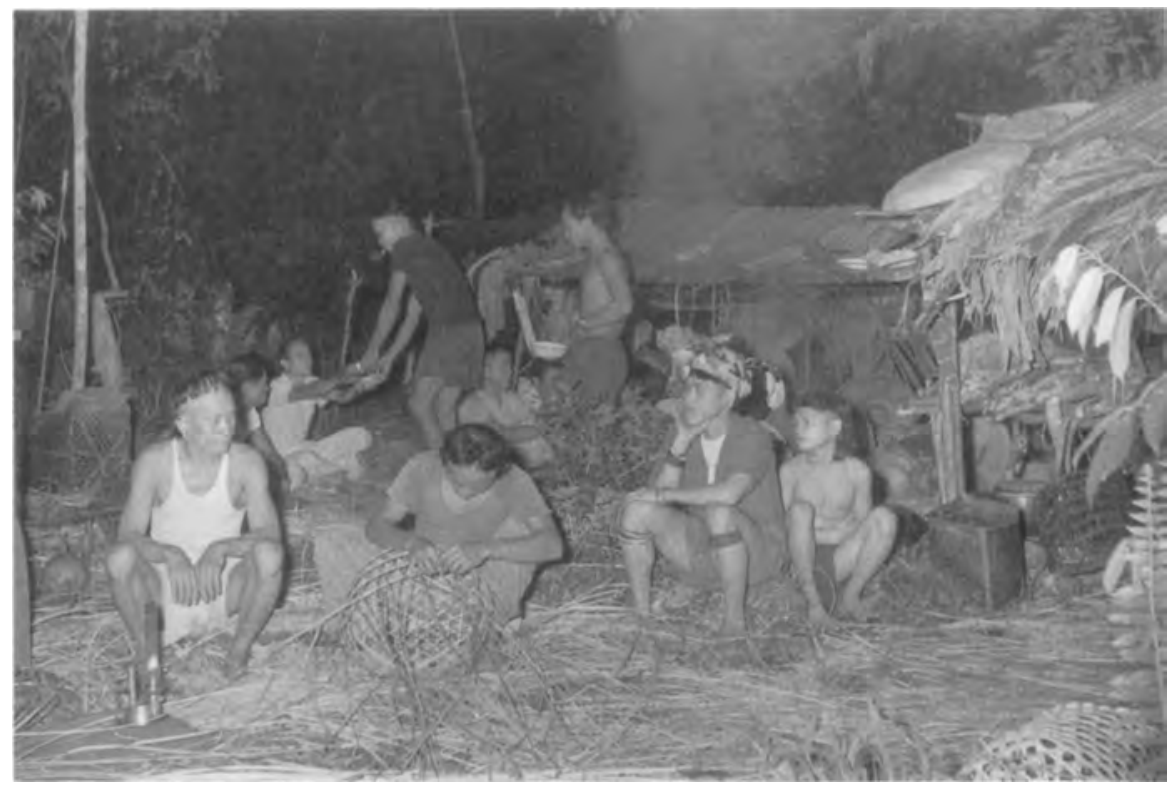

Plate 11. A Punan camp in Sarawak. Photo by Hedda Morrison. 


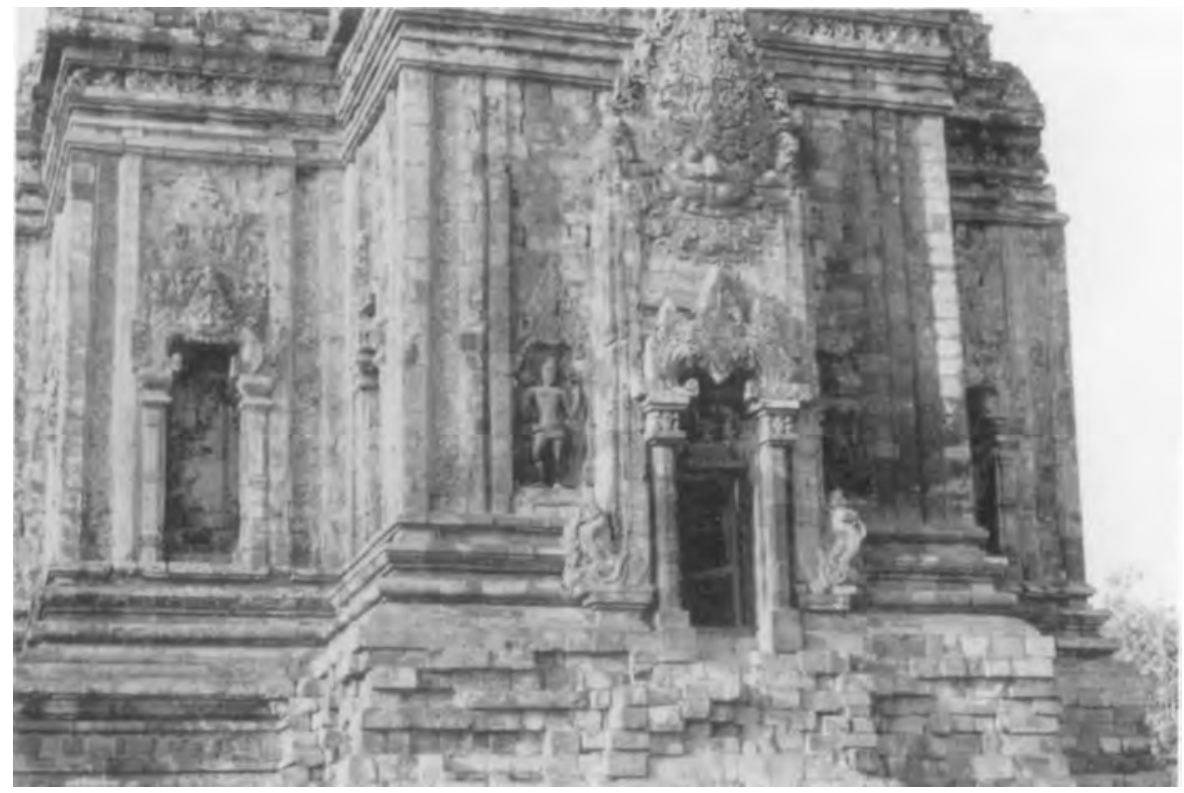

Plate 12. Hindu influence in Java: the ninth-century Candi Kalasan near Prambanan, central Java.

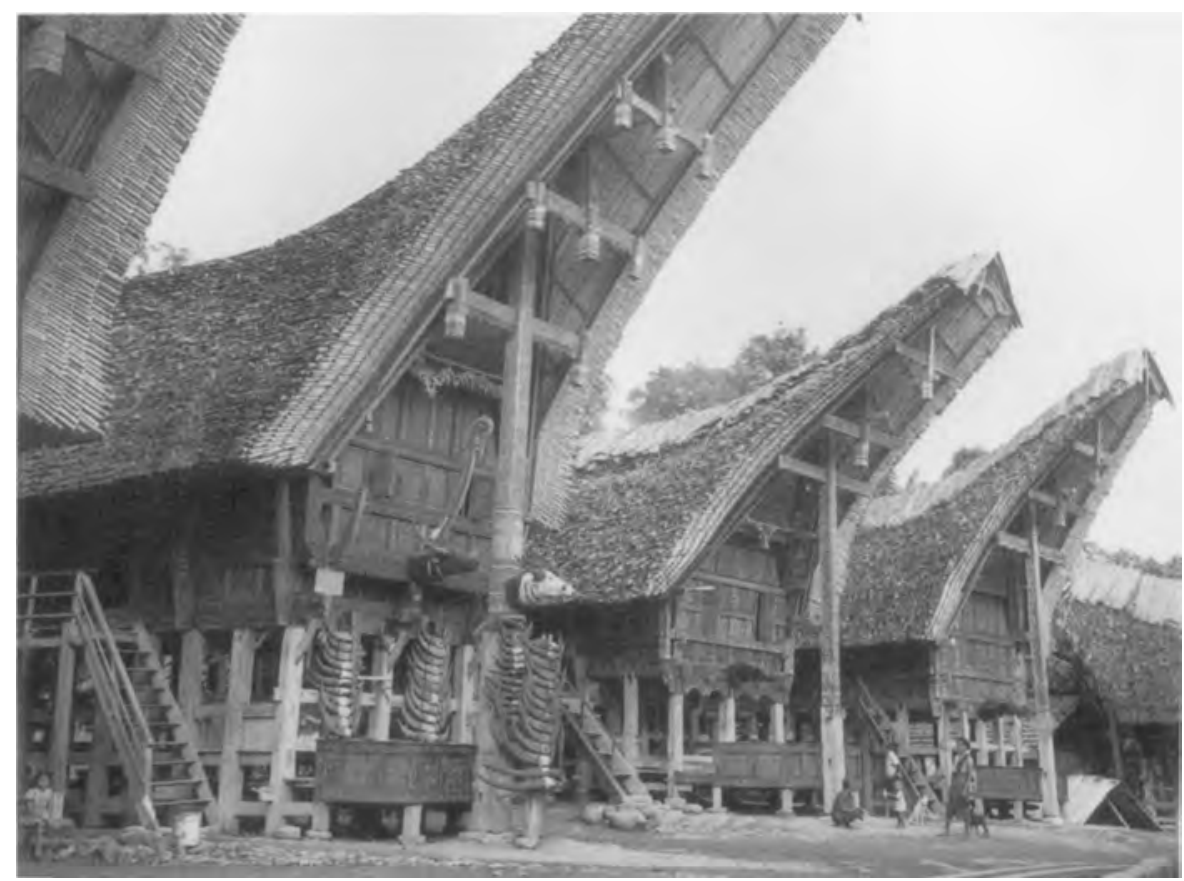

Plate 13. Toraja houses decorated with buffalo carvings and horns, Palawan village, central Sulawesi. Photo by Hedda Morrison. 


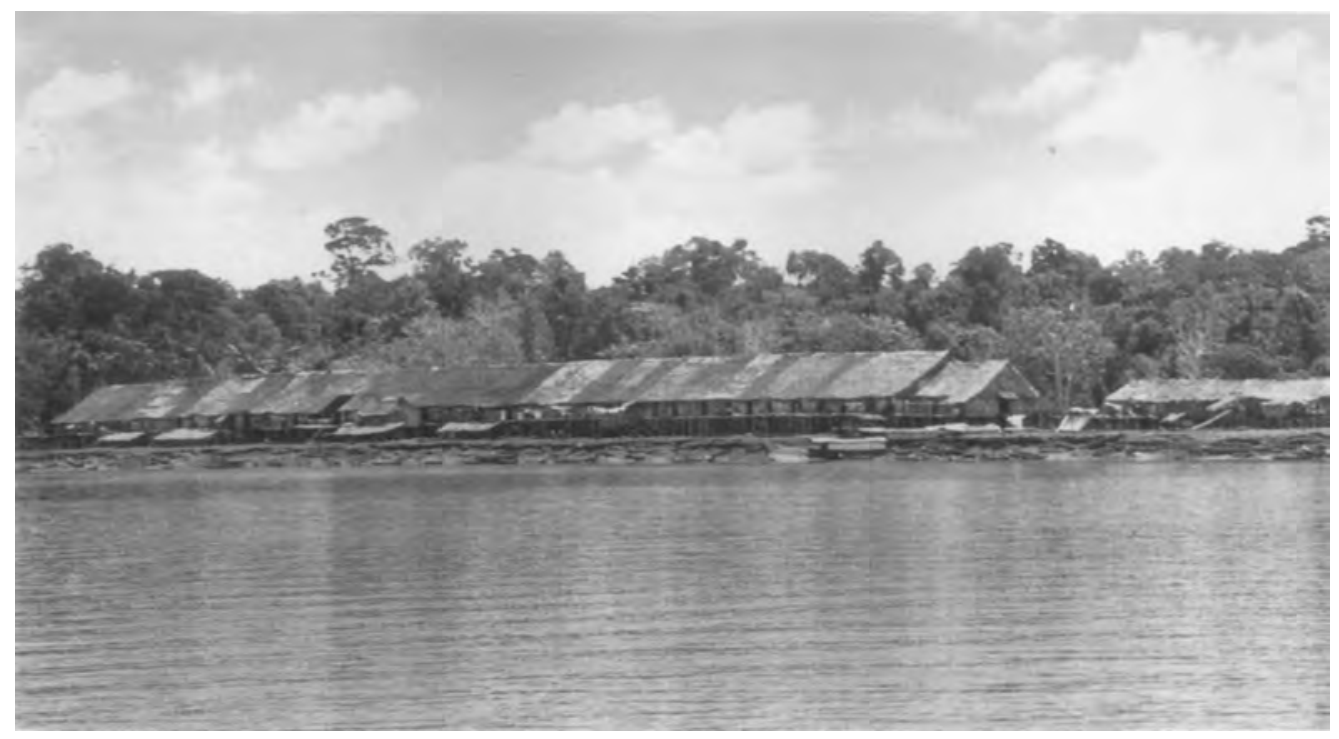

Plate 14. An Iban longhouse at Simanggang on the Batang Lupar, Sarawak. Photo by Hedda Morrison.

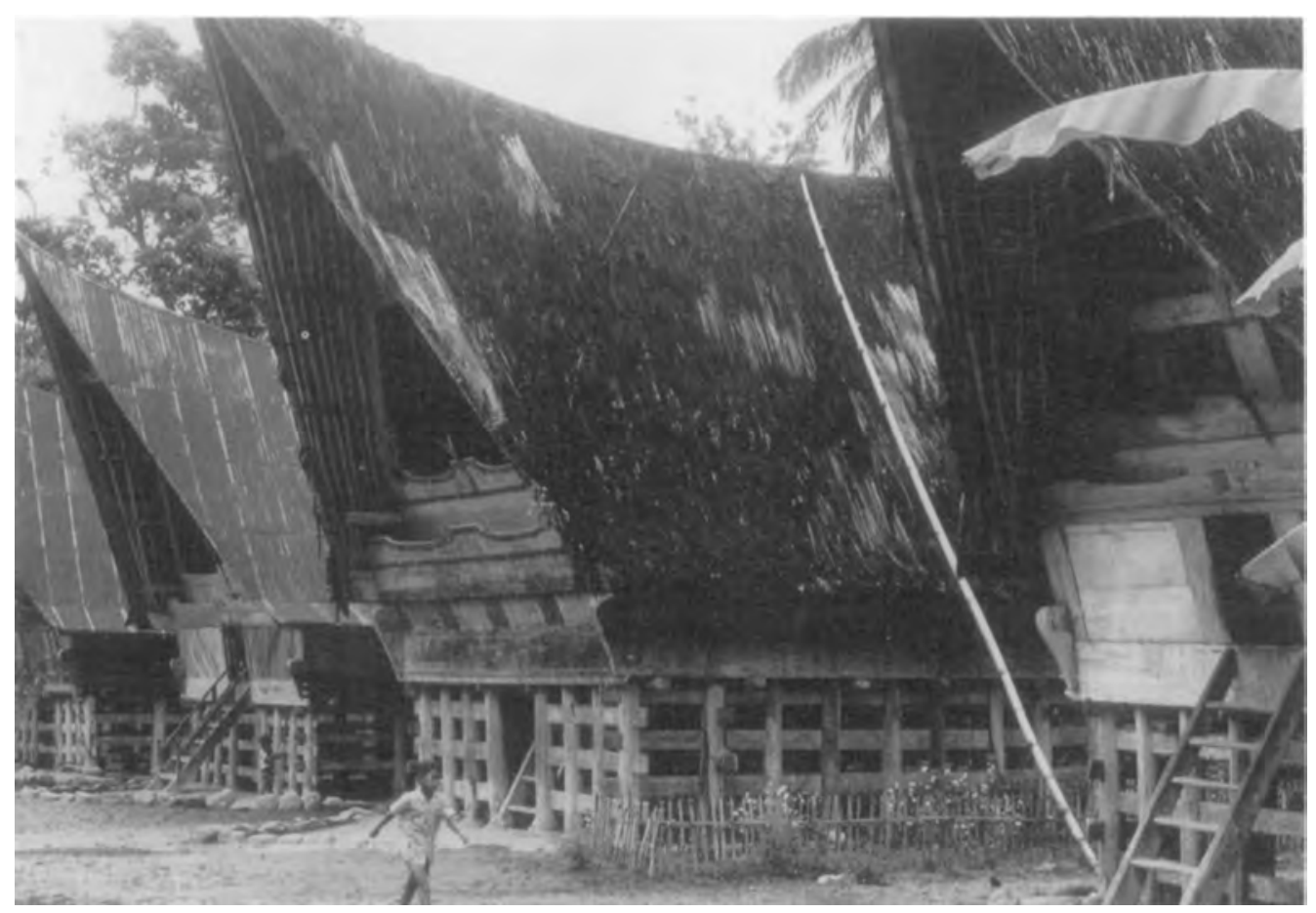

Plate 15. Batak houses near Lake Toba, northern Sumatra. 


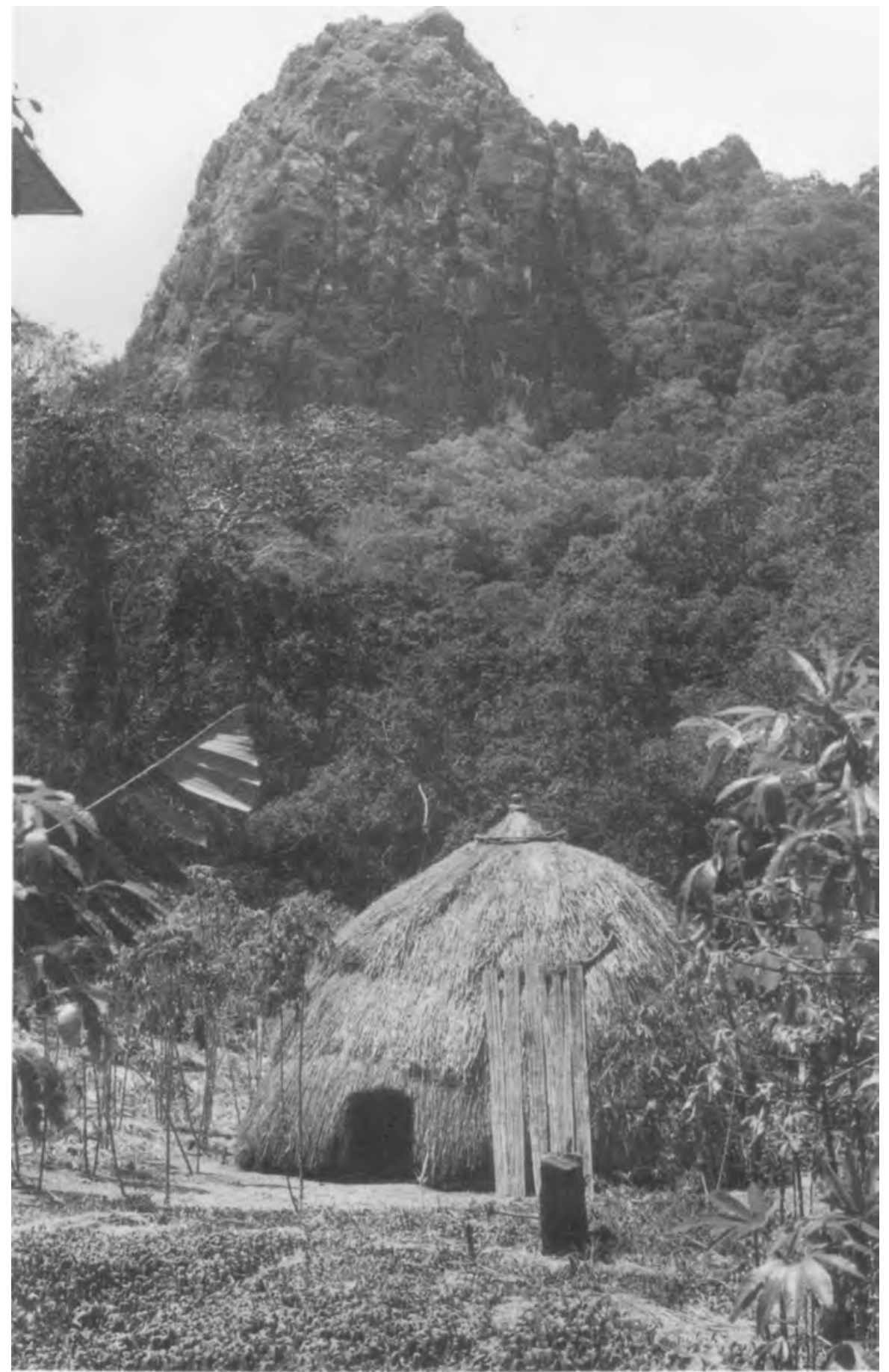

Plate 16. Circular Atoni house, South Amanuban, western Timor. Photo by James Fox. 


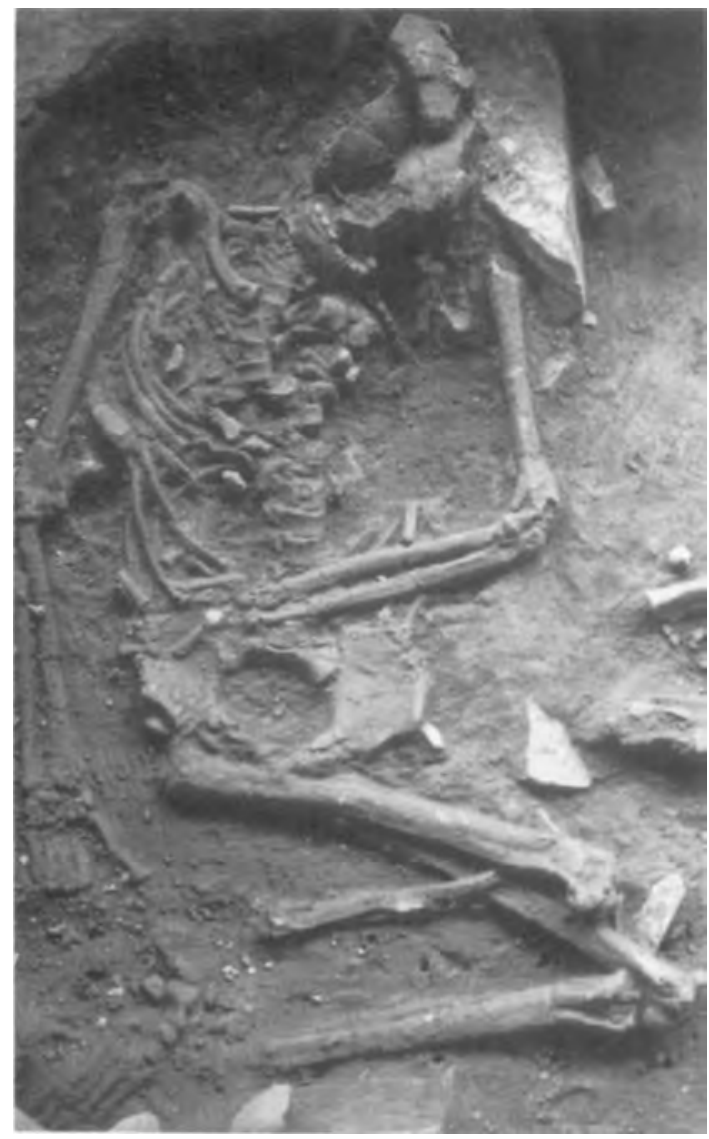

Plate 17. Left: Flexed burial of a young male with a stone pillow excavated at Gua Cha in 1979. Late Hoabinhian. Courtesy: National Museum of Malaysia.

Plate 18. Bottom: The 1994 excavations in deep Hoabinhian deposits in Gua Peraling, Kelantan. Courtesy: National Museum of Malaysia.

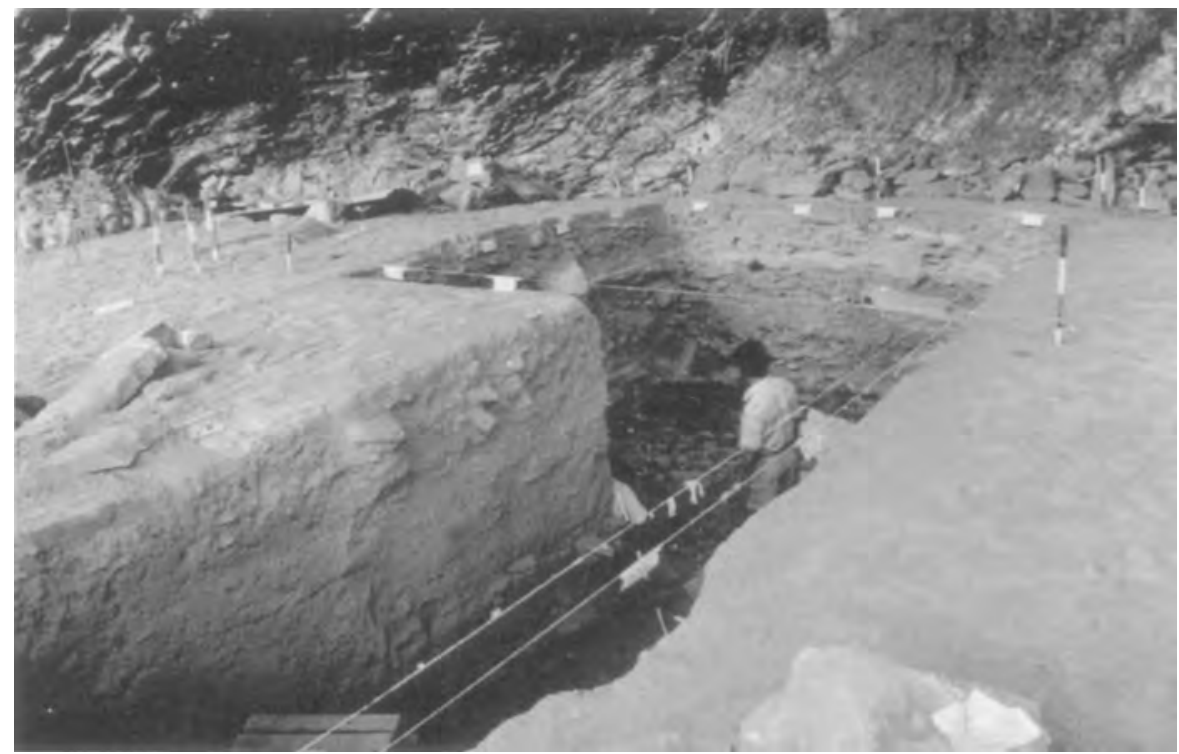



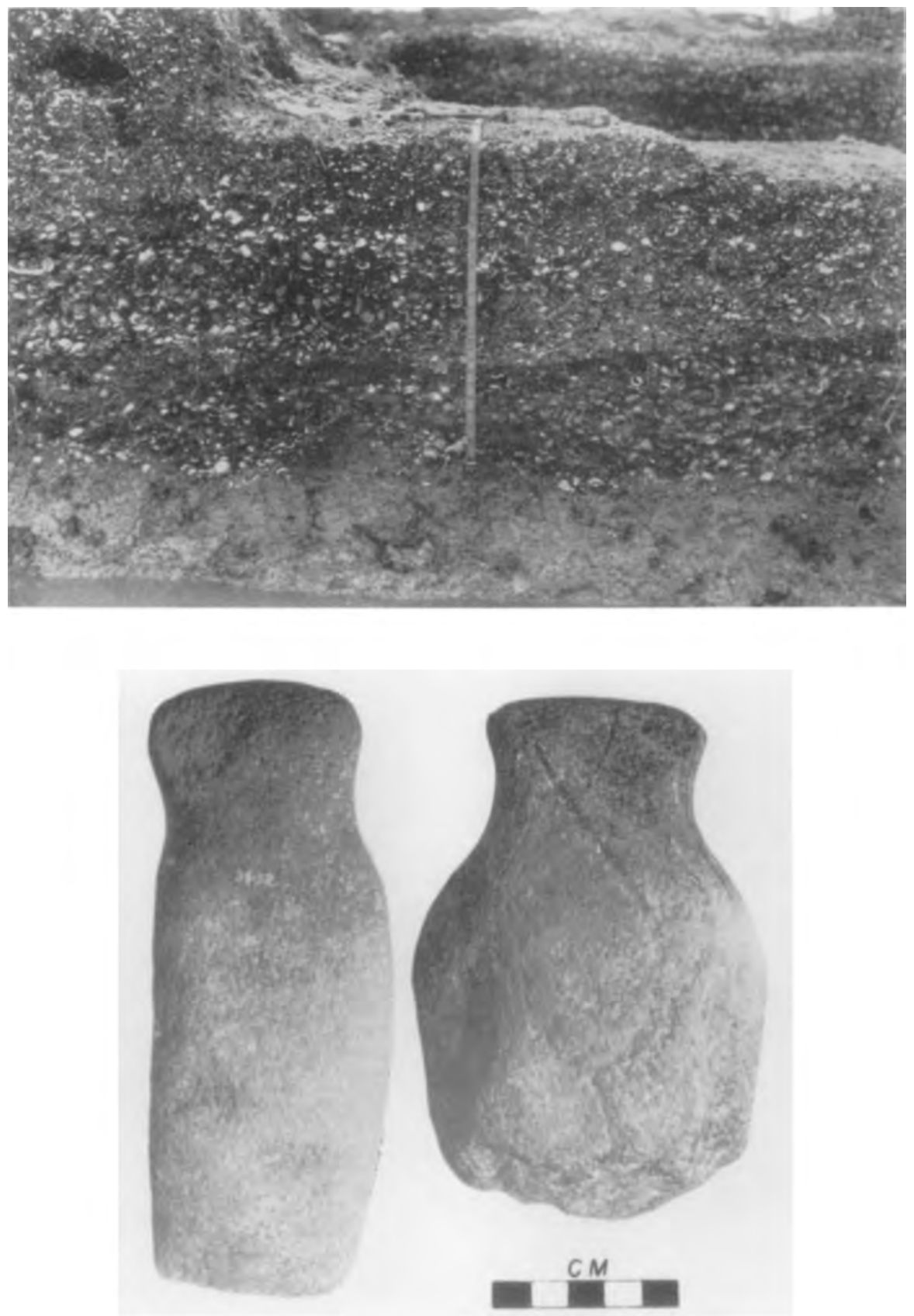

Plate 19. Top: basal section (about 60 centimeters deep) of Guar Kepah midden A, resting on clay. From Stein Callenfels 1936a. Courtesy: National Heritage Board, Singapore. Bottom: hammer-dressed and necked axes from Guar Kepah. From Tweedie 1953. Courtesy: Malaysian Branch of the Royal Asiatic Society. 


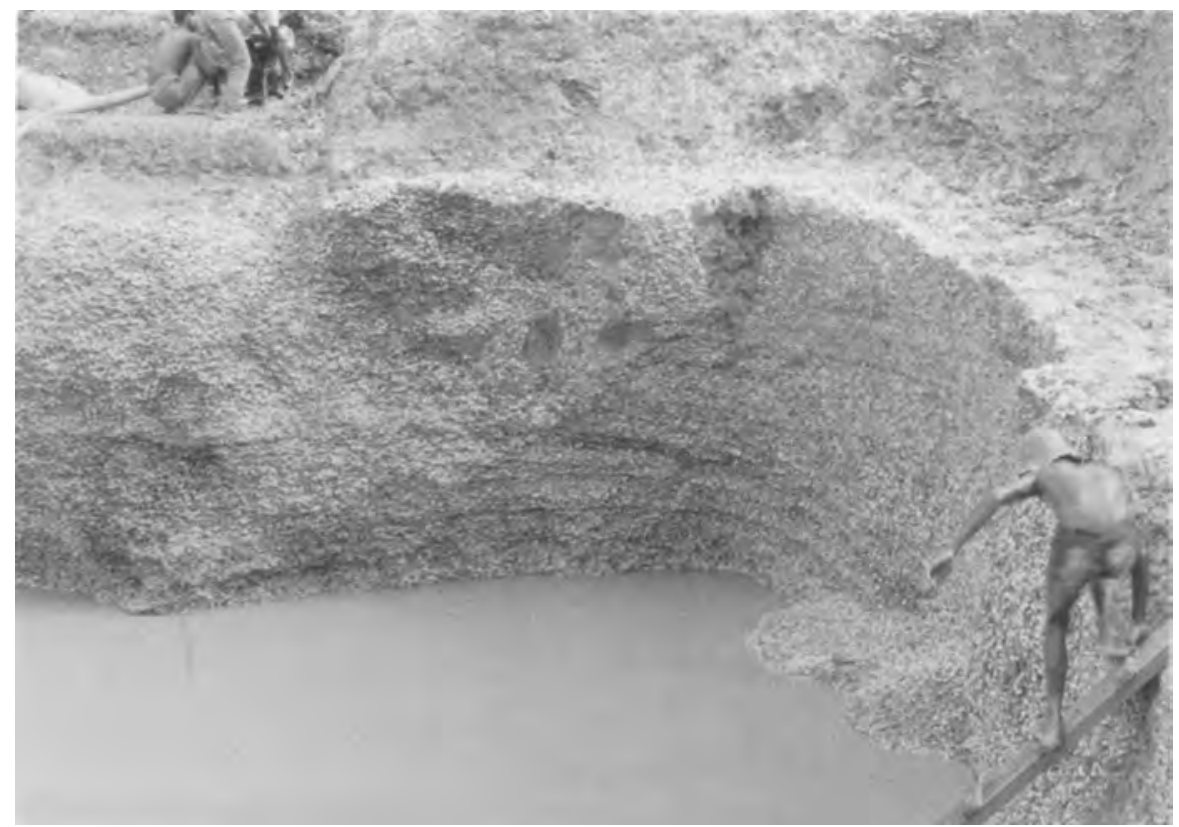

Plate 20. Hoabinhian shell midden being quarried for lime manufacture at Sukajadi, near Medan, northern Sumatra. Photo by lan Glover.

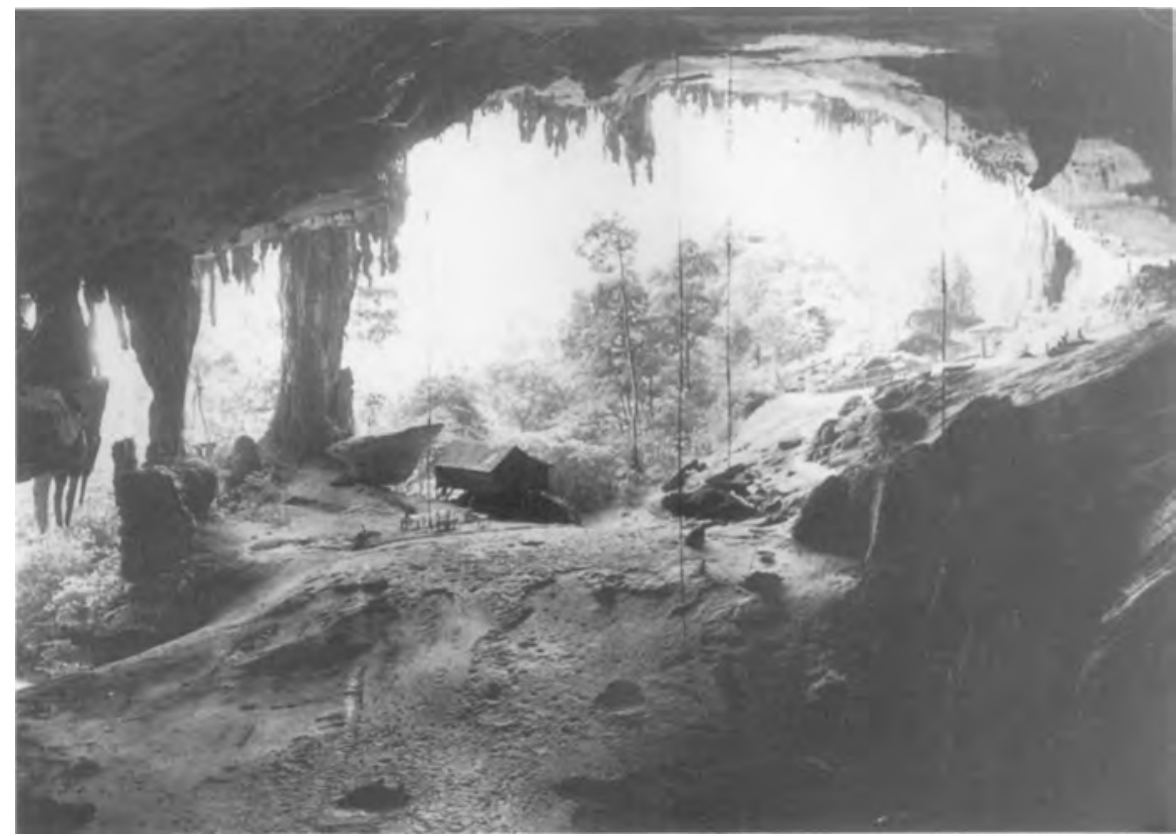

Plate 21. The West Mouth, Niah Caves. Harrisson's excavation area is at top right. Poles to roof are for bird's-nest collection. 


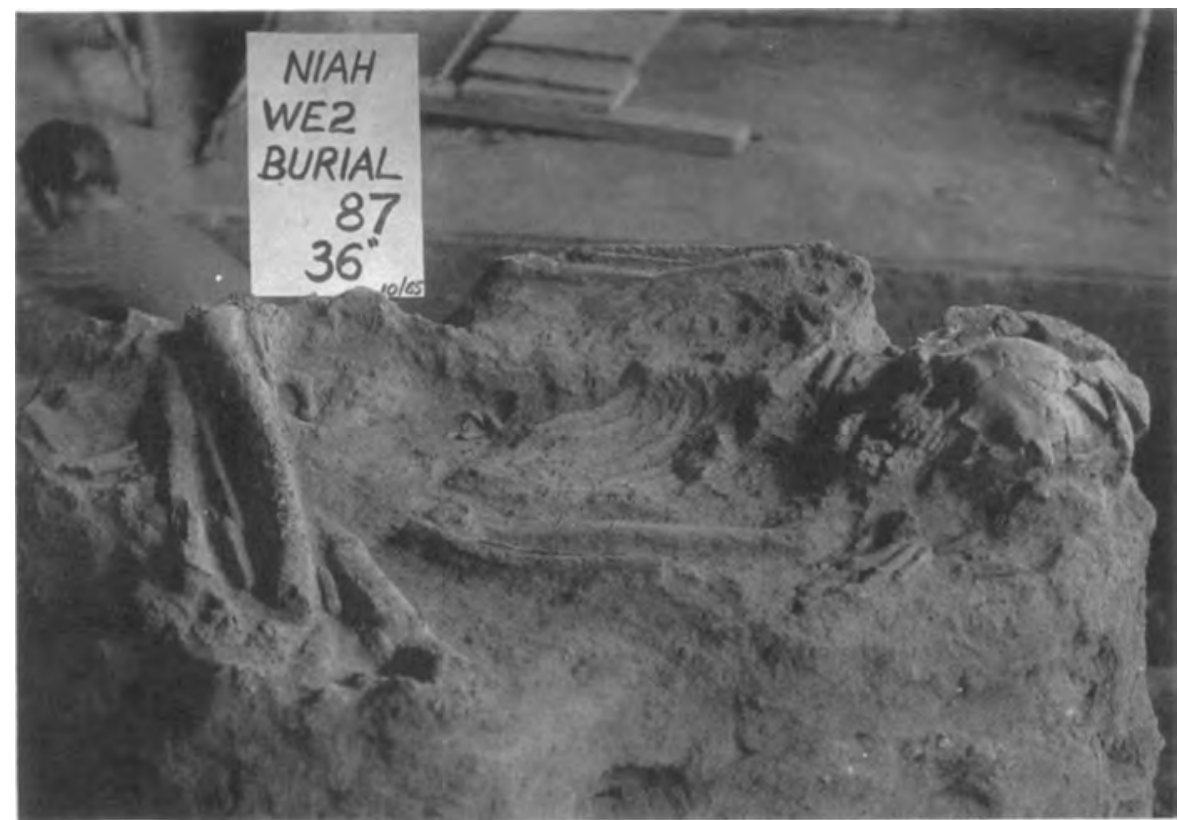

Plate 22. Flexed burial (undated) from a preceramic level at the West Mouth, Niah. Courtesy: Sarawak Museum.

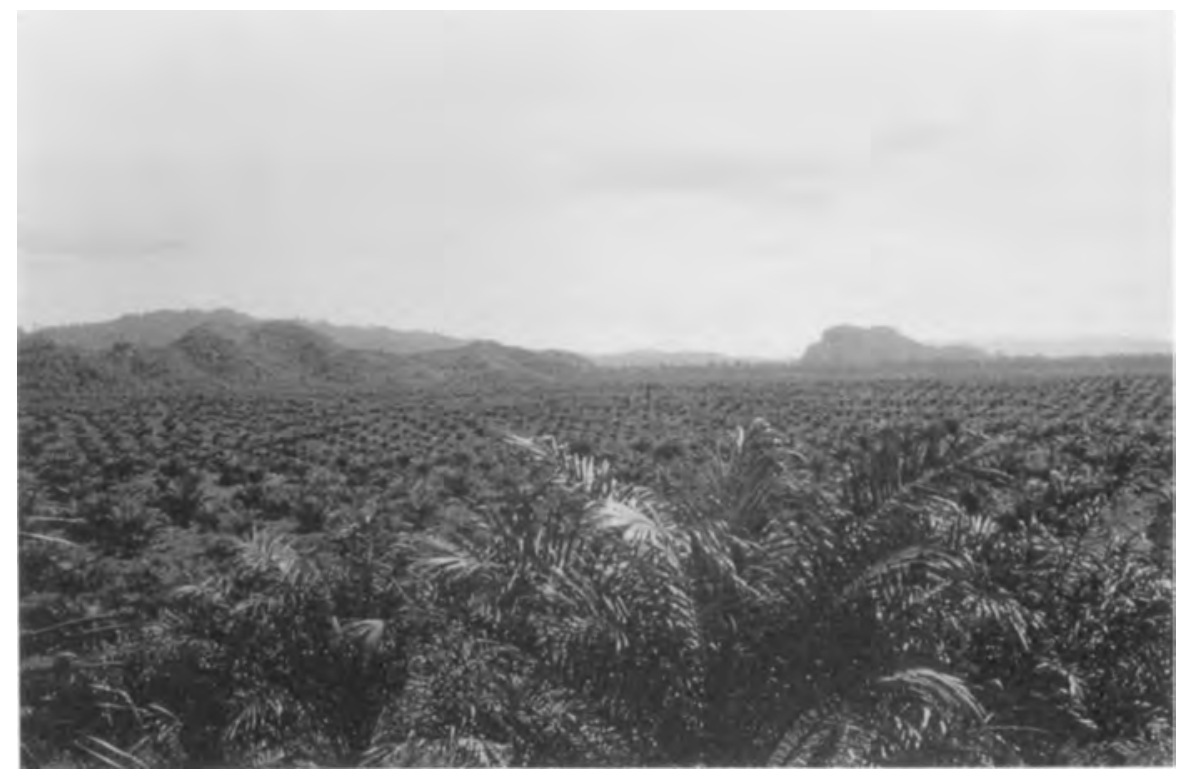

Plate 23. The old Tingkayu lake bed (now under oil palm plantations; this photo taken 1980), with the Baturong limestone massif just right of center. 


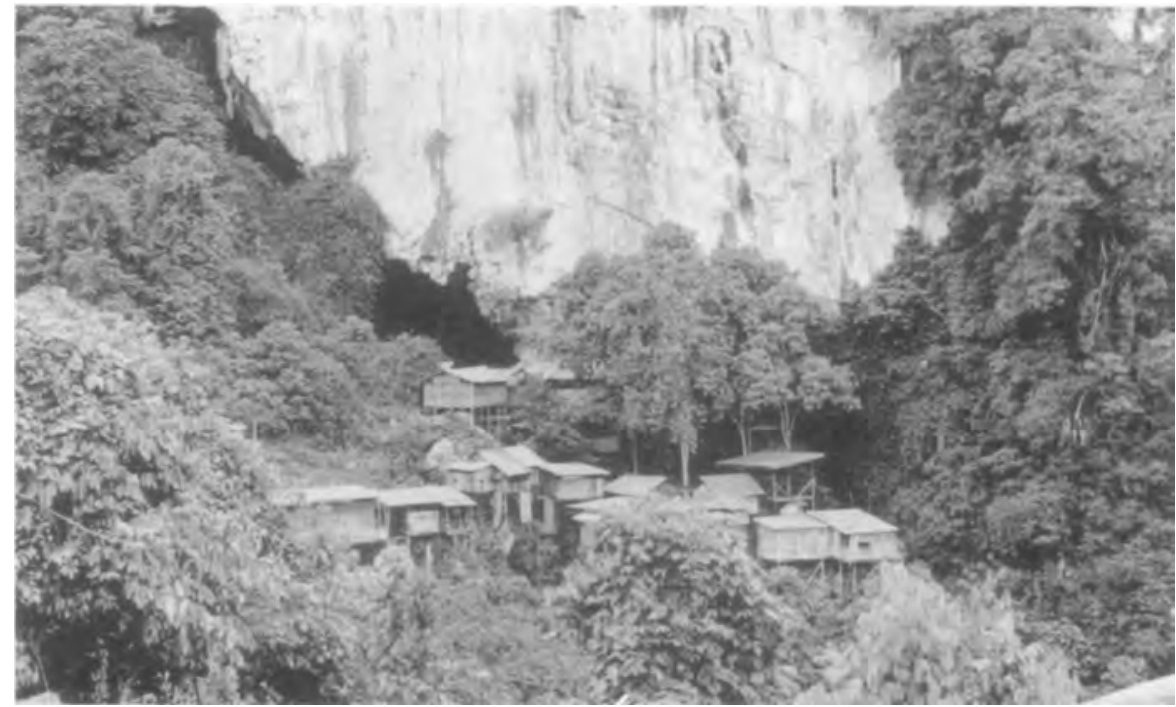

Plate 24. The Madai Caves; Agop Atas (MAD 1) lies just behind and above the village (which extends into the cave). Agop Sarapad (MAD 2) is the upper opening to the left.
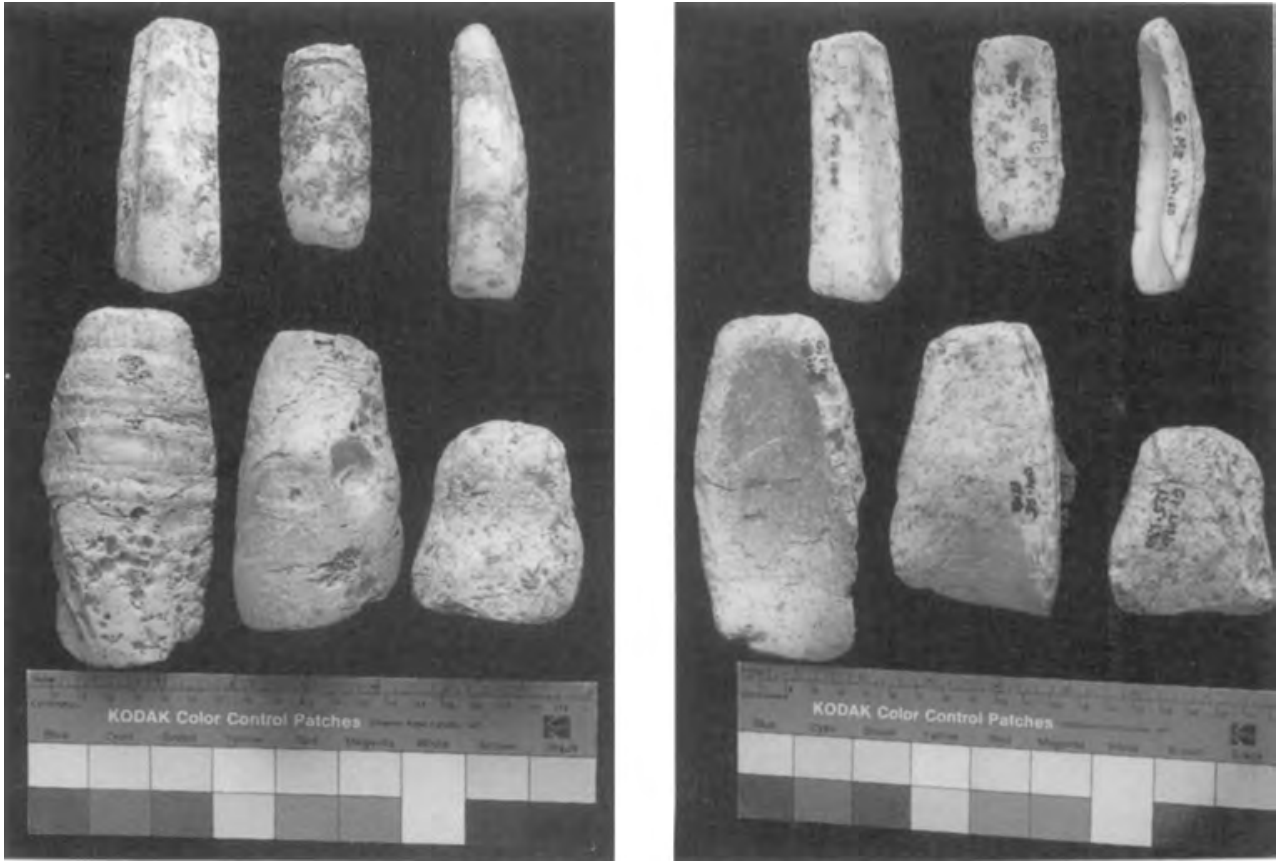

Plate 25. Tridacna (bottom) and Hippopus (top) shell adzes from Golo Cave and Buwawansi (center bottom row only), Gebe Island, northern Moluccas. Dated ca. 12,000 to $9000 \mathrm{BP}$. Scale in centimeters. 


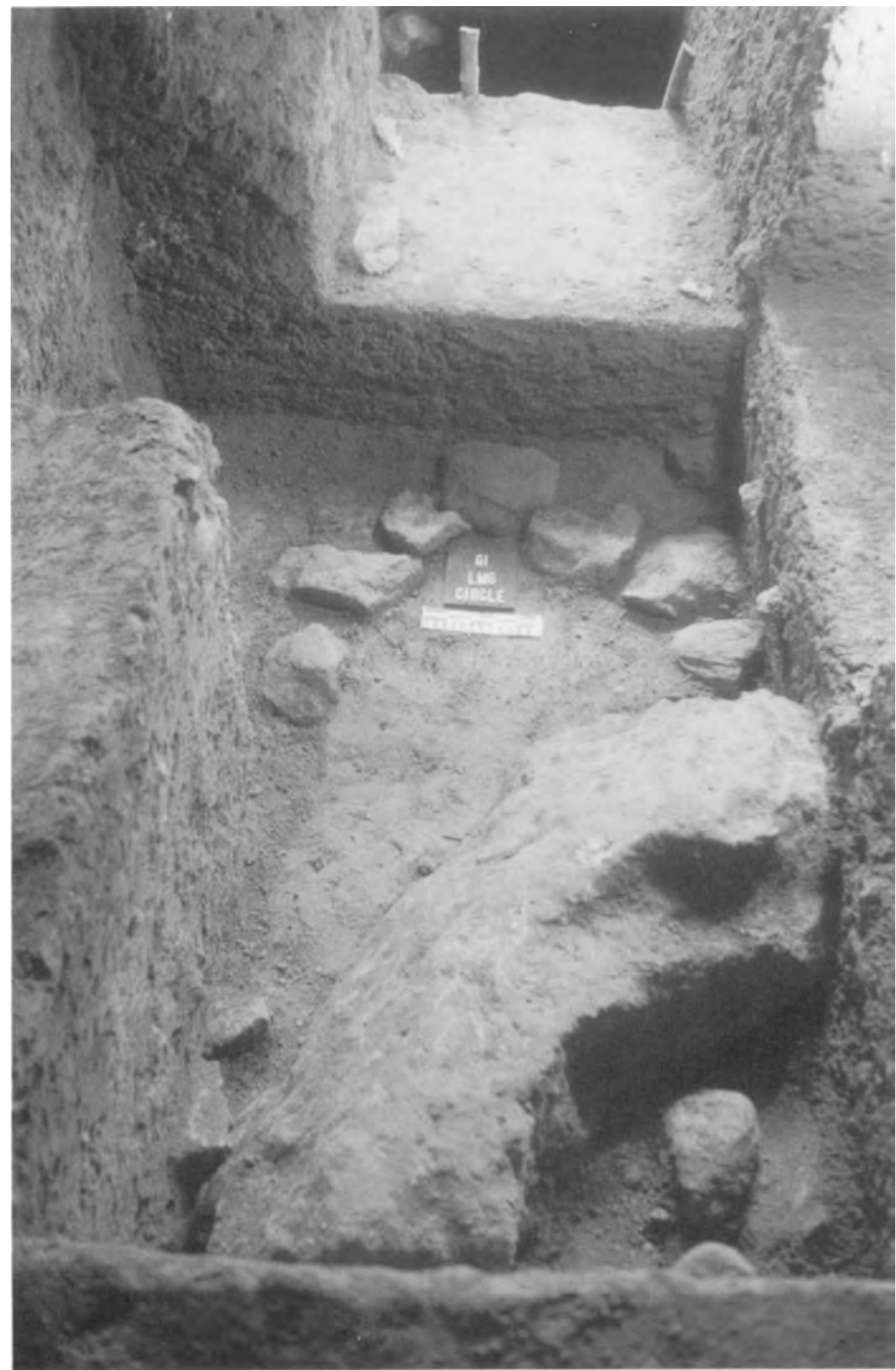

Plate 26. Semicircular setting of coral blocks in Golo Cave; inner diameter 90 centimeters. Dated ca. 12,000 to $10,000 \mathrm{BP}$. 


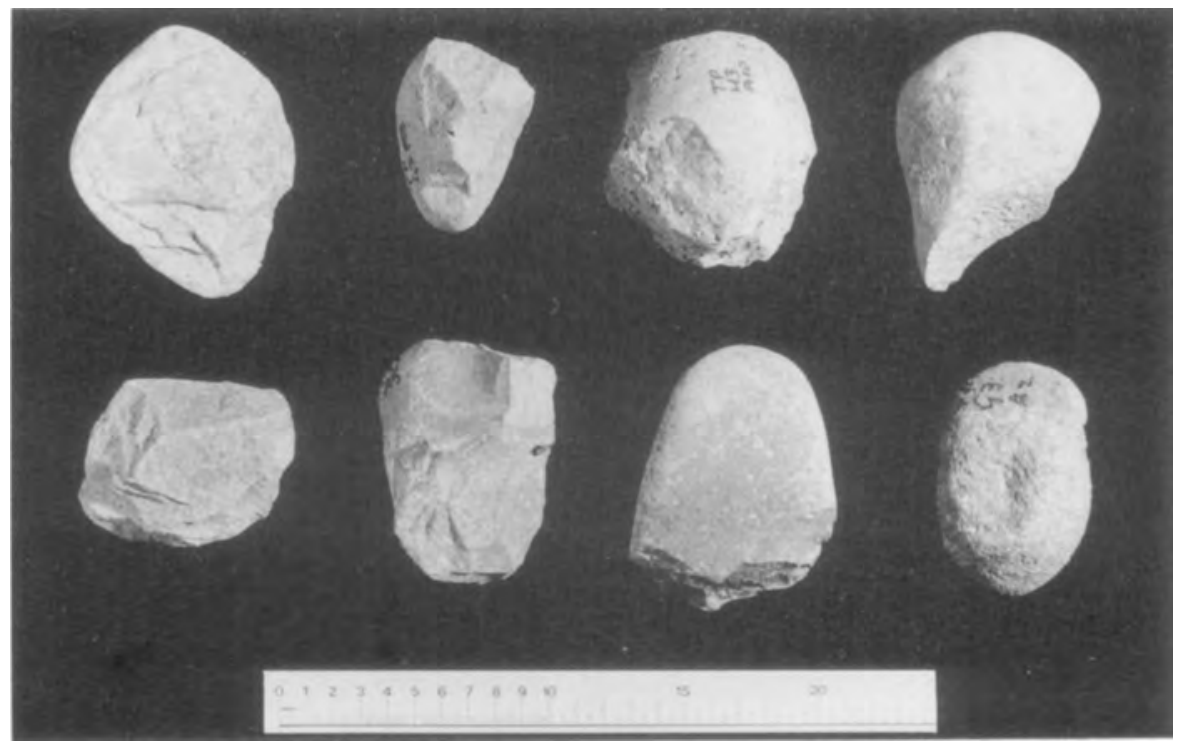

Plate 27. Tools made on volcanic beach pebbles from Tanjung Pinang and Daeo shelters, southern Morotai, northern Moluccas. Early Holocene. Scale in centimeters.

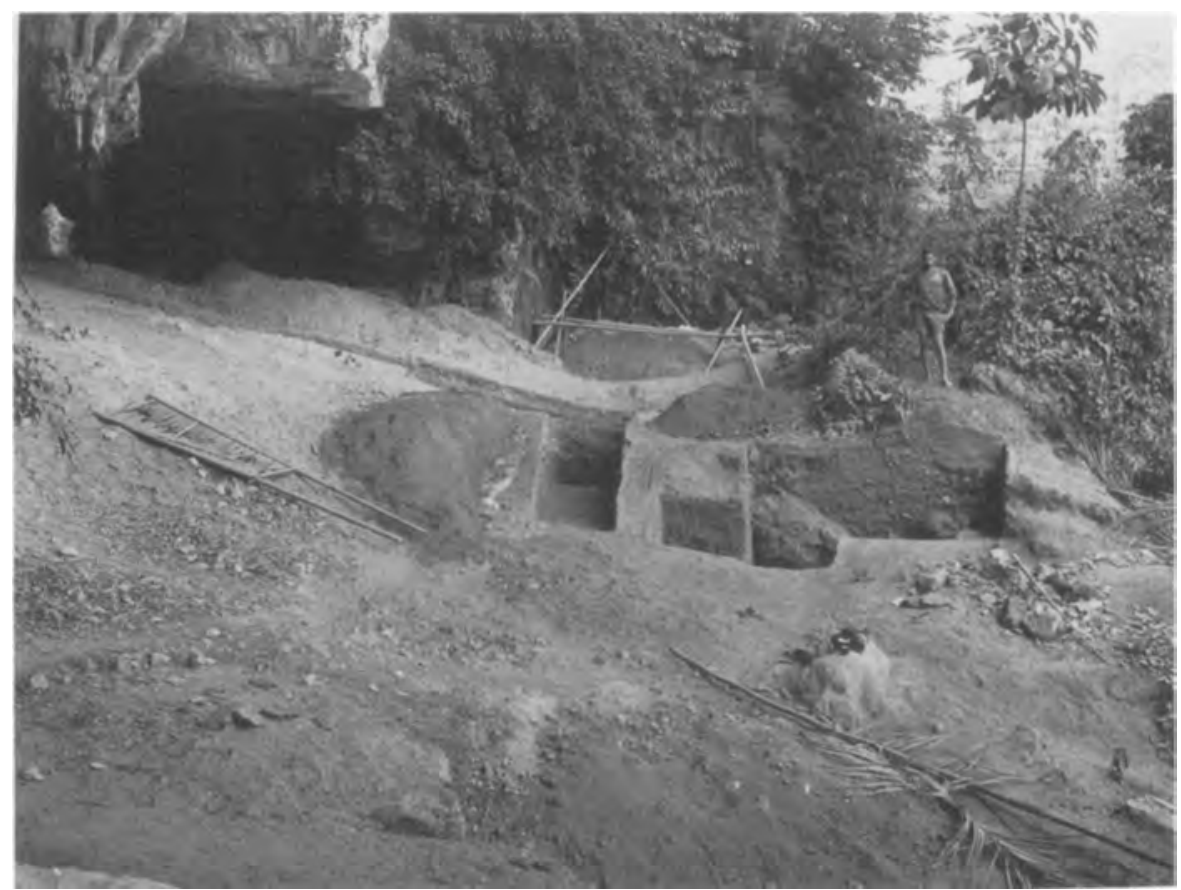

Plate 28. Excavations at Leang Burung 1 in 1969. Photo by John Mulvaney. 

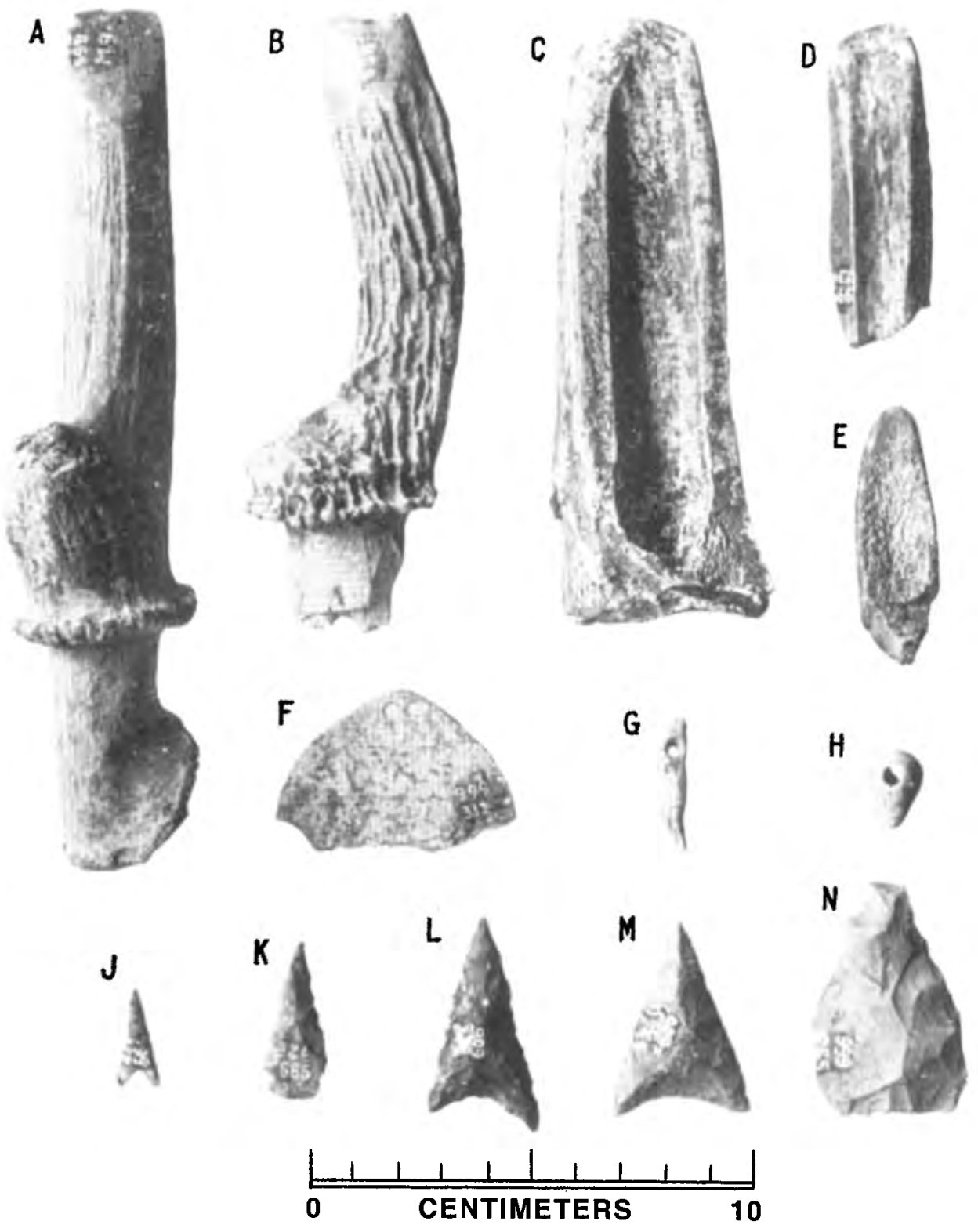

Plate 29. Tools from Gua Lawa Cave, eastern Java: (A-E) bone and antler tools; $(F-H)$ perforated shell and tooth pendants; $(J-N)$ stone points. From Heekeren 1972. Courtesy: Kluwer Academic Publishers. 


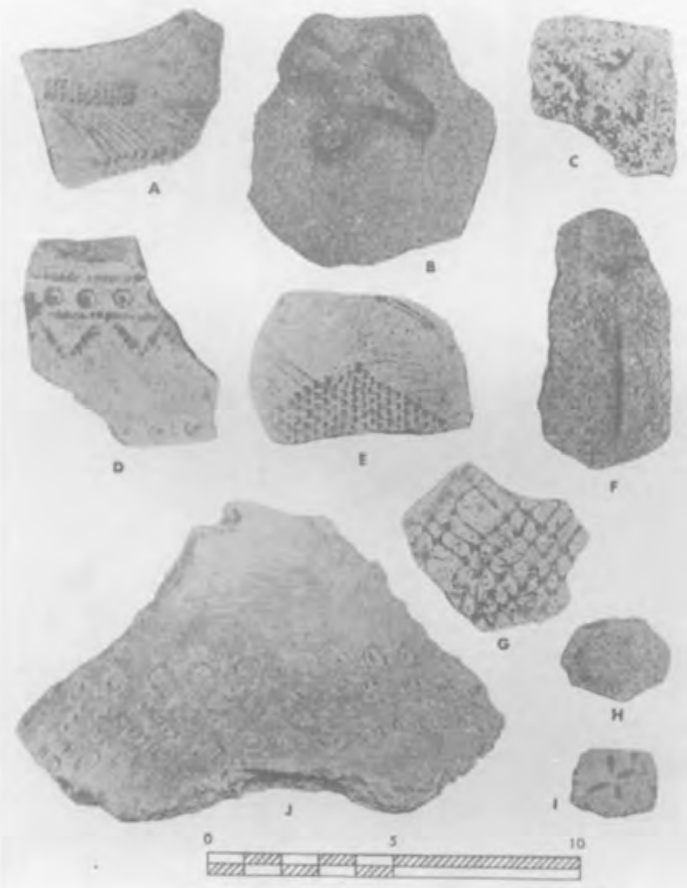

Plate 30. Left: Incised and stamped pottery from Ta-p'enk'eng (Yüan-shan culture). Scale in centimeters. From Chang 1969. Courtesy: Yale University, Department of Anthropology.

Plate 31. Bottom: General view of the Peinan 1987 excavations. Top left: boulder-paved house floors and low walls. Middle: a line of circular stone-walled storage structures. Right: slab graves. From Lien 1991. Courtesy: Lien Chaomei.

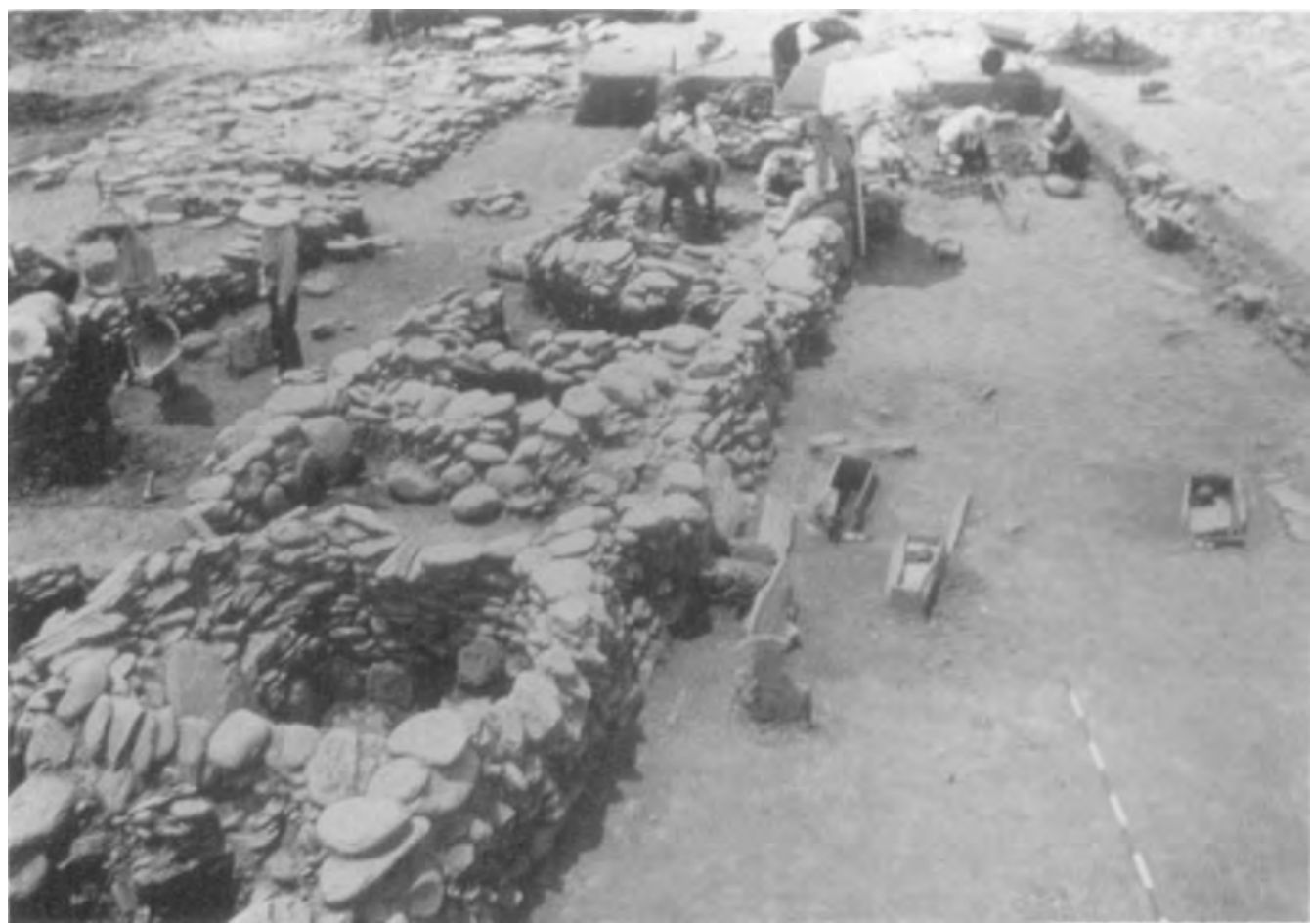




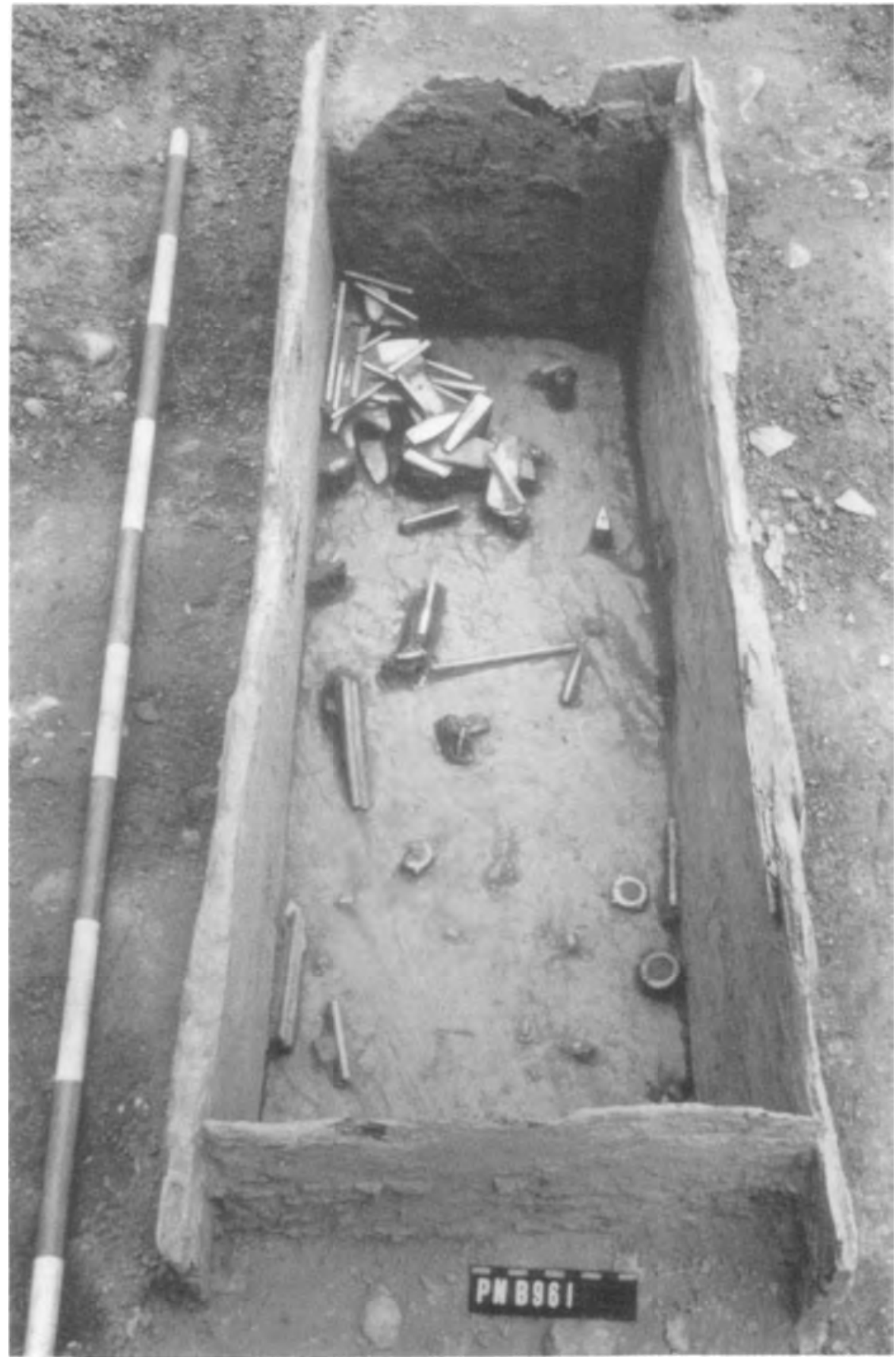

Plate 32. The jade contents (tubular beads, slotted earrings, adzes, and arrowheads) of an excavated slab grave at Peinan. This grave (PN B961) contained multiple burials. From Lien 1991. Courtesy: Lien Chaomei. 


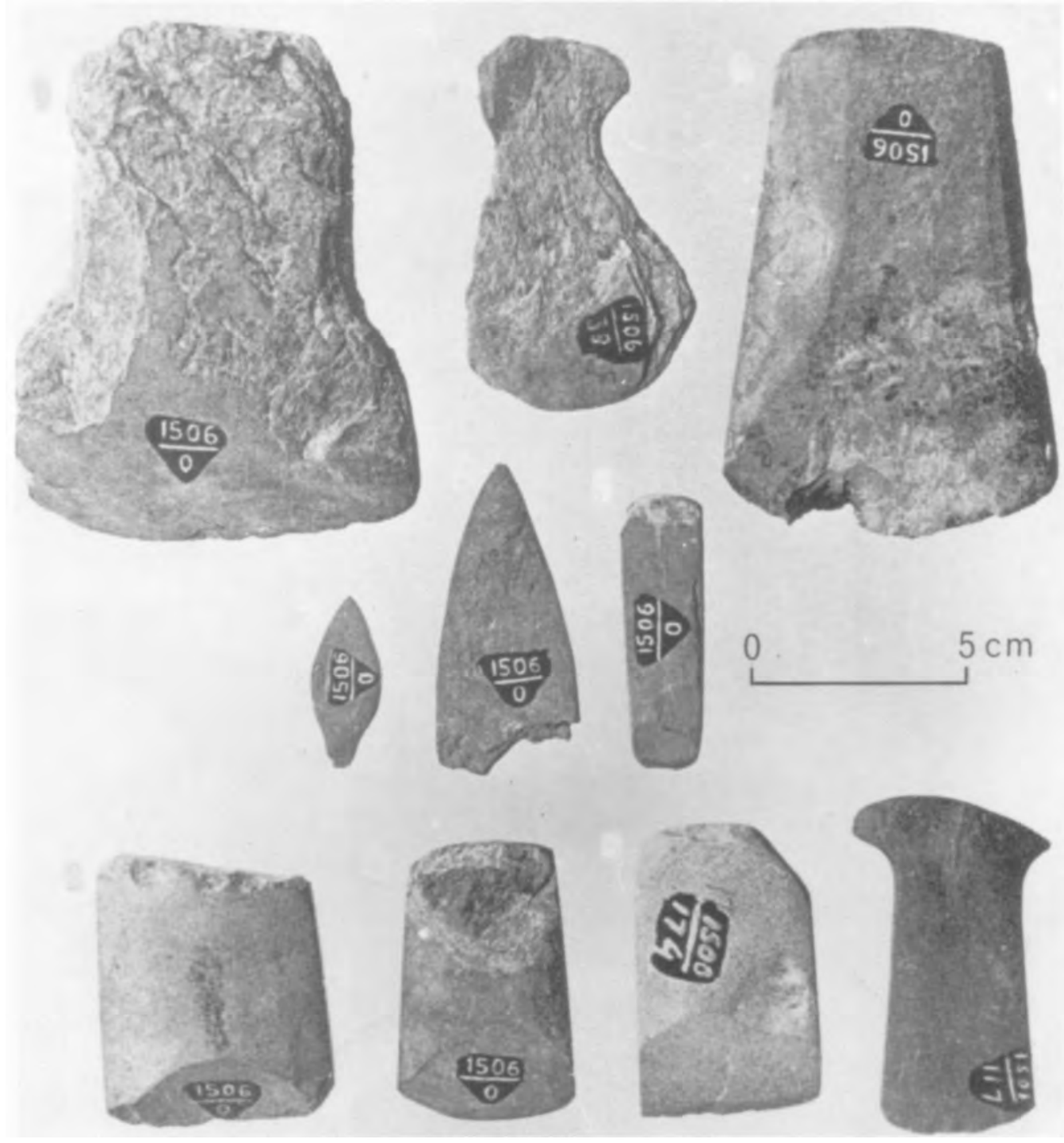

Plate 33. Stone adzes and points from Kalumpang, west-central Sulawesi. From Heekeren 1972. Courtesy: Kluwer Academic Publishers. 


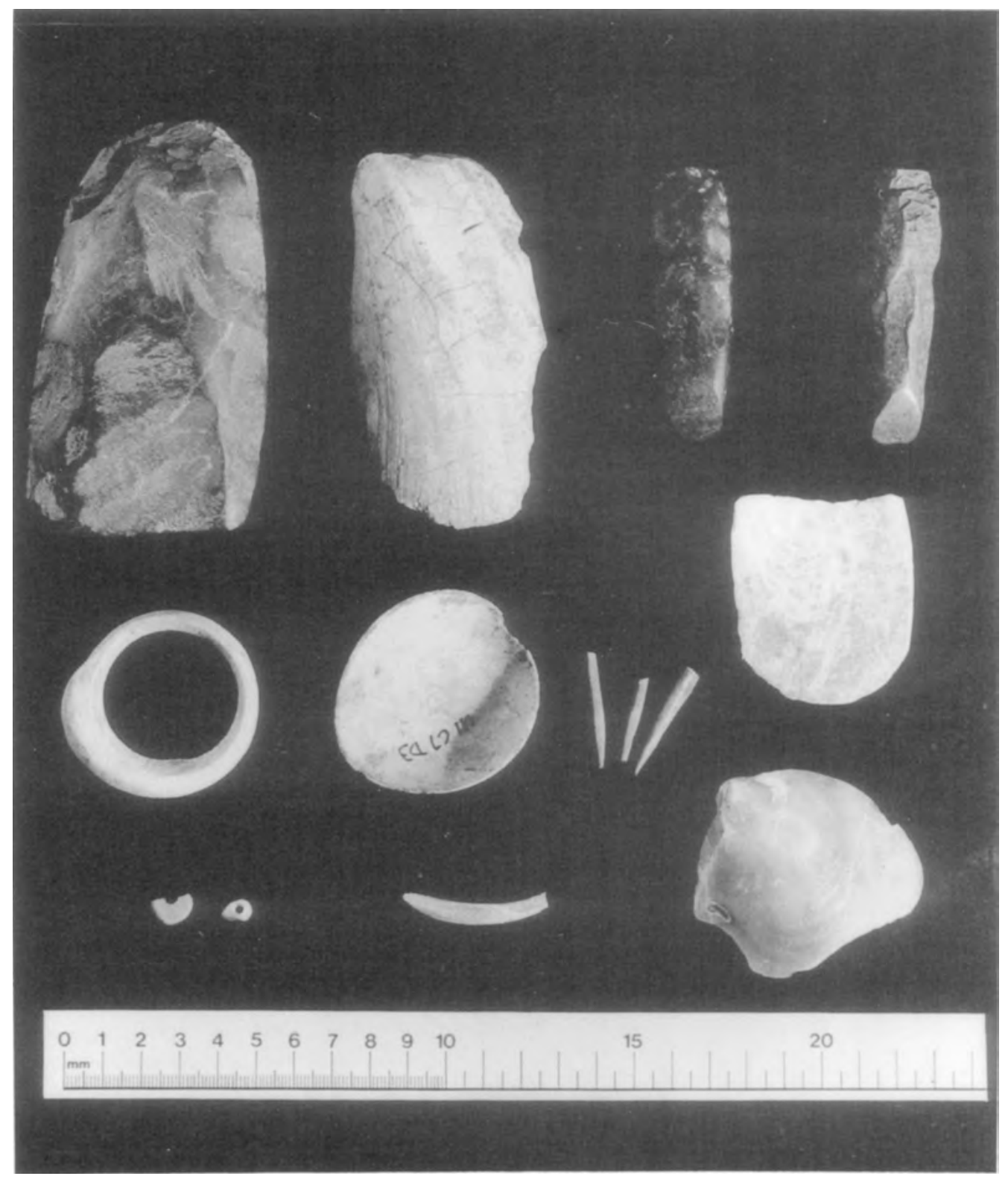

Plate 34. Artifacts from Uattamdi, Kayoa Island, and Buwawansi, Gebe Island, northern Moluccas, ca. $1500-500 \mathrm{BC}$ (all from Uattamdi unless stated). Top row, left to right: lenticularsectioned stone adze, shell adze, stone chisel with (at right) identically shaped stone chisel from Pitcairn Island, eastern Polynesia. Middle row: Conus shell armring from Buwawansi, cowrie shell disc, bone points, and pearl shell scraper. Bottom row: shell beads and Trochus armring fragment; pearl-shell scraper. 


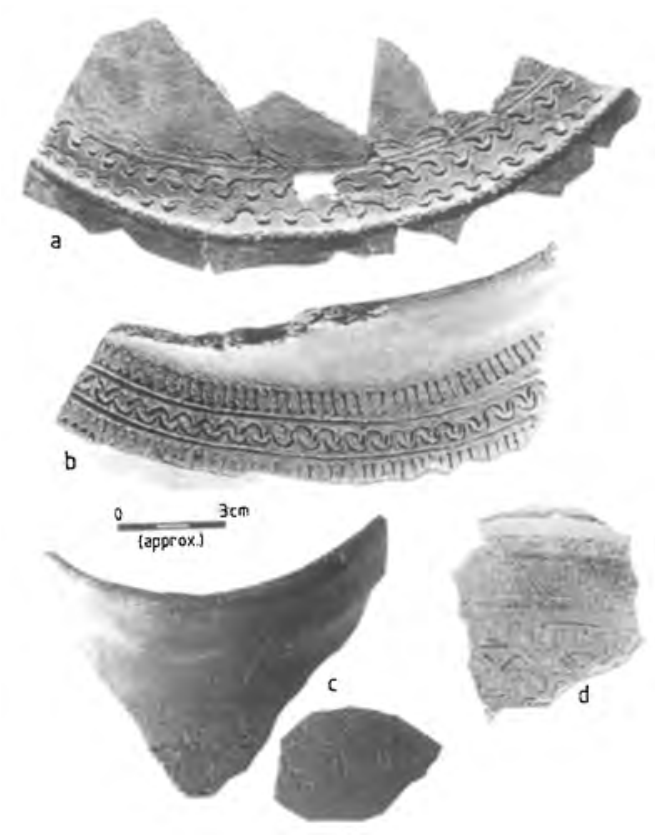

Plate 35. (a, b) Sherds with interlocked semicircle pattern; (a) from eastern Timor, ca. $1500 \mathrm{BC}$ to $\mathrm{AD} 500$, and (b) from Ulu Leang 2, southern Sulawesi (for similar patterns from Kalumpang see Fig. 7.13). (c) Redslipped sherds with incised arcade motif from Nikiniki I, western Timor, with related motif on a Lapita sherd from Watom Island, New Britain, ca. 1000 BC. Courtesy: lan Glover (a-c); Musée de l'Homme, Paris (d).

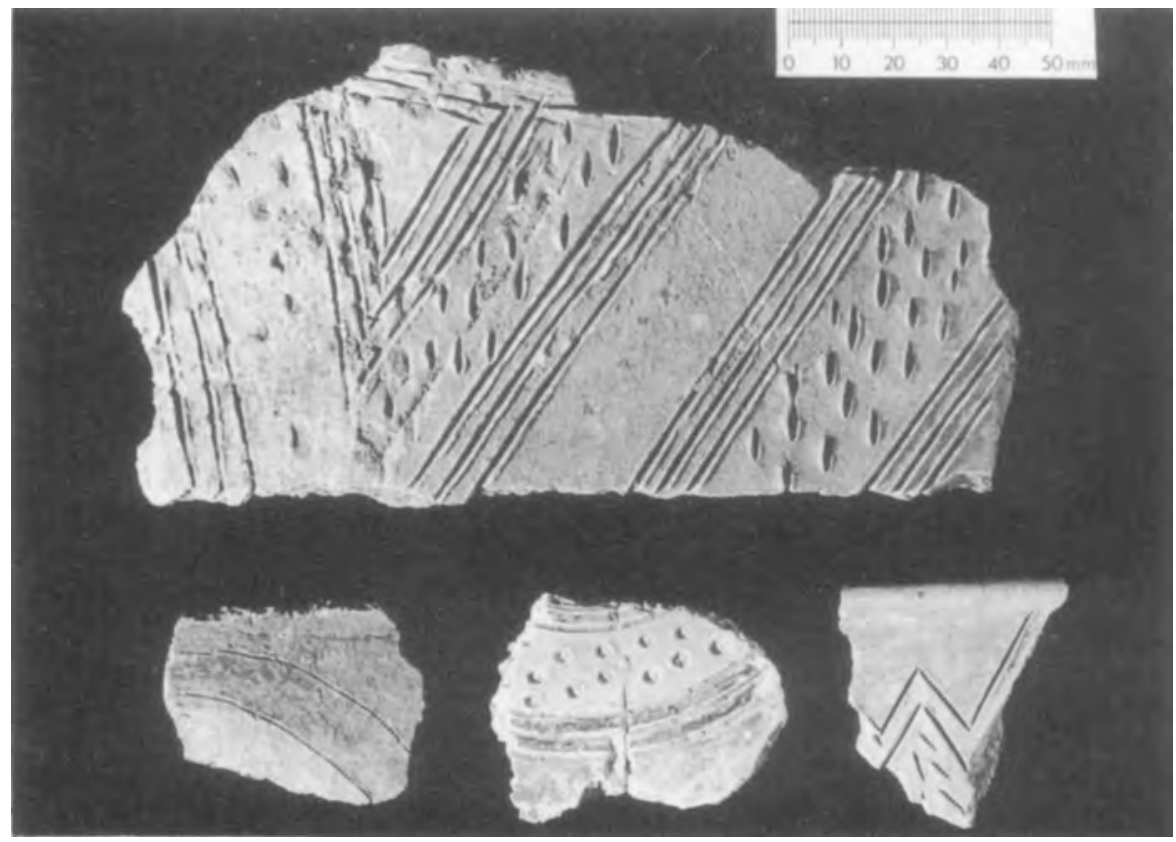

Plate 36. Sherds of three-color ware from Lubang Angin (the two at bottom right, both with red and/or black coloring not visible in photo); another from Niah (bottom left); and an uncolored sherd related to three-color ware from Niah (top). Courtesy: Sarawak Museum. 


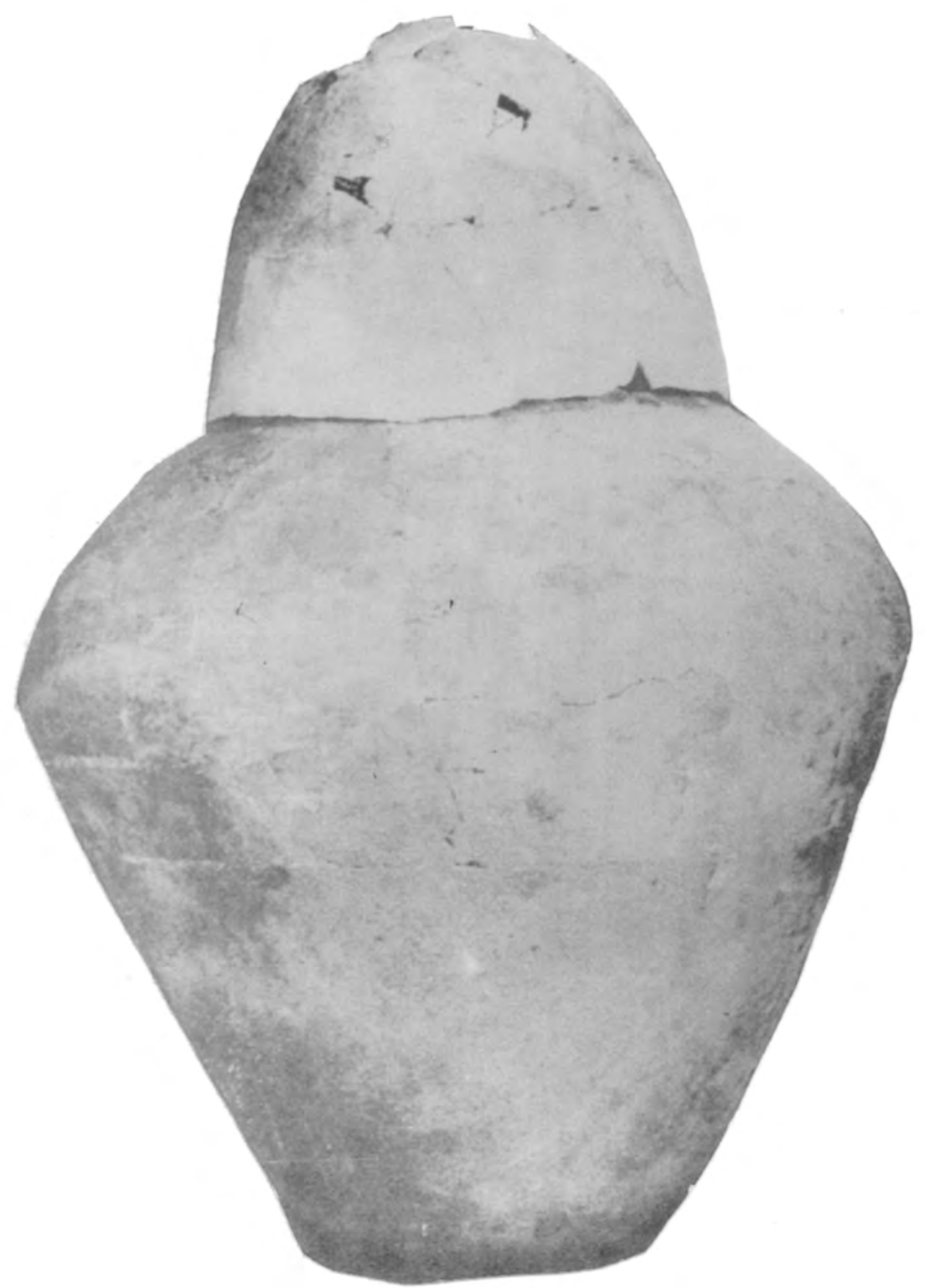

Plate 37. Lidded burial jar and lid (burial 159: Harrisson 1968) with carved-paddleimpressed decoration from the West Mouth, Niah. Height excluding lid, 69.5 centimeters. This jar was associated with burnt wood radiocarbon dated to ca. 1500 BC. Courtesy: Sarawak Museum. 

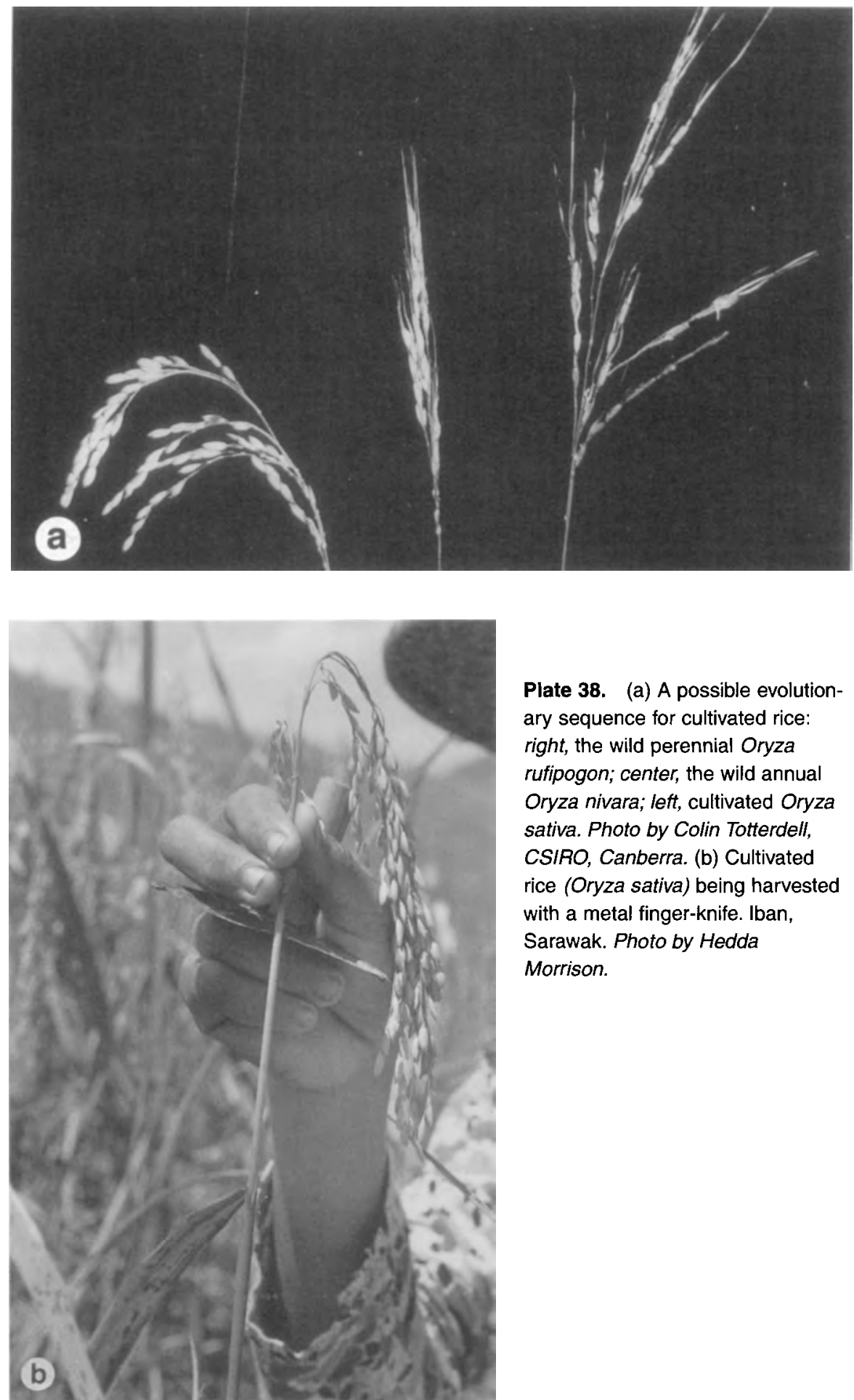

Plate 38. (a) A possible evolutionary sequence for cultivated rice: right, the wild perennial Oryza rufipogon; center, the wild annual Oryza nivara; left, cultivated Oryza sativa. Photo by Colin Totterdell, CSIRO, Canberra. (b) Cultivated rice (Oryza sativa) being harvested with a metal finger-knife. Iban,

Sarawak. Photo by Hedda Morrison. 

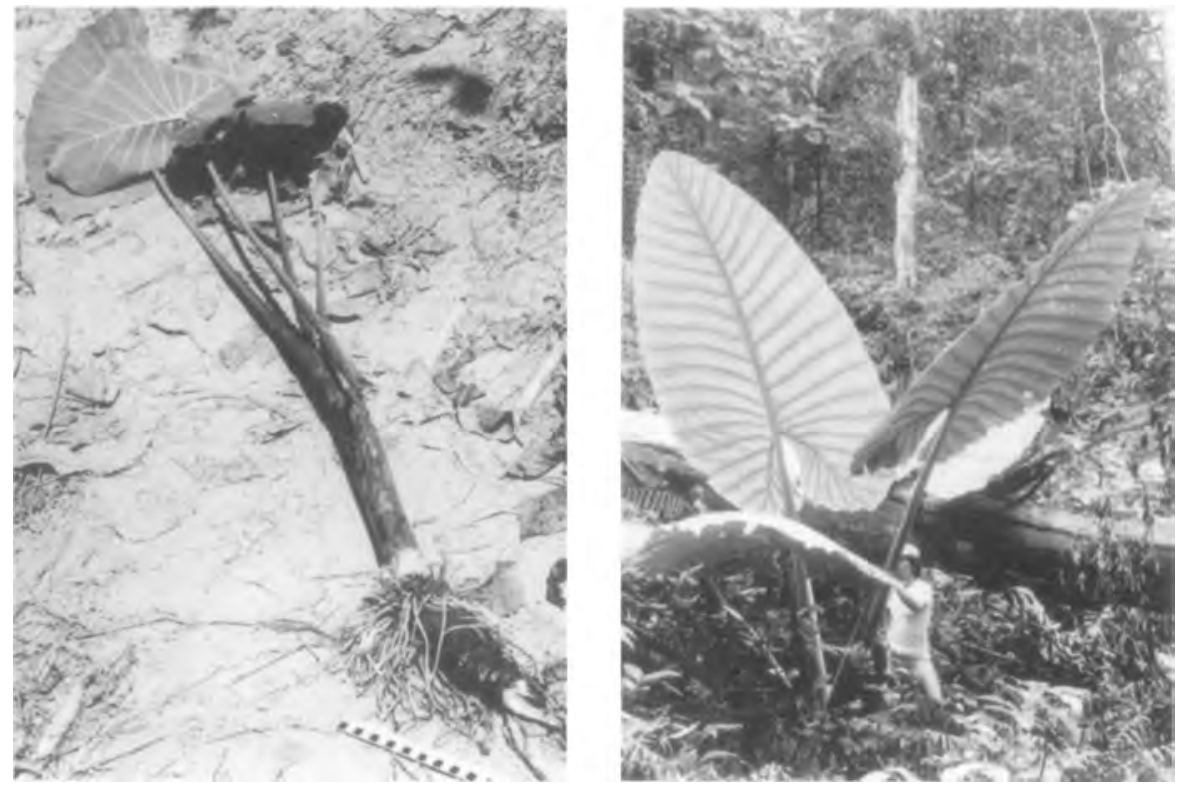

Plate 39. Left: wild or feral Colocasia esculenta (taro), Kelantan, Malaysia. The tuber at the base of the plant is the main edible part. Right: wild Alocasia macrorrhiza, Baturong Forest Reserve, Sabah.

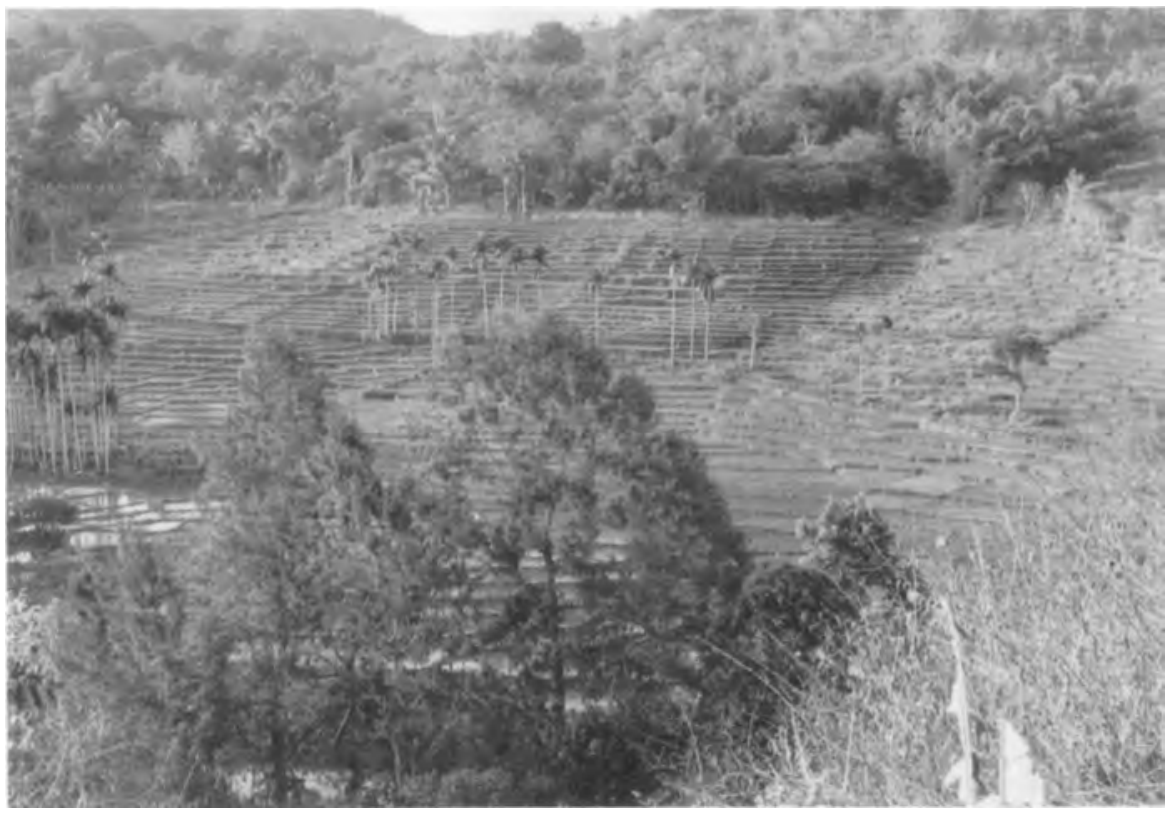

Plate 40. Terraced rice fields in South Amanuban, western Timor. Photo by James Fox. 


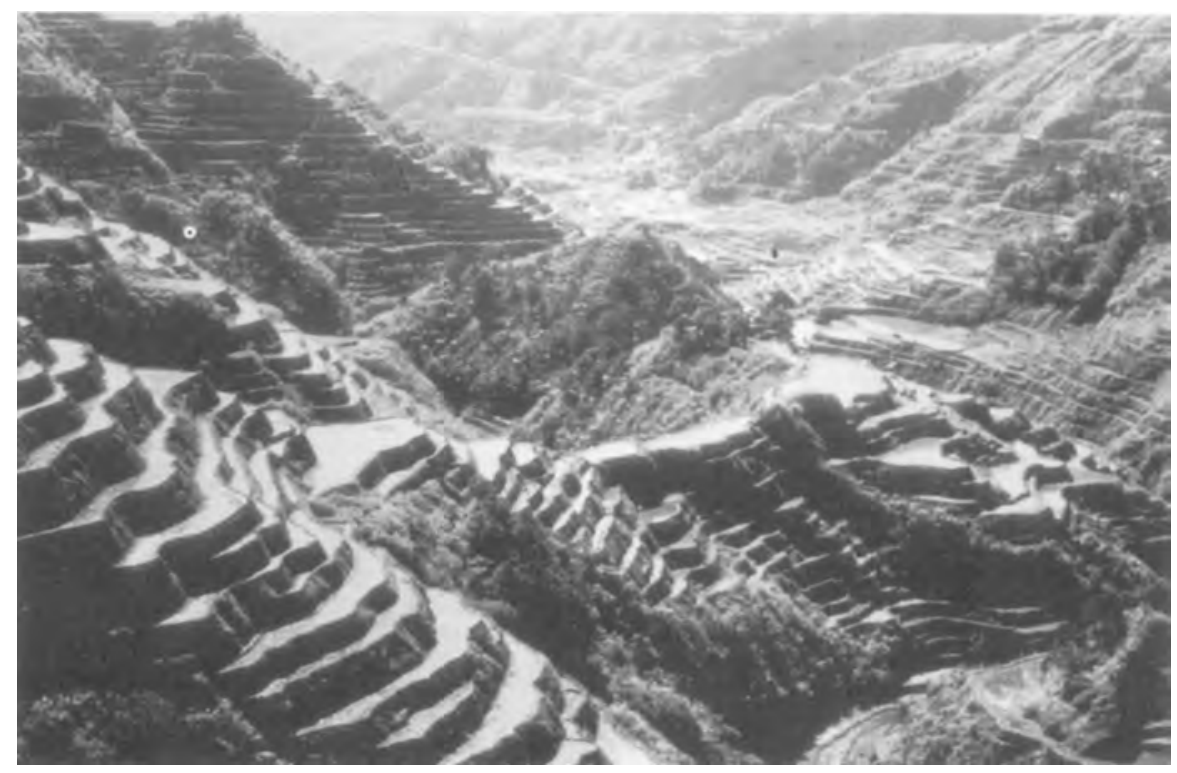

Plate 41. Ifugao rice terraces at Banaue, northern Luzon.

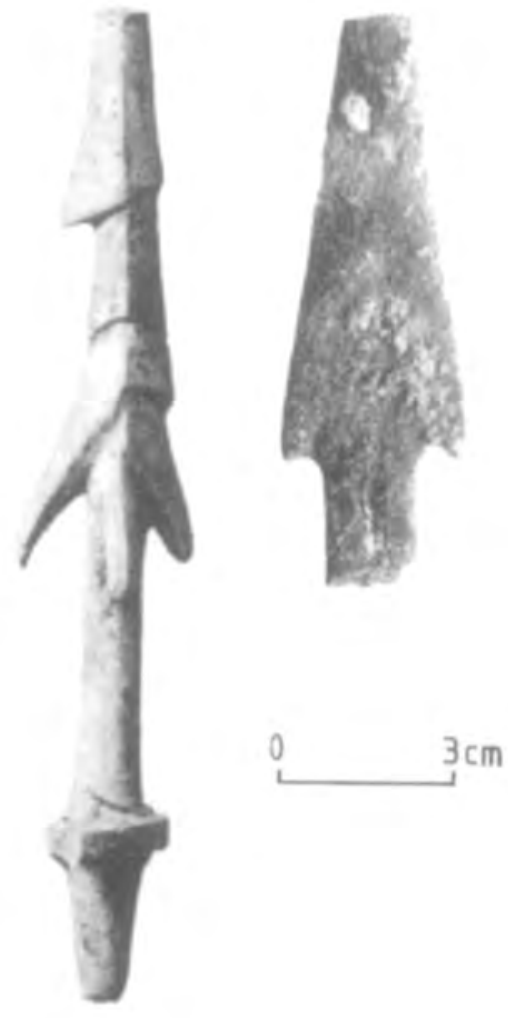

Plate 42. Bone points from Ban Kao (left) and Gua Kechil (right). From Sørensen and Hatting 1967; Dunn 1964. Courtesy: Munksgaard, Malaysian Branch of the Royal Asiatic Society. 

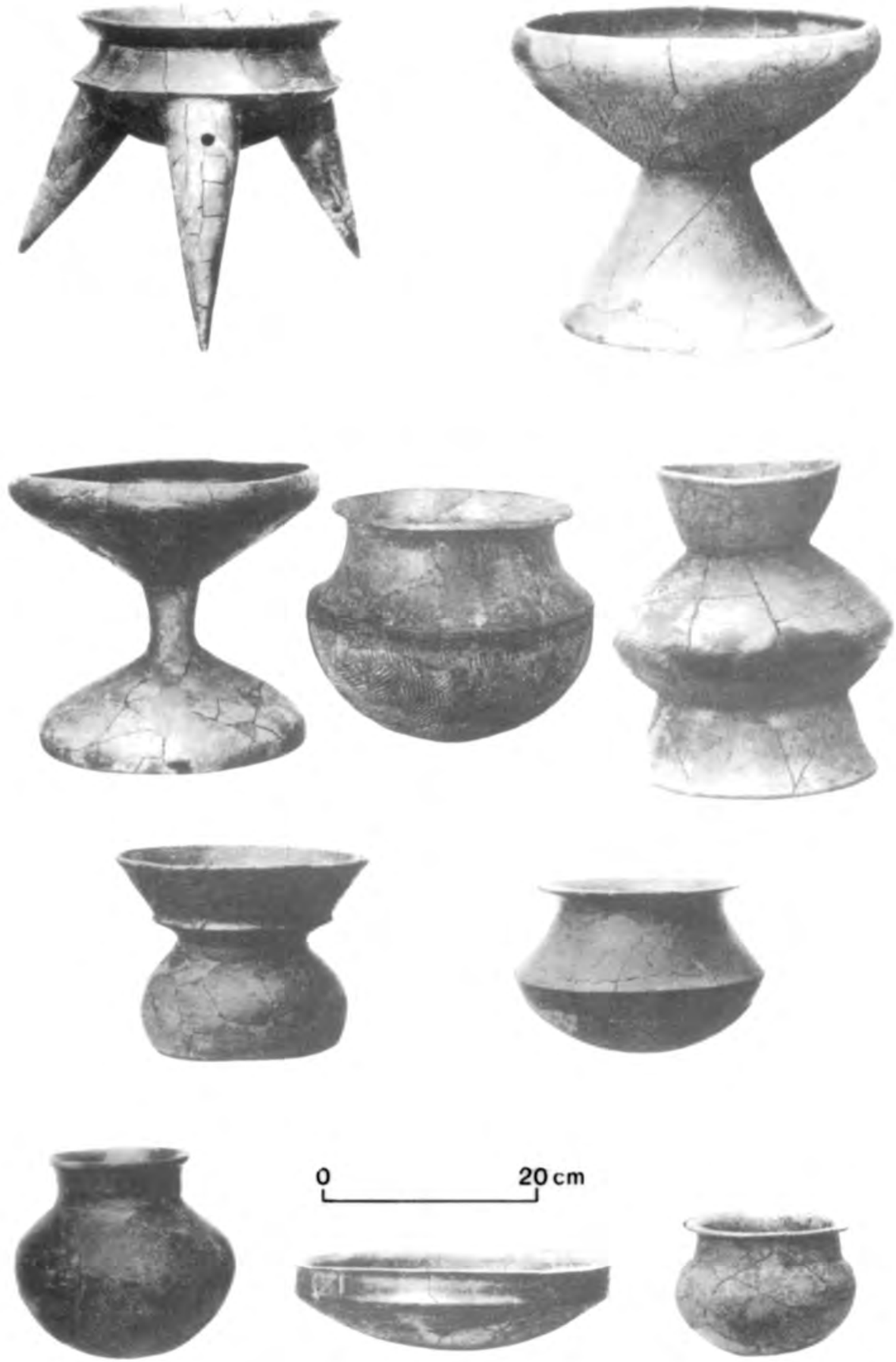

Plate 43. Neolithic pottery from Ban Kao, western Thailand. From Sørensen and Hatting 1967. Courtesy: Munksgaard. 


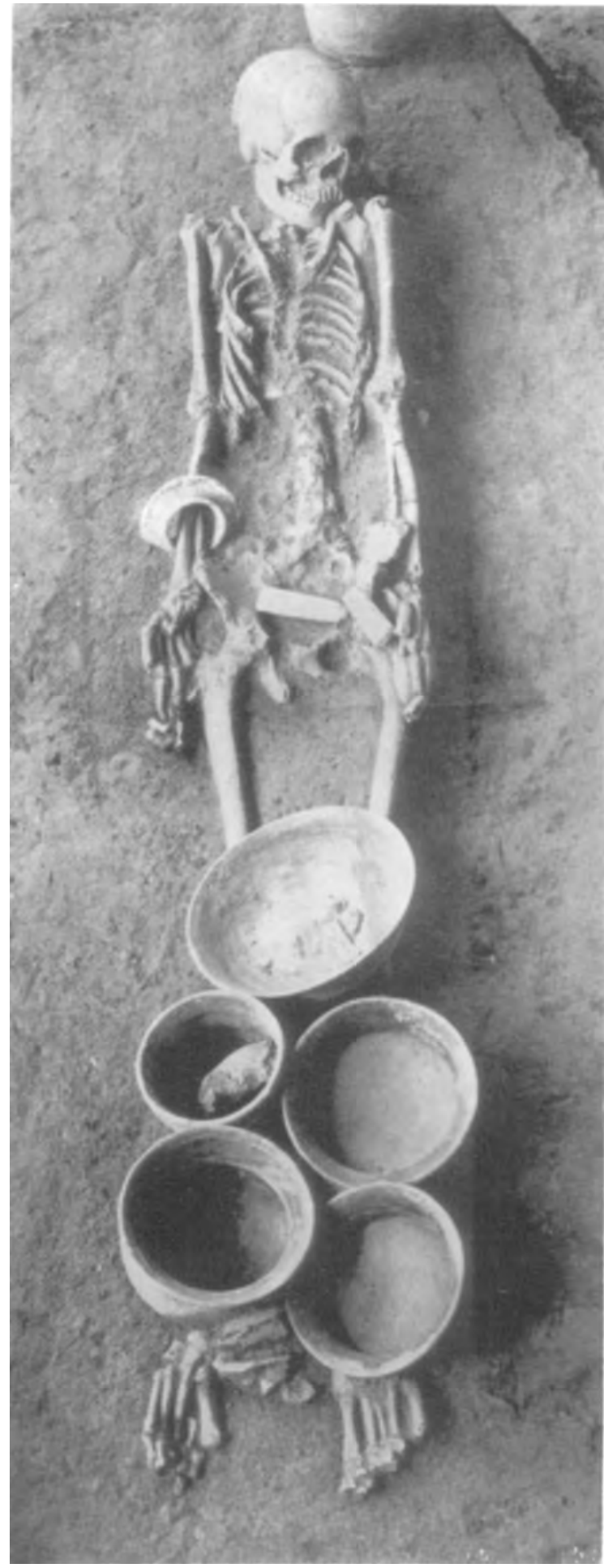

Plate 44. Neolithic burial of an adult female at Gua Cha, Kelantan, with pots at head and on legs (one of the latter contains a rat skull), a T-sectioned bracelet on the right forearm, a mussel-shell spoon in the left hand, and two quadrangular stone adzes on the pelvis. From Sieveking 1954 (burial 8). Courtesy: National Museum of Malaysia. 


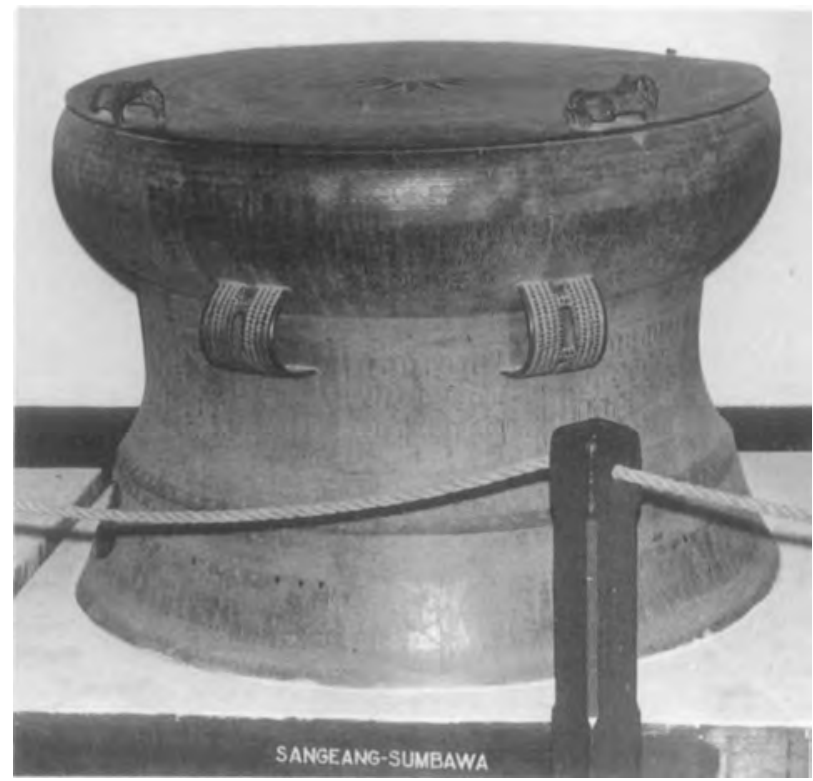

Plate 45. Bronze drum of Heger type I, 84 centimeters high, from Sangeang Island, Indonesia (the "Makalamau" drum). Courtesy: National Museum of Indonesia.

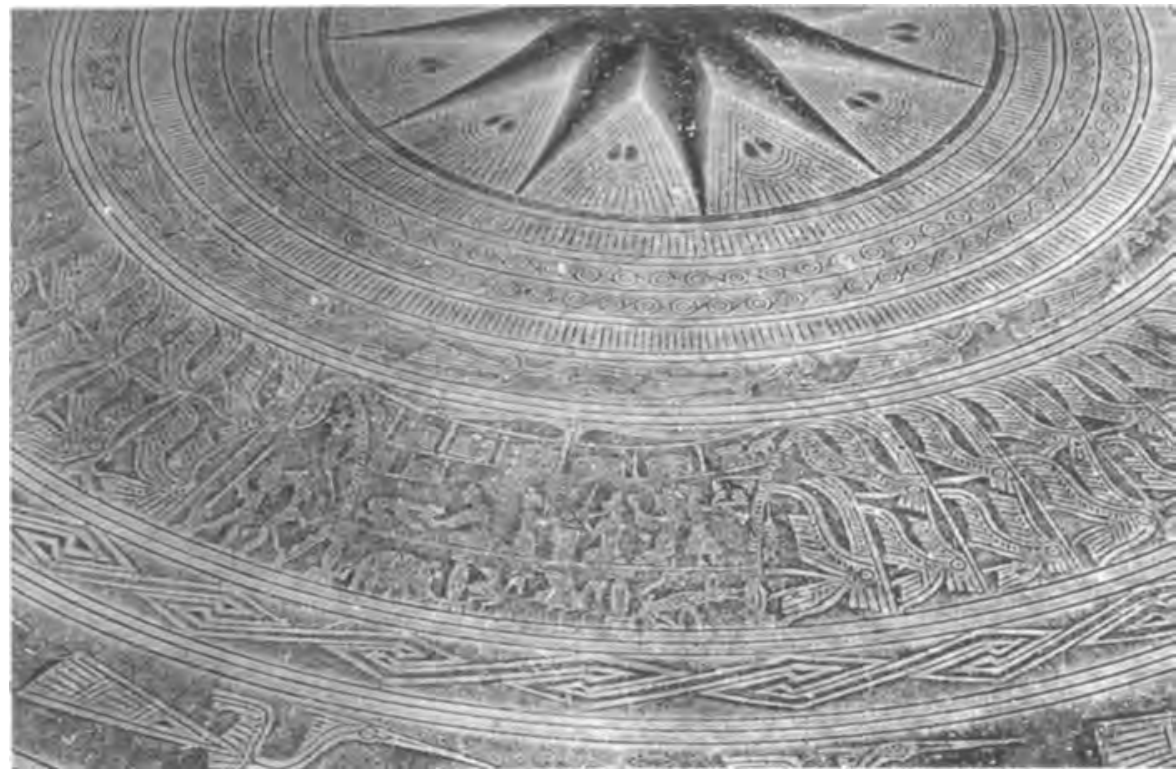

Plate 46. Part of the decorated tympanum of the "Makalamau" drum from Sangeang. This scene shows a pile dwelling with a saddle-shaped roof, partitioned attic (with a kettle drum at right), main floor, and basement. According to Heine Geldern (1947), the figures on the main floor may be in Chinese Han dynasty costumes. Scenes include paying homage and kneeling around a drum. The basement has a pig, two chickens, and a dog. Courtesy: National Museum of Indonesia. 

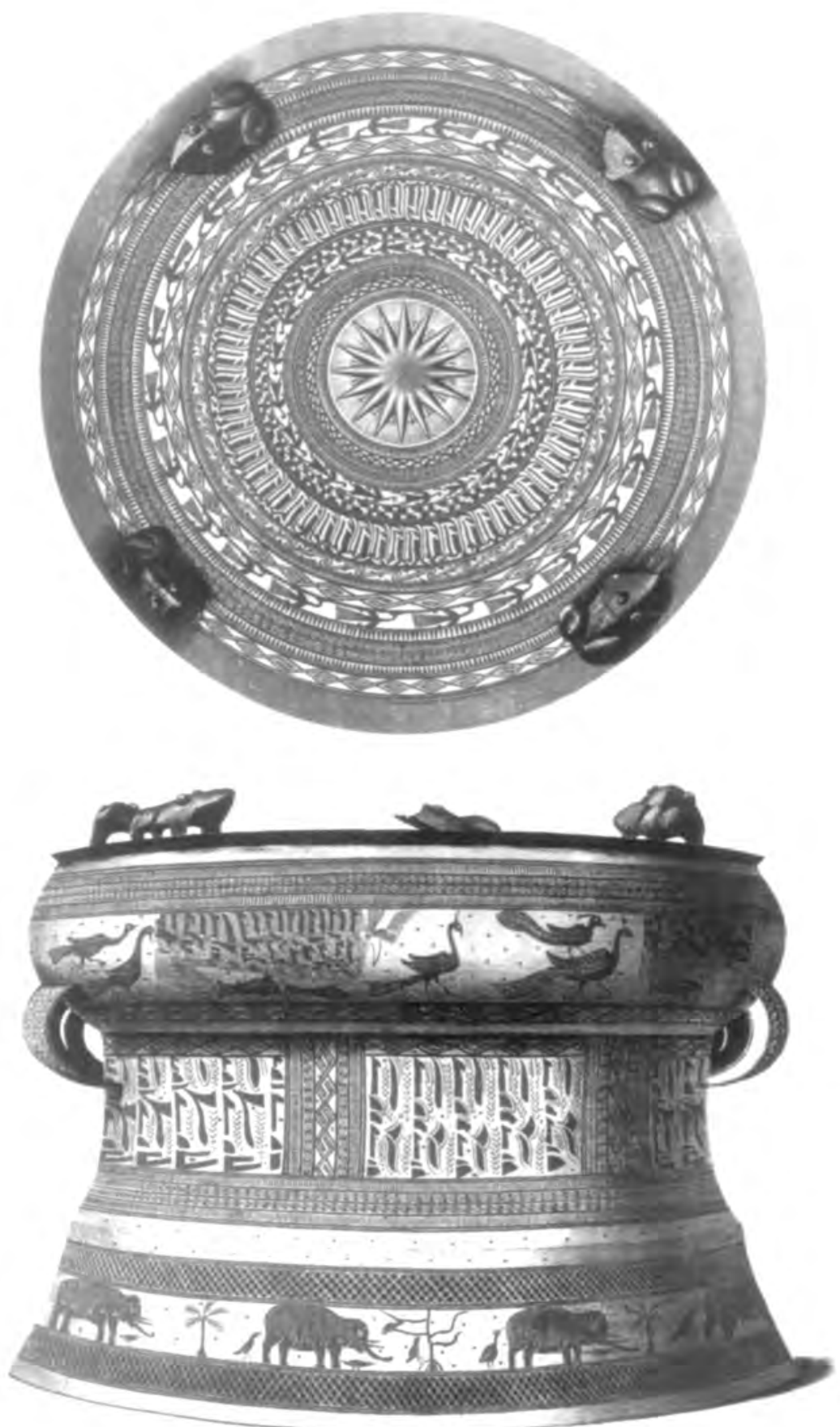

Plate 47. Top and side views of the Salayar drum, with its distinctive friezes of elephants and peacocks; 92 centimeters high, tympanum diameter 103 centimeters. From Schmeltz 1904. 

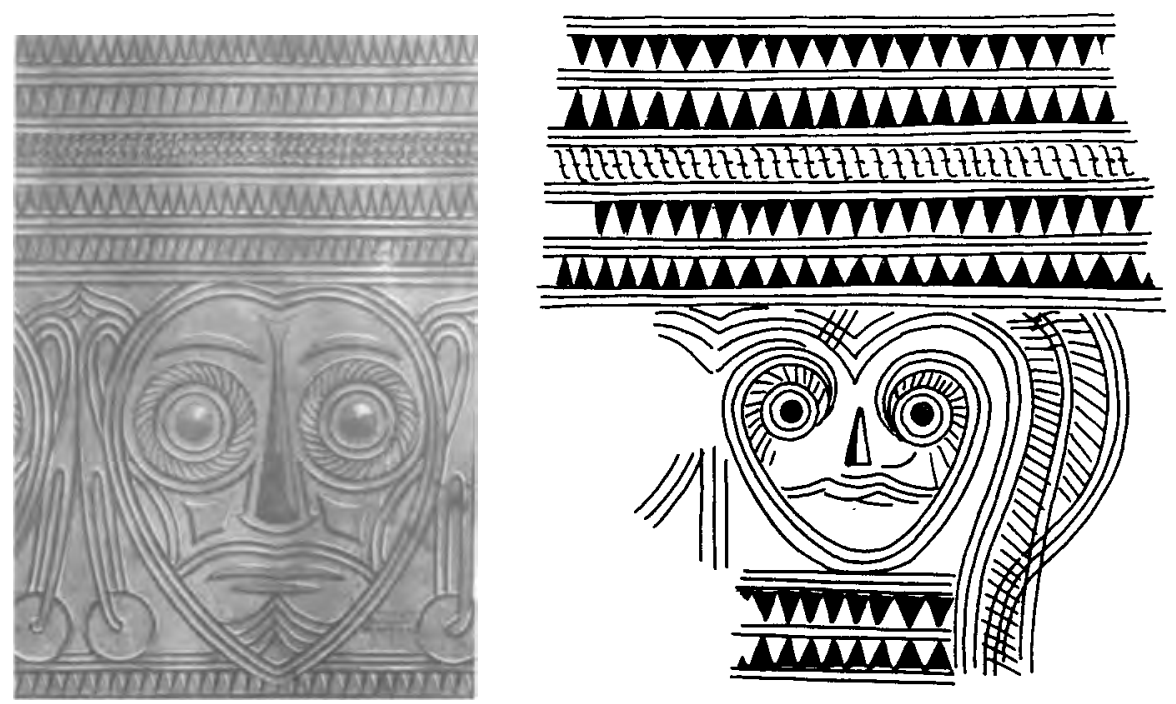

Plate 48. Left: human face design on the side of the Pejeng drum, flanked by rows of triangles identical to those on the Sembiran stamp. Right: similar design on the tuff stamp/mold from Manuaba. From Bernet Kempers 1988. Courtesy: A. A. Balkema.
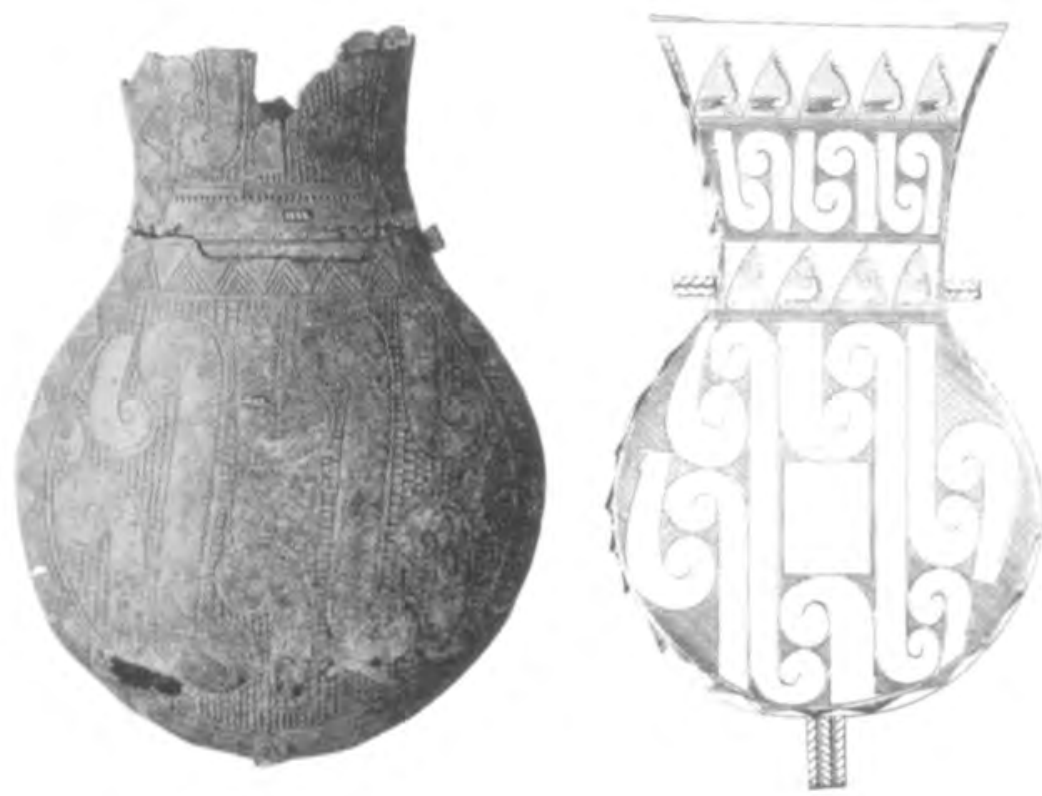

Plate 49. Bronze flasks from Indonesia. Left: from Kerinci, Sumatra, 51 centimeters high. Right: from Madura, 84 centimeters high (this specimen has a tin content of 15.2 percent). From Heekeren 1958. Courtesy: Kluwer Academic Publishers. 

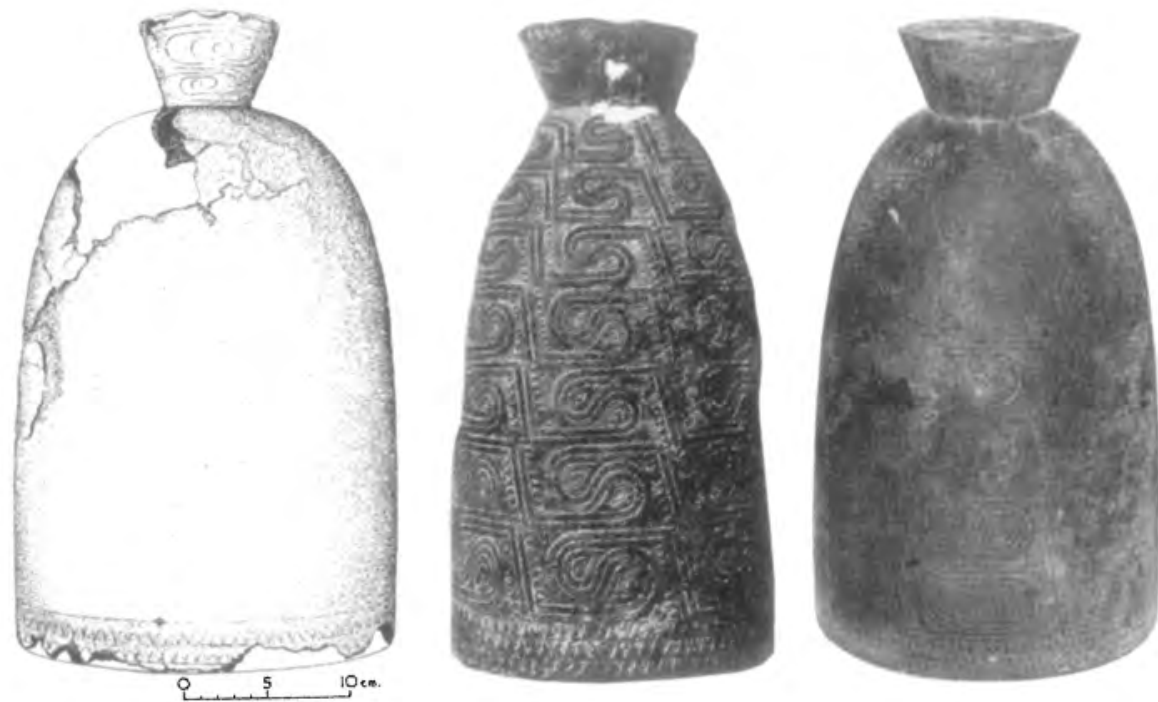

Plate 50. Left and center: bronze bells from Klang, Selangor (center specimen is 56.5 centimeters high). From Loewenstein 1956. Right: bronze bell 58 centimeters high from Kampong Pencu, Johor. From Adi 1983. Courtesy: Malaysian Branch of the Royal Asiatic Society; National Museum of Malaysia.

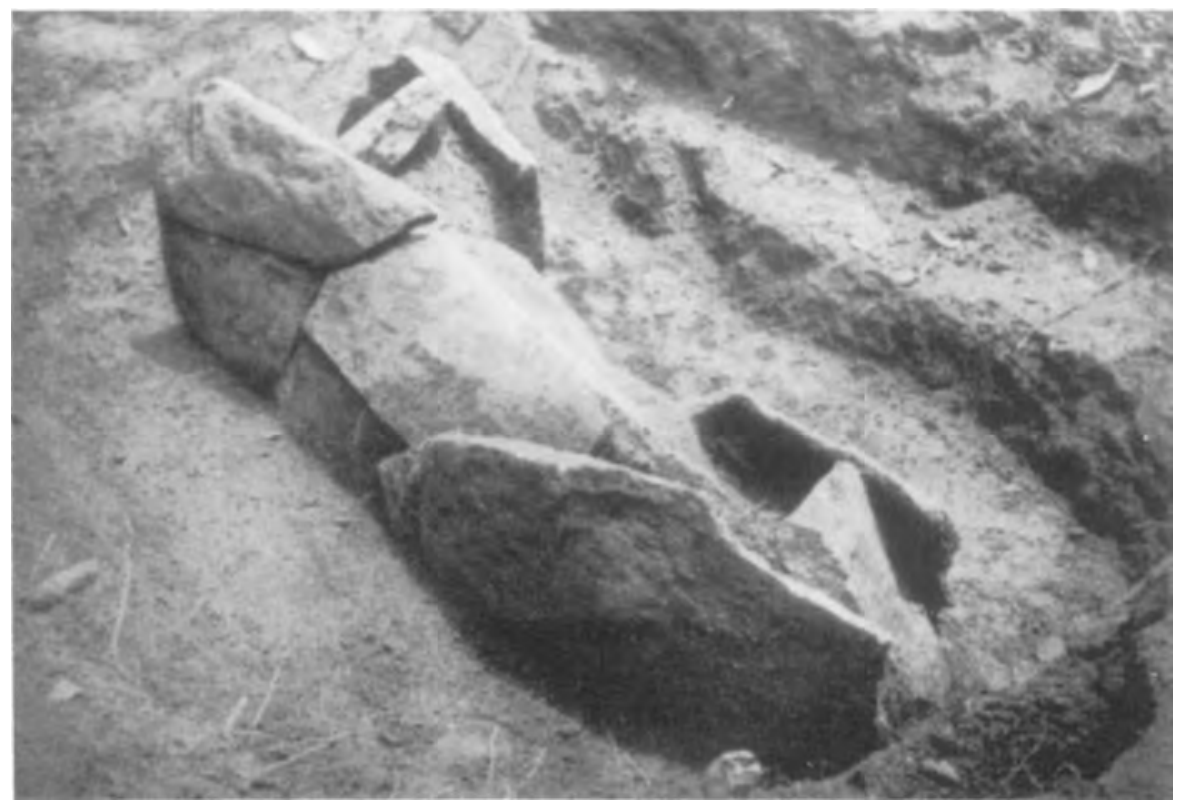

Plate 51. A slab grave 2.3 meters long excavated at Sungkai, southern Perak. From Collings 1937b. Courtesy: National Museum of Singapore. 


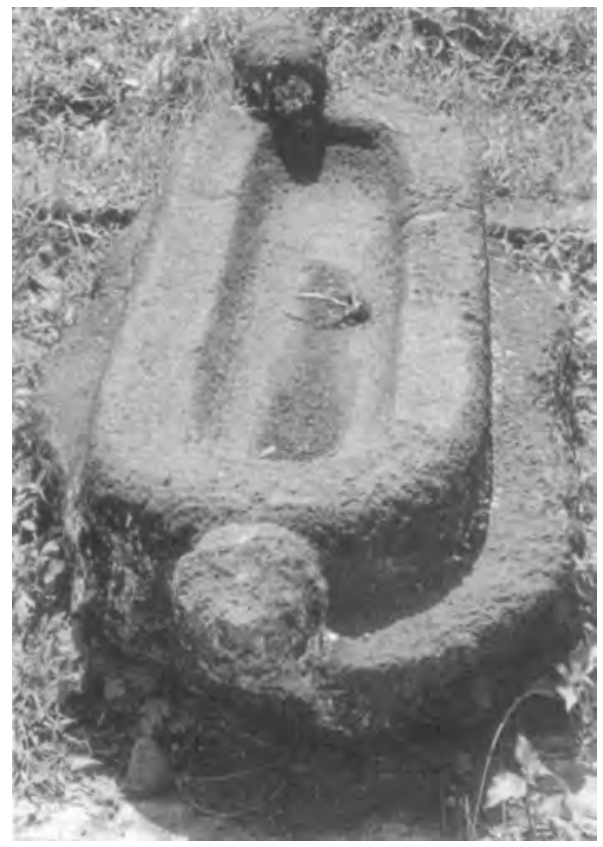

Plate 52. Left: stone trough with heads carved on ends, Pageralam, Pasemah. Below: mortar stone, Gunungmegang, Pasemah.

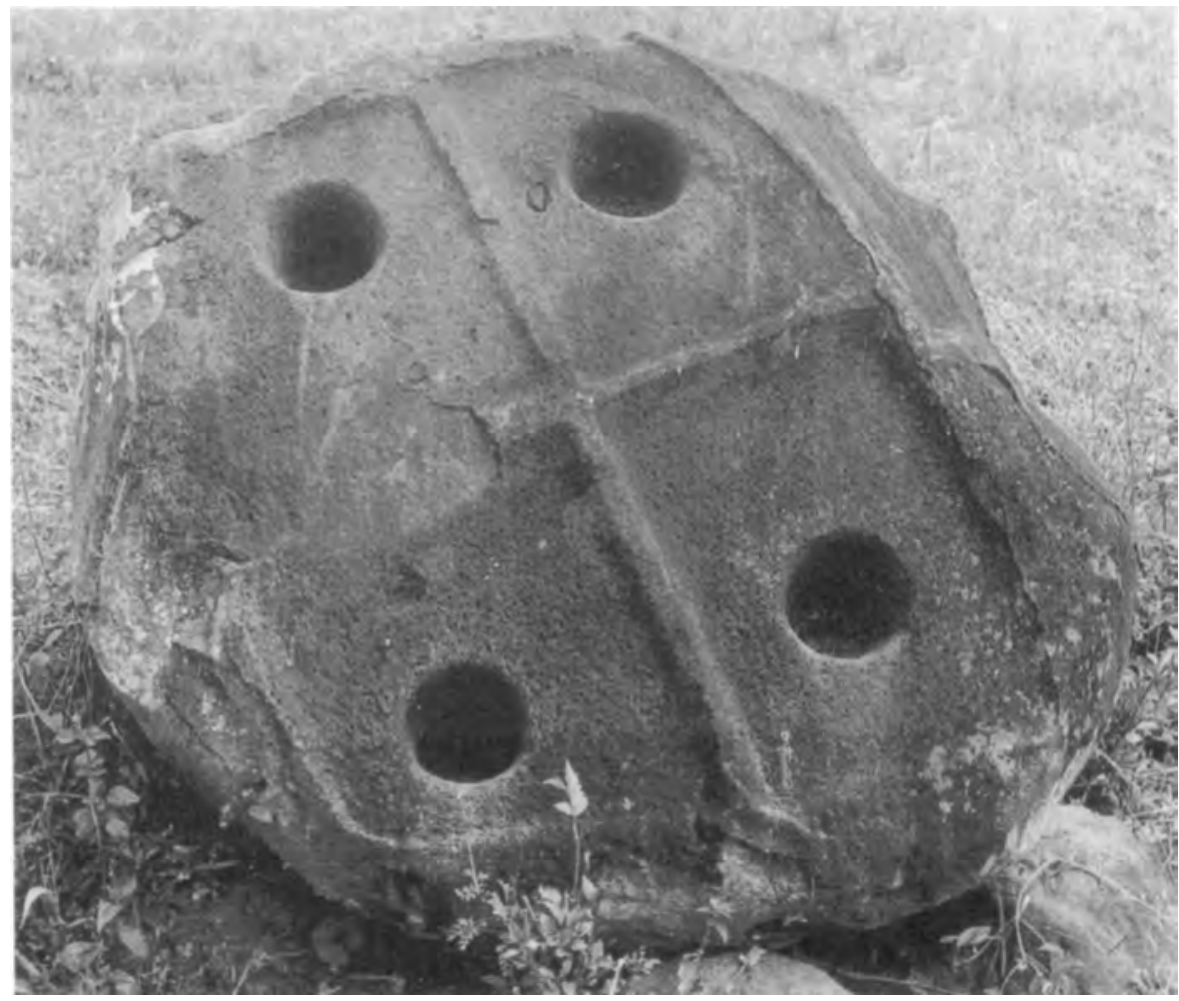




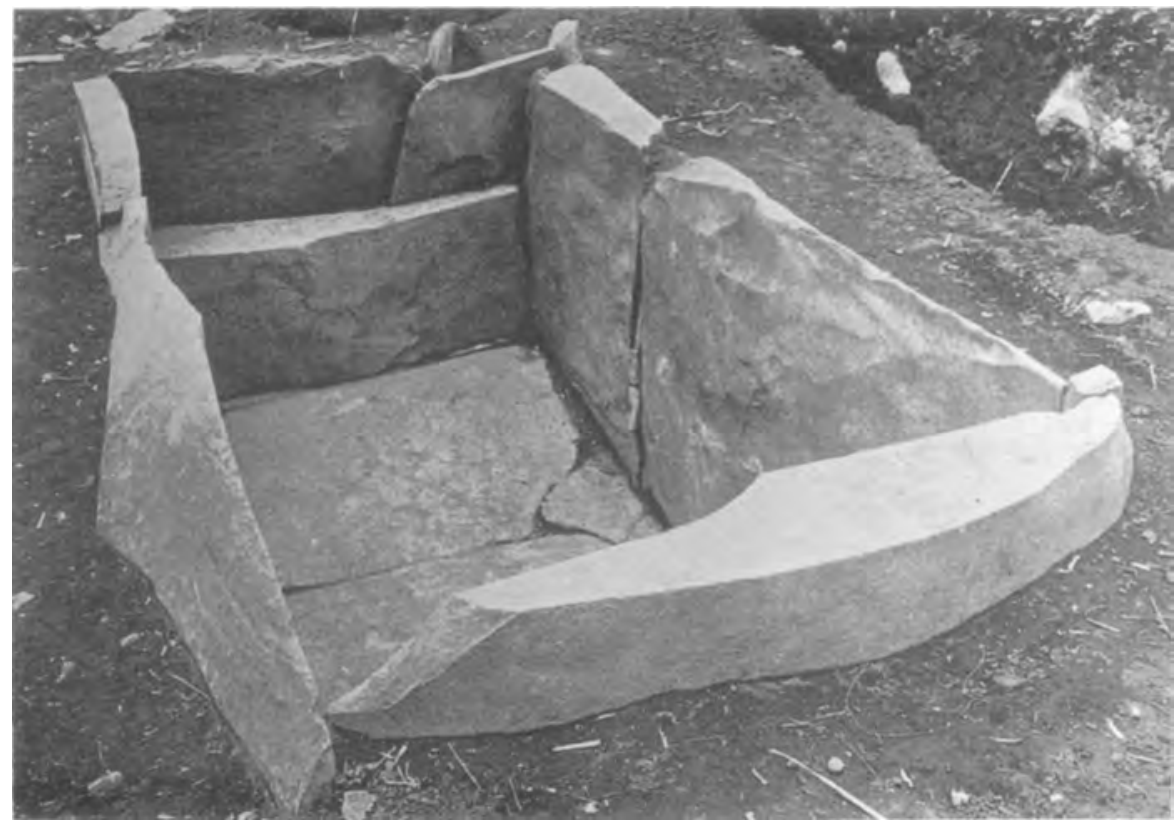

Plate 53. Excavated slab grave at Tegurwangi, Pasemah. From Hoop 1932. Courtesy: W. J. Thieme.

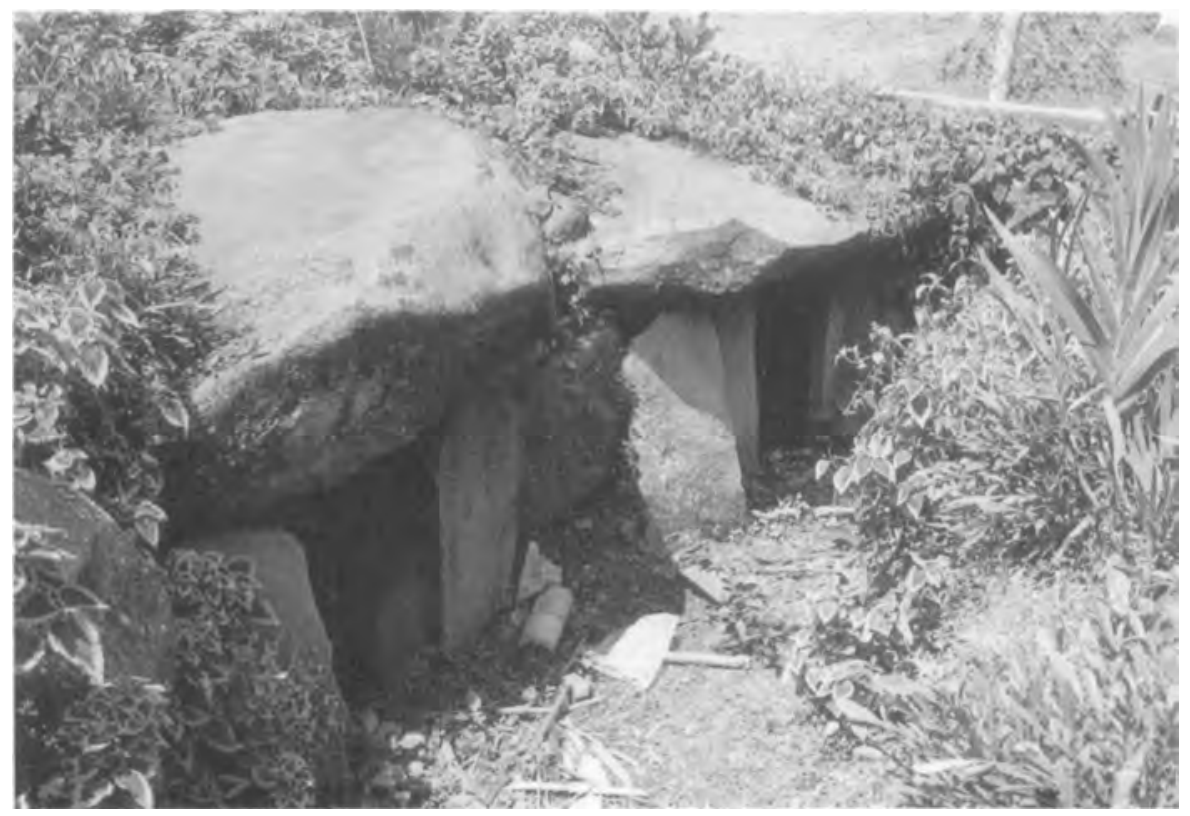

Plate 54. Stone chamber graves with massive capstones at Tanjung Ara, Pasemah. 


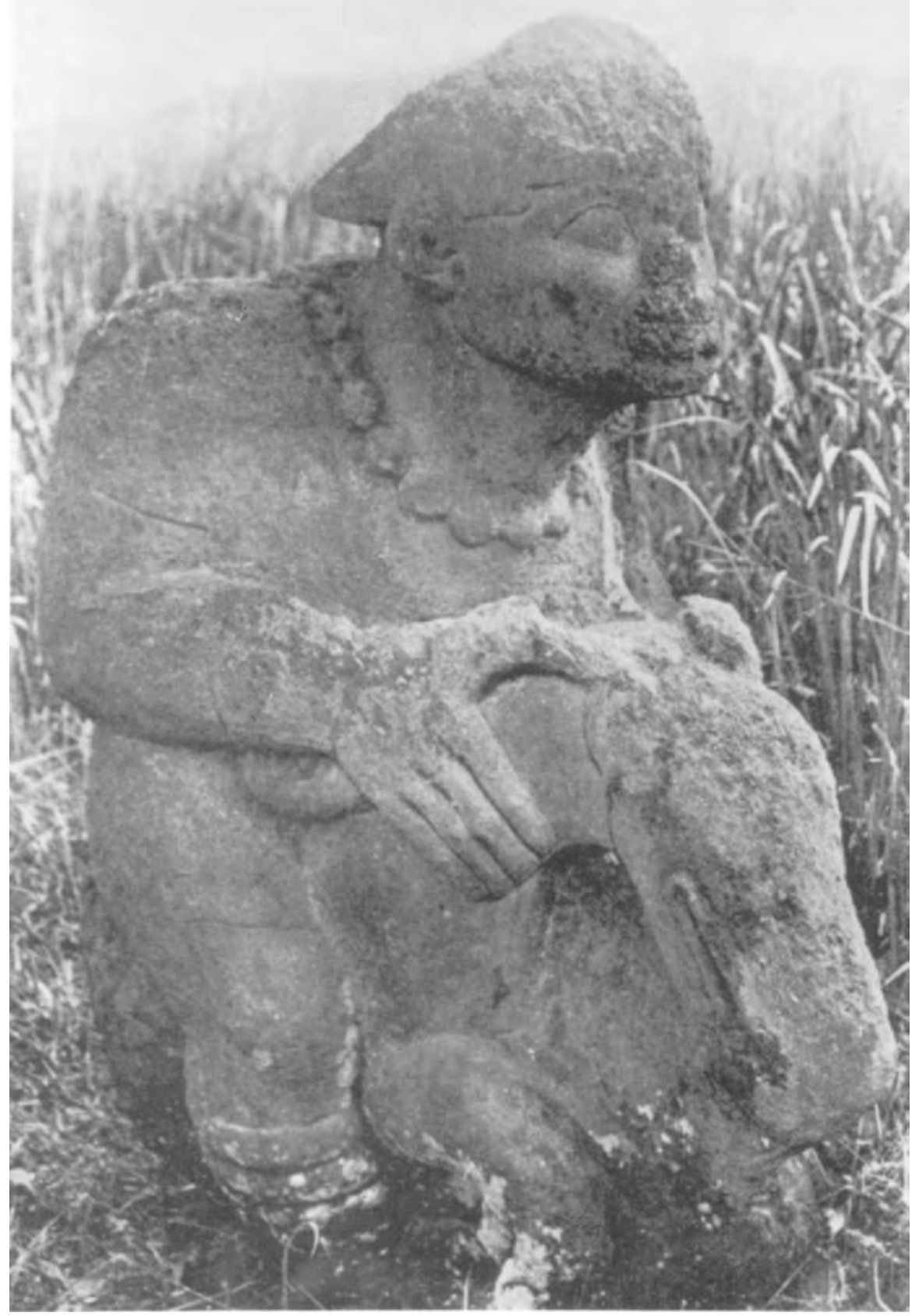

Plate 55. Man astride a buffalo, with necklace, helmet, and anklets, Pematang, Pasemah. From Hoop 1932. Courtesy: W. J. Thieme. 


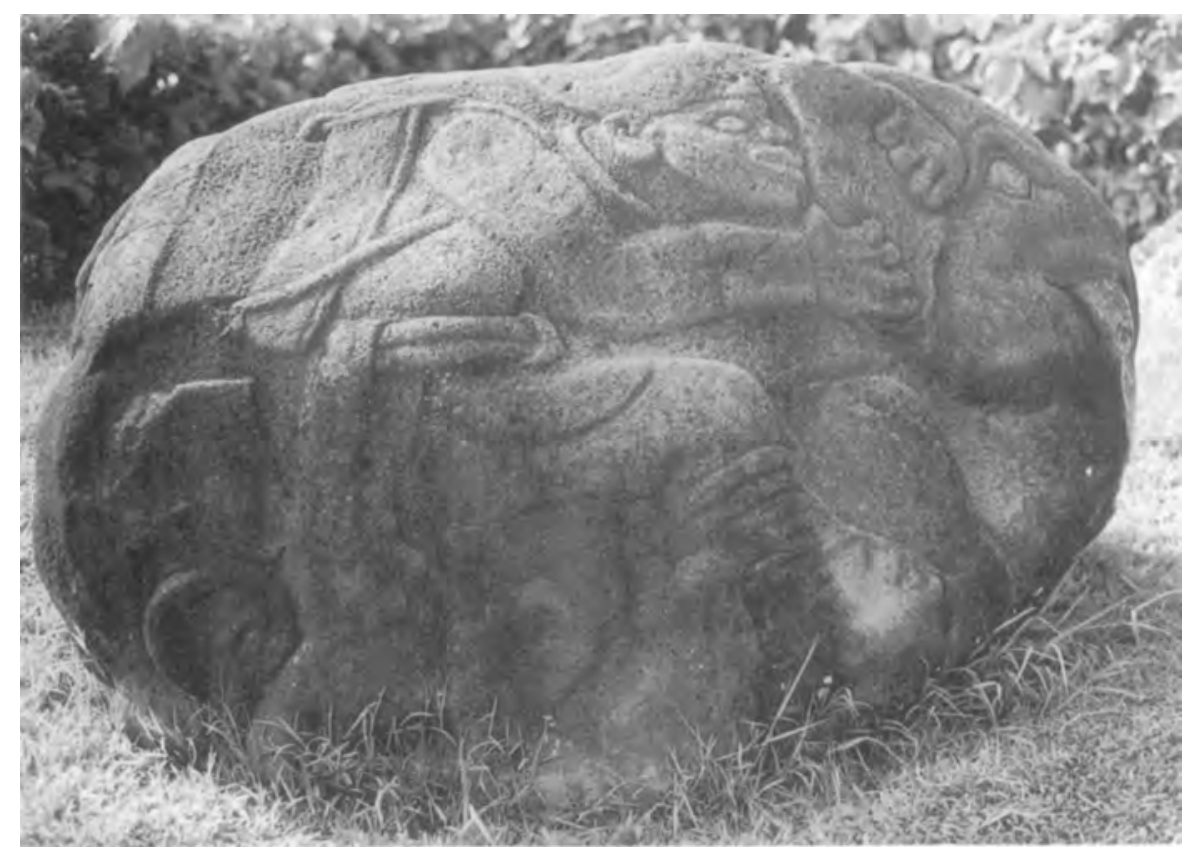

Plate 56. Relief carving of a man flanking an elephant, wearing anklets and carrying a drum of Heger type I. From Batugajah, Pasemah, but now in the museum grounds in Pelembang. For a similar carving at Wonotunggal in north-central Java see Satari 1981. 


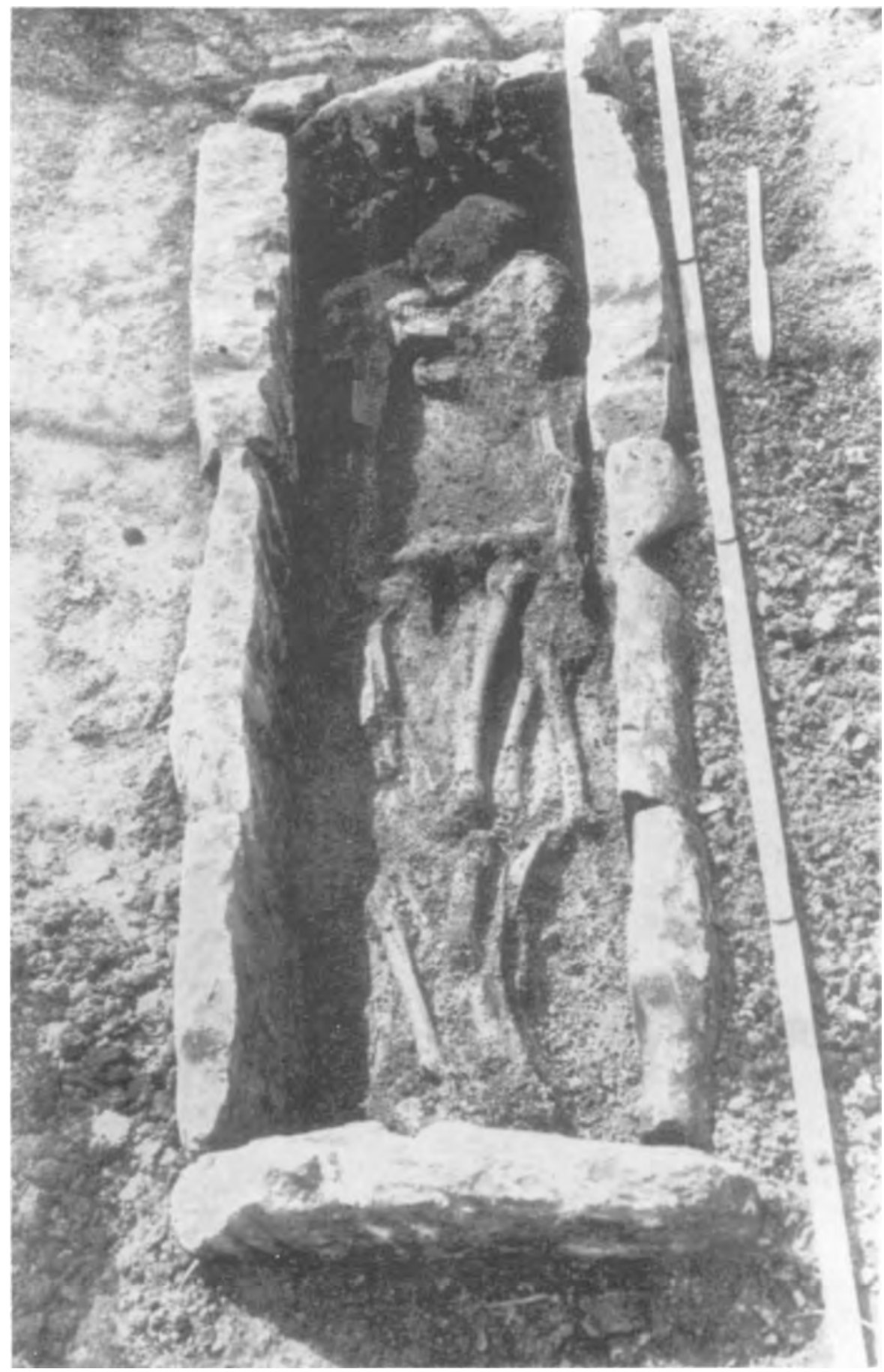

Plate 57. Excavated slab grave with extended burial from Bleberan, central Java. From Hoop 1935. 


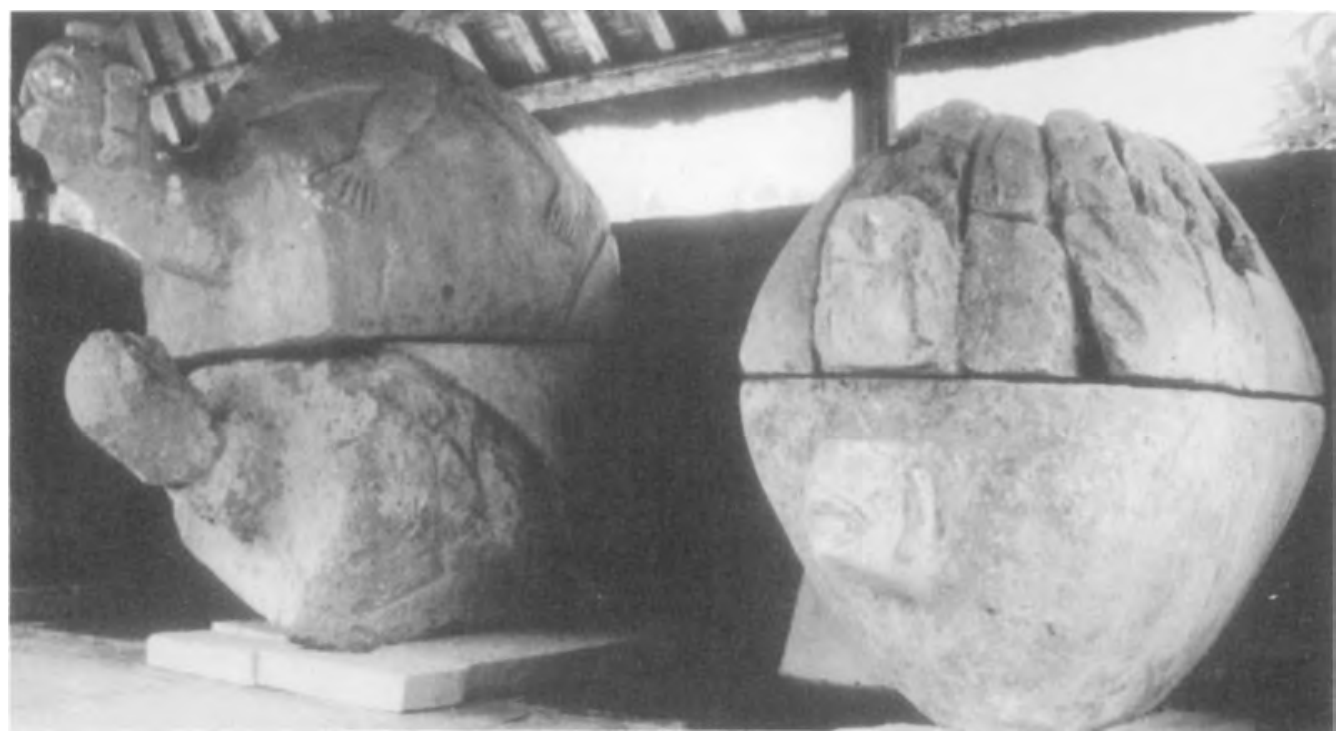

Plate 58. Balinese stone sarcophagi. Pejeng Museum.

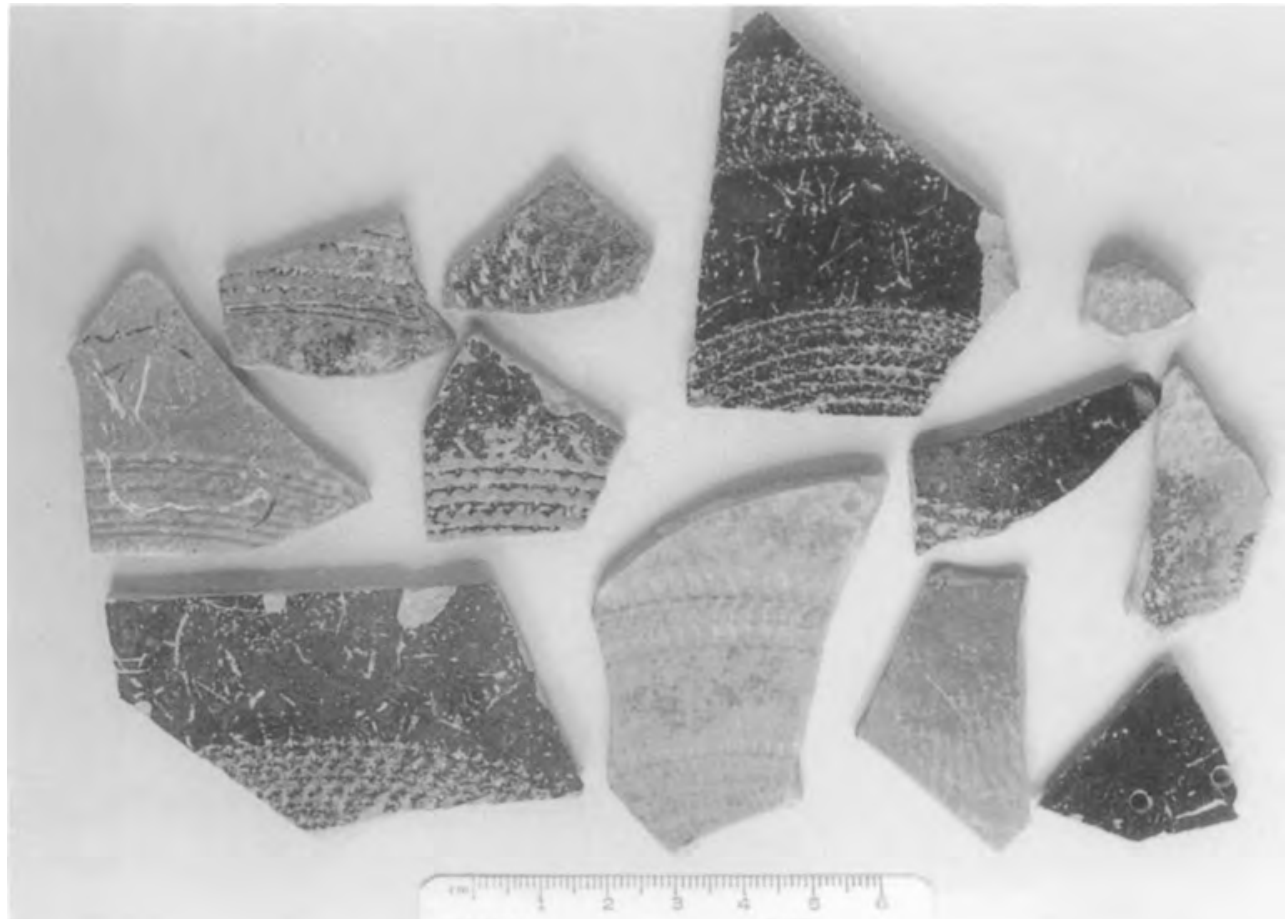

Plate 59. Sherds of Rouletted Ware from eastern India excavated at Sembiran in Bali, ca. $200 \mathrm{BC}$ to $\mathrm{AD} 200$. 

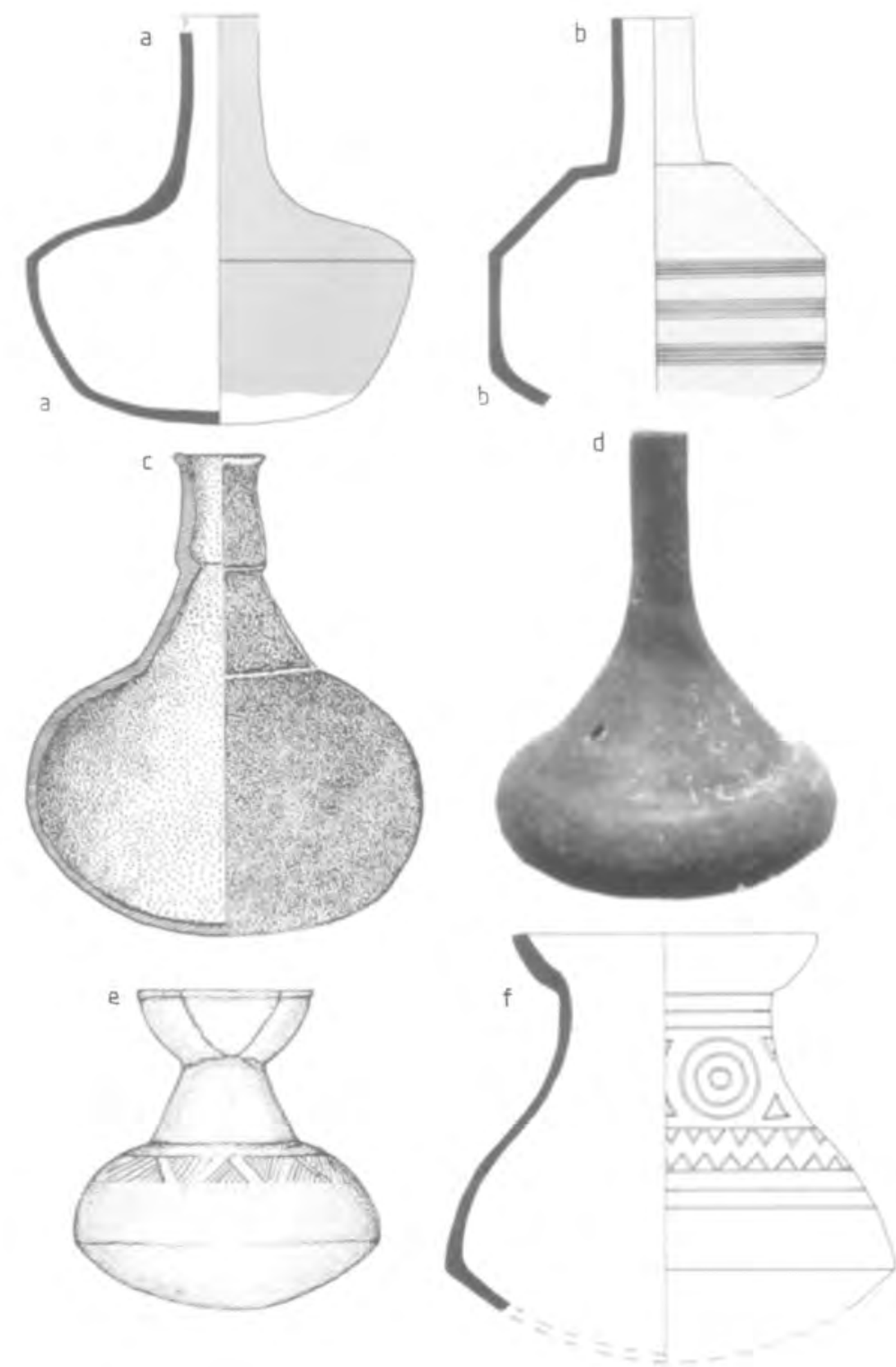

Plate 60. (a-d) High-necked flasks of the early Metal phase: (a) from Leang Buidane, Talaud (red-slipped), 18 centimeters high; (b) from Hagop Bilo, Sabah (red-slipped), 20 centimeters high; (c) from Gunung Piring, Lombok, 25 centimeters high; (d) from Anyar, Java (on footring), 29 centimeters high; (e, f) narrow-necked vessels of similar shape from Gunung Piring, Lombok (left, 15 centimeters high) and Leang Buidane, Talaud (right, 19 centimeters high). (c, e) From Gunadi et al. 1978; (d) from Heekeren 1956a. Courtesy: Indonesian National Research Center for Archaeology (c, e); Anthropos Institut (d). 

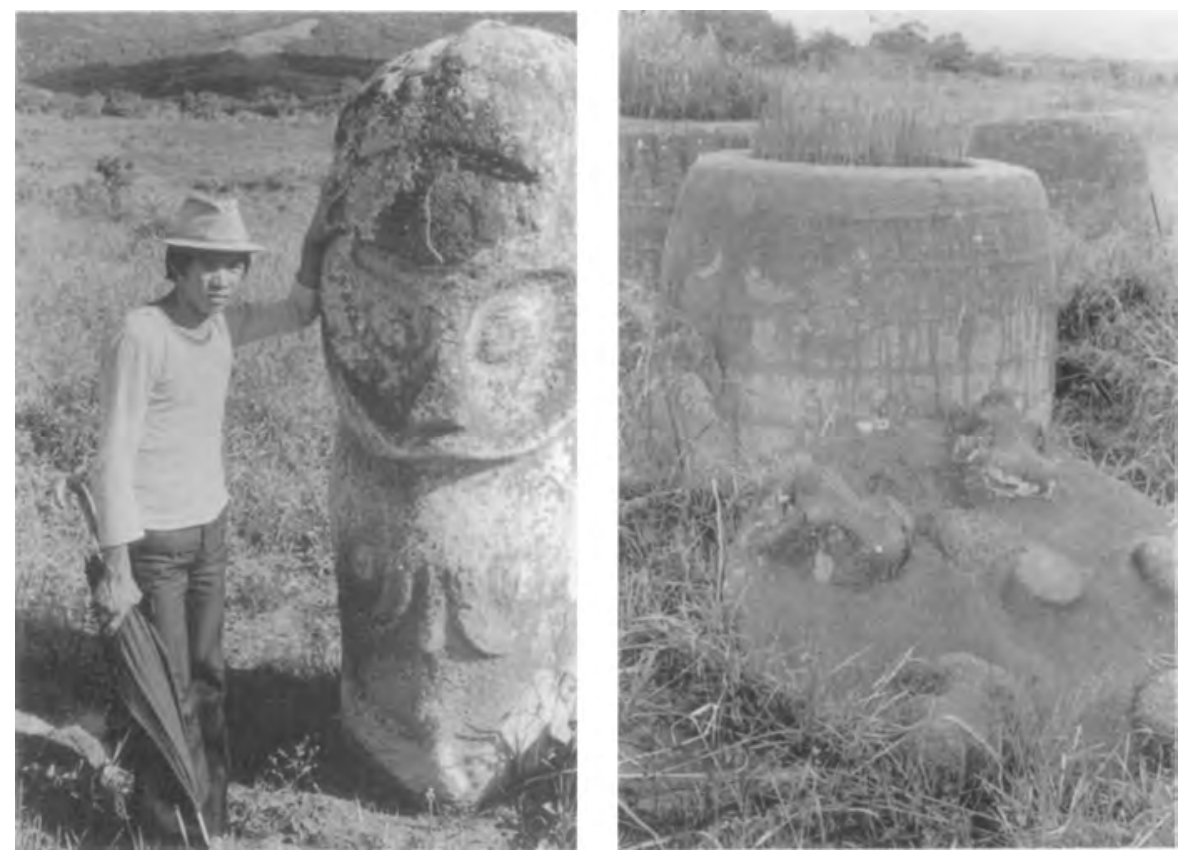

Plate 61. Large stone statue of a male (left) and stone burial jar with lid (the latter with relief quadrupeds) at Besoa, central Sulawesi. Photo: Derek Reid. 
Within the Southeast Asian Hoabinhian in general, the question of overlap with Neolithic assemblages characterized by pottery and fully ground stone adzes has always been a particularly vexed one, partly because the necessary stratigraphic details were simply not recorded in the earlier excavations. In northern Vietnam, as noted above, Hoabinhian tools do sometimes overlap with Neolithic assemblages of potsherds and stone adzes, as in the shell mound of Da But. So in some northern regions it is quite possible that the Hoabinhian did grade slowly into a fairly coherent and presumably agricultural array of Neolithic cultures. In Peninsular Malaysia, however, the situation appears to be different. According to Sieveking $(1954,1987)$, the Hoabinhian at Gua Cha was separated by a gap from the overlying Neolithic occupation, which commenced with a working floor for quadrangular cross-sectioned stone adzes and later continued with a series of burials that I will describe in Chapter 8 (the Neolithic occupation at Gua Cha was mainly associated with burial activity rather than true living-site occupation as in the Hoabinhian).

The 1979 work at Gua Cha tended to support Sieveking, although there can be no doubt that a few Hoabinhian tools do occur in the pottery-bearing upper layer from which the Neolithic burials were cut, despite their absence in the adze working floor. My own inclination here is to regard these tools as having been brought to the Neolithic surface during the course of intensive grave digging and then either reused or simply thrown away. They certainly do not occur amongst the Neolithic burial goods themselves, which do include several fully ground stone adzes. It seems, however, that Sieveking may originally have overemphasized his concept of a gap between the Hoabinhian and the Neolithic; a more likely explanation is that rapid cultural change took place in the region of the site and that this change, according to the skeletal evidence reviewed in Chapter 3, Section IIIB, involved no very major replacement of population. I strongly suspect that the whole of the Gua Cha sequence belongs to ancestral orang asli populations who, as I will document later, were brought fairly rapidly into the Neolithic world of the Malay Peninsula through some degree of Senoi immigration from the north commencing around $2000 \mathrm{BC}$.

Some of the complexity of the overall Peninsular Malaysian situation for the Hoabinhian can be estimated from a brief review of other sites. Another case of nonoverlap between Hoabinhian and Neolithic, similar to that for Gua Cha, is illustrated by Peacock (1971) for the unpublished excavations at Kota Tongkat in Pahang (here shown as Fig. 6.4). However, at the cave of Gua Kechil ("Small Cave"-modern spelling Gua Kecil), also in Pahang, Dunn (1964) found eight Hoabinhian tools together with cord-marked and plain pottery in a lower occupation layer about 40 centimeters thick. This was overlain by a layer with polished adzes and pottery similar to that of the main Neolithic layer at Gua Cha. Hoabinhian tools were absent in this upper layer, but the situation beneath 

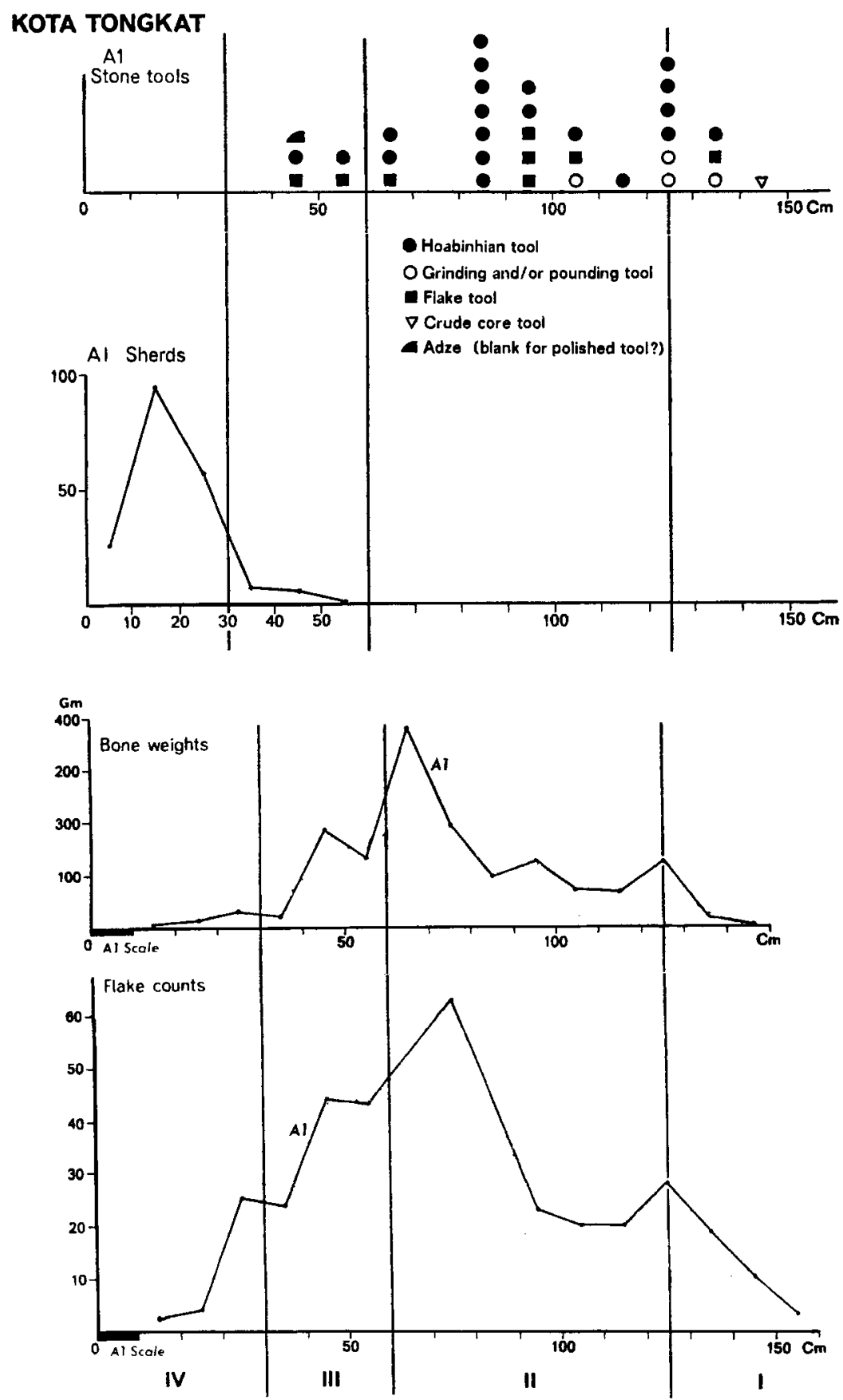

Fig. 6.4 Distributions of Hoabinhian tools, pottery, animal bone, and stone flakes by depth at Kota Tongkat, Pahang. From Peacock 1971. Courtesy: University of Sydney, B. A. V. Peacock. 


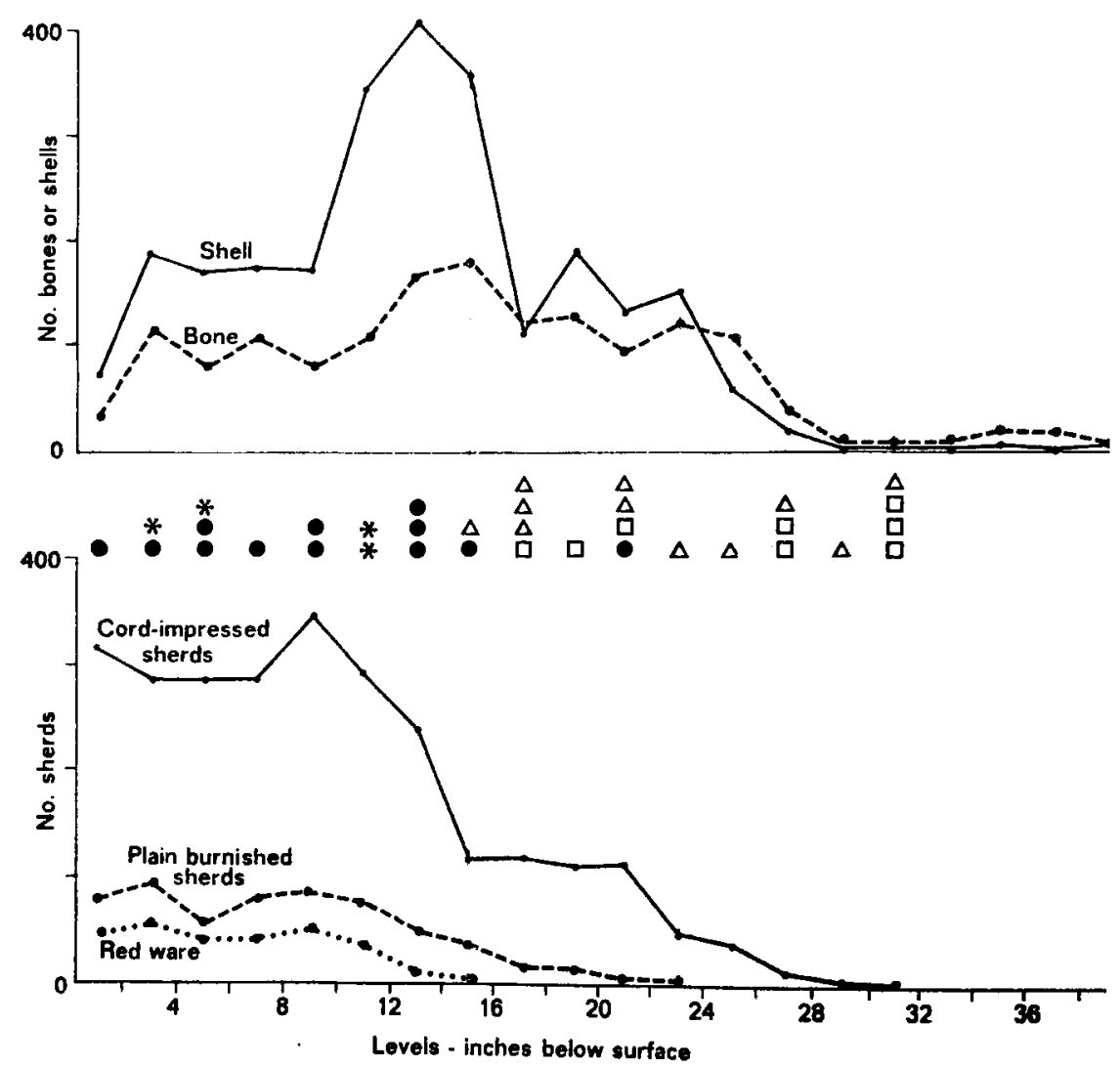

- Polished stone artifact

* Bone artifact

$\Delta$ Utilised flake

D Hoebinhian-like artlfact

After Dunn 1964 : 118, fig 5

Fig. 6.5 Apparent overlap between Hoabinhian tools and pottery at Gua Kechil, Pahang. From Peacock 1971. Courtesy: University of Sydney, B. A. V. Peacock.

could suggest an overlap situation of Hoabinhian tools and pottery (Fig. 6.5) that can certainly not be recognized in the sequence from Gua Cha. Nevertheless, Gua Kechil is a small site and the sample of artifacts is small; I have expressed some reservations about its real significance elsewhere (Bellwood 1993).

It may also be recalled that edge-ground tools are quite characteristic of later stages of the Hoabinhian (i.e., the Bacsonian) in Vietnam. In Malaysia they have been reported from old excavations at Gua Madu in Kelantan (Tweedie 1940) and from Gua Baik (Gol Ba'it, Stein Callenfels and Noone 1940) and Gua Kerbau (Stein Callenfels and Evans 1928) in Perak. At the last two sites they 
were reported as occurring down to the undated bases of the deposits. They are now recorded from the upper Hoabinhian layers in Gua Peraling in Kelantan (see below).

The most recent excavations in Peninsular Malaysia, undertaken since the first edition of this book was published, should add large quantities of data to the above reconstructions. Zuraina's excavations in Gua Gunung Runtuh in Perak (Zuraina 1994) have yielded a crouched burial of a Hoabinhian male laid on his back within a midden deposit dated between 11,000 and 7,500 BP on freshwater shell. The burial was of a person of Australomelanesian affinity with a deformed left arm and hand, aged forty to forty-five years. Zuraina points out that unifacial tools predominate in this site and that some links with the much older Kota Tampan assemblage are evident. Unfortunately, however, Gua Gunung Runtuh has almost no Neolithic deposits, so it does not inform about the post-Hoabinhian transition. Other recent excavations by Zuraina have been undertaken in the caves of Gua Sagu and Gua Tenggek in Pahang, the former with occupation from about 14,000 BP, and in Gua Teluk Kelawar (dated from 8,500 BP) and Gua Harimau, both near Lenggong in Perak (Zuraina 1991).

Two large rock shelters excavated in 1994 by Adi Taha-Gua Peraling on the Perias River and Gua Chawas in the hinterland of the Nenggiri-should also add much to questions about Hoabinhian technology and transitions to the Neolithic. The base of Chawas has currently not been reached with certainty, but Hoabinhian occupation here dates from at least 12,000 (freshwater shell) years ago. Above the Hoabinhian comes a rather sparse Neolithic deposit, present by ca. 3,000 years ago, with indications of virtually no overlap between the two. ${ }^{2}$ Gua Peraling (Plate 18) has about 3 meters of dense Hoabinhian occupation with masses of biface flaking debitage, large numbers of river pebble Hoabinhian bifaces, a few fragmentary burials, and large quantities of food remains (bone, shell) - all stratified beneath some rather disturbed Neolithic burials. Some of the Hoabinhian tools are edge-ground in the upper deposits. Analysis of the materials from these two sites is continuing, but Gua Peraling in particular promises an extremely detailed record of Hoabinhian activity during the early and middle Holocene. The deposits in Gua Chawas are especially rich in phytoliths, amongst which two species of banana, four of rattan, and three of bamboo have already been tentatively identified in layers dating between 12,000 and 5,000 BP (Doreen Bowdery; research in progress).

The Peninsular Malaysian shell middens now tell rather a sorry tale of destruction, although Adi (1983:53) has reported a recent discovery at Seberang Perak, near Teluk Anson in Perak, for which a date of about 6,000 BP is available from the middle of the deposit (Adi Taha, pers. comm.). It has also been known since $\mathbf{1 8 6 0}$ that large middens of marine/estuarine bivalves once occurred on old beach ridges in the mainland portion of the state of Pulau Pinang (formerly 
Province Wellesley). These have now been destroyed apart from small remnants, but the remains of three were excavated long ago by Stein Callenfels at a location about three miles inland called Guar Kepah (Stein Callenfels 1936a; the sites were then called Guak Kepah).

According to Stein Callenfels, these middens-originally up to 5 meters thick -contained hearths, secondary burials dusted with red ochre (one jaw was clas. sified as "Palae-Melanesian" by Mijsberg 1940; see Chapter 3, Section IIIB), pig and estuarine fish bones, Hoabinhian tools, necked and apparently hammerdressed axes, and small quantities of cord-marked and incised pottery (Plate 19). No stratigraphic order for these items was clearly established, but Tweedie (1953:69) thought that the pottery may have postdated the Hoabinhian tools. It appears that the Hoabinhian tools and the necked axes (often described as "ground," but I suspect from illustrations that they were simply hammerdressed) did belong together with the midden deposits, which presumably date from the present phase of sea level and thus somewhere within the Holocene. These sites clearly pose a number of unresolved problems; they appear to represent a coastal Hoabinhian adaptation and thus an aspect of Hoabinhian life that cannot be found in the inland shelters, but that may now be virtually lost as a result of the terminal Pleistocene rise in sea level and the activities of lime burners.

\section{Sumatra}

The only Hoabinhian sites found within the modern political boundaries of Indonesia lie inland from the northeastern coast of Sumatra for a distance of about 130 kilometers between Lhokseumawe and Medan (Witkamp 1920; Kupper 1930; Heekeren 1972:85-92; Brandt 1976; Glover 1978b; McKinnon 1991) (also see Fig. 3.2). Many of the sites are large shell middens up to 100 meters in diameter and 10 meters deep (Plate 20), with interstratified lenses of shells, soil, and ash. Most appear to be located at approximately present sea level on an early Holocene strandline that now lies between 10 and 15 kilometers inland, although some have their bases well below present sea level (e.g., see Sukajadi Pasar IX; McKinnon 1991:135). Most have been buried under sediments deposited along this rapidly aggrading coast during the past few millennia. None have been systematically excavated or dated, although a radiocarbon date of ca.7,500 BP has been reported from a point two-thirds of the way down the profile in the midden of Sukajadi Pasar III (McKinnon 1991:138). Unfortunately, most of the Sumatran middens have now been quarried for their shells-used in making cement-leaving behind huge holes in the ground that fill with water. Like the Pulau Pinang middens of Peninsular Malaysia, the Sumatran middens must be of Holocene date and may have been occupied at any time between 
10,000 and perhaps as recently as 3,000 years ago. The region has no caves or shelters, but other nonmidden Hoabinhian sites have been reported from inland terraces and flat limestone rises to about $\mathbf{1 5 0}$ meters above sea level.

Many archaeological collections have been made from the Sumatran middens over the years; these are described by Heekeren (1972). The majority of the tools appear to be unifacially flaked oval or elongated pebbles, often flaked all over one surface. Bifacial tools and edge-ground tools appear to be rather rare, as are retouched flakes. This industry thus gives the impression of being technologically simpler than that of the Peninsular Malaysian sites. Grindstones, mortars, red ochre, and human burials also occur in the middens, and faunal remains include elephant, rhinoceros, bear, deer, and presumably many smaller species. The shellfish illustrated by Heekeren (1972:Plate 36) appear to belong to the same estuarine species that once formed the Pulau Pinang middens. Pottery appears to be universally absent, at least in confirmed association with the Hoabinhian deposits.

\section{E. Further Comments on the Hoabinhian}

As reported from Peninsular Malaysia and Sumatra, the Hoabinhian seems to have had a coastal and inland hunting and gathering mode of economic orientation. I feel it is pushing the evidence too far to suggest a local development of agriculture in these regions. As I have stated, the Hoabinhian of the intermediate tropical zone in northern Vietnam and southern China may hold more significant evidence in this regard, although a lot more material needs to be excavated if this possibility is to be substantiated. The edge-grinding of stone tools is clearly an innovation from within the Hoabinhian cultural matrix, but the situation is not so clear for pottery, partly because shelter deposits are so prone to those types of hidden stratigraphic disturbance that will perhaps always cloud the issue.

Hoabinhian sites sensu stricto do not occur in the Indo-Malaysian islands outside northeastern Sumatra. I suspect they are present in Taiwan, perhaps in the so-called Ch'angpinian of the eastern coast (Sung 1979) and also perhaps in some aceramic assemblages of "chipped hoes" reported by Koyama (1977) from the western coast. In the Philippines, assemblages termed Hoabinhian have been reported by Kress (1977a, b) for Palawan and by Peterson (1974) for the Pintu shelter in northern Luzon, but the illustrations provided by Peterson (1974:Plate 1) do not convince me that the tools from this site are really any different from the contemporary pebble and flake industries characteristic of many of the other Indo-Malaysian islands. Naturally, signs of a gradation from the classic mainland Hoabinhian into the quite different stone tool expressions found in the islands might be expected, and it is possible that the Philippines 
and Sumatra are in such gradation areas. But my own experience from handling Peninsular Malaysian and Sumatran Hoabinhian stone tools is that they represent a dominance of pebble tools as opposed to flake tools, which sets them well apart from contemporary industries in all the island regions, including the Philippines.

\section{ISLAND SOUtheast asia: the later PEBBle and flake INDUSTRIES, WITH VARIATIONS}

In the Philippines, East Malaysia, and Indonesia the record of flaked stone tool production goes back to between 30,000 and 40,000 radiocarbon years ago, as it does in the Melanesian and Australian regions beyond. As noted in Chapter 3, the record appears to document a radiation of anatomically modern humans from Sundaland across the seas of eastern Indonesia into Australasia and western Oceania. The problem, however, is that radiocarbon dates of this antiquity could in fact be much older because small amounts of young radiocarbon can contaminate them in the direction of younger ages. This problem is currently a "hot topic" of debate in Australia, where archaeologists would like to know if the first colonists arrived 30,000 or more than 50,000 years ago (e.g., Roberts et al. 1994; Allen and Holdaway 1995). Currently, luminescence dating of sediments is being used to support the older ages back to 50,000 years or more in Australia, but this technique has never been applied in Southeast Asia. Common sense dictates that people were obviously somewhere (but not necessarily everywhere) in eastern Indonesia before Australia was first colonized, but "when" is a big question under current scientific analysis. In this chapter I can only give the radiocarbon dates as published. These go back for 40,000 years, but there is no guarantee that this is the true chronological basement.

The stone industries of Island Southeast Asia, beyond the Hoabinhian orbit, belong to a widespread pebble and flake technocomplex that was also-on present evidence-carried by at least one of the first populations to settle in Australia and New Guinea. The technocomplex includes the majority of the Indo-Malaysian flaked stone assemblages of the late Pleistocene and Holocene. In its most basic form it is characterized by varying proportions of simple pebble tools, cores, and flakes with nonstandardized shapes. As I have noted, the Hoabinhian tendency toward a dominance of pebble tools with regular bifacial or all-over unifacial working is not present. While this circumstance may reflect important cultural differences between mainland and islands, I suspect there may also be some geological reasons behind it. For instance, the blocky cherts and obsidians used more commonly in the island regions may have been more amenable to a flake tool technology than the rounded pebbles that occur in Mainland Southeast Asian rivers, although this is only a subjective opinion 
drawn from my own rather limited geological observations. In Indonesian islands where cherts and obsidians are not present but where beach or river pebbles are (such as the northern Moluccas-see below), the results, perhaps predictably, are often somewhat Hoabinhian-like.

The best way to visualize the prehistoric record of flaked stone tools within the last 40,000 years in the Indo-Malaysian islands is in terms of periodic and normally highly localized accretions to the basic pebble and flake technocomplex, which in its basic form underwent little change over this period. Thus, sporadic and short-lived occurrences of prepared-core, bifacial lanceolate, edgegrinding and blade technologies occur, each in a restricted region and over a different period of time. The final stage is of course the widespread appearance of fully ground adzes and axes together with pottery after $3000 \mathrm{BC}$. I will return to this in Chapter 7. However, it should be noted that the older flaked stone technologies often continued with no obvious changes until they finally faded in the face of metal tools from the late first millennium BC onward. The flaked stone traditions do not in themselves record the spread of an agricultural lifestyle in the region or even necessarily the arrival of new populations.

In organizing this chapter I have decided to proceed along geographical lines, discussing the best-known sequences from Borneo through eastern Indonesia. The industries with a blade or "microlithic" component, all dating within the last 7,000 years, are described separately in the final section.

\section{A. The West Mouth, Niah, Sarawak}

The huge West Mouth of the Niah Caves in Sarawak (Plate 21) contains the longest stratified record of human occupation in Island Southeast Asia. The caves themselves form a network of high and awe-inspiring passages, with an area of about 10.5 hectares, inside the Gunung Subis limestone massif near Niah in northern Sarawak. The system has many outlets, of which the West Mouth is the largest, being about 250 meters across and 60 meters high. Most of the system is floored with continuously deposited wet guano, but an area high and dry at the northern end of the West Mouth was used for habitation and burial from about 40,000 until perhaps 2,000 years ago or later. This area was excavated on a fairly massive scale by the late Tom Harrisson between 1954 and 1967.

Harrisson produced many impressionistic articles and a few detailed typological studies based on his research at Niah, but no proper plans or stratigraphic drawings were ever made. Zuraina (1982) later attempted to piece together some of the information that could be gleaned from the earlier records. In addition, other publications since 1967 by Barbara Harrisson, Lord Medway (Earl of Cranbrook), and by biological anthropologists have filled in many lacunae in the records of animal faunas and human burials. 
Harrisson's reconstructions of the cultural sequence at Niah were based partly on the idea that depths and ages could be correlated regularly across the site. However, the site has an uneven surface, and arbitrary levels of excavation up to 24 inches thick-plus a set of partially contradictory radiocarbon dates recorded only by depths below surface-clearly do not encourage much confidence in the finer details of the "Niah area phaseology" that he published and revised from time to time. His last version appeared in a 1970 paper, in which he favored a basal flake industry with pebble tools appearing intermittently and becoming edge-ground after about 10,000 years ago. I will consider the Neolithic tools and the pottery (the latter comes in at about $2000 \mathrm{BC}$ ) in Chapter 7, Section IIID, but wish now to examine the preceramic West Mouth sequence in terms of artifacts, faunas, and human burials.

\section{Artifacts at Niah}

The Niah industry is mainly of fairly coarse-grained rocks and comprises an unretouched array of chunks and chips, without coherent core forms and with few conchoidal flakes. There is little systematic retouch. Pebble tools also occur, but apparently not in the oldest levels. Bone spatulae and points do apparently occur to the base of the site, some made on pig tusks or mammal long bones (Harrisson and Medway 1962; Zuraina 1982:Appendix 3). Stone mortars and edge-ground pebble axes appear later in the sequence; dates unfortunately are not clear, but Zuraina suggests that both could have appeared somewhere between 20,000 and 10,000 years ago, although Harrisson preferred the latter date for the edge-ground axes.

The edge-grinding of pebble axes is of course a significant technological development (Hayden 1977). It occurs from about 30,000 years ago in Japan (Oda and Keally 1992), within the Hoabinhian in Vietnam and Malaysia, and from dates in excess of 20,000 years ago in northern Australia (Schrire 1982) and 14,000 in the New Guinea Highlands (Mountain 1983:94-95). Hence, if Niah is included, there are at least four apparently separate late Pleistocene occurrences, to which may be added possibly early Holocene appearances on Palawan in the Philippines (Kress 1977a; see also Peterson et al. 1979 for Luzon) and on Manus Island in northern Melanesia (Fredericksen et al. 1993). On Manus, and in Golo Cave on Gebe Island in the northern Moluccas, edge-grinding was also used on shell adzes after about 12,000 years ago (see Section F). The technique clearly precedes the development of blade technologies, even in Japan where blades appeared by perhaps 26,000 years ago, and it occurs in similar pebble- and flake-based industrial backgrounds in each region. At Niah it was probably adopted because of the difficulty of finding good stone for flaking, and it is totally absent in many contemporary or later Indo-Malaysian industries where good cherts were available (for instance, in neighboring Sabah).

Hence there are two rather contradictory aspects of the distribution of edge- 
grinding: on the one hand a widespread distribution around the eastern fringes of the Old World, but on the other hand a spotty occurrence within this territory (within Australia, for instance, it remained strangely restricted to the region of Arnhem Land until about 5,000 years ago). It thus seems that equal cases can be made for multiple independent development of the technique or for its diffusion from one source. The real answer may combine both processes.

\section{The Niah Economy}

The animal bones from the West Mouth indicate fairly eclectic hunting patterns. Medway (1977a) lists fifty-eight species of mammals found in the cave. Apart from the bats, which may have fallen naturally into the deposits, there are numerous species of rodents and insectivores, seven species of primates, eleven carnivores (excluding the Neolithic dog and a possibly imported tiger tooth from the top of the site), and ten other large native mammals (see also Harrison 1996). Wild pigs (Sus barbatus; Medway 1978) were the most popular prey throughout, together with porcupines, monkeys (four species), and orangutan; gibbons were virtually absent (Harrison 1996). Other large mammals from the oldest levels include giant pangolin (now extinct), Malay pangolin, Malayan tapir, deer, and bovids (Hooijer 1963; Medway 1977a). The Sumatran rhinoceros (Medway 1965) and the Malayan bear also make rare appearances at higher levels. In addition to these mammals, fish, birds, monitor lizards, snakes, and crocodiles were also brought into the cave.

In Chapter 1, Section IVE, I discussed some of the faunal evidence suggesting that the Niah region may have had a drier and more seasonal climate during the period of the last glacial maximum. Some of the larger mammals such as rhinoceros and bear seem to have been more common at this time, and during the early Holocene a number of species (such as orangutan, rhinoceros, monkeys, and rats; Medway 1978) commenced a slight decline in size. The Malayan tapir also declined into local extinction. Medway (1977a) attributes these Holocene changes to the spread of dense forest and its nonclearance by human agency, an explanation also favored by Harrison (1995). Estuarine shellfish also increased in numbers in both the West Mouth and neighboring Lobang Angus as a result of the rise in sea level and the encroachment of the coastline.

\section{Human Burials}

The biological affinities of the Niah human remains have been discussed in Chapter 3, Section IIIB, and I will only add cultural details here. The single "deep skull" (see Plate 10), associated by Tom Harrisson (1975b:161) with a radiocarbon age of about 40,000 years, was found together with some long bones under a large stone. Barbara Harrisson (1967:143) has stated that the dated charcoal was taken from directly above the skulI. Nevertheless, the fact 
that it lies some 125 centimeters below all other human remains in the site suggests burial from a higher level. The problem cannot really be solved because all deposits surrounding the skull have now been excavated away, although there is no reason to doubt a date at some time in the late Pleistocene.

The other burials from the preceramic levels comprised flexed, sitting, and disturbed fragmentary remains, similar in configuration to those from Hoabinhian sites. A number of radiocarbon dates on bone collagen have been published (Harrisson 1975b; Brooks et al. 1977), and these suggest that the burials in a sitting ("Buddhalike") posture date between 12,000 and 6,000 $\mathrm{BC}$, while the flexed ones (Plate 22) run from perhaps 9,000 BC onward. Several of the flexed burials occurred in later levels with pottery, so this mode is not of course a guarantee of an early date. Red ochre powder and traces of burning occurred on several burials (Harrisson 1967). Grave goods included an edge-ground pebble (unfortunately not with a dated burial), a rhinoceros femur pillow, a bone point, and an estuarine shell with red ochre. It should be noted that the only burial dated twice, number 147, has one published collagen determination of ca. 7,000 BP (Brooks et al. (1977) and another contradictory determination on bone from the same burial of ca. 13,600 BP (Harrisson 1975b).

The task of summarizing 35,000 years of preagricultural life around the Niah Caves is a difficult one. The flaked stone tool tradition reflects a poverty of raw material, and there are insufficient data to study trends in economy within these thirty-five millennia in any very useful way. The sequence clearly lacks the specialized flaking technologies that appeared in other nearby regions, yet the early adoption of edge-grinding suggests for Niah a fairly innovative prehistory somewhat unique to itself. It is also worth noting that Niah was probably almost 200 kilometers inland during the last glacial maximum, and that the cave of Gua Sireh in Sarawak, which has yielded a few riverine shellfish dated to ca. 20,000 years ago, was perhaps 500 kilometers inland (Ipoi and Bellwood 1991). This is important evidence in the light of the interior rain forest debate discussed in Chapter 5, Section I.

\section{B. Sites in Eastern Sabah (Northern Borneo)}

Between 1980 and 1987, an excavation project carried out in eastern Sabah under the aegis of the Sabah Museum in Kota Kinabalu documented a number of cave and open sites with deposits extending back for perhaps 30,000 years (Bellwood 1984, 1988). The situation of these sites is shown in Fig. 6.6; the caves and shelters are found in the Madai and Baturong limestone massifs, both of which contain networks of solution tunnels, some of which emerge into the open air as dry habitable locations (as in the Niah Caves). Baturong is in turn surrounded by a large area of water-laid deposits that are presumed to have 


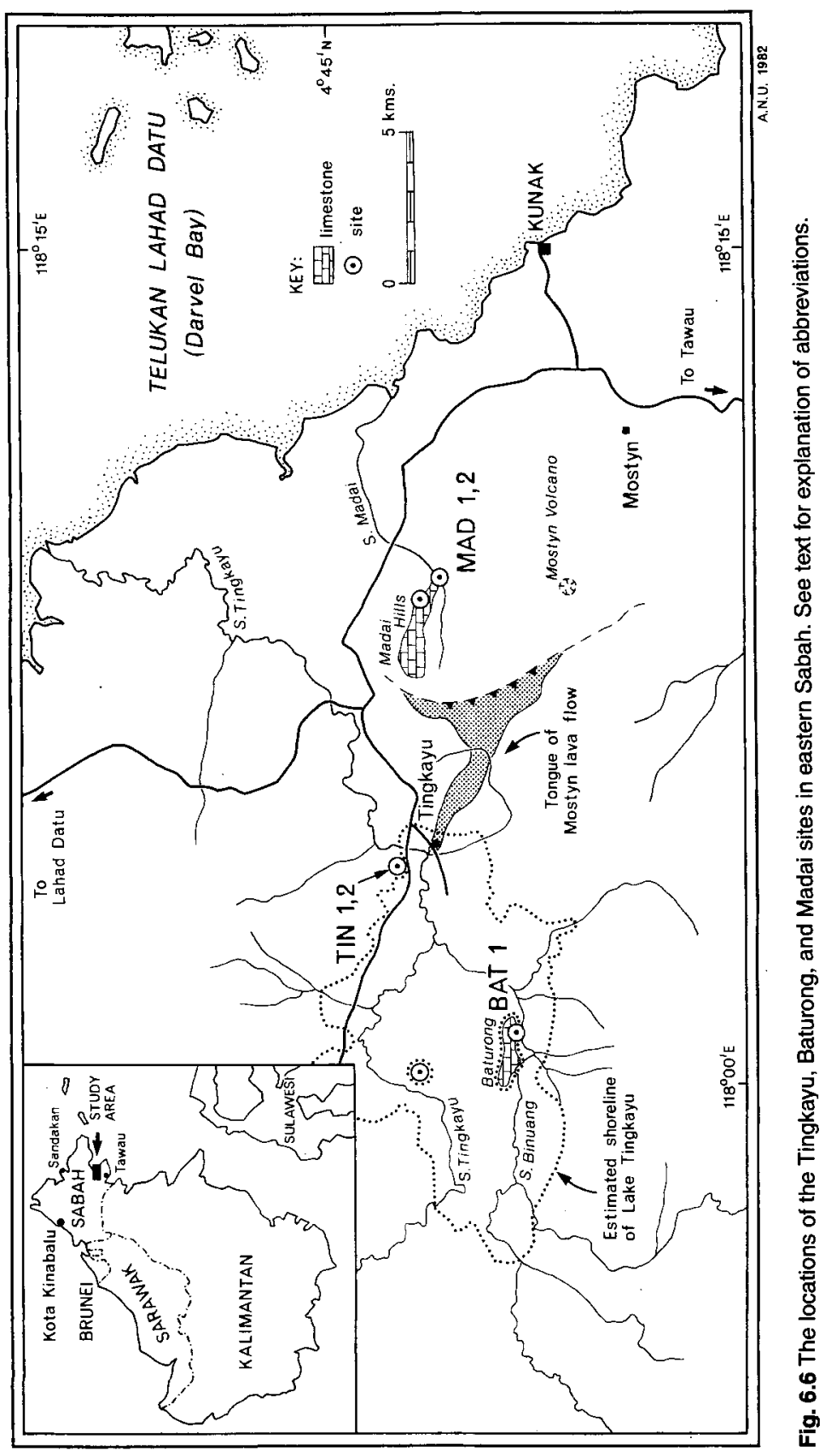


been laid down in the bed of an extinct lake formed by the damming of an old course of the Tingkayu River by a lava flow extruded from the flanks of nearby Mount Mostyn (Plate 23). Although these sites are near the coast now, the low sea level conditions of the late Pleistocene may have placed them up to 150 kilometers inland.

In Fig. 6.6 I have shown the approximate boundaries of the old Tingkayu lake, as identified by previous soil and geological surveys and by fieldwork undertaken in 1981. The lake covered perhaps 100 square kilometers before it drained away as the outlet of the Tingkayu River eroded a gorge just north of Tingkayu village. The date of formation of the lake is probably indicated by a radiocarbon determination of 28,000 years ago from charcoal sealed beneath the end of the lava flow, which outcrops into the side of the exit gorge. By 18,000 years ago the lake appears to have been partly or wholly drained away, as shown by the occurrence of sediments of backwater alluvial origin deposited beneath archaeological layers of this age in the Hagop Bilo shelter (Magee 1988). These dates are highly significant because a number of open sites lie directly on the shoreline of the old lake; on locational grounds they may be considered as contemporary with the lake, and thus between 28,000 and 18,000 years old.

The major lake-edge sites, labelled TIN 1 and 2 in Fig. 6.6, lie close together on a small promontory that juts into the old bed close to the lake outlet. TIN 1 has been mostly destroyed by the bulldozing that led to initial discovery of the site, but TIN 2-excavated in 1980, 1984, and 1987-contained a discrete manufacturing floor for the bifacial tools that characterize the Tingkayu industry. Unfortunately, the acid forest soil in which these sites lie has left no traces of bone or charcoal and there are no direct dates for the tools themselves.

If the Tingkayu stone industry (Fig. 6.7) really does belong to the Lake Tingkayu stage, it shows a unique level of skill for its time period in Southeast Asia. The tools are mostly made on a locally quarried tabular chert; the precise source is not known and may no longer exist, or it may be buried somewhere in the vicinity of the site. Many of the tabular blanks were worked into large bifaces and into smaller and quite remarkable lanceolate knives, the latter apparently representing the main goal of the manufacturing process. Only one lanceolate was found complete (in TIN 1: see Fig. 6.7, middle right); it has very fine surface flaking, but broken segments and points with varying degrees of finish are common. In fact, most of the bifaces in the site were found broken, occasionally in two parts that could be refitted; most complete specimens were presumably taken away for use elsewhere. In TIN 2 one excellent biface 14 centimeters long broke during manufacture and an artisan tried to continue flaking one of the parts into a smaller tool, but eventually gave up (Fig. 6.7, top left). The use-wear that occurs on a few bifaces suggests utilization of mainly the side edges, despite 

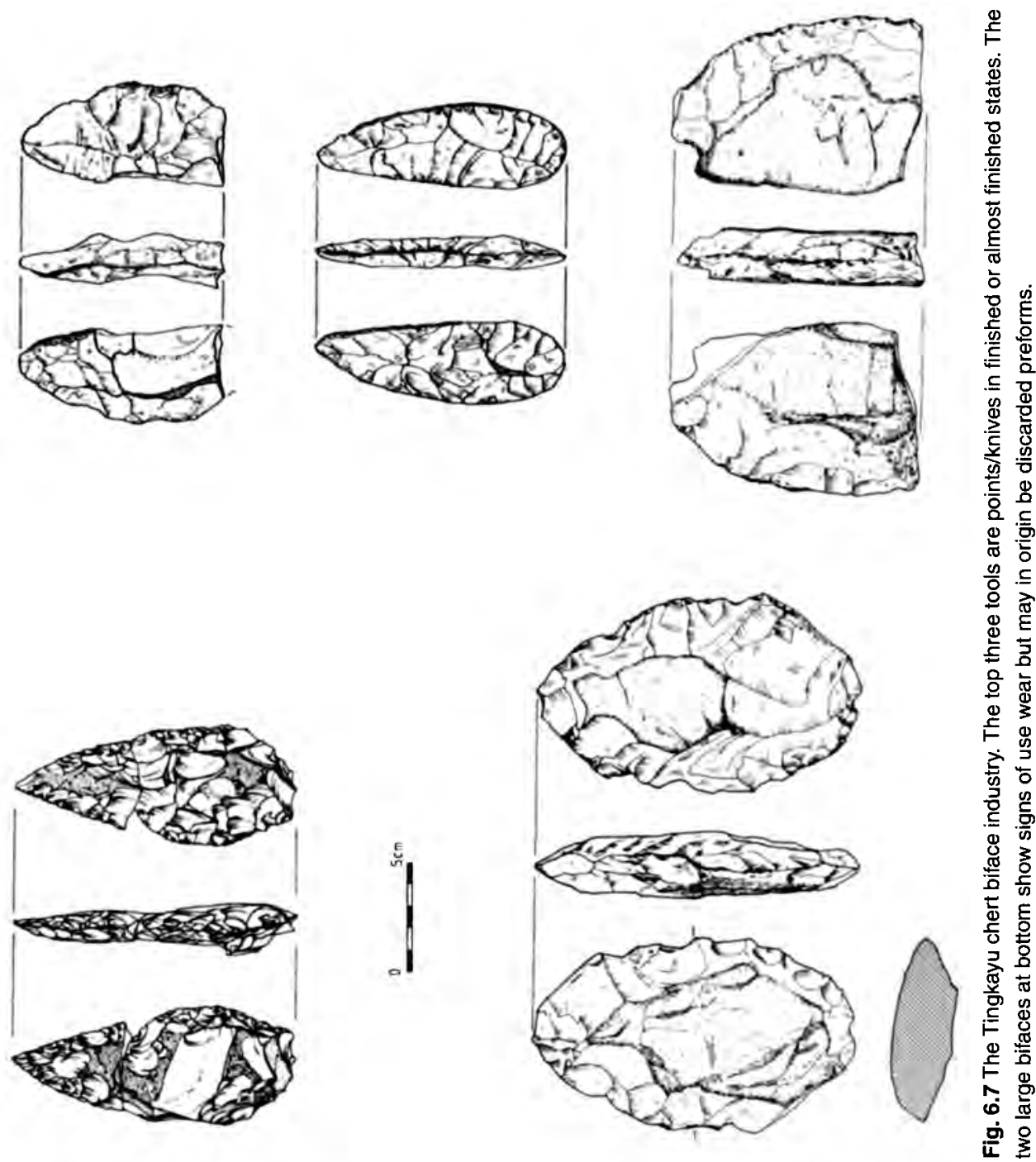

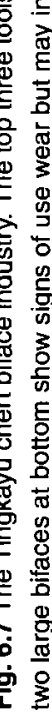


the overall pointed shapes. Hence, they could have served combined functions as both projectile points and knives.

The majority of the tools were found in the bulldozer-disturbed area of TIN 1 , but the stratigraphic evidence from TIN 2 strongly suggests a single unified industry. The sites have also yielded large numbers of bifacial reduction flakes, plus a steep-edged thumbnail scraper and a few flaked cores. In the first edition of this book I also referred to pebble tools and horsehoof cores (shown as Fig. $6.14 \mathrm{e}-\mathrm{j}$ in the first edition of this book), but these are all of a different kind of chert not found stratified in the excavated working floor at TIN 2. There is strong reason to suspect that many could have been made by heavy bulldozing equipment (Bellwood 1988:72). ${ }^{3}$ Alternatively, they could be from a layer of a different period originally above the TIN 1 site that has been totally disturbed. Unfortunately, it is not possible to check on this now, and the safest course is to remove these tools from consideration owing to the doubts about their provenance.

At present this bifacial industry is quite unique in the whole of Southeast Asia, except for one apparent lanceolate found years ago in a tin mine in Kedah in Peninsular Malaysia (Stein Callenfels 1936b). Although similar forms do occur in northern Australia, they all seem to postdate $4000 \mathrm{BC}$. It seems likely to me that this tradition was developed locally, perhaps to meet a specific need in this rather unusual lacustrine environment. In eastern Asia there are remote parallels for the lanceolates in the late Pleistocene (post-18,000 BP) Diuktai tradition of northeastern Siberia (Michael 1984; Yi and Clark 1983) and in several regions of Japan after about 18,000 years ago, but these occurrences are so distant that they can be no more than noted at the present time. The possibility of a link with the bifacial industry in the basal layer of Lang Rongrien in south Thailand is discussed on pages 160-161.

During the Lake Tingkayu period the Baturong massif formed a towering limestone island, and the rock shelters along the base of its southern cliff were all drowned. After the lake drained away the Tingkayu open sites were abandoned. In the shelter of Hagop Bilo (BAT 1 in Fig. 6.6) the basal and culturally sterile alluvial sediments were overlain by midden deposits in alluvium dating between about 18,000 and 12,000 years ago. These midden layers contain mainly three species of lacustrine gastropods, and marine shells are absent. The animal faunas (Cranbrook 1988a) include pig, sambhur deer, mouse deer, porcupine, monkey, rat, snake, tortoise, monitor lizard, birds, and other small unidentified species. The stone tools of this period lack the bifaces and comprise a fairly typical Indo-Malaysian pebble and flake industry with single- and multiplatform cores, utilized flakes, and flat-based and steep-edged, domed, scraperlike tools, all made of chert. Features of some interest include a class of long bladelike knives (Fig. 6.8), perhaps functional descendants of the Tingkayu lan- 

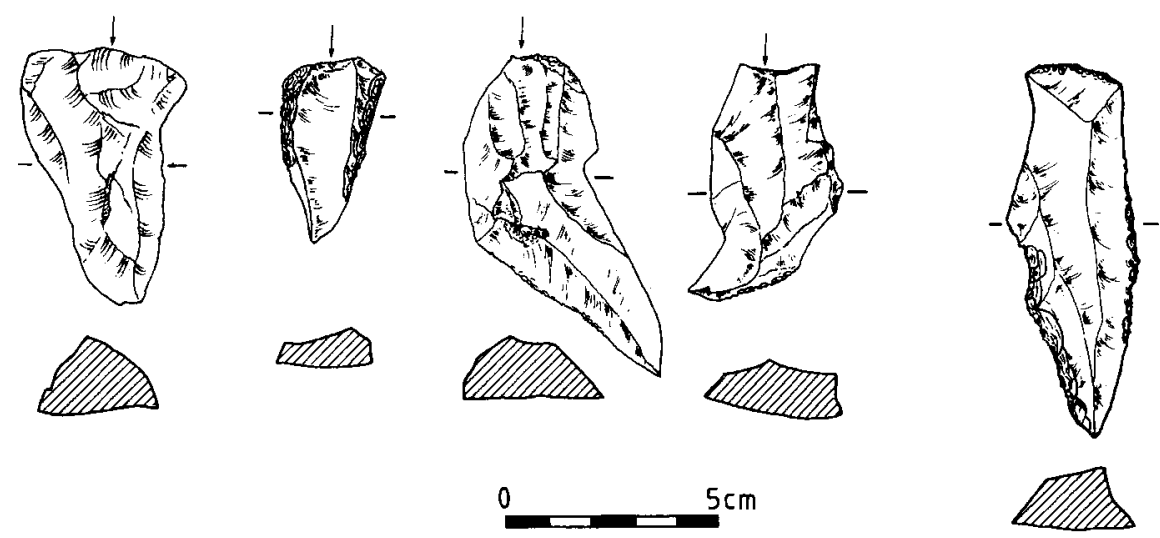

Fig. 6.8 Elongated bladelike chert flakes from Hagop Bilo.

ceolates, and the presence of an opal phytolith gloss on the working edges of some tools. This gloss is widely reported from other sites of this period and later in Southeast Asia (see Section IIIC below), but was absent in the Tingkayu industry where open situations may not have been conducive to its survivalhad it once existed. Another tool of interest from Hagop Bilo is a large bone spatula similar to those from Niah. Tablets of scratched red ochre were also recovered.

Soon after 12,000 years ago the Hagop Bilo shelter was in turn abandoned. The absence of marine shells in the deposits suggests that its inhabitants were mainly inland dwellers, as one might expect with the shoreline perhaps 100150 kilometers away. By 10,000 years ago, however, the Madai Caves (Plate 24) may have been coming within easy reach of the approaching coastal resources, and the cave users apparently moved seaward from Baturong to Madai at this time. The largest of the Madai Caves, Agop Atas (MAD l; see T. and B. Harrisson 1971 for earlier excavations here), today contains a substantial Idahan village occupied seasonally for bird's-nest collection. Above MAD 1 lies the smaller Agop Sarapad (MAD 2); both caves were intensively inhabited by hunters during the early Holocene, between about 10,000 and 7,000 years ago.

The early Holocene human deposits in MAD 1 lie in an acidic guano deposit; thus only stone tools survive, with some charcoal-all animal bones have totally dissolved. But the MAD 2 cave has much better conditions, for here the people deposited a large shell midden against the cave wall that has created and maintained its own alkaline environment; bone survives in quite good condition, although both caves are too damp for any plant organic matter to have survived. The MAD 2 midden thus tells the best story: It has yielded thousands of stone tools of local river-pebble chert-an industry similar to that from 
Hagop Bilo but lacking the bladelike knives (Fig. 6.9). There is a heavy emphasis on pebble tools, large steep-edged tools, multiplatform and horsehoof (singleplatform) cores, and utilized flakes, many of which have glossed edges. A number of large pitted anvils or grindstones occur, some coated with red ochre (Fig. 6.10). Hammerstones are also common, either for stone toolmaking or for food or ochre preparation on the anvils. The food remains in the midden include many shells of the estuarine mangrove shellfish Batissa and Anadara; clearly the inhabitants were now visiting the encroaching coast fairly frequently. Most shells, however, are of the three same riverine shellfish species that were eaten earlier at Hagop Bilo. The animals hunted were also similar, with the addition of larger creatures such as the orangutan, cattle, and rhinoceros; these appear to have been absent at Hagop Bilo, but the small sample size makes this uncertain. Remains of the wild dog Cuon alpinus and Javan rhinoceros have also been identified from Agop Sarapad by Cranbrook (1988b), and recent research by Terry Harrison (pers. comm.) has led to the identification of tiger. These are the only secure reports of these animals from prehistoric contexts in Borneo (Table 1.1), and they no longer live on this island.

After 7,000 years ago the two Madai caves were abandoned. I am unable to see any clear explanation for this, except to suggest that the inhabitants may have moved to a coastal location, or perhaps dwelt elsewhere in an unexcavated part of the cave system. However, there seem to be no other caves suitable for long-term habitation in the Madai massif; this implies strongly that the population did move away. For about 4,000 years the caves remained unoccupied; then a new and totally different cultural assemblage made its appearance. I will describe this in the next chapter.

\section{The Southwestern Arm of Sulawesi}

The southwestern arm of Sulawesi has produced one of the best preceramic sequences of late Pleistocene and Holocene stone tool working in the whole Indo-Malaysian Archipelago. I have already looked at the rather enigmatic opensite industry of the region around Cabenge (Chapter 2, Section IIID5). Archaeological excavations have also been carried out since early this century in caves and shelters in the towerlike karst topography that is particularly well developed in the Maros region inland near Ujungpandang (Makassar). Many sites here have produced assemblages belonging to the industry of backed flakes and microliths known as the Toalian, which I will describe in the next section. The Toalian postdates 7,000 years ago and overlaps with the appearance of pottery in the region. However, earlier assemblages are now known as a result of several seasons of fieldwork in the Maros shelters of Leang Burung 2 and Ulu Leang 1. 

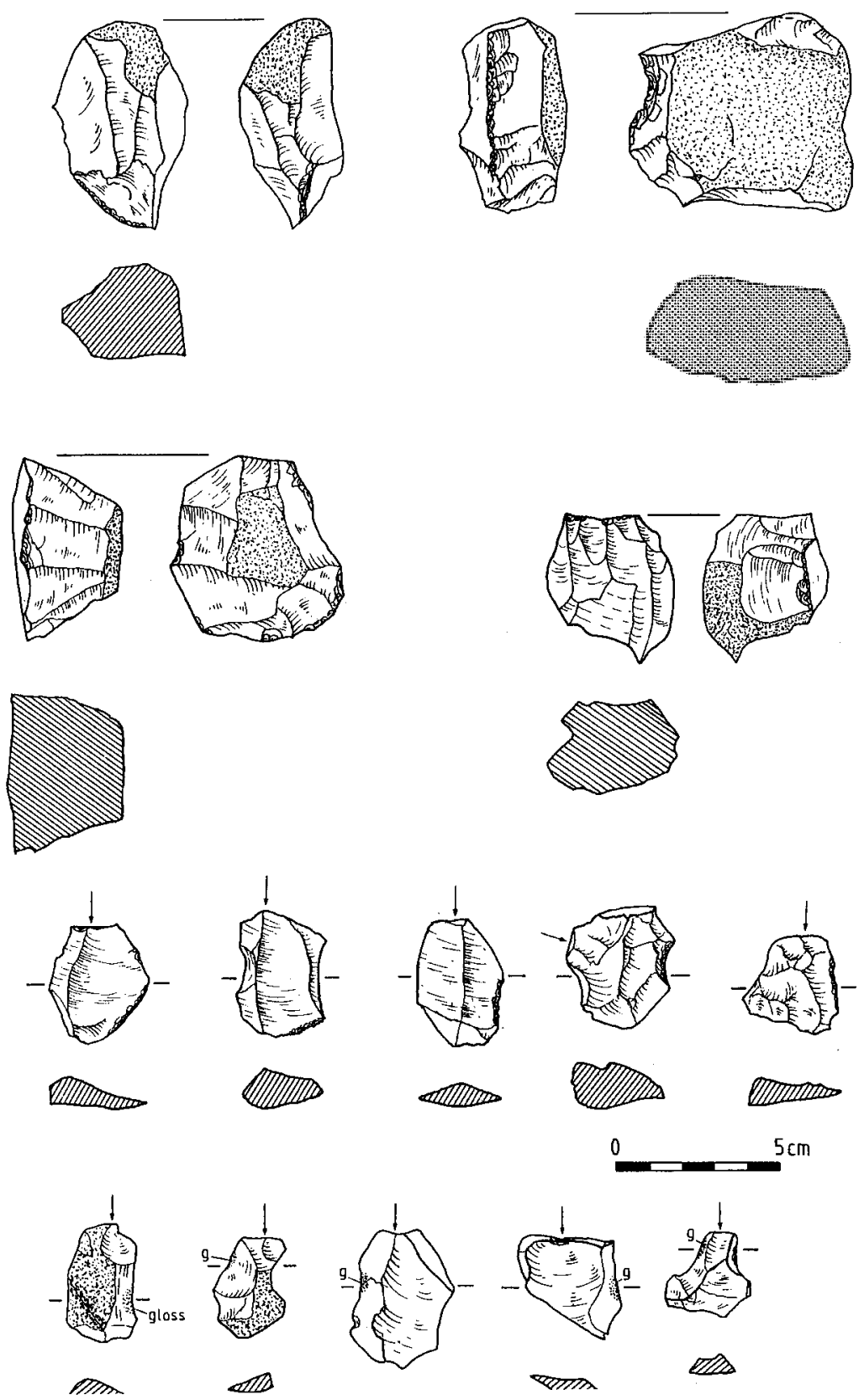

Fig. 6.9 The Agop Sarapad (MAD 2) chert industry. Top: pebble tools. Second row: cores. Third and fourth rows: flakes, some with edge gloss. Drawn by Lakim Kassim. 

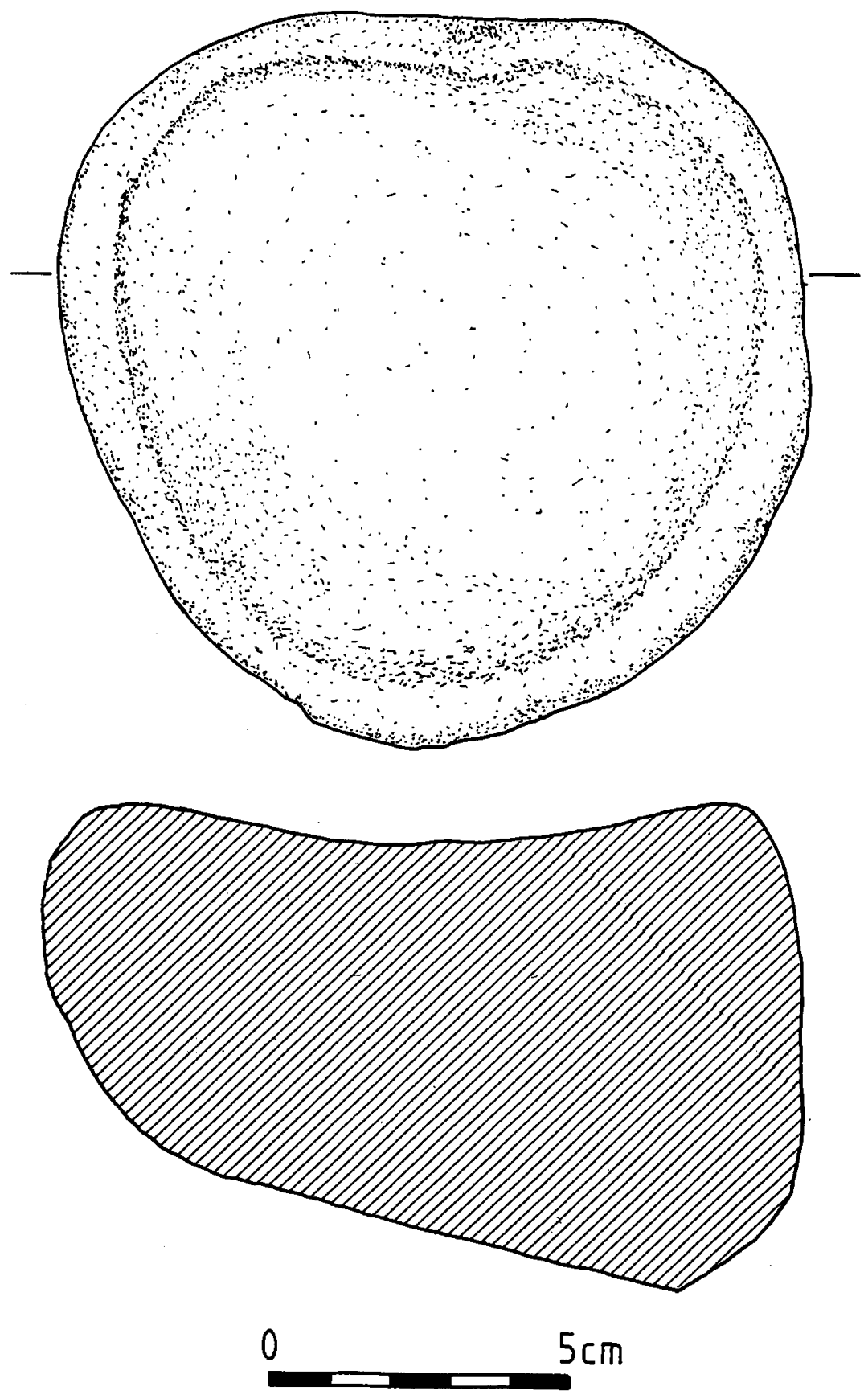

Fig. 6.10 Hollowed mortar of volcanic rock from Agop Sarapad. 

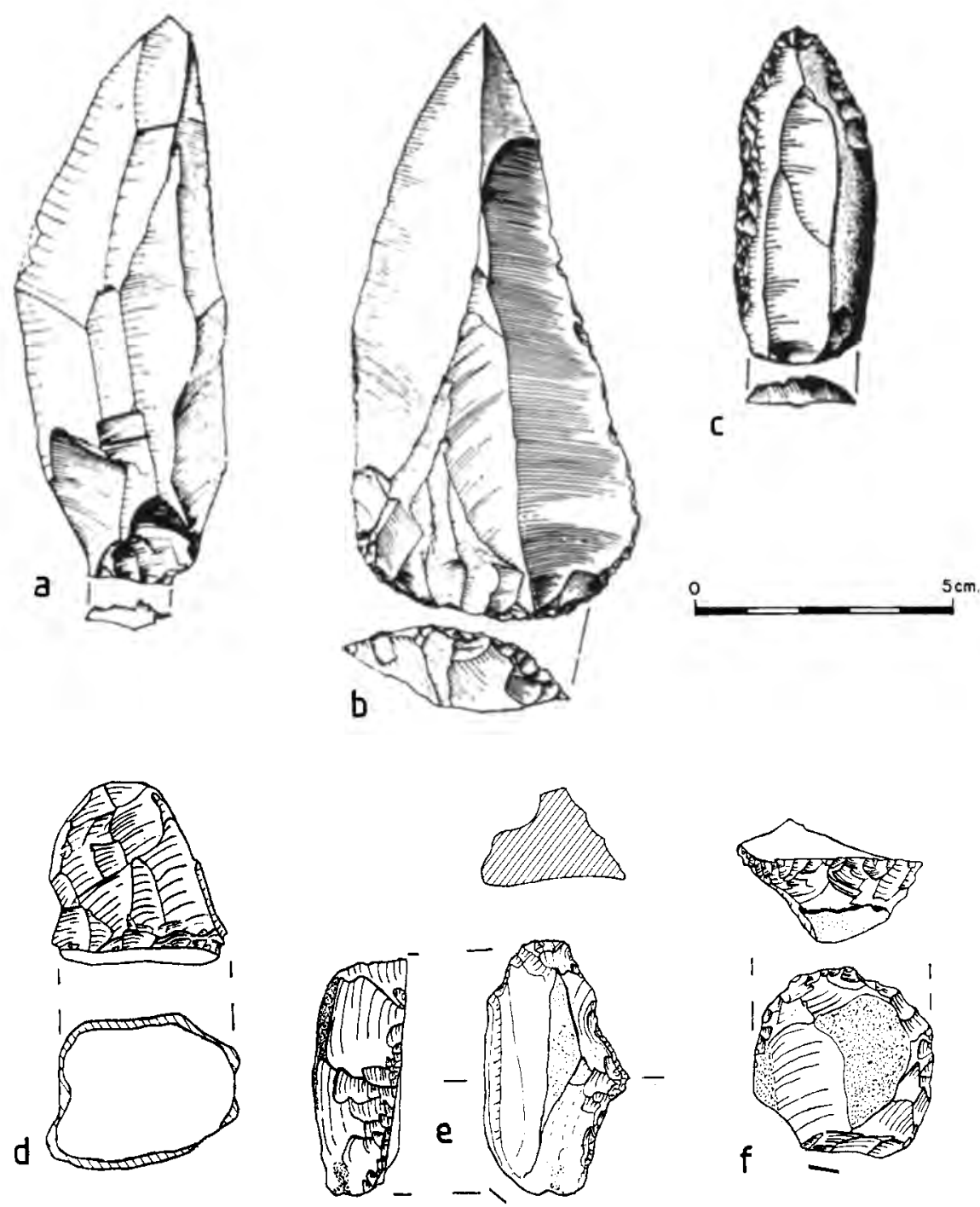

Fig. 6.11 Top: chert blades (item c has retouch) from Leang Burung 2. Bottom: "horsehoof" core (d) and flat-based, steep-edged tools (e, f) from Ulu Leang lower layers. From Glover 1977a. Courtesy: lan Glover.

Leang Burung 2 (Presland 1980; Glover 1981) has produced an industry characterized by unretouched flakes and small multiplatform cores of chert from levels dated between 29,000 and 17,000 years ago, according to radiocarbon determinations on freshwater shell. Some flakes have an edge gloss of a type found widely in this region (as in Hagop Bilo, above), suggested here to result 
from the cutting of stems or leaves (Sinha and Glover 1984), possibly for mats or baskets. In addition, there are at least four elongated bladelike flakes with faceted striking platforms (Fig. 6.11a, b). These are significant because they suggest a degree of conscious core preparation prior to flake removal that is not found in any other Southeast Asian industry of this period. There are, however, no recognizable prepared-platform cores in Leang Burung 2 and the technique seems here to have played quite a minor role. Its invention may have been independent of occurrences elsewhere-it does not appear to continue on into the Holocene period in the Sulawesi sites.

Also found in Leang Burung 2 are pieces of red ochre, but bone points are absent, as are fishbones and marine shells, as the sea was presumably very far from the site at this time. The industry seems to continue (after a possible gap) into the lower levels of the shelter of Ulu Leang 1 (Glover 1976). These date from the early Holocene and contain rare estuarine shells from the coast, which had approached to within 35 kilometers of the shelter by 6,000 years ago (Glover, E. 1990). In Ulu Leang there is a distinctive range of steep-edged domed tools and horsehoof-shaped cores of white chert, similar to the Agop Sarapad industry of the same date from Sabah (Fig. 6.11c, d, e). Bone spatulae also appear in basal Ulu Leang 1; this bone tool tradition is elaborated in the succeeding Toalian industry.

\section{The Northern Arm of Sulawesi: The Paso Midden}

The Paso shell midden (Bellwood 1976a) lies close to the shoreline of Lake Tondano, in the inland volcanic terrain of the Minahasa Peninsula. The midden is about 30 meters in diameter, averages 1 meter in depth, and consists of lenses of loose lacustrine shell interstratified with occupation layers. The latter contain an obsidian flake industry, bone points, red ochre, and prolific faunal remains. The site is radiocarbon dated to about $6500 \mathrm{BC}$.

The obsidian, collected locally, is vesicular and coarse; lumps were mostly smashed to obtain sharp chips and chunks, although flakes were struck individually from multiplatform cores as well. There are no pebble tools (one would perhaps not expect them in a raw material of this type), and no edge gloss has been observed, perhaps due to low visibility rather than total absence. A few chunks and flakes were retouched, often into steep-edged and high-backed forms like those of Agop Sarapad and basal Ulu Leang 1. The faunal remains from Paso and from the contemporary (pre-Toalian) layers at Ulu Leang 1 have been identified by Clason (1986, 1987). Pigs (Sus celebensis) were most popular in both sites and occurred with anoa, babirusa, macaque monkeys, rodents, and the two Sulawesi species of marsupial tree-dwelling cuscus (Phalanger celebensis and P. ursinus). The lake-edge situation of Paso allowed for considerable hunting 
of water birds (rails, coots, geese, ducks), pigeons, and doves, while the karstriverine situation of Ulu Leang led to more frequent catches of tortoises, snakes, and occasional fish. In neither assemblage are there indications of animal domestication.

\section{E. Eastern Timor and Flores}

From four caves in eastern Timor, Glover (1977a, 1986) has excavated an industry with basal dates of about 13,000 years ago. It continued with little change into the ceramic period, which began here during the late third or second millennium $\mathrm{BC}$. The tools are primarily chert flakes (there is also some obsidian), and the retouched forms are mainly steep-edged scrapers. A number of the unretouched flakes have an edge gloss, and there are also a few long, thick blades (Fig. 6.12). Basically, this Timorese industry has much in common with those just described from Sabah and Sulawesi, but it does seem to be a little distinctive in its predilection for long, bladelike artifacts.

The Timor fauna of the period prior to $2000 \mathrm{BC}$ is dominated by several species of extinct giant rats that survived until about 2,000 years ago, together with fruit bats, snakes, reptiles, fish, and shellfish (other placental mammal species
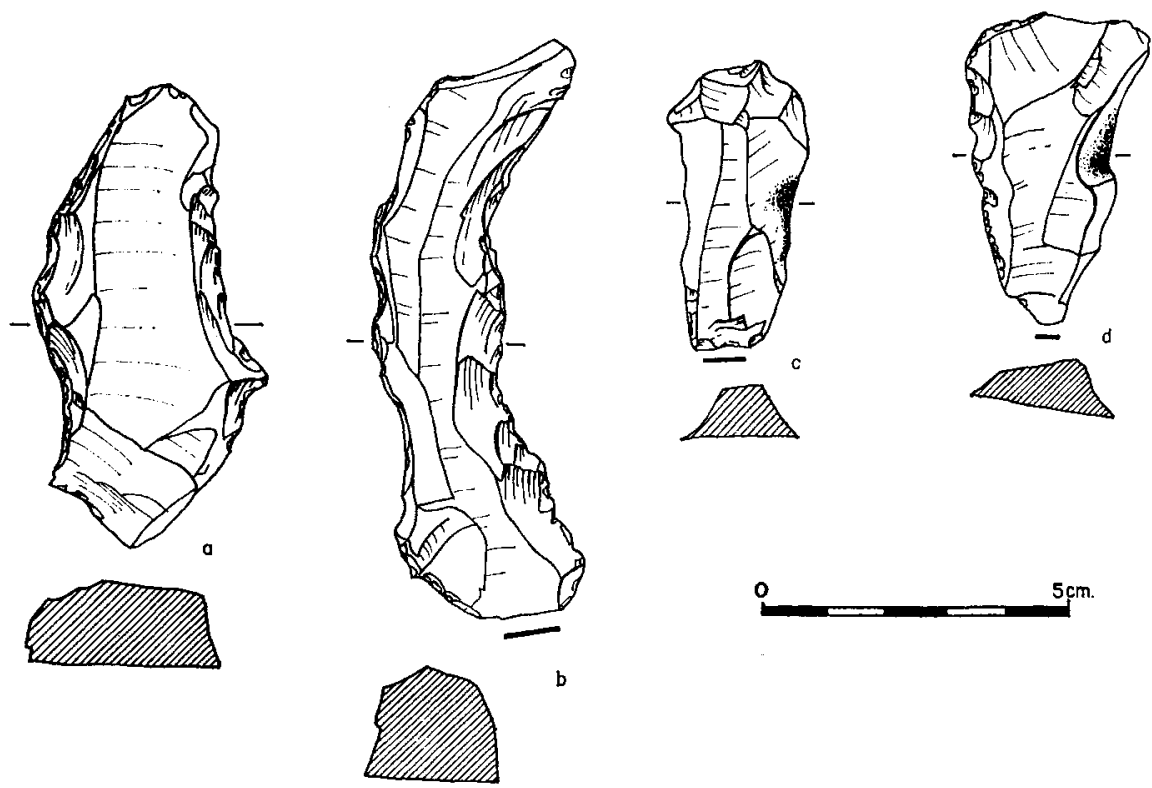

Fig. 6.12 Stone tools from Uai Bobo Cave 2, East Timor: (a, b) bladelike flakes with steep retouch; (c, d) flakes with edge gloss. Mid-Holocene. Courtesy: Ian Glover. 
such as pigs and deer were all introduced into Timor after $2500 \mathrm{BC}$ ). Remains of gathered plants in the early levels include seeds or fragments of the perennial cereal Job's tears, betel vine, areca nut (the ingredients of betel chewing), and candlenut (Aleurites) (Glover 1977b:18). An undated industry from several open sites on Flores may be related to the Timor material. I have already referred to these sites in Chapter 2, Section IIID5, as some components may be of considerable antiquity, possibly contemporary with an extinct species of Stegodon.

\section{F. The Northern Moluccas}

Since the first edition of this book was published, the record of early modern human colonizing activity has been expanded remarkably, at and just beyond the eastern fringe of the Indo-Malaysian Archipelago. Dates for the initial settlement of Australia are now being pushed beyond the 40,000-year "radiocarbon barrier," perhaps as far back as 60,000 years ago according to recent luminescence dates. Sites in Papua New Guinea indicate that some coastal regions of the island were settled by at least 35,000 years ago, and the Highlands by about 30,000 years ago. Cave sites in the Admiralty Islands, New Britain, New Ireland, and the northern Solomons also reveal that humans were crossing water barriers, in some cases perhaps out of sight of land, by 30,000 years ago.

These early maritime colonizers in the western Pacific made flake tools like those in contemporary sites in Indonesia, ate marine fish and shellfish, and by at least 20,000 years ago appear to have been trading small amounts of obsidian from New Britain to New Ireland. Although the navigational capabilities of these early settlers were perhaps not great-they were apparently unable to migrate beyond the Solomon Islands-they did achieve a 200-kilometer crossing from either New Guinea or New Ireland to the Admiralty Islands by at least 13,000 years ago. These were presumably intentional voyages, undertaken for purposes of colonization (for the new Melanesian data see Spriggs in press; Allen and Gosden 1992; Smith et al. 1993).

The ancestors of these Pleistocene pioneers must have come from Indonesia. Recent research here, on the islands of Halmahera, Morotai, and Gebe in the northern Moluccas, is helping to fill in some of the gaps that currently exist for late Pleistocene human activity between Sulawesi and Papua New Guinea (Bellwood et al. 1993). On Gebe, a sequence of human activity extending back for over 32,000 years has been recovered from two coastal caves called Golo and Wetef, located about 1 kilometer apart (for locations see Fig. 3.2). The lowest levels of both caves contain stone flakes, coral cooking stones, and burnt marine shells, but unfortunately little else. A major phase of human activity seems to have commenced in Golo cave (Wetef being still under analysis) around 12,000 years ago, when edge-ground adzes of Tridacna and Hippopus 
shell (Plate 25) appeared in the record and circular settings of coral blocks were placed on the cave floor (Plate 26). The latter represent the oldest examples of deliberate human construction yet found in the Indo-Malaysian Archipelago.

In the upper layers in both caves, between about 10,000 and 3,000 years ago, there is a sudden appearance of wallaby (Dorcopsis sp.) and cuscus (Phalanger alexandrae) bones, together with occasional bones of fish and reptiles. The wallaby appears to have been introduced to Gebe by human agency, but whether from Halmahera or New Guinea is not known. The cuscus may be an endemic Gebe species (Flannery and Boeadi 1995), but the absence of both the wallaby and the cuscus in the lower layers of the cave, despite excellent shellfish preservation, makes one wonder how long they have been on the island. Human introduction of both in the terminal Pleistocene seems to be a possibility. Bones of the two species also make an appearance in another Gebe cave called Um Kapat Papo at about 7,000 years ago. In Golo, these bones are found in association with many volcanic cooking stones, small bone points, and with an extended human burial that was placed in a shallow grave filled with soil mixed with powdered red ochre; many discarded faceted tablets of red ochre were found in the soil around and above the skeleton, but there were no grave goods. Finally, after about 3,000 years ago, pottery appears in the Golo record, and there is also a large series of adzes made on Cassis cornuta shell lips that find a parallel in sites of this age in the western Pacific. The Golo and Wetef sequences are still provisional, but it is evident that these sites will produce one of the longest and most detailed late Pleistocene records for any region of eastern Indonesia.

Another cave called Gua Siti Nafisah on the southern Halmahera mainland has preceramic levels of mid-Holocene date (ca. 5,500 to 3,000 BP), in which the same wallaby species as that found in Golo occurs together with an extinct bandicoot. Both animals became extinct on Halmahera only within the past 2,000 years, perhaps due to overhunting and dog predation. Surprisingly, Siti Nafisah has no flaked stone tools at all, only manuports (mainly cooking stones) and a few bone points; this is perhaps the only case in Southeast Asian archaeology where a site has turned out to have absolutely no flaked lithic technology, and the circumstance is a little hard to explain. Stone tools do occur, however, in two caves adjacent to each other on the southern coast of Morotai -Daeo 2 and Tanjung Pinang-that have a sequence extending back to 14,000 years ago. But here there are absolutely no ground-dwelling marsupials at all, only fish (interestingly confined to the Holocene layers when the sea was close to the sites), rodents, and cuscuses. The Morotai sites have a fairly amorphous stone industry made on flaked beach pebbles (Plate 27), again with volcanic cooking stones, bone points, and ochre.

These three Moluccan site complexes give much food for thought, partly 
because they are so variable in their marsupial records and their stone industries. Unlike the Melanesian sites, there is no good evidence for stone tool transport from island to island. The absence of marsupials (except cuscus) from Morotai is also a mystery given the possibility of the translocation of wallabies to Gebe and similar cases of animal translocation reported from Melanesia (Flannery and White 1991). One receives the impression of small, isolated groups of hunter-gatherers in the Moluccas, perhaps with economies based on sago and canarium nut exploitation, as well as coastal fishing and mammal hunting. The stone tool industries are very simple in technology, as they are in the Melanesian islands.

On the other hand, some hints of external contacts do occur after 15,000 years ago. For instance, the heavy Tridacna shell adzes found in Golo Cave are paralleled in contemporary layers in Pamwak Cave in the Admiralty Islands (Fredericksen et al. 1993) and also possibly in the southern Philippines (Ronquillo et al. 1993). The use of ochre tablets seems to have been fairly universal all over the archipelago by this time; the Golo examples are quite similar to those from Hagop Bilo in Sabah (above). It is also at about 14,000 years ago that we have the first evidence of a human presence on Morotai. In this regard, although the northern Moluccan islands are to some degree intervisible, we must beware of assuming that visibility need always mean accessibility. Some of the smallest islands like Gebe might only have been intermittently occupied, especially prior to 15,000 years ago. After this time it might be that people became better equipped for interisland travel on a regular basis, but further research on this question is required.

\section{F. Comments}

The above sections detail preceramic sites or site complexes for which there is ample information. Other sites of late Pleistocene to early Holocene date include the cave of Tianko Panjang in the Sumatran highlands near Lake Kerinci (Bronson and Asmar 1975), which has yielded unretouched obsidian flakes and chips dating from about 11,000 BP onward. In the Philippines we have the long industrial sequence of chert tools dating back for 30,000 years from the Tabon Caves on Palawan (Fox 1970, 1978), plus the preceramic industries from sites such as Musang Cave in northern Luzon (Thiel 1988-1989) and Balobok shelter in the Sulu Archipelago (Ronquillo et al. 1993). These Philippine industries all fit well within the Indo-Malaysian pebble and flake repertoire.

All the industries I have discussed (especially the cherts and obsidians) are characterized by flake production with stone hammers from multi- or singleplatform cores. Core smashing appears to have been as important as systematic 
flaking in some sites with poor-quality stone; this has been emphasized by Coutts and Wesson (1980) for the Philippines, where they refer rather colorfully to a category of "smash and grab" industries. Retouch is often steep and highdomed "scraper" forms are quite common, as is an edge gloss on sharp flake edges. Although flakes often have bladelike proportions, there is no sign of any systematic attempt at blade production until about 6,000 years ago (see below).

The basic core, flake, and pebble characteristics of these late Pleistocene and early Holocene preceramic industries, including the Hoabinhian, find fairly close parallels in the "core tool and scraper tradition" (Bowler et al. 1970) of Australia and the New Guinea Highlands, both of which were first settled from Indonesia before or around 40,000 years ago. These similarities are not surprising, and it is easy to misinterpret localized variations relating to raw materials as the results of cultural variation. On the other hand, some of the variation obviously does represent human intent, especially the technologies represented at Leang Burung 2 and Lake Tingkayu. Farther east there is also an unusual focus on large-waisted axelike tools in the Huon Peninsula of Papua New Guinea and in the Papua New Guinea Highlands, dating back in the case of the former location to perhaps 40,000 years ago (Groube et al. 1986). Such waisted tools have not yet been found in the Indo-Malaysian islands, but they appear occasionally and perhaps independently in some Hoabinhian sites (see Fig. 6.2).

\section{THE FLAKE AND BLADE TECHNOCOMPLEX OF THE MID- HOLOCENE}

In parts of the Philippines, Sulawesi, and Java there are a number of assemblages dated to after $5000 \mathrm{BC}$ that demonstrate regionally varied emphases on the production of small blades, and sometimes on other specialized tools such as "microliths" with blunted backs and small projectile points. In all cases these new technologies appear as accretions added to the old and continuing tradition of unprepared flake production.

In a previous book (Bellwood 1978:71), I quoted a definition by Morlan (1971:143) of blades as "elongate parallel-sided flakes with parallel arrises or parallel-sided facets on their dorsal faces. Blades are struck (by indirect percussion) from prepared, polyhedral cores." The Upper Paleolithic industries of much of the Old World were focused on the production of blades of this type, and in Japan, northern China, and northwestern India it is now clear that they were widespread by at least 20,000 years ago (Chen and Olsen 1990). In Island Southeast Asia and Australia, however, blades form only a small minority component of most assemblages in which they occur, and true cylindrical or conical blade cores are generally very rare. Many of the "blades" found in this region fall into Morlan's category of bladelike flakes, which are less symmetrical than 
true blades and which lack the parallel ridges. Nevertheless, because cores of a subprismatic shape do occur in small numbers and because 1 believe that both blades and bladelike flakes were produced intentionally in some sites, I will refer to the industries described in this section as the "flake and blade technocomplex."

At present there appear to be two kinds of industries within the flake and blade technocomplex: the unretouched blade industries of the Philippines and the Talaud Islands of northeastern Indonesia, and the backed flake-blade and microlithic industry-termed the Toalian-in southwestern Sulawesi. At the end of this section I will also consider some undated industries, perhaps related to the Toalian, that have been found on Java. The Philippines, Sulawesi, Java, Australia, and possibly southern Sumatra encompass the distribution of industries in this technocomplex; they seem to be completely absent in the preceramic from Borneo, eastern Indonesia, and New Guinea (although Borneo and Timor do have Neolithic blade industries; see Chapter 7).

\section{A. The Philippines and Talaud}

Industries in which a component of small unretouched blades is added to a continuing flake tradition occur in the Philippines and in the Talaud Islands south of Mindanao. In Duyong Cave, near Tabon Cave on Palawan, Fox (1970) excavated an industry with small bladelike flakes struck from what he originally termed "prepared cores," although he later stated that such cores were not present (Fox 1979:236). The tools occurred in a midden of marine and estuarine shell dated to the sixth millennium BC. Possibly similar industries have been described from Cebu by Tenazas (1985) and from a small island called Buad off the western coast of Samar by Cherry (1978). No clear chronologies are available for these sites, and both late preceramic and Neolithic associations seem likely. Blades account for up to 50 percent of the Buad collections, and some pieces have glossed edges or traces of a mastic used in hafting.

The most detailed information on an industry of this type comes from the rock shelter of Leang Tuwo Mane'e on Karakellang Island in the Talaud Islands (Bellwood 1976a, 1985). This shelter was originally cut by the sea into a cliff of coral limestone and was then uplifted to a habitable level by about $4000 \mathrm{BC}$. The basal deposits produced an industry comprising blades and bladelike flakes (about 50 percent of all flaked stone), together with some rather rudimentary prismatic cores, made on a grey chert (Fig. 6.13). Retouch is virtually absent, but some edge gloss occurs. Around $2000 \mathrm{BC}$ or later pottery appeared in the site, and at this time there was a surprising and unexplained change away from the grey chert toward a nodular brown chert used for the production of a much less refined flake industry that continued into the upper layers of the site. The blade 
a.

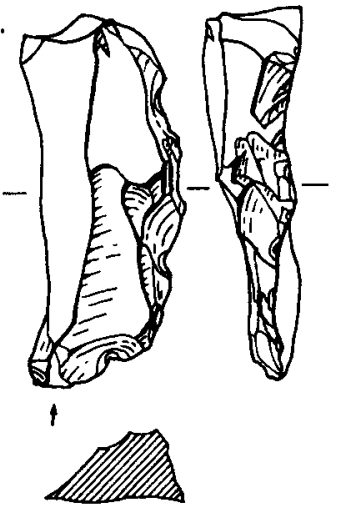

b.

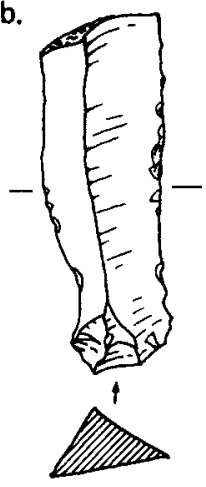

c.

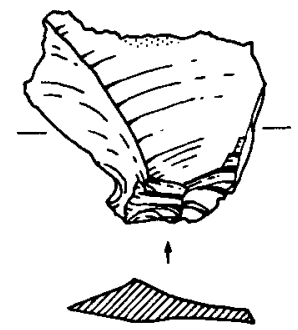

g.

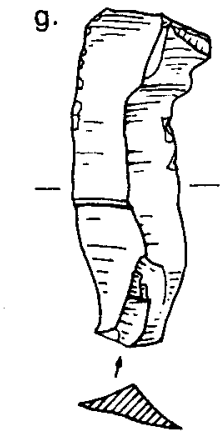

d.
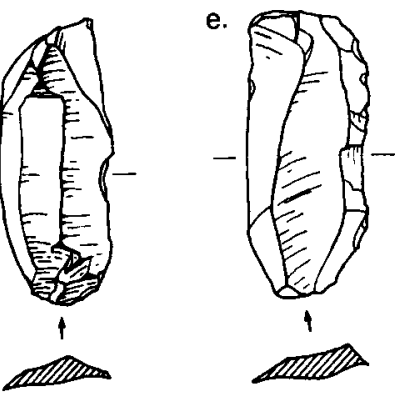

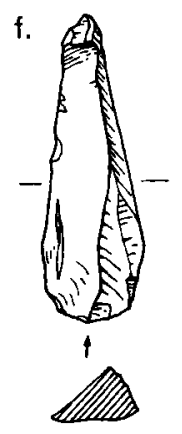

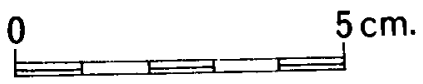

h.
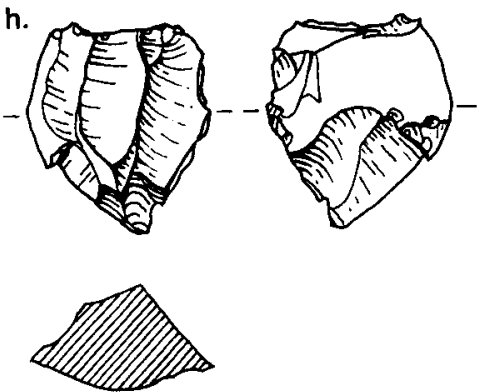

i.

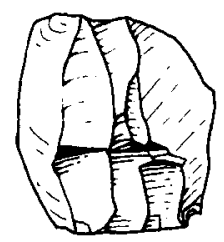

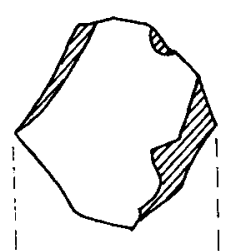

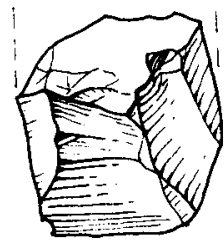

Fig. 6.13 Chert blades (a-g) and subprismatic cores from Leang Tuwo Mane'e, Talaud Isiands. Dated ca. fourth millennium Bc. Flake (c) has an edge gloss. 
industry is thus restricted mainly to the preceramic levels and the date of its actual appearance in Talaud cannot be determined from this site. Both preceramic and ceramic layers contained large numbers of shellfish of no less than ninety-four species from a wide range of reef and mudflat habitats (Heffernan 1980). Apart from pig in the ceramic layers, however, no mammal bones were present; the Talaud Islands had only a limited native fauna of rats, bats, and a species of marsupial phalanger (the latter being also potentially a translocated species).

\section{B. The Toalian}

The most important industry of the flake and blade technocomplex is undoubtedly the Toalian of southwestern Sulawesi, which apparently commenced during the fifth millennium $\mathrm{BC}$ with an array of microliths (small-backed flakes and geometrics) of types seemingly unique in the Indo-Malaysian Archipelago. Toalian assemblages have been excavated since 1902 from caves and shelters scattered across the southern half of the southwestern peninsula of Sulawesi. During the 1930 s and 1940 s some rudimentary typological successions were established by Stein Callenfels (1938) and Heekeren (1949). In Heekeren's last major summary (1972), by which time about twenty sites had been excavated, he felt justified in supporting a three-phase sequence commencing with plain blades, followed by a second phase with backed flakes and blades and geometric (crescentic and trapezoidal) microliths, and culminating finally in a third phase with bone points, serrated and hollow-based stone points, and pottery.

As a result of recent excavations there is now much more information on the Toalian. Two sites, Leang Burung 1 (Mulvaney and Soejono 1970, 1971; Chapman 1986) and Ulu Leang 1 (Glover 1976, 1977a, 1978a; Glover and Presland 1985), are located in the Maros limestone region north of Ujungpandang. Recent Indonesian excavations in caves in Pangkajene Kepulauan, north of Maros, promise to add further data (Anggraeni 1986).

Ulu Leang 1 has the most complete sequence. I have already considered the basal industry of flakes and steep-edged tools in this site, dated to the early Holocene (see Section IIC). The Toalian tool types (Fig. 6.14) appear in higher levels dated from before 6,000 years ago and occur within a continuing industry of flake tools and bone points, although the steep-edged tools fade in importance. The most important new tool type is a small flake or blade with straight or oblique blunting down one side and often around the butt (similar to a "backed blade" in Australian terminology). Some of these backed forms have distinctly crescentic or trapezoidal shapes and are commonly referred to as geometric microliths. The present trend (Chapman 1986; Glover and Presland 1985; Allen 1991b) is to refer to these new Toalian forms, backed blades and 

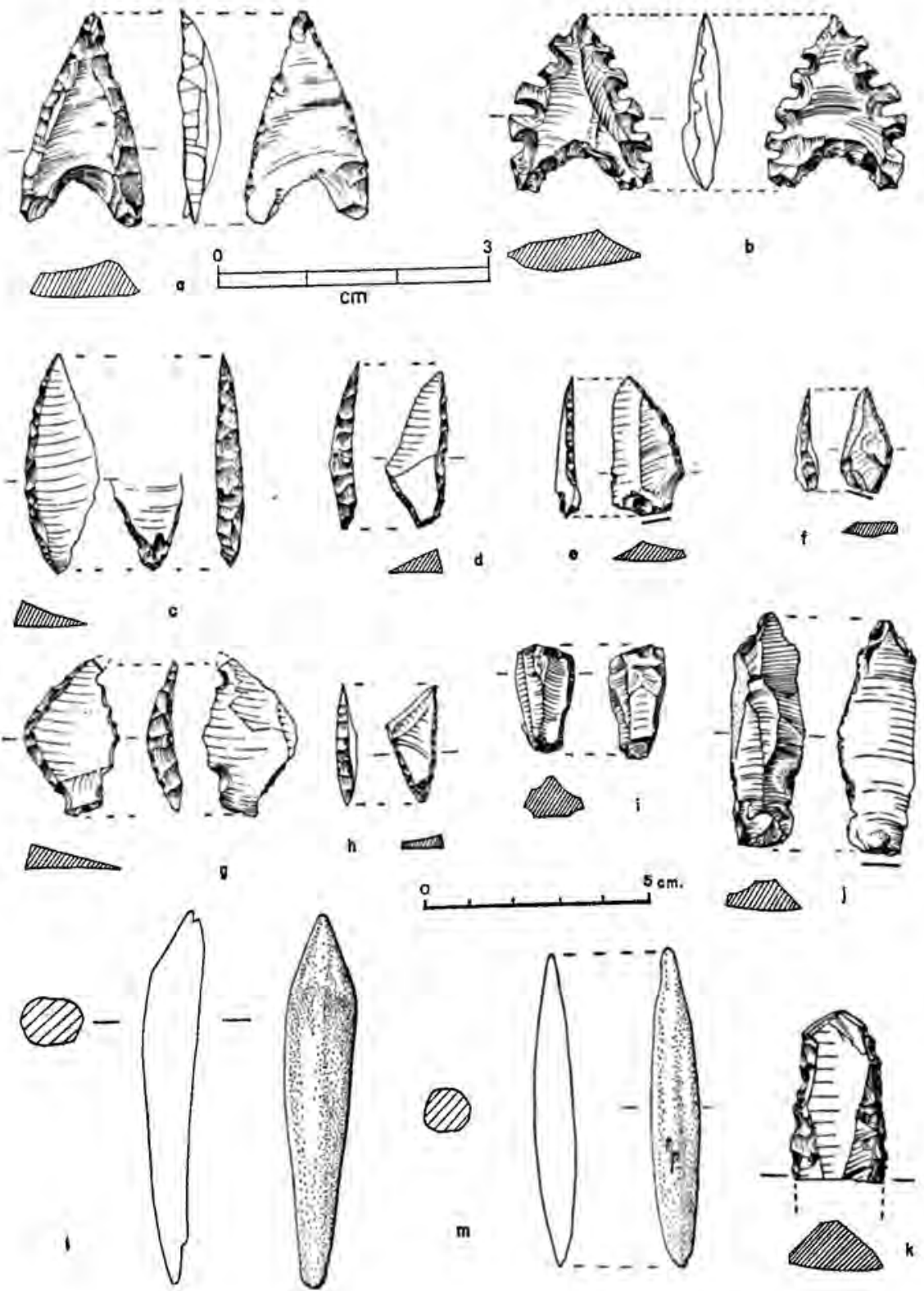

Fig. 6.14 Toalian stone tools from Uiu Leang: $(a, b)$ Maros points; $(c-h)$ small flakes and blades with blunted backs; (i) bipolar microcore; (j) blade with edge gloss; (k) retouched scraper; $(1, \mathrm{~m})$ bone points. Similar bone points are also widespread in the northern Moluccan sites. From Glover 1977a. Courtesy: lan Glover. 
geometrics alike, as microliths. Other artifacts that occur throughout the Ulu Leang sequence include glossed flakes, small bipolar cores, bone points, and bivalve shell scrapers (Willems 1939).

Sometime after 6,000 years ago another type of small stone implement, the "Maros" serrated and hollow-based point, appeared in the sequence. Maros points presumably served as arrowheads or spearheads and became common after 4,000 years ago, by which time pottery had already made its appearance in the Toalian caves (as at Ulu Leang 1). From a regional perspective the possibility thus arises that the Maros points were used by native hunters living in some kind of exchange relationship with adjacent Austronesian-speaking cultivators, as recent hunters have done from time to time in parts of Southeast Asia (see Chapter 5). It is not clear whether the Maros points represent indigenous innovation from a Toalian matrix or whether they represent an imported technology. If the latter, the source is unknown.

The site of Leang Burung 1 (Plate 28) is later in date than Ulu Leang: Its deposits appear to date within the last two millennia $\mathrm{BC}$. They have pottery throughout, meaning again that they are probably contemporary with the dispersal of agricultural peoples in the general region. As in upper Ulu Leang 1, microliths and Maros points are both present. Chapman (1986) stresses the importance of edge gloss in this site (on 24 percent of used tools) and also notes the absence of steep-edged tools, pebble tools, and edge-ground tools (the latter, to my knowledge, appear to be absent in all Toalian sites). At Leang Burung 1 the microliths (including the Maros points) comprise about 35 percent of all retouched tools, which in turn comprise only 6 percent of the total of stone artifacts. At Ulu Leang the corresponding percentages are 20 percent and 6 percent.

The Ulu Leang 1 sequence generally supports the essentials of Heekeren's second and third phases (i.e., backed microliths succeeded by Maros points), but the site has no earlier phase with plain blades. This need not mean that this earlier phase does not exist in all other Toalian sequences, for as I have shown it is present without later typological elaboration in the Talaud Islands. The question of intentional blade production in the Toalian is in fact a difficult one. Chapman feels that it was practiced at Leang Burung 1; about 10 percent of flakes here had blade proportions, although no prismatic cores were found. Heekeren (1972:Plate 63b) also illustrates a series of blades from the site of Panganreang Tudea (near the southern tip of the peninsula) that remind me closely of the blades from Talaud. However, Glover and Presland (1985) deny the existence of a blade technology at Ulu Leang. After analyzing the stone tools from the much older site of Leang Burung 2 (see Section IIC), together with those from Ulu Leang, Presland (1980) concluded that a blade technology was not evident in these particular Maros sites, although flakes did become smaller by 
about 20 percent in average size over the whole period represented (over 20,000 years). However, no one has challenged the significance of the sudden appearance of the new microlithic forms around 6,000 years ago. These raise the question of outside parallels, which I will consider later.

The Toalian industry continues on well into the pottery phase at most excavated sites. In itself it reveals no clear evidence for the agricultural economy that must surely have been developing in some parts of Sulawesi after 2000 BC, unless this is in some way reflected in the appearance of the Maros points. Even as late as AD 1000 the retouched and glossed flakes still occur at Batu Ejaya near the southern end of the peninsula (Chapman 1986), although most of the microliths have by now disappeared and the serrated Maros points appear to have been replaced by plainer round-based forms. The late survival of the Toalian here may document a continuing tradition of hunting and gathering, perhaps amongst indigenous non-Austronesian speakers contemporary with cultivators.

The economic evidence from the Toalian sites includes a range of hunted and gathered resources. Riverine shellfish are very common, and Glover (1977b: 52) found remains of wild seeds and nuts at Ulu Leang, although carbonized rice grains appeared in the site only after AD 500 (Glover 1985). In Section IID, I described the animal remains from the lower levels of Ulu Leang. The faunas from other Toalian sites (Hooijer 1950a) were drawn from a similar range of Sulawesi mammals: the two species of cuscus (Phalangeridae), macaque monkeys, civets, anoa, Sus celebensis, and babirusa. A small quantity of art found on the limestone walls of Toalian sites has been described by Heekeren (1950b, 1972:119-120), although none can be dated. Hand stencils and wild pigs were depicted in red ochre; the former are of great interest because of the worldwide occurrence and great antiquity of this motif. Some of the hand stencils, interestingly, lack one or more digits. Motifs including both hand and foot stencils, as well as depictions of animals and canoes, are reported by Anggraeni (1986) from the caves in Pangkajene Kepulauan, north of the Maros region.

\section{The Sampung Industry of Eastern Java}

This industry (Plate 29), called the "Sampung bone industry" by Heekeren (1972:92), is best known from a cave called Gua Lawa near the village of Sampung, between the Lawu and Liman volcanoes in central Java. The site was excavated by Stein Callenfels in 1931; although the methods were rather crude and the site obviously disturbed, he did provide a section drawing in his report (1932) that shows the vertical distribution of all the artifacts found within a 2-meter-thick occupation deposit. The lowest of three apparent levels of occupation produced a number of stone projectile points with hollow or round 
bases, but without the serrated edges characteristic of Maros points. Hollowed stone grindstones and spherical rubbing stones with traces of red ochre also appeared in this layer, but no records were published of the basic flaked stone industry that was also present.

Above the lowest level, and apparently extending over about half of the excavated area within the cave, Stein Callenfels found a lens of bone and antler points and spatulae, with more stone mortars, a few possibly downwarddisturbed potsherds, and a polished adze. Pottery, metal, and a general mixture of other material then occurred in the top layer, with four bone fishhooks. Flexed burials, at least one under a stone slab and including one child with a shell necklace, were indicated as stratified within the middle layer with the bone tools; all have been classified as Australomelanesian (see Chapter 3, Section IIIB).

At face value (and there is no other way to interpret this site now), the Gua Lawa sequence indicates a possibly preceramic industry of bone tools and stone arrowheads very similar to the nonserrated Toalian types, together with "flakes and blades without secondary working and many retouched shell scrapers" (Heekeren 1972:94). In a previous account of this site (Bellwood 1978:76), I included the bone tools with the top pottery-bearing layer, but then changed my mind in favor of a preceramic date in the first edition of this book. Now I am not so sure; some sherds occur to the base of the sequence and it is clear that the Maros points in Sulawesi overlap substantially with the Neolithic. Further research in this region of Java is required before the Gua Lawa bone tools and stone points can be dated with certainty.

The Gua Lawa fauna, apparently mainly from the middle layer with the bone tools (Dammerman 1934), comprised a broad range of mammals. Dammermann commented on the great predominance of teeth and leg bones of the animals represented-a taphonomically unusual circumstance for which no obvious explanation comes to mind-and gave the most numerous species as banteng cattle, followed by pigs (Sus scrofa rather than Sus verrucosus) and deer (Cervus, Muntiacus and Tragulus). Monkeys, Indian elephant, buffalo, and several large felines were also present. Four species in the site-Cervus eldi, elephant, clouded leopard, and wild water buffalo-are now locally extinct (see Chapter 1, Table 1.1). Palm civets and monitor lizards were also especially frequent.

The Gua Lawa type of industry is known from sites scattered all over the eastern end of Java. South of Gua Lawa a rock shelter in Gunung Cantalan produced round-based stone points and apparently many cranial bones of macaque monkeys (Heekeren 1972:99). Other sites occur to the north around Bojonegoro and inland from Tuban. Heekeren (1935-1936, 1937) also excavated three caves--Petpuruh, Sodong, and Marjan-near Puger in southeastern 
Java, about 200 kilometers east of Gua Lawa. These caves produced the same (apparently) preceramic assemblage of round-based stone points, bone tools, shell scrapers and rings, and flexed burials. Pebble tools and flakes of quartz, chert, and obsidian also occurred, together with hammerstones and grindstones. Heekeren seemed to favor Hoabinhian affinities for these industries, but I find this most unlikely and would rather stress their close similarities with the Toalian, except for the obvious absences in eastern Java of the backed flakes and geometrics. A similar mid-Holocene date also seems quite likely.

\section{Other Flake and Blade Industries in Java}

A large number of sites in western Java, especially on the Bandung Plateau, have produced an undated but presumably preceramic industry of obsidian (Bandi 1951; Heekeren 1972:133-137; Anggraeni 1976). The sites still await detailed archaeological investigation, and the existence of a definite blade element seems to be rather uncertain. The available illustrations, however, leave no doubt that backed flakes, round-based and unifacially retouched projectile points, and a few crescentic and trapezoidal geometrics are present (Fig. 6.15). The sites may in fact be mixtures of several time periods, for a number of more archaic domed and steep-edged tools also occur, but the overall impression is of an industry that may be fairly similar to the Toalian. Some surface-collected obsidians from sites around Lake Kerinci and Jambi in south-central Sumatra (Hoop 1940) may also contain points and microliths. Glover and Presland (1985) report backed crescents from some of these sites, although such forms were absent in the excavated Tianko Panjang Cave.

\section{E. The Flake and Blade Technocomplex in Broad Perspective}

Reasons for the appearances of these new flake and blade technologies, both in the Indo-Malaysian region and in Australia, are topics that cause considerable dispute amongst prehistorians. One important fact that should be noted is that in Island Southeast Asia and Australia, these new technologies are always grafted onto old ones. There are no wholesale replacements in fully preceramic contexts of flake industries by blade industries, nor are there good reasons from the stone tool evidence to invoke large-scale human expansions into the region prior to that of the agricultural Austronesians. It is nevertheless difficult to decide for individual regions whether the new technologies were developed independently or whether they were adopted from an outside source. In some cases the answer may be a combination of both processes. In company with Glover and Presland (1985) and Dortch (1977), I feel that some significance must be allocated to diffusion for the technologies of microlith manufacture 

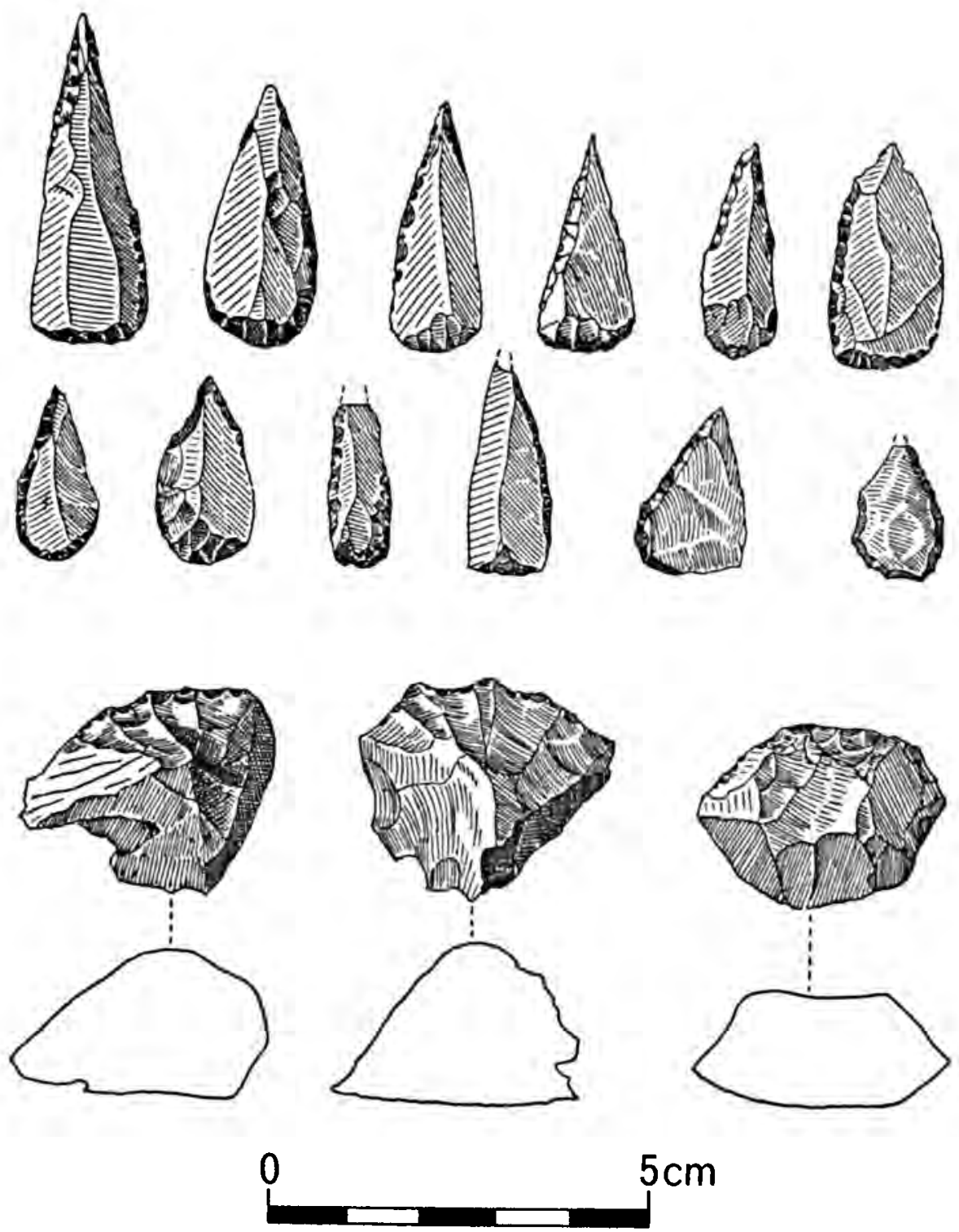

Fig. 6.15 Obsidian-backed points, geometrics, and steep-edged tools from the Bandung Plateau, western Java. From Bandi 1951. Courtesy: Museum für Völkerkunde, Basel. 
(backed blades and geometrics), although it should not be forgotten that the ability to produce elongated bladelike flakes was present at the sites of Leang Burung 1 and Hagop Bilo well before the end of the Pleistocene. Indeed, many Australian prehistorians today regard the "small tool industries" of Holocene Australia as local developments quite unrelated to anything in Indonesia (e.g., Hallam 1977; White and O'Connell 1982).

I rather doubt that these questions of independence versus diffusion will ever be resolved entirely, because stone tools of this kind-with the possible exception of the Maros points-do not carry a large component of cultural as opposed to purely functional information. But as far as backed blades and geometrics are concerned, the simple fact of coincidence in dates between Sulawesi and Australia can hardly be overlooked. The backed blades and geometrics so characteristic of later Australian prehistory appear there from about $3000 \mathrm{BC}$ onward (Mulvaney 1985). Heekeren (1972:125) actually suggested that these tool types diffused to Sulawesi from Australia, although the radiocarbon dates as known at present do not provide much support for this view.

Perhaps the most likely region of outside influence on the islands of Southeast Asia in terms of these flake and blade industries is Japan. India is only a remote possibility as the intervening Hoabinhian industries show no signs of any of the developments under consideration, although an India/Sri Lankan source of influence is favored by Allen (1991b). During the initial, early, and middle Jomon periods of Holocene Japan, there is a range of hollow-based projectile points and blade tools that look a little like some of the Southeast Asian examples, although geometric microliths of the Toalian types are to my knowledge not found there. An undated but presumably preceramic Holocene industry with some blades has recently been reported from a site near Guangzhou in southern China (Huang et al. 1982), but there seems to be no material of this type from Taiwan or the Ryukyu Islands. Nevertheless, Japan and southern China do provide a number of interesting though vague possibilities.

As with the appearance of edge-grinding, a development obviously quite unrelated to the appearance of the flake and blade industries, there are a lot of question marks when looking at the matter of origin. One cannot always rule out independent development easily, but in the case of the microliths a history of interaction and diffusion does seem to me to be more attractive. As always the question arises: Why do these expressions occur in some areas and not in others? 


\section{The Archaeological Record of Early Austronesian Communities}

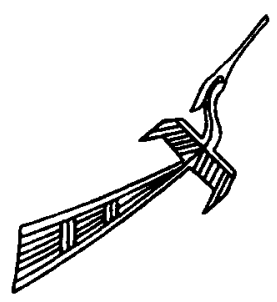

As demonstrated in Chapter 4, the reconstructions of comparative linguists indicate that the earliest identifiable Austronesian communities were located in Taiwan. Prior to the Austronesian colonization of this island, some degree of common linguistic ancestry with mainland Asian populations (especially ancestral Austroasiatic and Tai-Kadai speakers) is evident in macrofamily reconstructions. Beyond Taiwan, early Austronesian colonists later moved southward through the Philippines into Indonesia and Oceania.

These early Austronesian populations had economies based firmly on agriculture and maritime subsistence, some domesticated animals, and a technology that included canoes, well-constructed wooden houses, and probably pottery. The linguistic evidence can tell a great deal about the geographical patterning of Austronesian expansion and about certain adaptations that took place in the Austronesian lifestyle. It cannot, however, tell very much about absolute chronology or illustrate many stylistic details of material culture.

The provision of information in the latter categories is of course primarily the domain of archaeology, as the bulk of the next three chapters will show. This discipline can also provide some information that can be equated with the record of ethnolinguistic prehistory as derived from linguistics. For instance, the documented spread of pottery and polished stone adzes after $3000 \mathrm{BC}$ through parts of Island Southeast Asia is perhaps mainly a result of Austronesian settlement, although this is naturally impossible to prove in any absolute sense because pots and stones cannot talk. But such categories of material culture have dates, typological characteristics, and distributions that we can attempt to interpret.

To anticipate the following sections, I will set out here a brief version of my 
overall model for the later stages of Indo-Malaysian prehistory (Bellwood 1991, 1995a):

1. The Austronesian-speakers who expanded into the Indo-Malaysian Archipelago carried a fully agricultural economy and introduced pottery and a new repertoire of unibeveled stone adzes. However, woven in with this agricultural economy were continuing skills in terrestrial and maritime hunting and gathering. Linguistically, a presence of rice in the agricultural repertoire seems certain. Archaeologically, the evidence is less conclusive and I return to this problem below.

2. The pre-Austronesian inhabitants of the archipelago occasionally used edge-ground stone axes and shell adzes, but they did not use pottery. While they undoubtedly exploited many tubers and fruit trees, later to be of major importance as domesticates, they did not systematically cultivate these species. Had they done so, the present representation of non-Austronesian and non-Mongoloid populations in the major islands of Southeast Asia would, for demographic reasons, be much fuller.

3. Non-Austronesian hunters and gatherers survived in ever-diminishing numbers throughout the millennia of Austronesian expansion, but a noncereal agricultural economy might have developed independently in the New Guinea Highlands before this expansion took place. This could help to explain why Austronesian colonization of New Guinea was restricted only to marginal coastal locations.

4. During the millennia of expansion southward and into Oceania, the economies of Austronesian societies underwent a number of latitudinal and more localized ecological adaptations. Cereals were apparently replaced in eastern Indonesia by tubers and tree fruits. Some groups even specialized away from agriculture in the directions of terrestrial or maritime hunting and gathering (e.g., the Punans and Orang Laut).

5. During the millennium between $500 \mathrm{BC}$ and $\mathrm{AD} 500$, the archipelago was incorporated into wider South and East Asian spheres of interaction. Major introductions or developments of this period include metallurgy and probably domesticated cattle and water buffalo, together perhaps with a significant increase in commitment in certain areas (e.g., Northern Luzon, Java, Bali) to terraced and irrigated rice cultivation. Contacts with the civilizations of India and China also began during this period.

\section{THE ORIGINS OF AGRICULTURE}

There is now enough botanical, linguistic, and archaeological evidence to allow a partial reconstruction of the early stages of agricultural prehistory in China 
and Southeast Asia. This has not always been the case; until a few years ago it was fairly commonplace for geographers and botanists to offer purely hypothetical reconstructions that were always plausible but never testable. One was the well-known theory of Sauer (1952) that agriculture arose with the vegetative planting of tubers and other useful plants in the monsoon regions of Mainland Southeast Asia, especially in resource-rich coastal and riverine situations where varieties of useful plant species were available for many purposes as well as food. The literature that has grown up around Sauer's theory is too extensive to review here, but I must question (without necessarily entirely rejecting) two widely accepted corollaries of his views. One is that agriculture based on the vegetative planting of trees and tubers long preceded the cultivation of cereals in Southeast Asia. The other is that agriculture began in "affluent forager" conditions of leisure and plenty that allowed experimentation.

As far as the first corollary is concerned, I accept Gorman's suggestion (1977) that rice was one of the earliest plants to be domesticated in China and Mainland Southeast Asia. Archaeological evidence from China supporting this observation is now prolific and will be reviewed below; there is no direct archaeological evidence supporting an earlier stratum of noncereal cultivation, except in New Guinea, an area of only marginal relevance for early agriculture in Southeast Asia. However, it must be noted here that some of the oldest Neolithic sites in Southeast Asia have not yet produced direct evidence for rice (or for any other domesticated plants for that matter); such sites include Nong Nor in Thailand (ca. 2500 BC; O'Reilly 1995) and the sites of the Ta-p'en-k'eng culture in Taiwan (ca. 3000 в ; Tsang 1992). This problem could simply be one of sampling, but the possibility that rice was not universally grown by some of the earliest agricultural peoples to penetrate Southeast Asia must also be considered. I will return to this very important question later.

In addition, it is entirely plausible that hunter-gatherer populations such as the Hoabinhians practiced occasional protection and even some casual cultivation of forest tubers and fruit trees before systematic agriculture began. The main conceptual difference here is not between totally foraging and totally agricultural economies-few (perhaps no) populations in real life occupy such extreme poles. But a foraging economy with some casual cultivation does not necessarily carry the same evolutionary potential as an agricultural economy with some foraging. Those forager societies that eventually moved into systematic rather than casual agriculture were given unprecedented opportunities for demographic growth, especially if they grew cereals (as they no doubt did in China).

The second "affluence" corollary has long been favored by K. C. Chang $(1981,1986)$ for China and is currently popular amongst archaeological theoreticians in various forms for other parts of the world, centered mainly on con- 
cepts of competitive feasting as spurs to increased food production (e.g., Hayden 1990; Blanton and Taylor 1995). Of course, starving people are perhaps the least likely of any to "invent" agriculture, and relative affluence might have characterized many of the hunter-gatherers who first made the transition to agriculture. Even so, it is difficult to see affluence and social competition as having major causal roles in themselves, partly because so many ethnographic foragers have had such competitive economies without making any transition to agriculture at all (e.g., western North America).

Instead, the majority of published opinions in the 1990s regard the origin of agriculture as a reaction to encouraging and directed forms of stress, whether due to direct demographic pressures on resources or to less specific environmental or seasonal perturbations affecting resource availability (McCorriston and Hole 1991). One very common assumption is that hunter-gatherers will not successfully turn to cultivation-which intermittently requires higher inputs of labor and forward planning for land clearance, weeding, and harvestingunless they are forced to do so by an insistent and growing imbalance between population size and available food. The ethnographic record in Australia, Southeast Asia, and other regions makes this conclusion virtually unavoidable (Headland 1986; Bellwood 1996b).

This general stress-focused view has always seemed to me to be very plausible, but alone it can hardly explain why agriculture should have developedapparently independently-in a number of hearth regions in the Old and New Worlds around and immediately following the end of the Pleistocene. Since the first edition of this book was published, the whole topic of agricultural origins has snowballed into one of the biggest debates in archaeology, and of course the issues cannot be covered fully here-they demand a book in themselves (MacNeish 1992 represents one try, but with only limited applicability to eastern Asia). It is now evident that agriculture in Southwest Asia and perhaps China (I am reluctant to include other areas such as the Americas) evolved amongst "complex" terminal Pleistocene foragers who were already practicing some degree of sedentary life and food storage, and that it evolved in those regions where the plant species concerned were somehow "stressed" in supply, perhaps as a result of being at the edges of their distributional ranges and thus very vulnerable to minor environmental changes (for some discussion of these points see Bellwood 1990b, 1996a, b).

The record from Southwest Asia is the most detailed in the world for an early transition to agriculture. In general, last glacial environments in Southwest Asia seem to have been cold, relatively treeless, and perhaps too dry for large-grained annual grasses to have flourished widely. A major environmental change, the most major and most rapid in world history since the beginning of the last interglacial 130,000 years ago, then commenced with the spread of a warmer 
climate, apparently with a more dependable winter wet season, after about 14,000 years ago. The rapidity and magnitude of this change perhaps indicates why agriculture developed at this time rather than at various times back into the late Pleistocene. The result of this environmental change was a spread of annual grasses and nut-bearing tree species that allowed some groups, such as the Natufians of the Levant, to inhabit small but fairly permanent villages supported by food storage for lean seasons. Increasing sedentary settlement should then have allowed more frequent births, leading to an inexorable rise in population (as indicated by modern studies on recently settled hunter-gatherers; e.g., Gomes 1982 for settled Negritos in Malaysia). This in itself might have been sufficient to encourage groups to increase their grain yields by systematic cultivation, but a postulated shift back to a cooler and drier climate in the Levant after 11,000 B P (the "Younger Dryas") might have provided an extra stimulus in this regard. Soon after 10,500 years ago, the erstwhile Natufians were building large pre-pottery Neolithic villages such as Jericho, with some villages (Abu Hureyra, Ain Ghazal) attaining sizes over 10 hectares by 9,500 years ago. If this postulated trajectory is correct, the late Natufians were probably the first people in the world to cultivate grains-and we may almost know why (for references on the above see Bellwood 1996b).

\section{A. The Beginnings of Agriculture in China}

Note: The term China refers here to a geographical region, and not purely to the region currently inhabited by the Sinitic (Chinese-speaking) people. Some of the prehistoric societies of the region were perhaps ancestral to modern Chinese. Others, especially those to be described below, certainly were not and are better regarded as ancestral Southeast Asian in cultural affiliation.

It is quite difficult to apply the Southwest Asian model directly to eastern Asia because the necessary details in the archaeological record simply are not there, although this need not imply that a different model is required. Rice and millets were being cultivated in village settlements in central China by at least 8,000 years ago (Chang 1986; Yan 1991, 1992; Shih 1992; Jiang 1994), but in China the Neolithic as known to date begins with the presences of pottery and domestic animals, neither of which appeared in Southwest Asia until up to two millennia after the first evidence for plant cultivation. This suggests strongly that the indigenous roots of agriculture in China still remain to be discovered. Agriculture in China seems unlikely to be a simple transference from Southwest Asia as the crops, technologies, and people in the two regions are quite unrelated.

As in Southwest Asia, the climatic amelioration at the end of the Pleistocene in China involved a rise of temperature, approaching present levels by 13,000 
$\mathrm{BP}$, and also the development of a much stronger summer monsoon (see Chapter 1), which would have encouraged dispersal of the ancestors of the cultivated annual millets and rice, both summer monsoon crops (Whyte 1983; Oka 1988; Chang, T. T. 1989). Indeed, climatic conditions in China between 10,000 and 4,000 years ago were slightly warmer and wetter than now and would have been very favorable for summer rice crops in the swamps and lakes of the Yangzi valley (Chang, K. C. 1986:211-212). As in Southwest Asia, there is some evidence that the overall climatic amelioration was temporarily reversed by a Younger Dryas interval between 11,000 and 10,200 years ago, but whether this would have had any impact on the shift to cultivation, as currently claimed by some scholars for Southwest Asia (Moore and Hillman 1992), remains uncertain. Any temporary shortening of the summer growing season on the northern edge of wild rice distribution could, however, have been quite significant for populations targeting it as a food source.

Cultivated rice, Oryza sativa, was derived from the wild Oryza rufipogon with both annual and perennial habits according to Oka (1988), but via an intermediate wild annual form Oryza nivara according to T. T. Chang (1976b) (see Plate 38). According to Oka (1988:131-132), wild rice today grows only as far north as Fujian, but grew as far north as the Yangzi until the Song dynasty. In the warmer climatic conditions of the early Holocene we might expect wild rice to have grown well north into the region between the Yellow and Yangzi Rivers. It certainly appears to have been first domesticated in the swampy regions lining the middle and lower courses of the Yangzi River (Glover 1985; Yan 1991, 1992; Bellwood et al. 1992; Y. Wu 1996), and thus toward the edge of its natural range in a region where winter storage and synchronized annual planting would have been encouraged.

The morphological and genetic changes in rice-reduced shattering, greater synchronicity of germination and ripening, increased panicle (rather than grain) size, reduced awns and glumes, tendency to evolve self-pollination-were probably stimulated by the same kinds of harvesting and management activities that led to the related changes in wheat and barley in Southwest Asia (Oka 1988; T. T. Chang 1989; Thompson 1992). Such activities include the use of a sickle/ reaping knife, selection of nonshattering stock for planting, planting outside the areas of wild stands, and winter storage of seed leading to reduced selection pressures for tough protective glumes. According to Oka (1988:101), some of these changes could have taken place rapidly. The rice cultivation of Hemudu in Zhejiang Province (c. 5000 BC) is described by Yan (1992:121) as being already "of a fairly advanced form," and according to Li Kunsheng (1985) the Hemudu rice was already differentiated into indica and japonica varieties.

The overall trajectory of the oldest Chinese Neolithic cultures can be summarized as follows (for site locations, see Fig. 7.1): 


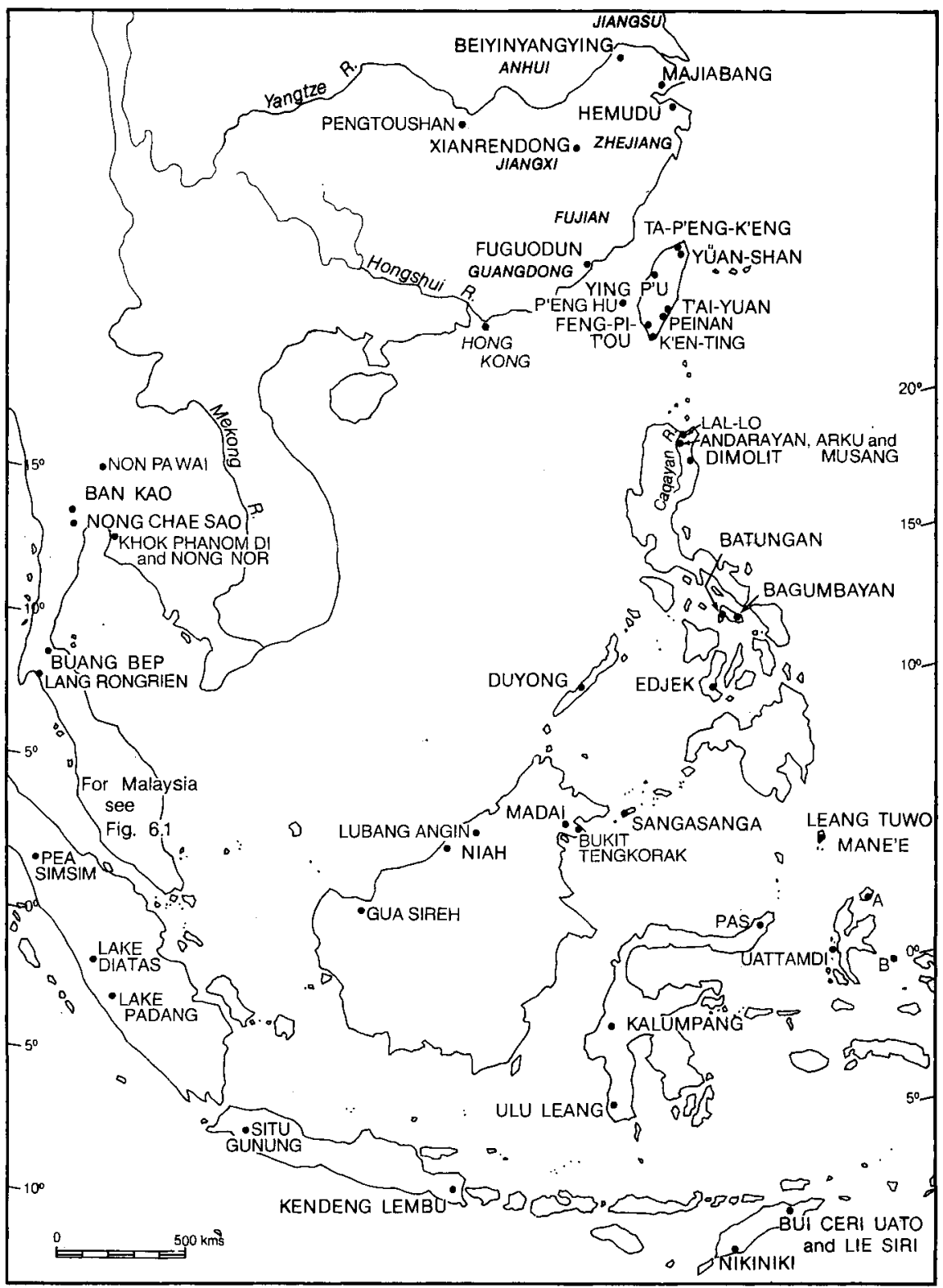

Fig. 7.1 Locations of sites described in Chapter 7. A = Tanjung Pinang; B = Buwawansi. 
1. $6000 \mathrm{BC}$ : A cluster of related millet-growing sites developed in the Yellow River basin (Peiligang, Cishan: Shih 1992, plus the newly discovered Pengtoushan rice-growing complex in the middle Yangzi (Yan 1992). From published data it is not clear whether we have here one primary development of agriculture with two minor sub-foci, or two independent developments, but numerous finds of rice in the Yellow River region indicate that all these groups were in some degree of contact Jiang 1994; Wu Yaoli 1996). Suggestions that some cave sites in southern China have older pottery than the above are suspect in the absence of well-verified open sites.

2. 5000 вС: The Yangshao, Dawenkou, Hemudu, and Majiabang cultures all developed in central China, plus assemblages distributed down the southern coast of China to Fujian and Guangdong (K. C. Chang 1995; Tsang 1992, 1995; and see Meacham 1984-1985a for some possible dates of this order for painted pottery assemblages from Hong Kong and Macau). The assemblages from coastal regions south of the Yangzi, including Taiwan, are all fairly closely related in terms of ceramic detail (e.g., red slip, cord marking, perforated pedestals, general shape repertoire, etc., not to mention the broad range of nonceramic material culture such as stone adzes and stone reaping knives ${ }^{1}$ ). However, direct evidence for rice cultivation is so far absent from the oldest sites in Guangdong, Taiwan, and Hong Kong, despite a definite presence by 3000 вс at the site of Shixia in Guangdong.

There can be no doubt that one of the most important sites excavated in recent years with respect to rice cultivation is Hemudu, which lies on a flat alluvial plain about 25 kilometers to the south of Hangzhou Bay in northeastern Zhejiang (Chekiang 1978; K. C. Chang 1986:208-224; Liu 1985). The bottom waterlogged layer of this site (layer 4 ) dates to between 5200 and perhaps 4900 $\mathrm{BC}$ and belonged to a village of rectangular timber houses (one being 7 meters wide by over 23 meters long) constructed with skillful mortice and tenon techniques and raised on rows of small timber piles. Large quantities of rice husks were found as temper in the sherds, and in one area of the excavation a solid mass of rice husks, grains, straw, and leaves formed a layer-perhaps once a threshing floor-with an average thickness of 40-50 centimeters. This rice was presumably cultivated in the alluvial soils around the site, possibly with a range of aquatic plants in low-lying swamps (H-L. Li 1983). Pigs accounted for 90 percent of all animal bones; it is assumed that they were domesticated, together with dogs and possibly water buffalo (domesticated fowl are known from other contemporary Chinese sites). The range of mammals hunted included deer, rhinoceros, elephant, and monkey; birds were hunted as well. An interesting occurrence amongst the plant remains is the gourd (Lagenaria siceraria), a plant of widespread importance in Southeast Asia and Oceania. Pollen of species 


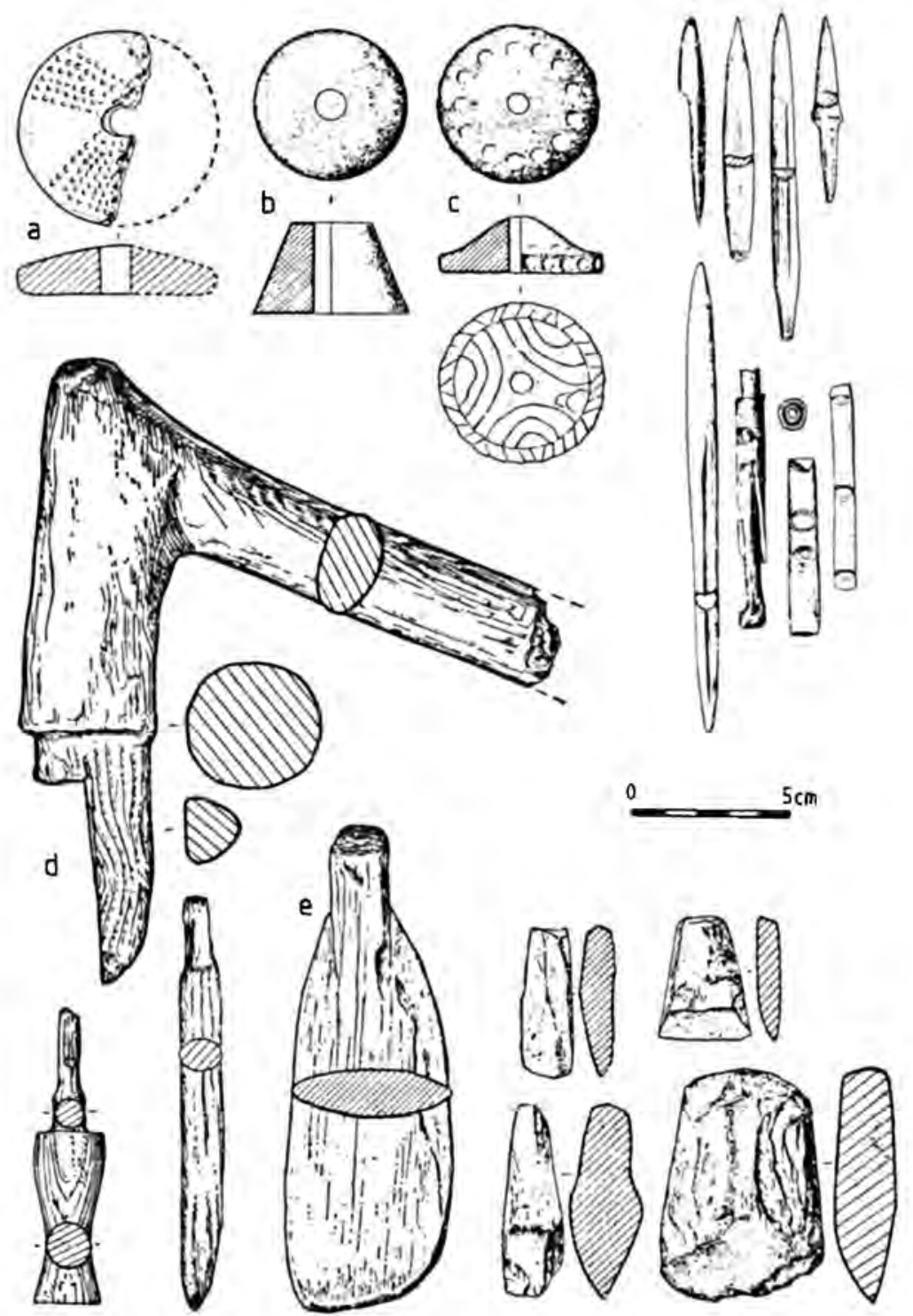

Fig. 7.2 Artifacts from Hemudu layer 4, ca. $5000 \mathrm{BC}$ : (a-c) perforated clay discs, possibly spindle whorls or ornaments (compare Fig. $7.6 f$ from Taiwan); (d) wooden adze handle; (e) wooden spade blade (?). Other objects shown are bone points and whistles (top right), wooden objects (bottom left) and stone adzes, including one with an incipient step (bottom right). From Chekiang 1978. 


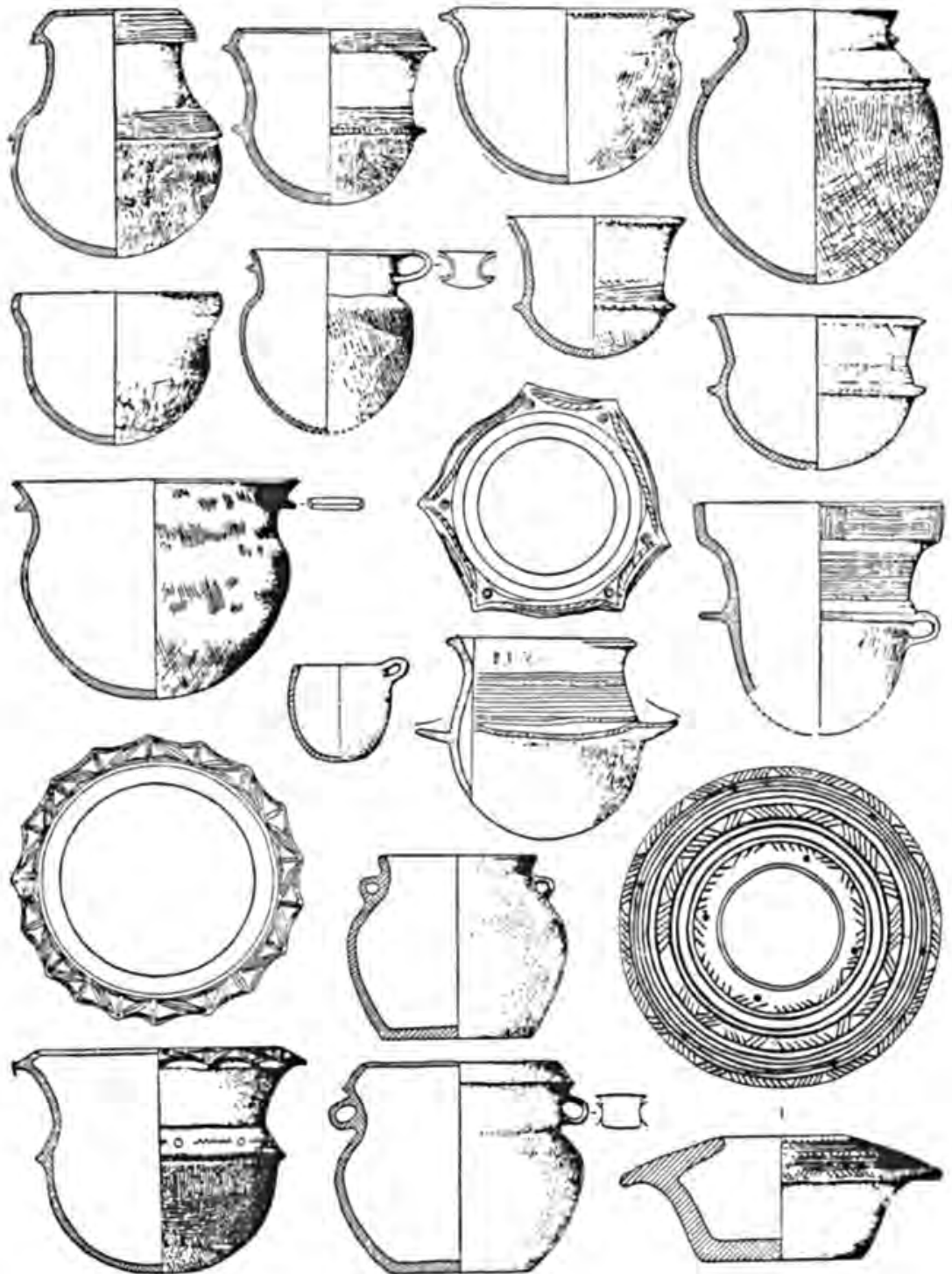

Fig. 7.3 Pottery from Hemudu layer 4, ca. 5000 BC. From Chekiang 1978.

found today only in Guangdong and Taiwan suggest that the climate may have been a little warmer then than now.

The material culture of Hemudu (Fig. 7.2 and Fig. 7.3) is particularly impressive. It includes hoes made from animal scapulae, bone tools and whistles, jade penannular earrings, and stone adzes with oval or quadrangular cross-sections, 
some resembling the stepped form of the Ta-p'en-k'eng culture of Taiwan (below). Knee-shaped adze hafts were found-this shape occurs in initial Jomon Japan and almost universally amongst the Austronesian peoples of Oceaniaand there are also some detachable wooden spade blades. Pottery items include spindle whorls and a range of round or flat-based, cord-marked vessels, the former often having carinated bodies and incised rims. Pot stands, pottery stoves, animal figurines, footed dishes, and lids also occur, and there are a few painted sherds. This pottery tradition shows that the totality of potting knowledge found in the early cultures of Taiwan and Island Southeast Asia was already present in this region a millennium before the beginnings of Austronesian expansion.

I have examined the consequences of the shift to agriculture in China elsewhere (Bellwood 1994, 1995b, 1996a, b). They were massive indeed if we examine both archaeological and linguistic sources of evidence, leading to a demographically driven dispersal of populations that encompassed the next five millennia and over half the world's surface (including Oceania). One part of this dispersal, as a peripheral offshoot from the southeastern part of the early Chinese agricultural domain, is represented by the Austronesians. Their Austroasiatic contemporaries in the Malay Peninsula are dealt with in Chapter 8.

\section{THE BEGINNINGS OF AUSTRONESIAN PREHISTORY}

I now wish to move straight into a review of the modern archaeological evidence pertinent to Austronesian dispersal. It is not my intention to review the outdated but historically interesting theories about stone adze types and waves of migration into the archipelago-readers will find these details in a previous book (Bellwood 1978:170-175, 207). Solheim (e.g., 1975, 1979a, b, 1984-1985) has also developed a nonlinguistic theory of Austronesian origins amongst mobile trading sea peoples in eastern Indonesia, with secondary movements up to the south Chinese coast and then eventual back movements of Malays and Chams from south China into the archipelago. A similar theory has been developed without reference to linguistic data by Meacham (1984-1985b, 1992). These theories differ from my own in several fundamental respects, as this chapter will indicate.

\section{A. The Prehistory of Taiwan}

Prior to the Neolithic, the island of Taiwan has only a limited record of flaked stone assemblages (the "Ch'angpinian"), probably related to the Hoabinhian, which survived in the south and east of the island until about $3000 \mathrm{BC}$ (Sung 1979; K-C. Li 1983a). ${ }^{2}$ (For recent reviews see K-C. Li et al. 1989; P. J-K. Li et al. 

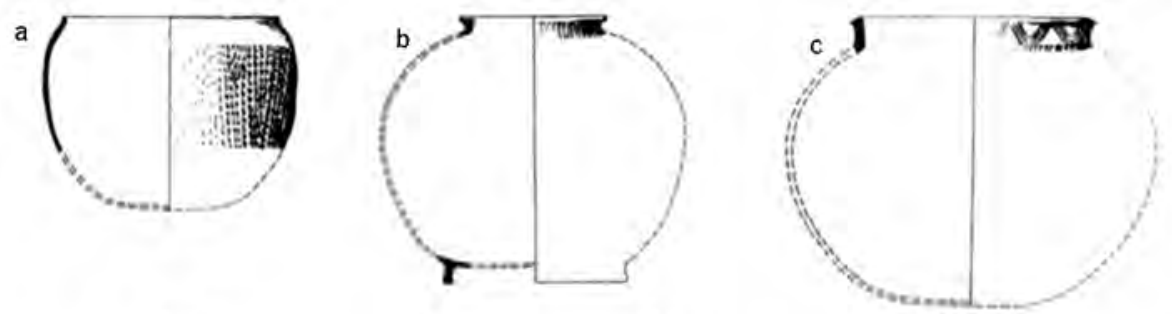

e
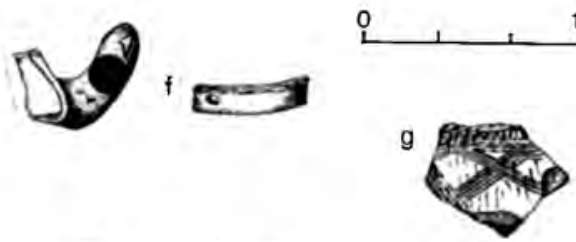

$15 \mathrm{~cm}$
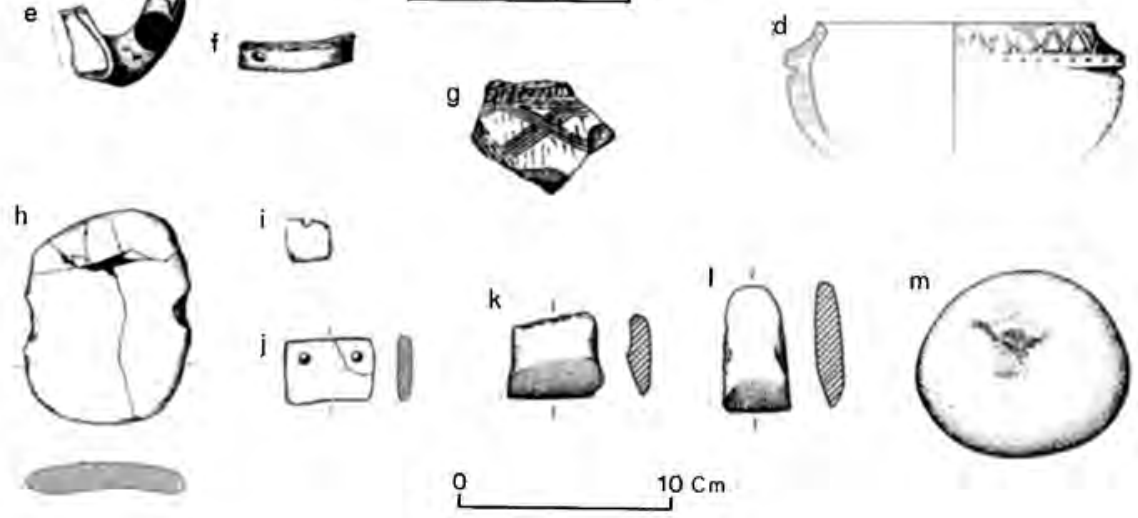

Fig. 7.4 Artifacts of the Ta-p'en-k'eng culture: $(a-g)$ cord-marked and incised pottery, including a lug (e) and a perforated ring-foot (f); (h) waisted "net sinker"; (i) perforated slate projectile point; (j) clay pendant (?); (k) stepped adze; (l) adze; (m) pitted pebble anvil. From Chang 1969. Courtesy: Yale University, Department of Anthropology.

1995.) The oldest Neolithic assemblages belong to a culture with cord-marked and incised pottery, termed the Ta-p'en-k'eng (henceforth TPK) culture by Chang (for details see K. C. Chang 1969, 1970, 1986; Chang et al. 1974; Dewar 1977). One of these sites (at Pa-chia-ts'un near T'ainan) has a single radiocarbon date of about $4300 \mathrm{BC}$, and the culture appears to have continued in existence in western Taiwan until about 2500 BC (Pearson 1989; Spriggs 1989:605). Characteristic artifacts (Fig. 7.4) include cord-marked globular pots with incised everted rims and occasional lug handles or ring feet (some with perforations), stone adzes with quadrangular cross-sections and occasionally with hafting steps, polished slate points, stone net sinkers, and possibly a stone bark-cloth beater (an artifact type also reported from Neolithic sites in southern China). The whole culture gives the appearance of having been introduced into the island fully formed.

Sites related to the TPK culture have been recently excavated by Tsang (1992; 1995) in the Pescadores (P'eng-hu) Islands, which lie 45 kilometers west of Taiwan and about 140 kilometers from the Chinese mainland. The first phase rep- 
resented here is the Kuo-yeh, dated from 3000 to $2500 \mathrm{BC}$. It contains a material culture of TPK type-without rice or reaping knives-that Tsang believes was brought by colonists from Taiwan. Rice (as husks in pottery), stone knives, and bone fishhooks make an appearance in the next phase, termed the Suo-kang, after 2500 в .

The economy of the TPK culture has been a matter of some obscurity as cereal remains have not yet been reported from the major excavated sites. The prehistory of millet in Taiwan is a complete mystery, even though linguistic reconstruction suggest a great antiquity for it (Zorc 1994:549). Nevertheless, given the importance of rice cultivation in the mainland province of Zhejiang at least a millennium before the Taiwanese Neolithic commenced, I would be most surprised if this crop was totally absent on the island before $2500 \mathrm{BC}$. Most authorities on Taiwanese prehistory (e.g., Tsang, above) believe that cereals were not introduced into the island until after $2500 \mathrm{BC}$, when stone reaping knives became common in the western region. However, the pollen diagram from the Sun-Moon Lake in the mountainous center of Taiwan indicates a marked increase in large grass pollen, second-growth shrubs, and charcoal particles soon after 3000 BC (Tsukada 1966, 1967). As this region is rather remote from the coastal areas where early agriculture must first have been established, then it follows that agriculture (if this is what the core records) must have appeared long before this date in Taiwan. More significantly, actual rice remains (apart from those of the Suo-kang phase, above) have been reported from the Chih-shan-yen site in Taipei (ca. 1500-2000 BC; Wang 1984), and rice impressions in pottery are reported from the K'en-ting site in the far south of the island (ca. $2500 \mathrm{BC}$; K-C. Li 1983a), although both of these sites belong to cultural phases apparently following the TPK.

The immediate origins of the TPK culture clearly lie on the Chinese mainland. K. C. Chang (1977) has drawn attention to TPK ceramic parallels with the shell-mound site of Fuguodun in nearby Fujian, dated between 5200 and 4200 BC. From here there are potsherds decorated with incised lines, impressed rows of semicircles, and zoned patterns within incisions made by dentate stamping with the toothed edge of an Anadara shell. More recently, both K. C. Chang (1995) and C-H. Tsang have looked in great detail at the question of TPK relations with the Chinese mainland and point to a very large number of pertinent sites, mostly shellmounds and some with evidence for stilt houses (Tsang 1992: 246; 1995). These sites include several in Guangdong (Tsang 1992:245-246), plus several farther north in Fujian (Xitou, Keqiutou, early Tanshishan), and there can be little doubt that the similarities indicate contact if not common population origin. Importantly for questions of navigational and canoe-construction technology, several of these sites are on small offshore islands (as are some of the Hemudu culture sites in Zhejiang). Whether these material culture 
similarities indicate borrowing by native Ch'angpinians of Taiwan from mainland sources, as suggested by Meacham (1995), or whether they represent an actual colonization of Taiwan by pre-Austronesians of mainland origin, is a question that will doubtless exercise the minds of archaeologists for some time to come. I have no doubts about my own opinions, which, as clearly stated above, give much credence to the results of comparative linguistics.

Pre-Austronesians therefore colonized Taiwan from Fujian or Guangdong, but when did their descendants, following the break-up of Proto-Austronesian, begin their moves into the Philippines? The answers to this question are to be found in the post-TPK cultures of Taiwan. By the late third millennium BC the TPK culture had apparently differentiated into at least three, perhaps four regional archaeological complexes, possibly already with initial linguistic differentiation into the several first-order Austronesian subgroups that are believed to exist on Taiwan (see Chapter 4). In the west and south of the island there are cultures with red cord-marked pottery, originally termed Lungshanoid by K. C. Chang (1969). These are best known from Chang's excavations at Feng-pit'ou and from the Choshui and Tatu River sites (including the site of Niu-mat'ou) in the west-center of the island (K. C. Chang et al. 1974; Dewar 1977; Stamps 1977), and also from sites with slightly different assemblages in the far south of the island (K'en-ting and O-luan-pi; K-C. Li 1983a, 1983b). Dates run from about 2500 to $500 \mathrm{BC}$. The Feng-pi-t'ou assemblage includes red cordmarked and painted pottery with a considerable elaboration of form, represented by tripods, high perforated ring feet, bottle forms, and the use of a slow wheel (Fig. 7.5). In addition there are clay spindle whorls, bone points, large

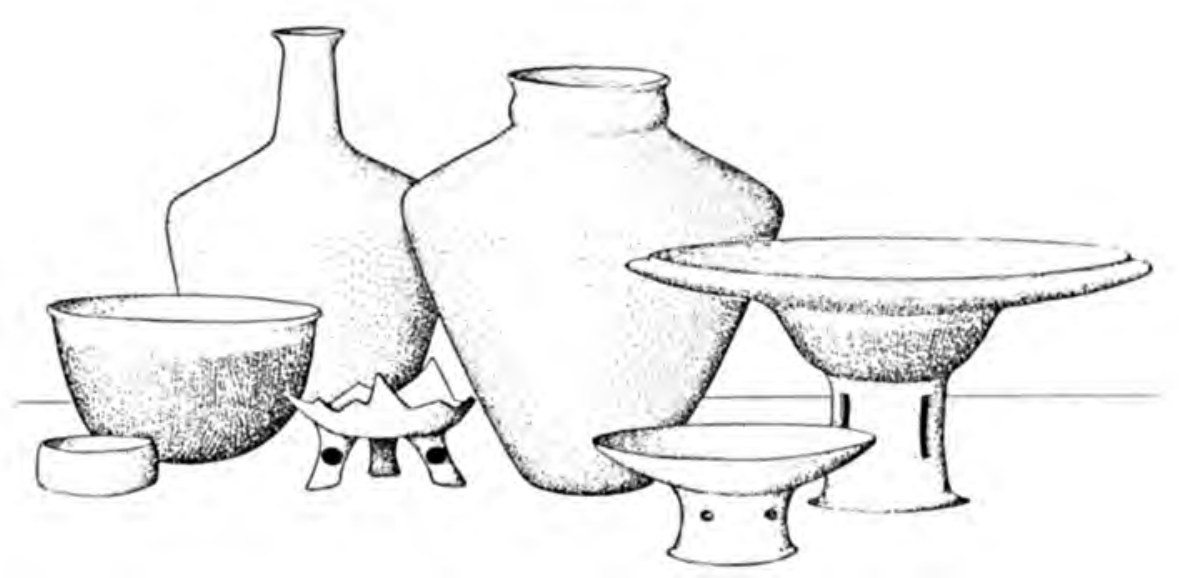

Fig. 7.5 "Fine red ware" from Feng-pi-t'ou, ca. 2000 BC. From Chang 1969. Courtesy: Yale University, Department of Anthropology. 
numbers of ground slate reaping knives and projectile points of coastal Chinese types, and stone hoes and adzes (untanged and shouldered). The postholes of part of a rectangular house were excavated at Feng-pi-t'ou, together with a burial stated to have Mongoloid affinities.

The Lungshanoid cultures of western Taiwan do not appear to be of direct significance for the settlement of the Philippine and Indonesian islands to the south. However, I think the situation may be different with the Yüan-shan culture of northern Taiwan, which was derived according to all authorities by local development from the preceding TPK culture (possibly via the Chih-shan-yen culture with its domesticated rice in the Taipei region; Wang 1984). Dates for Yüan-shan and Chih-shan-yen assemblages range from $2500 \mathrm{BC}$ into the first millennium BC. Yüan-shan pottery is characterized by globular vessels with ring feet and strap handles, decorated with some incision or punctation and red or brown slips (Plate 30). Cord marking and tripods are absent; this is quite significant because the oldest pottery assemblages in the Philippines and Indonesia also lack these features. In addition, the slate reaping knives are absent in the Yüan-shan culture, as they are again in the later sites to the south (although they are present in the west of the island). So if rice cultivation continued, people may have turned to bamboo knives, as used today for millet in highland Taiwan (see Note 1, pages 316-317).

Other Yüan-shan items include untanged, shouldered and stepped quadrangular adzes, slate projectile points, chipped stone hoes, stone bark-cloth beaters, and spindle whorls of clay (Fig. 7.6). The latter are of interest as they also occur in the red corded-ware sites of western Taiwan and in the southern Chinese Neolithic cultures; they suggest that a knowledge of weaving, perhaps using hemp fibers on a backstrap loom, was present. Domestic dogs are also claimed from some Yüan-shan sites, but the presence of domesticated pigs seems uncertain in the Taiwanese Neolithic (pig bones are present in many sites, but perhaps they were hunted).

Down the eastern coast of Taiwan, Pearson $(1968,1969)$ has investigated sites of the related T'ai-yuan culture; these are generally undated but they seem to be associated with stone-slab graves, sarcophagi, and uprights, together with Yüan-shan style pottery with the characteristic ring feet and loop handles. Since the first edition of this book was published, however, east coast Taiwan archaeology has been illuminated by some quite remarkable discoveries at the 40 - to 80-hectare village site of Peinan (Lien 1989, 1991, 1993). The excavations here took place owing to railway construction and have yielded remains of fifty house foundations and over 1,500 burials, dating mainly between 1500 and 800 $\mathrm{BC}$, but with an earlier and less well understood component related to the TPK culture beneath. The houses were constructed on rectangular stone pavements and laid out in rows, with adjacent rows of stone-walled storehouses (Plate 31 ). 

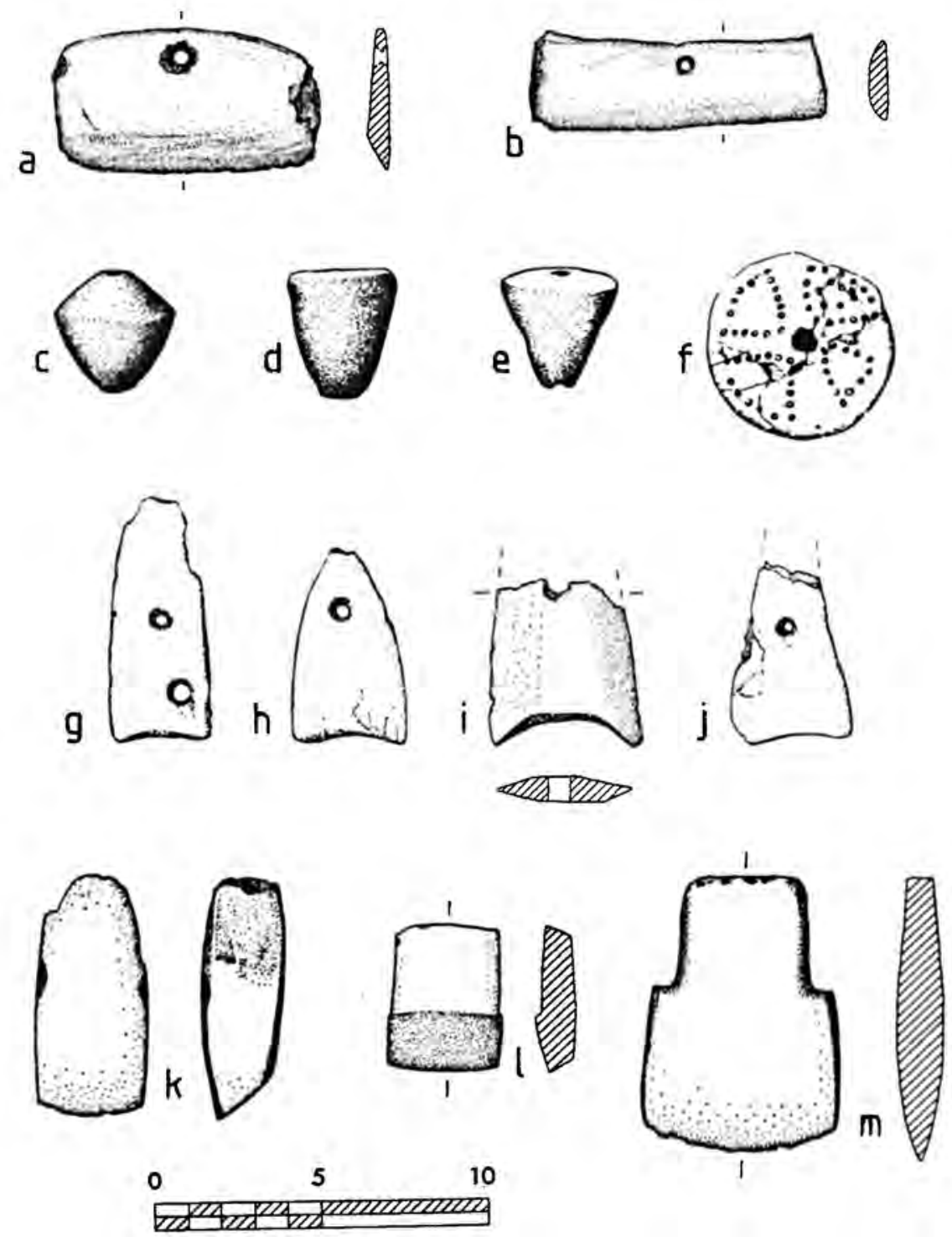

Fig. 7.6 Top two rows show artifacts from Feng-pi-t'ou: (a, b) stone knives; (c-e) baked clay spindle whorls; ( $f$ ) clay disc (compare Fig. 7.2a). Bottom two rows show artifacts from Ta-p'en-k'eng (Yüan-shan culture): ( $g-j)$ perforated stone points; (k) untanged adze; (l) stepped adze; (m) shouldered adze. Scale in centimeters. From Chang 1969. Courtesy: Yale University, Department of Anthropology. 
Some of the rows were separated by boulder walls, suggesting perhaps lineage divisions of some kind within the village plan. The floors of the dwelling houses sealed slab-lined burial cists (Plate 32), an arrangement indicating a definite interest in ancestor veneration on the part of the inhabitants. The graves, many multiple, revealed a high rate of infant and fetal death, for reasons at present unknown (Lien 1991: 344). ${ }^{3}$

The pottery from the Peinan graves is mainly a fine orange ware, sometimes red slipped, with no other forms of decoration. The most common form appears to be a jar with two vertical strap handles (like Yüan-shan pottery) and a ring foot. Spindle whorls, pig and dog figurines, and stone bark-cloth beaters also occur in the site. The grave goods include some remarkable items of Taiwan jade: tubular beads, bracelets, penannular earrings with circumferential projections (the so-called lingling-o, a very widespread form in Southeast Asia; see Chapter 9), anthropomorphic earrings (Fig. 7.7), and perforated projectile points. Most adults had four of their upper teeth extracted-canine and first incisor on each side-and stained teeth attest to betel chewing. Lien also suggests (1991: 350) that both rice and millet were cultivated. The Peinan culture is also claimed by Lien to have living representatives in the Paiwan peoples of eastern Taiwan.

Looking at Taiwanese prehistory from an Indo-Malaysian perspective, it is obviously the cultural phase prior to about $2000 \mathrm{BC}$ that is of most interest, since it is clear that Austronesian settlers had already moved into the Philippines and perhaps Indonesia by this time. The Peinan culture is thus a little late to be of direct interest, but its plain pottery style finds some affinity with the oldest pottery assemblages to the south. The TPK culture is of enormous importance as a potential record of the oldest stage of Austronesian society that can be identified on linguistic grounds (i.e., Initial Austonesian). Not only does this culture have clear mainland Chinese origins, but it also has what I believe are clear successors in the Yüan-shan and Peinan cultures and the earliest Neolithic cultures of the Philippines and Indonesia.

It is still uncertain when rice cultivation appeared within this cultural sequence; my suspicion is that rice remains will turn up in TPK sites eventually. Although most highland Austronesians of Taiwan grow hardier millets today (Setaria, Panicum, and the more recent Indian Sorghum and Eleusine; Chen 1968; Fogg 1983), it must be remembered that the warmer western lowlands where the oldest Neolithic sites occur are now entirely settled by Chinese rice growers. Other aboriginally cultivated plants in Taiwan include sugarcane and gourd, and it is highly likely that these were grown, with Setaria (foxtail) millet, by TPK societies as well; all are of at least Proto-Austronesian antiquity. Although coconuts and breadfruit grow in southeastern Taiwan today, it seems more than likely that these two tropical species were introduced later into the warmer parts of the island from the Philippines. 

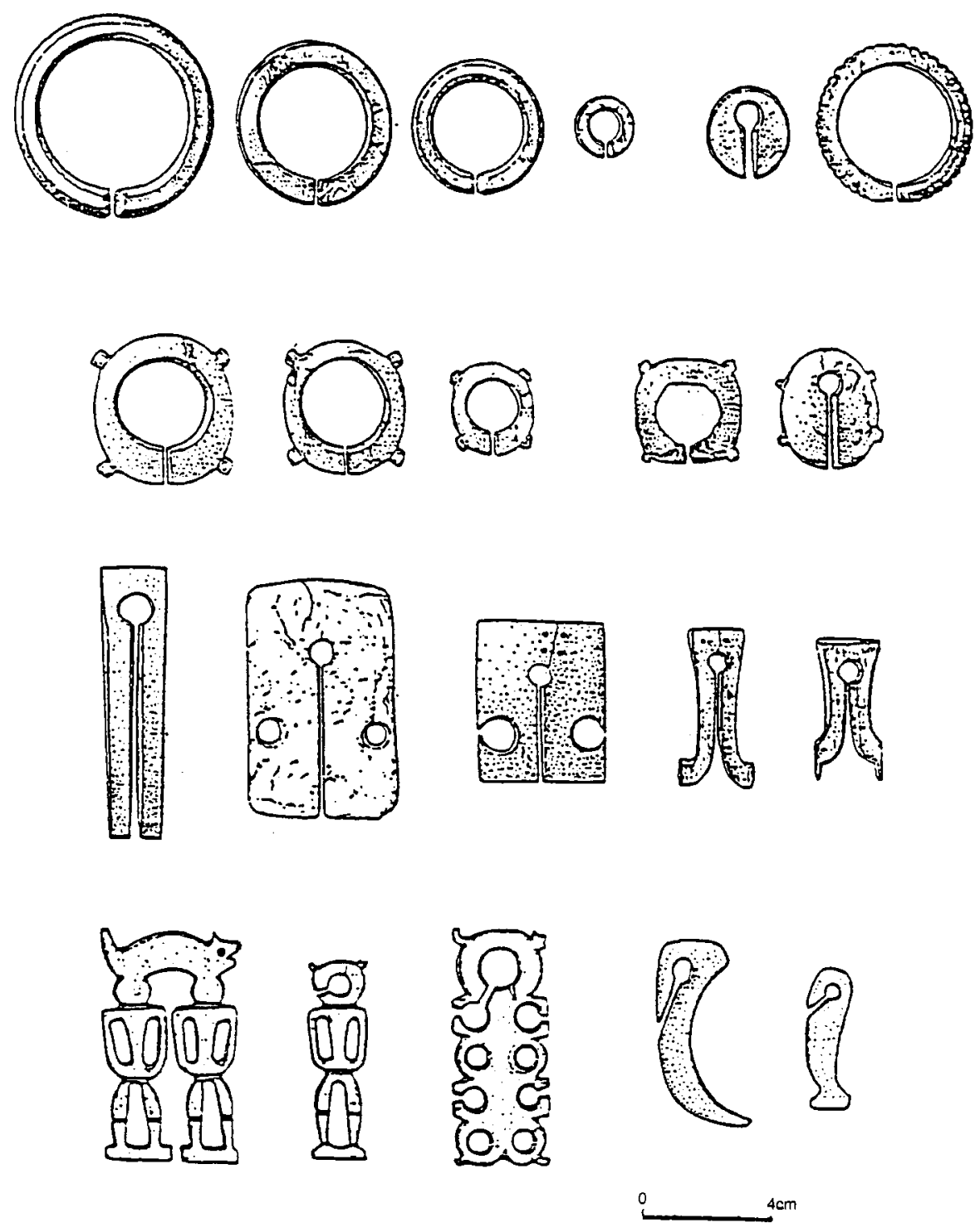

Fig. 7.7 Four major types of nephrite (jade) slotted earrings from the Peinan excavations, Taiwan. From Lien 1991. Courtesy: Lien Chaomei. 


\section{THE NEOLITHIC PHASE IN ISLAND SOUTHEAST ASIA AND WESTERN OCEANIA}

I will now consider the archaeological evidence for Neolithic assemblages in the Philippines and Indonesia, and will later expand this evidence into a larger picture of economic change and adaptation. However, I should first go back again to the Neolithic cultures of Taiwan between 3000 and $2000 \mathrm{BC}$ and note the material items present. These include quadrangular cross-sectioned adzes, bone and slate projectile points, and a pottery tradition that trends through time from a predominance of cord marking toward an emphasis on plain or slipped bodies (in the case of the Yüan-shan and Peinan cultures), with the continuing presence of incised, stamped circle, and punctate decoration and perforated ring feet. Other items include stone net weights, hoes, and possibly bones of domesticated pigs and dogs. Reaping knives and spindle whorls are not clearly present until after $2500 \mathrm{BC}$.

In the Philippines, northern Borneo, and many regions of eastern Indonesia, the oldest Neolithic pottery is characterized by simple forms with plain or redslipped surfaces, sometimes with perforated ring feet. This phase has no very clear internal divisions at present; it seems to continue everywhere with no marked breaks into the last 2,000 years. However, it is convenient to separate the post-500 $\mathrm{BC}$ cultures-those with metallurgy and predominantly incised pottery-from those that went before; these later cultures will be considered in Chapter 9. In the first edition of this book, I also separated a number of decorated pottery assemblages as "late Neolithic" and treated them separately, but subsequent research and dating has left little rationale for this decision. In this chapter I will cover all Neolithic sites in Island Southeast Asia, leaving the Malay Peninsula-a completely separate cultural entity from the islands during the Neolithic-for consideration in Chapter 8.

The most varied Neolithic assemblages occur in the northern Philippines, which is what could be expected given their closeness to Taiwan. The more southerly sites in Borneo, Talaud, the Moluccas, Sulawesi, and Timor show some degree of attenuation of material culture. Java and Sumatra are unclear in this respect because few presumed Neolithic sites there have been excavated-and none dated.

\section{A. The Philippines}

The Philippines reveal a widespread horizon of red-slipped pottery beginning perhaps around $2500 \mathrm{BC}$. In northern Luzon, an important open site called Dimolit has been excavated by Peterson (1974) on Palanan Bay in Isabela Province. The lower occupation level here has three rather widely spread radio- 


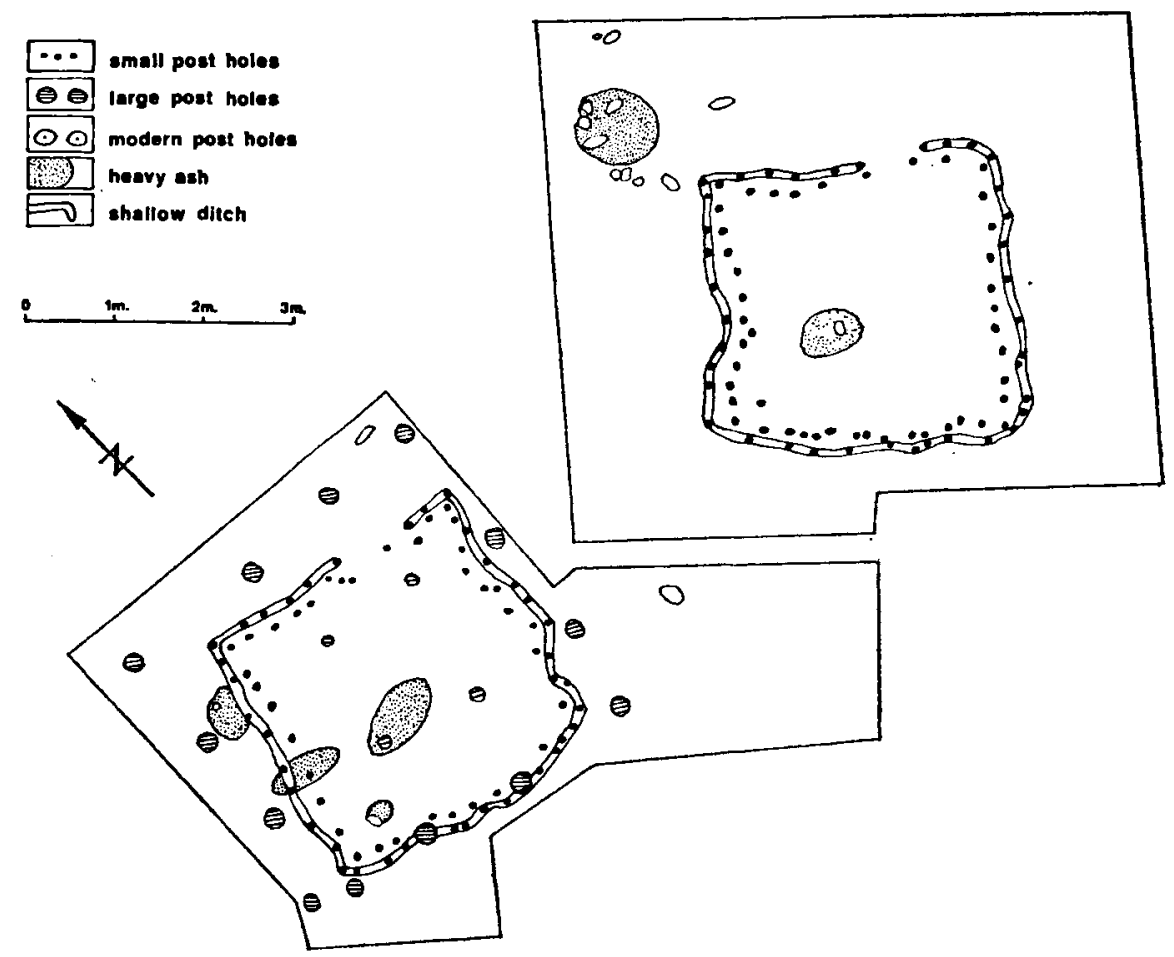

Fig. 7.8 House plans from Dimolit, northern Luzon. From Peterson 1974. Courtesy: University of Sydney.

carbon dates, but was probably occupied between 2500 and $1500 \mathrm{BC}$. Posthole settings for two $3 \times 3$-meter square houses were found, each with double walls, the outer post row being set in a slot (Fig. 7.8). The pottery is plain or red slipped and comprises globular or carinated vessels and dishes, some on ring feet with small, clustered perforations.

Many other sites in northern Luzon-located in the main valley and tributaries of the Cagayan River-have yielded types of pottery similar to Dimolit. The caves of Rabel and Laurente have yielded dates for it that might be as early as $2800 \mathrm{BC}$ (Spriggs 1989:593), and at Andarayan similar red-slipped pottery has been dated to $1500 \mathrm{BC}$, here with rice chaff temper, pottery stoves, and spindle whorls (Snow et al. 1986). Musang Cave (Thiel 1988-1989) has yielded similar plain and red-slipped pottery with ring feet. Another cave called Arku (Thiel 1986-1987a) produced a burial assemblage dated between 1500 BC and 0, with pottery similar to that from Musang. Other items from Arku, many clearly paralleled in Taiwanese Neolithic sites, include shell and stone beads, shell bracelets, two tattooing chisels of horn, penannular earrings of stone (including two 
of jade), shell, or pottery like those from Peinan, a stone bark-cloth beater, pottery spindle whorls, barbed bone points, and stone adzes. The burials were apparently primary or secondary and sometimes dusted with ochre or placed in jars.

Other artifacts with Taiwanese parallels, such as slate projectile points, have been found in surface collections in Luzon. It is apparent that excavations on this island are well on the way to demonstrating a significant Taiwan-northern Philippine axis of Neolithic continuity. This conclusion is reinforced by the discovery of a site called Sunget on Batan Island, between Taiwan and Luzon. Although Sunget has not yet been excavated or dated, the preliminary survey report (Kumamoto 1983:55-61) refers to discoveries of red-slipped pottery with ring feet and lug handles, perforated slate points, and stepped and shouldered adzes-all items closely paralleled in the Yüan-shan repertoire.

In addition, there are some sites in the northern and central Philippines that contain incised and dentate-stamped sherds as well as the universal plain or red-slipped wares. At Lal-lo and Magapit, in the lower valley of the Cagayan River in northern Luzon, there are many estuarine bivalve shell middens; they range in location from close to the river to nearby hilltops and in size up to 50 by 100 meters, with depths up to 3 meters. Many sporadic archaeological forays have been made on these middens, but unfortunately there is no overall summary available. However, the material culture is extremely interesting, not least because of the richness of incision and dentate stamping on some of the pottery, with high perforated ring feet and some lime infill of designs. Stepped stone adzes also occur, with dates apparently back to about $2000 \mathrm{BC}$ (Thiel 1984-1985, 1986-1987b; Aoyagi et al. 1991; Ogawa 1993). The richness of the dentate stamping, dated in the Magapit hilltop site to ca. $800 \mathrm{BC}$ by Aoyagi, is important for considering the affinities of the oldest pottery in the Micronesian and Melanesian islands (see the discussion on the Lapita culture below). Pottery very similar to that from the Cagayan middens is also reported from a disturbed burial cave excavated by Solheim (1968) in Batungan Mountain on the island of Masbate (central Philippines). A carbon date of about $900 \mathrm{BC}$ from an adjacent and perhaps slightly later cave may be pertinent for this, and the Batungan assemblage concerned (from cave 1) includes a quantity of red-slipped sherds from carinated vessels, with incised, dentate-stamped, and stamped-circle motifs much like those from the Yüan-shan repertoire on Taiwan (compare Plate 30 and Fig. 7.9). So far, this stamped and incised pottery decoration does not appear to be present in the oldest pottery sites in the Philippines, but this may simply be a sampling bias; much further work is needed on this question.

Moving toward the southern Philippines, Fox (1970) has excavated in Duyong Cave on Palawan a flexed and face-down burial of a male provided with a quadrangular-sectioned stone adze, four Tridacna shell adzes, two ear discs and 

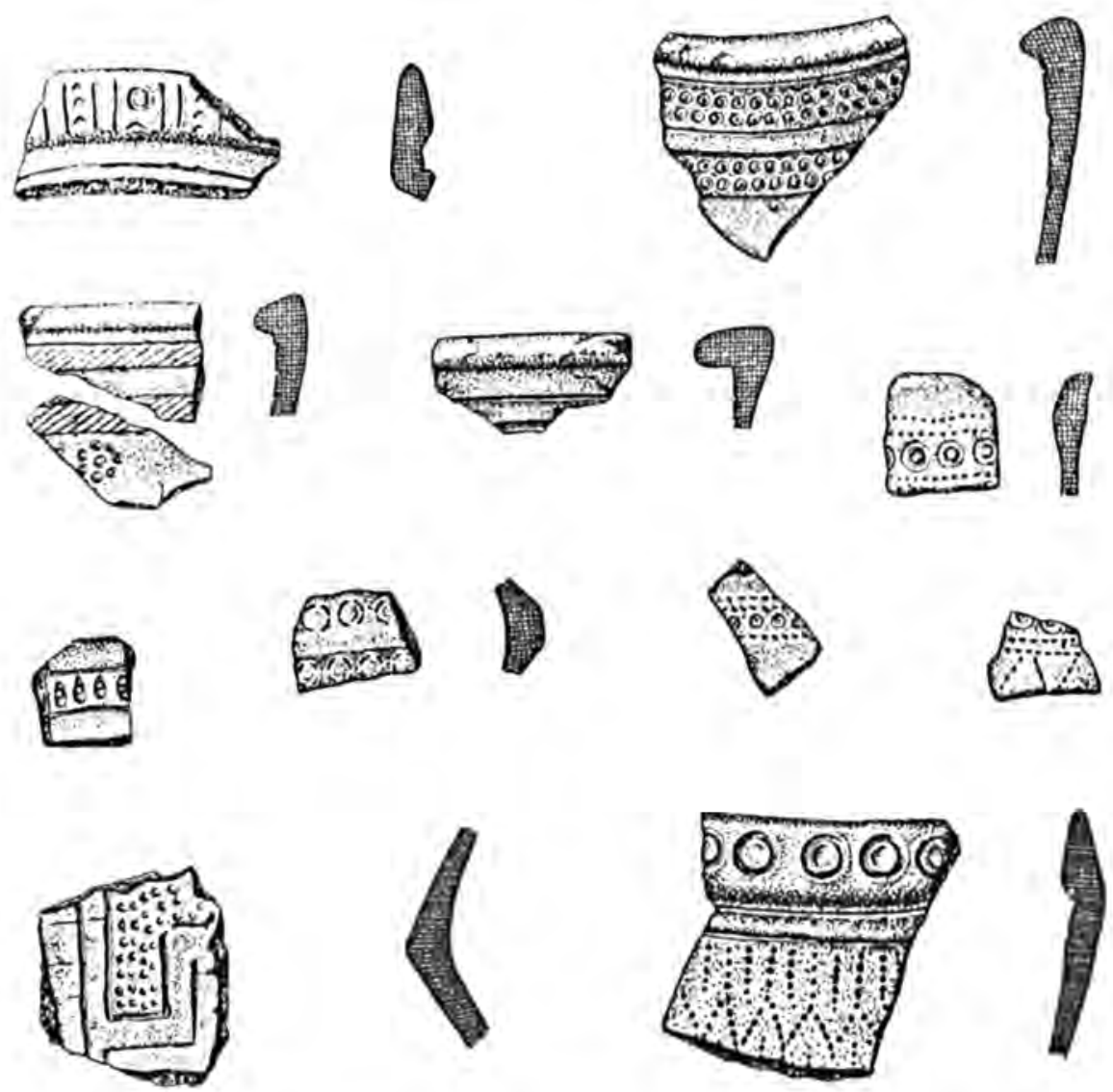

Fig. 7.9 Incised and stamped sherds from the Batungan Caves, Masbate. From Solheim 1968. Courtesy: Social Science Research Institute, University of Hawai'i.

a breast pendant made from perforated Conus shells (Fig. 7.10), and six Anadara shells that may have been used as lime containers for betel chewing (the skeleton also had betel-stained teeth). Charcoal from the burial pit was dated to about $3000 \mathrm{BC}$, and similar shell implements also occurred in a level in the cave deposits dated to about $4300 \mathrm{BC}$. This site is unusual in having no pottery and its early date suggests that the stone adze may have been traded from agricultural populations situated elsewhere into an indigenous hunting and gathering community. The shell tools may indicate a local tradition, a continuance perhaps of the preceramic tradition of shell adzes represented in the northern Moluccas (Chapter 6; see Plate 25).

Elsewhere in the Philippines, plain pottery is reported from shell midden deposits dated to about $2000 \mathrm{BC}$ in the Bagumbayan site on Masbate (Bay Peter- 

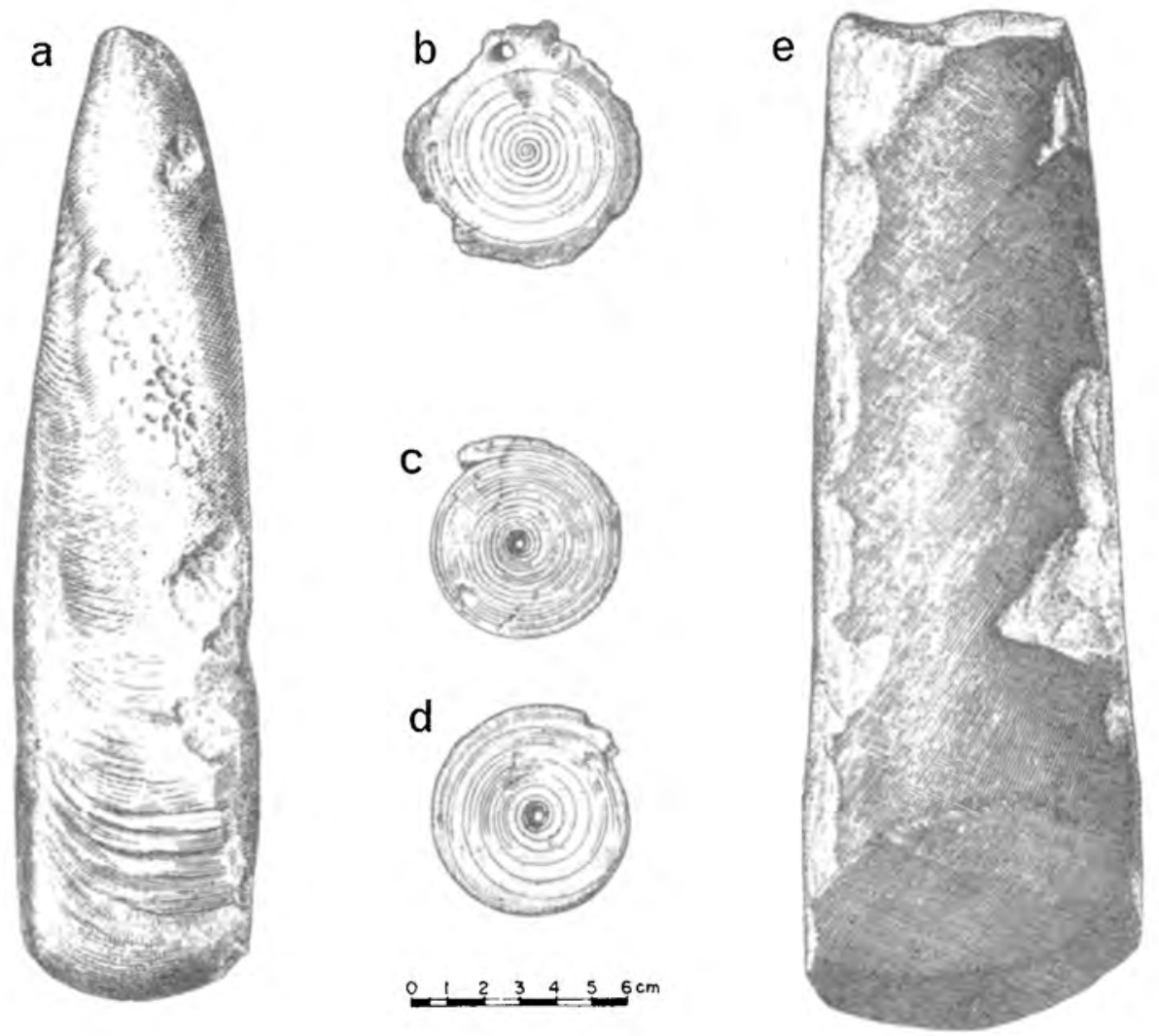

Fig. 7.10 Burial goods from Duyong Cave, Palawan, ca. 3000 BC (?): (a) shell adze; (b-d) Conus shell discs and pendant; (e) stone adze. From Fox 1970. Courtesy: National Museum of the Philippines.

sen 1982-1983), here with a few rice grains derived by flotation of the deposits, but considered intrusive by the excavator. Plain, slipped, and incised sherds occur at around the same date in the Edjek site on Negros (Hutterer and Macdonald 1982:223). Plain and red-slipped sherds also occur in the Balobok (Sangasanga) shelter in the Sulu Archipelago (Spoehr 1973; Ronquillo et al. 1993), but in this case with very uncertain dates.

In general, the Philippine Neolithic sites have continuing evidence for pig and deer hunting as well as for the use of flake tools (some with an edge gloss at Dimolit), but to my knowledge stone reaping knives are not found at all and the pottery spindle whorls do not occur south of Luzon. As noted for the Yüanshan and Peinan cultures of Taiwan, grain reaping may have been carried out with organic tools, or it may have faded away toward equatorial latitudes. The 
latter is an option that I will elaborate upon in due course. Weaving may have been replaced by bark-cloth production in many areas (a conclusion also reached by Ngo 1984-1985 to explain the shift away from cord-marked pottery to the south of Taiwan), but as weaving was so widespread ethnographically, an initial retraction followed much later by an expansion-perhaps with fibers such as cotton or abaca (Musa textilis)—might have occurred.

\section{B. Indonesia and Sabah}

Within Indonesia, the Leang Tuwo Mane'e shelter in the Talaud Islands has yielded plain and red-slipped sherds from thin-walled vessels with globular bodies and everted rims (Fig. 7.11 top) dating possibly from about $2500 \mathrm{BC}$ (Bellwood 1976a, 1981; the date comes from a single determination and is not very secure). Large numbers of shellfish continued to be discarded in the Neolithic layers in this site and the preceramic chert industry continued, but without the earlier tendency toward blade production (see Chapter 6, Section IIIA). Across the Sulawesi Sea in the cave of Agop Atas (Madai) in northern Borneo (see Chapter 6, Section IIB), the early Holocene pebble and flake industry was succeeded-after a long gap in occupation-by a pottery assemblage similar to that from Talaud (Fig. 7.11 bottom) with a continuing stone flake industry. This has been dated a little uncertainly-by thermoluminescence and radiocarbon-between 2000 and 500 BC (Bellwood 1988). After 500 BC the cave was again abandoned until the early Metal phase about 2,000 years ago.

Both Leang Tuwo Mane'e and Agop Atas produced little more than pottery, leading me in the earlier edition of this book to suggest an attenuation of material culture as Austronesian groups colonized southward. In 1987, excavations in the rock shelter of Bukit Tengkorak-formed amongst tumbled boulders on the rim of an extinct volcano near the town of Semporna in southeastern Sabah -have led me to change my mind somewhat (Bellwood 1989; Bellwood and Koon 1989). The lower layer in this shelter, dated between 1000 and 300 BC, yielded red-slipped pottery with plain or incised pedestals, a superbly decorated incised vessel with a lid (Fig. 7.12), and lots of shell items including adzes, beads, bracelets, and a possible fishhook shank, together with shell manufacturing debris. Stone tools included lava files, adze chips, a remarkable agate blade and awl industry made on prismatic cores and, perhaps most remarkable of all, small chips of obsidian from two sources: one unknown and the other being one of the Talasea sources in northern New Britain in Mela-nesia. The Talasea obsidian sources were used by Lapita people in the western Pacific, and the Bukit Tengkorak discovery of this material increases its distribution at ca. 1000 BC to 6,500 kilometers-from Borneo to Fiji-thus making it perhaps the most widely distributed material in the Neolithic world. 

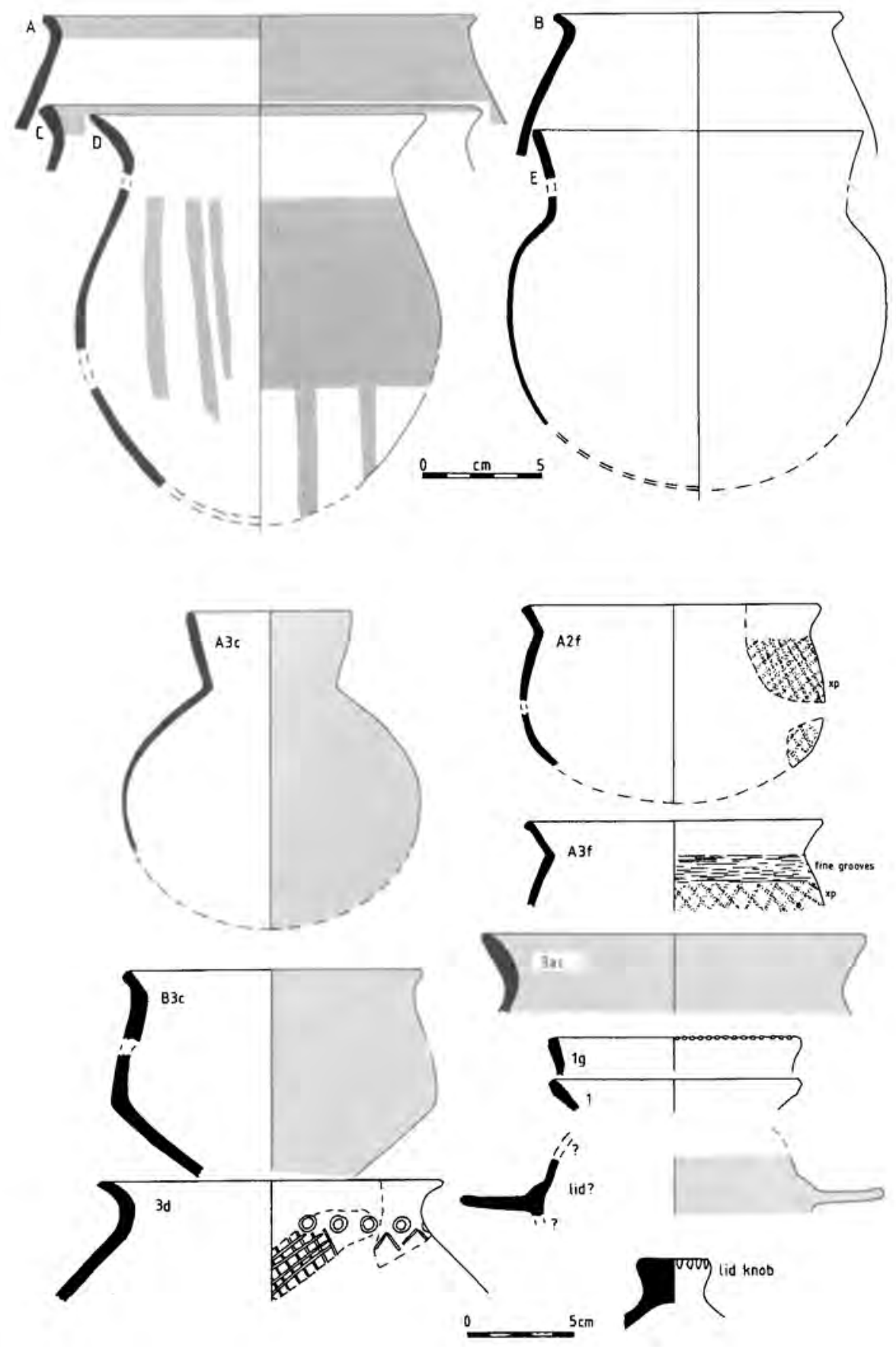

Fig. 7.11 Neolithic pottery from Leang Tuwo Mane‘e (top) and Agop Atas (bottom). 

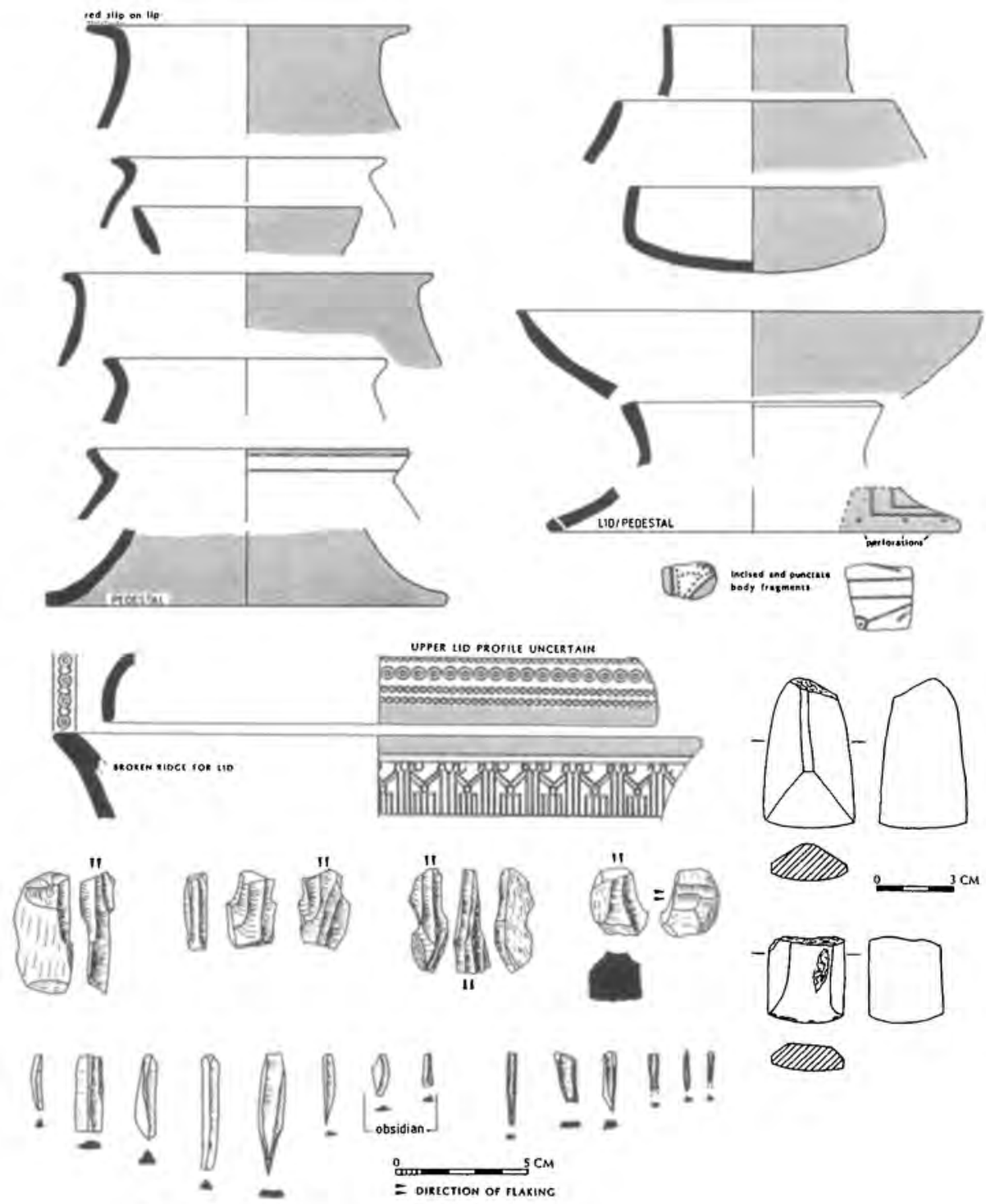

Fig. 7.12 Artifacts mainly from the lower layer ( 1000 to $300 \mathrm{BC}$ ) at Bukit Tengkorak, Sabah. Top: redslipped and incised pottery, including ring-feet. Bottom: agate microblades and awls, agate microcores, and obsidian chips. Bottom right: two trapezoidal sectioned stone adzes from uncertain stratigraphic contexts. 
The upper layer in Bukit Tengorak, also pre-Metal, produced more floridly decorated pottery with much incision, rim notching, cord marking, and paddle impression. Red slip faded in importance. Fragments of pottery stoves, an important artifact class known as far back as $4500 \mathrm{BC}$ in the Hemudu site in Zhejiang (Bellwood 1989:Plate 3), are quite common, as are decorated lids and pedestals. Talasea obsidian was no longer imported in this phase, which dates between $300 \mathrm{BC}$ and the early first millennium $\mathrm{AD}$, but the other shell and stone industries seem to have continued. Both phases at Bukit Tengkorak are rich in fish bones and these, plus the obsidian, pottery stoves (used ethnographically by sea nomads in the Sabah-Sulu region), and shell ornament manufacture indicate that the Bukit Tengkorak people were adept seafarers-and perhaps traders. Indeed, the agate prismatic blade industry is quite unique in Island Southeast Asia and, if not a local innovation, could reflect contact with an apparently contemporary region of similar microblade production in Guangdong, especially the site complex of Xiqiaoshan, near Guangzhou (Huang et al. 1982). The much poorer assemblage of this period from Agop Atas could thus represent a community of inland cultivators, people not a part of this interisland network of contacts.

Does the Bukit Tengkorak assemblage represent the kind of maritime-oriented tradition that should have characterized the earliest Austronesian colonists who moved into Oceania? The site has yielded no direct evidence for agriculture, but then neither have most others of this phase in Island Southeast Asia; the problem may have more to do with sampling and survival than a true absence.

The late-phase decorated pottery from Bukit Tengkorak has some perhaps superficial similarities with the pottery assemblage known for many years from the inland sites of Kalumpang and Minango Sipakko, which lie close together on the middle course of the Karama River in west-central Sulawesi. These two sites have produced perhaps the most remarkable pottery assemblages of any sites in Indonesia. Unfortunately, neither is dated, and Kalumpang-the most important-was investigated long ago by Stein Callenfels in 1933 and by Heekeren in 1949 (Heekeren 1950a, 1972:184-190; Sutayasa 1973). Both are open sites with no stratigraphic differentiation of the materials found, and the assemblages include quadrangular and lenticular-sectioned stone adzes, some with unusual waisted or knobbed profiles, ground slate projectile points similar to the Taiwanese Neolithic types (but without perforations), a stone bark-cloth beater, and some possible stone reaping knives (Plate 33). The pottery is especially remarkable-some of the motifs are shown in Fig. 7.13-and there are also some knobbed lids and ring feet with cut-out decoration. Of all the presumed pre-Metal assemblages excavated in Indonesia, this one-at least in its stone repertoire and some aspects of pottery decoration-seems to have the closest resemblance to the Neolithic assemblages of Taiwan. 


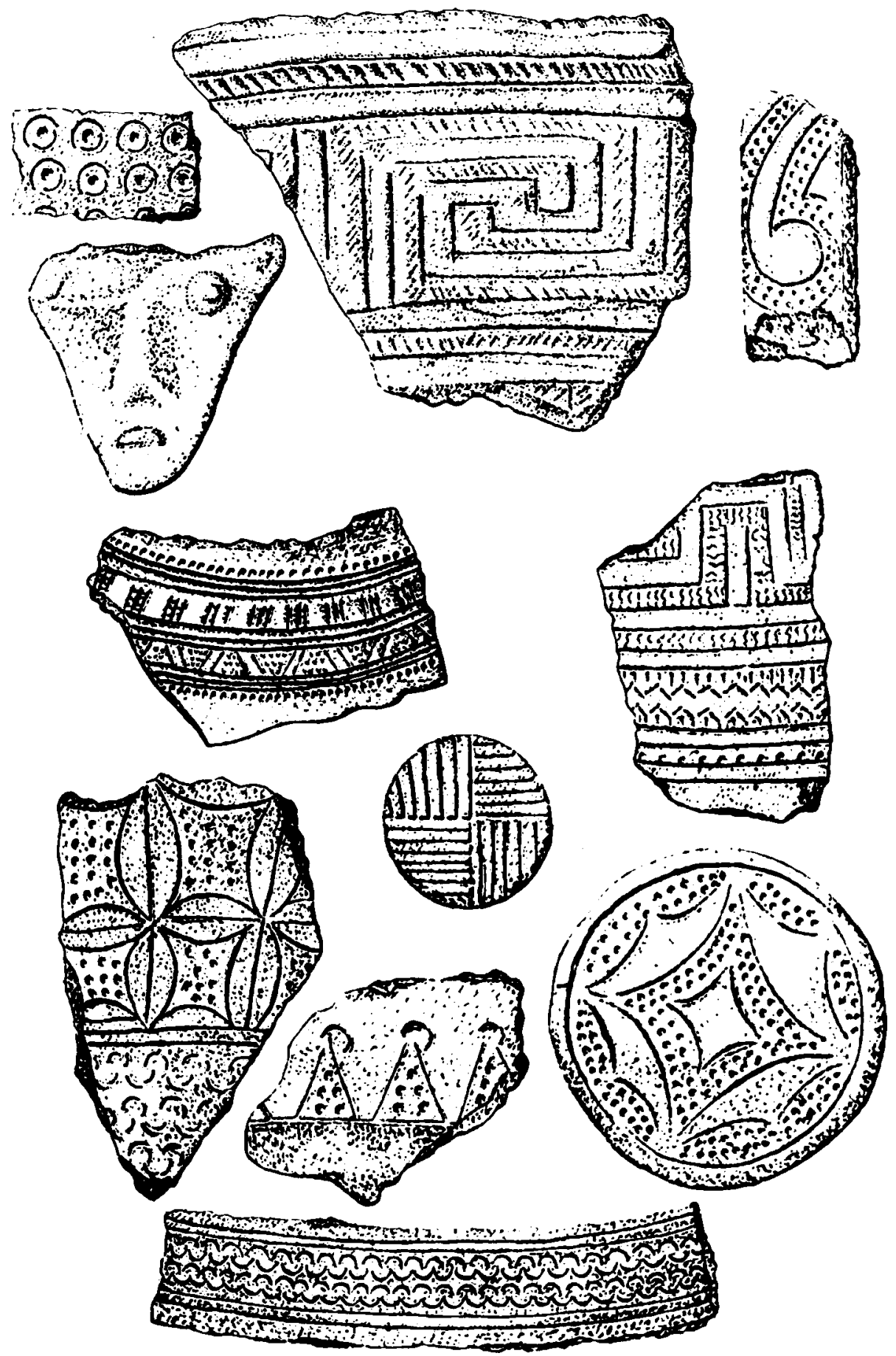

Fig. 7.13 Incised sherds and a modeled face from Kalumpang, west-central Sulawesi. From Heekeren 1972. Courtesy: Kluwer Academic Publishers. 
It is most unfortunate that these two sites have no dates, and it must be admitted that there are certain ceramic parallels with the late phase at Bukit Tengkorak, especially for the knobbed lids and some of the incised decoration, which could make a late Neolithic (post-300 BC) date for the Kalumpang material seem likely. However, given the Taiwanese parallels for the stone points and the Oceanic Lapita parallels for the pottery decoration, I would personally not be too surprised if an age of over 3,000 years can one day be demonstrated. As always, more research is needed.

In southwestern Sulawesi, pottery appears in small quantities in the upper layers of Toalian sites, perhaps here used by continuing hunter-gatherer populations. In the shelter of Ulu Leang, Glover (1976) has reported the first pottery at about $2500 \mathrm{BC}$ in a continuing Toalian industry with Maros points, although Bulbeck (1992:13), on the basis of some new radiocarbon dates on human bone from Leang Burung 1, is unwilling to date pottery here earlier than $1500 \mathrm{BC}$. The sherds here are of plain, unslipped globular cooking pots with everted rims.

In the northern Moluccas, the type of red-slipped and occasionally incised pottery typical of the Bukit Tengkorak early phase, Agop Atas, and Leang Tuwo Mane'e is also found in the excavated rock shelter of Uattamdi, on Kayoa Island to the west of Halmahera. The Uattamdi red-slipped pottery occurs in the lower layer of the site, beneath an upper layer of early Metal phase jar burials. It contains red-slipped pottery-some with painted stripes identical to examples from Leang Tuwo Mane'e (see Fig 7.11)-lots of shell beads, bracelets and spoons/ scrapers, a lenticular-sectioned stone adze and a stone chisel (plus an abundance of adze chips) (Plate 34). In addition, significantly, there are well-stratified bones of pig and dog-both of which were no doubt domesticated animals in this region. The whole assemblage is well dated between 1200 and $300 \mathrm{BC}$, after which it is replaced by the more elaborately incised early Metal phase pottery assemblage (Bellwood et al. 1993; Bellwood 1995c).

The shelter of Uattamdi also has a basal and culturally sterile beach sand dated to about $1300 \mathrm{BC}$; this could be taken to indicate that the makers of the red-slipped pottery were not occupying Kayoa Island at this time (a statement of course subject to a certain sampling proviso). Uattamdi has no obsidian and lacks indications of shell artifact manufacture-unlike Bukit Tengkorak-but its pottery assemblage is clearly closely related to those of the other sites just listed, to a degree that surely indicates close ethnic connection across this northeastern corner of Island Southeast Asia at ca. 1000 BC. Similar red-slipped pottery, in this case with some incised decoration, dates from about $900 \mathrm{BC}$ onward in the open site of Buwawansi, on Gebe Island to the east of Halmahera.

From caves in eastern Timor, Glover $(1977 a, 1986)$ has also reported similar but apparently unslipped pottery dated initially from somewhere between 2500 and 2000 BC. A few decorated sherds were also found in layers dated loosely be- 


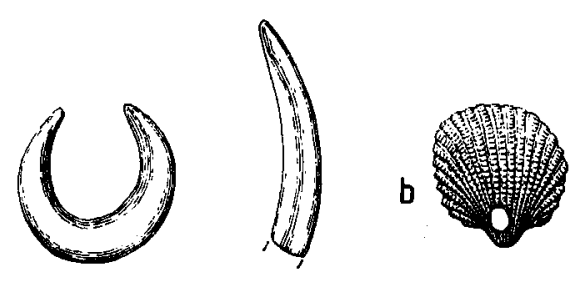

ב

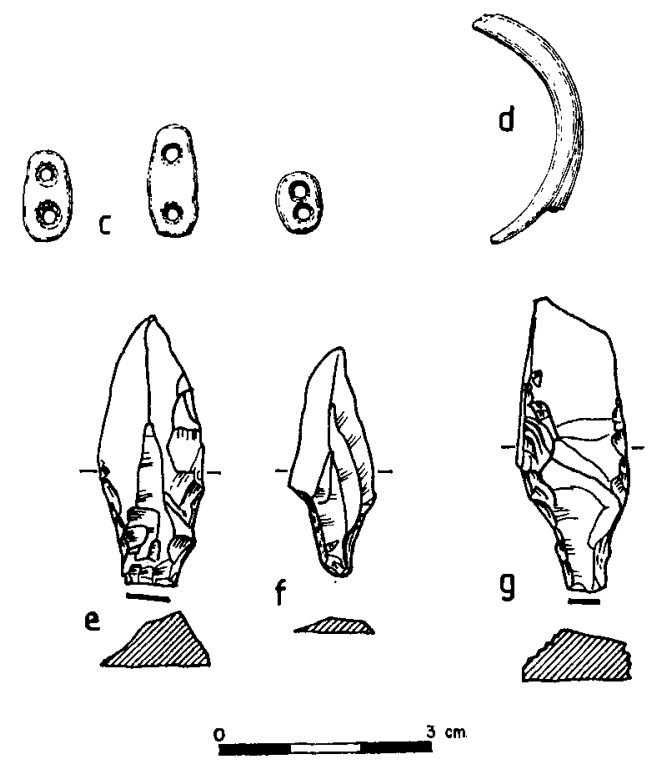

Fig. 7.14 Neolithic shell artifacts and tanged stone points from eastern Timor: (a) Trochus shell fishhooks from Bui Ceri Uato; (b) pierced Anadara shell from Uai Bobo 2; (c) Nautilus shell spacer beads from Bui Ceri Uato; (d) fragment of Trochus shell armlet from Uai Bobo 2; (e-g) tanged stone points from Uai Bobo I. From Glover 1977a. Courtesy: Ian Glover.

tween $1000 \mathrm{BC}$ and $\mathrm{AD} 500$; the patterns include incised hatched triangles and rows of interlocking semicircles like those from Kalumpang (Plate 35). The Timorese caves have also produced shell beads, bracelets, and one-piece angling hooks of Trochus shell (Fig. 7.14). As at Uattamdi, pig bones appear with the pottery, again surely domesticated here and derived from Sus scrofa of Java or $S$. celebensis of Sulawesi (Groves 1981, 1995). Even more surprisingly, Glover was able to show that cuscuses, civets, and macaques were also introduced at about the same time as the pigs. It is not clear whether these animals were tamed or wild at the time of introduction, but this evidence for transport of nondomesti- 
cated animals into the faunally impoverished islands of eastern Indonesia from Neolithic times onward is particularly interesting, especially in light of the much earlier evidence for marsupial translocation in the northern Moluccas. Although possibly historic introductions in many cases, the presence of macaques, civets, deer, and Javan porcupines on various other islands in the Lesser Sundas and Moluccas (Musser 1981; Groves 1984) and of the cassowary in Seram (Wallace 1962:300) should also be noted. Dog, cattle, and goats appear in the Timorese cave record after $1000 \mathrm{BC}$, but the goats and cattle could be more recent.

Plant remains from the Neolithic layers in Timor include the Polynesian chestnut (Inocarpus), bamboo, gourd, and (after $1000 \mathrm{BC}$ ) a single grain of foxtail millet (Glover 1977b). These Timorese finds are thus of great potential significance, since they allow the suggestion that an agricultural economy involving at least some form of pig husbandry and possibly millet cultivation was introduced to the island sometime around $2000 \mathrm{BC}$.

The early Neolithic sites I have described so far are really the only ones from which there is coherent dated information, with the exception of sites in Sarawak that I will consider in Section D below. For Sumatra there is little of a usable archaeological nature, and Java still remains something of a mystery. The problem for Java and Sumatra may be that Neolithic sites along former northern coastlines are now likely to be buried under many meters of alluvium and beneath the water table (like the Hoabinhian middens of Sumatra), and hence unavailable for archaeological research. ${ }^{4}$ Nevertheless, the enormous number of superbly manufactured quadrangular and pick adzes from Java (Duff types $2 \mathrm{~A}$ and 7A: Fig. 7.15), often made from semiprecious stones such as serpentine, agate, or chalcedony, suggests that Neolithic populations once occupied the island (although some of the finer adzes may actually be of early Metal phase date). The extensive working floors for adzes and stone bracelets found in several places in central and western Java add support to this view (Heine Geldern 1945); a detailed analysis of material from one such site located between the villages of Bomo and Teleng in south-central Java has been carried out by Tanudirjo (1991).

One working floor for quadrangular adzes, excavated at Kendeng Lembu in eastern Java (Heekeren 1972), produced sherds of thin, red-slipped vessels with round bases and everted rims. My own examination of this material in Jakarta suggests that it is related to the early red-slipped pottery of the Philippines and eastern Indonesia, but few details are available. Otherwise, a scatter of cordmarked and incised pottery finds, particularly from western Java, is summarized by Sutayasa (1973, 1979; see also Bellwood 1978:220-221). This material is undated and much of it could of course be long post-Neolithic. As far as Java is concerned, only the linguistic evidence suggests settlement by an Austronesian 


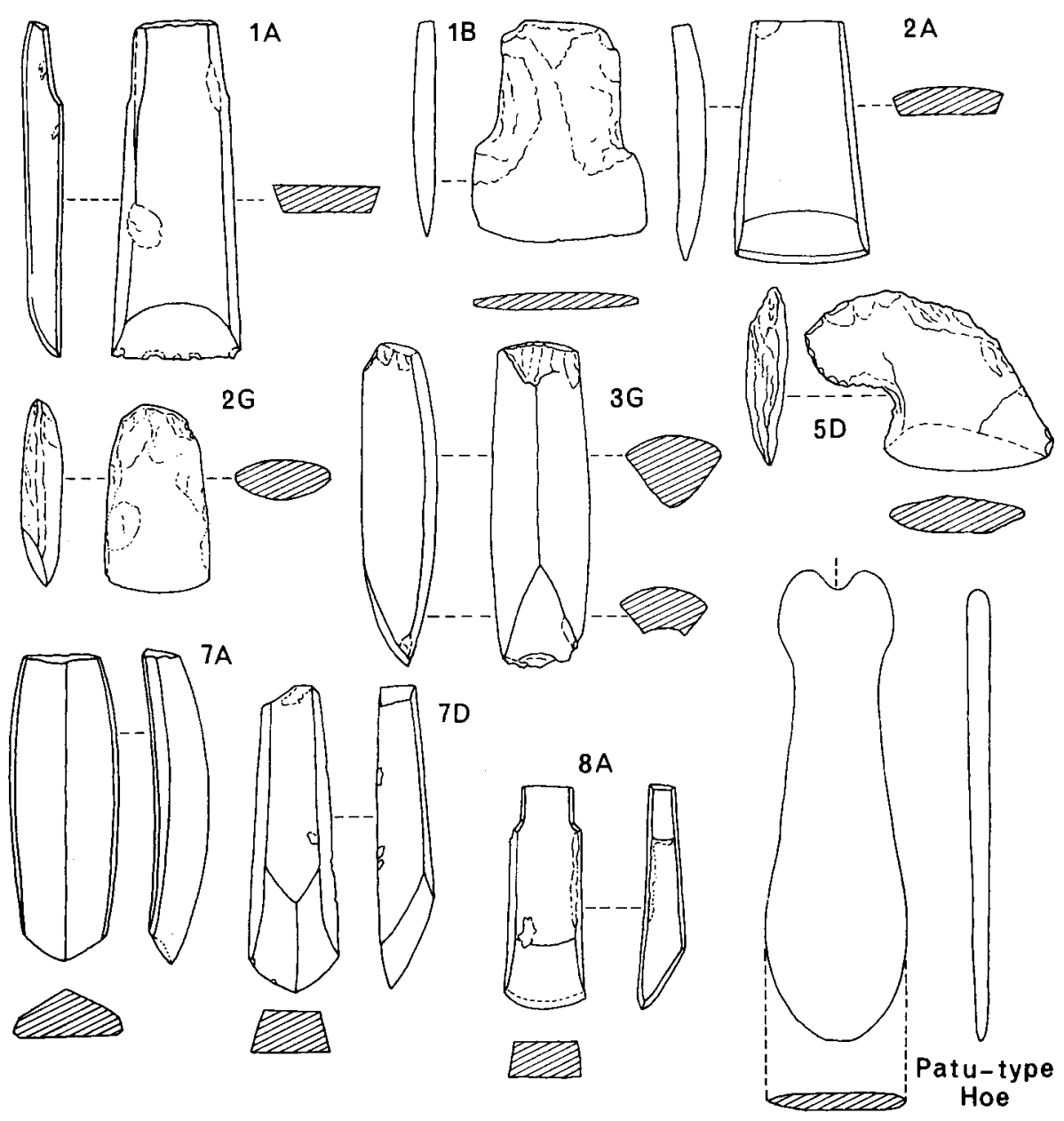

Fig. 7.15 Southeast Asian stone adzes, with the terminology used by Roger Duff (1970). Types, popular names, and provenances as follows: 1A stepped adze, Luzon; 1B shouldered adze, Kalumpang, Sulawesi; 2A quadrangular adze, Java; 2G oval or "round" adze, Vietnam; 3G Luzon; 5D Tembeling "knife," Pahang; 7A pick adze, Sumatra; 7D beaked adze, Peninsular Malaysia; 8A shouldered adze, Indochina; "patu-type hoe" from Taiwan. Not to scale. From Bellwood 1978.

population, expanding perhaps from Borneo-possibly as recently as $1000 \mathrm{BC}$ according to the estimate of one linguist (Blust 1984-1985:57).

To summarize some of the above, it is apparent that oldest dates for redslipped and plain pottery anywhere south of Taiwan are not much older than $2000 \mathrm{BC}$, except possibly for Luzon. Because Austronesian populations had already reached Samoa, in the central Pacific, by 1000 BC, it is beginning to look 
as if the Austronesian migrations, at least for the first 10,000 kilometers (Taiwan to Indonesia and eastward to Samoa), took place within a relatively short time scale within the second millennium BC. Another source of information, albeit very diffuse with respect to dating, comes from the pollen record of forest clearance. Pollen diagrams from highland swamps in northern and central Sumatra and western Java provide some interesting but rather equivocal evidence for forest clearance that may be related to settlement of these regions by cultivators, although unfortunately there are no archaeological records in direct association (for summaries see Flenley 1985a, 1985b, 1988; Maloney 1985, 1994).

For instance, a pollen core from Pea Sim Sim Swamp near Lake Toba in northern Sumatra $(1,450$ meters above sea level) indicates that some minor forest clearance could have started as early as $4500 \mathrm{BC}$, but the major phase, evidenced by an increase in large grass pollen, began during the first millennium вC. Lake Diatas near Padang (1,535 meters above sea level) in central Sumatra has yielded a similar sequence. The nearby Lake Padang core (950 meters above sea level) indicates swamp vegetation clearance and burning by about $2000 \mathrm{BC}$, and there is evidence here for an increasing protection of the useful Arenga palm species by 2,000 years ago. At Situ Gunung in western Java (1,015 meters above sea level) there is an increase of pandanus and fern spores-perhaps indicating some forest clearance-at about 2800 в . However, other Sumatran and Javan cores, admittedly from quite high altitudes, offer evidence for major forest clearance only after 1000 в (Flenley 1988; Stuijts 1993).

The overall pollen record therefore seems to suggest some intermittent forest clearance in Sumatra and Java occurring at high altitudes during the mid-Holocene, but with permanent clearance occurring only after about 3,000 years ago. As these records are all from highland areas, it may be reasonable to expect that cultivation in coastal lowlands began slightly earlier, but this remains uncertain. It is also apparent that the Pea Sim Sim dates for initial forest clearance are a little earlier than would be expected from the archaeological record alone. This may reflect the fact that hunter-gatherers are quite capable of burning forest in drought periods, even close to the equator, a circumstance made clear by the presences of charcoal particles in pollen cores going back well into the late Pleistocene in New Guinea (Haberle 1993) and by charcoal particles radiocarbon dated to about 10,000 years ago from soils in Brunei (Cranbrook and Edwards 1994:339). Given what we know of the chronology of dispersal of the Austronesians, it seems unlikely that systematic forest clearance for agriculture would have begun in Java and Sumatra much prior to $1500 \mathrm{BC}$, but new data could change this view. On the other hand, it is not impossible that Sumatra and western Borneo were settled by agricultural groups from southern Thailand or Malaysia before Austronesian settlement commenced; this possibility will be discussed further for Sarawak in Section D. 
Earlier agriculturalists, or at least intensive harvesters of tree crops, could also have occupied parts of eastern Indonesia before Austronesians arrived. The New Guinea Highlands clearly witnessed an independent transition to swamp agriculture-perhaps for taro-about 10,000 years ago (Golson 1977; Bellwood 1996b), and questions arise as to whether or not this system ever spread off the New Guinea mainland. Present indications are that it did not in its fully fledged swamp-cultivation form (Spriggs 1993), but van der Kaars (1991) notes an increase in palm pollen at about 6,000 years ago in Kau Bay, northern Halmahera. This could represent a record of indigenous intensification of arboriculture, with consequent population growth. Austronesians clearly were never able to settle this area successfully, for Papuan languages still dominate the northern Moluccas today.

\section{c. Western Oceania}

In the western Pacific, Austronesian colonists between 1500 and $1000 \mathrm{BC}$ left an extremely clear-cut trail of pioneer archaeological sites across about 6,500 kilometers of ocean and islands (many previously uninhabited), from the Admiralty Islands north of New Guinea to as far east as Samoa, in western Polynesia. This impressive migration probably correlates linguistically with the period of Proto-Oceanic (see Chapter 4, Section VB). Although much of western Melanesia had long been occupied by Papuan-speaking groups, it is clear that these tended to be settled mainly in the larger islands of New Guinea, the Bismarcks, and the Solomons. Many small islands, and all territories from perhaps New Caledonia and Vanuatu eastward (certainly including Fiji), were thus available for canoe-borne colonization by Austronesian groups.

The resulting Lapita culture, which represents colonization of virgin territory in most locations where it has been found beyond the Solomons, is generally well dated and well studied in terms of artifacts and economy. It suffers from few of the chronological problems that beset the often mixed and undated Neolithic assemblages from Island Southeast Asia. Lapita, therefore, can provide an excellent insight into its logical antecedents, which lie somewhere in the eastern regions of Indonesia or the Philippines (the linguistic evidence points to the Moluccas and western New Guinea) in the mid-second millennium вс. No one has yet located these antecedents very precisely, but even if many elaborations of ceramic decoration (such as dentate stamping) were developed within Melanesia itself, we now have clear evidence for the prior existence of cultures with similar economic, technological, and navigational skills in the Indo-Malaysian Archipelago (with Taiwan). A brief review of the Lapita culture, and its contemporary in the Mariana Islands, is thus offered here.

The Lapita evidence, when viewed from an Indo-Malaysian viewpoint, reveals 
quite clearly an integrated culture between 1500 and $1000 \mathrm{BC}$ (the later phases of disintegration are not of concern here) with the following archaeological features (for summaries see Kirch and Hunt 1988; Green 1991; Allen and Gosden 1992; Galipaud 1992; Spriggs 1995; Kirch 1995; Kirch 1997, Spriggs in press):

a. A range of coiled or slab-built vessels with volcanic or coral sand tempers, ranging in form from globular cooking pots through narrow-necked water jars to a variety of open bowls, some with flat bases. Some vessel profiles are sharply carinated, and other accessories include lug and strap handles, lids, and pedestals, the latter being most common in the earliest sites such as those in the Mussau Islands (ca. $1500 \mathrm{BC}$ ). Vessel surfaces are often red slipped, and the decoration, generally in zones around the upper surfaces of some of the vessels, includes a quite astonishing and intricate range of incised and dentate-stamped motifs (Plate 35d) of rectilinear, ${ }^{5}$ curvilinear, and even anthropomorphic forms (Spriggs 1990), the latter perhaps indicating a concern with ancestors common to all Austronesian populations. Later Lapita pottery tends to have simpler designs, and dentate stamping fades in popularity first in favor of incision, and eventually plain ware.

b. Economically, the Lapita culture was based on a mixed horticultural and maritime subsistence. Pigs, fowls, and dogs were all present; plant remains from waterlogged sites include taro, coconut, candlenut, pandanus, and canarium, all exploited by pre-Lapita populations in western Melanesia as well (Kirch 1989; Swadling et al. 1991; Loy et al. 1992). Village settlements, in some cases of stilt houses, occupied zones marked by sherdage, earth ovens, hearths, and other features averaging about one hectare in size (maximum seven hectares in the Mussau Islands) in coastal and small offshore island locations. A fairly healthy interisland exchange of obsidian and other tool stones was carried out, especially in western Melanesia between the Bismarck Archipelago and the Santa Cruz Islands. Rats (Rattus exulans) and occasional wallabies and cuscuses were transported, too.

c. Items of Lapita material culture apart from pottery include stone adzes (all untanged) with quadrangular and lenticular cross-sections, stone chisels, shell adzes, a range of shell ornaments (beads, armrings, necklace units), and one-piece bait fishhooks for angling. The shell fishhooks suggest a technological adaptation confined mainly to Oceania, but bait hooks of shell are also found in a few Indo-Malaysian Neolithic sites, especially in Taiwan and Timor.

The Mariana Islands of western Micronesia were also settled about 1500 BC (Craib 1993; Butler 1994; Rainbird 1994; Amesbury et al. 1996) by users of a thin-walled, red-slipped, and coral-sand-tempered pottery, formerly termed Marianas Redware but now perhaps better known as pottery of the Tarague Phase 
(1500 to $500 \mathrm{BC} ;$ Butler 1994). There are very strong indications that this pottery may be closely related to the Philippine and early Talaud/Moluccan redslipped assemblages described above, and all Micronesian archaeologists seem to agree that the Mariana Islands were settled from the Philippines or northeastern Indonesia by a separate and possibly slightly earlier movement than that indicated by Lapita in more southerly latitudes.

For Lapita itself, in Melanesia, there is less agreement, with many archaeologists who undertake research in the Melanesian region continuing to claim that Lapita origins reflect no significant contact with Indonesia at all and that all Pacific populations are derived in isolation from the early settler populations of western Melanesia in the Pleistocene. This view is to me completely at odds with the evidence from linguistics and genetics, and is rejected here in its entirety. The archaeological record alone is quite insufficient to prove or disprove movements of people in prehistory. Not only does the linguistic subgrouping of the Austronesian family make a Southeast Asian source for Oceanic Austronesian speakers almost unarguable (I say "almost," because there have of course been processes of population contact and influence at work in Melanesia during the past 3,000 years that obviously make the picture less than crystal clear), but we also have the striking fact that most Proto-Oceanic terms associated with agriculture are derived from Proto-Malayo-Polynesian forebears in Island Southeast Asia, rather than from the native Papuan languages of western Melanesia. Although Papuan peoples had developed some forms of agriculture before Austronesians arrived, especially in the interior of New Guinea, little of their knowledge seems to have been transmitted into those Lapita/Austronesian populations that moved eastward to settle Fiji and Polynesia. The "creolization" between Papuan and Austronesian cultures in western Melanesia occurred later.

\section{The Neolithic of Sarawak}

As 1 have noted, the Neolithic period of western Indonesia is virtually a total blank, despite the numerous reports of pottery from scattered sites in Java and Sumatra, often cord-marked or carved-paddle-impressed in contradistinction to that from eastern Indonesia (e.g., see Bronson and Asmar 1975 for Sumatra). Unfortunately, these assemblages are so far undated and generally resist any historical interpretation. The situation is different for Sarawak, however, which has a good sequence of Neolithic human activity covering the past 4,500 years from the cave of Gua Sireh inland from Kuching-a sequence illuminated also from the Niah Caves and from Lubang Angin Cave in the Gunung Mulu National Park. 


\section{Gua Sireh}

It will be remembered from Chapter 4 that the Land Dayak languages of western Sarawak have linguistic features identified as a possibly Aslian (Austroasiatic) substratum by Adelaar (1995). The work at Gua Sireh brings this observation into especial prominence, although it should be stressed that the bulk of the archaeological record during at least the past 3,000 years in Sarawak is likely to be of Austronesian affinity. Nevertheless, the earliest Neolithic assemblages of Gua Sireh have much in common with the Peninsular Malaysian and southern Thai Neolithic, perhaps more than they do with the red-slipped wares of Sabah and eastern Indonesia. This suggests that an Austroasiatic settlement of western Borneo (and perhaps also parts of Sumatra) could have occurred before the arrival of Austronesian populations in the region. The latter have come to dominate the linguistic scene, just as did their cousins who established the Chamic (Austronesian) enclaves in formerly Austroasiatic regions of southern Vietnam (see Chapter 9). ${ }^{6}$

The cave of Gua Sireh lies about 55 kilometers southeast of Kuching in western Sarawak, in the limestone massif of Gunung Nambi. It is flanked by flat alluvial terrain, used today and perhaps since about 4,500 years ago for the growing of rice. The site was first excavated by Harrisson and Solheim in 1959, then by Zuraina Majid in 1977, and most recently by Ipoi and Bellwood in 1989 (Ipoi 1993; Ipoi and Bellwood 1991). The Neolithic phase in Gua Sireh began about 4,500 years ago with the appearance of pottery with carved, cord-wrapped, or basketry-wrapped paddle-impressed surfaces. Other kinds of decoration such as red slip or incision/punctation were virtually absent. Some sherds had rice grain or husk inclusions, and a single rice grain in one sherd has been AMS radiocarbon dated to an age of ca. 4,500 years. This is a highly important discovery, supported by conventional $\mathrm{C} 14$ dates from the same layer and more recently by the discovery of many rice husk fragments in the soil of this phase (Sen 1995).

This date for rice from Gua Sireh is the oldest evidence for putatively domesticated rice in the Indo-Malaysian Archipelago and, as will be noted in Chapter 8 , it is roughly contemporary with the plentiful evidence for rice in some sites in central Thailand. Furthermore, the paddle-impressed pottery of Gua Sireh is very different from the red-slipped Neolithic pottery of Sabah, the Philippines, and eastern Indonesia (although some paddle-impressed pottery does occur in these latter regions during the early Metal phase). The early date, the rice temper, and the predominance of paddle impression on the pottery must open the possibility that the assemblage reflects the arrival in Sarawak of a Mainland Southeast Asian (Austroasiatic?) rather than an Austronesian population, although it 
should be noted that the mainland assemblages to be described in Chapter 8 are only similar to Gua Sireh-not identical. The Niah caves, farther to the northeast, also have paddle-impressed pottery similar to Gua Sireh.

\section{The Nlah Caves and Gunung Mulu}

I will now continue the Niah sequence where I left off in Chapter 6, Section IIA. According to the original reports of Harrisson (e.g., 1957, 1958, 1959, 1970), pottery first appeared in the West Mouth sequence at around 2500 or $2000 \mathrm{BC}$ together with quadrangular cross-sectioned adzes, which were preceded by an earlier lenticular-sectioned form. Spriggs (1989:603) has since pointed out many of the problems associated with the Niah C14 series and suggests that the oldest acceptable Neolithic date from Niah is ca. $1400 \mathrm{BC}$. However, the Niah pottery appears to be mainly of simple globular forms with plain or carved-paddle-impressed surfaces, and cord marking is rather rare (Solheim, Harrisson, and Wall 1959; Wall 1962; Zuraina 1982). The assemblage is similar to that from Gua Sireh, and may be of a similar commencement date.

In the Niah Caves, and also in another cave called Lubang Angin in the Gunung Mulu National Park (Ipoi 1993; Ipoi and Bellwood 1991), some very striking types of non-paddle-impressed pottery made an appearance after about
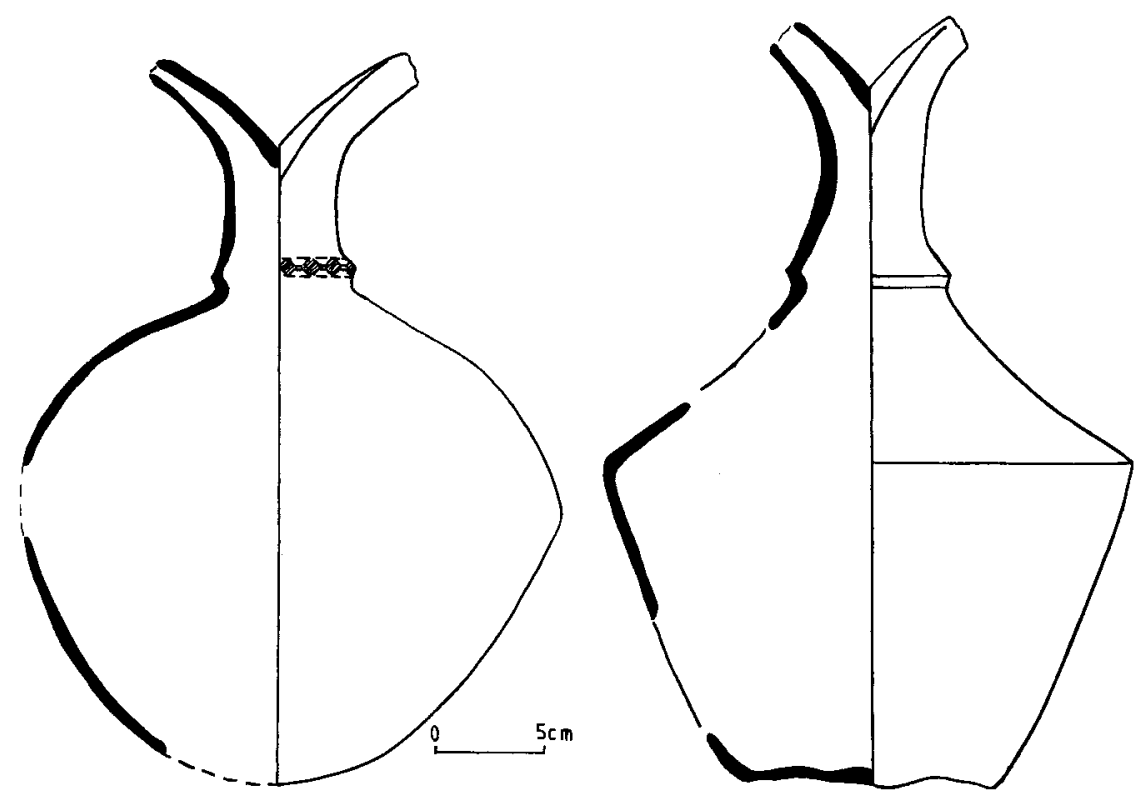

Fig. 7.16 Double-spouted vessels from Lobang Jeragan, near Niah. From Harrisson 1971. Courtesy: Malaysian Branch of the Royal Asiatic Society. 
$1000 \mathrm{BC}$, especially as grave goods. These vessel types, which do not occur in Gua Sireh, include closed water vessels with double spouts (Harrisson 1971) and elaborately incised and painted "three-color ware," which has painted or impressed designs enclosed within incised lines (Fig. 7.16, Plate 36). The doublespouted vessels are unique within Island Southeast Asia but do have distant parallels-possibly dated to about 2,000 years ago-in the Admiralty Islands to the north of Papua New Guinea (Kennedy 1982). Because a fragment of bronze of similar date has also been found in the Admiralties, presumably an import from somewhere in Indonesia (Ambrose 1988), this distant paralleI may reflect more than coincidence.

The 1989 excavations in the cave of Lubang Angin in Gunung Mulu National Park (about 160 kilometers southeast of Niah; Ipoi 1993; Ipoi and Bellwood 1991) yielded extended burials wrapped in bark cloth and laid in shallow pits in the floor of the cave; the grave goods seem to have been placed on the surface. This has led to much mixture within the deposits, but as the material culture is quite homogeneous the site might have been in use only for a relatively short period. The pottery contains some cord-impressed or carved-paddleimpressed vessels, together with double-spouted vessels identical to those from Niah, large carinated vessels of three-color ware decorated with red and black pigment (the third color being the surface of the pot) and complex designs of infilled incision, and two unusual deer heads attached to a pottery vessel of some unknown overall shape. The radiocarbon dates for Lubang Angin fall between about $700 \mathrm{BC}$ and $\mathrm{AD} 500$, and as glass beads and iron fragments were also found in the site it is possible that the assemblage spans the period (perhaps late first millennium $\mathrm{BC}$; see Chapter 9) when these items were first introduced to the region.

The Niah and Lubang Angin three-color ware is similar to some early Metal phase pottery from Sabah and especially the Philippines, where similar motifs are tentatively dated in Manunggul Cave A on Palawan to the early first millennium BC (Fox 1970). The Manunggul pottery, like the Niah three-color ware, has fairly exuberant incised curvilinear designs with punctate infillings. The three-color decoration also resembles that on pottery from the upper layer at Bukit Tengkorak in Sabah, dating after 300 вс (above), and also the undated assemblage from Kalumpang in western Sulawesi. The interesting possibility arises that this three-color pottery in these interior regions of Sarawak might be a record of the inland expansion of Austronesian populations from coastal locations some time in the early first millennium $\mathrm{BC}$.

As far as the overall sequence of Neolithic activity in the West Mouth at Niah itself is concerned, the general lack of information on habitation assemblages forces one to turn to burial types and their associations. During the Neolithic, an inner portion of the cave behind the area previously occupied in preceramic 
times was used for burial purposes. About 130 burials were excavated from this "cemetery sector" prior to 1967; cultural details have been described by Barbara Harrisson (1967). All graves are shallow and seem to belong to one continuous phase of activity dated quite uncertainly to some time between $3000 \mathrm{BC}$ and the first millennium $\mathrm{AD}$; dating was by a large series of thirty collagen or apatite radiocarbon dates from human bone reported by Brooks et al. (1977). However, these dates are of such extremely uncertain reliability and contain so many internal contradictions that I refer below only to dates determined on other materials, such as charcoal or shell.

The main burial types at Niah are as follows:

a. Extended burials, totaling sixty-eight, laid in shallow graves marked with stakes, with (in most cases) heads pointing into the cave. Many skeletons were coated with hematite or partially burnt, and it is interesting to note that a similar incomplete burning of corpses has been reported ethnographically for Land Dayak groups in Sarawak (Roth 1896:137). At Niah the bodies were placed in log coffins with plank lids or in cigar-shaped caskets of sewn bamboo strips, and in some cases they were also wrapped in pandanus mats before being placed in the containers. Some of the burials in bamboo caskets were provided with pillows of wood, matting, bamboo, or leaves. There are also traces of textiles with two burials that are probably quite late in the sequence. Associated artifacts, possibly grave goods, include a quadrangular adze, sherds of three-color ware, two bone rings, and a wooden disc-shaped earplug. Later graves in this series have glass beads and metal goods. Three radiocarbon dates from a mat and two wooden coffins (burials 75 and 60: Harrisson 1975b; Harrisson 1967:154) fall between about 1750 and $500 \mathrm{BC}$.

b. Cremations and burnt secondary burials. These two categories were separated by Barbara Harrisson, but it seems best to consider them together. Fifty-nine were found (twenty-six fully cremated and thirty-three less fully burnt), placed in small wooden coffins, pottery jars (Plate 37), or baskets. One was in a Chinese jar, presumably postdating AD 1000. Associated goods for the whole group included quadrangular adzes, shell rings and perforated discs, some double-spouted jar sherds, and a single copper object. Two burial jars are dated to about 1500 BC (burial 69, and from wood with burial 159; Harrisson 1968), and another burial jar to about 750 вс (burial 67). These dates for jar burial do seem a little early given the patterning elsewhere in the archipelago, but similar dates in the late second millennium $\mathrm{BC}$ for double-spouted sherds have been claimed from two other caves near Niah: Magala and Lobang Jeragan (Harrisson and Harrisson 1968; Harrisson 1971). The problem with Magala, however, is 
that the sherd concerned was found on the surface of the cave and not in definite association with the bone that was dated.

The Niah evidence thus presents some major problems. On the one hand there is a large series of radiocarbon dates, but mainly on bone collagen or apatite and of uncertain reliability. On the other hand there are large numbers of artifacts, but it is almost impossible to associate the vast majority of them in any convincing way with the dated bones. Nothing short of massive reexcavation, perhaps now impossible, can resolve this problem.

If the Niah dates are correct, the sequence there may indeed contain jar burials from the late second millennium BC in fully Neolithic contexts, as now appears to be the case in southern Vietnam (Ha Van Tan 1985b). I am also willing in principle to accept late second millennium вс dates for the doublespouted and three-color sherds, given the evidence from Lubang Angin. A few other comments can be added to the Niah story. It is possible that a small domestic dog was present in the Neolithic phase (Clutton Brock 1959; Medway 1977b) together with the domestic pig, although definitive evidence for the latter before the fifteenth century AD seems to be lacking (Medway 1973; Cranbrook 1979). Neither the dog nor the domesticated species of pig (Sus scrofa) is native to Borneo, and the native wild boar (Sus barbatus) appears never to have been domesticated. Otherwise, the economic evidence from the West Mouth suggests little real change from pre-pottery times, and it looks as if the site was used predominantly for burial during the Neolithic-and perhaps for occasional visits-rather than as a base for a sedentary agricultural population.

\section{AN INTEGRATED VIEW OF EARLY AUSTRONESIAN EXPANSION}

If the linguistic data from Chapter 4 plus a number of important botanical and ecological observations are added to the above archaeological record, then the course of Austronesian prehistory from the Initial Austronesian settlement of Taiwan (fourth millennium BC?) through about 1500 BC, when Neolithic voyagers were beginning the Austronesian settlement of Oceania, can be reconstructed.

During the late fifth or fourth millennium $\mathrm{BC}$, colonists from the mainland of southern China (probably Zhejiang or Fujian) settled Taiwan. Initial Austronesian languages were spoken on this island for several centuries (a millennium?) before further expansion took place. During the third millennium BC colonists moved into Luzon, and the Malayo-Polynesian subgroup now began its separation from the other primary subgroups of Austronesian that remained on Taiwan. The linguistic reconstruction for Proto-Austronesian (located on Taiwan) reveals an economy with domestic pigs and dogs and cultivated rice, 
millet, sugarcane (perhaps domesticated from the wild Chinese species Saccharum sinense; Daniels and Daniels 1993), yams, and Alocasia (Wolff 1994; Zorc 1994). The archaeological evidence adds pottery, weaving, and bark cloth, plus the stone and bone items (reaping knives, projectile points, adzes) already described for the Taiwan Neolithic.

By at least $2000 \mathrm{BC}$, Proto-Malayo-Polynesian began to break up, probably with settlement expanding in various directions into the southern Philippines, Borneo, Sulawesi, and the Moluccas. The Proto-Malayo-Polynesian vocabulary items presumably reflect the tropical environments of the Philippines, and important new items were added to the economic repertoire: the chicken, coconut, breadfruit, Colocasia (taro), banana, sago, betel chewing, and the addition of sails to canoes. Of course, none of these items can be proven definitely absent from the Proto-Austronesian vocabulary; all the linguistic evidence can say is that it cannot be demonstrated that they were present. Certainly it would not be at all surprising if taro and domestic fowl were present in the Taiwanese economy at around $3000 \mathrm{BC}$. But breadfruit, coconut, banana, and sago were most probably first incorporated into the Austronesian crop inventory in the tropics.

By about 2000 BC the Central-Eastern Malayo-Polynesians had expanded, presumably through the Moluccas, to at least as far as Timor. Navigational skills and sailing techniques must have been improving by this time-as the impending settlement of Oceania makes clear-and rapid coastal expansion was probably preferred to the more laborious process of settling island interiors, which might in some cases have sheltered hostile populations. Cereal cultivation declined to only minor importance in eastern Indonesia, and the Austronesian settlers of Oceania based their economy purely on tubers, tree fruits, and other vegetatively reproduced plants. I will now fill out the botanical background to these Austronesian adaptations by looking at some of the major crop plants and at the dynamics of shifting cultivation.

\section{A. Pice and Other Cereals}

As indicated earlier in this chapter, the earliest cultivated rices developed, according to the archaeological record, from wild annual or perennial forebears in central China (Plate 38a). It is apparent from studies of modern varieties that both the wild forms and the early varieties domesticated from them at this latitude would have been highly sensitive, during their growth and ripening cycles, to variations in day length (photoperiod) and sunshine incidence (Oka 1988). Flowering would have depended on latitudinally determined day length at the start of the dry season, and the ripening process would have required about forty-five days of dependable and adequate sunshine (Chandler 1979:44) and a 
precise progression of daylight durations (Oka and Chang 1960; Vergara 1976; IRRI n.d.). In effect these cultivars were genetically conditioned for successful growth cycles under specific latitudinal and climatic conditions.

Today, varieties with little or no photoperiod sensitivity have been developed that will grow successfully in equatorial or high latitudes, but rice yields are still highest in the intermediate tropical latitudes, especially where irrigation is carried out. They drop off (as do protein and starch contents) as one moves toward the equator (Fig. 7.17). For the early centuries of Austronesian expansion it may perhaps be assumed that all varieties were sensitive to daylight lengths, and any attempts to move them relatively quickly into equatorial latitudes would have met with either decreased harvests or no harvests at all (Spencer 1963:84). For instance, excessive cloudiness or rain during the ripening period, high night temperatures, and unvarying day lengths would in combination promote prolific vegetative growth, but grains would tend to be small and in many instances would probably never reach maturity (Oka 1975; J. H. Chang 1968). Grains might also ripen in the middle of an exceptionally wet period so that successful harvesting would be difficult or impossible.

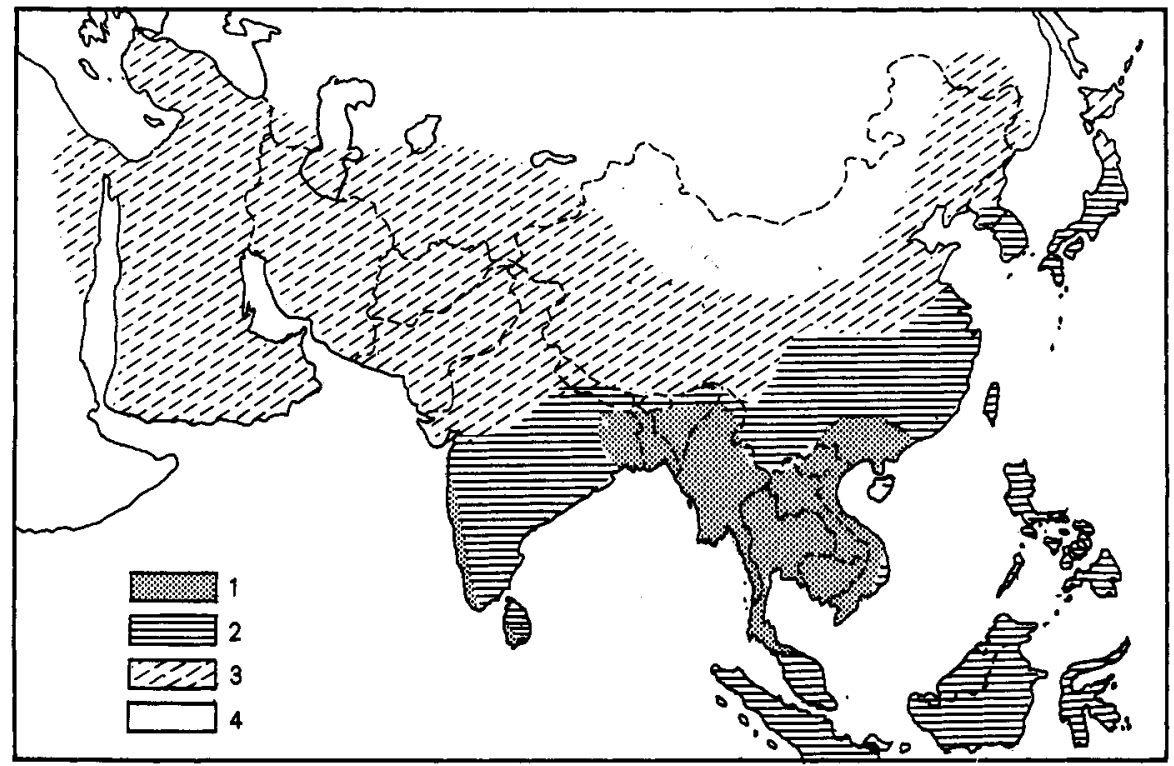

Fig. 7.17 Present-day relationships between rice cultivation and environment: (1) "home" area of rice where crop may be raised year after year without climatic modification; (2) important rice-producing areas where at least one parameter of climate is frequently less than ideal for successful crops; (3) areas where climate must be modified to produce a crop; (4) areas with no important rice production. From Huke 1976. Courtesy: International Rice Research Institute. 
All of this means, basically, that equatorial latitudes have never been the most suitable for rice cultivation. Many rice specialists have stressed the difficulties and drops in yields that result in changing from a monsoon to an equatorial climate (see especially Spencer 1963); I have also summarized the basic situation in more detail elsewhere (Bellwood 1980). As Austronesians moved south toward the equator and east toward Oceania, rice evidently dropped out of the crop repertoire until suitable southern hemisphere environments in Java and Bali were reached. This, at least, was a scenario I favored for the first edition of this book-a scenario basically driven by environmental factors-but now I think it needs slight modification.

The environmental factors are of course unarguable and undoubtedly of significance. But earlier in this chapter and in Chapter 5, I raised the possibilitywith Sather (1995) - that early Austronesian economies were varied and not entirely "standard agricultural." In other words, some groups probably grew rice in sedentary terrestrial circumstances, especially in Taiwan and the Philippines. Others adopted foraging, as some of the ancestral Punans of Borneo (Chapter 5, Section I). Still others favored maritime specialization and mobility, as perhaps the people of Bukit Tengkorak in the first millennium BC. It almost stands to reason that the latter type of adaptation would be the one most likely to lead to island colonization, and such people are perhaps the least likely to have had sedentary rice-growing lifestyles. It is also salutory to note that the earliest colonists of the Pacific Islands, from Lapita at ca. $1500 \mathrm{BC}$ through to the ends of Polynesia (especially New Zealand) at about 900 years ago, generally adopted mainly foraging economies in the first few centuries of settlement owing to the profusion of natural resources. Of course, this behavior often led to rapid faunal extinction, as in the case of the New Zealand moas (Anderson 1989). But these populations never abandoned agriculture entirely and were able to revert to it more and more in the later stages of prehistory.

The same might have happened in Island Southeast Asia. Those Austronesians who moved into Oceania abandoned rice cultivation and never thereafter recovered it. Their cousins in much of Malaysia and western Indonesia, if the historical and ethnographic records are any guides, recovered it with gusto (if indeed any of them ever dropped it entirely, which is perhaps unlikely). The archaeological record of rice cultivation, albeit faint, indicates this with clarity. Rice is now well attested in Taiwan and western Borneo by $2000 \mathrm{BC}$, so its cultivation was clearly known to many communities during the early millennia of Austronesian expansion. Sites in central Thailand also have domesticated rice at 2000 вC (Chapter 8). Although Hill (1977) suggested that rice was of minor importance in Peninsular Malaysia prior to the period of Funan (early first millennium AD), I suspect future archaeological research there will push dates for rice cultivation back to an earlier period as well. In eastern Indonesia, rice seems to 
have been less important than millet, yams, taro, and sago even as late as $\mathrm{AD}$ 1500 (Spencer 1966), although it clearly was grown in quantity in favorable lowland regions of the Philippines and Sulawesi (e.g., see Pelras 1981 for Makassar). However, it is almost universally of great importance today in the islands of western Indonesia, including equatorial Borneo and Sumatra, and may always have been so throughout Austronesian prehistory in the monsoon islands of Java and Bali.

Given the above observations, I would incline to the following view of the prehistory of Austronesian cereal cultivation. Firstly, the northern populations in Taiwan and Luzon have probably always cultivated rice and foxtail millet in varying proportions, although the failure to find direct evidence of rice in the oldest Neolithic sites of the TPK culture in Taiwan does raise some problems. Other cereals of Southeast Asian origin, such as the perennial Job's tears and the annual "Japanese barnyard" millet Echinochloa frumentacea (Li 1970), were probably also grown on a minor scale as well.

A second stage of adaptation followed with expansion, probably involving much coastal foraging mobility, into and beyond the southern Philippines after $2000 \mathrm{BC}$. The groups who moved toward Sulawesi and eastern Indonesia clearly dropped rice to the scale of a very minor crop but probably continued to grow Job's tears and some foxtail millet; I am unsure whether the latter would have suffered the same traumas as rice during an equatorial shift. Foxtail millet, of an apparently ancient and shattering variety, is still of importance in Halmahera (Ishige 1980), in central Sulawesi (Downs 1956), and in other parts of eastern Indonesia (Fig. 7.27). It has also retained considerable importance in Taiwan and Lan Yü (Botel Tobago Island; Arnaud 1974; Fogg 1983).

The other side of this second stage concerns those groups who moved toward western Indonesia, Peninsular Malaysia, and southern Vietnam. These appear to have concentrated more on rice and possibly Echinochloa (which does not occur in eastern Indonesia; see Fig. 7.18). Although millet has survived ethnographically amongst the Senoi of central Malaya, there is a chance that it may be a relatively recent introduction. Rice would undoubtedly always have been important in the ideal soil and climatic conditions of magnificently fertile Java and Bali. Nonphotoperiod-sensitive varieties better suited to an equatorial climate may already have developed by the time the first Austronesians began to settle Sumatra and the Malay Peninsula.

\section{B. The Tubers and Tree Fruits}

As far as eastern Indonesia and Oceania are concerned, the equatorial shift during the second stage described above clearly led to a dominance of fruits and tubers over cereals. Of the tubers, the most important are the aroids (Plate 39) 


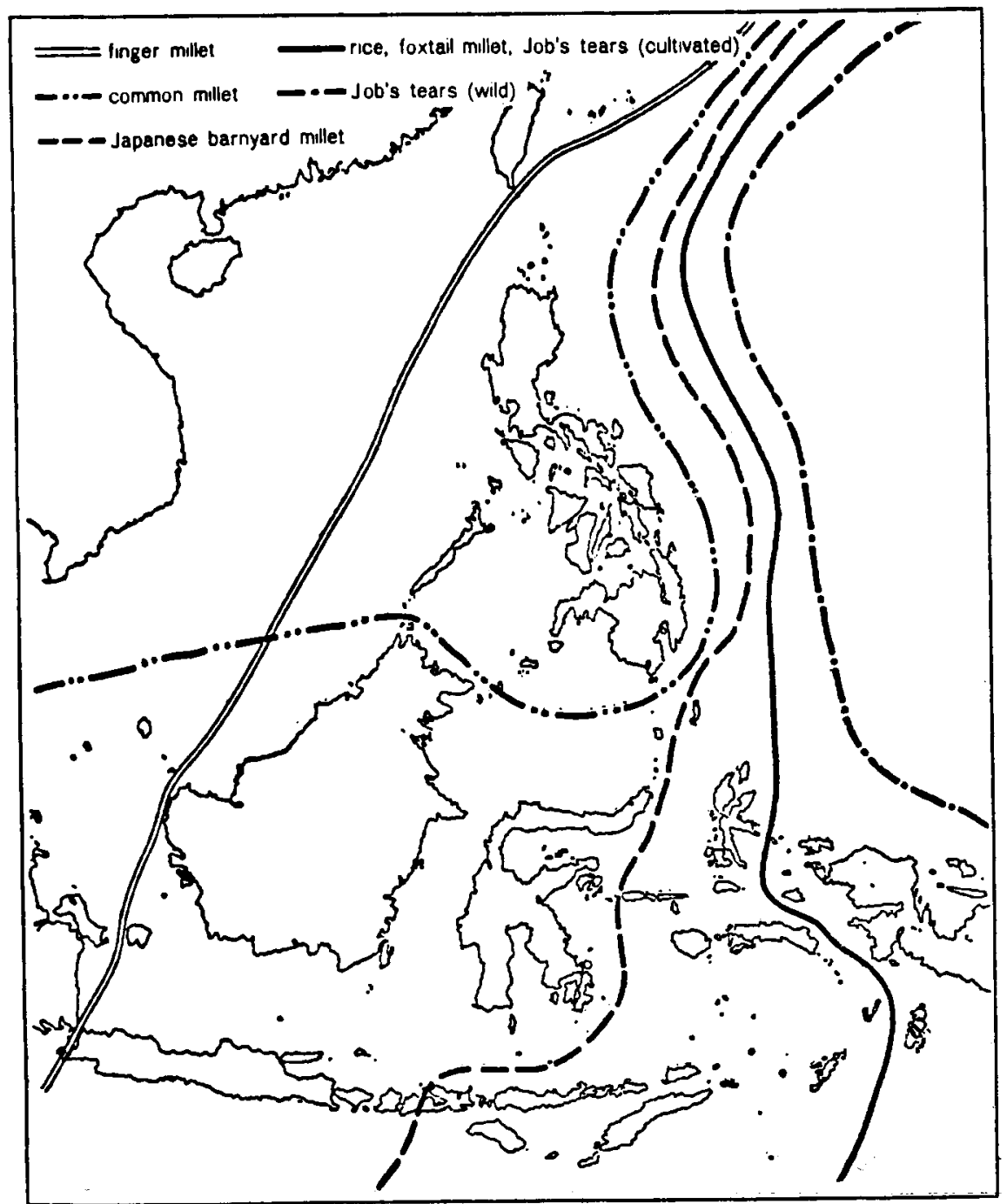

Fig. 7.18 The present eastern limits of cereal crops in the islands of Southeast Asia. Finger millet = Eleusine coracana, of Indian origin; common millet = Panicum miliaceum, of Chinese origin; Japanese barnyard millet = Echinochloa frumentacea; foxtail millet = Setaria italica; Job's tears = Coix lachryma-jobi. From Ishige 1980. Courtesy: National Museum of Ethnology, Osaka.

and the yams. The greater yam (Dioscorea alata) has its homeland in the monsoon regions of northern Mainland Southeast Asia (Burkill 1951) and may have been cultivated by Austronesians throughout their prehistory. Other species of yam were probably first domesticated in Sulawesi and the eastern IndonesiaMelanesia region (Coursey 1972, 1976). Today, yams survive as staples only in 
parts of Taiwan, Mentawai, and Banggai; wild yams still sustain some of the hunters and gatherers, such as the Negritos.

The taro (Colocasia) (Plate 39 left) poses a more difficult origin problem since it grows freely in a wild or feral state along river banks in many parts of Southeast Asia and India. Its homeland, if it ever had a restricted one, remains unknown (Barrau 1965; Yen and Wheeler 1968). Matthews (1995) has suggested the ancestor of cultivated taro is a wild form, Colocasia esculenta var. aquatilis, which grows widely from India to northern Australia and New Guinea; it could have been domesticated on more than one occasion independently. The same applies to the giant aroid Alocasia macrorrhizos, which has a similar widespread distribution (excluding Australia and New Guinea). Colocasia attained some importance in the islands of Southeast Asia; it has been reported as a traditional staple in regions as far apart as Nias, parts of northern Borneo and northern Luzon, and Lan Yü. In the latter island it is grown by the Yami using terraced wet-field techniques similar to those used widely in Oceania (Kano and Segawa 1956). Both the aroids and the yams have probably given way in the face of an expansion of rice cultivation in recent centuries, yet it is clear that they were both of major importance in eastern Indonesia prior to AD $\mathbf{1 5 0 0}$ (Spencer 1966).

Of the more important fruits, bananas were domesticated locally from Eumusa species in Indonesia and Malaysia and from Australimusa species in the Moluccas and New Guinea (Simmonds 1966). Wild forms of both still grow on Halmahera today. Coconuts, despite a rather uncertain ancestry, may well have been first domesticated (or at least systematically planted) in the IndonesianMelanesian region. Coconut remains are common in Lapita sites in Melanesia and coconuts from prehuman contexts have been excavated on Aneityum Island in Vanuatu, far out in the western Pacific (Hope and Spriggs 1982). Harries (1978) believes the round-fruited and thin-husked form of coconut most commonly grown today was first domesticated in Thailand or Island Southeast Asia, but indigenous domestication of a long-fruited and thick-husked variety in Island Melanesia is also possible.

The starch-yielding sago species (Metroxylon, Corypha, Arenga, and Caryota) are also native to equatorial Indonesia and Melanesia (Ruddle et al. 1978), while Eugeissona is restricted to Borneo and Peninsular Malaysia. All have localized roles in hunter-gatherer and agricultural subsistence in some swampy equatorial lowlands, particularly in eastern Indonesia and Melanesia (e.g., see Ohtsuka 1977; Ellen 1978; Ishige 1980). These trees, together with the breadfruit, pandanus, rambutan, durian, and other good fruit-bearing species, would all have been available for systematic exploitation by Austronesian societies expanding into equatorial Indo-Malaysian latitudes. Most were probably also exploited by previous hunting and gathering groups, but full domestication and the devel- 
opment of seedless varieties (as in the bananas and breadfruits) would have required at least some conscious selection and planting.

\section{Shifting Cultivation}

Shifting cultivation of cereals is still practiced widely in Island Southeast Asia today, in both equatorial and drier monsoon climatic regimes. The system obviously varies tremendously from place to place depending on a number of social and ecological factors, but it can support modern populations at densities of up to sixty persons per square kilometer (e.g., see Freeman 1955; C. Geertz 1963; Spencer 1966; Unesco 1978) or above in certain regions of high fertility such as Java (Chin 1977). Compared to the average densities of 0.005 to 0.12 persons per square kilometer given by Unesco (1978) for hunter-gatherers in tropical forests, these figures clearly reveal the demographic significance of this type of agriculture, even if they are small when compared to modern wet-rice population densities of up to 2,000 persons per square kilometer for Java.

Traditional systems of shifting cultivation in Island Southeast Asia normally require short cropping cycles of one or two years to be spaced with much longer fallow periods (e.g., see Freeman 1955; Rousseau 1977), when secondary forest can reestablish itself and shade out the thick-rooted grasses that would otherwise take over and render future cultivation difficult (Seavoy 1973b). The whole system depends a great deal on the nutrients provided by the burning of vegetation prior to planting; most nutrients in tropical forests are contained in the topsoil and vegetation rather than in the subsoil. If cropping continues for too long, the topsoil may be eroded away or depleted in nutrients, and grasslands requiring much more labor-intensive methods of cultivation will establish themselves (this has of course happened in many areas). Prolific weed growth can also promote frequent field shifts, since newly cleared plots require less weeding than old ones (Clarke 1976).

This type of field shifting may eventually require village movement, although it need not necessarily require territorial expansion. For instance, the Mnong Gar of Vietnam need to move their villages every seven years or so (Condominas 1980), but they do so in a cyclical fashion so that an abandoned locality will eventually be reoccupied after many years. The traditional system of the Kayan of Central Borneo appears to have been similar (Hose and McDougall 1912), with village moves occurring every twelve to fifteen years. However, the Iban of Sarawak provide a classic example of a unidirectional type of expansion that has allowed single bilek families to move as much as 300 kilometers in a single lifetime (Freeman 1955:25, 1970:286), through conscious selection of virgin rain forest for new ricefields that are used only for one or two years until weeds promote their abandonment. This phenomenal expansion is of course 
relatively recent and it has involved iron tools and an unlimited expanse of rain forest previously inhabited by only sparse populations, but it does still provide an idea of how rapidly Neolithic cultivators could have expanded into virgin agricultural territories when conditions were right. As the prehistory of Oceania shows, Stone Age people can clear forest quickly, perhaps by using techniques such as ring-barking rather than clear-felling.

Other features of modern shifting cultivation in Island Southeast Asia are also of interest for possible reconstructions of how such systems might have been managed in prehistory. For instance, most groups simply place seeds in holes made by digging sticks in the untilled ash and topsoil, and the system does not require tillage or plowing if it is maintained at a balanced level with sufficient forest regrowth during fallows. Grasslands and greatly increased population densities do of course require more intensive techniques of tillage and mulching, as in parts of the New Guinea Highlands, but in Island Southeast Asia it seems that intensification was directed much more toward the elaboration of wet-rice cultivaton, which I will discuss in more detail below. Most modern shifting cultivators also mix their crops in the fields; for instance, the Iban dibble in a few cucumber, pumpkin, and gourd seeds with the rice (Freeman 1955). This diversity may help to offset some of the risks associated with dependence on a single crop species and it may also help to discourage the depredations of crop pests and rats, which often tend to flourish in totally monocultural systems (e.g., see Takaya 1980 for modern rice cultivation and rat infestation in lowland Sumatra). Planting in holes rather than direct broadcasting also allows for more conscious selection of seeds and thus for the development of different varieties.

\section{THE StAGES Of AUSTRONESIAN AGRICULTURAL PREHISTORY}

In a previous paper (Bellwood 1980), I concluded a discussion of Austronesian agricultural prehistory by postulating four main stages of development. These stages can be reduced to three for purposes of the following discussion, in which I will expand on the types of cultivation practiced. The stages overlap in time, although they are successive in terms of commencement, and each has continued in some form to the present.

\section{A. Stage 1}

Stage 1 is the early phase of Austronesian expansion, centered in southern China, Taiwan, and the northern Philippines. Incorporating the pre-Austronesian Chinese mainland sites, this phase can be dated between 5000 and 2000 BC. Economies seem to have had both maritime and agricultural economies in 
varying proportions, the latter with a cereal component including rice and foxtail millet. As populations spread southward, rice might have continued to be grown in localized swamp or alluvial backswamp conditions similar to those in which its annual forebears originated. These were perhaps similar to those used by modern Borneo peoples such as the Lun Dayeh (Padoch 1985) and Kantu (Dove 1985), in which an initial labor investment can produce flooded fields on riverine flats or in swamps that are easy to maintain and consistently produce higher yields than dryland systems. However, as populations expanded in density, cultivation surely developed more toward the shifting dryland form. Millet can only be grown by dryland techniques, and the dry (or upland) rices were probably developed secondarily at this time by selection for thick and deep roots, loss of photoperiod sensitivity, and a tendency for early maturity to escape the effects of drought (T. T. Chang 1976a; 1989).

\section{B. Stage 2}

The Austronesian expansion toward the equatorial zone after $2000 \mathrm{BC}$ led to a partial replacement of the cereals by the ecologically better adapted tubers (especially the nonseasonal taro) and fruit or starch-bearing trees. The system of shifting cultivation also underwent changes. During Stage 1, plot preparation in the monsoon regions would have demanded a fairly complete clearance for cereals (which need full sunlight), with successful burning of vegetation. In the wetter equatorial zone, clearance would not have been so easy for a people with only stone tools. Vegetation grows prolifically throughout the year and the rain forest trees are more massive and could perhaps only be ring-barked. More importantly, heavy rain can make burning impossible (Freeman 1955; C. Geertz 1963). In Mindanao, yields can apparently double when a good dry period allows a burn (Yengoyan, in C. Geertz 1963:22). So there would be obvious pressures toward the development of cultivation systems requiring less forest clearance and more emphasis on trees and tubers that do not require such broad expanses of uninterrupted sunlight as cereals.

Systems of this type are still widespread in remoter parts of Indonesia and Melanesia today. The Nuaulu of equatorial Seram (Ellen 1978) cultivate taro, yams, bananas, and sago (wild sago stands are also exploited) in multicrop gardens where up to fifteen different species may be grown together (including sugarcane, manioc, coconut, and others). Because the region has no dependable dry season, up to ten burns may take place before planting can occur. If we subtract the iron tools these people now have, it is not difficult to see that largescale garden clearance would not have been a very viable option for Neolithic groups in such an environment. Another example comes from Mentawai, off 
the western coast of Sumatra, where sago, taro, and bananas are grown in swamps with very little clearing and no burning-the cut vegetation is simply used as a mulch (Mitchell and Weitzell 1983). Neither of these groups grows cereals at all, and 1 rather suspect that systems of this kind, which were eventually taken right through tropical Oceania, began to characterize Austronesian economic patterns increasingly after about $2000 \mathrm{BC}$ in the truly equatorial and ever-wet lowland zone.

It should also be remembered that agricultural systems based on tubers and tree fruits may have developed independently in New Guinea, although the only direct evidence for this at present relates to a tradition of swamp drainage for unknown cultigens (taro?) that commenced at about $7000 \mathrm{BC}$ in the Wahgi Valley, deep in the highlands of Papua New Guinea (Golson 1977, 1985). I have mentioned the role of New Guinea in this regard several times previously, and I have tried to make it clear that some parts of western Melanesia might already have been settled by agricultural groups before the period of Austronesian expansion (the biological and linguistic evidence both provide strong support for this viewpoint). However, while such developments may have been of profound significance for western Melanesia, there is no compelling evidence at the present time suggesting that they had any great effect on the development of agriculture in the Indo-Malaysian Archipelago.

\section{Stage 3}

By 3000 years ago most cultivation systems were probably still based on shifting and localized swamp cultivation, with a predominance of cereals in northern regions such as Taiwan and the northern Philippines, and perhaps in Java and some of the dry Lesser Sundas. Along the equator, cereal cultivation may have been of importance in the western islands of Borneo and Sumatra, but there is very good evidence that tuber and fruit dominance had long been developed in eastern Indonesia and, of course, in Oceania. Prior to 3,000 years ago it is possible that varieties of rice with low photoperiod sensitivity had developed within the archipelago and that these rices (like weaving and the backstrap loom) might have been undergoing geographical expansions in popularity through quite a long period. However, the main features of Stage 3 as recorded historically cannot really be stated to involve changes in crop dominance; instead it appears that a major but regionally localized shift toward intensive wet-rice cultivation in bunded fields (sawahs) occurred, contemporary with but perhaps independent of a similar shift to bunded field cultivation of taro in Oceania (Kirch and Lepofsky 1993). Such bunded or embanked fields for rice utilize water supplies derived either from wet-season rains (rainfed systems) or artifi- 
cial canals (irrigated systems). Both rainfed and irrigated systems can be laid out as a checkerboard network of bunds on flat land, or they may be terraced into slopes and even steep hillsides (Plates 40 and 41).

It is most unfortunate that wet-rice cultivation, a system that in both irrigated and rainfed forms has obviously transformed islands such as Java, Bali, and Luzon, has no clear archaeological or linguistic prehistory in the IndoMalaysian Archipelago. The massive success in the archaeological recognition of ancient rice fields through excavations and phytolith analyses in recent years in Japan suggests that modern technology might soon make some breakthroughs, but the fact remains that none have occurred yet. On present evidence, mainly based on guesswork, any great economic importance for this kind of cultivation prior to perhaps 2,500 years ago can hardly be assumed. Historical records indicate wet-rice cultivation in northern Vietnam from about $200 \mathrm{BC}$ (Wheatley 1965); here and in northern Thailand there is archaeological evidence that wet-rice cultivation in bunded fields may have developed during the Iron Age (after $500 \mathrm{BC}$ ), together with the use of water buffalo for plowing (Higham 1989:198-200). In Java the oldest inscriptions referring to irrigation (presumably for rice) date from the eighth century AD (Meer 1979). This is about as far as the direct evidence goes, and at this rather vague level it is clearly inappropriate to debate whether rainfed systems preceded canal-irrigated systems (or vice versa), or whether both forms developed together as a result of local differences in topography.

But there are other important points to note about wet rice. Most modern systems depend on iron and water buffalo for successful management, and this has led to the idea that wet rice must be an Iron Age phenomenon. This, of course, is not so, since the Polynesians managed quite well to develop and use similar wet taro systems with only stone tools and no traction animals. Nevertheless, there is no doubt that wet-rice cultivation as known today is mainly (but not entirely) related to large, dense populations and the iron-buffalo complex of technology. It is not commonly found in regions of light population and the system clearly flourishes best on fertile volcanic and alluvial soils, as in Java and Bali, where it was so closely tied historically with the Indianized civilizations that a major development during the first millennium AD must always remain likely (there is no good evidence against this possibility). Wet rice in these islands can support enormous populations as yields can be increased through more careful field preparation, transplanting, and continuous cropping through the year with irrigation (Geertz 1963). Wet rice also matures faster than dry rice. The sawahs themselves can be cropped indefinitely in many regions without fallows, partly because the irrigation waters carry nutrients and also because fern-dwelling algae in the sawahs are efficient fixers of atmospheric nitrogen. 
A major point here, of course, is that wet-rice cultivation on a given unit of land can feed many more people than dry rice grown by shifting cultivation, but the establishment of the necessary field systems does require a great deal of initial labor. So it is hardly surprising that many shifting cultivators would continue with swiddening unless obliged to intensify, perhaps owing to population pressure (e.g., see Seavoy 1973a for Kalimantan), or in the face of managerial demands for increased production to support a state or bureaucratic apparatus. The Indianized civilizations may well have been able to enforce such managerial demands in Java and Bali, but explanations of this type hardly suffice for wet-rice cultivation by the remote Kelabits and Lun Dayeh of inland Borneo (Schneeberger 1979; Padoch 1983), or by the peoples of the northern interior of Luzon whose magnificent terraces are amongst the most spectacular anywhere in eastern Asia (see Plate 41).

The Ifugao terraces of Luzon (Conklin 1980) comprise 20,000 kilometers of embanked terraced fields, of which 7,000 kilometers are stone faced. They support a relatively small-scale bilateral society with densities of between 100 and 250 persons per square kilometer. About one-half of Ifugao subsistence needs are also provided by shifting cultivation, and land does not seem to be in short supply. According to Reid (1994d), the linguistic terminology for rice agriculture and terracing in northern Luzon descends from Proto-Nuclear Cordilleran, a language that might have existed 1,500 to 2,000 years ago. There is as yet no archaeological evidence to support this rough date, but it seems not unreasonable. The terraces are clearly not associated in any way with the presence of a centralized authority or a master plan; they have probably been developed piecemeal by wealthy but tribally organized families of high status, able to command sufficient labor for construction and maintenance. The mountainous Luzon terrain is obviously also suited to terracing in an aesthetic sense, and one cannot entirely discount the importance of such a factor.

Perhaps I can draw one moral from this story: Wet-rice irrigation can exist on a large scale without bureaucratic intervention of the type associated with the ancient canal irrigation systems of Mesopotamia or northern China. This, as pointed out by Bray (1986) for southern China, is because wet-rice irrigation can operate as a piecemeal family or small group system, as with the subak irrigation corporations of Bali. There is, therefore, a potentially optative basis to the origins of wet-rice cultivation; the system need not always reflect the demands of a state organization and a dense population, although history of course shows that this has often been the case. Seemingly aberrant cases such as Luzon and central Borneo are really rather hard to explain without at least a partial model of free choice-and perhaps emulation.

Prior to AD 1500, sawah rice may have been the limit of intensification in the Indo-Malaysian Archipelago, although I should perhaps mention the intensive 
tapping of the juices of the lontar palm (Borassus sundaicus) in Roti and Savu in eastern Indonesia (Fox 1977). In addition, the modern story of the opening of the lowland swamps of Sumatra, Kalimantan, and Irian Jaya to rice cultivation (Collier 1979; Tsubouchi 1980) shows just how productive such apparently useless environments can become, but this is a technology-based and organizational option that probably would not have been at all attractive to, or even feasible for, prehistoric Austronesian cultivators. 


\section{The Archaeological Record of Early Agricultural Communities in Peninsular Malaysia}

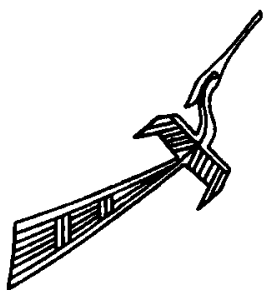

In this chapter we examine the spread of agricultural peoples down the Malay Peninsula from the north. This spread occurred approximately contemporaneously with (beginning perhaps slightly before) the major dispersal of the Austronesians in the second millennium $\mathrm{BC}$, and involved the ancestors of the present Austroasiatic-speaking Senoi populations of southern Thailand and Malaysia. As discussed in Chapters 4 and 5, these populations are now surrounded and impinged upon by Austronesian and Thai speakers, but they still exist today in greatest numbers in the interior of Peninsular Malaysia.

In order to understand the Peninsular Neolithic it is necessary to commence with the rich agricultural settlements of southern Thailand, several of which are now firmly dated from about 2500 BC onward. Indeed, one could extend this section into a discussion of the whole topic of early agricultural population expansion through the mainland of Southeast Asia, a topic already broached by several authors (Bellwood 1992; Higham and Thosarat 1994; Higham 1996a). I do not have space here to follow this temptation, but will only note, with Higham, that an early agricultural expansion from a homeland region in the northern part of Mainland Southeast Asia and southern China can perhaps be recognized in the dispersal of a distinctive type of ceramic decoration-found in the oldest regional assemblages-focused on incised zones infilled with stamped (shell-edge, dentate, punctate) impression. This kind of decoration is dated between the mid-third and mid-second millennia $\mathrm{BC}$ in many sites in southern China, Vietnam, and Thailand (Rispoli 1992). ${ }^{1}$ We are about to meet it again in central Thailand and the Malay Peninsula, where the Neolithic phase can be considered to have continued after $2500 \mathrm{BC}$ until the local spread of copper metallurgy during the first millennium вс. 
The huge habitation and burial mound of Khok Phanom Di, which now lies on the inland edge of an extensive alluvial plain about 50 kilometers east of Bangkok, is one of the richest and most impressive pre-Bronze sites ever excavated in Southeast Asia (Higham and Bannanurag 1990, 1991; Higham et al. 1992; Higham and Thosarat 1994). The site is 200 meters in diameter and has almost 7 meters of archaeological deposit dating between 2000 and $1400 \mathrm{BC}$. When first occupied it lay close to a mangrove shore-perhaps with freshwater ponds that could be modified for rice growing-but today the site is far inland as a result of a slight fall in sea level combined with alluviation resulting from inland forest clearance for agriculture. The basal of the three major excavated phases yielded 104 burials (mostly extended) in clusters, some wrapped in bark cloth and dusted with red ochre. One of the most striking features of this early phase is the tragically high fetal and infant death rate, comprising 42 percent of all burials (Higham et al. 1992:47), possibly caused by the ravages of malaria (cf. the high infant death rate for the contemporary burial ground of Peinan in Taiwan: Chapter 7, Section IIA).

Grave goods of this early phase at Khok Phanom Di include shell beads and bracelets, stone adzes, and well-crafted pottery; the finest vessels have cordmarked or burnished surfaces and horizontal zones of incised and infilled decoration of the type referred to above (Fig. 8.1). The people of Khok Phanom Di grew rice in large quantities (Thompson 1992)-it occurs as husk temper and impressions in pottery-used harpoons and fishhooks of bone, and ate large quantities of marine foods such as fish, shellfish, crabs, and turtles. Domestic dogs were present. Pig bones are fairly rare and it is not clear if pigs were domesticated, but bones of a species of jungle fowl that would not have been native to this region suggest that domesticated poultry might have been present.

In the middle level of the site, dating presumably to early in the second millennium BC, a number of richly provided burials made an appearance. Two women were buried under an apparent mortuary hut with a floor that was replastered with clay forty-three times. Another woman was buried under a large pile of the unfired clay cylinders from which pots are made, together with strings of beads-120,787 shell disc beads in total-over her chest, and lots of fine pottery vessels. Evidently she was a potter of high status. A child, perhaps a member of the same family, was buried near her with similar high-status goods. These wealthy burial assemblages indicate that the society was perhaps becoming ranked on a genealogical basis, and females in particular seem to have enjoyed high positions. Individual burial zones seem also to have been used by members of the same family through several generations, according to skeletal analyses.

The date of appearance of rice cultivation in this part of Thailand is uncertain. Rice phytoliths from a core drilled near Khok Phanom Di have been dated 

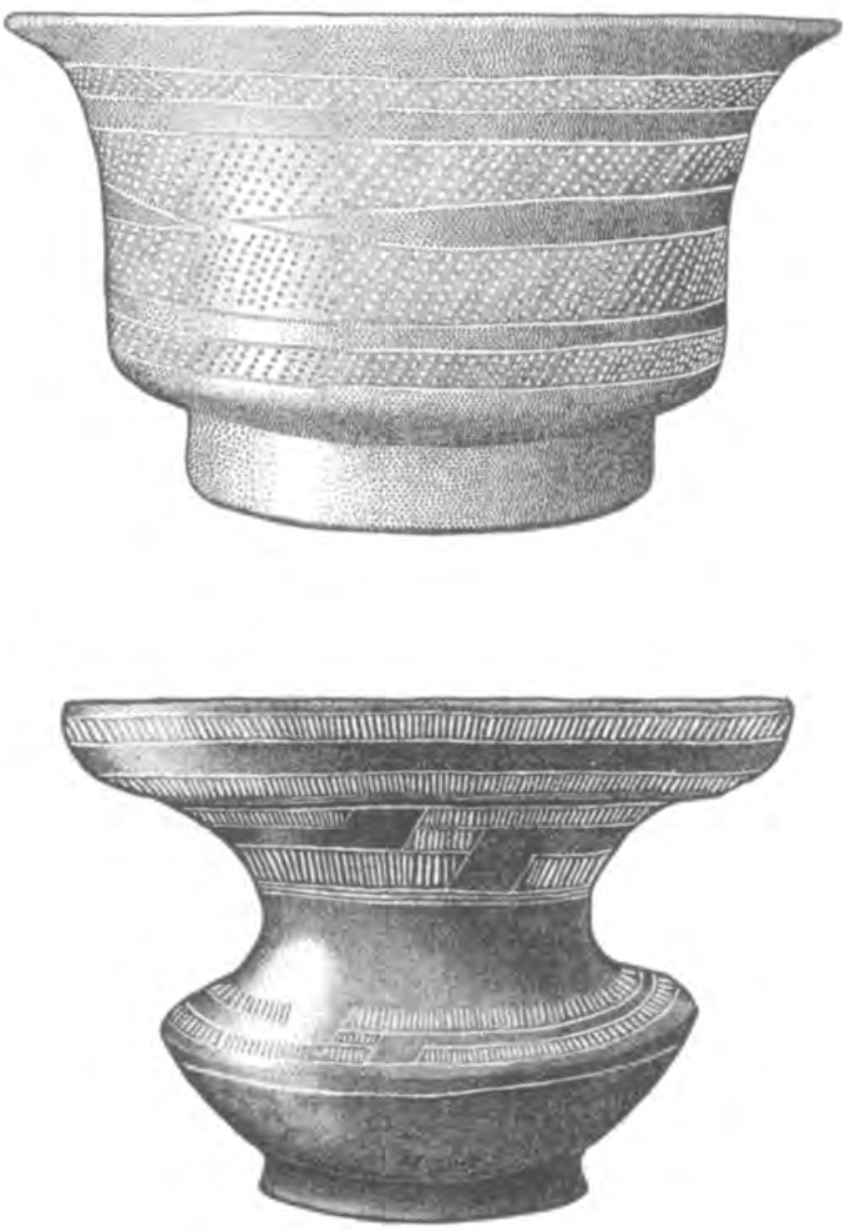

Fig. 8.1 Pottery with incised and stamped zones of decoration from Khok Phanom Di, central Thailand, 2000-1500 BC. Top, 19.5 centimeters high; bottom, 21 centimeters high. Courtesy: Charles Higham.

to about 2500 вС (Kealhofer and Piperno 1994), but these could be from wild rice growing naturally in the coastal swamps.The site of Nong Nor, which lies 20 kilometers south of Khok Phanom Di and overlaps in date with the beginning of occupation there, also shares a similar material culture (including pottery), but has no evidence for rice at all in its lowest layer ( $O^{\prime}$ Reilly 1995). Neither does the site of Non Pa Wai Phase I, located near Lopburi in the Chao Phraya Basin, which has yielded a burial assemblage from the late third millen- 
nium BC (V. Pigott: pers. comm.). This is puzzling, but the absence could be explained in the case of Nong Nor if it was a seasonal camp for specialized resource collection rather than an agricultural village. The whole issue regarding the point at which rice first appears in regional archaeological records is clearly a most important one, as discussed at length in Chapter 7.

The main significance of Khok Phanom Di for Malay Peninsula prehistory is that it supported a rich and populous society with an economy focused on rice cultivation and maritime resources, albeit living in an environment initially plagued by an apparently high incidence of malaria. The site was finally abandoned about $1400 \mathrm{BC}$ after the sea had retreated far from its vicinity. It is worth noting that as the sea retreated, so too did the infant death rate, but the proportion of people dying in later childhood rather than infancy increased with time. This suggests that malaria itself was the major killer in the early phase, but later it decreased in intensity as the environment became less swampy and mosquitoes presumably decreased in numbers, so that more people survived early childhood only to die from anemia. This change appears to reflect the presence of abnormal hemoglobins in the blood of the Khok Phanom Di population, selected for because of the resistance they give to malaria for those people with heterozygous alleles at the relevant genetic loci (Tayles 1994).

Khok Phanom Di was therefore abandoned because of a declining locational advantage, rather than simply to escape disease. Throughout its history, however, it is clear that the birth rate there was quite high, presumably to compensate for the high infant and child death rate. Under such cultural circumstances, populations who were able to move away from malarial swamps into situations of lower infant mortality might have been able to support very high rates of demographic growth, similar perhaps to those revealed by studies of modern Orang Asli populations in Peninsular Malaysia (Fix 1977; Gomes 1982). It was thus presumably no coincidence that the period between 2000 and 1500 BC also saw the Neolithic colonization of the Malay Peninsula.

Elsewhere in central Thailand, the assemblages from Nong Nor and Khok Phanom Di are paralleled quite closely in the site of Ban Kao, in the valley of the Kwae Noi River in Kanchanaburi Province, northwest of Bangkok (Sørensen and Hatting 1967; Sørensen 1972, 1985, 1988). The burial assemblage here dates from the late third or second millennium $\mathrm{BC}$; it postdates Hoabinhian assemblages that have been dated in the nearby caves of Khao Talu and Heap to as recently as $2500 \mathrm{BC}$ (Pookajorn 1990). It comprises extended burials with a range of grave goods including untanged stone adzes, barbed bone harpoon or spear points (Plate 42), shell beads and bracelets, and finely made cord-marked pottery with an unusual predilection for high pedestal or tripod supports (Plate 43). The skeletal remains from the Ban Kao burials are similar to those of modern Southeast Asian Mongoloid populations (Sangvichien et al. 1969). The 


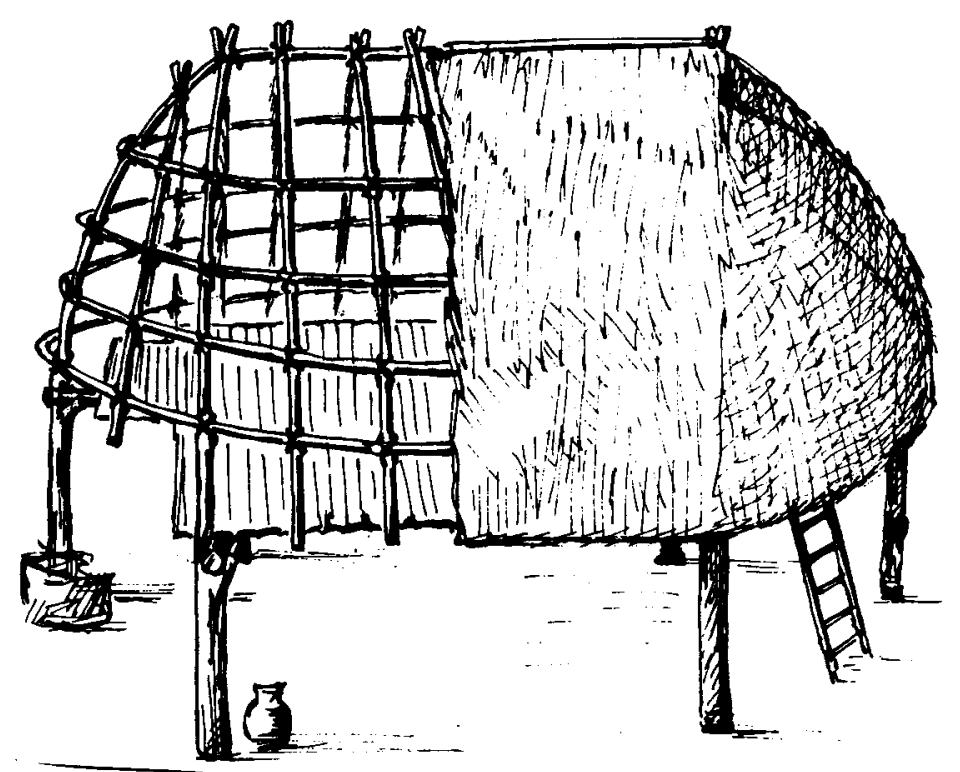

Fig. 8.2 One possible reconstruction for the postholes of a house excavated at Nong Chao Sao, western Thailand. Ban Kao culture. House length ca. 9.5 meters. From Henricksen 1982. Courtesy: Per Sørensen.

habitation layers of the site have also produced many other important categories of Neolithic technology, including shouldered adzes, stone bracelets, bone fish hooks and combs, and baked clay bark-cloth beaters and spindle whorls (the latter for spinning-possibly cotton thread?). One site called Nong Chae Sao, located south of Ban Kao, has yielded the postholes of a small raised-floor house (Henricksen 1982) (Fig. 8.2), and there is some evidence that these people may have had domesticated pigs and fowl. So far there is no direct evidence for rice at Ban Kao, but its presence must be assumed given its importance at Khok Phanom Di. Ban Kao has also yielded stone reaping knives that might have been used for rice.

By 2000 в , assemblages related to those from Khok Phanom Di and Ban Kao begin to appear in sites all down the Malay Peninsula, southward for 1,500 kilometers, into central Peninsular Malaysia. Sørensen (1972) referred to this whole phenomenon as the "Ban Kao Culture," and I used this term myself in the first edition of this book. Today, it is more apparent that a degree of regional variation must be recognized, although the homogeneity of the whole archaeological phenomenon is still very striking.

The pottery from the Ban Kao burials was divided by Sørensen into two chronological groups: an early one with many vessels with tripod feet, ring feet, or 
high pedestals, and a later one with plainer round or flat-based forms. Fragments of the cord-marked tripod-footed vessels that characterize the early phase (ca. 2000-1500 BC) have now been found in about twenty sites right down the Malay Peninsula, in both southern Thailand and Malaysia (Bellwood 1993:47). At the site of Jenderam Hilir in Selangor, many such tripods-virtually identical to those from Ban Kao-have been found during tin mining operations and have been radiocarbon dated to ca. $2000 \mathrm{BC}$ (Leong 1990, 1991). This date suggests that the spread of the Ban Kao type of assemblage was very rapid from north to south.

In Peninsular Malaysia there are no known open burial grounds like Khok Phanom Di and Ban Kao; most assemblages come from burials in caves, mostly located in limestone massifs in the central and northern parts of the country. The Malaysian Neolithic pottery as a whole is quite similar to that from the Thai sites; some was made on a slow wheel, the same Ban Kao tripod and footed forms and vessel shapes occur, and most surface decoration is cord marked or burnished (Peacock 1959). The distinctive tripods, with round holes to allow air to escape during firing, have been found (as well as at Jenderam Hilir) unstratified in the caves of Gua Berhala in Kedah (Fig. 8.3), Gua Bintong in Perlis (Peacock 1964a), and in the open site of Kampung Dusun Raja in the interior of Kelantan (Adi 1993). The Jenderam Hilir unstratified finds also include untanged quadrangular and shouldered adzes, a Tembeling knife (see below), and two wooden oars carbon dated to within the first millennium BC (Batchelor 1977; wherein the site is referred to as Dengkil).

At Gua Cha in Kelantan (see Chapter 6, Section IA for the Hoabinhian Phase in this site) the Neolithic deposits began, according to Sieveking (1954), with a discrete working floor for quadrangular adzes found in his Cutting 1 . Human

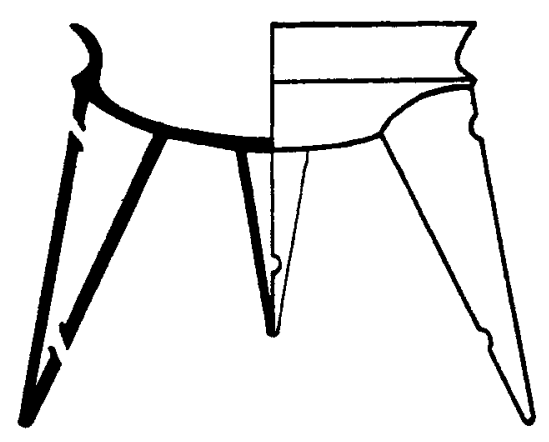

Fig. 8.3 Reconstructed tripod vessel about 28 centimeters high from Gua Berhala, Kedah. From Peacock 1959. Courtesy: University of Hawai'i Press. 

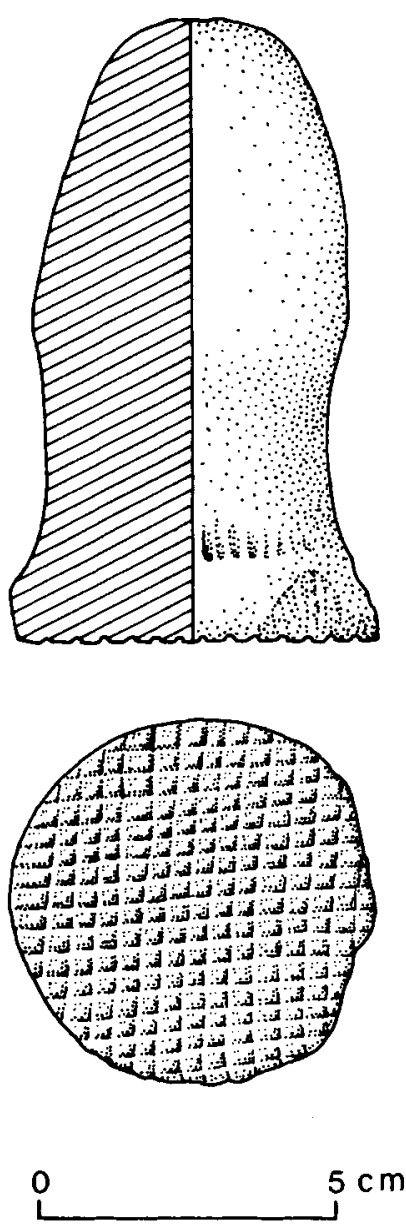

Fig. 8.4 Cylindrical stone bark-cloth beater from Gua Cha. After Sieveking 1956c.

burials were then placed in the shelter. Sieveking believed in 1954 that the burials belonged to two separate periods: an earlier one with fairly crude cordmarked vessels, and a later one with the more elaborate pottery of Ban Kao type. In a more recent paper (Sieveking 1987), he has revised his opinion to favor a single Neolithic phase, during which time the shelter was used almost exclusively for burial rather than habitation. This exclusive burial use characterizes most of the Neolithic cave assemblages in Malaysia and reinforces the view that a shift to a village-based lifestyle had already occurred in riverine locations adjacent to the caves.

Most of the Gua Cha burials were in extended positions. They were buried with grave goods (Plate 44) that included D- or T-sectioned stone bracelets, quadrangular-sectioned adzes and a single beaked adze (see Chapter 7, Fig. 7.15, 
7D), a cylindrical stone bark-cloth beater (Fig. 8.4), shell bead necklaces, and shell spoons. The T-sectioned bracelets in particular are distinctive artifact types paralleled in many Neolithic and Bronze-Iron Age sites across the mainland of Southeast Asia, from Khok Phanom Di through the Bronze-Iron Age graves of Yunnan. The Gua Cha pottery presents the most complete Neolithic assemblage known from Peninsular Malaysia; it includes footed, round, and flat-based forms (no tripods) with a predominance of cord-marked decoration (Fig. 8.5). Many vessels have burnished upper surfaces and definite slow-wheel striations, and some are red slipped. Another cave in Kelantan called Gua Musang has vessel forms similar to Gua Cha (Tweedie 1940), and the collections of the National Museum in Kuala Lumpur have sherds with an almost identical red slip from sites as widespread as Gua Cha and Gua Musang in Kelantan, Gua Bintong in Perlis (Collings 1937a), Gua Kajang in Perak (Evans 1918), Gua Kelawar in Kedah (Collings 1936), and Gua Kechil in Pahang.

Another important but unstratified Neolithic collection comes from a cave in the limestone massif of Bukit Tengku Lembu in Perlis (Sieveking 1962) (Fig. 8.6). The pottery here is of the same fabric and manufacture as that from Gua Cha, but forms apparently localized to this region include beakers on high splayed feet and flat-based, bell-mouthed jars. Sherds of a vessel of Indian manufacture, of a south Indian form of the period ca. $200 \mathrm{BC}$ to AD 200 (Bronson 1979:330; see also Wheeler et al. 1946:58 for parallel form 18C from Arikamedu in Tamil Nadu), have been found in the site, but its precise association with the rest of the assemblage remains uncertain. However, it seems likely that the Bukit Tengku Lembu assemblage is later in time than that from Gua Cha. Bukit Tengku Lembu has also yielded a bone gouge, a stone bracelet, and some very fine beaked adzes and untanged quadrangular adzes with splayed cutting edges.

As well as the dates from Jenderam Hilir, the Gua Cha Neolithic assemblage is probably dated by a single radiocarbon determination to about 1500-1000 BC (Adi 1985). There is also a carbon date of about $1800 \mathrm{BC}$ for cord-marked pottery from a cave called Gua Harimau in Perak (Dunn 1966), and results of recent excavations in this site by Zuraina Majid (1991) are awaited. The site of Gua Kechil in Pahang, which has lower levels with a few Hoabinhian tools and cordmarked pottery associated together (Dunn 1964), has a non-Hoabinhian upper level with pottery in the same tradition as that from Gua Cha, dated to sometime in the fourth millennium BC by a single radiocarbon date with an 800 -year error range (Dunn 1966). However, this date seems to be too old for the upper Gua Kechil assemblage. The site has also produced two bone projectile points (one sharply tanged, see Plate 42) and untanged adzes with quadrangular and lenticular cross-sections, but none of this material demands a date on typological grounds older than the other Malaysian Neolithic sites. Given these 

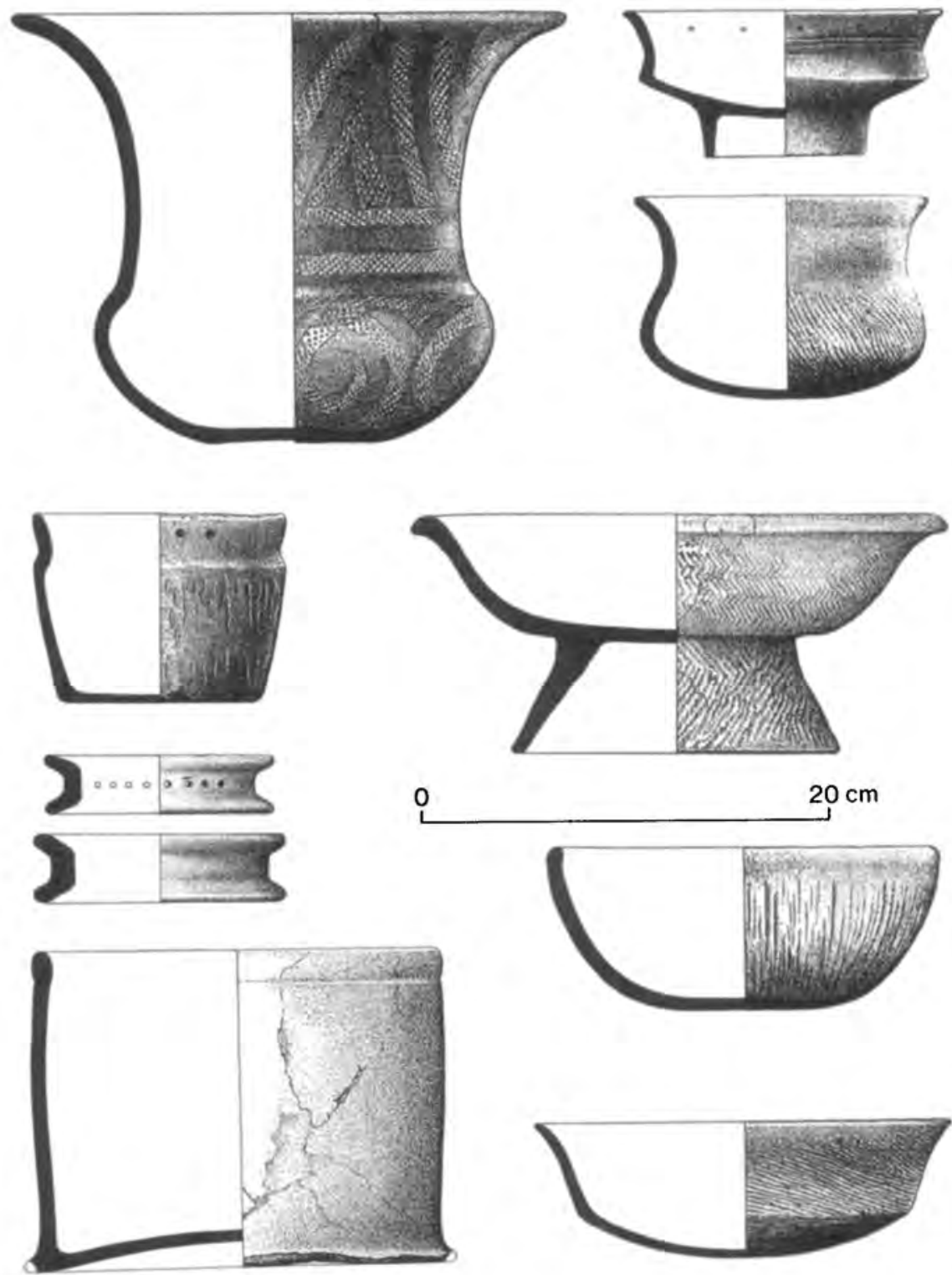

0
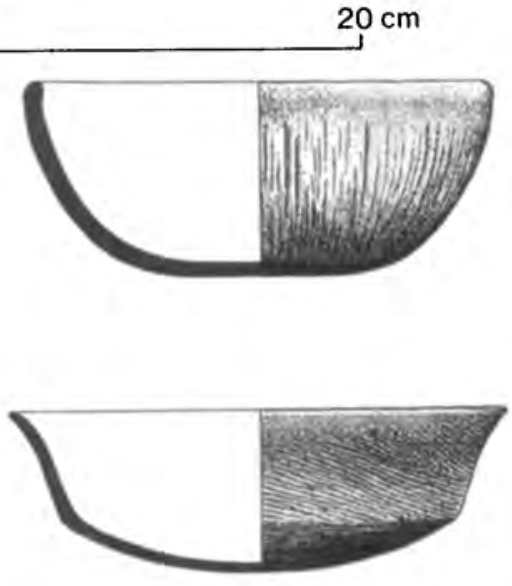

Fig. 8.5 Cord-marked, incised, and punctate pottery (top left), and red-slipped pottery (bottom left) from Gua Cha, Kelantan. There are also two pot stands at center left. From Sieveking 1954. Courtesy: National Museum of Malaysia. 

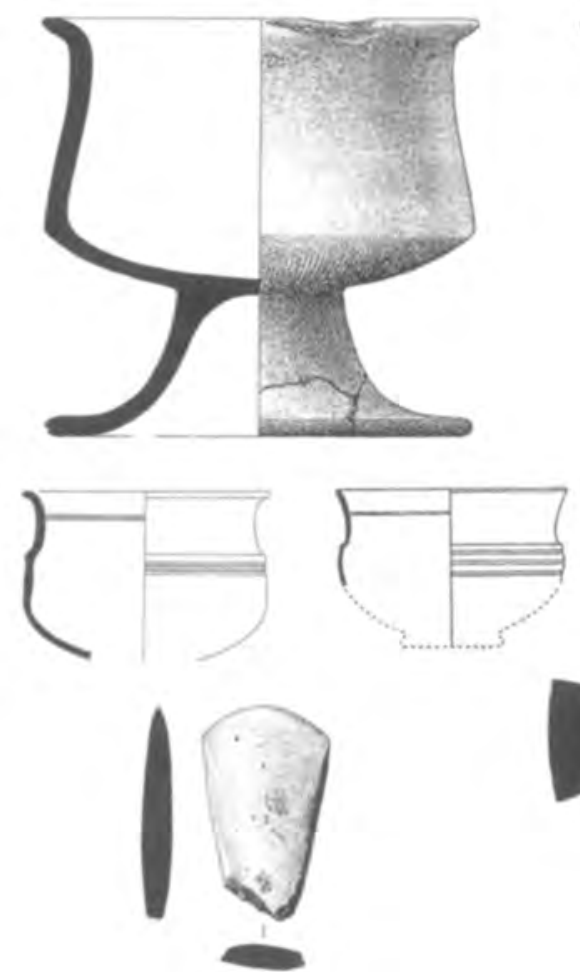
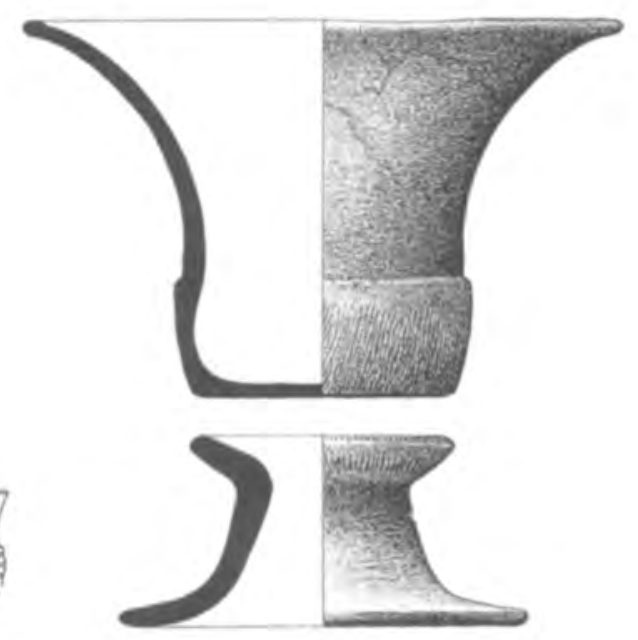

,
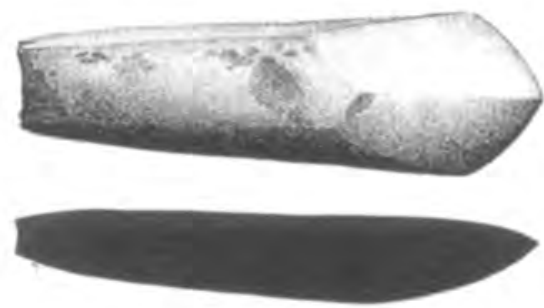

Fig. 8.6 Pottery and stone adzes from Bukit Tengku Lembu, Perlis. To same scale; top left vessel is 17.7 centimeters high. Beaked adze at bottom right. Center left: vessels of Arikamedu type $18 \mathrm{C}$ from Bukit Tengku Lembu (left) and Arikamedu (after Wheeler et al. 1946); these two items not to scale. From Sieveking 1962. Courtesy: National Museum of Malaysia.

pointers, one can perhaps place the whole Peninsular Malaysian Neolithic within the last two millennia $\mathrm{BC}$.

There are other Neolithic sites in central and northern Peninsular Malaysia that do not fit so well within the general bounds of the Ban Kao culture (the southern half of the country remains fairly blank). I have already referred to the unusual necked axes and pottery from the shell mounds at Guar Kepah in the state of Pulau Pinang (see Chapter 6, Section IA), and I should also refer to an open site of uncertain character partly excavated by Evans (1928a, 1931a) in the alluvium of the Tembeling River at Nyong in Pahang. Artifacts were found here scattered through a 4-meter thickness of alluvium; they included several of the distinctive stone "Tembeling knives" (see Chapter 7, Fig. 7.215, 5D)—an unusual tool that may best be regarded as a side-hafted axe or adze, but that in some cases may have served as a reaping knife. There is also another possible stone 
reaping knife with two perforations. Other items include adzes of quadrangular and beaked forms, a cylindrical bark-cloth beater, and fragments of stone bracelets and a discarded center ring. The stone assemblage is in fact well paralleled at Gua Cha, and there are also similar sherds of cord-marked and red-slipped pottery, some made on a slow wheel. However, a few spouts and lugs (both absent in Ban Kao pottery) suggest that the assemblage may not all belong to one period.

\section{ThE SIGNIFICANCE OF THE BAN KAO CULTURE AND ThE MALAY PENINSULAR NEOLITHIC}

Explanations for the Ban Kao culture and its southward extension in anthropological and historical terms will necessarily be rather complex, given the high degree of anthropological and biological variation still found in the peninsula. Prior to $2000 \mathrm{BC}$ the region was occupied by Hoabinhian foragers who may be considered ancestral to the Semang Negritos, and perhaps to a lesser degree to the Senoi, who have a greater degree of Southern Mongoloid biological affinity than the Semang (Saha et al. 1995). The southward expansion of Neolithic assemblages was most probably by movement of people rather than by trade or superficial diffusion, and in virtually all sites apart from Gua Kechil the transition from Hoabinhian to Neolithic is a relatively sharp one (see Chapter 6). This major change seems to have been associated with the introductions of agriculture and Austroasiatic languages to southern Thailand and Malaysia. The Semang have clearly at some time in their past adopted Austroasiatic languages, and the languages of both the Semang and the Senoi populations are today classified in a subgroup termed Aslian, which retains distant relationships with Mon and Khmer.

The ancestry of the Senoi, if this historical reconstruction is accepted, may thus be quite closely correlated with the expansion of Neolithic cultures down the Malay Peninsula at around $2000 \mathrm{BC}$. Continuity from local populations cannot be ignored, however, and skeletons from both Hoabinhian and Neolithic contexts at Gua Cha show no marked signs of any phenotypic population change across the cultural boundary. Presumably, therefore, the skeletons from both periods in this site can be considered ancestral Senoi to some degree, assuming that the Senoi really are the descendants of the Neolithic populations of the Peninsula-an assumption that seems logical enough, even if impossible to demonstrate clearly. However, Gua Cha is a remote interior site; one might expect the evidence for biological change to be a little sharper in more accessible and densely populated coastal regions, should large skeletal series ever be found there.

Whatever the exact situation, it is clear that prior to the first arrival of Aus- 


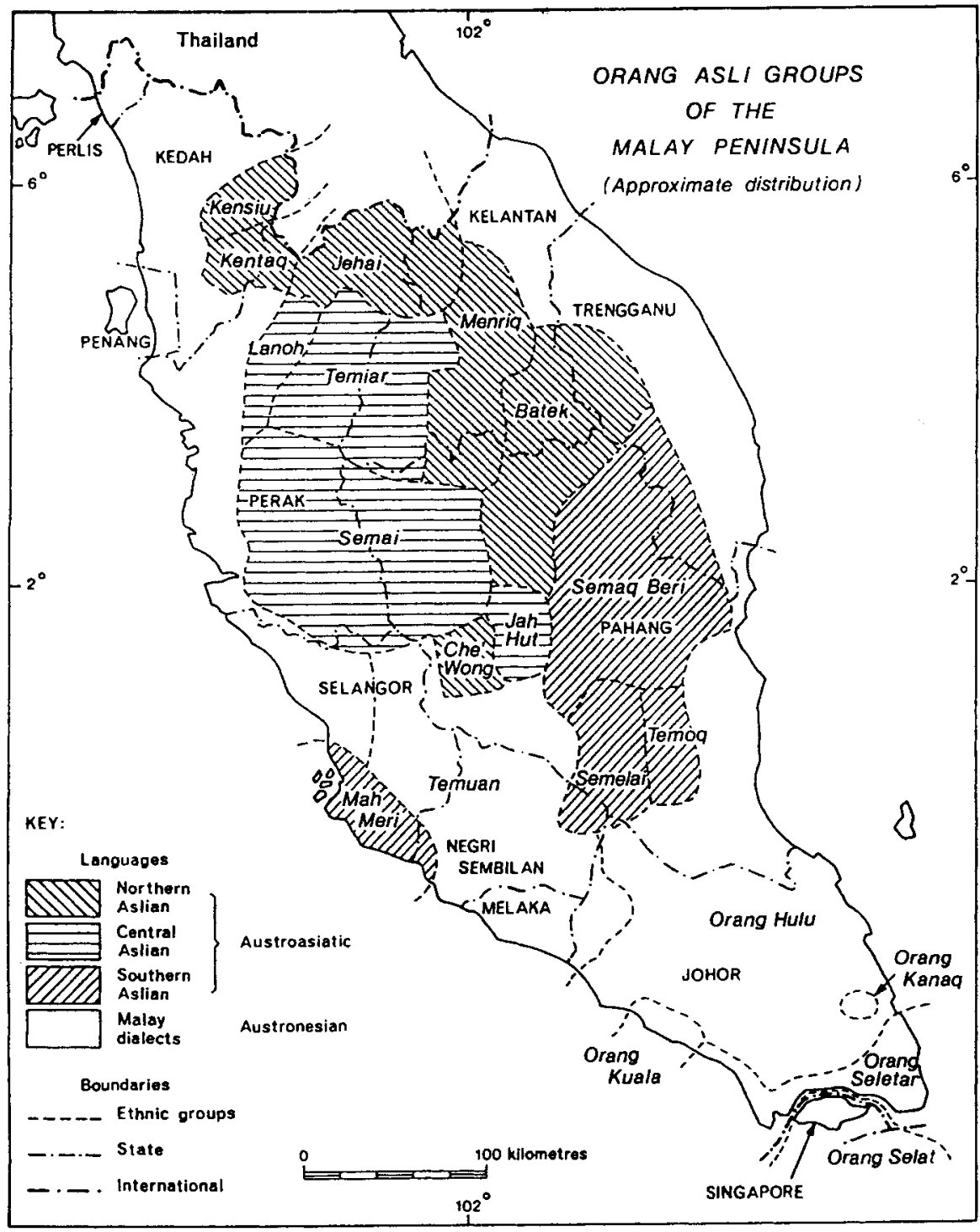

Fig. 8.7 The distribution of Aslian languages and Malay dialects (excluding Malay proper, which is now spoken over most of the peninsula except for the interior Temiar, Semai, and Jehai regions). Courtesy: Geoffrey Benjamin, drawn by Joan Goodrum. 
tronesian-speaking peoples in Peninsular Malaysia, perhaps during the first millennium $\mathrm{BC}$, the Austroasiatic populations of the lowlands would have been firmly established in an agricultural mode of production for at least a millennium-perhaps longer.

A linguistic reconstruction of the course of prehistory in Peninsular Malaysia has been proposed by Benjamin (1976), who suggests that the Negritos (Northern Aslian speakers in the Mon-Khmer subgroup, Austroasiatic family; Fig. 8.7) have always retained their mobile hunting and gathering lifestyle. This suggests that they have presumably not been associated with Malaysian Neolithic developments, and from this viewpoint can be regarded as the most direct descendants of the Hoabinhians. The Central and Southern Aslian-speaking Senoi appear to have undergone more rapid linguistic diversification than the Negritos; the Central Aslians owing to their sedentary endogamous social pattern based on agriculture and the development of corporate cognatic descent groups; and the Southern Aslians owing to a growing involvement in trade with and influence from the more recent Austronesian settlers of the coastal parts of the Peninsula (see also Benjamin 1986). The later emphasis on trade in the southern part of the peninsula gave rise to a different and more assimilatory trend, mostly from Austroasiatic into Austronesian in language terms, epitomized by such "Malayized" groups as the Austronesian-speaking ("Aboriginal Malay") Temuan. Prior to the nineteenth century, the interior parts of Peninsular Malaysia were probably occupied entirely by Aslian speakers (Benjamin 1976; Dunn 1975). 


\section{The Early Metal Phase: A Protohistoric \\ Transition toward Supra-Tribal Societies}

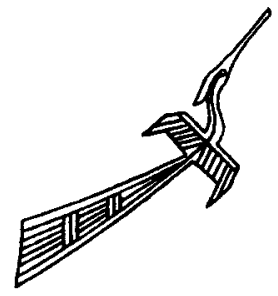

The early Metal (or Paleometallic) phase correlates with the introduction of new technologies and trade items to the Indo-Malaysian Archipelago from Vietnamese, Indian, and Chinese sources. In addition, it overlaps chronologically with and merges into the period of the developing Indianized states during the first millennium AD. In this sense it seems quite reasonable to regard it as basically protohistoric.

However, the difficulties attending any attempt to gauge the real significance of this phase are considerable. Most of the older reports contain little more than lists of undated artifacts, and the major cultural changes presumed to have taken place at this time remain virtually undocumented. My own research in Sabah and eastern Indonesia has provided a partial chronology for certain localized and regional aspects of the phase as a whole, but the vast bulk of the archipelago has not yet received the attention it deserves and it is still very difficult for Southeast Asian archaeologists to gain access to radiocarbon dating facilities. Perhaps the best contribution to future studies this chapter can make will be to review the material in related groups that can in certain cases be elevated to the status of localized archaeological cultures.

The early Metal phase commenced with the introduction of copper-bronze ${ }^{1}$ and iron artifacts and their manufacturing technologies, presumably together (there is no separate "Bronze Age") and almost certainly from immediate sources on the Southeast Asian mainland during the last few centuries BC. I will take $500 \mathrm{BC}$ as an arbitrary starting point, although I personally do not feel that any metal in the archipelago can be conclusively dated as early as this; future research may push this date closer to $200 \mathrm{BC}$. Artifacts and assemblages attributed to this phase of course exclude those that can unequivocally be associated 
with the historical Indianized or Islamic states, although many assemblages considered to be "Bronze-Iron Age" in the terminology of Heekeren (1958) could quite easily be fully historical in this sense. However, this is usually hard to prove owing to poor documentation and dating. It should be emphasized that pre-Indianized styles of metal and other artifacts undoubtedly continued in production well after the first appearance of Indian bronzes and inscriptions in the archipelago, and for many of the remoter eastern regions and Borneo it would be quite acceptable to continue the early Metal phase into ethnographic times, as in the case of the small bronze drums (moko) of Javanese or Balinese manufacture used in Alor (Du Bois 1944). For practical reasons such a diffuseness of ending might cause this chapter to lengthen into another book, so I will draw an arbitrary termination at AD 1000 and thus leave the archaeology of the China trade, Islam, and the Malay sultanates out of consideration.

\section{THE DONG SON CULTURE OF NORTHERN VIETMAM}

Bronze working in northern Vietnam commenced around the middle of the second millennium BC and is associated with the Dong Dau and succeeding Go Mun phases of Vietnamese archaeologists (Ha Van Tan 1980; Hoang and Bui 1980; Higham 1989; Higham 1996b). Together with central and northeastern Thailand, this region has the earliest evidence for bronze working in Southeast Asia and in recent years there has been considerable discussion about the origins of the tradition, which has no preceding copper phase. Ultimate origins are of no real concern for an understanding of Island Southeast Asian prehistory, and because the issue is by no means resolved it will not be followed here. However, the types of bronze artifacts involved in the millennium prior to $500 \mathrm{BC}$ include socketed axes and spearheads, shaft-hole sickles (in Vietnam), tanged spearheads and arrowheads, and other small items such as knives, fishhooks, and bracelets. At some time between 500 and $300 \mathrm{BC}$, according to recent carbon dates, the classic Dong Son phase of Vietnamese protohistory began, with its bronze drums, high-status burials, and the first appearance of iron.

The Dong Son archaeological assemblages are of considerable importance because the earliest metal goods found in the Indo-Malaysian Archipelago are generally of this type, rather than of direct Indian or Chinese inspiration. Bronzes of Dong Son style are found widely in Mainland Southeast Asia and southern China, but stylistic and compositional homogeneity, especially of the drums, suggests an outstanding dominance of Dong Son workshops on northern Vietnamese soil at this time. ${ }^{2}$ The drums are of Heger type I (Plate 45) and the finest and oldest examples found in Vietnam have remarkable decorative friezes of human, animal, and geometric ornament (Bernet Kempers 1988; Pham Huy Thong 1990). Such friezes occur-albeit with considerable simplifi- 


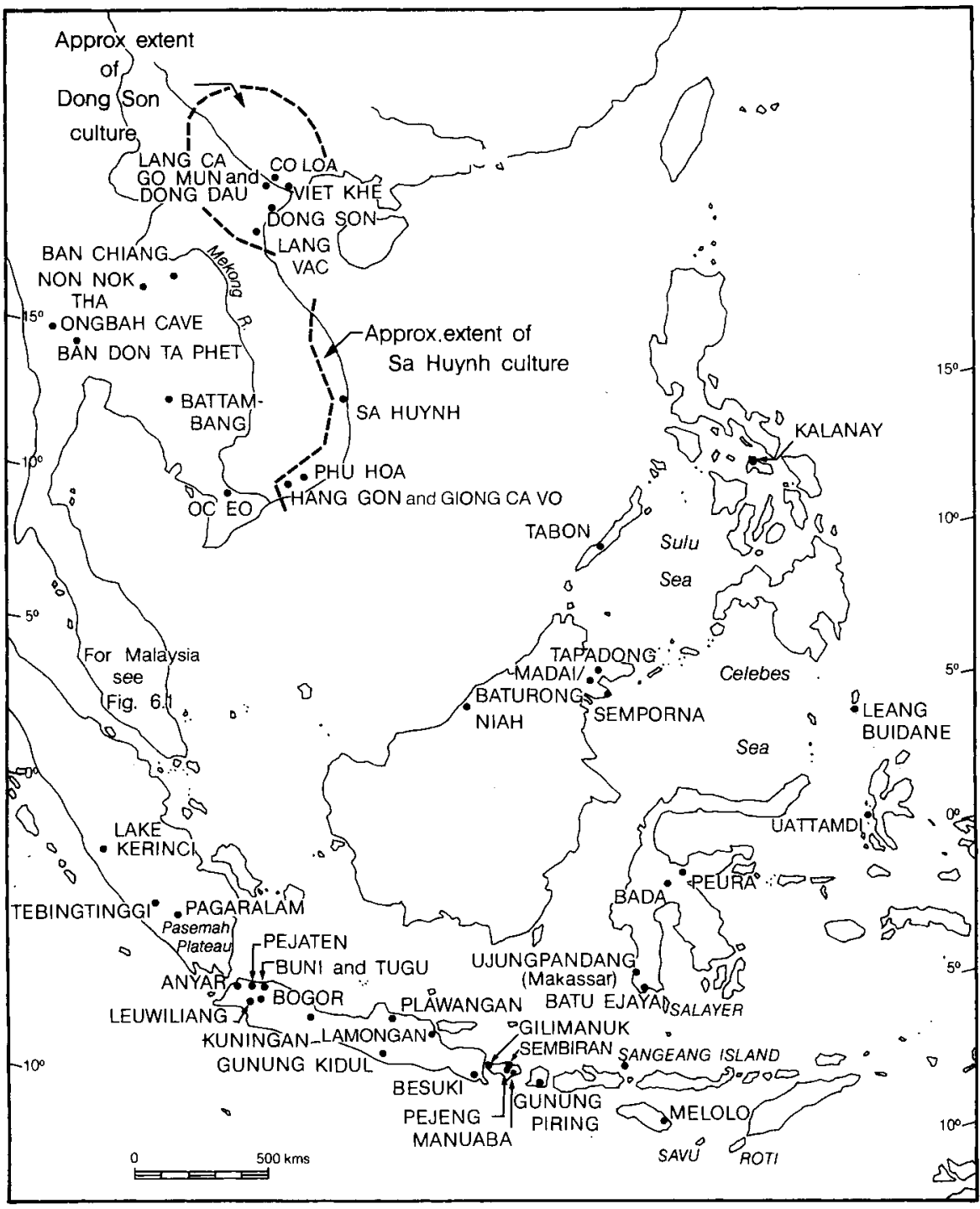

Fig. 9.1 Locations of sites described in this chapter.

cation and schematization-in all the later drums of this type, including those exported to Indonesia and Malaysia.

The range of other Dong Son bronze goods, excavated from such sites as the Dong Son settlement itself (Janse 1958) and more recently from burials at Viet Khe, Lang Ca, and Lang Vac, includes bowls and situlae (small buckets), miniature drums and bells, socketed axes with splayed or "boot-shaped" blades, sock- 
eted hoes, socketed or tanged arrowheads and spearheads, daggers with anthropomorphic handles (see Fig. 9.7), bracelets, belt hooks, and many other items of more idiosyncratic interest. One massive drum unearthed at Co Loa contained ninety-six socketed bronze plowshares. Iron is rather rare, but there are a few spearheads and at least one cast iron hoe, the latter of possible Chinese manufacture. Imperial Chinese domination of northern Vietnam overlapped with the later stages of the Dong Son culture and there are some undoubted Chinese imports in a number of the northern Vietnamese sites, but these occurrences cannot be used to support the derivation of Dong Son bronze metallurgy as a whole from China. The local genius expressed in the drum, situla, and axe forms, plus the importance of lost wax casting (a technique only rarely used at this time in China), indicate quite clearly that northern Vietnam was a vital center of bronze metallurgy that had a dramatic impact on many other regions of Southeast Asia. So too did the contemporary bronzeworking traditions of Yunnan, to the west. The basic Dong Son and Yunnan artifact styles overlap only marginally with those of metropolitan China and not at all with the contemporary bronze and iron industries of India, which lack the emphasis on socketed hafting. However, the rather limited iron industry of Dong Son could have an immediate Chinese origin.

The Dong Son culture has a number of other features that merit attention. I will be returning to certain purely artifactual aspects repeatedly in this chapter, but it is also of great importance to realize that this culture was centrally involved in a transition to a highly stratified and partly urbanized society: The outer earthen ramparts of the Dong Son fortified center of Co Loa near Hanoi enclose about 600 hectares. This society had an economy based on intensive rice production, presumably in rainfed or irrigated bunded fields with plows and buffalo traction. The intensified production supported an upper ruling echelon whose wealthy burials have been found in many sites and who in turn were able to support a degree of craft specialization associated in many other areas with literate civilizations. It is therefore not surprising that such professionally made items as the magnificent bronze drums, and perhaps Vietnamese techniques for the manufacture of lesser bronze tools and weapons, should have had such an impact on the contemporary societies of Indonesia and Malaysia.

\section{I1. THE SA HUYNH CULTURE OF SOUTHERM VIETMAM}

I will now move southward to examine a different mainland culture that may also have been involved in the transmission of metalworking techniques-particularly of iron-to the islands of Southeast Asia. The Sa Huynh culture of southern Vietnam belonged to an Austronesian-speaking (Chamic) population 
of Indo-Malaysian origin that appears to have settled this region from either Peninsular Malaysia or Borneo (see Chapter 4, Section VA). When this settlement took place is uncertain, but the event may be documented by the Sa Huynh culture itself: It appeared in mature form around $600 \mathrm{BC}$, although possibly ancestral assemblages from the late second millennium $\mathrm{BC}$ are now being reported by Vietnamese archaeologists. Prior to the Sa Huynh culture or its immediate ancestor, southern Vietnam was presumably occupied entirely by Austroasiatic-speaking populations. The Chams developed the important Indianized civilization of Champa during the first millennium $\mathrm{AD}$, but later succumbed to the pressures of Vietnamese expansion and now survive as minority hill peoples.

From an Indo-Malaysian point of view, it is significant that the Chams of late prehistory were the closest resident Austronesian groups to the northern Vietnamese centers of metallurgy. Given their ethnic affiliations, they were undoubtedly in a central position to introduce new metalworking techniques acquired on the mainland, particularly of iron, to the Indo-Malaysian Archipelago. However, their direct contacts with the bronzeworking centers of the Dong Son region seem to have been rather limited-only seven Heger I drums have been found in the south of Vietnam, out of a total of 130 recorded for the whole country by 1990 . These items might thus have been transmitted into the archipelago by other routes, perhaps through Thailand and Peninsular Malaysia or directly by sea.

Assemblages of the Sa Huynh culture known to date have come mostly from jar burial sites. This is a custom that may have been brought by the first Chamic settlers themselves from the Indo-Malaysian islands, especially if the Niah and Tabon dates for jar burial from the late second and early first millennia $\mathrm{BC}$ onward can be relied upon. Generally speaking, pottery-jar burial was not characteristic of the Dong Son or other contemporary Mainland Southeast Asian cultures. Where it does occur, as at the Dong Son site of Lang Vac in northern Vietnam (Ha Van Tan 1980:133), influence from Chamic sources may be suspected. The stone burial jars of northern Laos (Colani 1935) do not seem, on present evidence, to represent a likely region of origin for the Sa Huynh or other Indo-Malaysian jar burial traditions (although research in the Plain of Jars is currently in progress).

Major Sa Huynh sites occur in coastal regions from central Vietnam southward to the Mekong delta; the type site is Sa Huynh itself, but there are others farther south in the Mekong delta region at Hang Gon, Phu Hoa, and Giong Ca Vo, and farther north of Sa Huynh around Da Nang (for summaries see Bellwood 1978:191-194; Ha Van Tan 1980:136-137; Fontaine 1979, 1980; Nguyen Duy Ty 1991; Dang and Vu 1995). The finer details of this culture are not of concern here, and I will return to the whole question of jar burial in Indonesia 

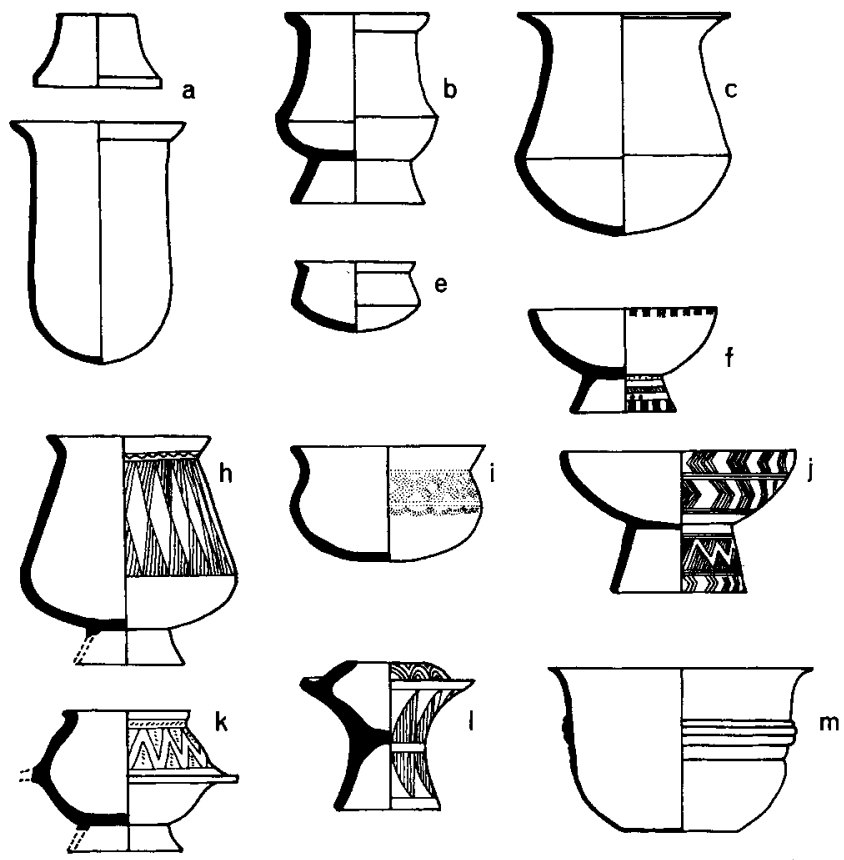

$\mathrm{t}$

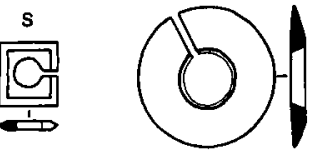

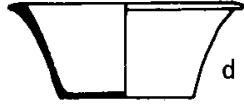
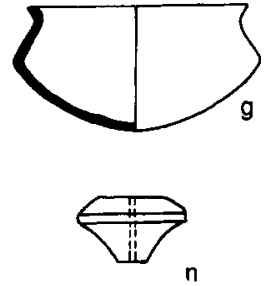

n
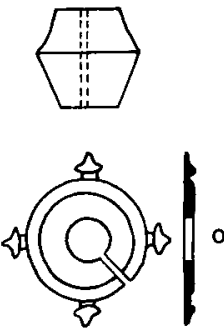

u

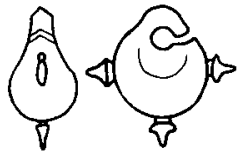

Fig. 9.2 The Sa Huynh assemblage, after Parmentier 1924: (a) large burial jar 77 centimeters high with lid; (b-1) pottery vessels between 14 and 21 centimeters in diameter, decorated by incision or punctation; $(\mathrm{m})$ bronze vessel 9 centimeters in diameter; $(\mathrm{n})$ clay spindle whorls 3.5 centimeters in diameter; (o, s- $u$ ) stone earrings- $(0)$ and $(u)$ are of lingling-o type-diameters 2-5 centimeters; $(p)$ iron hoe 17 centimeters long; $(q, r)$ faceted carnelian beads 19 and 15 millimeters long. From Bellwood 1978.

and East Malaysia in more detail below. It should be noted, however, that the Sa Huynh burial jars and the associated accessory vessels with their incised and shell-edge stamped zones of decoration (Fig. 9.2) are paralleled quite closely in the early Metal phase jar burial assemblages of the Philippines, northern Borneo, and the Sulawesi Sea region of northern Indonesia. These links have been strengthened by the discovery of almost identical knobbed pennanular stone earrings (the so-called lingling-o) and of a special kind of earring or pendant with two animal heads (presumably deer) in a number of sites in Thailand, Vietnam, Palawan, and Sarawak ${ }^{3}$ (Loofs-Wissowa 1980-1981; and Zuraina 1982 for Niah) (Fig. 9.3). At the large jar burial site of Giong Ca Vo, near Ho Chi Minh 

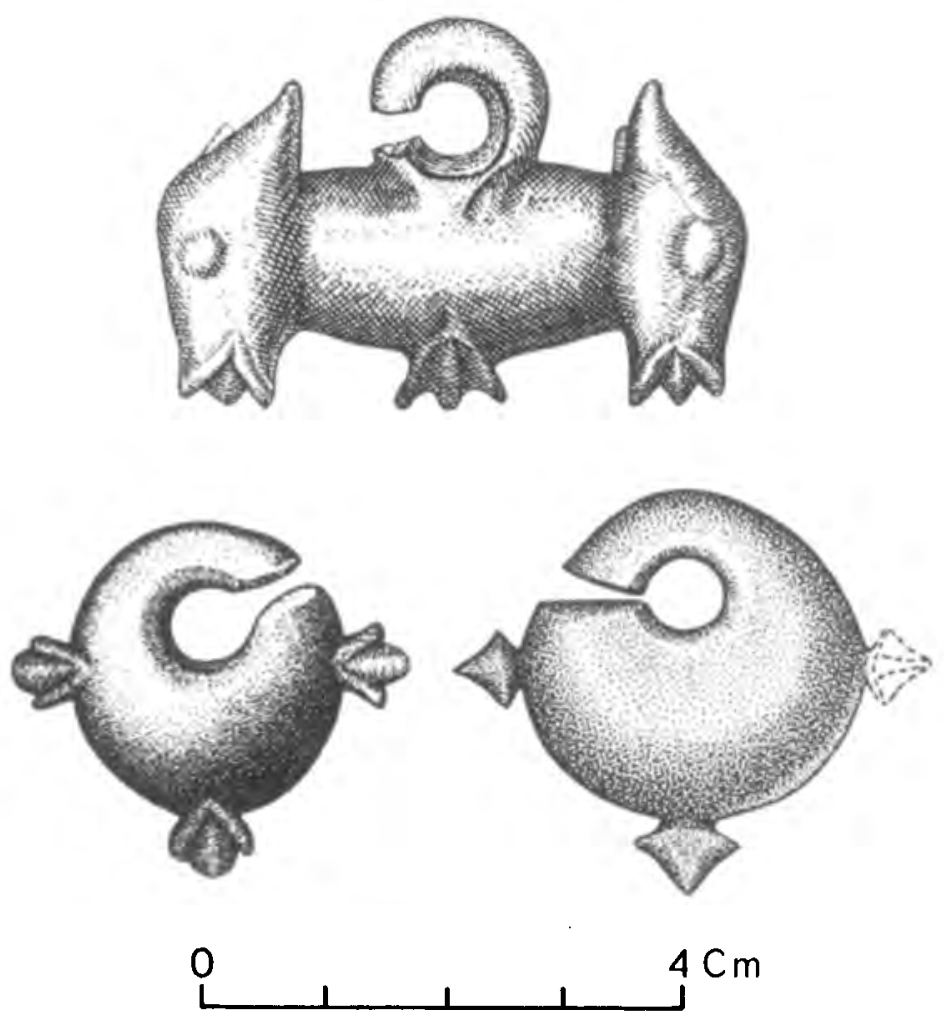

Fig. 9.3 Stone earrings (the bottom two of lingling-o type) from the Tabon Caves, Palawan. Compare similar ornaments in Figures 7.7 and 9.2. From Fox 1970. Courtesy: National Museum of the Philippines.

City, eighteen of these animal-headed pendants made of glass were found (Nguyen Kim Dung 1995).

These connections between southern Vietnam and the Borneo-Philippine region in the early Metal phase (and possibly the preceding Neolithic) may be important when considering the evidence in the Sa Huynh sites for iron metallurgy. The iron repertoire as a whole includes many socketed tools such as spades, picks, and axes and there are also unsocketed sickles, tanged knives, spindle whorls, rings, and spiral bracelets. A sword of possible Chinese manufacture was found at Hang Gon (Saurin 1973), and there is a possibility that the technology of ironworking was introduced to the area from a Chinese source, although I suspect that a lot of metallurgical analysis will need to be done before this question can be settled. As with Dong Son, neither the bronze nor the iron goods from the Sa Huynh sites resemble Indian models. 
In general, the Sa Huynh sites reveal a greater usage of iron than the Dong Son sites. Sa Huynh bronzes are mainly decorative items rather than tools and weapons (i.e., bracelets, bells, and small vessels). There are also some rare gold beads and silver wire (e.g., see Fontaine and Hoang 1975 for Phu Hoa). Most sites have glass, banded agate, and a range of carnelian beads (round, cigarshaped, or faceted) that have generally been considered to be of Indian origin (see Fig. 9.2q, r), although dates of 1200-800 BC for carnelian beads at Nong Nor (Higham 1996b) and of 700-500 BC from Nil Kham Heng (A. Weiss; pers comm), both in central Thailand, make a more local origin for these an attractive alternative. The site of Giong $\mathrm{Ca}$ Vo has produced evidence for local glassworking dated to ca. 400 BC (Nguyen 1995), but Glover (1990a:36-37) also reports radiocarbon dates in the fourth century $\mathrm{BC}$ for Indian beads in the site of Ban Don Ta Phet in central Thailand, so contacts with the Indian subcontinent might also have been underway by this time (see below).

Carbon dates from Phu Hoa, Hang Gon, and Giong Ca Vo suggest an overall date range for the Sa Huynh culture between $600 \mathrm{BC}$ and 0 . Phu Hoa does have some comb-incised pottery similar to that from the Funanese site of Oc Eo (early to mid-first millennium $A D$ ), so perhaps an overall date range from 600 BC until well into the first millennium AD, thus overlapping with the civilizations of Champa and Funan, will one day be demonstrated for the mature (Iron Age) Sa Huynh culture as a whole. Internal phases still await definition.

\section{THE ROLE OF INDIA}

The bulk of the Paleometallic sites in the Indo-Malaysian Archipelago overlap in date with the historical evidence for the earliest historical trading states and the succeeding Indianized kingdoms in the western part of the region. It is necessary here to expand a little on the brief survey of this topic given in Chapter 5, Section II. Of direct Chinese contact with the archipelago prior to AD 1000 there is little to report, except to note that northern Vietnam was made a protectorate of the Chinese Han Empire in $111 \mathrm{BC}$ and a province in AD 43, that a quantity of Han dynasty pottery has been found in uncertain contexts in southern Sumatra (Hoop 1940; Heine Geldern 1945; Orsoy de Flines 1969), and that Chinese Buddhist pilgrims were traveling to India by sea via Indonesia from the fifth century AD onward. But Chinese trade goods in any quantity are generally very rare in the archipelago prior to the Song dynasty (see Chapter 5, Note 2).

Indian enterprise, however, presents a very different picture. Sanskrit and Tamil literary references to Southeast Asia may go back as far as the third century BC (Wheatley 1961:Chapter 11). By AD 70 there is evidence that cloves from the Moluccas were reaching Rome (Miller 1969:49). ${ }^{4}$ Between the first and fifth centuries AD a number of small indigenous trading "states" (or emporia) 


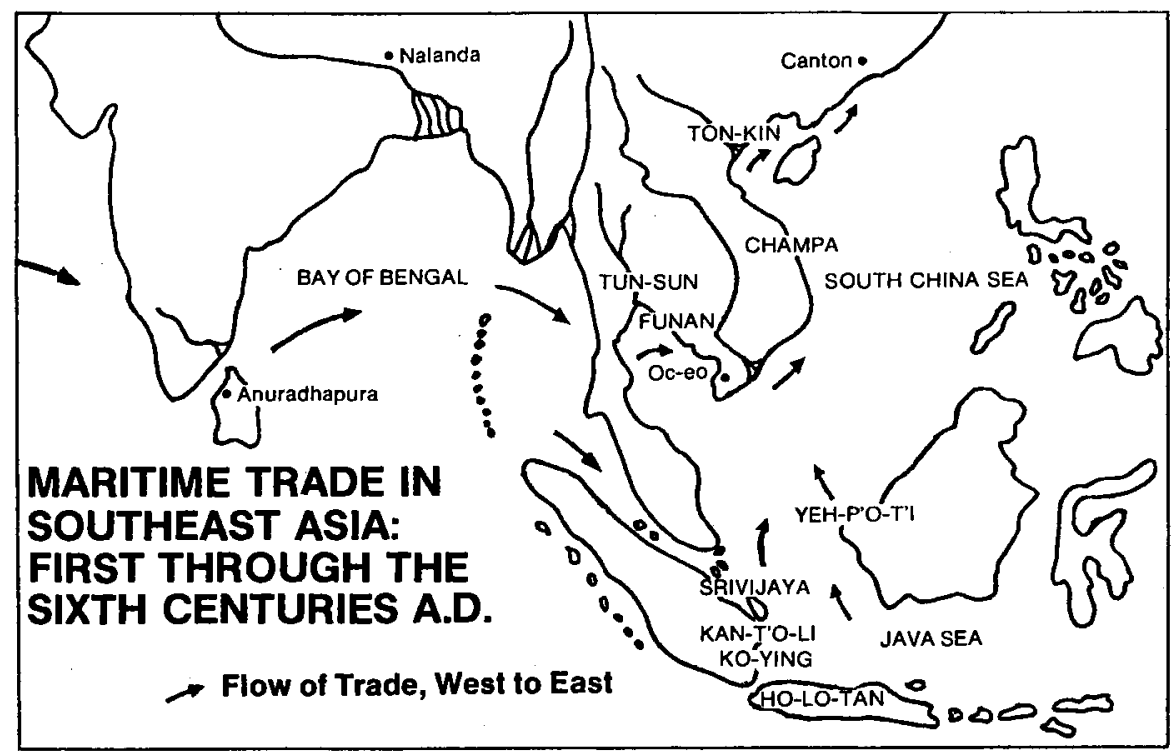

Fig. 9.4 Maritime trade in Southeast Asia, first to sixth centuries AD. From Hall 1980. Courtesy: K. R. Hall.

developed in southern Indochina and in the northern part of the Malay Peninsula (Fig. 9.4). In the first few centuries AD there appears to have been a land portage for trade goods from the Andaman Sea to the Gulf of Thailand across the narrow Kra Isthmus (at the head of the Thai-Malaysian Peninsula), but by the fifth century a lot of the traffic was using the more southerly sailing route through the Strait of Malacca. This change may have caused the decline of the trading state of Funan and the consequent rise of Champa in southern Vietnam, and also of a contemporary Buddhist kingdom in Kedah (Nik Hassan Shuhaimi and Othman Yatim 1990). By the fifth century it is also apparent that extensive areas of the western Indo-Malaysian Archipelago were becoming increasingly important links in the trade routes. Austronesian-speaking crews may have been in control of much of the shipping (Wolters 1979; Hall 1980, 1985)a circumstance that no doubt played a large role in the settlement of Madagascar by perhaps AD 700 (Adelaar 1995).

Archaeological evidence for Indian-Southeast Asian trade contact in the period from about $200 \mathrm{BC}$ to $\mathrm{AD} 500$ is now much stronger than it was when the first edition of this book was published, especially for Thailand and Bali (Glover 1990a; Ardika and Bellwood 1991). The latter island will be considered later. I will simply note here, as one of the most noteworthy mainland examples, the presence of Roman and Indian imported items dated from the second century 
$\mathrm{AD}$ onward at OC Eo in southern Vietnam; the Indian items include a Gandharan Buddha head, seals, rings, bronzes, and carnelian and agate beads. There are also second-century AD Roman coins and some Chinese later Han dynasty bronze mirror fragments (Coèdes 1947; Malleret 1959-1963; Christie 1979a; Wheatley 1983). Indian or local raw materials might also have been worked here into glass and stone beads in Indian styles, as perhaps in the Malay Peninsular sites of Khlong Thom in Krabi and Kuala Selinsing in Perak (Veraprasert 1987; Lamb 1965). In addition, Oc Eo has a number of ornaments of tin that could have been imported from Thailand, Laos, or Peninsular Malaysia. Indeed, some of the earliest small states on the Malay Peninsula-particularly in its narrower Thai portion-may have developed partly on the proceeds of an export of tin, as well as of the forest products and spices that bulk larger in the historical records (e.g., see Wolters 1967; Dunn 1975; Hall 1985). Gold was probably also important as a stimulus for early trade (Miksic 1990).

\section{BRONZE ARTIFACTS OF DONG SON AND LOCAL STYLES FROM THE SUNDA ISLANDS AND PENINSUlap malaysia}

A large number of artifacts of precise Dong Son affinity, especially Heger type I drums, have survived in villages or turned up as chance finds without coherent archaeological contexts in the Malay Peninsula and the Sunda chain of Indonesia. There are now six fragmentary Heger I drums known from Peninsular

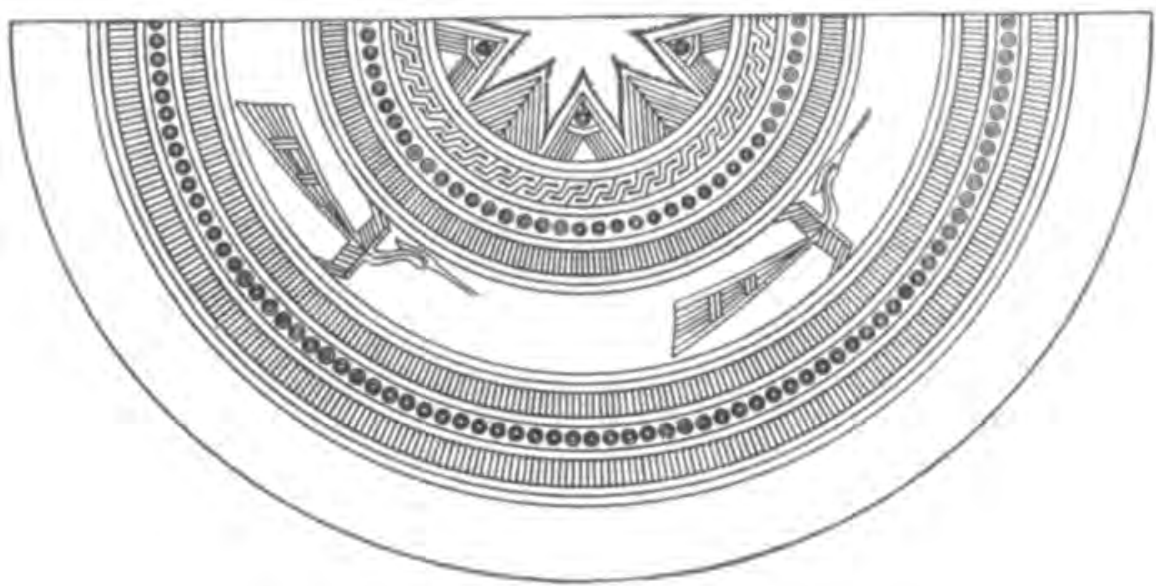

Fig. 9.5 The Heger type I drum from Klang: a semidiagrammatic rendering of the decoration on one half of the tympanum. From Loewenstein 1956. Courtesy: Malaysian Branch of the Royal Asiatic Society. 
Malaysia; the Klang (Fig. 9.5) and Tembeling (Batu Pasir Garam) fragments were both dated to the second century BC on stylistic grounds by Loewenstein (1956), and two other damaged drums excavated from beneath a possible burial mound at Kampong Sungei Lang in Selangor have been carbon dated rather uncertainly from an associated wooden plank between about $500 \mathrm{BC}$ and AD 200 (Peacock 1964b, 1979). There are also two further drum fragments from Kuala Trengganu on the east coast.

No fewer than fifty-six drums or parts thereof are known from the Sunda chain of Indonesia, mostly from Java, Sumatra, and the southern Moluccas, with examples occurring as far east as the Kai Islands south of western New Guinea, and also the Bird's Head of West New Guinea itself (see Bernet Kempers 1988 for a list). Some of the more significant Indonesian examples include the "Makalamau" drum from Sangeang Island near Sumbawa (Plate 46), with its figures in possible Han dynasty and Kushan (northern Indian) or Satavahana (central Indian) costumes (Heine Geldern 1947); the drum from Kai with its deerand tiger-hunting frieze; and that from Salayer (Plate 47) with its elephants and peacocks (Schmeltz 1904). All these are scenes that would presumably have been unfamiliar to the inhabitants of the eastern Indonesian islands where the drums eventually came to rest, so on these grounds alone it is clearly most unlikely that they were cast locally (although Imamura 1993 suggests that some of the youngest Heger I drums might have been cast in Indonesia). Indeed, in terms of style and a frequent high lead content, it looks as if most of these Peninsular Malaysian and Indonesian drums were manufactured in Vietnam, many during the period of Chinese domination after the second century BC (Bernet Kempers 1988). Heine Geldern (1947) suggested that the Sangeang drum might have been cast in Indianized Funan as late as AD 250.

One interesting observation by Bernet Kempers is that all drums found east of Bali have four frogs cast in relief around their tympana; another is that the rather disintegrated patterns on the Indonesian drums-derived from the warrior friezes and boat motifs on the oldest Vietnamese drums-suggest that they are relatively late in date of manufacture (see also Imamura 1993). Bernet Kempers describes how each Heger I drum was cast in one piece: Wax slabs laid over a clay core were impressed with the boat and procession patterns using clay or stone molds, while some of the more naturalistic patterns, such as the house scenes, were incised individually into the wax. The wax was then sealed in a clay outer mold held in place by driven "spacers," and melted out prior to the pouring of the molten bronze. This cire perdu (lost wax) method can still be seen in use in Mandalay (Burma) for casting temple Buddha statues and miniature bronze drums.

In terms of distribution within the Indo-Malaysian Archipelago, it may be important to stress that Heger I drums have mainly been found in Peninsular 
Malaysia and in the islands of the Sunda chain (Sumatra, Java, Bali, and the Lesser Sundas/southern Moluccas). Two unpublished drums have recently been found at Kota Waringin in West Kalimantan and there are three pieces from the Bird's Head of New Guinea, but none have ever been reported from the rest of Borneo, Sulawesi, the northern Moluccas, or the Philippines. This distribution does of course overlap in the west with that of the earliest recorded Indian contact, and it may be that many of these exotic bronzes were transported secondarily, long after their dates of manufacture, within the trade (especially spice trade) networks of the earliest historical states in the Malay Peninsula and western Indonesia. Loofs-Wissowa (1991) has suggested that they were bestowed on local chiefs as symbols of kingship and authority by politico-religious authorities in Vietnam, and that the boat scenes on the sides represent fertility-focused boat races rather than the more commonly favored identification as "ships of the dead" carrying souls into the afterlife. On the other hand, Bernet Kempers (1988) suggested that many could have been carried into Indonesia by refugees from the bloodshed of early Sinicized Vietnam. Whetever the mechanisms of their dispersal, it would be clearly unwise to regard all of the Heger I drums as inherently "prehistoric."

A number of other copper or bronze artifacts found in Indonesia might also represent imports from Vietnam. There can be no certainty of this, but outstanding finds of Dong Son affinity that attract attention in this regard include the male statuette similar to a Dong Son dagger handle (Fig. 9.6) from Satus near Bogor, a miniature Heger I drum from Cibadak in western Java (Heekeren 1958), and a lidded bronze vessel with Dong Son circle and tangent decoration found in the drum burial at Lamongan in East Java (Bintarti 1985a). While details of date and composition for these artifacts are insufficient to prove a mainland Asian origin, it nevertheless seems possible that they were transmitted, like the Heger I drums, as status items into the chiefly lineages of the Sunda Islands. Indeed, the early Metal phase sarcophagi of Bali (see below) have yielded large numbers of socketed tools with heart-shaped blades-possibly small axes or projectile points-of a type recently shown to have been manufactured in very large numbers between 700 and $500 \mathrm{BC}$ in the copperworking site of Nil Kham Heng in central Thailand (Weiss; pers comm). In the absence of metallurgical analysis, however, this cannot prove importation into Bali; it should also be noted that Sumatra and Java do have many small deposits of copper ore, albeit not clearly utilized in prehistory (Bronson 1992), and Peninsular Malaysia and Bangka/Belitung of course have ample supplies of tin.

A fairly rapid result of this external introduction of bronze goods and technology into the Indo-Malaysian Archipelago was clearly the actual establishment of local metalworking centers. There have been several finds of stone or terra-cotta valves from the molds used for casting copper and bronze axes from 

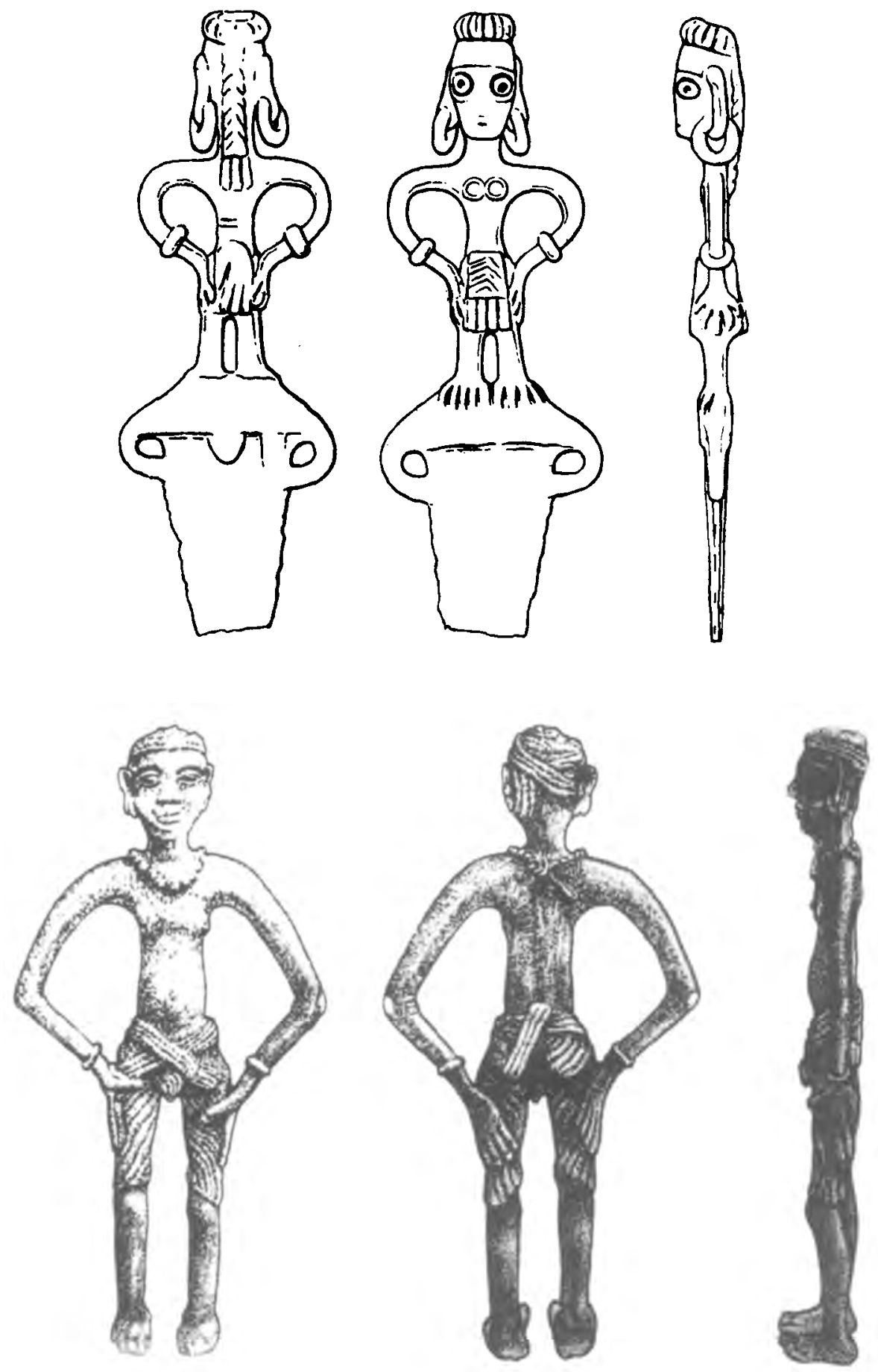

Fig. 9.6 Top: bronze dagger handle from Dong Son, 11 centimeters high. From Goloubew 1929. Courtesy: Ecole Française d'Extrême-Orient. Bottom: copper or bronze statuette from Satus, near Bogor, 25 centimeters high. From Heekeren 1958. Courtesy: Kluwer Academic Publishers. 
sites in Java, Sabah, and the Talaud Islands, all of which show quite conclusively that some casting of either local or imported raw materials was being carried out during the early to middle first millennium AD. Socketed bronze axes exist in large numbers, some with incised decoration in typical Dong Son style (Fig. 9.7). There is also the important fragment of a volcanic tuff stamp for impressing the wax mold for a bronze drum of Pejeng type (below) from Sembiran in Bali, dated by Indian imports to the first two centuries AD. This is clear evidence that some local casting was being carried out at this time (Ardika and Bellwood 1991).

Evidence for the beginnings of one or more Indonesian casting traditions can be seen in a number of quite splendid copper or bronze objects that are not in a classic Dong Son style. In one group is the almost 2-meter-high hourglassshaped drum from Pejeng in Bali (first recorded by Rumphius in 1690), together with a number of similar but smaller Pejeng-type drums from this island and Java that have been placed in a stylistic sequence by Bernet Kempers (1988; see also Bintarti 1990). Some of these drums were clearly made in Bali because, apart from the Sembiran stamp fragment, three more decorated stamp pieces are known from the village of Manuaba (Plate 48). The Sembiran stamp fragment carries part of a running triangular motif like that on the Pejeng drum itself. Pejeng-type drums have also been found in direct association with Heger I drums at Kradenanrejo (Lamongan) and near Semarang in Java. One Pejengtype drum was found by villagers digging a well at Pacung near Sembiran in Bali, and this appears to be from a layer contemporary with the one that yielded the Sembiran stamp fragment. The Pacung drum, like the others of this type, has its body and tympanum cast separately (McConnell and Glover 1990); in this specimen the tympanum is of bronze, the body of copper.

Other items related in style and characterized, like the Pejeng drum, by the use of a face mask in a dominant position in the decoration include an unusual flask from Ujungpandang (Makassar), a ceremonial axe from Savu in eastern Indonesia (Bintarti 1981a), and two (originally three; one is now lost) ceremonial axes from Roti (Fig. 9.8), although one of the latter does show a typical Dong Son tangent and circle motif. A second stylistic group, in this case of flasks and large clapperless bells, is distinguished by an unusual and elaborate type of incised spiral decoration that is again outside the Dong Son repertoire. The flasks, of which a total of six are now known (Glover 1992), include specimens from Kerinci and Lampung in Sumatra, from Madura, and from Kandal in Cambodia (the last two almost identical; Plate 49 shows that from Madura). The bells include one from Battambang in Cambodia, three (one now lost) from Klang in Selangor, and a newly discovered example from Kampong Pencu near the Muar River in Johor (Plate 50). The Muar bell has been dated by thermoluminescence-on a fragment of the clay casting core preserved in its narrow 


$$
\frac{801}{801}
$$




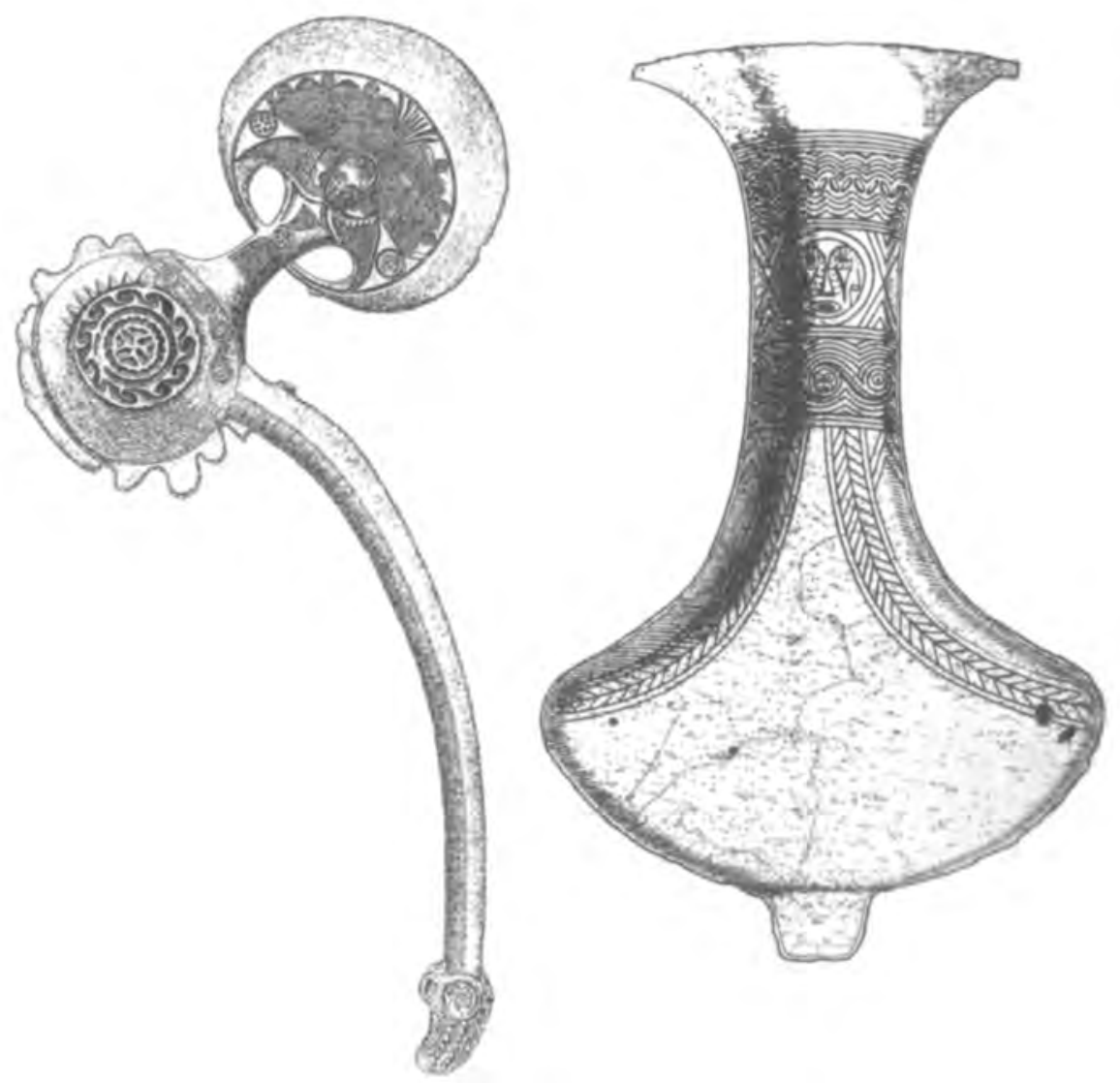

Fig. 9.8 Left: bronze ceremonial axe from Roti, 77 centimeters maximum length. Right: bronze flask, possibly from southern Sulawesi (purchased in Ujungpandang), 70 centimeters high. From Heekeren 1958. Courtesy: Kluwer Academic Publishers.

neck-to less than 1,800 years old (Adi 1983:61). Although this date is not very precise, at least it does not contradict a view that these bells and flasks belonged to a first-millennium AD casting tradition that was not Vietnamese, but that might have been of ultimate Vietnamese technological derivation. Indeed, a bronze bell that might conceivably represent a prototype for these specimens was excavated by Janse at Dong Son (Loewenstein 1956:Fig. 22). For these bells and flasks there is a huge region of potential manufacture from Cambodia to Madura, and a bell very similar to the ones described has also been found at Dabona in Yunnan.

Other Indonesian bronzes of possible local manufacture include the statuettes and knobbed bracelets from Bangkinang in southern Sumatra (Heekeren 
1958:Plates 5 and 9) and the remarkable bronze canoe model from Dobo village on Flores (Bintarti 1985b:64). There are also daggers or short swords with iron blades and bronze handles from Prajekan (Heekeren 1958:Plate 11) and Lumajang (Cselik 1986) in Java, reminiscent of a widespread tradition in Iron Age sites in Yunnan, Vietnam, and Thailand. One also comes from Gilimanuk in Bali (see below).

\section{THE SLAB GRAVES AND IRON INDUSTRY OF PENINSULAR MALAYSIA}

Apart from the drums and bells already considered, the early Metal phase in Peninsular Malaysia is also associated with a number of slab-lined graves (Plate 51), presumably for extended inhumations (although bones always seem to have dissolved) found in southern Perak and northern Selangor (Evans 1928b, 1931b; Collings 1937b; Linehan 1951; Adi 1993). The general range of associated artifacts from the graves includes glass and carnelian beads, a stone barkcloth beater, a bronze bowl, and a most unusual industry of iron tools. The pottery is wheel made, either plain or slipped with a thick resinous coat and usually with impressed lip patterns. Similar pottery was also found with part of a socketed iron spearhead under the mound that contained the two Sungei Lang bronze drums (Peacock 1979). One recently discovered grave in Selangor has produced Chinese pottery evidently of Tang date (seventh to tenth centuries AD).

The iron industry has been found in several other sites apart from the slab graves, and there appears to be a unity in terms of style and the associations of different tool forms in hoards that occur commonly in Peninsular Malaysia. The forms have been clearly described by Sieveking (1956a) and include axes (some with very long shafts, known colloquially as tulang mawas), knives, and sickles, all with shaft holes. In addition, there are some socketed spearheads and tanged knives (Fig. 9.9). The tools appear to be of low-carbon steel, according to Sieveking, which suggests that the iron was roasted purposefully in a bed of charcoal after the initial smelting process. Tools of this type were also found with the Klang bells as well as with the dated Sungei Lang drums.

Sieveking saw the Peninsular Malaysian iron industry as having an origin in the socketing tradition for bronze tools represented in the Dong Son culture of Vietnam, a tradition he believed was transmitted in iron toward Peninsular Malaysia via southern Vietnam after the first century AD. At the time he was writing there was little archaeological evidence to support such a connection, but recent excavations have produced the highly respectable socketed iron industry of the Sa Huynh culture of southern Vietnam (Section II), and from just above the top of the Malay Peninsula in southwestern Thailand there are prolific iron industries from Ongbah Cave and Ban Don Ta Phet. Ongbah Cave 


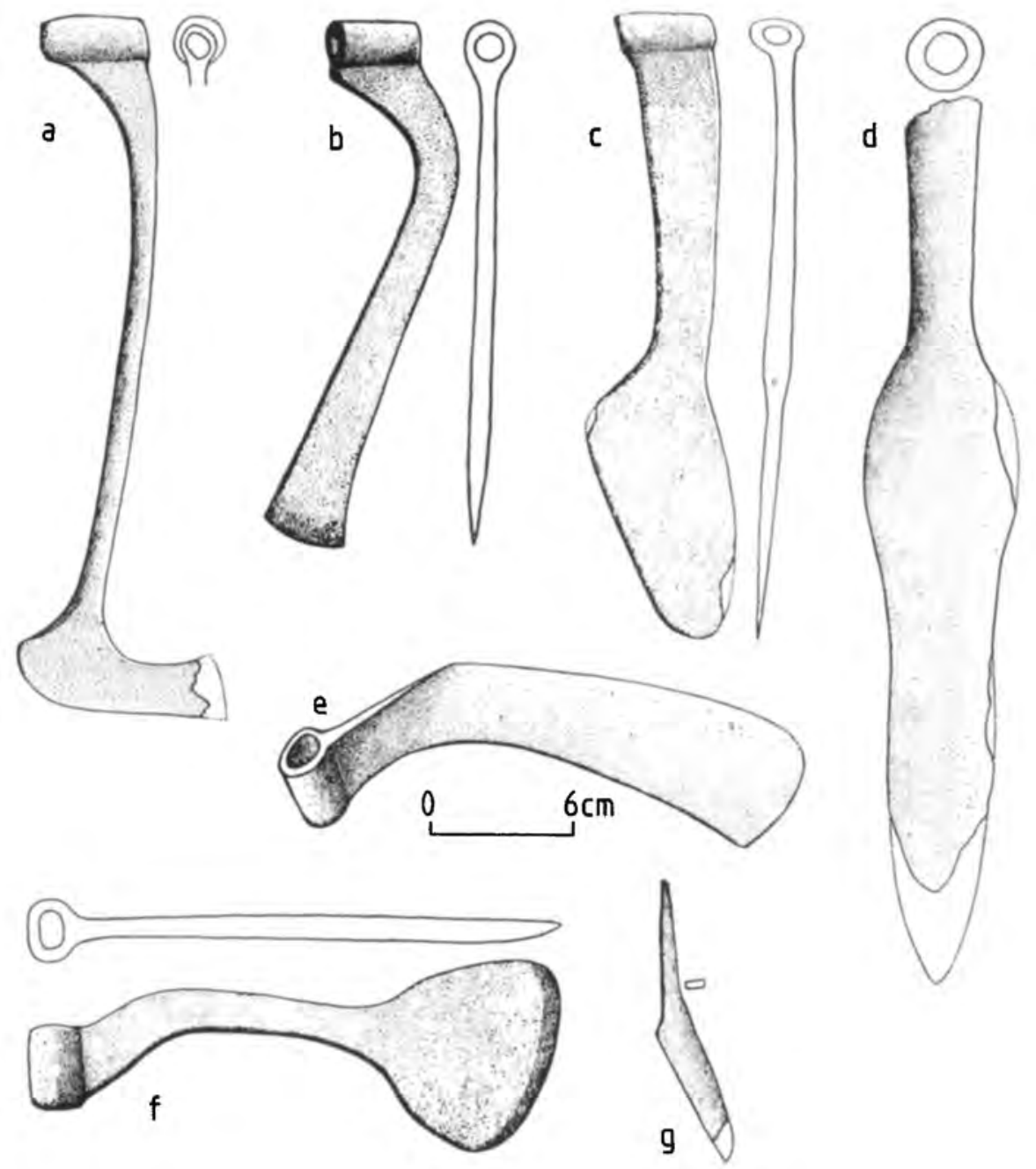

Fig. 9.9 The iron industry of the Peninsular Malaysian early Metal phase: (a) tulang mawas from Tersang, Raub, Pahang; (b, f) shaft-hole axes from Bukit Jong, Pahang; (c) shaft-hole knife (?) from Tanjong Rambutan, Perak; (d) socketed spearhead from Bukit Jati, Klang, Selangor; (e) shaft-hole sickle from Bukit Jong; (g) tanged knite from Batu Kurau, Perak. From Sieveking 1956a. Courtesy: Malaysian Branch of the Royal Asiatic Society. 
(Sørensen 1973, 1988) has yielded a tanged iron industry in association with wooden boat-shaped coffins, Heger I drums, and radiocarbon dates of about 200 BC. Ban Don Ta Phet (Glover 1990b) is an open burial ground, presumably for extended burials, although-as with the Peninsular Malaysian cists-no bone has survived. The iron industry here, which has a larger socketed component than that from Ongbah and also a shaft-hole sickle or billhook a little like the Peninsular Malaysian ones, is associated with a rich array of Indian carnelian and etched agate beads, and appears to date between 2,400 and 2,000 years ago (Glover 1990a).

One feature of the early Mainland Southeast Asian iron industries is that they are all characterized by different and localized tool forms, especially in terms of hafting. This localization is more marked than in the case of the bronze industries, where there is the integrating phenomenon of the long-distance trade of status items such as drums and other decorated ceremonial objects. Iron objects give an impression of being localized in style to regional industries and hence manufactured on the spot for use as everyday tools and weapons with only limited trade or status value. This naturally suggests that ironworking was a process that could be carried out easily by small, local communities and that knowledge of its manufacture spread rapidly from about 2,000 years ago, as the superior economic potential and easier availability of this metal-compared to bronze-was realized.

In Peninsular Malaysia, the iron tools and slab graves are apparently contemporary with a rather enigmatic coastal site at Kuala Selinsing in Perak. The original assemblage from here was excavated by Evans (1932) and included a wheelmade, comb-incised ware like that from Oc Eo in Vietnam (early to middle first millennium AD), a carnelian seal with an inscription in Pallava script, evidence for local blue glass and agate bead manufacture, glass bracelets, and lead slag. The site was evidently an estuarine pile village; it has produced human burials in canoe-shaped coffins (Sieveking 1956b). Beck (Appendix in Collings 1937b) dated the monochrome glass beads to between AD 1 and 400 (cf. Francis 1990). Recently, excavations at Kuala Selinsing have been started again by Nik Hassan Shuhaimi (1991; Davison 1991), who was unable to locate the original Evans site but confirms that the location as a whole consists of a series of mounds of earth and shell in a mangrove swamp, possibly deposited under the floors of pile dwellings.

According to current information, the Kuala Selinsing sites were inhabited between $200 \mathrm{BC}$ and $\mathrm{AD} 1000$. Nik Hassan Shuhaimi has recovered Middle Eastern pottery dating between the sixth and tenth centuries, locally made incised and paddle-impressed pottery, shell bracelets, tin ear pendants, and extended burials laid under sherd sheets. The latter occur in many central and northeast- 
ern Thai sites and could be indicators of some degree of Austroasiatic linguistic affinity, as the Mon language seems to have been widely spoken in the northern Malay Peninsula prior to Malay expansion (Stargardt 1983; Benjamin 1987). Kuala Selinsing has also yielded lots of valuable economic information, including bones of pig, dog, and chicken, plus remains of coconuts, gourds, bamboo, areca nut, pandanus and bamboo mats, rice husks, and part of a dugout canoe. The whole site complex might thus have been the residence of a local community in frequent touch with the trade network linking India and China at this time via the Straits of Malacca (for the archaeological record of some of the lashed-lug and dowelled boats that perhaps serviced this trade, see Manguin 1989).

A final point to note about the Peninsular Malaysian early Metal phase industries is that they are presumably associated-unfortunately to an unknowable degree-with the Austronesian coastal settlement of the region (Bellwood 1993). Linguistically (see Chapter 4, Section VA), an origin for the Malays, and also for the other smaller Orang Melayu Asli groups such as the Temuan and Jakun (Carey 1976), may perhaps be located in Sumatra (where slab graves also occur) or western Borneo during the first millennium BC. But there is little archaeological evidence to throw light on this, and the whole period between the Malaysian Neolithic as represented at Gua Cha and the Indianized monastery and temple monuments of Kedah, which mainly postdate AD 800 (Nik Hassan Shuhaimi and Othman Yatim 1990), is in need of further research.

\section{THE EARLY METAL PHASE IN SUMATRA, JAYA, AND BALI}

In this section I will describe the slab graves and stone monuments of southern Sumatra and Java, the stone sarcophagi of eastern Java and Bali, and a number of other important excavated early Metal phase assemblages in western Indonesia. I do not intend to go deeply into the question of megaliths and will not be concerned with such matters as the oft-proposed unity of megalithic cultures (e.g., Christie 1979b), or with Heine Geldern's theories that different types of megaliths can be ordered into "older" and "younger" strata (Heine Geldern 1937, 1945). Most of the celebrated ethnographic "megalithic" cultures of Indonesia -on Nias, amongst the Bataks of northern Sumatra, in parts of Borneo, in central and southern Sulawesi, and in some of the Lesser Sunda Islands (see Feldman 1985; Newton and Barbier 1988 for recent reviews)-are all of recent or ethnographic date and have no archaeologically documented antecedents. It is perhaps time for prehistorians to join the ethnologists and art historians in examining the antecedents of these recent cultures, but so far there has been little progress in this respect. 


\section{A. Sumatra}

One of the main concentrations of prehistoric stone monuments in Indonesia lies on the 70-kilometer-long Pasemah Plateau around Pagaralam in southern Sumatra (Hoop 1932; Heekeren 1958:63-79). A fairly simple megalithic tradition ("dolmens," mortars, and upright stones, the latter often in single or double alignments) occurs widely in the adjacent Lampung district at the southern tip of the island (Sukendar 1979). The Pasemah monuments, however, are quite striking and have attracted attention since 1850 . The structures include groups and alignments of upright stones, stone blocks with carefully hollowed cuplike mortars, troughs with human heads carved on their ends (Plate 52), simple terraced platforms (often referred to as "graves"), "dolmens" of uncertain function with large capstones, slab graves (some in the form of massive underground chambers), and some remarkable stone carvings of humans and animals.

The slab graves excavated by Hoop (1932) at Tegurwangi (Plate 53) contained large numbers of glass beads and a few metal objects-copper or bronze spirals, a gold pin, and a corroded iron lance-that cannot in themselves be closely dated. As in the Peninsular Malaysian slab graves, the acid soils had dissolved all traces of bone. One of the Tegurwangi graves and several megalithic chamber graves at Tanjungara (Bie 1932) (Plate 54) and Kotaraya Lembak (Soejono 1991) still preserved on discovery traces of polychrome wall paintings showing human figures and water buffalo. One of the newly discovered chamber graves at Kotaraya Lembak has a quite remarkable frontal figure of a cockerel in fighting stance painted in four colors (Caldwell in press) (Fig. 9.10).

The Pasemah human and animal statues are carved in relief or in the round on large stone blocks in a dynamic style. Men are shown riding on elephants or buffalo (Plate 55), wearing bracelets, anklets, helmets with peaks at the back, loincloths, tunics, and earplugs. Necklaces of oblong plaques and what appear to be faceted beads are also shown. Animal and human heads are often carved in considerable detail, while bodies are often disproportionately small or simply left out, depending perhaps on the original shape of the stone. Some reliefs also show combat themes of men fighting tigers or snakes, although the elephants and water buffalo are more often in situations demonstrating human control and possibly domestication or taming.

The most important chronological indicators on these carvings are the Heger I drums shown on the Batugajah and Airpurah reliefs (Plate 56), painted on a Kotaraya Lembak chamber wall (Soejono 1991:19) and possibly once shown on an incised carving on a rock outcrop near Tegurwangi (Caldwell in press). These could indicate a date in the early or middle first millennium AD, although some may overlap in time with the period of the Srivijaya trading state on the plains to the east around Palembang (i.e., after AD 670). 


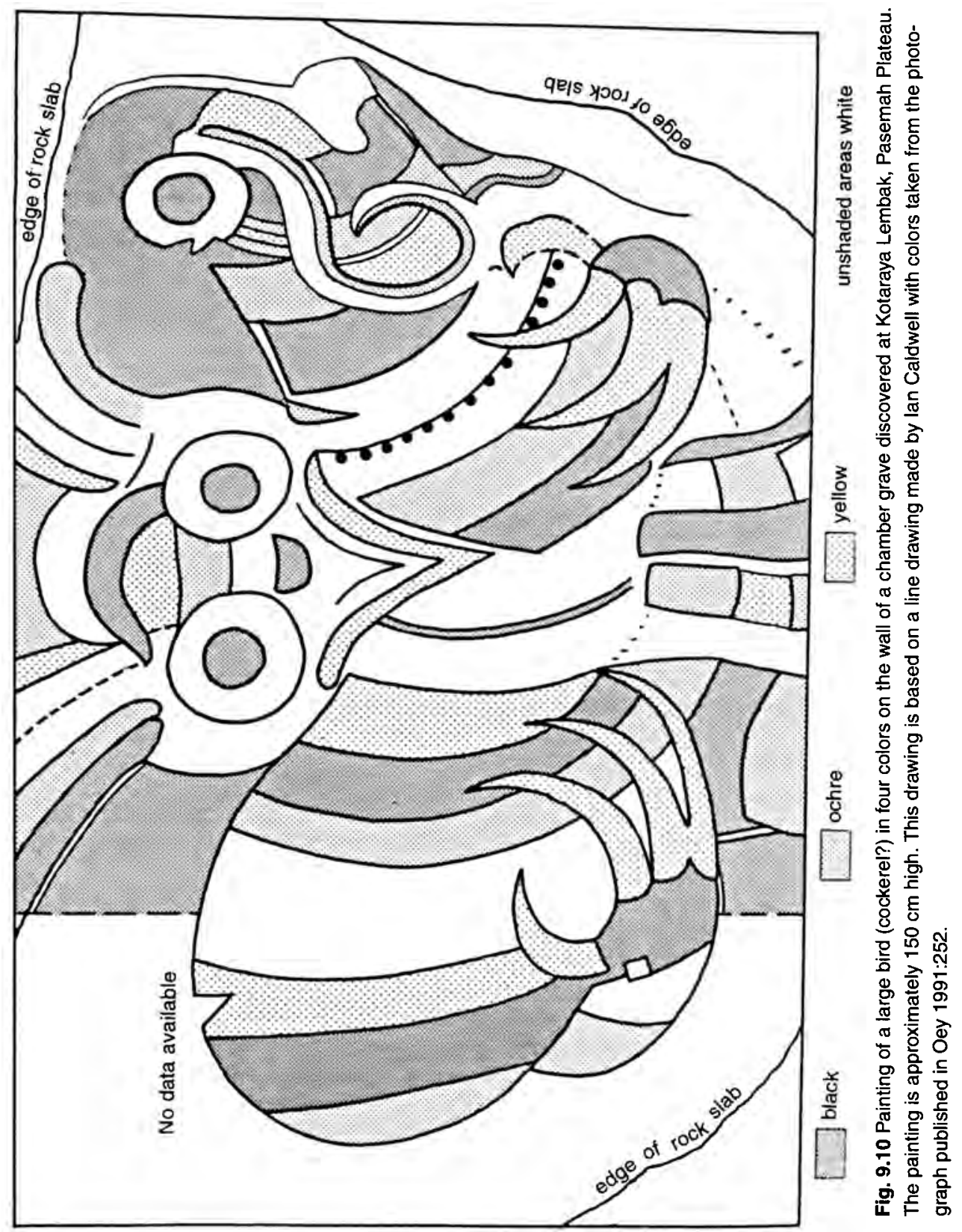




\section{B. Java}

In Java, many sites have produced early Metal phase assemblages in association with slab graves or with the more elaborate carved sarcophagi that occur from eastern Java through Bali to Sumbawa and Sumba (Soejono 1969, 1982b; Glover 1979). Only the slab graves at Kuningan in western Java appear, rather uncertainly, to lack metal associations (Kosasih et al. 1986), and all other sites appear to belong to the first millennium $\mathrm{AD}$ or later. Knowledge of the Javanese slab graves is vague (for some data see Heekeren 1958:46-54; Suryanto 1985; PPAN 1992), although Hoop (1935) did present a clear report of some excavated around Gunung Kidul near Wonosari in central Java. Well-preserved examples of these at Kajar and Bleberan (Plate 57) produced evidence for extended burials with a lot of iron tools (mainly tanged knives, a dagger, axes, and chisels), bronze rings, and beads of glass and faceted carnelian (Fig. 9.11). The slab graves and carved sarcophagi examined by Heekeren (1931) near Besuki in eastern Java produced no coherent archaeological assemblages, but similar monuments at Pakauman were apparently associated with Chinese imported ceramics of ninth century AD date or later. Therefore, like the slab graves of Peninsular Malaysia, many of these graves might have overlapped in date with the Javanese historical civilizations, as Hoop thought possible for the Gunung Kidul sites.

There are also a number of localities in western Java with complexes of stone-paved terraces and platforms that appear to belong to a pre-Indic architectural tradition. Bintarti (1981b) has described an excellent example at Gunung Padang, south of Cianjur (see also Sukendar 1985b), and others occur in the northwestern corner of the island at Lebak Sibedug (Hoop 1932:Plate 204) and Arca Domas (Tricht 1929). These structures probably served as open-air temples or gathering places, rather like the marae of Polynesia. Stone human statues of fairly simple shape, which many authors have called "Polynesian," are also known from localities widely distributed through Java and Bali (Mulia 1980; Sutaba 1997). Unfortunately, it is impossible to date these monuments and statues, although one statue of this type near Bandung carries an inscription, possibly secondary, of AD 1341 (Suleiman 1976:8). The apparent Polynesian similarities are either coincidental or may reflect some form of shared early Austronesian architectural and artistic inheritance.

Apart from the research on the stone graves and other monuments, which have always attracted much archaeological attention in western Indonesia, there have been a number of excavations in other types of sites belonging to the early Metal phase in Java. As I will show later, the practice of jar burial was predominant mainly in the more easterly parts of Indonesia, but flexed primary burials in jars have been uncovered with inhumation burials at Anyar in western Java (Heekeren 1956a; Sukendar et al. 1982), and jar burials also occur at Tebing- 


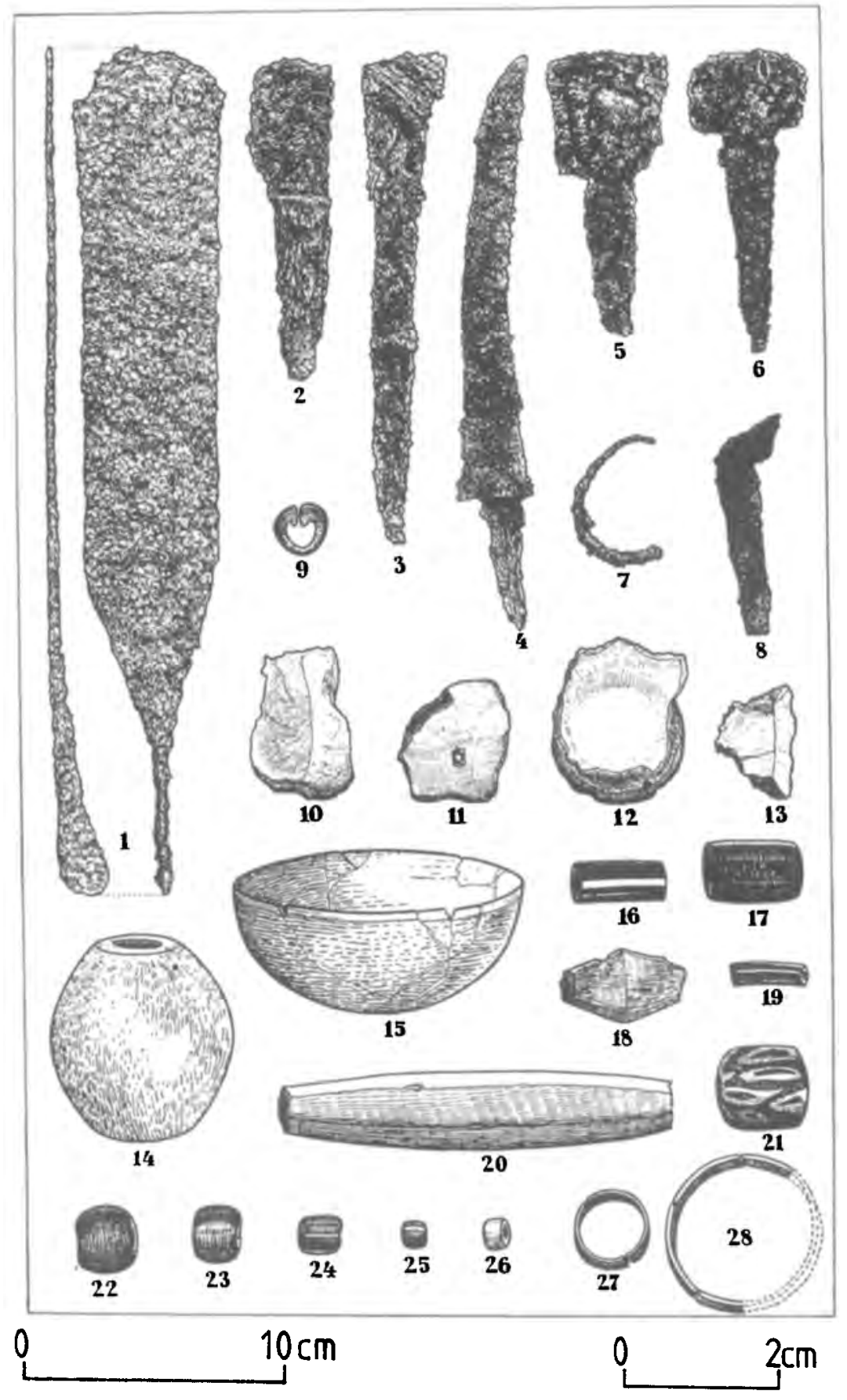

Fig. 9.11 Artifacts from slab graves at Gunung Kidul, central Java. The tanged iron tools include a kris (dagger: no. 4), and a range of stone and glass beads is shown. Left-hand scale is for 1-13 and 15. Right-hand scale is for 14 and 16-28. From Hoop 1935. 
tinggi in southern Sumatra (Heekeren 1958:83). Excavations at Plawangan in north-central Java (Sukendar and Awe 1981) have produced an interesting mixture of flexed or extended inhumations and burials of both adults and children in jars with inverted-vessel lids. In one case a flexed child skeleton was placed inside an upturned Heger I drum together with pottery, a bronze spearhead and bracelet, glass beads, and gold eye and mouth covers (Soejono 1991). The assemblage from Plawangan also includes iron knives, a bronze fishhook and ring, and incised and stamped pots placed at the heads and feet of the extended burials.

At another north Javanese site called Kradenanrejo, near Lamongan, a child was placed inside a drum of Pejeng type with a Heger I drum on top as a cover, with carnelian, glass and faceted gold beads, a bronze container with Dong Son circle and tangent ornamentation (Section IV), gold umbrellashaped ornaments (hints of an overlap with Buddhism?), two bronze cups, and various other iron and bronze items (Bintarti 1985a; unfortunately few of these items have ever been illustrated, and the Pejeng drum was destroyed when found).

Other Javanese sites that have produced important early Metal phase assemblages include Leuwiliang, near Bogor, where alignments of grave goods from now-dissolved inhumations include an anthropomorphic bronze pendant (Soejono 1984: Foto 77) and a face mask of an unidentified precious metal (PPAN 1988), and Pejaten, to the south of Jakarta (Sutayasa 1979), where bakedclay casting molds for bronze axes and knives are apparently radiocarbon dated to before $\mathrm{AD} 200$. None of these sites can as yet be placed within a coherent reconstruction of Javanese prehistory, and the most urgent requirements are for a fuller publication record and many more well documented radiocarbon dates.

The so-called Buni cultural complex of looted graves on the western coast of northern Java (Sutayasa 1972, 1973, 1979) also has obvious future potential for archaeological investigation. Brief records of the grave goods, apparently found with extended burials, include gold and carnelian beads, stone adzes, undefined metal artifacts, and a range of carved-paddle-impressed and incised pottery with a variety of forms, including ring-footed vessels, high-necked flasks, and knobbed lids. The Buni complex is of great significance because it has produced three flat-based vessels of the distinctive south Indian Rouletted Ware; one each from the sites of Kobak Kendal, Cibutek, and Cibango (Walker and Santoso 1977) (Fig. 9.12). Another location at Batujaya to the east of Buni has produced another sherd of Rouletted Ware (McKinnon et al. 1994). One site at Rengasdengklok has also produced gold eye and mouth covers of a type found also in Balinese sites of the same date (see below, and Miksic 1990). Because this rather exciting material has been found in the same part of western Java as the oldest 

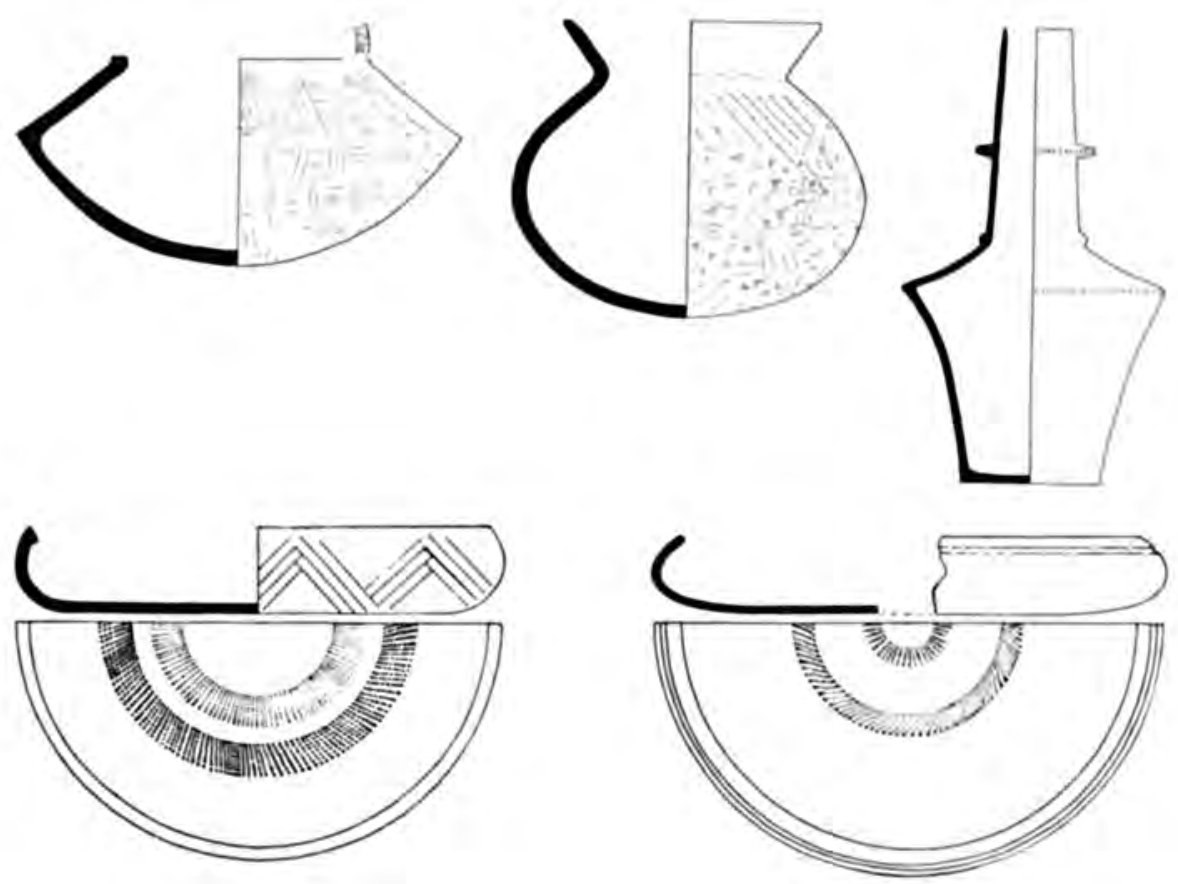

Fig. 9.12 Pottery from the Buni complex, northwestern Java. Top: two paddle-impressed vessels and a high-necked flask (not to scale, but the flask is 30 centimeters high). From Summerhayes and Walker 1982. Bottom: Rouletted Ware from Kobak Kendal (left) and Cibutak (right), both ca.28 centimeters in diameter. From Walker and Santoso 1977. Courtesy: M. Walker.

Sanskrit inscriptions (e.g., the Purnavarman inscription at Tugu, possibly of fifth-century date; Noorduyn and Verstappen 1972), it is apparent that the Buni sites, despite their tragic looting, may contain information directly relevant to the initial period of contact between India and Java, presumably in the first few centuries $\mathrm{AD}$. This period has recently been highlighted by discoveries on Bali, especially at the site of Sembiran, to which we turn below.

\section{Bali}

Bali is renowned for its highly distinctive sarcophagi, carved from soft tuff or breccia, found mainly at sites in the central and southern interior of the island (Heekeren 1955; 1958:54-58; Soejono 1977, 1995; Sukarto 1979; Ardika 1987). These have separate bodies and high, domed lids and usually have knobbed projections on their ends, sometimes carved into human or turtlelike heads (Plate 58). A range of sizes was produced to accommodate both flexed and extended burials. The grave goods include glass and carnelian beads, some rather 
indeterminate iron objects, unusual ornaments and finger-sheaths made of spiraled bronze wire (e.g., see Soejono 1977:Foto 67-70), and socketed bronze tools with crescentic and heart-shaped blades (Ardika 1987), the latter being closely paralleled in mid-first millennium BC contexts in central Thailand (see Section IV). At Gilimanuk in western Bali, two of these stone sarcophagi-one with a lid shaped like a buffalo, the other with a stylized design apparently based on female genitalia-have been excavated in contexts perhaps dating to about 1,500 to 2,000 years ago (Soejono 1995).

Gilimanuk is an important coastal burial site that has produced extended burials and jar burials (some in double mouth-to-mouth jars) with associated pottery and bronzes like those from some of the sarcophagus sites (Soejono 1979; Santoso 1985). Other Gilimanuk grave goods include a tanged iron spearhead, an iron dagger with a bronze handle and beads of gold, glass, and carnelian. Gilimanuk and a sarcophagus at Pangkungliplip have both produced gold eye and mouth covers, like the Buni example mentioned above. The assemblage is clearly contemporary with those of Buni and Sembiran and thus falls somewhere between $200 \mathrm{BC}$ and $\mathrm{AD} 300$, a range solidly confirmed by nine C14 dates from the site listed by Bronson and Glover (1984:41).

No actual Indian material has yet been reported from Gilimanuk, but this is certainly not the case for the site of Sembiran, near Tejakula on the north coast of Bali (Ardika 1991; Ardika and Bellwood 1991). Here, evidence for early Indian contacts with Bali has recently come to light in prolific quantities. Excavations through coastal alluvial sediments down to the water table at about 3.5 meters depth have yielded many pieces of Rouletted Ware (Plate 59), together with sherds of Arikamedu (Tamil Nadu) molded vessels of types 10 , 18, and 141 (Wheeler et al. 1946), a black-slipped sherd with a scratched line of Kharoshthi or Brahmi characters, the above-mentioned fragment of a tuff stamp for decorating the wax preform of a Pejeng type drum (Section IV), and local pottery of incised and impressed types similar to those from Gilimanuk. Some of the pottery, especially a black-slipped ware of uncertain origin (an import?), has rice chaff temper. Rice phytoliths in the soils of the site indicate that this cereal was grown during the period of occupation (Doreen Bowdery, pers comm).

Sembiran and the Buni complex bring back the question of the origins and date of the initial Indian contacts with western Indonesia. On the matter of date, it has already been noted that Indian beads occur in Mainland Southeast Asian sites, such as Ban Don Ta Phet and Giong Ca Vo, dated to the fourth century BC. The Rouletted Ware (which has so far been found outside India/Sri Lanka only in Indonesia and Vietnam) was originally dated to the first and second centuries AD by Wheeler et al. (1946), owing to its association with Roman imports at Arikamedu. Begley $(1983,1986)$ has since pushed this dating back 
into the second century BC. Rouletted Ware has been recovered from excavations along the whole eastern coast of the subcontinent, from Anuradhapura in Sri Lanka (Deraniyagala 1986) to sites as far north as Sisupalgarh in Orissa and Chandraketugarh in West Bengal. It is in these northerly sites that very distinctive etched agate beads occur in late first millennium $\mathrm{BC}$ contexts-beads of a type that have also been found in sites of the early Metal phase in Talaud and the Philippines. The sites with Rouletted Ware in south India and Sri Lanka also have utilitarian pottery decorated with carved-paddle-impressed and stamped patterns (Ray 1997) that find suggestive parallels in the pottery of the Buni complex, Sembiran, and Gilimanuk. It would be premature to suggest a definite link between India and Indonesia from this type of pottery on present evidence, and Solheim (1990) has for many years favored a southern Chinese origin for the Southeast Asian paddle-impressed wares as a whole (which belong in his "Bau-Malay" pottery complex). But I believe that the possibility of an Indian link does deserve careful consideration.

One further point about this burgeoning evidence for Indian trading contact with Indonesia in the centuries around the birth of Christ concerns its impact on those regions beyond the "core" Indianized regions of Sumatra, Java, and Bali. It is worth repeating that the far-flung distribution of Heger I drums might have had much to do with these activities, especially via those trade networks that surely extended from Java and Bali into the Moluccas for cloves, nutmeg, and other spices destined for the Mediterranean, India, and China. But, more than this, it has been my observation during many years of fieldwork in Indonesia-a difficult observation to substantiate in a brief text-that the pottery assemblages all over the Indo-Malaysian Archipelago in the centuries of the early Metal phase are remarkably similar, whether they be from Java, Bali, Talaud, or Halmahera (cf. Diniasti 1986). The Papuan-speaking peoples of northern Halmahera even appear to have first adopted pottery making at this time, perhaps as a result of increasing trade contacts. Some of these Indonesian "outer-island" assemblages will now be reviewed.

\section{THE EARLY METAL PHASE IN EAST MALAYSIA AND EASTERN INDONESIA}

The early Metal phase in the northern and eastern parts of the Indo-Malaysian Archipelago is now becoming better known as a result of a number of recent cave and open-site excavations. In the northeast, many sites have yielded evidence for a fairly homogeneous tradition of secondary burial in large jars or pottery bone boxes. In the Lesser Sundas, primary and jar burials often occur together, as in Java and Bali. The pottery assemblages of this phase all demonstrate fairly close relationships, and the period as a whole-with its copper/ 


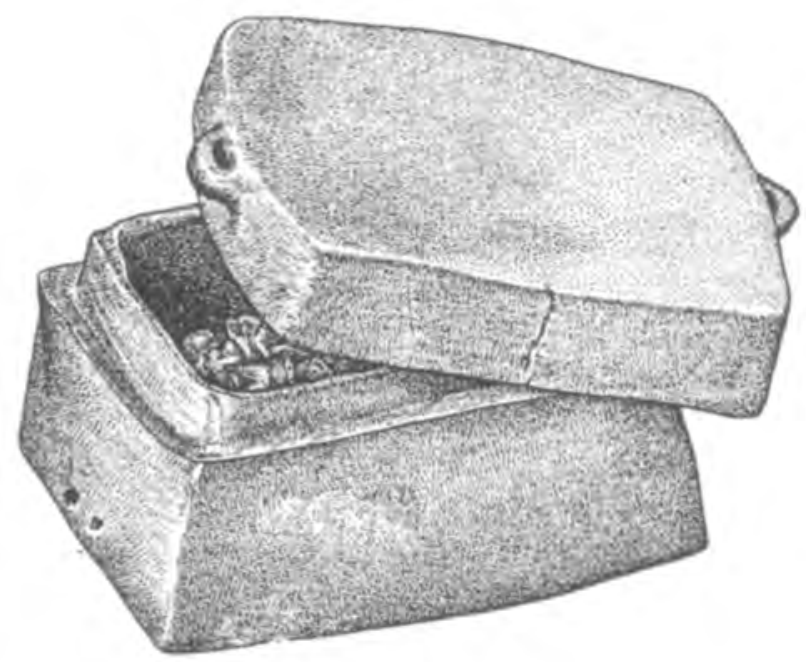

Fig. 9.13 Pottery bone box containing human teeth from Bato Puti Cave, Tabon Cave complex, Palawan. From Fox 1970. Courtesy: National Museum of the Philippines.

bronze and iron associations-may be dated from about $200 \mathrm{BC}$ to AD 1000. The tradition of jar burial continued after AD 1000 into ethnographic times in some remoter parts of Borneo, Sulawesi, and the Philippines, but in association with imported Chinese and Southeast Asian glazed ceramics that lie outside the upper chronological limits of this chapter.

The jar burial tradition is seen at its most elaborate in the islands around the Sulawesi and Sulu Seas (northern Borneo, Talaud, central and southern Philippines) and in parts of the Lesser Sundas-especially Sumba. In the former region it involved the placing of previously exposed secondary burials in large jars or bone boxes provided with lids (Fig. 9.13). Small accessory vessels were placed in or around the jars, together with beads, bracelets, and metal artifacts, to list the most common grave goods. The jars were placed either on the floors of fairly remote caves (in which situations they have invariably become smashed and their contents mingled) or in pits dug into open sites (where they have normally survived fairly complete).

In general, the tradition of jar burial gave way fairly rapidly to extended inhumation burial in those regions influenced by the Indianized and the later Islamic states, and also in those parts of the Philippines closest to the Chinese trade networks. The whole tradition thus achieved its apogee in the first millennium AD. In eastern Sabah and the Philippines, the second millennium witnessed a widespread change to a tradition of log-coffin burial. 


\section{A. The Talaud Islands and Northern Moluccas}

The first jar burial site to be discussed is the small cave of Leang Buidane on Salebabu Island in the Talaud group of northeastern Indonesia (Bellwood 1976a, 1976b, 1981). The jar burials here were originally placed on the floor of the cave, but were smashed-presumably deliberately (Bellwood 1981:71)—in antiquity. The bone containers comprised a range of large globular jars with round bases and occasional tripod or ring feet, together with flat-based cylindrical vessels and roughly rectangular pottery boxes (Fig. 9.14). All these large containers appear originally to have been lidded. The human bones (Bulbeck 1978) were mainly of young individuals under forty years of age. The ratio of thirty-six individuals (based on teeth) to a minimum of thirty-two large vessels suggests that only one individual was placed in each container. The bones were mainly skulls, mandibles, and limb bones-pelvic bones and vertebrae appear to have been discarded or lost. The teeth revealed some evidence for betel staining, occasional tooth evulsion for females during life, and a Mongoloid morphology presumably directly ancestral to that of the present population. In my original reports I dated this site between $\mathrm{AD} 700$ and 1200 according to a number of rather oblique chronological indicators, but as a result of my more recent work in Sabah I now incline to an earlier commencement date.

The accessory vessels and other items found with the remains of the jar burials form a homogeneous stylistic group and define a Buidane culture that appears to have been current throughout Talaud for much of the first millennium AD. The small pots include round-based carinated vessels with quite elaborate horizontal zones of incised decoration (Fig. 9.15), distinctive high-necked flasks with a polished red slip, and a range of cooking vessels. The carinated vessels in particular have an angular cross-sectioned rim that is also characteristic of this phase in Sabah. The Buidane ceramic range is also very closely paralleled in a southern Philippine site visited in the 1920s by the Guthe Expedition, but unfortunately in a now unknown location (Solheim 1964b:94-101, the "unknown site"). However, the concept of a Buidane culture is best confined to the Talaud Islands themselves, since all the sites of this period reveal a gradual falloff in similarity with distance across the whole region, from the central Philippines to Sumba. At present it would be unwise to class the material at a level greater than that of the individual region or island group.

The other artifacts found in Leang Buidane include shell bracelets and beads, part of a glass bracelet, beads of agate and carnelian, coral flask stoppers, and a penannular pottery earring. The stone beads are particularly interesting: The majority are either spherical or elongated faceted red carnelians with a precision in drilling that probably indicates an Indian origin, although the shapes are chronologically complacent and belong to types common in both India and 

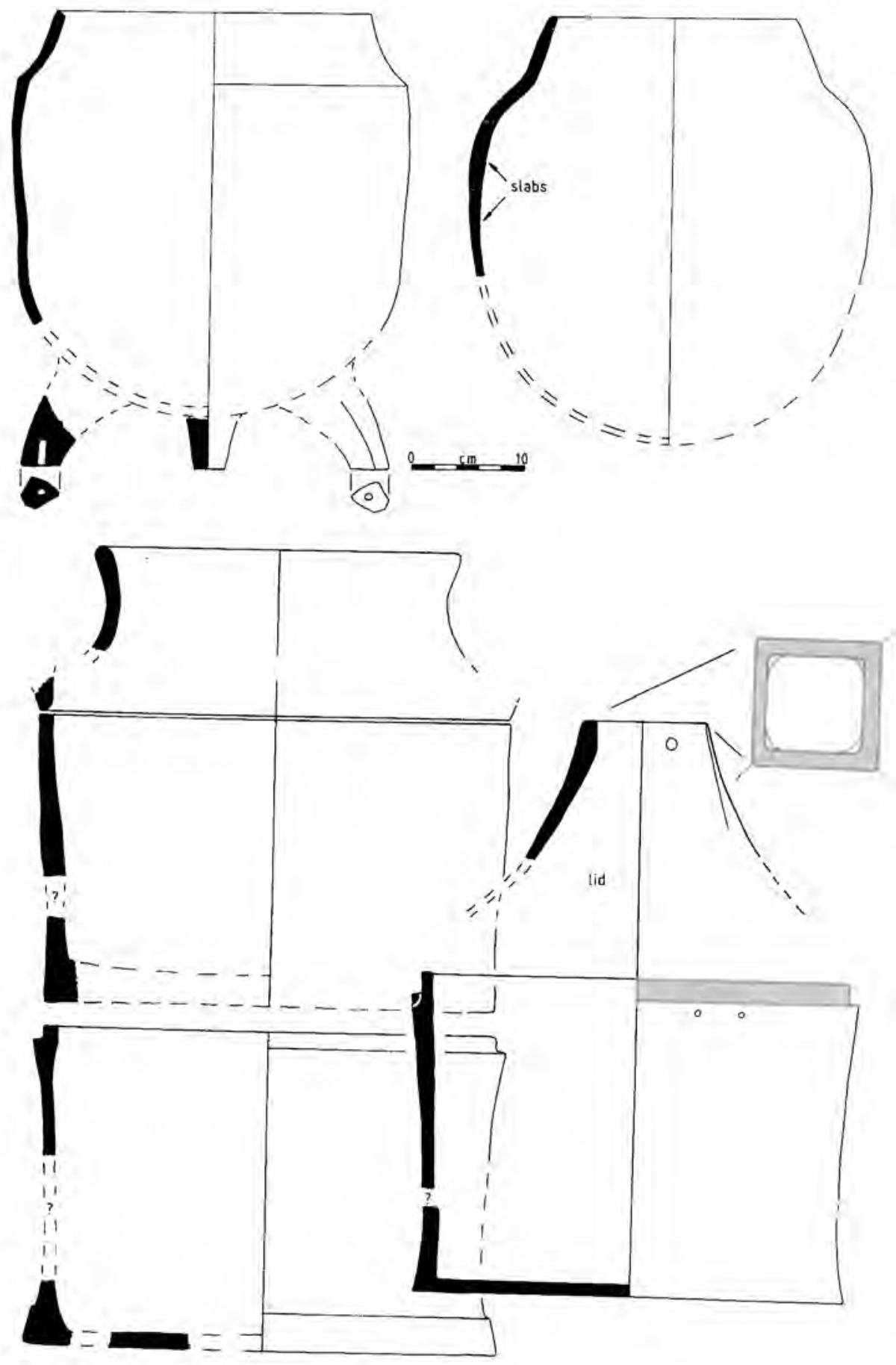

Fig. 9.14 Large burial jars from Leang Buidane, Talaud. The lower three vessels are cylindrical with vertical walls, and two have lids. Shading $=$ red slip. 

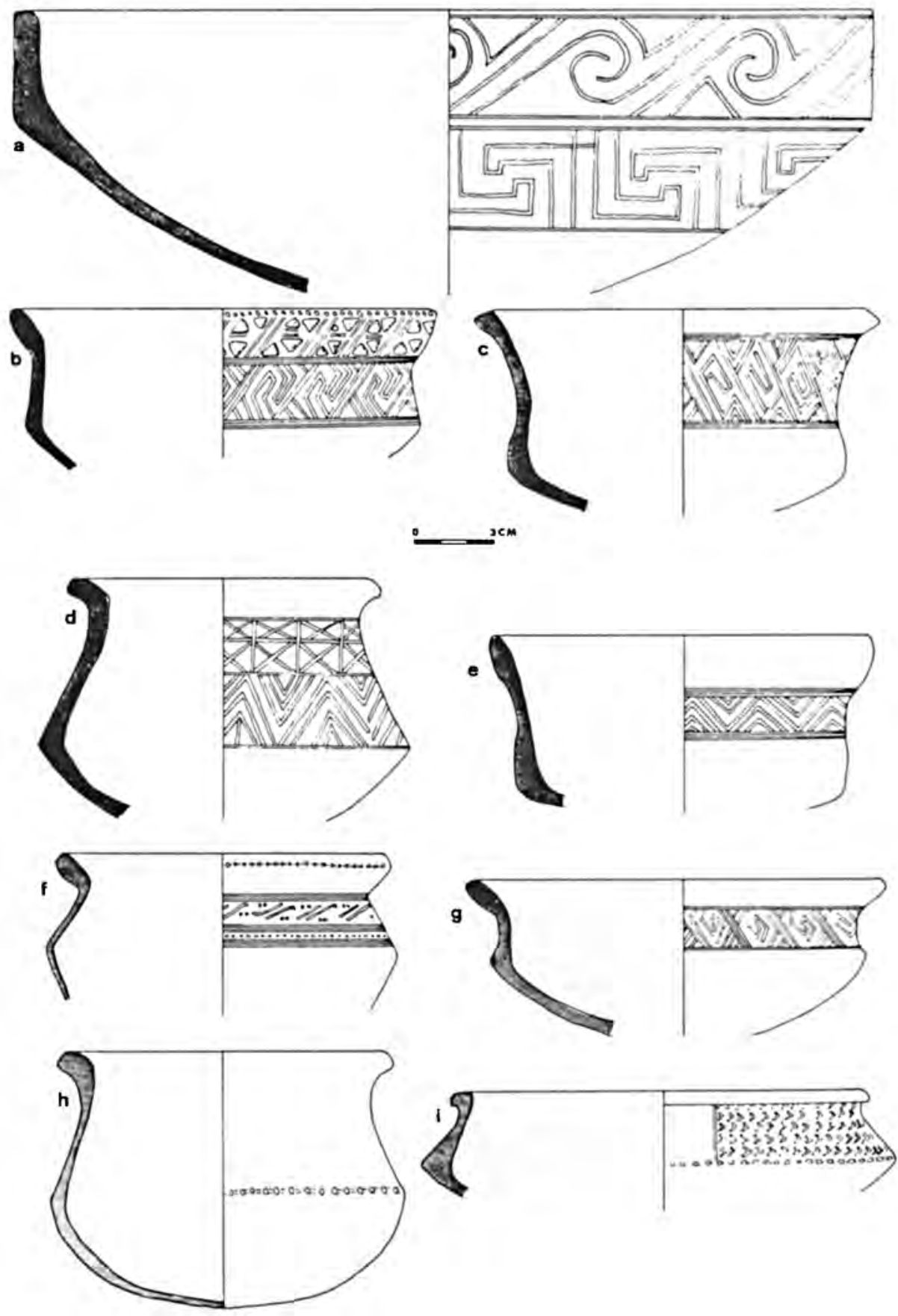

Fig. 9.15 Small carinated vessels from Leang Buidane with friezes of incised geometric decoration; (a) is probably a lid; ( $g$ ) and (i) have red-slipped interiors. 


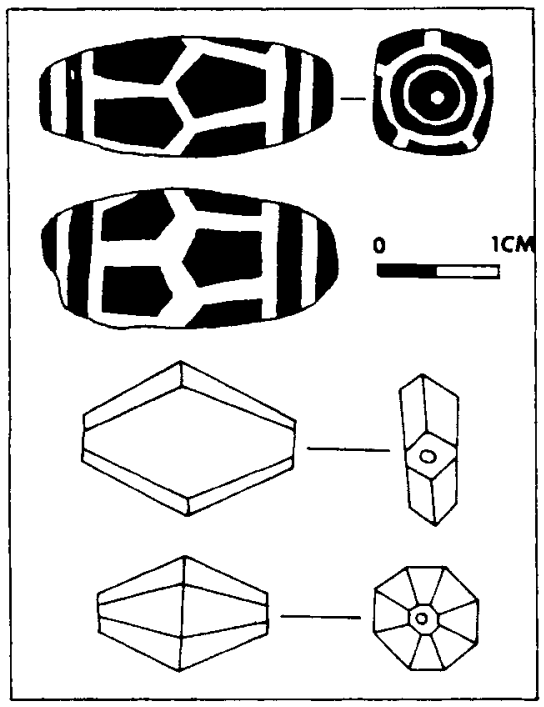

Fig. 9.16 Indian beads from Leang Buidane. Top: white-etched black agate, with identical specimen beneath from Sirkap Mound, Taxila, Pakistan (ca. first century AD). Bottom: flattened lozenge and octagonal bicone of red carnelian. From Bellwood 1976a (Taxila bead after Beck 1941:PI. II,17).

Southeast Asia throughout the past 2,000 years. However, there are three black agate beads with designs etched in white (Fig. 9.16) that are paralleled very precisely in late first millennium BC layers in major Gangetic and Indus sites such as Hastinapura (Lal 1954-1945:Plate LV), Taxila (Dikshit 1952:35), Kausambi and Chandraketugarh. There is also a banded agate bead of a type common from the Harappan onwards. The etched agates support the above-mentioned evidence from Sembiran for contact between India and Indonesia as early as 200 $\mathrm{BC}$, although in a location as remote as Talaud the beads might have been in circulation for many years prior to their eventual burial.

Leang Buidane has also produced metal artifacts; there are a number of indeterminate pieces of iron, and the copper or bronze objects include bracelet fragments, a bronze cone, and a copper socketed axe. Three baked-clay valves of the bivalve moIds for axes and other cuprous objects were also found, indicating that metal casting was carried out locally, although this may have been confined to recasting of artifacts that were originally imported. In general, the Buidane metallurgy fits conformably within the range reported from this date in Sabah and the Philippines, and the copper- and bronzeworking seems to have been restricted to bivalve mold techniques, without the use of wax.

In the northern Moluccas, fragmentary jar burials have been excavated in the cave of Uattamdi on Kayoa Island, associated with glass beads, fragments of iron and bronze, undated Chinese coins, and large reef shells, all apparently placed as grave goods. Dates for this assemblage run from about AD 1 to 1200 (Bellwood et al. 1993). Other regions of the northern Moluccas have produced 
incised pottery of early Metal phase affinity, radiocarbon dated to the first millennium $\mathrm{AD}$, associated with secondary skull burials in the rock shelter of Tanjung Pinang on Morotai and found in habitation deposits in the cave of Gua Siti Nafisah on Halmahera and the open site of Buwawansi on Gebe. This material is still being analyzed, but it appears that pottery making began in the Papuan-speaking regions of north Halmahera at this time, perhaps, as noted above, because of the increasing frequency of trade contact emanating from the western regions of Indonesia.

\section{B. Eastern Sabah}

In Chapter 7, Section IIIB, I discussed the Neolithic pottery assemblages found in Bukit Tengkorak and Agop Atas. Bukit Tengkorak has not produced any metal, but the cave of Agop Atas in the Madai massif (Bellwood 1988) has produced a habitation layer of the early Metal phase that is firmly dated by radiocarbon to the early to middle first millennium $\mathrm{AD}$. This layer is separated by an interval of nonoccupation from the underlying Neolithic layer. The cave was clearly intensively inhabited in the early Metal phase; several of the layers con-
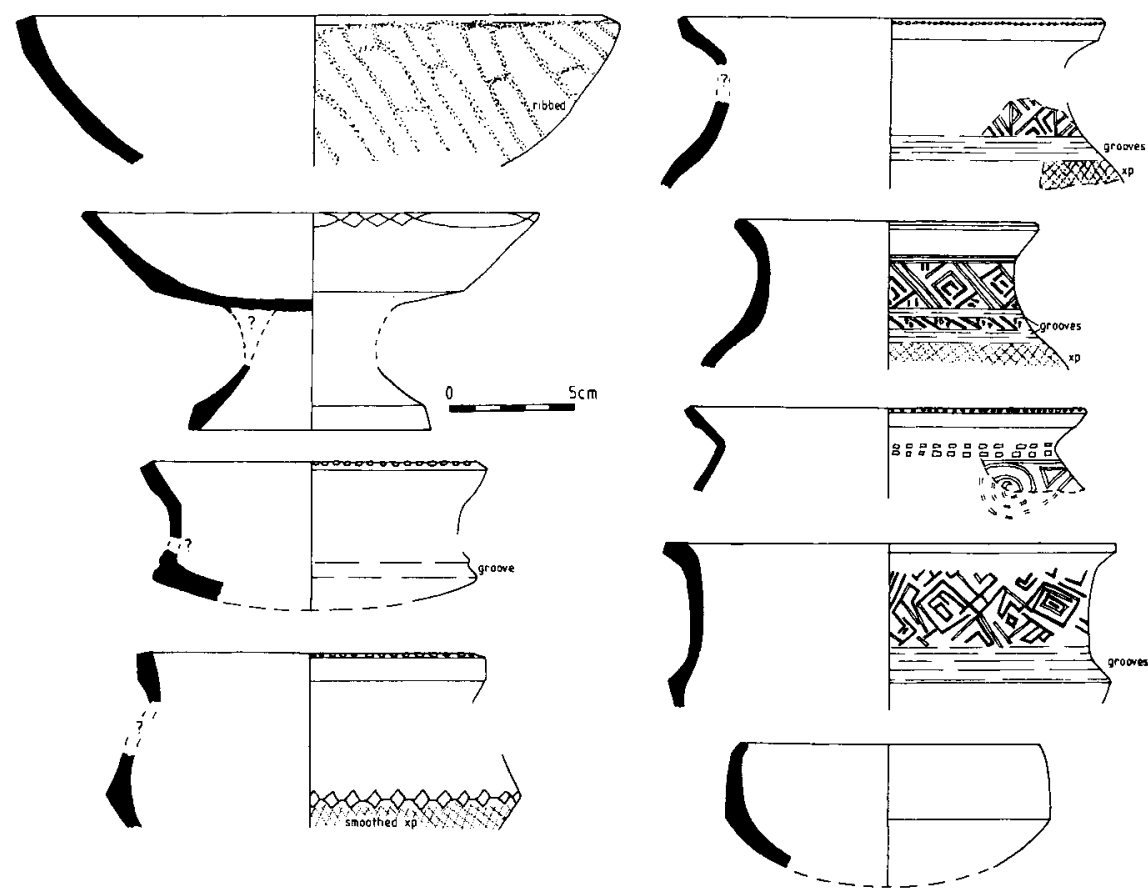

Fig. 9.17 A selection of vessels from Agop Atas. ( $x p=$ checkerboard paddle-impressed). 
tain postholes, perhaps for sleeping platforms. The pottery (Fig. 9.17) has basically the same rim and vessel forms as Leang Buidane, and the repertoire of incised decoration, carved-paddle and corded impression, and burnished red slip is quite similar between the two sites. The distinctive red-slipped and highnecked flasks also occur at Agop Atas and in another contemporary assemblage from Hagop Bilo shelter in the nearby Baturong massif (Plate 60b). Other objects found in Agop Atas include copper or bronze fragments, a forged iron tanged spearhead, a small iron knife, and a few carnelian beads-the latter of somewhat cruder manufacture than those from Buidane.

Jar burial assemblages of this phase in Sabah occur in Pusu Samang Tas Cave in a remote part of the Madai massif (T. and B. Harrisson 1971) and in a cave in the Tapadong massif on the Segama River (Harrisson 1965). The Tapadong assemblage has produced eleven stone adzes with trapezoidal cross-sections, plus a copper or bronze socketed axe and a casting valve of soft stone. This find demonstrates that local casting was carried out, as in Talaud, although stone adzes clearly continued in use well into the early Metal phase. Adzes identical to those from Tapadong are also known from surface collections in Mindanao (Lynch and Ewing 1968) and from the upper layer (late Neolithic, post-500 BC) at Bukit Tengkorak.

The overall significance of the well-dated Agop Atas and Buidane assemblages is that they demonstrate the existence of iron and copper/bronzeworking, together with imported Indian beads and a style of decorated pottery known to many authors as "Sa Huynh Kalanay" (after Solheim 1964a, 1967), from a date probably quite early in the first millennium $A D$. The beads and the available carbon dates make it difficult to push the beginnings of the early Metal phase around the Sulawesi Sea back before AD 1, although the traditions of jar burial and the friezes of incised decoration on pottery apparently go back into the late Neolithic in Sarawak and Palawan (Chapter 7, Section IIIC).

\section{The Central and Southern Philippines}

The best-known ceramic and metal (copper/bronze and iron) complex of the early Metal phase in the central Philippines is undoubtedly that termed Kalanay by Solheim (1964b), from his excavations in Kalanay Cave on Masbate and from earlier collections made by the Guthe Expedition. This material is not of direct concern here, although it should be noted that the whole range shows considerable similarity to the Agop Atas and Buidane assemblages.

The extensive complexes of caves on the west-central coast of Palawan (including the Tabon Caves) have produced prolific jar burial assemblages that still remain only partially published (Fox 1970; Kress 1978). Much of this material is virtually identical to that from Agop Atas and Buidane, although detailed 
comparison remains impossible. Fox placed the beginnings of the jar burial tradition at Tabon with the Manunggul chamber A assemblage, which he dated to the beginning of the first millennium $\mathrm{BC}$ on the basis of two radiocarbon dates. This assemblage contained no metal, and Fox was therefore willing-perhaps rightly-to place in it the Neolithic. The pottery is especially fine and appears to lack the sharply carinated forms of the Tabon early Metal phase. However, it does have at least one pottery coffin and some vessels have red-painted curvilinear designs enclosed by incised lines-a technique well represented in the three-color ware from Sarawak, in early Metal assemblages in Sabah, and also in the Sa Huynh culture in southern Vietnam.

Although Fox suggested that copper and bronze objects first appeared in the Palawan sequence at about $500 \mathrm{BC}$, the only dated site for the early Metal phase at Tabon is Manunggul chamber $B$, which has a radiocarbon date of about 200 BC. This assemblage produced iron, glass bracelets, glass and carnelian beads, and also five acid-etched agate beads similar to those from Leang Buidane. Copper or bronze items occur in other jar burial caves in the area and include socketed axes and spearheads, a tanged and barbed arrowhead, and a possible barbed harpoon. Axe casting molds, gold beads, and jade lingling-o earrings have also been found. In general, I suspect that the Tabon jar burial sequence after analysis will resemble the sequence from the Sabah sites and belong mainly in the first millennium AD.

Another central Philippine site worthy of mention is the open jar burial site of Magsuhot on Negros Island, where Tenazas (1974) excavated three large burial jars placed side by side in a large pit lined with broken potsherds. Pottery of Kalanay type, human and animal figurines of baked clay, and bones of pig and chicken were found with the jar burials, which were sealed in by an earth fill. Only one jar contained human bones-of a young woman and two children. Another pit contained an enormous lidded burial jar with a weight of 52 kilograms, together with a pottery bone box and no fewer than seventy accessory vessels. The burial jar was connected to the surface by a tube of stacked pots; it contained an iron knife but no identifiable bones. This site is of interest because it reveals the wealth of information that can survive with undisturbed jar burial sites and-while undated-it would fit well into a middle or late first millennium AD context.

\section{Southern Indonesia and Sulawesi}

The most remarkable of the jar burial sites of Indonesia is undoubtedly the large urnfield of Melolo on eastern Sumba. This open site was first investigated during the 1920s and 1930s and most recently in 1985 (Bintarti 1986). It has produced an alignment of large, close-set burial urns with round-based vessels as 
lids, containing fragmentary secondary burials, stone adzes, stone and shell beads, shell bracelets, and small accessory pots of which the only kind adequately reported is an elegant high-necked flask with incised geometric and anthropomorphic designs filled in with a white paint (Heekeren 1956b) (Fig. 9.18). It appears that some of these flasks were provided with a burnished red slip.

Some metal items have been found in the recent excavations, so Heekeren's (1972:191) classification of the site as Neolithic appears to be incorrect. Some of the Melolo flat-based flasks are paralleled precisely in the Buni pottery of western Java (see Fig. 9.12), and the flask forms in general are paralleled in a number of other sites of the early Metal phase, including Sembiran in Bali. I suspect that this may be a significant observation for future research because the form is not, to my knowledge, found in any Indo-Malaysian assemblage (outside Taiwan) that can conclusively be considered as Neolithic. Indeed, the high-necked flask, often with a globular body and sometimes a burnished red slip, can perhaps be regarded as a clear marker of the early Metal phase; for example, at Leang Buidane in Talaud, at Agop Atas and Hagop Bilo in Sabah, at Gunung Piring on Lombok, at Leang Bua on Flores, at Batu Ejaya in southern Sulawesi, at Gilimanuk and Sembiran on Bali, and at Anyar in western Java (Plate 60). The form clearly transcends local cultural areas, and at Gilimanuk, Leang Bua, and Gunung Piring it is found in association with inhumation rather than jar burials.

Melolo is really the only jar burial site in eastern Indonesia that merits close attention at present, although a related site called Lambanapu in eastern Sumba is currently under investigation. However, there are some further generalizations about the early Metal phase in the archipelago as a whole that can be made. First, it is clear that the sites around the Sulawesi and Sulu Seas-the Tabon Caves, the "Kalanay" sites, and the sites of eastern Sabah and Talaud-do share quite closely related pottery assemblages with iron and copper/ bronze during the first millennium AD. Jar burial is the predominant rite in this region; another common characteristic is the small pottery bone box.

In southern Indonesia the picture becomes rather more confused and here comprises the relatively "pure" jar burial site at Melolo, the mixed-rite sites such as Anyar, Plawangan, Gilimanuk, and also Gunung Piring on Lombok, which appears to have only inhumations. Outside these two regions, on the islands of Sumatra, Borneo (except Sabah), Sulawesi, and the central and southern Moluccas there are unfortunately many blanks, although jar burial assemblages from caves in southern Sulawesi (Mulvaney and Soejono 1970, 1971) have bone boxes and pottery loosely related to the Tabon-Sabah-Talaud sites that may date within the first millennium AD. The pottery from the jar burial site of Ulu Leang 2 in the Maros region of southern Sulawesi (Andrews and Glover 1986) has produced pottery with zones of densely incised decoration like some from 


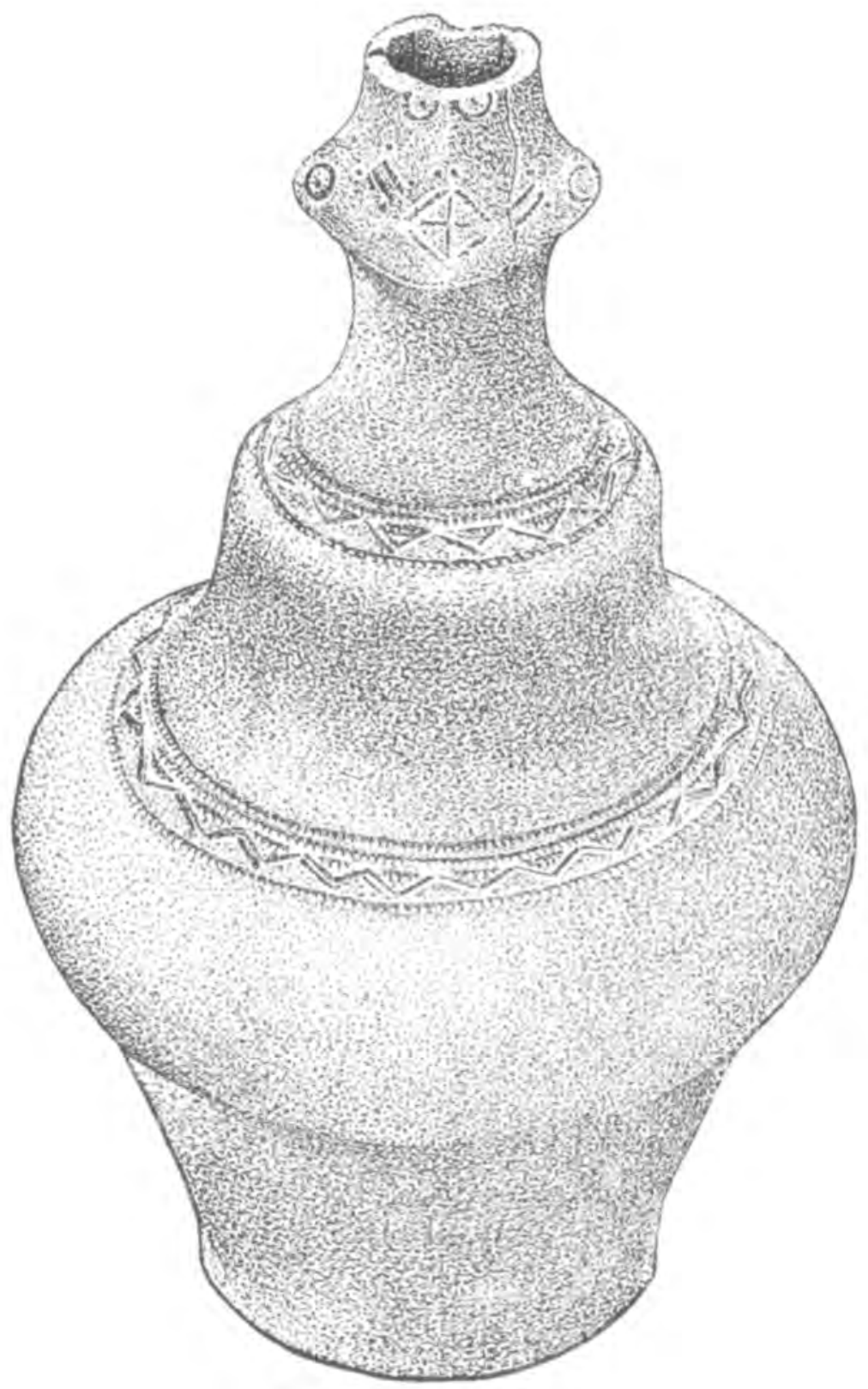

Fig. 9.18 Flask with human face carved on neck from Melolo, Sumba. From Heekeren 1956b. Courtesy: Indonesian National Research Center for Archaeology. 
Sembiran in Bali. However, it is clear that even the most basic cultural and chronological framework for the early Metal phase does not yet exist for the great bulk of the archipelago; until this is established, understanding of the period is unlikely to advance very far.

The same observation still applies to the remarkable complex of large stone jars and human statues in central Sulawesi (Kaudern 1938) (Plate 61). I have little to add to my previous description of these monuments (Bellwood 1978: 228), and dates are still elusive, but an extensive survey in the Bada district to the west of Lake Poso (Sukendar 1980) has brought to light more stone jars and statues and demonstrated their association with iron and carved-paddleimpressed pottery. However, the absence of a chronological scheme for Sulawesi prehistory subsequent to the Toalian industry makes accurate dating virtually impossible, and the apparent absence of Chinese pottery can only suggest a date older than 500 years.

\section{E. The Origins of the Jar Burial Tradition}

I have already discussed the evidence, of varying reliability, indicating that jar burial was practiced in late Neolithic contexts at Niah in Sarawak and in the Tabon Caves. Possible commencement dates fall in the late second and early first millennia $\mathrm{BC}$; if these dates are correct, they provide strong grounds for regarding the tradition as an indigenous development in Island Southeast Asia. However, it must still be realized that the bulk of the jar burial sites are certainly not older than $200 \mathrm{BC}$.

Of course, no conclusive evidence exists to support a watertight case for or against a local origin of the jar burial tradition, but some comparative observations may be of interest. Although infant burial in jars occurs in some prehistoric Chinese and Thai sites, it is apparent that the Southeast Asian mainland prehistoric sequence (outside parts of Laos and southern Vietnam) is almost totally devoid of this tradition and normally stressed extended inhumation in post-Hoabinhian contexts. ${ }^{5}$ On the other hand, a coherent tradition of jar burial does occur in the late Jomon and Yayoi periods of southwestern Japan (1000 BC to $A D 300$ ), where it appears that bones were often placed in two jars laid horizontally mouth-to-mouth (Mori 1956; Chard 1974). Although this pattern is not to my knowledge found in the Indo-Malaysian Archipelago, there are records of vertical mouth-to-mouth jar burials on Batan Island between Luzon and Taiwan (Solheim 1960), at Plawangan on Java (Sukendar and Awe 1981), and at Gilimanuk on Bali (Soejono 1969:Plate 24). In addition, the Yayoi pottery style, which is different in many respects from that of the preceding Jomon periods, does include flasks, cutouts in ring feet, red-slipped surfaces, and incised scroll patterns that overlap to some extent with the repertoire of the early Metal 
phase in the Philippines. While I would not suggest Japan as a source for the Indo-Malaysian jar burials, I do feel that some degree of contact between the two archipelagic regions may have taken place from the late first millennium BC onward.

In India there is a widespread tradition of mouth-to-mouth urn burial in Chalcolithic sites spread across the country from Karnataka to West Bengal, but of more relevance is the tradition of burial in single upright urns found in association with the Black and Red pottery of the Iron Age cultures of southern India and Sri Lanka. Many specific features of this tradition-the practice of secondary burial with grave goods in the urns, the use of bone boxes and legged coffins, and the occasional occurrence of stone jar lids (as in some Philippine sites) - clearly do have definite Indo-Malaysian parallels. The historical and archaeological data already reviewed indicate that Indian contact with Southeast Asia was taking place from perhaps the middle of the first millennium $B C$ onward, so some exchange of ideas may have been occurring. However, it is also apparent that the basic artifact forms, especially in metal and pottery, differ so considerably between the two regions that an actual Indian source for the Indo-Malaysian jar burials would be unthinkable. I am therefore still strongly inclined to keep to my previous conclusion (Bellwood 1978:213) that the Austronesian jar burial tradition was an indigenous development. 


\section{A Final Overview}

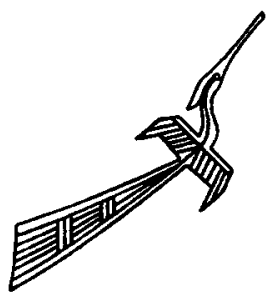

I wish now to reiterate a number of outstanding questions and to review my conclusions on those aspects of the prehistory of the Indo-Malaysian Archipelago that I regard as having central significance for its overall human story. Few of the major problems will ever be elucidated and explained to the satisfaction of all scholars; hence perhaps the attraction and vitality of the multifaceted discipline of prehistory. Many pieces of the total jigsaw will doubtless be added in the future by devoted analyses of stone tools, words, and skulls, but the whole will probably always remain a sum of more than its individual archaeological, linguistic, and biological parts.

If we commence at the remote and misty beginning, there are obviously many questions concerning Homo erectus that have scarcely even begun to be answered. Did these hominids travel with the stegodons to Sulawesi and the Lesser Sundas? Did they with certainty make stone tools? Did they belong to one single chronospecies? Did they become extinct or do some of their genes still survive in the modern populations of the region? What were the effects of the long periods of isolation of Java on the culture and biology of these populations? Were these early hominids restricted to monsoon forest and parkland environments, or were they able to colonize the equatorial rain forests as well?

There are also major biological questions that concern more recent populations. For instance, if anatomically modern humans entered the archipelago from outside, what was their source? Did they mix genes with local Homo erectus populations and, if so, what chronology was involved? As will by now be realized, most of these questions-while clear enough to comprehend when stated baldly-in fact elude simple answers and perhaps always will do so. The disciplines that investigate the deep human past are very limited in scope and de- 
pendent upon sparse and often unreliable sets of data. One could take the pessimistic approach and state that the more we learn, the less we know; how can we understand Homo erectus when we often do not understand the root causes of so many aspects of our own modern human behavior?

Such pessimism would not be entirely justified. We can see this by simply comparing the pattern of prehistory that we dimly grasp today with the virtual lack of any grasp whatsoever a century ago. Furthermore, Homo erectus might still be a dim figure in the fog, but the picture sharpens surprisingly as we enter radiocarbon-dateable time, and becomes ever sharper with leaps and bounds as we enter the past 5,000 years of agriculturalist dispersal, by which time the linguistic record can be brought to bear in full force.

For the period after 40,000 years ago there is much sharper evidence for human movement both out from and into the archipelago. Ancestral AustraloMelanesian populations were able to expand into western Melanesia and Australia, and many of the Wallacean islands were first settled at about this time. It also looks as if newcomers entered the archipelago from the Asian mainland on many occasions long before the period of Austronesian expansion. The evidence is sparse, but it includes a small amount of biological evidence (Chapter 3, Section IIID) and possibly certain archaeological assemblages such as the Tingkayu lanceolates and the later flake and blade industries. All these hints point to the east Asian mainland and Japan, rather than to the Indian subcontinent, as sources of such movements.

Environmentally related questions also arise. I have suggested that the equatorial and densely forested regions were always less important for human settlement than the more open intermediate tropical belts that have a long dry season. This contrast seems to hold for all periods-from early hunting and gathering through the Neolithic to the present day, when it is of course crystal clear. But were the interior equatorial rain forests really inhabited to any major extent only from the end of the last glaciation onward? The evidence from Malaysia, Sumatra, and Borneo lends support to such a view, but we still need to know what happened to these lowland Sundaland rain forests in the earlier glacial periods. Were they reduced in extent or broken up by "dry season corridors"? If so, was there periodic Pleistocene occupation of these drier zones? Furthermore, did the expansion of rain forest and the rise in sea level in the early Holocene cause the observed Sundaland cases of animal extinction, orless likely, in my view-were human hunters partly to blame? The fauna of Sundaland would have been habituated to a human presence for a million years or more and would accordingly have been prone to human avoidance. The naive faunas of Wallacea and Australia/New Guinea, on the other hand, would have been completely unhabituated when first confronted by hungry humans. Hence one can cite the apparent-if elusive-evidence in Australia for a fairly 
rapid human extermination of giant marsupials (Flannery 1994) and, in Wallacea, perhaps stegodons as well.

The early Holocene climatic amelioration leads on to further questions, since this was apparently more marked and rapid on a worldwide scale than any climatic and environmental change that had occurred in the previous 100,000 years. I regard this change as having been crucial for the radiation of annual cereals in certain key regions of the northern hemisphere, and of course for their ultimate domestication. One offshoot of this economic transition in the southern Chinese region was the phenomenon of Austronesian expansion after $4000 \mathrm{BC}$. As I have tried to make clear, I do not regard the Indo-Malaysian region itself as a zone of pristine agricultural origins, although it is obvious that many useful trees and tubers were brought under systematic cultivation there. The situation for New Guinea appears to be different because agriculture apparently evolved independently there in unique highland environments, thus sowing at least some of the seeds of Papuan demographic resistance to Austronesian expansion, seeds which kept New Guinea as a Papuan-speaking heartland through all of its prehistory.

There is also the question of the significance of the postglacial rise in sea level, which I-together with other scholars-once regarded as a stimulus for demographic crowding in Sundaland and for ultimate and local developments there toward agriculture (Bellwood 1978:422). I have long since changed my mind about this, although the idea has been revived by Thiel (1987). Basically, I doubt that the mangrove coasts of late glacial Sundaland ever supported large populations; and even if they did, the sea level rise would have increased rather than reduced the extent of coastline (Chapter 1, Section IVD). The drowning of Sundaland might have been a major environmental event on a geomorphological scale, but there is really no good evidence that human populations were particularly affected by it on a short-term basis. One result of the sea level rise might have been the increased visibility of human occupation in the interior of the Malay Peninsula after 12,000 years ago, but this is more likely to be a result of decreased distance from the sea than population crowding.

It will by now be clear that I regard the main period of Austronesian expansion between 3000 and $1000 \mathrm{BC}$ as the foundation for major biological, linguistic, and cultural changes in the prehistory of the archipelago. I have reviewed the agricultural background to this expansion, and also the economic changes that early Austronesian groups underwent as they expanded southward toward and across the equator. I choose the term expansion with some care, as I do not think there is good evidence for long-distance migration of the Polynesian type until the Oceanic islands were being settled after $1500 \mathrm{BC}$. The expansion surely approximated the "wave of advance" model postulated by Ammerman and Cavalli-Sforza (1984) for Europe, but with a coastal emphasis and with the pro- 
viso that early Austronesian populations might have followed a coastal "hopping" strategy rather than settling all of island A in nice neat order before moving on to settle island B.

The Austronesian dispersal was also quite a slow affair in overall terms; after all, it required almost 4,000 years for Austronesians to reach New Zealand and Madagascar if $3000 \mathrm{BC}$ is to be taken as a reasonable date for the first movements southward from Taiwan. But within this overall time span there seems to have been a phase of very rapid and continuous colonizing activity between 2000 and $1000 \mathrm{BC}$, when we find the first archaeological traces of Neolithic colonists all the way from the Philippines through eastern Indonesia and into Melanesia, western Polynesia, and western Micronesia. This is an impressive colonization, perhaps the most rapid, successful, and widespread in the history of humanity prior to the recent dispersals from Europe. Why did it occur over this geographical segment so quickly? I no longer think agriculture alone drove it, although agriculture might have been the ultimate driver whose foot coaxed the Austronesian "ark" into first gear. But by the time Austronesians moved beyond the Philippines they were undoubtedly getting into high gear, colonizing purposefully and extremely skillfully, searching in the case of the remote Pacific Islands for colonizing opportunities in order to establish new founderfocused lineages of high status (Bellwood 1996c).

I have also mentioned viewpoints that are in opposition to mine and that postulate foundation developments of the Southern Mongoloid phenotype and the Austronesian languages in the Indo-Malaysian Archipelago itself, or even (in the case of the languages) in western Melanesia. I look forward to future debate on these matters and have expressed my own views forcefully on several occasions. Such hypotheses convince me less as time goes by and as stronger evidence accumulates in support of a major phenomenon of Austronesian population dispersal out of the southern China/Taiwan regions.

My opinion on the overall "shape" of Indo-Malaysian prehistory should by now be apparent (Fig. 10.1). Basically, I see few indications of major cultural change in the region prior to the period of Austronesian expansion, apart from those kinds of regional variation in stone toolmaking techniques that are perhaps no more than one would expect given the time span involved and the inherent capacity of modern humans to communicate and occasionally to innovate. With the exception of periodic trickles of new peoples and occasional items of cultural baggage, I doubt whether the archipelago witnessed any major replacements of population or dramatic spurts of local development from the period of first appearance of anatomically modern humans into the early Holocene. Early voyaging across the narrow waterways of Wallacea and western Melanesia does not seem to have led to any particularly marked spurt in the rate of cultural development. 


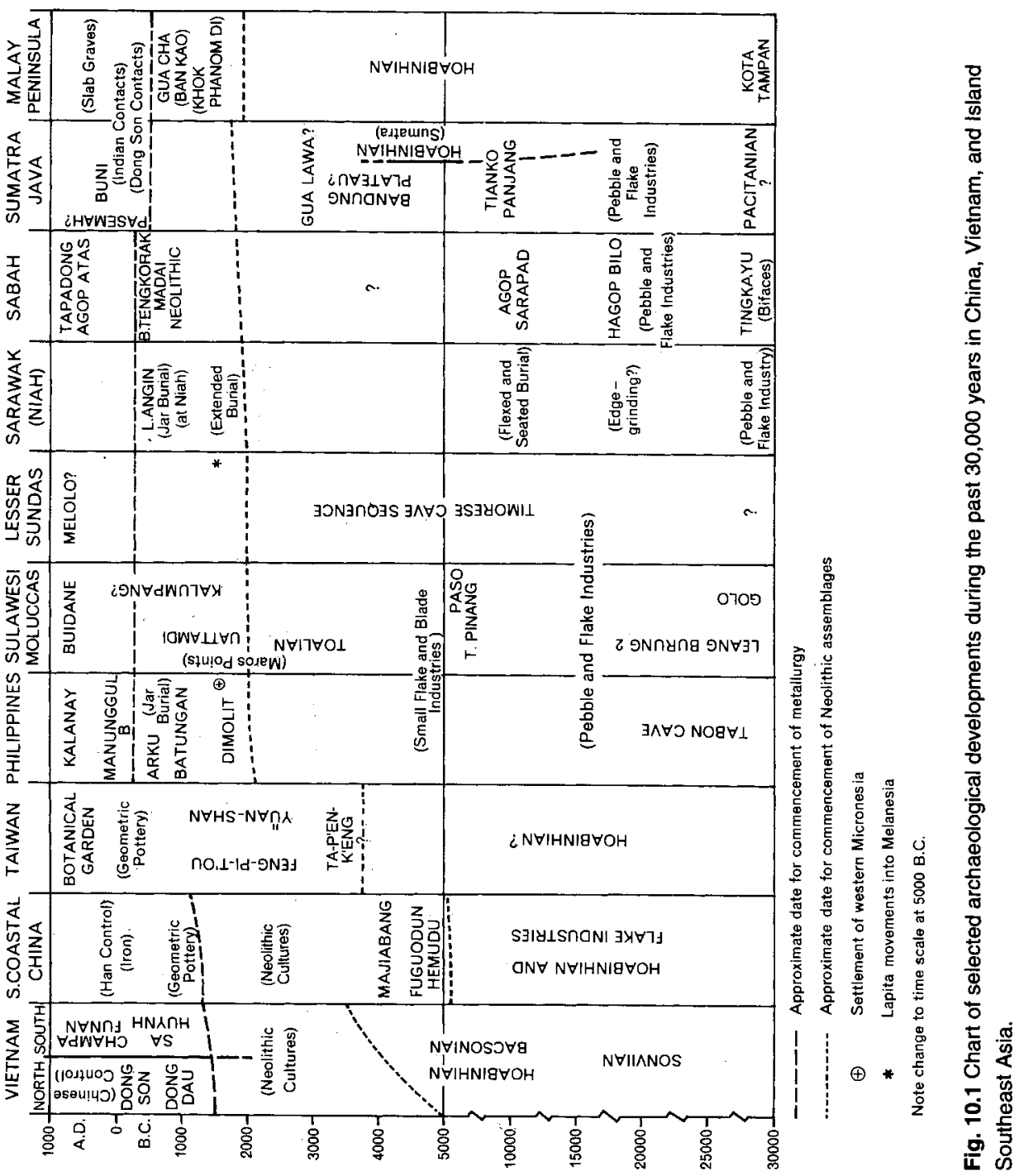


However, the long preagricultural millennia of relative stability came to a dramatic close in the period of Austronesian expansion. As agriculturalists by virtue of prior cultural developments outside the Indo-Malaysian region, the Austronesians had a culturally inbuilt and demographically founded drive for expansion that eventually took them to Easter Island and New Zealand. Agriculture on the whole allows for much higher population densities than hunting and gathering, and it is clearly an economic system that can utilize and even encourage the breeding of an increasing labor force in the form of children and adolescents. Although populations of agriculturalists that inhabit constrained geographical ranges have been forced either to control population growth or to intensify production in the past, it is clear that the early Austronesians, like the nineteenth-century Iban of Sarawak, did not face such constraints. Confronted by fertile environments with good agricultural potentials occupied only by hunters and gatherers-except in certain locations such as western Melanesia and parts of mainland Asia-they "chose" expansion. Many of their descendants are still expanding their geographical ranges at the ultimate expense of forest hunters and gatherers in some regions to this day.

There are also two observations of Oceanic significance that arise from my review of earlier Austronesian prehistory in the Indo-Malaysian Archipelago. The first is that the Austronesian societies of eastern Indonesia and Melanesia were heavily affected (more so than those in the west and north of Indonesia) by two-way contact with preexisting non-Austronesian societies. It is not yet clear whether agriculture had spread from the independent New Guinea source region into eastern Indonesia prior to Austronesian settlement. If it had, it would go far toward explaining the biological and cultural variation observed in this region. A second observation must be that the spread of the ancestral Polynesian Lapita culture through Melanesia after about 1600 BC correlates in linguistic terms with the period of Proto-Oceanic. Hence the problems that arose through acceptance of an erroneous date of $3000 \mathrm{BC}$ for Proto-Oceanic (Bellwood 1978:423) - thereby having the ancestral Polynesians somehow "lost" in a millennium and a half of archaeological void in Melanesia-can now be forgotten. The original problem lay in an unwillingness to recognize the rapidity of change in the Austronesian languages of this region.

As far as the later phases of Austronesian prehistory are concerned, I have little to add to my opinions as presented in Chapter 9. Clearly, it is important to find out more about the development and spread of metallurgy in the Archipelago. There is still little coherent information about the archaeology and the societies of western Indonesia just prior to the period of intensive Indian influence in the first millennium AD. Questions concerning the evolution of complex societies provide just as much stimulus and excitement for prehistorians as do questions concerning the origins of agriculture, and I believe that the former 
represent perhaps the largest untapped field of research facing Indonesian-and especially Javanese-prehistorians at the present time.

Finally, what of the future for prehistoric research in the Indo-Malaysian Archipelago? First, it is becoming obvious that high-quality archaeological excavations with full publication of data are absolutely essential. It is also essential for archaeologists from the various countries of the region to have access to the results of each other's research. Language and political barriers sometimes intervene in hindering such access, but international associations such as SPAFA ${ }^{1}$ and the Indo-Pacific Prehistory Association ${ }^{2}$ help to overcome such problems. Secondly, new data must be assessed against well-formulated models of IndoMalaysian prehistory that are based on the whole Southeast Asian and Oceanic scene, and that draw (when applicable) on information from archaeology, historical sources, linguistics, biological anthropology, cultural anthropology, and the natural sciences. I hope that I have presented such a model in this book, and that it will encourage, guide, and also be modified by future research. 


\section{Notes}

\section{ONE The Environmental Background: Present and Past}

1. In this book, the term Borneo is used for the whole island, and Kalimantan for the Indonesian portion. The Malaysian portions are called Sarawak and Sabah. Brunei is a separate nation.

2. Climatic changes in the New Guinea Highlands might have been rather more severe than those in the islands of Indonesia. For instance, Stuijts (1993) suggests a glacial maximum treeline drop of only 500 meters for west Java. Van der Kaars (1991) suggests a drop of only 350 meters for Halmahera, and Maloney (1985) suggests 350 meters for the Lake Toba region of Sumatra. On the other hand, Rind and Peteet (1985) review several tropical data sets suggesting treeline falls of 900 to 1,200 meters in various parts of the world. Clearly, local circumstances can vary greatly.

3. Flannery (1994) has recently presented a case, albeit rather hypothetical, for much more catastrophic patterns of extinction by early human populations of the "naïve" (i.e., unaccustomed to humans) animal faunas of Late Pleistocene Wallacea and Australasia.

\section{Two Homo erectus in Sundaland}

1. Note added in proof: For a new claim that the Ngandong skulls may be only between 25,000 and 55,000 years old, based on uranium series and electron spin resonance of bovid teeth, see Swisher et al. 1996.

\section{THREE Indo-Malaysians of the Last 40,000 Years}

1. It is apparent from current research that this deletion contains several lineages in terms of nucleotide substitutions and may even have arisen independently in more than one population (Redd et al. 1995; Melton et al. 1995).

\section{F OU Recent Indo-Malaystan Prehistory: According to the Languages}

1. Variations in opinions about how many Austronesian languages actually exist reflect issues over differentiating dialects from separate languages (see Wurm and Laycock 1961, who document this problem for New Guinea). Dyen (1965a) classified speech systems as dialects of one language if they share 70 percent or over of basic vocabulary and gave a figure of under 500 separate languages for Austronesian. Most other linguists use a figure of 80 percent for the division. Tryon (1995a) gives the figure of 1,200 .

2. Family tree diagrams tend to suggest that populations split asunder forever at points of subgroup separation. Perhaps they did in some remote Oceanic situations, but on large or adjacent landmasses, languages clearly did not differentiate by such a 
simple process. Varying rates of intercommunication mean that some subgroups are "clear-cut" and others are not, for technical reasons relating to the nonoverlapping distributions of innovations. This is a matter clearly discussed, for those interested, by Pawley and Ross (1995), who differentiate between innovation-defined and innovation-linked subgroups.

3. Proto-Austronesians could have used pots and sailing canoes too, but cognates have not survived. In this regard, it is important to note that proto-language vocabularies can record presences, but cannot guarantee absences. Proto-Iroquoian has no word for knee (Mithun 1984). Iroquoians without knees are hard to imagine.

4. Old Malay (from the seventh century) and Old Javanese (from the ninth century) do occur on inscriptions in Sumatra and Java (earlier inscriptions are all in Sanskrit), but these are too limited to assist in more than purely local reconstructions. The Cham language of Vietnam also occurs in inscriptions back to the fourth century (Marrisson 1975).

\section{FIV The Patterns of History and Ethnography}

1. It should be noted that Semang band exogamy differs fundamentally from the situation of band endogamy for the Punan forest nomads of Borneo (Sellato 1994). Band exogamy is not an automatic feature of a hunter-gatherer lifestyle.

2. The National Museum in Jakarta contains several Han pottery vessels from locations in southern Sumatra. In addition, many Chinese bronze items, some perhaps dating from the later part of the Zhou Dynasty (prior to $221 \mathrm{BC}$ ), are in private collection in London-reputedly from looted graves in the vicinity of Lumajang in eastern Java. The question of when Chinese contact with Indonesia first began is still open, but it needs to be firmly stated that trade contacts were perhaps very rare before the Tang Dynasty (AD 618-906).

\section{sIX The Hoabinhians and Their Island Contemporaries}

1. Recent research on the Toba eruption sequence suggests that the last major one occurred 74,000 rather than 30,000 years ago (Chesner et al. 1991; Rampino and Self 1992). Toba ash from Selangor has also been dated to 68,000 years ago by fission track dating. At present it is not clear how these new determinations affect the suggested age of 34,000 to 30,000 years ago for Kota Tampan, but the possibility of course arises that the site could be much older than hitherto claimed by Zuraina.

2. Although not specifically relevant for the period covered by this book, the top layer of Gua Chawas yielded abundant evidence for the firing of Buddhist votive tablets impressed with Bodhisattva and Avalokitesvara images, which were placed in large numbers in minor caves and crevices in the vicinity. The art has Srivijayan affinities and appears to be about 800 to 1,000 years old. This is the first time such evidence has been found so far inland in the Malay Peninsula; similar tablets are known from coastal locations in southern Thailand.

3. This suggestion is not as silly as it sounds. Pebbles embedded in road surfaces can be flaked into excellent tools by truck wheels, and quarry rock-crushing machines also make excellent blades and blade cores! 


\section{SEVEN The Archaeological Record of Early Austronesian Communities}

1. As in Southwest Asia, the oldest Neolithic sites in the Yellow River basin have reaping knives or sickles of stone, shell, and even pottery (Chang 1986:93). However, in the earliest Neolithic societies in southern China-Pengtoushan in Hunan and Hemudu layer 4 in Zhejiang, dating from 7000 to $5000 \mathrm{BC}$-such knives are absent, as they are in most prehistoric sites in Southeast Asia (including the TPK in Taiwan). Perhaps bamboo knives were used, as rice was certainly present in quantity at both Pengtoushan and Hemudu. Like any cereal, rice can be shaken into a basket or hand stripped, but if the people of Pengtoushan and Hemudu did this there would presumably have been no selection for nonshattering domesticated varieties (cf. Wilke et al. 1972; Hillman and Davies 1990). Today, reaping knives (termed "finger" knives) of metal are used widely in Southeast Asia, including Indonesia (Miles 1979). These allow individual harvesting of panicles without prior weeding and also selection of ripe panicles as they mature (Plate $38 \mathrm{~b}$ ). However, there is no archaeological record for these tools in Southeast Asia.

2. Wade-Giles place-name spellings have been retained for Taiwan in this section, but Pinyin is used for the People's Republic of China.

3. Compare the similarly very high numbers of infants and fetuses in early Neolithic graves at Khok Phanom Di in central Thailand, here due to malaria (Higham and Thosarat 1994; and see page 258 of this volume).

4. The site of Sembiran in Bali, only 2,000 years old, is buried under 3.5 meters of alluvium, and this is on the narrow north coastal plain of an island much smallerparticularly in terms of extent of coastal plains - than either Java or Sumatra. According to H. D. Tjia (1980), deltaic regions of northern Java grow seawards at an average rate of 200 meters per year. Compare also the burial of the Sumatran middens (see Plate 20).

5. A red-slipped and incised vessel with decoration precisely paralleled in some Lapita sites (see Plate 35c) was found by Alfred Buhler in his 1935 excavations in the disturbed cave of Nikiniki I in western Timor (Glover 1972b). Tanged blades found in the same site have been dated between 2,300 and 1,200 years ago by Glover (1972a:226), according to the results of his own excavations in other sites (Fig. $7.14 \mathrm{e}-\mathrm{g}$ ), but the vessel itself is not precisely datable.

6. It should be pointed out that Vietnamese, an Austroasiatic language, has since replaced the Chamic languages through much of their former distribution. Language replacements can go back and forth!

\section{E10нт The Archaeological Record of Early Agricultural Communities in Peninsular Malaysia}

1. It is also found, presumably not coincidentally, in the Neolithic pottery from Sarawak, Kalumpang (Sulawesi), and the Philippines; see Chapter 7, Section IIID. 


\section{NIN E The Early Metal Phase: A Protohistoric Transition toward Supra- Tribal Societies}

1. Copper and bronze in archeological contexts cannot be differentiated by visual inspection alone, and my general use of the term "bronze" may be imprecise in some circumstances, especially in Indonesia. Many so-called bronze objects may simply be of copper, but without analysis it is pointless to speculate.

2. Questions concerning the origins of the drum tradition are not of concern for the Indo-Malaysian Archipelago, but most scholars favor either a Vietnamese (e.g., Pham Huy Thong 1990; Bernet Kempers 1988) or a Yunnan (e.g., Nitta 1985; Sørensen 1988) origin. Others are not so certain (Imamura 1993). For details of the magnificent bronzes, including Heger I drums, from Yunnan, see Rawson 1983.

3. Animal-headed pendants also occur as far west as central Thailand (Glover 1990b: 166) and the lingling-o ear pendants are typical of the Peinan site in Taiwan (see Chapter 7, Section IIA) and other sites in southern China. Such items of personal adornment were probably traded widely.

4. See Pliny, Natural History, Book XII, paragraph 30;

There is also in India a grain resembling that of the pepper, but larger and more brittle, called the caryophyllon.... It is imported here for the sake of its scent (Rackham 1952:22-23).

5. Note added in proof: Pottery jar burials have recently been excavated in Iron Age (late first millennium BC-early first millennium AD) contexts in northeastern Thailand (Nitta 1996; Indrawooth 1997).

\section{TEN A Final Overview}

1. SPAFA was originally the acronym for Special Program in Archaeology and the Fine Arts, an organization now termed the SEAMEO Regional Center for Archaeology and Fine Arts. SPAFA is organized through the Southeast Asian Ministers of Education Organization (SEAMEO) for archaeologists from ASEAN countries (currently Thailand, Laos, Vietnam, Malaysia, Singapore, Indonesia, Brunei). Its main office is in Bangkok. It publishes conference proceedings and the SPAFA Journal.

2. The Indo-Pacific Prehistory Association is run from the Department of Archaeology and Anthropology, Australian National University, Canberra ACT 0200, Australia. It holds conferences every four years (most recent ones: Philippines 1985, Japan 1987, Indonesia 1990, Thailand 1994, Malaysia 1998) and publishes the IPPA Bulletin. 


\section{References}

\section{ABBREVIATIONS}

$\begin{array}{ll}\text { A } & \text { Antiquity } \\ \text { AJPA } & \text { American Journal of Physical Anthropology } \\ \text { AO } & \text { Archaeology in Oceania } \\ \text { AP } & \text { Asian Perspectives } \\ \text { APAO } & \text { Archaeology and Physical Anthropology in Oceania } \\ \text { APAS } & \text { Asian and Pacific Archaeology Series } \\ \text { BIPPA } & \text { Bulletin of the Indo-Pacific Prehistory Association } \\ \text { BPA } & \text { Berita Penelitian Arkeologi (Jakarta: Pusat Penelitian Arkeologi Nasional) } \\ \text { BRB } & \text { Borneo Research Bulletin } \\ \text { BRM } & \text { Bulletin of the Raffles Museum, Singapore } \\ \text { BTLV } & \text { Bijdragen tot de Taal-, Land- en Volkenkunde } \\ \text { CA } & \text { Current Anthropology } \\ \text { EATQN } & \text { East Asian Tertiary and Quaternary Newsletter } \\ \text { FMJ } & \text { Federation Museums Journal } \\ \text { JAM } & \text { IUrnal Arkeologi Malaysia } \\ \text { JFMSM } & \text { Journal of the Federated Malay States Museums } \\ \text { JHE } & \text { Journal of Human Evolution } \\ \text { JHKAS } & \text { Journal of the Hong Kong Archaeological Society } \\ \text { JMBRAS } & \text { Journal of the Malaysian Branch of the Royal Asiatic Society } \\ \text { ISEAS } & \text { Journal of Southeast Asian Studies } \\ \text { MQRSEA } & \text { Modern Quaternary Research in Southeast Asia } \\ \text { OL } & \text { Oceanic Linguistics } \\ \text { PQCS } & \text { Philippine Quarterly of Culture and Society } \\ \text { PL } & \text { Pacific Linguistics Series, Department of Linguistics, Research School of } \\ & \quad \text { Pacific and Asian Studies, Australian National University } \\ \text { SMI } & \text { Sarawak Museum Journal } \\ \text { TITLV } & \text { Tijdschrift voor Indische Taal-, Land- en Volkenkunde } \\ \text { TKNAG } & \text { Tijdschrift van het Koninklijk Nederlands Aardrijkskundig Genootschap } \\ \text { WA } & \text { World Archaeology } \\ & \\ & \end{array}$

Adelaar, K. A. 1989. Malay influence on Malagasy: Linguistic and culture-historical inferences. $O L 28(1): 1-46$. 1992. Proto-Malayic. PL Series C-119.

1995. Borneo as a cross-roads for comparative Austronesian linguistics. In P. Bellwood, J. J. Fox, and D. Tryon (eds.), The Austronesians, 75-95. 
Adhyatman, S. 1984. Kunjungan ke Bukit Siguntang, Palembang, Sumatera Selatan. Amerta 8:25-33.

Adi Haji Taha. 1983. Recent archaeological discoveries in Peninsular Malaysia 19761982. JMBRAS 56(1):47-63.

1985. The re-excavation of the rockshelter of Gua Cha, Ulu Kelantan, West Malaysia. FMJ 30.

1993. Recent archaeological discoveries in Peninsular Malaysia (1991-1993). JMBRAS 66(1):67-83.

Adi Haji Taha, and Zulkifli Jaafar. 1990. A preliminary report on archaeological research and excavation at Gua Kelawar, Sungei Siput, Perak. JAM 3:111-124.

Aigner, J. 1978a. Pleistocene faunal and cultural stations in South China. In F. IkawaSmith (ed.), Early Palaeolithic in South and East Asia, 129-160.

. 1978b. Important archaeological remains from North China. In F. IkawaSmith (ed.), Early Palaeolithic in South and East Asia, 163-232.

1981. Archaeological Remains in Pleistocene China. Munich: Verlag C. H. Beck.

Akazawa, T., K. Aoki, and T. Kimura (eds.) 1992. The Evolution and Dispersal of Modern Humans in Asia. Tokyo: Hokusen-sha.

Alexander, J. 1993. The Lahanan longhouse. In J. J. Fox (ed.) 1993:30-43.

Alkire, W. 1972. Concepts of order in Southeast Asia and Micronesia. Comparative Studies in Society and History 14(4):484-493.

Allen, H. 1991a. Stegodonts and the dating of stone tool assemblages in Island Southeast Asia. AP 30:177-192.

. 1991b. A review of the late Pleistocene/early Recent stone tool assemblages of Java. BIPPA 11:36-47.

Allen, J., J. Golson, and R. Jones, eds. 1977. Sunda and Sahul. London: Academic.

Allen, J., and C. Gosden, eds. 1992. Report of the Lapita Homeland Project. Canberra: Department of Prehistory, Research School of Pacific Studies, Occasional Papers in Prehistory 20.

Allen, J., and S. Holdaway. 1995. The contamination of Pleistocene radiocarbon determinations in Australia. A 69:101-112.

Ambary, H. M. 1981. Notes on research on sites from the Srivijaya period. In S. Suleiman (ed.), Studies on Srivijaya, 1-12. Jakarta: Pusat Penelitian Arkeologi Nasional.

Ambrose, W. 1988. An early bronze artefact from Papua New Guinea. A 62:483-491.

Amesbury, J., D. R. Moore, and R. L. Hunter-Anderson. 1996. Sea level change in the Marianas: Recent excavations at Chalan Piao, Saipan, Micronesia. BIPPA 15: 53-69.

Ammerman, A., and L. L. Cavalli-Sforza. 1984. The Neolithic Transition and the Genetics of Population in Europe. Princeton: Princeton University Press.

Andaya, B., and L. Andaya. 1982. A History of Malaysia. London: Macmillan.

Andel, T. H. van, G. R. Heath, T. C. Moore, and D. F. R. McGeary. 1967. Late Quaternary history, climate and oceanography of the Timor Sea. American Joumal of Science 265:737-758.

Anderson, A. 1989. Prodigious Birds. Cambridge: Cambridge University Press. 
Anderson, D. 1987. A Pleistocene-early Holocene rockshelter in peninsular Thailand. National Geographic Research 3:184-198.

1990. Lang Rongrien Rockshelter. Philadelphia: University of Pennsylvania Museum.

Andrews, P. 1984. On the characters that define Homo erectus. In P. Andrews and J. L. Franzen (eds.), The Early Evolution of Man, 167-178. Frankfurt: Courier Forschungsinstitut Senckenberg No. 69.

Andrews, R., and I. Glover, 1986. Ulu Leang 2: An Iron Age jar burial cave in South Sulawesi, Indonesia. Indonesia Circle 40:46-94.

Anggraeni, N. 1976. Peninggalan-peninggalan prasejarah di sekitar danau Cangkuang (Leles). Kalpataru 2:55-70.

1986. Alat-alat batu dari Pangkep, Sulawesi Selatan. In Pertemuan Ilmiah Arkeologi IV, Vol. I, 246-272. Jakarta: Pusat Penelitian Arkeologi Nasional.

Aoyagi, Y., et al. 1991. Excavations at Lal-lo shell middens (3). Journal of Sophia Asian Studies 9:49-137.

Appell, G. N. 1978. The Rungus Dusun. In V. T. King (ed.), Essays on Borneo Societies, 143-171.

Ardika, I. W. 1987. Bronze artefacts and the rise of complex society in Bali. Unpublished M.A. Thesis, Australian National University.

1991. Archaeological research in northeastern Bali, Indonesia. Unpublished Ph.D. Thesis, Australian National University.

Ardika, I. W., and P. Bellwood. 1991. Sembiran: The beginnings of Indian contact with Bali. A 65:221-232.

Arnaud, V. 1974. La culture du millet chez les Yami. Journal d'Agriculture Tropicale et de Botanique Appliquée 21:275-311.

Arnold, G. 1958. Nomadic Penan of the Upper Rejang (Plieran), Sarawak. JMBRAS 31(1):40-82.

Ashton, P. S. 1972. The Quaternary geomorphological history of western Malesia and lowland forest phytogeography. In Ashton, P. and M. (eds.), The Quaternary Era in Malesia, 35-49.

Ashton, P. and M., eds. 1972. The Quaternary Era in Malesia. University of Hull, Department of Geography, Miscellaneous Series 13.

Association of Japanese Agricultural Scientific Societies ed. 1975. Rice in Asia. Tokyo: University of Tokyo Press.

Atlas. 1938. Atlas van Tropisch Nederland. The Hague: Nijhoff.

Atlas. 1980. Atlas of Primitive Man in China. New York: Van Nostrand Reinhold.

Audley-Charles, M. G. 1981. Geological history of the region of Wallace's Line. In T. C. Whitmore (ed.), Wallace's Line and Plate Tectonics, 24-35.

1987. Dispersal of Gondwanaland: Relevance to evolution of the angiosperms. In T. C. Whitmore (ed.), Biogeographical Evolution of the Malay Archipelago, 5-15.

Audley-Charles, M. G., and D. Hooijer. 1973. Relation of Pleistocene migrations of pygmy stegodonts to island arc tectonics in eastern Indonesia. Nature 241: 197-198. 
Azis, B. S. 1981. Tradisi kapak perimbas di Indonesia. Amerta 4:11-21.

Baba, H., and S. Narasaki. 1991. Minatogawa Man, the oldest type of modern Homo sapiens in East Asia. The Quaternary Research (Japan) 30:221-230.

Bailey, R. C., et al. 1989. Hunting and gathering in tropical rain forest: Is it possible? American Anthropologist 91:59-82.

Baker, P. T. 1992. Human adaptations to the physical environment. In Cambridge Encyclopaedia of Human Evolution, 46-51. Cambridge University Press.

Ballinger, S. W., et al. 1992. Southeast Asian mitochondrial DNA analysis reveals genetic continuity of ancient Mongoloid migrations. Genetics 130:139-152.

Bandi, H. G. 1951. Die obsidianindustrie der umgebung von Bandung in Westjava. In Südseestudien, 127-161. Basel: Museum für Volkerkunde.

Barbujani, G., and R. Sokal. 1990. Zones of sharp genetic change in Europe are also linguistic boundaries. Proc. National Academy of Sciences (USA) 87:18161819.

Barnard, N., ed. 1972. Early Chinese Art and Its Possible Influence in the Pacific Basin (3 vols.). New York: Intercultural Arts Press.

Barrau, J. 1965. Histoire et préhistoire horticoles de l'Oceanie tropicale. Journal de la Société des Océanistes 21:55-78.

Barth, F. 1952. The southern Mongoloid migration. Man 52:5-8.

Bartlett, H. H. 1934. The Sacred Edifices of the Batak of Sumatra. University of Michigan Museum of Anthropology, Occasional Contribution 4.

Bartstra, G.-J. 1974. Notes about Sangiran. Quartär 25:1-12.

- 1976. Contributions to the Study of the Palaeolithic Patjitan Culture of Java, Indonesia. Leiden: Brill.

-1977. Walanae formation and Walanae terraces in the study of South Sulawesi. Quartär 27-8:21-30.

1978a. The Patjitan culture: A preliminary report on new research. In

F. Ikawa-Smith (ed.), Early Palaeolithic in South and East Asia, 29-36.

1978b. Recent Palaeolithic research in Java. MQRSEA 4:63-70.

. 1978c. Note on new data concerning the fossil vertebrates and stone tools in the Walanae valley in South Sulawesi. MQRSEA 4:71-72.

1982. The river-laid strata near Trinil. MQRSEA 7:97-130.

- 1983. The vertebrate-bearing deposits of Kedungbrubus and Trinil, Java, Indonesia. Geologie en Mijnbouw 62:329-338.

Bartstra, G.-J., and Basoeki. 1989. Recent work on the Pleistocene and the Palaeolithic of Java. CA 30:241-243.

Bartstra, G.-J., Basoeki, and B. Santoso. 1976. Solo valley research 1975. MQRSEA 2: 23-36.

Bartstra, G.-J., and D. A. Hooijer. 1992. New finds of fossil vertebrates from Sulawesi, Indonesia. Lutra 35:113-122.

Bartstra, G.-J., D. A. Hooijer, B. Kallupa, and M. Anwar Akib. 1991-1992. Notes on fossil vertebrates and stone tools from Sulawesi, Indonesia. Palaeohistoria 33/ 34:1-18. 
Bartstra, G.-J., S. G. Keates, Basoeki, and Bahru Kallupa. 1991. On the dispersion of Homo sapiens in eastern Indonesia: The Palaeolithic of South Sulawesi. CA 32: 317-320.

Bartstra, G.-J., S. Soegondho, and A. van der Wijk. 1988. Ngandong man: Age and artefacts. JHE 17:325-337.

Batchelor, B. C. 1977. Post Hoabinhian coastal settlement indicated by finds in stanniferous Langat River alluvium near Dengkil, Selangor, Peninsular Malaysia. FMJ 22.

1979. Discontinuously rising late Cenozoic sea levels, with special reference to Sundaland, Southeast Asia. Geologie en Mijnbouw 58:1-20.

Bay-Petersen, J. 1982-1983. Excavations at Bagumbayan, Masbate, central Philippines: An economic analysis. AP 25/2:1-22.

Beatty, A. 1990. Society and Exchange in Nias. Oxford: Clarendon Press.

Beck, H. C. 1941. The Beads from Taxila. Archaeological Survey of India, Memoir 65.

Begley, V. 1983. Arikamedu reconsidered. American Journal of Archaeology 87:461481.

1986. From Iron Age to early Historical in the archaeology of South India. In J. Jacobsen (ed.), Essays in the Archaeology of India and Pakistan, 297-319. New Delhi: American Institute of Indian Studies.

Bellwood, P. 1976a. Archaeological research in Minahasa and the Talaud Islands, north-eastern Indonesia. AP 19:240-288.

. 1976b. The significance of excavated bronze objects and casting moulds from the Talaud islands. In N. Barnard (ed.), Ancient Chinese Bronzes and Southeast Asian Metal and other Archaeological Artefacts, 413-420. Melbourne: National Gallery of Victoria.

1978. Man's Conquest of the Pacific. Auckland: Collins.

1980. Plants, climate and people: The early horticultural prehistory of Indonesia. In J. J. Fox (ed.), Indonesia: The Making of a Culture, 57-74.

. 1981. The Buidane culture of the Talaud islands. BIPPA 2:69-127.

1984. Archaeological research in the Madai-Baturong region, Sabah. BIPPA 5:

38-54.

1984-1985. A hypothesis for Austronesian origins. AP 26:107-117.

. 1985. Holocene flake and blade industries of Wallacea and their predecessors. In V. N. Misra and P. Bellwood (eds.), Recent Advances in Indo-Pacific Prehistory, 197-206.

. 1988. Archaeological Research in South-Eastern Sabah. Kota Kinabalu: Sabah Museum Monograph 2.

1989. Archaeological investigations at Bukit Tengkorak and Segarong, southeastern Sabah. BIPPA 9:122-162.

. 1990a. From late Pleistocene to early Holocene in Sundaland. In C. Gamble and O. Soffer (eds.), The World at 18,000 $\mathrm{BP}$, Vol. 2, 255-263. London: Unwin Hyman.

- 1990b. Foraging towards farming. Review of Archaeology 11/2:14-24. 
1991. The Austronesian dispersal and the origin of languages. Scientific American 265(1):88-93.

1992. Southeast Asia before history. In N. Tarling (ed.), The Cambridge History of Southeast Asia, Vol I, 55-136. Cambridge: Cambridge University Press.

1993. Cultural and biological differentiation in Peninsular Malaysia: The last 10,000 years. $A P$ 32:37-60.

- 1994. An archaeologist's view of language macrofamily relationships. OL 33: 391-406.

- 1995a. Austronesian prehistory in Southeast Asia: Homeland, expansion and transformation. In P. Bellwood, J. J. Fox, and D. Tryon (eds.), The Austronesians, 96-111.

1995b. Early agriculture, language history and the archaeological record in China and Southeast Asia. In Conference Papers on Archaeology in Southeast Asia, 11-22. University of Hong Kong Museum and Art Gallery.

1995c. Archaeological research in the Northern Moluccas: An interim report. SE Asian Archaeology Newsletter 7:3-12.

- 1996a. Early agriculture and the dispersal of the Southern Mongoloids. In T. Akazawa and E. Szathmary (eds.), Prehistoric Mongoloid Dispersals, 287-302. Tokyo: Oxford University Press.

- 1996b. The origins and spread of agriculture in the Indo-Pacific region. In D. Harris (ed.), The Origins and Spread of Agriculture and Pastoralism in Eurasia, 465-498. London: University College Press.

1996c. Hierarchy, founder ideology and Austronesian expansion. In J. J. Fox and C. Sather (eds), Origins, Ancestry and Alliance: Explorations in Austronesian Ethnography, 18-40. Canberra: Department of Anthropology, Research School of Pacific and Asian Studies, Australian National University.

1996d. Phylogeny and reticulation in prehistory. $A$ 70: 881-890.

- (in press). The archaeology of Papuan and Austronesian prehistory in the Northern Moluccas, Eastern Indonesia.

Bellwood, P., J. J. Fox, and D. Tryon, eds. 1995. The Austronesians: Comparative and Historical Perspectives. Canberra: Department of Anthropology, Research School of Pacific and Asian Studies, Australian National University.

Bellwood, P., R. Gillespie, G. B. Thompson, J. S. Vogel, I. W. Ardika, and I. Datan. 1992. New dates for prehistoric Asian rice. $A P$ 31:161-170.

Bellwood, P., and P. Koon. 1989. Lapita colonists leave boats unburned. A 63:613-622.

Bellwood, P., and M. Omar. 1980. Trade patterns and political developments in Brunei and adjacent areas, AD 700-1500. Brunei Museum Journal 4/4:155-179.

Bellwood, P., A. Waluyo, Gunadi, G. Nitihaminoto, and G. Irwin. 1993. Archaeological research in the northern Moluccas. BIPPA 13:20-33.

Bemmelen, R. W. van. 1949. The Geology of Indonesia (2 vols). The Hague: Government Printing Office.

Benedict, P. K. 1942. Thai, Kadai and Indonesian: A new alignment in Southeastern Asia. American Anthropologist 44:576-601.

1975. Austro-Thai Language and Culture. New Haven: HRAF Press. 
Benjamin, G. 1976. Austroasiatic subgroupings and prehistory in the Malay Peninsula. In P. N. Jenner, L. C. Thompson, and S. Starosta (eds.), Austroasiatic Studies, 37-128. Honolulu: University of Hawai'i Press.

. 1986. In the long term: Three themes in Malayan cultural ecology. In K. L. Hutterer, A. T. Rambo, and G. Lovelace (eds.), Cultural Values and Human Ecology in Southeast Asia, 219-278. Ann Arbor: Center for South and Southeast Asian Studies, University of Michigan.

- 1987. Ethnohistorical perspectives on Kelantan's prehistory. In Nik Hassan Shuhaimi (ed.), Kelantan Zaman Awal, 108-153. Kota Bharu: Muzium Negara Kelantan.

Bergh, G. van den, B. Mubroto, Fachroel Aziz, P. Sondaar, and J. de Vos. 1996a. Did Homo erectus reach the island of Flores? BIPPA 14:27-36.

Bergh, G. van den, J. de Vos, P. Sondaar, and Fachroel Aziz. 1996b. Pleistocene zoogeographic evolution of Java (Indonesia) and glacio-eustatic sea level fluctuations: A background for the presence of Homo. BIPPA 14:7-21.

Bergman, P. A. M., and P. Karsten. 1952. Fluorine content of Pithecanthropus and of other species from the Trinil fauna. Koninklijke Nederlandse Akademie van Wetenschappen, Proceedings Series B, vol. 55:150-152.

Bergsland, K., and H. Vogt. 1962. On the validity of glottochronology. CA 3:115-153. Bernet Kempers, A. J. 1959. Ancient Indonesian Art. New Haven: Harvard University Press.

1988. The kettledrums of Southeast Asia. MQRSEA 10.

Bhatia, D., S. Easteal, and R. L. Kirk. 1995. A study of genetic distance and the Austronesian/Non-Austronesian dichotomy. In P. Bellwood, J. J. Fox, and D. Tryon (eds.), The Austronesians, 181-191.

Bickerton, D. 1990. Language and Species. Chicago: University of Chicago Press.

Bie, C. W. P. de. 1932. Verslag van de ontgraving der steenen kamers in de doesoen Tandjoeng Ara, Pasemah-Hoogvlakte. TITLV 72:626-635.

Bijlmer, H. J. T. 1929. Outlines of the Anthropology of the Timor-Archipelago. Weltevreden: G. Kolff.

Bilsborough, A. 1973. A multivariate study of evolutionary change in the hominid cranial vault and some evolutionary rates. JHE 2:387-404.

Binford, L. R. 1983. In Pursuit of the Past. London: Thames and Hudson.

Binford, L., and C. K. Ho. 1985. Zhoukoudian: The cave home of Beijing man? CA 26:413-442.

Bintarti, D. D. 1981a. The bronze object from Kabila, West Sabu. Aspek-Aspek Arkeologi Indonesia 8. Jakarta: Pusat Penelitian Arkeologi Nasional.

—. 1981b. Punden berundak di Gunung Padang, Jawa Barat. Amerta 4:28-37. 1985a. Analisis fungsional nekara perunggu dari Lamongan, Jawa Timur. In Pertemuan Ilmiah Arkeologi III, 68-80. Jakarta: Pusat Penelitian Arkeologi Nasional.

1985b. Prehistoric bronze objects in Indonesia. BIPPA 6:64-73. 1986. Lewoleba sebuah situs masa presejarah di Pulau Lembata. In Pertemuan Ilmiah Arkeologi IV, Vol. Ila, 73-91. Jakarta: Pusat Penelitian Arkeologi Nasional. 
1990. Pejeng type bronze kettledrums. Kalpataru 9:190-205.

Birdsell, J. B. 1949. The racial origin of the extinct Tasmanians. Records of the Queen Victoria Museum, Launceston 2:105-122.

1967. Preliminary data on the trihybrid origins of the Australian Aborigines. APAO 2:100-155.

1972. Human Evolution. Chicago: Rand McNally.

1977. The recalibration of a paradigm for the first peopling of Greater Australia. In J. Allen et al. (eds.), Sunda and Sahul, 113-168.

-1993. Microevolutionary Patterns in Aboriginal Australia. New York: Oxford University Press.

Blanton, R. E., and J. Taylor. 1995. Patterns of exchange and the social production of pigs in Highland New Guinea. Journal of Archaeological Research 3:113146.

Blust, R. A. 1976. Austronesian culture history: Some linguistic inferences and their relations to the archaeological record. WA 8:19-43.

- 1977. The Proto-Austronesian pronouns and Austronesian subgrouping: A preliminary report. Working Papers in Linguistics 9:2. Department of Linguistics, University of Hawai'i.

- 1978. Eastern Malayo-Polynesian: A subgrouping argument. In S. A. Wurm and L. Carrington (eds.), Second International Conference on Austronesian Linguistics, fascicle 1:181-234.

-1980. Early Austronesian social organization: The evidence of language. $C A$ 21:205-247.

. 1981a. Variation in retention rate among Austronesian languages. Paper presented at the Third International Conference on Austronesian Linguistics, Bali, January 1981.

. 1981b. The reconstruction of Proto-Malayo-Javanic: An appreciation. BTLV 137:456-469.

- 1981c. Linguistic evidence for some early Austronesian taboos. American Anthropologist 83:285-319.

. 1982. The linguistic value of the Wallace Line. BTLV 138:231-250.

- 1984-1985. The Austronesian homeland: A linguistic perspective. AP 26: 45-67.

. 1988. Malay historical linguistics: A progress report. In M. T. Ahmad and Z. M. Zaim (eds.), Rekonstruksi dan Cabang-Cabang Bahasa Melayu Induk, 1-34. Kuala Lumpur: Dewan Bahasa dan Pustaka.

— 1991. The Greater Central Philippines hypothesis. OL 30:73-129.

- 1993. Central and Central-Eastern Malayo-Polynesian. OL 32:241-293. . 1994a. Austronesian sibling terms and culture history. In A. K. Pawley and M. Ross (eds.), Austronesian Terminologies, Continuity and Change, 3172.

1994b. The Austronesian settlement of mainland Southeast Asia. In K. L. Adams and T. Hendak (eds.), Papers from the 2nd Annual Meeting of the Southeast Asian Linguistic Society, 25-83. Arizona State University: Program for Southeast Asian Studies. 
1995a. The position of the Formosan languages: Method and theory in Austronesian comparative linguistics. In P J-K. Li et al. (eds.), Austronesian Studies Relating to Taiwan, 585-650.

1995b. The prehistory of the Austronesian-speaking peoples: A view from language. Journal of World Prehistory 9:453-510.

1996. Beyond the Austronesian homeland: The Austric hypothesis and its implications for archaeology. In W. H. Goodenough (ed.), Prehistoric Settlement of the Pacific, pp. 117-140. Philadelphia: American Philosophical Society.

Boaz, N. T. 1977. Paleoecology of early Hominidae in Africa. Kroeber Anthropological Society Papers 50:37-62.

Bosch, F. D. K. 1961. The problem of the Hindu colonization of Indonesia. In F. D. K. Bosch, Selected Studies in Indonesian Archaeology, 1-22. The Hague: Nijhoff.

Bowler, J. M., R. Jones, H. Allen, and A. G. Thorne. 1970. Pleistocene human remains from Australia: A living site and human cremation from Lake Mungo, western New South Wales. WA 2:39-60.

Brace C. L. 1964. A non-racial approach towards the understanding of human diversity. In M. Montagu (ed.), The Concept of Race, 103-152. New York: Free Press. 1976. Tooth reduction in the Orient. $A P$ 19:203-219.

1980a. Tooth-size and Austronesian origins. In P. Naylor (ed.), Austronesian Studies: Papers from the 2nd Eastern Conference on Austronesian languages, 167180. Michigan Papers on South and Southeast Asia 15.

- 1980b. Australian tooth-size clines and the death of a stereotype. $C A 21$ : 141-164.

Brace, C. L., and R. J. Hinton. 1981. Oceanic tooth-size variation as a reflection of biological and cultural mixing. CA 22:549--569.

Brace, C. L., X. Q. Shao, and Z. B. Zhang. 1984. Prehistoric and modern tooth size in China. In F. H. Smith and F. Spencer (eds.), The Origins of Modern Humans, 485516. New York: Liss.

Brace, L., D. P. Tracer, and K. D. Hunt. 1991. Human craniofacial form and the evidence for the peopling of the Pacific. BIPPA 12:247-269.

Braches, F., and R. Shutler. 1983-1984. Early vertebrates and the theory of the emergence of Java. Southeast Asian Studies Newsletter 13:1-2; 16:1-3. Singapore: British Institute in South-East Asia.

Brain, C.K. 1978. Some aspects of the South African Australopithecine sites and their bone accumulations. In C. Jolly (ed.), Early Hominids of Africa, 131-161. London: Duckworth.

Brandt, R. W. 1976. The Hoabinhian of Sumatra: Some remarks. MQRSEA 2:49-52.

Bräuer, G. 1992. The origin of modern Asians. In T. Akazawa et al. (eds.), The Evolution and Dispersal of Modern Humans in Asia, 401-414.

Bräuer, G., and F. H. Smith, eds. 1992. Continuity or Replacement: Controversies in Homo Sapiens Evolution. Rotterdam: Balkema.

Bray, F. 1986. The Rice Economies. Oxford: Blackwell.

Broecker, W. S. 1995. Chaotic climate. Scientific American, November 1995:44-50.

Broecker, W. S., and G. H. Denton. 1990. What drives glacial cycles? Scientific American, January 1990:49-56. 
Broecker, W. S., et al. 1988. New evidence from the South China Sea for an abrupt termination of the last glacial period. Nature 333:156-158.

Bronson, B. 1979. The late prehistory and early history of central Thailand with special reference to Chansen. In R. B. Smith and W. Watson (eds.), Early South East Asia, 315-336.

- 1992. Patterns in the early Southeast Asian metals trade. In I. Glover, P. Suchitta, and J. Villiers (eds.), Early Metallurgy, Trade and Urban Centres in Thailand and Southeast Asia, 63-114. Bangkok: White Lotus.

Bronson, B., and T. Asmar. 1975. Prehistoric investigations at Tianko Panjang Cave, Sumatra. AP 18:128-145.

Bronson, B., and I. Glover. 1984. Archaeological radiocarbon dates from Indonesia. Indonesia Circle 34:37-44.

Bronson, B., and J. C. White. 1992. Southeast Asia. In R. W. Ehrich (ed.), Chronologies in Old World Archaeology, Vol. 2, 475-515. Chicago: University of Chicago Press.

Brooks, S. T., R. Hegler, and R. H. Brooks. 1977. Radiocarbon dating and palaeoserology of a selected burial series from the Great Cave of Niah, Sarawak, Malaysia. AP 20:21-31.

Brosius, P. 1988. A separate reality. BRB 20/2:81-105.

- 1990. After Duwagan. Ann Arbor: Center for South and Southeast Asian Studies, University of Michigan.

Brothwell, D. R. 1960. Upper Pleistocene human skull from Niah Caves, Sarawak. SMJ 9:323-349.

Brown, D. E. 1970. Brunei: The Structure and History of a Bornean Malay Sultanate. Brunei Museum.

1978. Brunei in contemporary perspective. SMJ 47:135-160.

Brown, P. 1987. Pleistocene homogeneity and Holocene size reduction: The Australian human skeletal evidence. AO 22:41-66.

Brues, A. M. 1977. People and Races. New York: Holt, Rinehart and Winston.

Budhisampurno, S. 1985. Kerangka manusia dari Bukit Kelambai Stabat, Sumatera Utara. In Pertemuan Ilmiah Arkeologi III, 955-984. Jakarta: Pusat Penelitian Arkeologi Nasional.

Buettner-Janusch, J. 1966. Review of Coon, C. S. 1966. AJPA 25:182-188.

Bui Vinh. 1991. The Da But culture in the Stone Age of Viet Nam. BIPPA 10:127-131. Bulbeck, D. 1978. Analysis of a skeletal assemblage from Leang Buidane, Talaud Islands, Indonesia. Unpublished B.A. honors thesis, Australian National University.

1981. Continuities in Southeast Asian evolution since the late Pleistocene. Unpublished M.A. thesis, Australian National University.

- 1982. A re-evaluation of possible evolutionary processes in Southeast Asia since the late Pleistocene. BIPPA 3:1-21.

1992. A tale of two kingdoms. Unpublished Ph.D. thesis, Australian National University.

Burkill, I. H. 1951. The rise and decline of the greater yam in the service of man. Advancement of Science 7:443-448. 
Burrett, C., N. Duhig, R. Berry, and R. Varne. 1991. Asian and South-western Pacific continental terranes derived from Gondwana, and their biogeographical significance. Australian Systematic Botany 4:13-24.

Butler, B. 1994. Early prehistoric settlement in the Mariana Islands. Man and Culture in Oceania 10:15-38.

Butlin, N. G. 1993. Economics and the Dreamtime. Cambridge: Cambridge University Press.

Caldwell, I. (in press). A rock carving and a newly discovered stone burial chamber at Pasemah, Sumatra. Manuscript.

Cann, R. L., M. Stoneking, and A. C. Wilson. 1987. Mitochondrial DNA and human evolution. Nature 325:31-36.

Carey, 1. 1976. The Orang Asli. Kuala Lumpur: Oxford University Press.

Casparis, J. G. de. 1975. Indonesian Palaeography. Leiden: Brill.

Cavalli-Sforza, L. L. 1986. African Pygmies: An evaluation of the state of research. In L. L. Cavalli-Sforza (ed.), African Pygmies, 361-426. Orlando: Academic.

Cavalli-Sforza, L. L., P. Menozzi, and A. Piazza. 1994. The History and Geography of Human Genes. Princeton: Princeton University Press.

Chandler, R. F. 1979. Rice in the Tropics. Boulder, Colorado: Westview Press.

Chang, J. H. 1968. The agricultural potential of the humid tropics. Geographical Review 58:333-361.

Chang, K. C. 1969. Fengpitou, Tapenkeng and the Prehistory of Taiwan. New Haven: Yale University Publications in Anthropology 73.

1970. Prehistoric archaeology of Taiwan. AP 13:59-78.

. 1972. Neolithic cultures in the coastal areas of Southeast China. In

N. Barnard (ed.), Early Chinese Art and Its Possible Influence in the Pacific Basin, vol. 2, 431-458.

-1977. A new prehistoric ceramic style in the southeastern coastal area of China. AP 20:179-182.

- 1981. The affluent foragers in the coastal areas of China. Senri Ethnological Studies 9: 177-186.

- 1986. The Archaeology of Ancient China. Fourth ed. New Haven: Yale University Press.

- 1995. Taiwan Strait archaeology and the Protoaustronesians. In Li, P. J-K. et al. (eds.), Austronesian Studies Relating to Taiwan, 161-184.

Chang, K. C., and collaborators. 1974. Man in the Choshui and Tatu River valleys in central Taiwan. AP 17:36-55.

Chang, T. T. 1976a. The rice cultures. Philosophical Transactions of the Royal Society of London, Series B, 275:143-155.

. 1976b. The origin, evolution, cultivation, dissemination and diversification of Asian and African rices. Euphytica 25:425-441.

1983. The origin and early cultures of the cereal grains and food legumes. In

D. N. Keightley (ed.), The Origins of Chinese Cultivation, 65-94.

- 1989. Domestication and the spread of the cultivated rices. In D. Harris and

G. Hillman (eds.), Foraging and Farming, 408-417. 
Chapman, V. 1986. Inter-site variability in South Sulawesi. $A O$ 21:76-84.

Chappell, J. 1982. Sea levels and sediments: some features of the context of coastal archaeological sites in the tropics. AO 17:69-78.

1983. A revised sea-level curve for the last 30,000 years from Papua New Guinea. Search 14:99-101.

. 1994. Upper Quaternary sea levels, coral terraces, oxygen isotopes and deepsea temperatures. Journal of Geography (Japan) 103(7):828-840.

Chappell, J., A. Omura, T. Esat, M. McCulloch, J. Pandolfi, Y. Ota, and B. Pillans. 1996. Reconciliation of late Quaternary sea levels derived from coral terraces at Huon Peninsula with deep-sea oxygen isotope records. Earth and Planetary Science Letters 141:227-237.

Chappell, J., and N. Shackleton. 1986. Oxygen isotopes and sea level. Nature 324: $137-140$.

Chappell, J., and B. G. Thom. 1977. Sea levels and coasts. In J. Allen et al. (eds.), Sunda and Sahul, 275-292.

Chard, C. S. 1974. Northeast Asia in Prehistory. University of Wisconsin Press.

Chasen, F. N., and M. W. F. Tweedie, eds. 1940. Proceedings of the Third Congress of Prehistorians of the Far East. Singapore: Government Printer.

Chekiang Province and Chekiang Provincial Museum. 1978. Excavations (first season) in YuYao County, Chekiang Province. K'ao Ku Hsüeh Pao, 1978, 1:94, 106-107 (English sections).

Chen, Chi-lu. 1968. Material Culture of the Formosan Aborigines. Taipei: Taiwan Museum.

Chen, L. Z., et al. 1992. Genetic affinities of Oceanic populations based on RFLP and haplotype analysis. Human Biology 64:1-15.

Chen Chun and J. W. Olsen. 1990. China at the last glacial maximum. In P. Soffer and C. Gamble (eds.), The World at 18000 BP, Vol. 2, 276-295. London: Unwin Hyman.

Cheng, Te-k'un. 1969. Archaeology in Sarawak. Cambridge University Press.

Cherry, R. 1978. An analysis of the lithic industry of Buad Island, Samar. PQCS 6:3-80.

Chesner, C. A., et al. 1991. Eruptive history of Earth's largest Quaternary caldera (Toba, Indonesia) clarified. Geology 19:200-203.

Chin, S. C. 1977. Shifting cultivation-a need for greater understanding. SMJ 46: 107-128.

Chivers, D. J. 1977. The lesser apes. In Prince Rainier III and G. H. Bourne (eds.), Primate Conservation, 539-598. London: Academic Press.

Chretien, C. D. 1956. Word distribution in southeastern Papua. Language 32:88-108.

Christie, A. H. 1979a. Lin-i, Fu-nan, Java. In R. B. Smith and W. Watson (eds.), Early Southeast Asia, 281-287.

- 1979b. The megalithic problem in South East Asia. In R. B. Smith and W. Watson (eds.), Early Southeast Asia, 242-252.

Christie, J. W. 1984-1985. Trade and early state formation in Maritime Southeast Asia: Kedah and Srivijaya. Jebat 13:43-56.

1985. On Po'ni: The Santubong sites of Sarawak. SMJ 24:77-89.

- 1986. Negara, mandala and despotic state: Images of early Java. In D. G. Marr 
and A. C. Milner (eds.), Southeast Asia in the 9th to 14th Centuries, 65-94. Singapore: Institute of Southeast Asian Studies.

Clark, J. D. 1992. African and Asian perspectives on the origins of modern humans. Philosophical Transactions of the Royal Society of London B337:201-215.

Clarke, W. C. 1976. Maintenance of agriculture and human habitats within the tropical forest ecosystem. Human Ecology 4:247-259.

Clason, A. T. 1986. The faunal remains of Paso in northern Sulawesi, Indonesia. In Archaeozoologia, Mélanges, 35-62. Paris: La Pensée Sauvage.

— 1987. Late Pleistocene/Holocene hunter-gatherers of Sulawesi. Palaeohistoria 29:67-76.

Clausen, I. H. S. 1989. Cranial capacity in Homo erectus. In G. Giacobini (ed.), Hominidae, 217-220. Milan: Jaca.

Climate and Rice. 1976. Los Baños, Philippines: International Rice Research Institute.

Clutton Brock, J. 1959. Niah's Neolithic dog. SMJ 9:143-145.

Coèdes, G. 1947. Fouilles en Cochinchine: Le site de Go Oc Eo, ancien port du Royaume Fou-nan. Artibus Asiae X:193-199.

COHMAP Members. 1988. Climatic changes of the last 18,000 years. Science 241: 1043-1051.

Colani, M. 1935. Megalithes du Haut-Laos (2 vols.). Paris: Publications de l'Ecole Française d'Extrême-Orient nos. 25, 26.

Cole, F. C. 1945. Peoples of Malaysia. New York: Van Nostrand.

Collier, W. L. 1979. Social and Economic Aspects of Tidal Swamp Land Development in Indonesia. Canberra: Development Studies Centre Occasional Paper 15, Australian National University.

Collings, H. D. 1936. Report of an archaeological excavation in Kedah, Malay Peninsula. $B R M$ Series B, I(1):5-16.

1937a. An excavation at Bukit Chuping, Perlis. BRM Series B, I(2):94-119.

- 1937b. Recent finds of Iron Age sites in southern Perak and Selangor. BRM Series B, I(2):75-92.

Condominas, M. 1980. The Mnong Gar of Vietnam. In D. R. Harris (ed.), Human Ecology in Savanna Environments, 209-251. London: Academic Press.

Conklin, H. C. 1980. Ethnographic Atlas of Ifugao. New Haven: Yale University Press.

Coon, C. S. 1962. The Origin of Races. London: Jonathan Cape. 1966. The Living Races of Man. London: Jonathan Cape.

Cooper, J. M. 1941. Temporal Sequence and the Marginal Cultures. Washington, D.C.:

Catholic University of America, Anthropological Series 10.

Coppens, Y. 1994. East side story: The origin of humankind. Scientific American, May 1994:62-69.

Cortesão, A. ed. 1944. The Suma Oriental of Tomé Pires. London: Hakluyt Society.

Coursey, D. G. 1972. The civilizations of the yam. APAO 7:215-233.

. 1976. Yams. In N. W. Simmonds (ed.), Crop Plant Evolution, 70-74. London: Longmans.

Coutts, P. J. F., and J. P. Wesson. 1980. Models of Philippine prehistory: A review of the flaked stone industries. PQCS 8:203-259.

Covey, C. 1984. The earth's orbit and the Ice Ages. Scientific American 250(2):42-50. 
Craib, J. 1993. Early occupation at Unai Chulu, Tinian. BIPPA 13:116-134.

Cranbrook, Earl of (formerly Lord Medway). 1979. A review of domesticated pig remains from archaeological sites in Sarawak. SMJ 48:79-86.

1988a. Report on bones from the Madai and Baturong Cave excavations. In

P. Bellwood, Archaeological research in South-Eastern Sabah, 142-154.

- $1988 \mathrm{~b}$. The contribution of archaeology to the zoogeography of Borneo. Fieldiana Zoology, new series 42.

Cranbrook, Earl of, and D. S. Edwards. 1994. Belalong: A Tropical Rainforest. London: Royal Geographical Society.

Crowley, T. 1992. An Introduction to Historical Linguistics. Auckland: Oxford University Press.

Cselik, A. 1986. Technology of a bimetallic sword from Java. Indonesian Circle 40:6567.

Cunningham, C. E. 1967. Soba: An Atoni village of West Timor. In Koentjaraningrat (ed.), Villages in Indonesia, 63-89. New York: Cornell University Press.

Cuong, N. L. 1986-1987. An early Hoabinhian skull from Vietnam. BIPPA 7:30-35.

Curtis, G. H. 1981. Establishing a relevant time scale in anthropological and archaeological research. Philosophical Transactions of the Royal Society of London, Series B, 292:7-19.

Dahl, O. C. 1951. Malagache et Maanyan. Oslo: Egede Instituttet.

1973. Proto-Austronesian. Scandinavian Institute of Asian Studies. London: Curzon Press.

Dalrymple, R. E. 1984. The waruga burial tombs of North Sulawesi. Hemisphere 28(5): 267-272.

Dammerman, K. W. 1934. On prehistoric mammals from the Sampoeng Cave, central Java. Treubia XIV:477-486.

Dang Van Thang and Vu Quoc Hien. 1995. Excavation of Giong Ca Vo site. Khao Co Hoc for 1995, no. 2:3-19.

Daniels, J., and C. Daniels. 1993. Sugarcane in prehistory. AO 28:1-8.

Darlington, P.J. 1957. Zoogeography: The Geographical Distribution of Animals. New York: Wiley.

Davison, G. 1991. Animal remains from the protohistoric community at Kuala Selinsing, Perak. JAM 4:95-102.

Day, M. H., and T. L. Molleson. 1973. The Trinil femora. In M. H. Day (ed.), Human Evolution, 127-154. London: Taylor and Francis.

Dentan, R. K. 1968. The Semai. New York: Holt, Rinehart and Winston.

- 1975. If there were no Malays, who would the Semai be? Contributions to Asian Studies 7:50-64.

Deraniyagala, S. U. 1986. Excavations in the Citadel of Anuradhapura. Ancient Ceylon 6:39-47.

Dewar, R. E. 1977. Archaeology as Ecological Anthropology in Central Taiwan. Ann Arbor: University Microfilms International.

Dickerson, R. E. 1928. Distribution of Life in the Philippines. Manila: Bureau of Printing. Diffloth, G. 1979. Aslian languages and Southeast Asian prehistory. FMJ 24:3-18. 
1994. The lexical evidence for Austric, so far. OL 33:309-322.

Dikshit, M. G. 1952. Beads from Ahichchhatra, U. P. Ancient India 8:33-63.

Diniasti, A. 1986. Incised potteries from some Palaeometallic sites in Indonesia. In Pertemuan Ilmiah Arkeologi IV IIb:83-88.

Dobby, E. H. G. 1976. Southeast Asia. Eleventh ed. University of London Press.

Dortch, C. E. 1977. Early and late stone industrial phases in Western Australia. In R. V. S. Wright (ed.), Stone Tools as Cultural Markers, 104-132.

Dove, M. R. 1985. Swidden Agriculture in Indonesia. Berlin: Mouton.

Downs, R. E. 1955. Head hunting in Indonesia. BTLV 111:40-70.

. 1956. The Religion of the Bare'e-Speaking Toradja of Central Celebes. The Hague: Excelsior.

Du Bois, C. 1944. The People of Alor. Minneapolis: University of Minnesota Press.

Duff, R. 1970. Stone Adzes of Southeast Asia. Christchurch: Canterbury Museum Bulletin 3.

Dunn, F. L. 1964. Excavations at Gua Kechil, Pahang. IMBRAS 37(2):87-124.

1966. Radiocarbon dating of the Malayan Neolithic. Proceedings of the Prehistoric Society 32:352-353.

- 1975. Rain Forest Collectors and Traders. Malaysian Branch, Royal Asiatic Society, Monograph 5.

Dunn, F. L., and D. F. Dunn. 1977. Maritime adaptations and the exploitation of marine resources in Sundaic Southeast Asian prehistory. MQRSEA 3:1-28.

Duplessy, J. C. 1982. Glacial to interglacial contrasts in the northern Indian Ocean. Nature 295:494-498.

Dutton, T. 1995. Language contact and change in Melanesia. In P. Bellwood, J. J. Fox, and D. T. Tryon, (eds.), The Austronesians, 192-213.

Dutton, T., and D. Tryon, eds. 1994. Language Contact and Change in the Austronesian World. Berlin: Mouton de Gruyter.

Duy, N., and N. Q. Quyen. 1966. Early Neolithic skulls in Quynh Van, Nghe An, North Vietnam. VertebrataPalAsiatica 10:49-57.

Dyen, I. 1962. The lexicostatistical classification of the Malayopolynesian languages. Language 38:38-46.

-1965a. A lexicostatistical classification of the Austronesian Languages. International Journal of American Linguistics Memoir 19.

. 1965b. Formosan evidence for some new Proto-Austronesian phonemes. Lingua 14:285-305.

- 1971. Malagasy. In T. Seboek (ed.), Linguistics in Oceania, Vol. 1, 211-239. Current Trends in Linguistics Vol. 8. The Hague: Mouton.

-1975. Linguistic Subgrouping and Lexicostatistics. The Hague: Mouton. 1995. The internal and external classification of the Formosan languages. In

P. J-K. Li et al. (eds.), Austronesian Studies Relating to Taiwan, 455-520.

EATQN. 1992. East Asian Tertiary and Quaternary Newsletter, Vol. 15.

Eder, J. F. 1987. On the Road to Tribal Extinction. Berkeley: University of California Press. Egerod, S. 1991. Far Eastern languages. In S. M. Lamb and E. D. Mitchell (eds.), Sprung from Some Common Source, 205-231. Stanford University Press. 
Ellen, R. F. 1978. Nuaulu Settlement and Economy. The Hague: Nijhoff. Endicott, K. 1979. Batek Negrito Religion. Oxford: Clarendon Press.

- 1984. The economy of the Batek of Malaysia. Research in Economic Anthropology 6:29-52.

Endicott, K., and P. Bellwood. 1991. The possibility of independent foraging in the rain forest of Peninsular Malaysia. Human Ecology 19:151-185.

Evans, I. 1918. Preliminary report on cave explorations near Lenggong, Upper Perak. JFMSM 7(4):227-234.

1928a. On a find of stone implements associated with pottery. JFMSM 12(5): 133-135, 143-144.

1928b. On slab-built graves in Perak. JFMSM 12(5):111-120.

1931a. Excavations at Nyong, Tembeling River. JFMSM 15(2):51-62.

1931b. A further slab-built grave at Sungkei, Perak. JFMSM 15(2):63-64.

. 1932. Excavations at Tanjong Rawa, Kuala Selinsing, Perak. JFMSM 15(3):79_ 134.

Feldman, J., ed. 1985. The Eloquent Dead. Los Angeles: UCLA Museum of Cultural History.

Fernandez, C. A., and F. Lynch. 1972. The Tasaday: Cave-dwelling food-gatherers of South Cotobato, Mindanao. Philippine Sociological Review 20:277330.

Fix, A, 1977. The demography of the Semai Senoi. Ann Arbor: Museum of Anthropology, University of Michigan, Anthropological Papers 62.

—. 1984. Genetic structure of the Semai. In M. H. Crawford and J. H. Mielke (eds.), Current Developments in Anthropological Genetics, Vol.2, 179-204. New York: Plenum.

Flannery, T. 1994. The Future Eaters. Melbourne: Reed.

Flannery, T., P. Bellwood, P. White, A. Moore, Boeadi, and G. Nitihaminoto. 1995. Fossil marsupials (Macropodidae, Peroryctidae) and other mammals of Holocene age from Halmahera. Alcheringa 19:17-25.

Flannery, T., and Boeadi. 1995. Systematic revision within the Phalanger ornatus complex (Phalangeridae: Marsupalia). Australian Mammalogy 18:35-44.

Flannery, T., and P. White. 1991. Animal translocation. National Geographic Research and Exploration 7:96-113.

Flenley, J. R. 1979. The Equatorial Rainforest: A Geological History. London: Butterworths.

1985a. Man's impact on the vegetation of Southeast Asia: The pollen evidence. In V. N. Misra and P. S. Bellwood (eds.), Recent Advances in Indo-Pacific Prehistory, 297-306.

- 1985b. Quaternary vegetational and climatic history of Island Southeast Asia. MQRSEA 9:55-64.

1988. Palynological evidence for land use changes in South-East Asia. Journal of Biogeography 15:185-197.

Flenley, J. R., and R. J. Morley. 1978. A minimum age for the deglaciation of Mt. Kinabalu, East Malaysia. MQRSEA 4:57-62. 
Fogg, W. H. 1983. Swidden cultivation of foxtail millet by Taiwan aborigines. In D. N. Keightley (ed.), The Origins of Chinese Civilization, 95-116.

Foley, W. A. 1980. History of migrations in Indonesia as seen by a linguist. In J. J. Fox (ed.), Indonesia: The Making of a Culture, 75-80.

. 1986. The Papuan Languages of New Guinea. Cambridge: Cambridge University Press.

Fontaine, H. 1979. A note on the Iron Age in southern Vietnam. JHKAS 8:91-98.

- 1980. On the extent of the Sa Huynh culture in continental Southeast Asia. AP 23:67-70.

Fontaine, H., and Thi Than Hoang. 1975. Nouvelle note sur le champ de jarres funéraires de Phu Hoa. Bulletin de la Société des Etudes Indochinoises 51:7-74.

Forman, S. 1977. East Timor: Exchange and political hierarchy at the time of the European discoveries. In K. L. Hutterer (ed.), Economic Exchange and Social Interaction in Southeast Asia, 97-112. Ann Arbor: Michigan Papers on South and Southeast Asia 13.

Forth, G. R. 1981. Rindi. The Hague: Nijhoff.

Fox, J. J. 1977. Harvest of the Palm. Cambridge: Harvard University Press.

1985 Possible models of early Austronesian social organisation. AP 26:35-44. 1993 Inside Austronesian Houses. Canberra: Dept. of Anthropology, Research School of Pacific and Asian Studies, Australian National University.

. 1994. Who's who in Ego's generation. In A. K. Pawley and M. Ross (eds.), Austronesian Terminologies, Continuity and Change, 127-140.

Fox, J. J., ed. 1980. Indonesia: The Making of a Culture. Canberra: Research School of Pacific Studies, Australian National University.

Fox, R. B. 1953. The Pinatubo Negritos. Philippine Journal of Science 81:173-414.

- 1970. The Tabon Caves. Manila: National Museum Monograph 1. 1978. The Philippine Palaeolithic. In F. Ikawa-Smith (ed.), Early Palaeolithic in South and East Asia, 59-85.

- 1979. The Philippines during the first millennium BC. In R. B. Smith and W. Watson (eds.), Early South East Asia, 227-241.

Frake, C. O. 1956. Malayo-Polynesian land tenure. American Anthropologist 58:170173.

Francis, P. 1990. Glass beads in Asia, Part II: Indo-Pacific beads. AP 29:1-24.

Francisco, J. R. 1965. On the date of the coming of the Indian influences in the Philippines. Philippine Historical Review 1:136-152.

Fredericksen, C., M. Spriggs, and W. Ambrose. 1993. Pamwak rockshelter: A Pleistocene site on Manus Island, Papua New Guinea. In M. Smith, M. Spriggs, and B. Fankhauser (eds.), Sahul in Review, 144-154.

Freeman, D. 1955. Iban Agriculture. London: Her Majesty's Stationery Office.

- 1960. The Iban of western Borneo. In G. P. Murdock (ed.), Social Structure in Southeast Asia, 65-87.

1970. Report on the Iban. London: Athlone Press.

. 1981. Some Reflections on the Nature of Iban Society. Canberra: Australian National University, Department of Anthropology. 
Fried, M. 1975. The Notion of Tribe. Menlo Park: Cummings.

Friedlaender, J. 1987. The Solomon Islands Project. Oxford: Clarendon Press.

Gajdusek, D. C. 1970. Physiological characteristics of Stone Age man. Engineering and Science 33:26-33, 56-62.

Galipaud, J.-C., ed. 1992. Poterie Lapita et Peuplement. Nouméa: ORSTOM.

Gates, R. R. 1961. The Melanesian dwarf tribe of Aiome, New Guinea. Acta Geneticae Medicae et Gemellologiae (Rome) 10(3):277-311.

Gee, H. 1995. Uprooting the family tree. Nature 373:15.

Geertz, C. 1963. Agricultural Involution. Berkeley: University of California Press.

- 1972. The wet and the dry: Traditional irrigation in Bali and Morocco. Human Ecology 1:23-39.

1980 Negara. Princeton: Princeton University Press.

Geertz, H. 1963. Indonesian cultures and communities. In R. McVey (ed.), Indonesia, 24-96. New Haven: Yale University Press.

Glinka, J. 1978. Gestalt und Herkunft: Beitrag zur Anthropologischen Gliederung Indonesiens. St. Augustine bei Bonn: Studia Instituti Anthropos 35.

- 1981. Racial history of Indonesia. In H. Suzuki (ed.), Rassengeschichte der Mensheit 8, Lieferung Asien I: Japan, Indonesien, Ozeanien, 79-113. München: Oldenbourg.

Glover, E. 1990. The molluscan fauna of Ulu Leang 1 cave. In 1. and E. Glover (eds.), Southeast Asian Archaeology 1986, 29-36. Oxford: BAR International Series 561.

Glover, 1. C. 1972a. Excavations in Timor (2 vols.). Unpublished Ph.D. thesis, Australian National University.

- 1972b. Alfred Buhler's excavations in Timor: A re-evaluation. Art and Archaeology Research Papers 2:117-142. Institute of Archaeology, University of London.

- 1976. Ulu Leang cave, Maros: A preliminary sequence of post-Pleistocene cultural development in South Sulawesi. Archipel 11:113-154.

1977a. The late Stone Age in eastern Indonesia. WA 9:42-61.

1977b. Prehistoric plant remains from Southeast Asia, with special reference to rice. In M. Taddei (ed.), South Asian Archaeology, 7-37. Naples: Istituto Universitario Orientale.

1978a. Survey and excavation in the Maros district, South Sulawesi, Indonesia. BIPPA 1:60-102.

1978b. Report on a visit to archaeological sites near Medan, Sumatra. BIPPA 1:56-60.

1979. The late prehistoric period in Indonesia. In R. B. Smith and W. Watson (eds.), Early South East Asia, 167-184.

- 1981. Leang Burung 2: An Upper Palaeolithic rock shelter in South Sulawesi, Indonesia. MQRSEA 6:1-38.

1985. Some problems relating to the domestication of rice in Asia. In V. N. Misra and P. S. Bellwood (eds.), Recent Advances in Indo-Pacific Prehistory, 265-274.

1986. Archaeology in Eastern Timor. Canberra: Department of Prehistory, Research School of Pacific Studies, Terra Australis 11. 
1990a. Early Trade Between India and South-East Asia. Second Edition. Hull: Centre for South-East Asian Studies, University of Hull.

. 1990b. Ban Don Ta Phet: The 1984-5 excavation. In I. and E. Glover (eds.), Southeast Asian Archaeology 1986, 139-184. Oxford: BAR International Series 561.

1992. Large bronze urns in Southeast Asia. In I. Glover (ed.), Southeast Asian Archaeology 1990, 209-226. University of Hull, Centre for South-East Asian Studies.

Glover, I. C., and E. A. Glover. 1970. Pleistocene flaked stone tools from Timor and Flores. Mankind 7:88-90.

Glover, I. C., and G. Presland. 1985. Microliths in Indonesian flaked stone industries. In V. N. Misra and P. S. Bellwood (eds.), Recent Advances in Indo-Pacific Prehistory, 185-195.

Golson, J. 1977. No room at the top: Agricultural intensification in the New Guinea Highlands. In J. Allen et al. (eds.), Sunda and Sahul, 601-638.

- 1985. Agricultural origins in Southeast Asia: A view from the east. In V. N. Misra and P. S. Bellwood (eds.), Recent Advances in Indo-Pacific Prehistory, 307314.

Gomes, A. G. 1982. Ecological Adaptation and Population Change: Semang Foragers and Temuan Horticulturalists in West Malaysia. Honolulu: East-West Center, EastWest Environment and Policy Institute, Research Report 12.

Gonda, J. 1973. Sanskrit in Indonesia. Second edition. New Delhi: International Academy of Indian Culture.

Goodenough, W. 1955. A problem in Malayo-Polynesian social organization. American Anthropologist 57:71-83.

Gorman, C. F. 1970. Excavations at Spirit Cave, North Thailand: Some interim interpretations. AP 13:79-108.

- 1971. The Hoabinhian and after: Subsistence patterns in Southeast Asia during the latest Pleistocene and early Recent periods. WA 2:300-320.

- 1977. A priori models and Thai prehistory. In C. A. Reed (ed.), Origins of Agricluture, 321-356.

Goudie, A. S. 1983. Environmental Change. Oxford: Clarendon Press.

Gould, R. A. 1977. Ethnoarchaeology; or where do models come from? In R. V. S. Wright (ed.), Stone Tools as Cultural Markers, 162-168.

- 1980. Living Archaeology. Cambridge: Cambridge University Press.

Grace, G. W. 1959. The position of the Polynesian languages within the Austronesian (Malayo-Polynesian) family. International Joumal of American Linguistics Memoir 16.

-1985. Oceanic subgrouping: Retrospect and prospect. In A. Pawley and L. Carrington (eds.), Austronesian linguistics at the 15th Pacific Science Congress, 1985:1-18. PL Series C-88.

Green, R. C. 1991. The Lapita Cultural Complex: Current evidence and proposed models. BIPPA 11:295-305.

Grimes, C. E., and K. R. Maryott. 1994. Named speech registers in Austronesian lan- 
guages. In T. Dutton and D. Tryon (eds.), Language Contact and Change in the Austronesian World, 275-322.

Groube, L. 1989. The taming of the rainforests: A model for late Pleistocene forest exploitation in New Guinea. In D. Harris and G. Hillman (eds.), Foraging and Farming, 292-304.

Groube, L., et al. 1986. A 40,000-year-old human occupation site at Huon Peninsula, Papua New Guinea. Nature 324:453-455.

Groves, C. P. 1976. The origin of the mammalian fauna of Sulawesi. Zeitschrift fur Säugetierkunde 41:201-216.

1981. Ancestors for the Pigs. Canberra: Australian National University, Department of Prehistory.

1984. Of mice and men and pigs in the Indo-Australian Archipelago. Canberra Anthropology 7:1-19.

1985. Plio-Pleistocene mammals in island South-east Asia. MQRSEA 9:43-54.

1987. On the cuscuses (Marsupalia: Phalangeridae) of the Phalanger orientalis group from Indonesian territory. In M. Archer (ed.), Possums and Opossums: Studies in Evolution, 569-579. Sydney: Surrey Beatty.

1989. A Theory of Human and Primate Evolution. Oxford: Clarendon Press.

1995. Domesticated and commensal mammals of Austronesia and their histories. In P. Bellwood, J. J. Fox, and D. Tryon (eds.), The Austronesians, 152163.

Gudschinsky, S. 1964. The ABC's of lexicostatistics (glottochronology). In D. Hymes (ed.), Language in Culture and Society, 612-623. New York: Harper and Row.

Gunadi Nitihaminoto, Harry Truman Simandjuntak, Suwarno, Timbul Hartono, and Budijanto. 1978. Laporan Ekskavasi Gunung Piring (Lombok Selatan). BPA 17.

Ha Van Tan. 1978. The Hoabinhian in the context of Vietnam.Vietnamese Studies 46:127-197.

1980. Nouvelles recherches préhistoriques et protohistoriques au Vietnam. Bulletin de l'Ecole Française d'Extrême-Orient 68:113-154.

1985a. Late Pleistocene climate in Southeast Asia: New data from Vietnam. MQRSEA 9:81-86.

- $1985 \mathrm{~b}$. Prehistoric pottery in Viet Nam and its relationships with Southeast Asia. AP 26/1:135-146.

1991 New researches on the Stone Age sites previously excavated by French archaeologists in Viet Nam. In Recherches en Archéologie en Thailande. Deuxième Symposium Franco-Thai, 166-171. Bangkok: Silpakorn University.

Haberle, S. 1993. Pleistocene vegetation change and early human occupation of a tropical mountainous environment. In M. Smith, M. Spriggs, and B. Fankhauser (eds.), Shahul in review, 109-122.

1994 Anthropogenic indicators in pollen diagrams: Problems and prospects for late Quaternary palynology in New Guinea. In J. Hather (ed.), Tropical Archaeobotany, 172-201. London: Routledge.

Habgood, P. J. 1986. The origin of the Australians: A multivariate approach. AO 21: 130-137. 
1992. The origin of anatomically modern humans in east Asia. In G. Bräuer and F. H. Smith (eds.), Continuity or Replacement, 273-288.

Hagelberg, E. 1994. DNA from ancient Easter Islanders. Nature 369:25-26.

Haile, N.S. 1968. The Quaternary geomorphic history of North Sarawak. SMJ 16: 277-281.

1973. The geomorphology and geology of the northern part of the Sunda shelf. Pacific Geology 6:73-90.

Hall, D. G. E. 1968. A History of Southeast Asia. Third edition. London: Macmillan.

Hall, K. R. 1976. State and statecraft in early Srivijaya. In K. R. Hall and J. K. Whitmore (eds.), Explorations in early Southeast Asian history, 61-105. Ann Arbor: Michigan Papers on South and Southeast Asia 11.

1977. The coming of Islam to the archipelago: A reassessment. In K. L. Hutterer (ed.), Economic exchange and social interaction in Southeast Asia, 213232. Ann Arbor: Michigan Papers on South and Southeast Asia 13.

. 1980. The origin of maritime trade in Southeast Asia. The Elmira Review 2: 35-43.

- 1985 Maritime Trade and State Development in Early Southeast Asia. Sydney: Allen and Unwin.

Hall, R. 1995. Reconstructing Cenozoic SE Asia. [Animated version of the following entry, which can be located in the World Wide Web (Internet) at http:// glsun.gl.rhbnc.ac.uk/seasia/welcome.html]

—. 1996. Reconstructing Cenozoic SE Asia. In R. Hall and D. Blundell (eds.), Tectonic Evolution of Southeast Asia, 153-184. London: Geological Society Special Publication 106.

Hallam, S. J. 1977. The relevance of Old World archaeology to the first entry of man into the New World: Colonization as seen from the Antipodes. Quaternary Research 8:128-148.

Hamilton, W. 1979. Tectonics of the Indonesian region. U.S. Geological Survey, Professional Paper 1078.

Hammen, T. van der, and M. L. Absy. 1994. Amazonia during the last glacial. Palaeogeography, Palaeoclimatology, Palaeoecology 109:247-261.

Hanbury-Tenison, R. 1980. Mulu: The Rainforest. London: Weidenfeld and Nicholson.

Hanihara, T. 1990. Affinities of the Philippine Negritos with Japanese and the Pacific populations I. Journal of Anthropological Society of Nippon 98:13-27.

- 1993. Craniofacial features of Southeast Asians and Jomonese. Anthropological Science 101:25-46.

Haq, B. K., W. A. Berggren, and J. A. van Couvering. 1977. Corrected age of the Pliocene-Pleistocene boundary. Nature 269:483-488.

Harihara, S., et al. 1992. Frequency of a 9-bp deletion in the mitochondrial DNA among Asian populations. Human Biology 64:161-166.

Harries, H. C. 1978. Malesian origin for a domestic Cocos nucifera. In P. Baas (ed.), The Plant Diversity of Malesia, 351-357. Netherlands: Kluwer.

Harris, D. R., ed. 1980. Human Ecology in Savanna Environments. London: Academic Press. 
Harris, D., and G. Hillman, eds. 1989. Foraging and Farming. London: Unwin Hyman. Harrison, T. 1996. The palaeoecological context at Niah Cave, Sarawak: Evidence from the primate fauna. BIPPA 14:90-100.

Harrisson, B. 1967. A classification of Stone Age burials from Niah Great Cave, Sarawak. SMJ 15:126-200.

- 1968. A Niah Stone-Age jar-burial C14 dated. SMJ 16:64-66.

Harrisson, B., and T. Harrisson. 1968. Magala: A series of Neolithic and Metal Age burial grottos at Sekaloh, Niah, Sarawak. JMBRAS 41(2):148-175.

Harrisson, T. 1957. The Great Cave of Niah. Man 57:161-166.

- 1958. Niah: A history of prehistory. SMJ 8:549-595.

-1959. New archaeological and ethnological results from Niah Caves, Sarawak. Man 59:1-8.

1962. Borneo death. BTLV 118:1-41. 151-159.

1970. The prehistory of Borneo. AP 13: 17-46.

- 1971. Prehistoric double-spouted vessels excavated from Niah caves, Borneo. JMBRAS 44(2):35-78.

- 1975a. Tampan: Malaysia's Palaeolithic reconsidered. MQRSEA 1:53-70.

- $1975 b$. Early dates for "seated" burial and burial matting at Niah caves, Sara wak. AP 18:161-165.

- 1978. Recent status and problems for Palaeolithic studies in Borneo and adjacent areas. In F. lkawa-Smith (ed.), Early Palaeolithic in South and East Asia, 37-57.

Harrisson, T., and B. Harrisson. 1971. The Prehistory of Sabah. Kota Kinabalu: Sabah Society.

Harrisson, T., D. A. Hooijer, and Lord Medway. 1961. An extinct giant pangolin and associated mammals from Niah Caves, Sarawak. Nature 189:166.

Harrisson, T., and Lord Medway. 1962. A first classification of prehistoric bone and tooth artefacts. SMJ 10:335-362.

Harvey, M. 1982. Subgroups in Austronesian. In A. Halim, L. Carrington, and S. A. Wurm (eds.), Papers from the Third International Conference on Austronesian Linguistics, Vol. 2, 47-99. PL Series C75.

Haudricourt, A. G. 1954. Les origines asiatiques des langues Malayo-Polynesiennes. Journal de la Société des Océanistes 10:180-183.

Hayden, B. 1977. Sticks and stones and edge-ground axes. In J. Allen et al. (eds.), Sunda and Sahul, 73-109.

- 1979. Palaeolithic Reflections. Canberra: Australian Institute of Aboriginal Studies.

- 1990. Nimrods, pescators, pluckers and planters. Journal of Anthropological Archaeology 9:31-69.

Headland, T. 1986. Why Foragers Do Not Become Farmers. Ann Arbor: University Microfilms International. 1987 The wild yam question: How well could independent hunter-gatherers live in a tropical rain forest ecosystem? Human Ecology 15:463-491. 
1988. Ecosystemic change in a Philippine tropical rainforest and its effect on a Negrito foraging society. Tropical Ecology 29:121-135.

Headland, T., ed. 1992. The Tasaday Controversy. Washington: American Anthropological Association.

Headland, T., and L. A. Reid. 1989. Hunter-gatherers and their neighbours from prehistory to the present. $C A 30: 43-66$.

- 1991. Holocene foragers and interethnic trade. In S. Gregg (ed.), Between Bands and States, 333-340. Southern Illinois University, Center for Archaeological Investigations.

Heaney, L. R. 1985. Zoogeographic evidence for middle and late Pleistocene land bridges to the Philippine Islands. MQRSEA 9:127-144.

- 1986. Biogeography of mammals in Southeast Asia: Estimates of rates of colonization, extinction and speciation. Biological Journal of the Linnean Society 28:127-165.

Heekeren, H. R. van. 1931. Megalithische overblijfselen in Besoeki, Java. Djawa 11: $1-18$.

1935-1936. Prehistorisch grottenonderzoek in Basoeki (Java). Djawa 15:123$129 ; 16: 187-193$.

1949. Rapport over de ontgraving van de Bola Batoe, nabij Badjo (Bone, Zuid-Celebes). Oudheidkundig Verslag for 1941-1947:89-108.

- 1950a. Rapport over de ontgraving te Kamasi, Kalumpang (West CentralCelebes). Oudheidkundig Verslag for 1949:26-48.

1950b. Rock paintings and other prehistoric discoveries near Maros (South West Celebes). Laporan Tahunan for 1950:22-35. Jakarta: Dinas Purbakala Republik Indonesia.

1955. Proto-historic sarcophagi on Bali. Berita Dinas Purbakala 2:1-15. Jakarta.

1956a. Notes on a proto-historic urn-burial site at Anjar, Java. Anthropos 51: 194-201.

— 1956b. The urn cemetery at Melolo, East Sumba. Berita Dinas Purbakala 3. Jakarta.

- 1958. The Bronze-Iron Age of Indonesia. The Hague: Nijhoff. 1972. The Stone Age of Indonesia. Second edition. The Hague: Nijhoff.

Heffernan, K. 1980. Molluscan resources and Talaud economy: Ecological and cultural parameters in the study of refuse. Unpublished B.A. honors thesis, Australian National University.

Heine Geldern, R. von. 1932 Urheimat und früheste Wanderungen der Austronesier. Anthropos 27:543-619.

1937. L'art prébouddique de la Chine et de I'Asie du Sud-Est et son influence en Océanie. Revue des Arts Asiatiques 11:177-206.

- 1945. Prehistoric research in the Netherlands Indies. In P. Honig and F. Verdoorn (eds.), 129-167.

- 1947. The drum named Makalamau. India Antiqua 1947:167-179. Leiden.

Henriksen, M. A. 1982. The first excavated prehistoric house site in Southeast Asia.

In K. G. Izikowitz and P. Sørensen (eds.), The house in East and Southeast Asia, 17-24. Copenhagen: Scandinavian Institute of Asian Studies Monograph 30. 
Hickson, S. J. 1889. A Naturalist in North Celebes. London: J. Murray.

Higham, C. 1989. The Archaeology of Mainland Southeast Asia. Cambridge: Cambridge University Press.

1996a. Archaeology and linguistics in Southeast Asia: Implications of the Austric hypothesis. BIPPA 14:110-118.

1996b. The Bronze Age of Southeast Asia. Cambridge: Cambridge University Press.

Higham, C., and R. Bannanurag. 1990. The Excavation of Khok Phanom Di. Vol. 1. London: Society of Antiquaries.

Higham, C. and R. Bannanurag, eds. 1991. The Excavation of Khok Phanom Di. Vol. 2, Part 1. London: Society of Antiquaries.

Higham, C., R. Bannanurag, G. Mason, and N. Tayles. 1992. Human biology, environment and ritual at Khok Phanom Di. WA 24:35-54.

Higham, C., and R. Thosarat. 1994. Khok Phanom Di. Fort Worth: Harcourt Brace.

Hill, A. V. S., and S. Serjeantson, eds. 1989. The Colonization of the Pacific: A Genetic Trail. Oxford: Clarendon Press.

Hill, R. D. 1977. Rice in Malaya. Kuala Lumpur: Oxford University Press.

Hillman, G., and M. Davies. 1990. Measured domestication rates in wild wheats and barley. Journal of World Prehistory 4:157-222.

Hislop, J. A. 1954. Notes on the migration of bearded pig. FMJ 1 and 2:134-137.

Hoang, X. C., and V. T. Bui. 1980. The Dong Son culture and cultural centres in the Metal Age of Vietnam. AP 23:55-66.

Hobart, A., U. Ramseyer, and R. Leeman. 1996 (forthcoming). The Peoples of Bali. Oxford: Basil Blackwell.

Hoffman, C. 1986. The Punan: Hunters and Gatherers of Borneo. Ann Arbor: UMI Research Press.

Holloway, R. 1981. The Indonesian Homo erectus brain casts revisited. AJPA 55:503521.

Holloway, R. L. 1980. Indonesian "Solo" (Ngandong) endocranial reconstructions. AJPA 53:285-295.

Honig, P., and F. Verdoorn, eds. 1945. Science and Scientists in the Netherlands Indies. New York: Board for the Netherlands, Surinam and Curaçao.

Hooijer, D. A. 1950a. Man and other mammals from Toalean sites in southwestern Celebes. Verhandelingen der Koninklijke Nederlandse Akademie van Wetenschappen, Afdeling Natuurkunde, Tweede Sectie 46(2):1-158.

- 1950b. Fossil evidence of Australomelanesian migrations in Malaysia? South Western Journal of Anthropology 6:416-422.

1952. Australomelanesian migrations once more. South Western Journal of Anthropology 8:472-477.

1956. The lower boundary of the Pleistocene in Java and the age of Pithecanthropus. Quaternaria 3:5-10.

- 1963. Further "Hell" mammals from Niah. SMJ 11:196-200.

1967-1968. Indo-Australian pygmy elephants. Genetica 38:143-162.

- 1968. The middle Pleistocene fauna of Java. In G. Kurth (ed.), Evolution und Hominization, 86-90. Second ed. Stuttgart: Gustav Fischer. 
1975. Quaternary mammals east and west of Wallace's Line. MQRSEA 1:3746.

1981. What, if anything new, is Stegodon sumbaensis Sartono? MQRSEA 6:89-90. 1983. Facts and fiction around the fossil mammals of Java. Geologie en Mijnbouw 62:339-343.

Hoop, A. N. van der. 1932. Megalithic Remains in South-Sumatra. Zutphen: Thieme.

- 1935. Steenkistgraven in Goenoeng Kidoel. TITLV 75:83-100.

1940. A prehistoric site near Lake Kerinchi, Sumatra. In F. N. Chasen and M. W. F. Tweedie (eds.), Proceedings of the Third Congress of Prehistorians of the Far East, 200-204.

Hope, G. S. 1980. Historical influences in the New Guinea flora. In P. van Royen (ed.), The Alpine Flora of New Guinea, 223-248. Vaduz: J. Cramer.

Hope, G. S., and M. Spriggs. 1982. A preliminary pollen sequence from Aneityum Island, southern Vanuatu. BIPPA 3:88-94; 4:v.

Hose, C. 1926. Natural Man: A Record from Borneo. London: Macmillan.

Hose, C., and W. McDougall. 1912. The Pagan Tribes of Borneo (2 vols.). London: Macmillan.

Hoskins, J. A. 1986. So my name shall live. BTLV 142:31-51.

Howell, F. C. 1994. A chronostratigraphic and taxonomic framework of the origins of modern humans. In M. H. and D. V. Nitecki (eds.), Origins of Anatomically Modern Humans, 253-319.

Howells, W. W. 1970. Anthropometric grouping analysis of Pacific peoples. APAO 5: 192-217.

1973a. Cranial variation in man. Cambridge: Harvard University, Papers of the Peabody Museum no. 67.

1973b. The Pacific Islanders. New York: Scribner's.

. 1976. Physical variation and history in Melanesia and Australia. AJPA 45: 641-650.

1977. Hominid fossils. In W. W. Howells and P. J. Tsuchitani (eds.), Palaeoanthropology in the Peoples' Republic of China. Washington, D.C.: National Academy of Science.

1989. Skull shapes and the map. Cambridge: Harvard University, Papers of the Peabody Museum no. 79.

Huang Wanpo, et al. 1996. Early Homo and associated artefacts from Asia. Nature 378:275-278.

Huang Weiwen, Li Chunchu, Wang Honshou, and Huang Yukun. 1982. Re-examination of a microlithic site at Xiqiaoshan, Nanhai County, Guangdong. CA 23: $487-492$.

Hudson, A. B. 1970. A note on Selako: Malayic Dayak and Land Dayak languages in western Borneo. SMJ 18:301-318.

Hudson, A. B., and J. M. Hudson. 1978. The Ma'anyan of Paju Epat. In V. T. King (ed.), Essays on Borneo Societies, 215-232.

Hughen, K. A., J. T. Overpeck, L. C. Peterson, and S. Trumbore. 1996. Rapid climatic changes in the tropical Atlantic region during the last deglaciation. Nature 380:51-54. 
Huke, R. 1976. The geography and climate of rice. In Climate and Rice 31-50. International Rice Research Institute.

Hutchison, C. S. 1989. Displaced terranes of the southwest Pacific. In Z. Ben-Avraham (ed.), The Evolution of the Pacific Ocean Margins, 161-175. Oxford: Clarendon Press.

Hutterer, K. L. 1976. An evolutionary approach to the Southeast Asian cultural sequence. CA 17:221-242.

- 1977. Reinterpreting the Southeast Asian Palaeolithic. In J. Allen et al. (eds.), Sund a and Sahul, 31-71.

Hutterer, K. L., and W. K. Macdonald, eds. 1982. Houses Built on Scattered Poles. Cebu City: University of San Carlos.

Hyodo, M., et al. 1993. Magnetostratigraphy of hominid fossil bearing formations in Sangiran and Mojokerto, Java. Anthropological Science 101:157-186.

Ikawa-Smith, F., ed. 1978. Early Palaeolithic in South and East Asia. The Hague: Mouton.

Imamura, K. 1993. Two traditions of Heger I type bronze drums. Japan Society of Southeast Asian Archaeology 13:113-130.

Indrawooth, P. 1997. The practice of jar burial in the Mun and Chi valleys. BIPPA 16: 149-152.

Ipoi, D. 1993. Archaeological excavations at Gua Sireh (Serian) and Lubang Angin (Gunung Mulu National Park), Sarawak, Malaysia. SMJ 45, Special Monograph 6.

Ipoi, D., and Bellwood, P. S. 1991 Recent research at Gua Sireh (Seriasn) and Lubang Angin (Gunung Mulu National Park), Sarawak. BIPPA 11:386-405.

IRRI. n.d. Effects of Temperature and Photoperiodism on Rice Growth. International Rice Research Institute, Slide-Tape Instructional Unit GM-5. Los Baños, Philippines.

Isaac, G. 1977a. Squeezing blood from stones. In R. V. S. Wright (ed.), Stone Tools as Cultural Markers, 5-12.

1977b. Olorgesailie. University of Chicago Press.

. 1980. Casting the net wide. In L-K. Konigsson (ed.), Current Argument on Early Man, 226-251.

Ishige, N., ed. 1980. The Galela of Halmahera. Osaka: Senri Ethnological Studies 7.

Jacob, T. 1967a. Some Problems Pertaining to the Racial History of the Indonesian Region. Utrecht: Drukkerij Neerlandia.

1967b. Racial identification of the Bronze Age human dentitions from Bali, Indonesia. Journal of Dental Research 46:903-910.

- 1972. The problem of head hunting and brain eating among Pleistocene men in Indonesia. APAO 7:81-91.

- 1978a. New finds of Lower and Middle Pleistocene hominines from Indonesia. In F. Ikawa-Smith (ed.), Early Palaeolithic in South and East Asia, 13-22.

$1978 \mathrm{~b}$. The puzzle of Solo Man. MQRSEA 4:31-40.

- 1979. Hominine evolution in South East Asia. APAO 14:1-10.

. 1980. The Pithecanthropus of Indonesia: Phenotype, genetics and ecology. In L-K. Konigsson (ed.), Current Argument on Early Man, 170-179.

Jacob, T., R. P. Soejono, L. G. Freeman, and F. H. Brown. 1978. Stone tools from mid PIeistocene sediments in Java. Science 202:885-887. 
Jacobs, M. 1974. Botanical panorama of the Malesian archipelago. In UNESCO, Natural Resources of Humid Tropical Asia, 263-294.

Janse, O.R. T. 1958. The Ancient Dwelling Site of Dong Son (Thanh-Hoa, Annam). Bruges: St. Catherine Press.

Jawan, J. A. 1992. Between tradition and change. BRB 24:24-32.

Jett, S. C. 1970. The development and distribution of the blowgun. Annals of the Association of American Geographers 60:662-688.

Jia Lanpo. 1980. Early Man in China. Beijing: Foreign Languages Press.

- 1989. On problems of the Beijing-Man site: A critique of new interpretations. CA 30:200-205.

Jiang, Q. 1994. Phytolith analysis and the origin of rice agriculture research in China. The Phytolitharien Newsletter 8/3:6-7.

Johanson, D. C. 1980. Early African hominid phylogenesis: A re-evaluation. In L-K. Konigsson (ed.), Current Argument on Early Man, 1980:31-69.

Johanson, D. C., and M. A. Edey. 1981. Lucy. London: Granada.

Kaars, W. A. van der. 1989. Aspects of late Quaternary palynology of eastern Indonesian deep sea cores. Netherlands Journal of Sea Research 24:495-500.

1991. Palynology of eastern Indonesian marine piston-cores: A late Quaternary vegetational and climatic record for Australasia. Palaeogeography, Palaeoclimatology, Palaeoecology 85:239-302.

Kahler, H. 1978. Austronesian comparative linguistics and reconstruction of the earlier forms of the language. In S. A. Wurm and L. Carrington (eds.), Second International Conference on Austronesian Linguistics, fascicle 1:3-18.

Kano, T., and K. Segawa. 1956. An Illustrated Ethnography of Formosan Aborigines. Vol. l: The Yami. Tokyo: Maruzen.

Katili, J. A. 1974. Geological environment of the Indonesian mineral deposits. Bandung: Direktorat Geologi, Publikasi Teknik, Seri Geologi Ekonomi 7.

- 1975. Volcanism and plate tectonics in the Indonesian island arcs. Tectonophysics 26:165-188.

-1991. Tectonic evolution of eastern Indonesia and its bearing on the occurrence of hydrocarbons. Marine and Petroleum Geology 8:70-83.

Kaudern, W. 1938. Megalithic Finds in Central Celebes. Ethnographical Studies in Celebes, vol. V. Göteborg: privately published by the author.

Kealhofer, L., and D. Piperno. 1994. Early agriculture in Southeast Asia: Phytolith evidence from the Bang Pakong Valley, Thailand. A 68:564-572.

Keers, W. 1948. An Anthropological Survey of the Eastern Little Sunda Islands. Amsterdam: Koninklijke Vereeniging Indisch Instituut.

Keightley, D. N., ed. 1983. The Origins of Chinese Civilization. Berkeley: University of California Press.

Kelly, K. M. 1990. Gm polymorphisms, linguistic affinities, and natural selection in Melanesia. CA 31:201-220.

Kennedy, J. 1982. Archaeology in the Admiralty Islands: Some excursions. BIPPA 3: 22-35.

Kennedy, K. A. R. 1977. The deep skull of Niah. AP 20:32-50. 
Kennedy, R. 1937. A study of Indonesian civilization. In G. P. Murdock (ed.), Studies in the Science of Society Presented to Albert Galloway Keller, 268-297. New Haven: Yale University Press.

Kern, H. 1889/1917. Taalkundige gegevens ter bepaling van het stamland der Maleisch-Polynesische volken. In H. Kern, Verspreide Geschriften, vol. 6, 1917: 105-120. The Hague: Nijhoff.

Kim, Byung-mo. 1982. Megalithic Cultures in Asia. Seoul: Hanyang University Press.

King, V. T. 1982. Ethnicity in Borneo: An anthropological problem. Southeast Asian Journal of Social Science 10:23-43.

- 1993. The Peoples of Borneo. Oxford: Basil Blackwell.

- ed. 1976. Migration, warfare and culture contact in Borneo: A critique of ecological analysis. Oceania 46:306-327.

-, ed. 1978. Essays on Borneo Societies. Oxford: Oxford University Press.

Kingdon, J. 1993. Self-made Man and His Undoing. London: Simon and Schuster.

Kirch, P. V. 1989. Second millennium вP arboriculture in Melanesia. Economic Botany 43:225-240.

. 1995. The Lapita culture of western Melanesia in the context of Lapita origins and dispersal. In P. J-K. Li et al. (eds.), Austronesian Studies Relating to Taiwan, 1995: 255-294.

1997. The Lapita People. Oxford: Blackwell.

Kirch, P. V., and T. L. Hunt, eds. 1988. Archaeology of the Lapita Cultural Complex. Seattle: Burke Museum.

Kirch, P. V., and D. Lepofsky. 1993. Polynesian irrigation: Archaeological and linguistic evidence for origins and development. AP 32:183-204.

Kirk, R. L. 1982. Microevolution and migration in the Pacific. In Human Genetics Part A: The Unfolding Genome, 215-225. Proceedings of the VI International Congress of Human Genetics, Jerusalem, 1981. New York: Liss.

Kirk, R. L. 1986. Human genetic diversity in South-East Asia and the western pacific. In D. F. Roberts and G. F. de Stefano (eds.), Genetic Variation and Its Maintenance, 111-133. Cambridge: Cambridge University Press.

Kirk, R. L., and A. G. Thorne, eds. 1976. The Origin of the Australians. Canberra: Australian Institute of Aboriginal Studies.

Koenigswald, G. H. R. von. 1949. Vertebrate stratigraphy. In R. W. van Bemmelen, 91-93.

- 1951. Introduction. In F. Weidenreich, Morphology of Solo Man, 211-221. 1952. Evidence of a prehistoric Australomelanesoid population in Malaya and Indonesia. South Western Journal of Anthropology 8:92-96.

- 1956. Meeting Prehistoric Man. London: Scientific Book Club. 1958. Remarks on the prehistoric fauna of the Great Cave. SMJ 8:620-626.

Koenigswald, G. H. R. von, and A. K. Ghosh. 1973. Stone implements from the Trinil beds. Koninklijk Nederlands Akademie van Wetenschappen, Proceedings Series B 76: 1-34.

Koenigswald, G. H. R. von, and F. Weidenreich. 1939. The relationship between Pithecanthropus and Sinanthropus. Nature 144:926-929. 
Konigsson, L-K., ed. 1980. Current Argument on Early Man. Oxford: Pergamon Press.

Kosasih, S., N. Anggraeni, and D. D. Bintarti. 1986. Survei di daerah Kuningan, Tahap 1, 1981. BPA 36: 27-46.

Koyama, S. 1977. Archaeological surveys at the sites of Shih-pa-chang and Ta-chiuyüan, Nant'ou, Taiwan. Institute of History and Philology, Academia Sinica, Special Publication 70:349-387. Taipei.

Krantz, G. S. 1980. Climatic Races and Descent Groups. North Quincy, MA: Christopher Publishing House.

Kress, J. 1977a. Contemporary and prehistoric subsistence patterns on Palawan. In W. Wood (ed.), Cultural-ecological perspectives on Southeast Asia, 29-48. Ohio University, Center for International Studies, Southeast Asia series no. 41. 1977b. Tom Harrisson, North Borneo and Palawan. AP 20:75-86. 1978. The ceramics from Pilanduk cave and Sa'agung rockshelter, Quezon Municipality, Palawan Island. AP 21:58-85.

Krien, P., ed. 1992. The SBS World Guide. Melbourne: Text Publishing Company.

Kuchikura, Y. 1993. Wild yams in the tropical rain forest. Man and Culture in Oceania 9:103-126.

Kukla, G. 1977. Pleistocene land-sea correlations. 1: Europe. Earth-Science Reviews 13: 307-374.

1981. Pleistocene climates on land. In A. Berger (ed.), Climatic Variations and Variability: Facts and Theories, 207-232. Dordrecht: Reidel.

Kulke, H. 1990. Indian colonies, Indianization or cultural convergence? In H. Schulte Nordholt (ed.), Onderzoek in Zuidoost-Asië, 8-32. Leiden: Vakgroep Talen en Culturen van Zuidoost-Asië en Oceanië.

Kumamoto, University of. 1983. Batan Island and Northern Luzon. University of Kumamoto (Japan), Faculty of Letters.

Kumar, A. 1979. Developments in four societies over the sixteenth to eighteenth centuries. In H. Aveling (ed.), The Development of Indonesian Society: From the Coming of Islam to the Present Day, 1-44. Brisbane: Queensland University Press.

Kupper, H. 1930. Palaeolitische werktuigen uit Atjeh, Nord Sumatra. TKNAG 47:985989.

Kutzbach, J. E. 1981. Monsoon climate of the early Holocene. Science 214:59-61.

Lal, B. B. 1954-1955. Excavations at Hastinapura and other explorations in the Upper Ganga and Sutlej basins. Ancient India 10-11:5-151.

Lamb, A. 1965. Some observations on stone and glass beads in early Southeast Asia. JMBRAS 38(2):87-124.

Lamb, H. H. 1982. Reconstruction of the course of post-glacial climate over the world. In A. Harding (ed.), Climatic Change in Later Prehistory, 11-32. Edinburgh University Press.

Leach, E. R. 1954. Political Systems of Highland Burma. London: Bell.

Leakey, M. 1981. Tracks and tools. Philosophical Transactions of the Royal Society of London, Series B, 292:95-102.

Lebar, F. M. (ed.). 1972. Ethnic Groups in Insular Southeast Asia. Vol. l: Indonesia, Andaman Islands and Madagascar. New Haven: HRAF Press. 
Lebar, F. M., G. C. Hickey, and J. K. Musgrave. 1964. Ethnic Groups of Mainland Southeast Asia. New Haven: HRAF Press.

Le Gros Clark, W. E. 1964. The Fossil Evidence for Human Evolution. University of Chicago Press.

Leinders, J. J. M., F. Aziz, P. Y. Sondaar, and J. de Vos. 1985. The age of the hominidbearing deposits of Java: State of the art. Geologie en Mijnbouw 64:167-173.

Leong Sau Heng. 1990. A tripod pottery complex in Peninsular Malaysia. In I. and E. Glover (eds.), Southeast Asian Archaeology 1986, 65-76. Oxford: BAR International Series 561.

1991. Jenderam Hilir and the mid-Holocene prehistory of the west coast plain of Peninsular Malaysia. BIPPA 10:150-160.

Leur, J. C. van. 1967. On early Asian trade. In J. C. van Leur (ed.), Indonesian Trade and Society, 1-144. The Hague: van Hoeve.

Lewis, D. 1988. People of the Source. Dordrecht: Foris.

Li, H-L. 1970. The origins of cultivated plants in Southeast Asia. Economic Botany 24: 3-19.

. 1983. The domestication of plants in China: Ecogeographical considerations. In D. H. Keightley (ed.), The Origins of Chinese Civilization, 21-64.

Li Kunsheng. 1985. The origin of Asiatic rice cultivation. Bulletin of the Ancient Orient Museum 7:93-112.

Li, K-C. 1983a. K'en-ting: An Archaeological Laboratory near Southern Tip of Taiwan. Ann Arbor: University Microfilms International.

1983b. Report of archaeological investigations in the O-Luan-Pi Park at the southern tip of Taiwan. Taipei: Department of Anthropology, National Taiwan University.

Li, K-C., K-C. Chang, A. P. Wolf, and A. C. Yin, eds. 1989. Anthropological studies of the Taiwan area: Accomplishments and prospects. Taipei: Department of Anthropology, National Taiwan University.

Li, P. J-K. 1983. Male and female forms of speech in Atayal. Paper given at 15 th Science Congress, Dunedin.

. 1985. The position of Atayal in the Austronesian family. In A. Pawley and L. Carrington (eds.), Austronesian Linguistics at the 15th Pacific Science Congress, 257-280. PL Series C88.

Li, P. J-K., D-A. Ho, Y-K. Huang, C-W. Tsang, and C-Y. Tseng, eds. 1995. Austronesian Studies Relating to Taiwan. Taipei: Academia Sinica, Institute of History and Philology, Symposium Series 3.

Lie-Injo, L. E. 1976. Genetic relationships of several aboriginal groups in Southeast Asia. In R. L. Kirk and A. G. Thorne (eds.), The Origin of the Australians, 277-306.

Lien, Chaomei. 1989. The interrelationship of Taiwan's prehistoric archaeology and ethnography. In K-C. Li et al. (eds.), Anthropological Studies of the Taiwan Area, 173-192.

- 1991. The Neolithic archaeology of Taiwan and the Peinan excavations. BIPPA 11:339-352.

1993. Pei-nan: A Neolithic village. In G. Burenhult (ed.), People of the Stone Age, 132-133. San Francisco: Harper. 
Lincoln, G. A. 1975. Bird counts either side of Wallace's Line. Journal of the Zoological Society of London 177:349-361.

Linehan, W. 1951. Traces of a Bronze Age culture associated with Iron Age implements in the regions of Klang and the Tembeling, Malaya. JMBRAS 24(3): $1-59$.

Linsley, B. K. 1996. Oxygen-isotope record of sea level and climate variations in the Sulu Sea over the past 150,000 years. Nature 380:234-237.

Littlefield, A., L. Lieberman, and L. T. Reynolds. 1982. Redefining race: The potential demise of a concept in physical anthropology. CA 23:641-656.

Liu Jun. 1985. Some observations on the arhaeological site of Hemudu, Zhejiang Province, China. BIPPA 6:40-45.

Liu, T., and M. Ding. 1983. Discussion on the age of "Yuanmou Man." Acta Anthropologica Sinica 2(1):48-58.

Loeb, E. M. 1935. Sumatra. Wiener Beiträge zur Kulturgeschichte und Linguistik III.

Loeb, E. M., and J. O. M. Broek. 1947. Social organization and the longhouse in Southeast Asia. American Anthropologist 49:414-425.

Loewenstein, J. 1956. The origin of the Malayan Metal Age. JMBRAS 29(2):5-78.

Loofs-Wissowa, H. 1980-1981. Prehistoric and protohistoric links between the Indochinese Peninsula and the Philippines. JHKAS 9:57-76.

1991. Dongson drums: Instruments of shamanism or regalia? Arts Asiatiques 46:39-49.

Loomis, W. F. 1967. Skin pigment regulation of vitamin biosynthesis in man. Science 157:501-506.

Lovejoy, C. O. 1970. The taxonomic status of the Meganthropus mandibular fragments from the Djetis beds of Java. Man (new series) 5:228-236. 1981. The origin of man. Science 211:341-350.

Loy, T., M. Spriggs, and S. Wickler. 1992. Direct evidence for human use of plants 28,000 years ago. A 66:898-912.

Lum, J. K., et al. 1994. Polynesian mitochondrial DNAs reveal three deep maternal lineage clusters. Human Biology 66:567-590.

Lynch, F. X., and J. F. Ewing. 1968. Twelve ground stone implements from Mindanao, Philippine Islands. APAS 2:7-20.

Mabbett, I. W. 1977. The "Indianization" of Southeast Asia. JSEAS 8:1-14, 143-161.

Maceda, M. N. 1974. Artificial cranial deformation. In First Regional Seminar on Southeast Asian Prehistory and Archaeology, 27-36. Manila: National Museum of the Philippines.

Macintosh, N. W. G. 1978. The Tabon Cave mandible. APAO 13:143-159.

Macintosh, N.W. G., and S. L. Larnach. 1976. Aboriginal affinities looked at in world context. In R. L. Kirk and A. G. Thorne (eds.), The Origin of the Australians, 113-126.

Macknight, C. C. 1973. The emergence of civilization in South Celebes and elsewhere. In A. Reid and L. Castles (eds.), Pre-Colonial State Systems in Southeast Asia, 126-135. Malaysian Branch, Royal Asiatic Society, Monograph 6.

MacNeish, R. 1992. The Origins of Agriculture and Settled Life. Norman: University of Oklahoma Press. 
Magee, J. 1988. Oriented soil samples from the Tingkayu Basin. In P. Bellwood, Archaeological research in South-eastern Sabah, 31-37.

Maglio, V.J. 1973. Origin and evolution of the Elephantidae. Transactions of the American Philosophical Society 63(3):1-149.

Malleret, L. 1959-1963. L'Archéologie du Delta du Mekong (4 vols.). Paris: Ecole Française d'Extrême-Orient.

Maloney, B. K. 1985. Man's impact on the rainforests of West Malesia: The palynological record. Journal of Biogeography 12:537-558.

-1994. The prospects and problems of using palynology to trace the origins of tropical agriculture: The case of Southeast Asia. In J. Hather (ed.), Tropical Archaeobotany, 139-171. London: Routledge.

Manguin, Y. 1989. The trading ships of insular Southeast Asia: New evidence from Indonesian archaeological sites. In Pertemuan Ilmiah Arkeologi V, Vol. 1, 200220. Jakarta: Pusat Penelitian Arkeologi Nasional.

. 1992. Excavations in South Sumatra 1988-90. In I. Glover (ed.), Southeast Asian Archaeology 1990, 63-74. University of Hull: Centre for Southeast Asian Studies.

Manning, A., E. E. McKinnon, and F. E. Treloar. 1980. Analysis of gold artefacts from the Kota Cina site, near Medan, Sumatra. JMBRAS 53(2): 102-116.

Marcus, L. F., and W. S. Newman. 1983. Hominid migrations and the eustatic sea level paradigm: A critique. In P. M. Masters and N. C. Flemming (eds.), Quaternary Coastlines and Marine Archaeology, 63-86. London: Academic Press.

Maringer, J., and Th. Verhoeven. 1970a. Die oberflächenfunde aus dem Fossilgebiet von Mengeruda und Olabula auf Flores, Indonesien. Anthropos 65:530-546.

-1970b. Die Steinartefakte aus der Stegodon-Fossilschicht von Mengeruda auf Flores. Anthropos 65:229-247.

Maringer, J., and J. Verschuuren. 1981. Zum Paläolithikum der Insel Timor, Indonesien. Anthropos 76:584-588.

Marrison, G. E. 1975. The early Cham language and its relationship to Malay. IMBRAS 48(2): 52-60.

Marschall, W. 1968. Metallurgie und frühe Besiedlungsgeschichte Indonesiens. Ethnologica 4:31-263.

1980. Enggano and Nias. In W. Stohr (ed.), Art of the Archaic Indonesians, 1942. Geneva: Musée d'Art et d'Histoire.

Massing, A. W. 1981. The journey to paradise. BRB 13(2):85-104.

Matisoff, J. A. 1991. Sino-Tibetan linguistics: Present state and future prospects. Review of Anthropology 20:469-504.

Matsu'ura, S. 1982. A chronological framing for the Sangiran hominids. Bulletin of the National Science Museum, Tokyo, Series D (Anthropology) 8:1-53.

Matthews, P. 1995. Aroids and the Austronesians. Tropics 4:105-126.

Mayr, E. 1945. Wallace's Line in the light of recent zoogeographic study. In P. Honig and F. Verdoorn (eds.), Science and Scientists in the Netherlands Indies, 241-250.

McCabe, R., and J. Cole. 1989. Speculations on the late Mesozoic and Cenozoic evoIution of the Southeast Asian margin. In Z. Ben-Avraham (ed.), The Evolution of the Pacific Ocean Margins, 143-160. Oxford: Clarendon Press. 
McConnell, J., and I. Glover. 1990. A newly found bronze drum from Bali, Indonesia: Some technical considerations. MQRSEA 11:1-38.

McCorriston, J., and F. Hole. 1991. The ecology of seasonal stress and the origins of agriculture in the Near East. American Anthropologist 93:46-69.

McDonald, P. 1980. An historical perspective to population growth in Indonesia. In J. J. Fox (ed.), Indonesia: The Making of a Culture, 81-94.

McKinley, R. 1978. Pioneer expansion, assimilation and the foundation of ethnic unity among the Iban. SMJ 47:15-28.

McKinnon, E. E. 1991. The Hoabinhian in the Wampu/Lau Biang Valley of northeastern Sumatra: An update. BIPPA 10:132-142.

McKinnon, E. E., H. Djafar, and M. P. Soeroso. 1994. Tarumanagara? A note on discoveries at Batujaya and Cibuaya, West Java. Unpublished paper given at 15 th Congress of the Indo-Pacific Prehistory Asssociation, Chiang Mai.

Meacham, W. 1984-1985a. C14 dating of pottery. JHKAS 11:108-111.

. 1984-1985b. On the improbability of Austronesian origins in South China. AP 26:89-106.

1995. Austronesian origins and the peopling of Taiwan. In P. J-K. Li et al. (eds.), Austronesian Studies Relating to Taiwan, 227-253.

Medway, Lord. 1965. Niah cave animal bone VIII: Rhinoceros in late Quaternary Borneo. SMJ 12:77-82.

- 1972. The Quaternary mammals of Malesia: A review. In P. and M. Ashton (eds.), The Quaternary Era in Malesia, 63-83.

-1973. The antiquity of domesticated pigs in Sarawak. IMBRAS 46(2): 167-178.

1977a. The Niah excavations: And an assessment of the impact of early man on mammals in Borneo. AP 20:51-69.

- 1977b. The ancient domestic dogs of Malaysia. IMBRAS 50(1):14-27.

- 1978. The wild pig remains from the West Mouth, Niah Cave. SMJ 25:21-39.

Meer, N. C. van Setten van der. 1979. Sawah Cultivation in Ancient Java. Canberra: Australian National University Press.

Mellars, P., and C. Stringer, eds. 1989. The Human Revolution. Edinburgh University Press.

Melton, T., et al. 1995. Polynesian genetic affinities with Southeast Asian populations as identified by mitochondrial DNA analysis. American Journal of Human Genetics, 57:403-414.

Merimee, T. J., J. Zapf, and E. R. Froesch. 1981. Dwarfism in the Pygmy. New England Journal of Medicine 305(17):905-908.

Metcalf, P. 1982. A Borneo Journey into Death. Philadelphia: University of Pennsylvania Press.

Metcalfe, I. 1996. Pre-Cretaceous evolution of SE Asian terranes. In R. Hall and D. J. Blundell (eds.), Tectonic evolution of Southeast Asia, 97-122. London: Geological Society Special Publication 106.

Metzner, J. K. 1982. Agriculture and Population Pressure in Sikka, Isle of Flores. Canberra: Australian National University Press.

Michael, H. N. 1984. Absolute chronologies of late Pleistocene and early Holocene cultures of northeastern Asia. Arctic Anthropology 21/2:1-68. 
Mijsberg, W. A. 1932. Recherches sur les restes humaines trouvés dans les fouilles des abris-sous-roche de Goea Lawa à Sampoeng et des sites préhistoriques a Bodjonegoro. In Hommage du Service Archéologique des Indes Neérlandaises au Ir Congrès des Préhistoriens d'Extrême-Orient à Hanoi, 39-54. Batavia: Albrecht.

. 1940. On a Neolithic Palae-Melanesian lower jaw fragment found at Guak Kepah, Province Wellesley, Straits Settlements. In F. N. Chasen and M. W. F. Tweedie (eds.), Proceedings of the Third Congress of Prehistorians of the Far East, 100-118.

Miksic, J. 1985. Traditional Sumatran trade. Bulletin de l'Ecole Française d'ExtrêmeOrient 74:423-467.

1990. Old Javanese Gold. Singapore: Ideation.

Miksic, J. N. 1979. Archaeology, Trade and Society in Northeast Sumatra. Ann Arbor: University Microfilms International.

Miles, D. 1972. Land, labour and kin groups among Southeast Asian shifting cultivators. Mankind 8:185-197.

1979. The finger knife and Occam's razor. American Ethnologist 6:223-243.

Miller, J. I. 1969. The Spice Trade of the Roman Empire. Oxford: Clarendon Press.

Misra, V. N., and P. S. Bellwood, eds. 1985. Recent Advances in Indo-Pacific Prehistory. New Delhi: Oxford and IBH.

Mitchell, A., and V. Weitzell. 1983. Monkeys and men in the land of mud. Hemisphere 27(5):308-314.

Mithun, M. 1984. The Proto-Iroquoians. In M. K. Foster et al. (eds.), Extending the Rafters. Albany: State University of New York Press.

Mizukoshi, M. 1971. Regional divisions of monsoon Asia by Koppen's classification of climate. In M. M. Yoshino (ed.), Water Balance of Monsoon Asia: A Climatological Approach, 259-273. Honolulu: University of Hawai'i Press.

Mohr, E. C. J. 1945. Climate and soil in the Netherlands Indies. In P. Honig and F. Verdoorn (eds.), Science and Scientists in the Netherlands Indies, 250-254.

Molengraaff, G. A. F. 1921. Modern deep-sea research in the East Indian Archipelago. Geographical Journal 57:95-118.

Moore, A., and G. C. Hillman. 1992. The Pleistocene to Holocene transition and human economy in Southwest Asia: The impact of the Younger Dryas. American Antiquity 57:482-494.

Moore, J. 1994. Putting anthropology back together again. American Anthropologist 96:925-948.

Mori, T. 1956. Archaeological study of jar-burials in Eneolithic Japan. In Proceedings 4th Far-Eastern Prehistory and the Anthropology Division of the 8th Pacific Science Congresses Combined, part 1, fascicle 2, section 1, 225-245. Quezon City.

Morlan, V. J. 1971. The preceramic period of Japan: Honshu, Shikoku and Kyushu. Arctic Anthropology 8:136-170.

Morley, R. J., and J. R. Flenley. 1987. Late Cenozoic vegetational and environmental changes in the Malay Archipelago. In T. C. Whitmore (ed.), Biogeographical Evolution of the Malay Archipelago, 50-59.

Mountain, M-J. 1983. Preliminary report of excavations at Nombe rockshelter, Simbu Province, Papua New Guinea. BIPPA 4:84-99. 
Movius, H. L. 1944. Early man and Pleistocene stratigraphy in South and East Asia. Cambridge: Harvard University, Papers of the Peabody Museum 19(3).

. 1948. The Lower Palaeolithic cultures of southern and eastern Asia. Transactions of the American Philosophical Society (new series) 38(4):329-420.

. 1955. Palaeolithic archaeology in southern and eastern Asia, exclusive of India. Cahiers d'Histoire Mondiale 2:157-182, 520-553.

Mulia, R. 1980. Beberapa catatan tentang arca-arca yang disebut arca tipe Polinesia. In Pertemuan Ilmiah Arkeologi 599-646. Jakarta: Pusat Penelitian Arkeologi Nasional.

Muller, J. 1975. Pollen-analytical studies of peat and coal from northwest Borneo. MQRSEA 1:83-86.

Mulvaney, D. J. 1970. The Patjitanian industry: Some observations. Mankind 7:184187.

1985. Australian backed-blade industries in perspective. In V. N. Misra and P. S. Bellwood (eds.), Recent Advances in Indo-Pacific Prehistory, 211-217.

Mulvaney, D. J., and R. P. Soejono. 1970. The Australian-Indonesian archaeological expedition to Sulawesi. AP 13:163-178.

1971. Archaeology in Sulawesi, Indonesia. A 45:26-33.

Murdock, G. P. 1960a. Social Structure. New York: Macmillan.

- 1964. Genetic classification of the Austronesian languages: A key to Oceanic culture history. Ethnology 3:117-126.

—, ed. 1960b. Social Structure in Southeast Asia. New York: Viking Fund Publication in Anthropology 29.

Musser, G. G. 1981. The giant rat of Flores and its relatives east of Borneo and Bali. Bulletin of the American Museum of Natural History 169: article 2.

1982. The Trinil rats. MQRSEA 7:65-86.

1987. The mammals of Sulawesi. In T. C. Whitmore (ed.), Biogeographical Evolution of the Malay Archipelago, 73-93.

Naerssen, F. H. van. 1977. Economic history in early Indonesia. In F. H. van Naerssen and R. C. de Iongh, The Economic and Administrative History of Early Indonesia, 1-84. Leiden: Brill.

Nance, J. 1975. The Gentle Tasaday. New York: Harcourt Brace Jovanovitch.

Needham, R. 1954. Penan and Punan. JMBRAS 27(1):73-83.

Neel, J. V. 1967. Genetic structure of primitive human populations. Japanese Journal of Human Genetics 12:1-16.

Nei, M., and A. K. Roychoudhury. 1993. Evolutionary relationships of human populations on a global scale. Molecular Biology and Evolution 10:927-943.

New Researches into Prehistory of Viet Nam. 1988. Ha Noi: Archaeological Institute of the Social Sciences Commission of the Socialist Republic of Viet Nam.

Newsome, J., and J. R. Flenley. 1988. Late Quaternary vegetational history of the Central Highlands of Sumatra. Journal of Biogeography 15:555-578.

Newton, D., and J.-P. Barbier. 1988. Islands and Ancestors. New York: Metropolitan Museum of Art.

Ngo, T.P. 1984-1985. Some archaeological questions of the North Vietnamese coastal areas in relation to Austronesian origins. AP 26:147-152. 
Nguyen Duy Ty. 1991. Nouvelles recherches sur le culture de Sa Huynh au Viet Nam. In Recherches en Archéologie en Thaï. Deuxième Symposium Franco-Thai. Bangkok: Silpakorn University.

Nguyen Kim Dung. 1995. Ornaments from jar burial sites in Can Gio district, Ho Chi Minh City. Khao Co Hoc for 1995, no. 2:27-46.

Nicholl, R., ed. 1975. European Sources for the History of the Sultanate of Brunei in the Sixteenth Century. Brunei Museum.

Nicolaisen, I. 1976. The Penan of Sarawak. Folk 18:205-236. 1977-1978. The dynamics of ethnic classification: A case study of the Punan Bah of Sarawak. Folk 19-20:183-200.

Nik Hassan Shuhaimi. 1991. Recent research at Kuala Selinsing, Perak. BIPPA 11:141152.

Nik Hassan Shuhaimi and Othman Yatim. 1990. Antiquities of Bujang Valley. Kuala Lumpur: Museum Association of Malaysia.

Nik Hassan Shuhaimi, et al. 1990. Tapak Presejarah Gua Bukit Ta'at Hulu Terengganu. JAM 3:1-14.

Nimmo, H. 1967. Review of Sopher, D. 1965. Philippine Studies 15:209-212.

. 1968. Reflections on Bajau history. Philippine Studies 16:32-59.

. 1972. The Sea People of Sulu. London: Intertext.

Ninkovitch, D., and L. H. Burckle. 1978. Absolute age of the base of the hominidbearing beds in eastern Java. Nature 275:306-308.

Nitecki, M. H. and D. V., eds. 1994. Origins of Anatomically Modern Humans. New York: Plenum.

Nitta, E. 1985. Pre-Heger I bronze drums from Yunnan. Shiroku 18.

-1996. Comparative study on the jar burial traditions in Vietnam, Thailand and Laos. Kagoshima University Historical Science Reports 43: 1-19.

Nix, H. A., and J. D. Kalma. 1972. Climate as a dominant control in the biogeography of northern Australia and New Guinea. In D. Walker (ed.), Bridge and Barrier, 61-91. Canberra: Australian National University Press.

Noorduyn, J., and H.T. Verstappen. 1972. Purnavarman's river-works near Tugu. BTLV 128:298-307.

Nooy-Palm, H. 1979 The Sa'dan Toraja. The Hague: Nijhoff.

Nothofer, B. 1975. The Reconstruction of Proto-Malayo-Javanic. The Hague: Nijhoff.

- 1985. The subgrouping of the languages of the Javo-Sumatra Hesion: A reconsideration. BTLV 141:288-302.

1986. The Barrier Island languages in the Austronesian language family. In FOCAL II: Papers from the 4th International Conference on Austronesian Linguistics, 87-109. PL Series C94.

1988. A discussion of two Austronesian subgroups: Proto-Malay and ProtoMalayic. In M. T. Ahmad and Z.M. Zaim (eds.), Rekonstruksi dan CabangCabang Bahasa Melayu Induk, 34-58. Kuala Lumpur: Deman Bahasa dan Pustaka.

1991. Current interpretations of Western Malayo-Polynesian linguistic prehistory. BIPPA 12:388-397.

- 1994. The relationships between the languages of the Barrier Islands and the 
Sulawesi-Philippine languages. In T. Dutton and D. Tryon (eds.), Language Contact and Change in the Austronesian World, 389-410.

Oda, S., and C. Keally. 1992. The origin and early development of axe-like and edgeground stone tools in the Japanese Palaeolithic. BIPPA 12:23-31.

Oey, E., ed. 1991. Sumatra I. Singapore: Periplus.

Ogawa, H. 1993. Lal-lo shell middens on the lower Cagayan River. Japan Society for Southeast Asian Archaeology 13:64-66.

Ohtsuka, R. 1977. The sago-eaters: An ecological discussion with special reference to the Oriomo Papuans. In J. Allen et al. (eds.), Sunda and Sahul, 465-492.

Oka, H-l. 1975. The origin of cultivated rice and its adaptive evolution. In Rice in Asia 21-34. University of Tokyo Press. 1988. Origin of Cultivated Rice. Amsterdam: Elsevier.

Oka, H-I., and W. T. Chang. 1960. Survey of variations in photoperiodic response in wild Oryza species. Botanical Bulletin, Academia Sinica 1:1-14. Taipei.

Ollier, C. D. 1985. The geological background to prehistory in island Southeast Asia. MQRSEA 9:25-42.

Olsen, J. W., and S. Miller-Antonio. 1992. The Palaeolithic in southern China. AP 31: 129-160.

Omar, M. 1981. Archaeological Excavations in Protohistoric Brunei. Brunei Museum.

Omoto, K. 1981. The genetic origins of the Philippine Negritos. CA 22:421-422.

- 1987. Population genetic studies in the Philippines. Man and Culture in Oceania 3 (Special Issue):33-40.

Oppenoorth, W. F. F. 1932. Homo javanthropus een Pleistocene mensch van Java. Dienst van den Mijnbouw, Wetenschappelijke Mededelingen 20:49-63.

- 1936. Een prehistorisch cultuur-centrum langs de Solo-Rivier. TKNAG 53: 399-411.

Orban-Segebarth, R., and F. Procureur. 1983. Tooth size of Meganthropus palaeojavanicus. JHE 12:711-720.

Orchiston, D. W. 1978. The supposed Javan affinities of the tula "adze-flake" from Australia. MQRSEA 4:1-18.

Orchiston, D. W., and W. G. Siesser. 1982. Chronostratigraphy of the Plio-Pleistocene fossil hominids of Java. MQRSEA 7:131-150.

O'Reilly, D. 1995. An archaeological analysis of the initial occupation phase at Nong Nor, Thailand. Unpublished M.A. Thesis, University of Otago, New Zealand.

Orsoy de Flines, E. W. van. 1969. Guide to the Ceramic Collection. Second ed. Jakarta: Museum Pusat.

Padoch, C. 1983. Agricultural practices of the Kerayan Lun Dayeh. BRB 15(1):30-33.

- 1985. Labor efficiency and intensity of land use in rice production: An example from Kalimantan. Human Ecology 13:271-289.

Pallesen, A. K. 1985. Culture Contact and Language Convergence. Manila: Linguistic Society of the Philippines.

Parkin, R. 1991. A Guide to Austroasiatic Speakers and their Languages. Honolulu: University of Hawai'i Press.

Parmentier, H. 1924. Dêpots de jarres à Sa Huynh (Quang-ngai, Annam). Bulletin de l'Ecole Française d'Extrême-Orient 24:325-343. 
Pawley, A. K. 1974. Austronesian languages. Encyclopaedia Britannica. Fifteenth ed., Macropaedia 2:484-494.

1981. Melanesian diversity and Polynesian homogeneity: A unified explanation for language. In K. Hollyman and A. K. Pawley (eds.), Studies in Pacific Languages and Cultures, 269-309. Auckland: Linguistic Society of New Zealand.

-1995. C. L. Voorhoeve and the Trans New Guinea Phylum hypothesis. In

C. Baak, M. Bakker, and D. van der Meij (eds.), Tales from a Concave World, 83123. Leiden University: Department of Languages and Cultures of South-East Asia and Oceania.

Pawley, A. K., and M. Pawley. 1994. Early Austronesian terms for canoe parts and seafaring. In A. K. Pawley and M. Ross (eds.), Austronesian Terminologies, Continuity and Change, 329-362.

Pawley, A. K., and M. Ross. 1993. Austronesian historical linguistics and culture history. Review of Anthropology 22:425-459.

1995. The prehistory of the Oceanic languages: A current view. In P. Bellwood, J. J. Fox, and D. Tryon (eds.), The Austronesians, 39-74.

, eds. 1994. Austronesian Terminologies, Continuity and Change. PL Series C-127.

Peacock, B. A. V. 1959. A short description of Malayan prehistoric pottery. AP 3:121156.

1964a. The Kodiang pottery cones. FMJ 9:4-18.

. 1964b. A preliminary note on the Dong-s'on bronze drums from Kampong Sungei Lang. FMJ 9:1-3.

1971. Early cultural development in South-East Asia with special reference to the Malay Peninsula. APAO 6:107-123.

- 1979. The later prehistory of the Malay Peninsula. In R. B. Smith and W. Watson (eds.), Early South East Asia, 199-214.

Pearson, R. 1968. Archaeological investigations in eastern Taiwan. AP 12:137-156. . 1969. Archaeological survey in south-eastern Taiwan. Bulletin of the Institute of Archaeology, Academia Sinica 30:317-330. Taipei.

- 1989. Taiwan and its place in East Asia prehistory. In K-C. Li et al. (eds.), Anthropological Studies of the Taiwan Area, 1989:111-142.

Pelras, C. L. 1981. Célèbes-sud avant l'Islam selon les premiers témoignages étrangers. Archipel 21:153-184.

(in press). The Buginese. Oxford: Basil Blackwell.

Perry, W. J. 1918. The Megalithic Culture of Indonesia. Manchester University Press.

Peterson, J. T. 1978. The Ecology of Social Boundaries. University of Illinois Press.

Peterson, W. 1974. Summary report of two archaeological sites from north-eastern Luzon. APAO 9:26-35.

Peterson, W. and the University of Philippines Field School. 1979. Archaeological research in the Novaliches Watershed, Philippines. AP 22:120-139.

Pfeffer, P. 1974. Fauna of humid tropical Asia. In UNESCO, Natural Resources of Humid Tropical Asia, 295-306.

Pham Huy Thong, ed. 1990. Dong Son Drums in Viet Nam. Ha Noi: Viet Nam Social Science Publishing House. 
Pham Ly Huong. 1994. Radiocarbon dates of the Hoabinh culture in Vietnam. Vietnam Social Sciences 5(43):9-21.

Pietrusewsky, M. 1984. Metric and non-metric cranial variation in Australian Aboriginal populations compared with populations from the Pacific and Asia. Canberra: Australian Institute of Aboriginal Studies, Occasional Papers in Human Biology 3.

Pietrusewsky, P., et al. 1992. Modern and near modern populations of Asia and the Pacific: A multivariate craniometric interpretation. In T. Akazawa et al. (eds.), The Evolution and Dispersal of Modern Humans in Asia, 531-558.

Pirazzoli, P. A., et al. 1991. Quaternary raised coral-reef terraces on Sumba Island, Indonesia. Science 252:1834-1836.

Polak, B. 1975. Character and occurrence of peat deposits in the Malaysian tropics. MQRSEA 1:71-82.

Polunin, I. 1953. The medical natural history of Malayan aborigines. Medical Journal of Malaya 8:55-175.

Polunin, I., and H. A. Sneath. 1953. Studies of blood groups in Southeast Asia. Journal of the Royal Anthropological Institute 83:215-251.

Pookajorn, S. 1990. Hoabinhian cave excavations in Ban Kao District, West Thailand. In I. and E. Glover (eds.), Southeast Asian Archaeology 1986, 11-28. Oxford: BAR International Series 561.

- 1994. Recent archaeological data of human activities and environmental changes during the late Pleistocene to middle Holocene in southern Thailand and Southeast Asia. Unpublished ms.

Pope, G. G. 1984. The antiquity and palaeoenvironment of the Asian Hominidae. In R. O. Whyte (ed.), The Evolution of the Asian Environment, Vo1. II, 822-847. Centre of Asian Studies, University of Hong Kong.

- 1985. Taxonomy, dating and palaeoenvironment: The palaeoecology of the early Far Eastern hominids. MQRSEA 9:65-80.

__ 1992. Replacement versus regionally continuous models: The palaeobehavioural and fossil evidence. In T. Akazawa et al. (eds.), The Evolution and Dispersal of Modern Humans in Asia, 3-14.

Postma, A. 1991. The Laguna copper-plate inscription. BIPPA 11:160-171.

PPAN. 1988. Rapat Evaluasi Hasil Penelitian Arkeologi III. Jakarta: Pusat Penelitian Arkeologi Nasional.

- 1992. Laporan Kegiatan Penelitian Arkeologi selama Pelita III. Jakarta: Pusat Penelitian Arkeologi Nasional.

Prentice, M. L., and G. H. Denton. 1988. The deep-sea oxygen isotope record, the global ice sheet system and human evolution. In F. Grine (ed.), Evolutionary History of the Robust Australopithecines, 383-404. New York: Aldine de Gruyter.

Presland, G. 1980. Continuity in Indonesian lithic traditions. The Artefact 5:19-45. Rackham, H. 1952. Pliny: Natural History, Vol. IV. London: Heinemann.

Rainbird, P. 1994. Prehistory in the tropical northwest Pacific. Journal of World Prehistory $8: 293-349$. 
Rambo, A. T. 1988. Why are the Semang? In A. T. Rambo, K. Gillogly, and K. L. Hutterer (eds.), Ethnic diversity and the control of natural resources in Southeast Asia, 19-35. Ann Arbor: Michigan Papers on South and Southeast Asia 32.

Rampino, M. R., and S. Self. 1992. Volcanic winter and accelerated glaciation following the Toba super-eruption. Nature 359:50-52.

Rangin, C., L. Jolivet, M. Pubellier, and the Tethys Pacific Working Group. 1990. A simple model for the tectonic evolution of Southeast Asia and Indonesia region for the past 43 m.y. Bulletin de la Société Géologique de France VI(6):889905.

Rausa-Gomez, L. 1967. Sri Vijaya and Madjapahit. Philippine Studies 15:63-107.

Rawson, J. 1983. The Chinese Bronzes of Yunnan. London: Sidgwick and Jackson.

Ray, H. P. 1991. In search of Suvarnabhumi. BIPPA 10:357-365.

- 1997. The emergence of urban centres in Bengal: Implications for the late prehistory of Southeast Asia. BIPPA 16:43-48.

Redd, A. J, et al. 1995. Evolutionary history of the COII/tRNALys intergenic 9 b.p. deletion in human mitochondrial DNAs from the Pacific. Molecular Biology and Evolution 12:604-615.

Reed, C. A., ed. 1977. Origins of Agriculture. The Hague: Mouton.

Reid, A. 1980. The structure of cities in Southeast Asia. JSEAS 11(2):235-250.

Reid, L. A. 1982. The demise of Proto-Philippines. In A. Halim et al. (eds.), Papers from the Third International Conference on Austronesian Linguistics, Vol, 2, 201216. PL Series C-75. 1984-1985. Benedict's Austro-Tai hypothesis: An evaluation. AP 26:19-34.

- 1987. The early switch hypothesis. Man and Culture in Oceania 3 (Special Issue):61-70.

1994a. Possible Non-Austronesian Lexical elements in Philippine Negrito languages. $O L$ 33:37-72.

1994b. Morphological evidence for Austric. $O L$ 33:323-344.

1994c. Unravelling the linguistic histories of Philippine Negritos. In T. Dutton and D. Tryon (eds.), Language Contact and Change in the Austronesian World, $443-476$.

1994d. Terms for rice agriculture and terrace building in some Cordilleran languages of the Philippines. In A. K. Pawley and M. Ross (eds.), Austronesian Terminologies, Continuity and Change, 363-388.

Reynolds, T. 1993. Problems in the Stone Age of South-east Asia. Proceedings of the Prehistoric Society 59:1-16.

Rightmire, J. P. 1990. The Evolution of Homo erectus. Cambridge: Cambridge University Press.

Rind, D., and D. Peteet. 1985. Terrestrial conditions at the last glacial maximum. Quaternary Research 24:1-22.

Rispoli, F. 1992. Preliminary report on the pottery of Tha Khae, Lopburi, central Thailand. In I. Glover (ed.), Southeast Asian Archaeology 1990, 129-142. University of Hull, Centre for South-East Asian Studies.

Robbins, A. H. 1991. Biological Perspectives on Human Pigmentation. Cambridge: Cambridge University Press. 
Robequain, C. 1954. Malaya, Indonesia, Borneo and the Philippines. London: Longmans, Green.

Roberts, R., R. Jones, and M. Smith. 1994. Beyond the radiocarbon barrier in Australian prehistory. $A$ 260:611-615.

Robinson, J. T. 1968. The origin and adaptive radiation of the Australopithecines. In G. Kurth (ed.), Evolution und Hominization, 150-175. Stuttgart: Gustav Fischer.

Rogers, A. R., and L. B. Jorde. 1995. Genetic evidence on modern human origins. Human Biology 67:1-36.

Ronquillo, W., et al. 1993. The 1992 archaeological reexcavation of the Balobok rockshelter. Journal of Historiographical Institute 18:1-40. Okinawa Prefectural Library, Japan.

Ross, M. D. 1988. Proto Oceanic and the Austronesian languages of Melanesia. PL Series C-98.

. 1994. Areal phonological features in north-central New Ireland. In T. Dutton and D. Tryon (eds.), Language Contact and Change in the Austronesian World, 551-572.

1995a. Some current issues in Austronesian linguistics. In D. Tryon (ed.), Comparative Austronesian Dictionary, Part 1, Fascicle 1: An introduction to Austronesian studies, 45-120. Berlin: Mouton de Gruyter.

-1995b. Reconstructing Proto-Austronesian verbal morphology: Evidence from Taiwan. In P. J-K. Li et al (eds.), Austronesian Studies Relating to Taiwan, 727-792.

Roth, H. L. 1896. The Natives of Sarawak and North Borneo (2 vols.). London: Truslove and Hanson.

Rousseau, J. 1977. Kayan agriculture. SMJ 46:129-158.

- 1978. The Kayan. In V. T. King (ed.), Essays on Borneo Societies, 78-91.

- 1987 Kayan land tenure. BRB 19:47-55.

- 1990 Central Borneo. Oxford: Clarendon.

Ruddle, K., D. Johnson, P. K. Townsend, and J. D. Rees. 1978. Palm Sago. Canberra: Australian National University Press.

Ruhlen, M. 1987. A Guide to the World's Languages. Volume 1: Classification. Stanford University Press.

Rutter, O. 1929. The Pagans of North Borneo. London: Hutchinson.

Sagart, L. 1994. Proto-Austronesian and Old Chinese evidence for Sino-Austronesian. OL 33:271-308.

Saha, N., et al. 1995. Population genetic study among the Orang Asli (Semai Senoi) of Malaysia. Human Biology 67:37-57.

Sangvichien, S., P. Sirigaroon, J. B. Jorgensen, and T. Jacob. 1969. Archaeological Excavations in Thailand. Vol. 3, part 2: The Prehistoric Thai Skeletons. Copenhagen: Munksgaard.

Santa Luca, A. P. 1980. The Ngandong fossil hominids. New Haven: Yale University Publications in Anthropology 78.

Santoso, S. 1985. The pottery from Gilimanuk, Bali. BIPPA 6:46-54.

Sartono, S. 1969. Stegodon timorensis: A pygmy species from Timor. Koninklijk Nederlands Akademie van Wetenschappen, Proceedings Series B 72:192-202. 
1973. On an additional Stegodon timorensis Sartono. Geological Survey of Indonesia, Publikasi Teknik, Seri Palaeontologi 5:1-10.

1975. Implications arising from Pithecanthropus VIII. In R. H. Tuttle (ed.), Palaeoanthropology, 327-360.

1976. Genesis of the Solo terraces. MQRSEA 2:1-21.

1979a. The age of the vertebrate fossils and artefacts from Cabenge in South Sulawesi. MQRSEA 5:65-82.

$1979 \mathrm{~b}$. The discovery of a pygmy stegodont from Sumba. MQRSEA 5:57-64.

1979c. The stratigraphy of the Sumbung Mekan site in central Java. MQRSEA

5:83-88.

1991. A new Homo erectus skull from Ngawi, east Java. BIPPA 11:23-35.

Satari, S. 1981. New finds from north-central Java. SPAFA Digest 2(2):23-28.

Sather, C. 1985. Boat crews and fishing fleets. Contributions to Southeast Asian Ethnography 4:165-214.

1990. Trees and tree tenure in Paku Iban society. Borneo Review 1:16-40.

1995. Sea nomads and rainforest hunter-gatherers. In P. Bellwood, J. J. Fox, and D. Tryon (eds.), The Austronesians, 229-268.

Sauer, C. O. 1952. Agricultural Origins and Dispersals. New York: American Geographical Society.

Saurin, E. 1973. La champ de jarres de Hang Gon près Xuan Loc (Sud Viêt-Nam). Bulletin de l'Ecole Française d'Extrême-Orient 60:329-358.

Schmeltz, J. D. E. 1904. Einige vergleichende Bemerkungen über die Kesseltrommel von Saleyer. Internationales Archiv für Ethnographie 16:158-161.

Schneeberger, W. F. 1979. Contributions to the Ethnography of Central Northeastern Borneo. Bern University: Institute of Ethnology.

Schneider, W. M. 1978. The Selako Dayak. In V. T. King (ed.), Essays on Borneo Societies, 59-77.

Schnitger, F. M. 1964. Forgotten Kingdoms in Sumatra. Reprint of 1939 edition. Leiden: Brill.

Schrire, C. 1982. The alligator rivers: Prehistory and ecology in western Arnhem Land. Canberra: Department of Prehistory, Australian National University, Terra Australis 7.

Schulte Nordholt, H. G. 1971. The Political System of the Atoni of Timor. The Hague: Nijhoff.

Scott, W. H. 1968. Prehispanic Source Materials for the Study of Philippine Prehistory. Manila: University of Santo Tomas Press.

Scrivenor, J. B., I. H. Burkill, M. A. Smith, H. K. A. Shaw, P. W. Richards, and F. E. Zeuner. 1942-1943. A discussion of the biogeographic division of the IndoAustralian archipelago. Proceedings of the Linnaean Society of London 154:120165.

Seavoy, R. E. 1973a. The transition to continuous rice cultivation in Kalimantan. Annals of the Association of American Geographers 63:218-225.

. 1973b. The shading cycle in shifting cultivation. Annals of the Association of American Geographers 63:522-528. 
Sellato, B. 1994. Nomads of the Borneo Rainforest. Honolulu: University of Hawai'i Press. Sémah, A-M. 1982a. Variations de la vegetation au Plio-Pleistocene sur les sites de Sangiran et Sambungmacan par l'analyse pollinique. $1^{\text {er }}$ Congrès International de Paléontologie Humaine, 1982:559-577. Nice.

Sémah, A-M. 1982b. A preliminary report on a Sangiran pollen diagram. MQRSEA 7:165-170.

Sémah, F. 1982. Pliocene and Pleistocene geomagnetic reversals recorded in the Gemolong and Sangiran domes (central Java). MQRSEA 7:131-150.

- 1986. Le peuplement ancien de Java: Ebauche d'un cadre chronologique. L'Anthropologie 90:359-400.

Sémah, F., S. Sartono, Y. Zaim, and T. Djubiantono. 1981. A palaeomagnetic study of Plio-Pleistocene sediments from Sangiran and Simo (central Java). MQRSEA 6:103-110.

Sémah, F., A-M. Sémah, T. Djubiantono, and T. Simanjuntak. 1992. Did they also make stone tools? JHE 23: 439-445.

Sen, A. 1995. Borneo's history in a handful of husks. New Scientist 1972:10.

Serjeantson, S. W., R. L. Kirk, and P. B. Booth. 1983. Linguistic and genetic differentiation in New Guinea. JHE 12:77-92.

Serjeantson, S. W., D. R. Ryan, and A. R. Thompson. 1983. The colonization of the Pacific: The story according to human leucocyte antigens. American Journal of Human Genetics 34:904-918.

Shackleton, N. J. 1977. Carbon-13 in Uvigerina: Tropical rainforest history and the equatorial Pacific carbonate dissolution cycles. In N. R. Anderson and A. Malahoff (eds.), The Fate of Fossil Fuel $\mathrm{CO}_{2}$ in the Oceans, 401-427. New York: Plenum.

1982. The deep-sea sediment record of climatic variability. Progress in Oceanography 11:199-218.

- 1987. Oxygen isotopes, ice volume and sea level. Quaternary Science Reviews 6: 183-190.

Shackleton, N. J., and N. D. Opdyke. 1973. Pacific core V28-238. Quaternary Research 3:39-55.

1977. Oxygen isotope and paleomagnetic evidence for early northern hemisphere glaciation. Nature 270:216-219.

Shih, X-B. 1992. Neolithic cultural systems in China. In C. M. Aikens and Song Nai Rhee (eds.), Pacific Northwest Asia in Prehistory, 125-132. Pullman: Washington State University Press.

Shutler, R., and J. C. Marck. 1975. On the dispersal of the Austronesian horticulturalists. APAO 10:81-113.

Shutler, R., M. J. Head, D. J. Donohue, A. J. Jull, M. M. Barbetti, S. Matsu'ura, J. de Vos, and P. Storm. 1994. Wadjak AMS bone apatite C14 dates. Paper given at 15 th Congress of the Indo-Pacific Prehistory Association, Chiang Mai.

Siesser, W. G., and D. W. Orchiston. 1978. Micropalaeontological re-assessment of the age of Pithecanthropus mandible C from Sangiran, Indonesia. MQRSEA 4: 25-30. 
Sieveking, G. de G. 1954. Excavations at Gua Cha, Kelantan 1954. Part 1. FMJ 1 and 2:75-143.

- 1956a. The Iron Age collections of Malaya. JMBRAS 29(2):79-138.

- 1956b. Recent archaeological discoveries in Malaya (1955). JMBRAS 29(1): 200-211.

- $1956 \mathrm{c}$. The distribution of stone bark-cloth beaters in prehistoric times. JMBRAS 29(3):79-85.

- 1962. The prehistoric cemetery at Bukit Tengku Lembu, Perlis. FMJ 7:25-54.

-1987. Gua Cha in the light of recent research. In Nik Hassan Shuhaimi (ed.), Kelantan Zaman Awal, 73-86. Kota Bharu: Muzium Negeri Kelantan.

Sim, R., and A. Thorne. 1990. Pleistocene human remains from King Island, Southeastern Australia. Australian Archaeology 31:44-51.

Simanjuntak, T., and F. Sémah. 1996. A new insight into the Sangiran Flake Industry. BIPPA 14:22-26.

Simmonds, N. W. 1966. Bananas. London: Longman.

Simmons, R. T. 1962. Blood group genes in Polynesians and comparisons with other Pacific peoples. Oceania 32:198-210.

- 1976. The biological origin of the Australian Aboriginals. In R. L. Kirk and A. G. Thorne (eds.), The Origin of the Austronesians, 307-328.

Simpson, G. G. 1977. Too many Lines: The limits of the Oriental and Australian zoogeographical regions. Proceedings of the American Philosophical Society 121:107120.

Sinha, P., and I. C. Glover. 1984. Changes in stone tool use in Southeast Asia 10,000 years ago. MQRSEA 8:137-164.

Slamet-Velsink, I. E. 1995. Emerging Hierarchies. Leiden: KITLV Press.

Smith, F. H. 1992. The role of continuity in modern human origins. In G. Bräuer and F. H. Smith (eds.), Continuity or Replacement, 145-156.

Smith, M., M. Spriggs, and B. Fankhauser, eds. 1993. Sahul in review. Canberra: Department of Prehistory, Research School of Pacific Studies, Occasional Papers 24.

Smith, R. B., and W. Watson, eds. 1979. Early South East Asia. New York: Oxford University Press.

Snell, C. A. R. D. 1938. Menschelijke Skeletresten uit de Duinformatie van Java's Zuidkust nabij Poeger. Surabaya: G. Kolff.

- 1948. Human skulls from the urn-field of Melolo, East Sumba. Acta Neerlandica Morphologiae Normalis et Pathologicae 6(3):1-20.

Snow, B. E., et al. 1986. Evidence of early rice cultivation in the Philippines. Philippine Quarterly of Culture and Society 14:3-11.

Soejono, R. P. 1969. On prehistoric burial methods in Indonesia. Bulletin of the Archaeological Institute of the Republic of Indonesia 7. 1977. Sarkofagus Bali dan Nekropolis Gilimanuk. Jakarta: Pusat Penelitian Purbakala dan Peninggalan Nasional.

. 1979. The significance of excavations at Gilimanuk (Bali). In R. B. Smith and W. Watson (eds.), Early South East Asia, 185-198. 
1982a. New data on the Palaeolithic industry in Indonesia. In ler Congrès International de Paléontologie Humaine, 78-92. Nice.

1982b. On the megaliths in Indonesia. In B-M. Kim (ed.), Megalithic Cultures in Asia, 73-98. Seoul: Hanyang University Press.

. 1984. Jaman Prasejarah di Indonesia. Sejarah Nasional Indonesia Vol. 1. Jakarta: Balai Pustaka.

1987. Stone tools of Palaeolithic type in Lombok. Man and Culture in Oceania 3 (Special Issue):81-104.

1991. Important prehistoric discoveries in Indonesia. JAM 4:16-22. 1995. A late prehistoric burial system in Indonesia: Additional notes on Gilimanuk, Bali. In Conference Papers on Archaeology in Southeast Asia, 180-190. Hong Kong: University of Hong Kong Museum and Art Gallery.

Solheim, W. G. II. 1960. Jar burial in the Babuyan and Batanes Islands and in central Philippines, and its relation to jar burial elsewhere in the Far East. Philippine Journal of Science 89(1):115-148.

- 1964a. Pottery and the Malayo-Polynesians. CA 5:360, 376-384, 400-403. 1964b. The Archaeology of Central Philippines. Manila: Bureau of Printing.

. 1967. Two pottery traditions of late prehistoric times in Southeast Asia. In

F. S. Drake (ed.), Symposium on Historical, Archaeological and Linguistic Studies on Southern China, Southeast Asia, and the Hong Kong Region, 15-22. Hong Kong University Press.

- 1968. The Batungan cave sites, Masbate, Philippines. APAS 2:21-62.

1975. Reflections on the new data of Southeast Asian prehistory: Austronesian origins and consequence. AP 18:146-160.

1979a. New data on late Southeast Asian prehistory and their interpretation. JHKAS 8:73-87.

1979b. A look at "L'art prébouddhique de la Chine et de l'Asie du Sud-Est et son influence en Océanie" forty years after. AP 22:165-205.

- 1980. Searching for the origins of the orang asli. FMJ 25:61-76.

1984-1985. The Nusantao hypothesis. AP 26:77-88.

1990. Earthenware pottery, the T'ai and the Malay. AP 29:25-36.

1996. The Nusantao and north-south dispersals. BIPPA 15:101-109.

Solheim, W. C. II, B. Harrisson, and L. Wall. 1959. Niah "Three Colour Ware" and related prehistoric pottery from Borneo. AP 3:167-176.

Sondaar, P. Y. 1981. The Geochelone faunas of the Indonesian archipelago. MQRSEA 6:111-119.

. 1984. Faunal evolution and the mammalian biostratigraphy of Java. In

P. Andrews and P. L. Franzen (eds.), The Early Evolution of Man, 219-236. Frankfurt: Courier Forschungsinstitut Senckenberg no. 69.

Sopher, D. E. 1965. The Sea Nomads. Singapore: National Museum.

Sørensen, P. 1972. The Neolithic cultures of Thailand (and north Malaysia) and their Lungshanoid relationships. In N. Barnard (ed.), Early Chinese Art and Its Possible Influence in the Pacific Basin, vol. 2, 459-506.

1973. Prehistoric iron implements from Thailand. AP 16:134-173. 
1985. Agricultural civilizations. In C. Flon (ed.), The World Atlas of Archaeology. London: Mitchell Beazley.

vations. London: Curzon.

Sørensen, P., and T. Hatting. 1967. Archaeological Excavations in Thailand. Vol. 2: Ban Kao. Copenhagen: Munksgaard.

Spencer, J. E. 1963. The migration of rice from mainland Southeast Asia into Indonesia. In J. Barrau (ed.), Plants and the Migrations of Pacific Peoples, 83-90. Honolulu: Bishop Museum Press.

- 1966. Shifting Cultivation in Southeast Asia. Berkeley: University of California Press.

Spoehr, A. 1973. Zamboanga and Sulu. Ethnology Monograph 1, Department of Anthropology, University of Pittsburgh.

Spriggs, M. 1981. Vegetable kingdoms. Unpublished Ph.D. thesis, Australian National University.

1989 The dating of the Island Southeast Asian Neolithic. $A$ 63:587-612.

. 1990. The changing face of Lapita. In M. Spriggs (ed.), Lapita design, form and composition, 83-122. Canberra: Department of Prehistory, Research School of Pacific Studies, Occasional Papers 19.

1993. Pleistocene agriculture in the Pacific: Why not? In M. Smith, M. Spriggs, and B. Fankhauser (eds.), Sahul in review, 137-143.

- 1995. The Lapita Culture and Austronesian prehistory in Oceania. In P. Bellwood, J. J. Fox, and D. Tryon (eds.), The Austronesians, 112-133.

(in press). The Island Melanesians. Oxford: Basil BlackwelI.

St. John, S. 1974. Life in the Forests of the Far East. Reprint of 1862 original, 2 vols. Kuala Lumpur: Oxford University Press.

Stamps, R. B. 1977. An archaeological survey of the P'uli basin, west central Taiwan. Institute of History and Philology, Academia Sinica, Special Publication 70: 237-301. Taipei.

Stargardt, J. 1983. Satingpra I. Oxford: BAR International Series S158.

Starosta, S. 1995. A grammatical subgrouping of Formosan languages. In P. J-K. Li et al. (eds.), Austronesian Studies Relating to Taiwan, 683-726.

Steenis, C. G. G.J.van. 1961. Preliminary revisions of some genera of Malaysian Papilionaceae I. Reinwardtia 5:419-456.

- 1965. Concise plant-geography of Java. In C. A. Backer and R. E. Bakhuizen van den Brink (eds.), Flora of Java, vol. 2, 1-72. Groningen: Noordhoff.

Stein Callenfels, P. V. van. 1932. Note préliminaire sur les fouilles dans l'abri-sousroche du Guwa Lawa à Sampung. In Hommage du Service Archéologique des Indes Néerlandaises au ler Congrès des Prehistoriens d'Extrême-Orient à Hanoi, 25-29. Batavia: Albrecht.

- 1936a. An excavation of three kitchen middens at Guak Kepah, Province Wellesley. BRM Series B l(1):27-37.

. 1936b. A remarkable stone implement from the Malay Peninsula. BRM Series B $1(1): 38-40$. 
1936c. L'industrie osseuse de Ngandong. L'Anthropologie 46:359-362.

1938. Mededeelingen het Proto-Toaliaan. TITLV 68:579-584.

Stein Callenfels, P. V. van, and I. H. N. Evans. 1928. Report on cave excavations in Perak. JFMSM 12(6): 145-160.

Stein Callenfels, P. V. van, and H. D. Noone. 1940. A rock-shelter excavation at Sungei Siput, Perak. In F. N. Chasen and M. W. F. Tweedie (eds.), Proceedings of the Third Congress of Prehistorians of the Far East, 1940:119-125.

Stoneking, M., et al. 1990. Geographic variation in human mitochondrial DNA from Papua New Guinea. Genetics 124:171-133.

Storm, P., and A. J. Nelson. 1992. The many faces of Wadjak Man. AO 27:37-46.

Stringer, C. 1992. Replacement, continuity and the origin of Homo sapiens. In G. Bräuer and F. H. Smith (eds.), Continuity or Replacement, 9-24.

Stuijts, I., J. C. Newsome, and J. R. Flenley. 1988. Evidence for late Quaternary vegetational change in the Sumatran and Javan highlands. Review of Palaeobotany and Palynology 55:207-216.

Stuijts, I. M. 1993 Late Pleistocene and Holocene Vegetation of West Java, Indonesia. Rotterdam: Balkema.

Stutterheim, W. F. 1956. Some remarks on pre-Hinduistic burial customs in Java. In W. F. Stutterheim, Studies in Indonesian Archaeology, 63-90. The Hague: Nijhoff.

Sudjoko. 1981. Ancient Indonesian technology. Aspek-Aspek Arkeologi Indonesia 9. Jakarta: Pusat Penelitian Arkeologi Indonesia.

Sukarto, K., and M. M. Atmodjo. 1979. Notes on a protohistoric sarcophagus at Selasih in Bali. Majalah Arkeologi 2(4):61-74.

Sukendar, H. 1979. Laporan penelitian kepurbakalaan Daerah Lampung. BPA 20.

- Laporan penelitian kepurbakalaan di Sulawesi Tengah. BPA 25.

. 1985a. Peninggalan tradisi megalitik di Daerah Cianjur, Jawa Barat. Jakarta: Pusat Penelitian Arkeologi Nasional.

- 1985b. The living megalithic tradition in eastern Indonesia. BIPPA 6:55-63.

Sukendar, H., and R. D. Awe. 1981. Laporan penelitian Terjan dan Plawangan, Jawa Tengah. BPA 27.

Sukendar, H., R. I. Panggabean, and R. D. Awe. 1982. Laporan survei Pandeglang dan Ekskavasi Anyar, Jawa Barat 1979. BPA 28.

Suleiman, S. 1976. Monuments of Ancient Indonesia. Jakarta: National Research Center of Archaeology.

Summerhayes, G., and M. Walker. 1982. Elemental analysis and taxonomy of prehistoric pottery from western Java. In W. Ambrose and P. Duerden (eds.), Archaeometry: An Australasian Perspective, 60-67. Canberra: Department of Prehistory, Research School of Pacific Studies.

Sung, W-H. 1979. Prehistoric Taiwan. JHKAS 8:88-91.

Supomo, S. 1995. Indic transformation. In P. Bellwood, J. J. Fox, and D. Tryon (eds.), The Austronesians, 291-313.

Suryanto, D. 1985. Kubur peti batu Kidangan dalam perbandingan. In Pertemuan Ilmiah Arkeologi III, 142-148. Jakarta: Pusat Penelitian Arkeologi Nasional. 
Sutaba, I. M. 1976. Megalithic traditions in Sembiran, north Bali. Aspek-Aspek Arkeologi Indonesia 4. Jakarta: Pusat Penelitian Arkeologi Nasional.

- 1997. Preliminary notes on ancestor statues in Bali. BIPPA 16 (forthcoming).

Sutayasa, I. M. 1972. Notes on the Buni pottery complex, northwest Java. Mankind 8:182-184.

- 1973. The study of prehistoric pottery in Indonesia. Nusantara 4:67-82.

-1979. Prehistory in west Java, Indonesia. The Artefact 4:61-75.

Suzuki, H., and K. Hanihara. 1982. The Minatogawa Man. Tokyo: University of Tokyo Press.

Suzuki, P. 1959. The Religious System and Culture of Nias, Indonesia. The Hague: Exelsior.

Swadesh, M. 1964. Linguistics as an instrument of prehistory. In D. Hymes (ed.), Language in Culture and Society, 575-584. New York: Harper and Row.

Swadling, P., N. Araho, and B. Ivuyo. 1991. Settlements associated with the inland Sepik-Ramu Sea. BIPPA 11:92-112.

Swisher, C. C., G. H. Curtis, T. Jacob, A. G. Getty, A. Suprijo, and Widiasmoro. 1994. Age of the earliest known hominids in Java, Indonesia. Science 263:1118-1121.

Swisher, C. C., W. J. Rink, S. C. Anton, H. P. Schwartz, G. H. Curtis, A. Suprijo, and Widiasmoro. 1996. Latest Homo erectus of Java: potential contemporaneity with Homo sapiens in Southeast Asia. Science 274:1870-1874.

Takaya, Y. 1980. The agricultural landscape of the Komering River of south Sumatra. In Y. Tsubouchi et al. (eds.), South Sumatra, article 4.

Tanudirjo, D. 1991. Some behavioural aspects of the Bomo-Teleng stone adze workshop site in east Java. Unpublished M.A. thesis, Australian National University.

Tarling, N., ed. 1992. The Cambridge History of Southeast Asia (2 vols.). Cambridge: Cambridge University Press.

Tasa, G. L., et al. 1985. Reflectometer reports on human pigmentation. CA 26:511512.

Tayles, N. 1994. The health of prehistoric people in coastal central Thailand. Paper presented at 15th Congress of the Indo-Pacific Prehistory Association, Chiang Mai.

Teeter, K. V. 1963. Lexicostatistics and genetic relationships. Language 39:638-648.

Tenazas, R. 1974. A progress report on the Magsuhot excavations in Bacong, Negros Oriental. PQCS 2:133-155.

- 1985. A note on stone and shell implements from late Palaeolithic and Neolithic sites in Carcar, Cebu, Philippines. In V. N. Misra and P. Bellwood (eds.), Recent Advances in Indo-Pacific Prehistory, 207-209.

Terra, H. de. 1943. Pleistocene geology and early man in Java. Transactions of the American Philosophical Society 32:437-464.

Terrell, J. 1981. Linguistics and the peopling of the Pacific Islands. Journal of the Polynesian Society 90:225-258.

Theunissen, B., J. de Vos, P. Sondaar, and F. Aziz. 1990. The establishment of a chronological framework for the hominid-bearing deposits of Java: A historical survey. In L. F. Laporte (ed.), Establishment of a geologic framework for palaeoanthropology, 39-54. Geological Society of America Special Paper 242. 
Thiel, B. 1984-1985. Austronesian origins and expansion: The Philippines archaeological data. AP 26:119-130.

.1986-1987a. Excavations at Arku Cave, northeast Luzon, Philippines. AP 27:229-264.

1986-1987b. Excavations at the Lal-lo shellmiddens, northeast Luzon, Philippines. $A P$ 27:71-94.

. 1987. Early settlement of the Philippines, eastern Indonesia and AustraliaNew Guinea: A new hypothesis. CA 28:236-241.

- 1988-1989. Excavations at Musang Cave, northeast Luzon, Philippines. AP 28:61-82.

Thommeret, J., and Y. Thommeret. 1978. 14C datings of some Holocene sea levels on the north coast of the island of Java. MQRSEA 4:51-56.

Thompson, G. B. 1992. Archaeobotanical investigations at Khok Phanom Di, central Thailand. Unpublished Ph.D. thesis, Australian National University.

Thorne, A. G. 1980a. The arrival of man in Australia. In A. Sherratt (ed.), The Cambridge Encyclopaedia of Archaeology, 96-100. Cambridge University Press.

. 1980b. The longest link in human evolution in Southeast Asia and the settlement of Australia. In J. J. Fox (ed.), Indonesia: The Making of a Culture, 3544.

Thorne, A. G., and M. Wolpoff. 1981. Regional continuity in Pleistocene hominid evolution. AJPA 55:337-350.

- 1992. The multiregional evolution of humans. Scientific American, April 76-83.

Thurgood, G. 1994. Tai-Kadai and Austronesian: The nature of the historical relationship. OL 33:345-368.

Tillotson, D. 1994. Who invented the Dayaks? Unpublished Ph.D. thesis, Australian National University.

Tjia, H. D. 1980. The Sunda shelf, Southeast Asia. Zeitschrift für Geomorphologie 24: 405-427.

1987. Tectonics, volcanism and sea level changes during the Quaternary in Southeast Asia. In N. Thiramongkol (ed.), Economic Geology, Tectonics, Sedimentary Processes and Enviromment of the Quatemary in Southeast Asia, 3-22. Bangkok: Department of Geology, Chulalongkorn University.

Tobias, P. V. 1981. The emergence of man in Africa and beyond. Philosophical Transactions of the Royal Society of London, Series B, 292:43-55.

1992. The environmental background of hominid emergence. Human Evolution 6: 129-142.

Tobias, P. V., and G. H. R. von Koenigswald. 1964. A comparison between the Olduvai hominines and those of Java, and some implications for hominid phylogeny. Nature 204:515-518.

Trevor, J. C., and D. R. Brothwell. 1962. The human remains of Mesolithic and Neolithic date from Gua Cha, Kelantan. FM/ 7:6-22.

Tricht, B. van. 1929. Levende antiquiteiten in west Java. Djawa 9:43-120.

Tryon, D. T. 1995a. The Austronesian languages. In D. Tryon (ed.), Comparative Austronesian Dictionary. Part 1, Fascicle 1: An introduction to Austronesian studies, 5-44. Berlin: Mouton de Gruyter. 
1995b. Proto-Austronesian and the major Austronesian subgroups. In P. Bellwood, J. J. Fox, and D. Tryon (eds.), The Austronesians, 17-38.

Tsang, C-H. 1992. Archaeology of the P'eng-hu Islands. Taipei: Academia Sinica, Institute of History and Philology.

1995. New archaeological data from both sides of the Taiwan Strait. In P. J-K.

Li et al (eds.), Austronesian Studies Relating to Taiwan, 185-226.

Tsubouchi, Y., N. Iljas, Y. Takaya, and A. Rasjid Hanafiah, eds. 1980. South Sumatra: Man and Agriculture. Kyoto University, Center for Southeast Asian Studies.

Tsukada, M. 1966. Late Pleistocene vegetation and climate in Taiwan (Formosa). Proceedings of the National Academy of Sciences 55:543-548. Washington, D.C.

. 1967. Vegetation in subtropical Formosa during the Pleistocene glaciations and the Holocene. Palaeogeography, Palaeoclimatology and Palaeoecology 3:49-64.

Turner, C. G. II. 1983. Sinodonty and Sundadonty. In R. S. Vasilievsky (ed.), Late Pleistocene and Early Holocene Cultural Connections of Asia and America, 72-76. Novosibirsk: USSR Academy of Science, Siberian Branch.

- 1987. Late Pleistocene and Holocene population history of East Asia based on dental variation. AJPA 73:305-321.

1989. Teeth and prehistory in Asia. Scientific American 260/2:88-96.

-1990. Major features of Sundadonty and Sinodonty. AJPA 82:295-317.

1992. The dental bridge between Australia and Asia. AO 27:143-152.

Turner, C. G. Il, and D. R. Swindler. 1978. The dentition of New Britain West Nakanai Melanesians. AJPA 49:361-372.

Tuttle, R. H., ed. 1975. Palaeoanthropology: Morphology and Palaeoecology. The Hague: Mouton.

Tweedie, M. W. F. 1940. Report on excavations in Kelantan. JMBRAS 18(2): 1-22.

. 1953. The Stone Age in Malaya. JMBRAS 26(2): 1-90.

Tyler, D. 1992. A taxonomy of Javan hominid mandibles. Human Evolution 6: 401420.

Umbgrove, J. H. F. 1949. Structural History of the East Indies. Cambridge: Cambridge University Press.

UNESCO. 1974. Natural Resources of Humid Tropical Asia. Paris.

UNESCO. 1978. Tropical Forest Ecosystems. Paris.

Uytterschaut, H. 1992. A morphological study of some old and new Pleistocene discoveries from Java. BIPPA 12:1-9.

Veraprasert, M. 1987. Khlong Thom: An ancient bead manufacturing location and an ancient entrepôt. In SPAFA final report: Seminar in prehistory of Southeast Asia, 323-331. Bangkok: SPAFA.

Vergara, B. S. 1976. Physiological and morphological adaptability of rice varieties to climate. In Climate and Rice, 67-86.

Verstappen, H. T. 1975. On Palaeo climates and landform development in Malesia. MQRSEA 1:3-36.

Voorhoeve, C. L. 1988. The languages of the North Halmaheran Stock. PL Series A76:181-209.

1994. Contact-induced change in the non-Austronesian languages in the 
North Moluccas, Indonesia. In T. Dutton and D. Tryon (eds.), Language Contact and Change in the Austronesian World, 649-674.

Vos, J. de., S. Sartono, S. Hardja-Sasmita, and P. Y. Sondaar. 1982. The fauna from Trinil, type locality of Homo erectus: A reinterpretation. Geologie en Mijnbouw 61: 207-211.

Vos, J. de, and P. Y. Sondaar. 1982. The importance of the Dubois collection reconsidered. MQRSEA 7:35-64.

Vovin, A. 1994. Is Japanese related to Austronesian? OL 33:369-390.

Walker, D. 1980. The biogeographical setting. In J. J. Fox (ed.), Indonesia: The Making of a Culture, 21-34.

WaIker, D., and A. de G. Sieveking. 1962. The Palaeolithic industry of Kota Tampan, Perak, Malaya. Proceedings of the Prehistoric Society 28:103-139.

Walker, M. J., and S. Santoso. 1977. Romano-Indian rouletted pottery in Indonesia. AP 20:228-235.

Wall, L. 1962. Earthenwares: Prehistoric pottery common to Malaya and Borneo. SMJ 10:417-427.

Wallace, A. R. 1962. The Malay Archipelago. Originally published 1869. New York: Dover.

Wang, S. C. 1984. The Neolithic Site of Chih-shan-yen. Taipei Municipal Cultural Heritage Commission, Taiwan.

Warren, J. F. 1981 The Sulu Zone 1768-1898. Singapore: Singapore University Press.

Wasson, R. J., and R. M. Cochrane. 1979. Geological and geomorphological perspectives on archaeological sites in the Cagayan valley, northern Luzon,the Philippines. MQRSEA 5:1-26.

Watanabe, N., and D. Kadar, eds. 1985. Quaternary Geology of the Hominid Fossil Bearing Formations in Java. Bandung: Geological Research and Development Center.

Waterson, R. 1990. The Living House. Oxford: Oxford University Press.

Weidenreich, F. 1945. Giant early man from Java and South China. Anthropological Papers of the American Museum of Natural History, vol. 40, part 1.

1946. Apes, Giants and Man. University of Chicago Press.

1951. Morphology of Solo Man. Anthropological Papers of the American Museum of Natural History, vol. 43, part 3.

Wetmore, A. 1940. Avian remains from the Pleistocene of central Java. Journal of Palaeontology 14:447-450.

Wheatley, P. 1961. The Golden Khersonese. Kuala Lumpur: University of Malaya Press.

- 1965. Discursive scholici on recent papers on agricultural terracing and on related matters pertaining to northern Indochina. Pacific Viewpoint 6:123144.

. 1983. Nagara and Commandery. University of Chicago, Department of Geography, Research Paper, 207-208.

Wheeler, R., A. Ghosh, and Krishna Deva. 1946. Arikamedu: An Indo-Roman trading station on the east coast of India. Ancient India 2:17-124.

White, J. P., and J. F. O'Connell. 1982. A Prehistory of Australia, New Guinea and Sahul. Sydney: Academic Press. 
White, T. D., G. Suwa, and B. Asfaw. 1994. Australopithecus ramidus, a new species of early hominid from Aramis, Ethiopia. Nature 371:306-312.

Whitmore, T. C. 1975. Tropical Rainforests of the Far East. Oxford: Clarendon Press.

- 1981. Palaeoclimate and vegetation history. In T. C. Whitmore (ed.), Wallace's Line and Plate Tectonics, 36-42.

—

don Press.

Whyte, R. O. 1983. The evolution of the Chinese environment. In D. N. Keightley (ed.), The Origins of Chinese Civilization, 3-20.

Wilke, P. J., et al. 1972. Harvest selection and domestication in seed plants. $A$ 46: 203-209.

Willems, W. 1939. Merkwaardige praehistorische schelpartefacten van Celebes en Java. Cultureel Indiä 1:181-185.

Wilson, A. C., and R. Cann. 1992. The recent African genesis of humans. Scientific American, April 1992:68-73.

Wilson, P. 1980. Man, the Promising Primate. New Haven: Yale University Press.

Winstedt, R. 1953. The Malays: A Cultural History. Third ed. London: Routledge and Kegan Paul.

Winters, N. J. 1974. An application of dental anthropological analysis to the human dentition of two early Metal Age sites, Palawan, Philippines. AP 17:28-35.

Witkamp, H. 1920. "Kjokkenmoddinger" ter oostkust van Sumatra. TKNAG 37:572574.

Wolff, J. 1994. The place of plant names in reconstructing Proto Austronesian. In A. K. Pawley and M. Ross (eds.), Austronesian Terminologies, Continuity and Change, 511-540.

. 1995. The position of the Austronesian languages of Taiwan within the Austronesian group. In P. J-K. Li et al (eds.), Austronesian Studies Relating to Taiwan, 521-584.

Wolpoff, M. H. 1980. Palaeoanthropology. New York: Knopf.

. 1984. Evolution in Homo erectus: The question of stasis. Paleobiology 10:389406.

1985. Human evolution at the peripheries: The pattern at the eastern edge. In P. V. Tobias (ed.), Hominid Evolution: Past, Present and Future, 355-365. New York: Liss.

Wolpoff, M. H., A. G. Thorne, F. H. Smith, D. Frayer, and G. Pope. 1994. Multiregional evolution: A world-wide source for modern human populations. In M. H. and D. V. Nitecki (eds.), Origins of Anatomically Modern Humans, 175-199.

Wolpoff, M. H., X. J. Wu, and A. G. Thorne. 1984. Modern Homo sapiens origins: A general theory of hominid evolution involving the fossil evidence from East Asia. In F. H. Smith and F. Spencer (eds), The Origins of Modern Humans, 411483. New York: Liss.

Wolters, O. W. 1967. Early Indonesian Commerce: A Study of the Origins of Srivijaya. Ithaca: Cornell University Press. 
1979. Studying Srivijaya. JMBRAS 52(2):1-32.

Woo, J-K. (see also Wu Rukang). 1980. Palaeoanthropology in the New China. In

L-K. Konigsson (ed.), Current Argument on Early Man, 182-206.

Wood, B. A. 1978. Human Evolution. London: Chapman and Hall.

-1992. Origin and evolution of the genus Homo. Nature 355:783-790.

1994. The oldest hominid yet. Nature 371:280-281.

Wood, J. 1978. Population structure and genetic heterogeneity in the upper Markham Valley of Papua New Guinea. AJPA 48:463-470.

Wouden, F. A. E. van. 1968. Types of Social Structure in Eastern Indonesia. Translation of 1935 original edition. The Hague: Nijhoff.

Wright, R. V. S., ed. 1977. Stone Tools as Cultural Markers. Canberra: Australian Institute of Aboriginal Studies.

Wu Rukang. 1982. Recent advances of Chinese palaeoanthropology. Hong Kong University Press, Occasional Papers Series no. 2.

Wu Xinzhi. 1996. Mosaic evolution of humankind in China. BIPPA 15:225-228.

Wu Yaoli. 1996. Prehistoric agriculture of rice in the Yellow River Valley. BIPPA 15:223-224.

Wurm, S. A. 1978. The emerging linguistic picture and linguistic prehistory of the southwestern Pacific. In W. C. McCormack and S. A. Wurm (eds.), Approaches to Language: Anthropological Issues, 191-221. The Hague: Mouton.

- 1982. Papuan Languages of Oceania. Tübingen: Gunter Narr Verlag.

- 1983. Linguistic prehistory in the New Guinea area. JHE 12:25-35.

Wurm, S. A., and L. Carrington, eds. 1978. Second International Conference on Austronesian Linguistics. 2 fascicles. PL Series C-61.

Wurm, S. A., and S. Hattori, eds. 1983. Language Atlas of the Pacific Area, Part II. Canberra: Australian Academy of the Humanities.

Wurm, S. A., and D. C. Laycock. 1961. The question of language and dialect in New Guinea. Oceania 32:128-143.

Yan Wenming. 1991. China's earliest rice agriculture remains. BIPPA 10:118-126.

- 1992. Origins of agriculture and animal husbandry in China. In C. M. Aikens and Song Nai Rhee (eds.), Pacific Northeast Asia in Prehistory, 113-124. Pullman: Washington State University Press.

Yan, Z., and N. Petit-Maire. 1994. The last $140 \mathrm{ka}$ in the Afro-Asian arid/semi-arid transitional zone. Palaeogeography, Palaeoclimatology, Palaeoecology 110:217-233.

Yen, D. E. 1977. Hoabinhian horticulture: The evidence and the questions from northwest Thailand. In J. Allen et al. (eds.), Sunda and Sahul, 567-600.

Yen, D. E., and J. Nance, eds. 1976. Further Studies on the Tasaday. Makati, Philippines: Panamin Foundation.

Yen, D. E., and J. M. Wheeler. 1968. Introduction of taro into the Pacific: The indications of chromosome numbers. Ethnology 7:259-267.

Yi, S., and G. A. Clark. 1983. Observations on the Lower Palaeolithic of Northeast Asia. CA 24:181-202.

Zingeser, M. R. 1979. The "Pithecanthropus $I V$ " diastemata: Malocclusion in a fossil man. JHE 8:523-525. 
Zorc, R. D. 1986. The genetic relationships of Philippine languages. In FOCAL II: Papers from the 4th International Conference on Austronesian Linguistics, 1986: 147-173. PL Series C-94.

1994. Austronesian culture history through reconstructed vocabulary (an overview). In A. K. Pawley and M. Ross (eds.), Austronesian Terminologies, Continuity and Change, 541-595.

Zuraina Majid. 1982. The West Mouth, Niah, in the prehistory of Southeast Asia. SMI 31, Special Issue no. 2.

1990. The Tampanian problem resolved. MQRSEA 11:71-96.

. 1991. Prasejarah Malaysia: Sudahkah Zaman Gelap Menjadi Cerah? Penang; Universiti Sains Malaysia.

- ed. 1994. The Excavation of Gua Gunung Runtuh. Malaysia: Department of Museums and Antiquity.

Zuraina Majid, and H. D. Tjia. 1988. Kota Tampan, Perak: The geological and archaeological evidence for a late Pleistocene site. IMBRAS 61:123-134. 


\section{Index}

The italicized numbers in the index are figure numbers.

Aceh, Acehnese, 120-121, 143

Admiralty Islands, 173, 187, 189, 234, 239

adzes (stone), 111, 155, 165, 169, 172, 197, 201-240 passim, 256-265 passim, 292, 304, Plates 19, 32, 33, 34,44

Aeta. See Negritos

Africa, 33, 75-76, 90-93, 133; human evolution in, 39-54 passim

Agathis, 14

Agop Atas, 180-181, 224-229, 301-304, Plate 24

Agop Sarapad, 180-185, Plate 24

agriculture, 11-13, 111, 146, 155, 172, 196, 201-254 passim; irrigation, 139, $147,202,208,250-254,271$, Plate 40 ; origins of, 131-133, 162, 170, 201-211, 234, 236, 310; and population expansions, 70-71, 87, 91, 94$95,112,127,255,258,265,311-313$; shifting, 148, 248-251, 253; and tooth size, 88-89

Agta. See Negritos

Ailuropoda, 61

Ainu, 72, 79, 83, 93

Airpurah, 288

Alocasia, 109, 242, 247, Plate 39

Alor , 25, 124-126, 269

American Indians, 75-79, 204

Andamanese, 71, 131

Aneityum, 247

Anoa, 24, 185

Anuradhapura, 295

Anyar, 290, 304, Plate 60

Arca Domas, 290

Ardipithecus, 40
Areca nut. See betel

Arikamedu, 262, 264, 294

Arku Cave, 220-221

Arnhem Land, 174

arrowheads. See bows and arrows; projectile points

Aslian languages, 97, 117, 130-131, 237, 265,267

Atayal (Atayalic), 115

Atoni, 149-150

Australia, 3-16, 22, 33-36, 67, 187, 204, 247, 309; biological anthropology, 72, 76, 78-95 passim, 126; stone industries, 54-56, 155, 171-174, 179, 190-191, 198-200. See also Australoids

Australoids, 54, 70-95 passim Australo-Melanesians, 70-95 passim, 168-169, 309

Australopithecines, $39-48$ passim, 51

Austric, 112

Austroasiatic languages, 73, 95, 97, 112, $117,121,129,147,150,155,158$, $201,211,237,267,271,276,287$, 317

Austronesians, 1, 3, 136, 155, 158, 198, $309,313-315$; archaeological correlates, 116, 123, 201-202, 211, 217, 227-251 passim, 267, 271, 287, 290, 311 ; biological correlates, 73-74, 8895; ethnographic correlates, 128-154 passim; linguistic history, 97-127 passim, 255, 287; Proto Austronesian, 99-113, 150-154 passim, 117, 214, 217, 241-242, 316. See also Malayo Polynesian languages axes. See adzes 
Austro-Tai, 111-112

Ayta. See Negritos

Babirusa, 24, 185

Bacsonian industry, 161-162, 168

Bada, 306

Bagumbayan, 222

Bajaus, 135-136, 141

Baksoko River, 64

Bali, Balinese, 8, 12, 24, 71, 87, 105, 109, 120, 130, 138-139, 154, 202, 244, 252-253, 269, 276-295 passim, 304, 306, Plates 48, 58, 59

Balobok, 189

banana, 168, 247-248, 250-251

Banaue, Plate 41

Banda Arc. See Sunda-Banda Arc

Banda Sea, 8

bandicoot, 188-189

Ban Don Ta Phet, 275, 284, 286, 294

Bandung, 198, 290

Banggai, 247

Bangka, 279

Bangkinang, 283

Banjarmasin, 140

Ban Kao (site and culture), 258-267 passim, Plates 42,43

barkcloth (production and beaters), 150 , $212,215,217,221,224,227,239$, $242,256,259,8.4,262,265,284$

Bataks, 122, 138, 143, 148, 150-152, 287, Plate 15

Batan, 221, 306

bats, $16,174,186,193$

Battambang, 281

Batu Ejaya, 196, 304

Batugajah, 288, Plate 56

Batujaya, 292

Batungan, 221-222

Baturong, 175-181

Bau-Malay pottery, 295

beads, 146, 217, 220, 229, 235, 239, 256, 258, 262, 275-277, 284, 286, 290297, 302-304, Plates 32, 55 bears, 26-27, 163, 170, 174

Belitung, 279

bells (bronze), 281, 283, Plate 50

Berawan, 152

Besoa, Plate 61

Besuki, 290

betel, 108, 111, 135, 152, 187, 217, 222, $242,287,297$

birds, 1.5, 133, 174, 179-180, 185, 208

Bismarck Archipelago, 105-106, 123, 234-235

blade industries (including bladelike flakes), 172-173, 179-180, 184-200 passim, 224, 7.12, 227

Bleberan, 290, Plate 57

blowpipe, 130, 150-152

bone tools, 61 , 66, 161-163, 173-198 passim, 209-210, 214, 219, 256-258, Plates 29, 34

Borneo, 315, Plates 21-24; environmental features, 3-16 passim, 38, 157158, 224-227, 254; languages, 105 , 117-122, 271, 287; Neolithic and early Metal phases, 219, 224-227, 232, 237-242, 244, 251, 269, 273274, 279, 287, 296, 304, Plates 36, 37; peoples of, 129, 131-152 passim, 248-249, 253, Plates 7, 8, 11, 14; Pleistocene, Pliocene in, 23-38 passim; stone industries, $58,64,172$ 181, Plate 10. See also Sabah; Sarawak Borobodur, 137-138

Botel Tobago. See Lan Yü

bovids, 1.5, 26-27, 38, 52, 106, 111-112, $152,157,163,174,181,197,202$, 231,315 . See also water buffalo

bows and arrows, 108, 111, 135. See also projectile points

bracelets, 217, 220, 229-231, 256-304 passim, Plate 44

Brahmans, 138-139

breadfruit, $150,217,242,248$

bronze (and copper), 239, 255, 268-303 passim, 317 
Brunei, 3, 140-142, 148, 233

Buad Island, 191

Buddhism, Buddhist civilizations, 74, 137-139, 275-276, 292, 316

buffalo. See water buffalo

Bugis (Buginese), 130, 140

Bukit Tengkorak, 136, 224-229, 239, 244, 301-302

Bukit Tengku Lembu, 262-264

Buni, 292-295, 304

burials, 84-86, 174; cremations, 175 ,

240-241; ethnographic methods,

151-152; inhumations (prehistoric),

163-197 passim, 215-216, 221,

239-241, 256-261 passim, 284, 286,

290-293, 296, 304, Plates 17, 22 ,

44, 57; jar burials (prehistoric), 85 ,

175, 221, 229, 240-241, 272-273,

290-307 passim, 318, Plates 37 ,

61. See also megaliths and mega-

lithic traditions; sarcophagi; slab

graves

Burma, 147, 158, 161

Buru, 7

Buwawansi, 229, 301, Plate 34

Cabenge, 24-25, 66-67, 181

Cagayan Valley, 57, 67, 220-221

Calabrian fauna, 17

Cambodia (Kampuchea), 161, 281

Canarium, 235

candlenut (Aleurites), 187, 235

canoes, 108, 111, 201, 213, 242, 283, 287

Carpentarians, 93

cassowary, 231

cattle. See bovids

Cebu, 191

Cham (Chamic, Champa), 120-121,

137, 142-143, 211, 237, 271-276,

316-317

Chamorro, 105

Chandraketugarh, 295, 300

chert, 171-173, 177-198 passim, 224 chicken, 108, 208, 235, 242, 256, 259, 287-289, 303, Plate 46

Chih-shan-yen, 213, 215

China, 3, 9, 24, 52, 253, 255, 311; agricultural prehistory, 112, 202-211; civilizations of and influences on Southeast Asia, 74, 136-141, 146, 202, 268-270, 274-278, 284, 287, 295, 316; evolution and biological anthropology, 40, 53-54, 77-94 passim; Holocene archaeology, 158, 161-162, 170, 200-214 passim, 217, 249-250, 306; languages, 97, 106, 110-112; Pleistocene industries, 30 , 59-63, 190

chopper/chopping tool industries, 5758

Choshui River, 214

Cibadak, 279

Ci Julang fauna, 17

Cishan, 208

civets, $38,197,230-231$

coconuts, 217, 235, 242, 247, 250, 287

Co Loa, 271

Colocasia. See taro

copper. See bronze

cotton, 224, 259

crocodiles, 174

cuscus. See Phalanger

Da But, 162, 165

Dabona, 283

Daeo, 188

Dawenkou, 208

deer, 23, 26-27, 37-38, 52, 61, 163, 170, $174,179,187,208,223,231,239$, 273,278

descent terminology, 132, 135, 139 , 142-154 passim, 197, 217, 267

Deutero-Malays, 74-75, 128

Dimolit, 219-220

Dingcun, 61-63

Dipterocarps, 16, 32

Diuktai, 179 
Dobo, 284

dogs: Canis and domestic dogs, 108, $111,116,174,208,215-219,229$, 231, 235, 241, 256, 287, Plate 46; Cuon, 26-27, 37-38, 181

Dong Dau, 269

Dong Son, 269-283 passim, 292, Plates $45-47$

drums (bronze), 146, 277-295 passim, 318 , Plates $45-47,56$

Dusun, 141, 145

Duyong Cave, 191, 221-223

earrings (and earplugs), 217, 220-221, $7.10,273,288$, Plate 32

edge-ground tools, $155,160-162,167-$ 175 passim, $187-188,195,200$ 201

Edjek, 223

elephants, 14, 1.5, 23-27, 37-38, 170, $197,208,278,288$, Plates 47,56

Enggano, 115, 122, 150, 152

extinctions, 36-38, 188-189, 244, 309

Feng-pi-t'ou, 214-215

Fiji, 102, 115, 224, 234, 236

fish, fishing, 16, 108-111, 134-135, 169 , $174,185-188,227,256$

fishhooks, 197, 213, 224, 230, 235, 256, $259,269,292$

Flores, 24-25, 57, 67, 74, 86, 143-144, $150,187,284,304$

Formosan. See Taiwan, languages fowls. See chicken

Fuguodun, 213

Fujian, 206, 208, 213-214, 241

Funan, 137, 244, 275-278

Galela, 74

Gayo, 122, 143

Gebe, 173, 187-189, 229, 301

Gehe, 61-63

Geochelone, 24-25

gibbons, 25, 28, 31, 163, 174
Gigantopithecus, 48

Gilimanuk, 87, 284, 294-295, 304-306

Giong Ca Vo, 272-275, 294

glaciations (and interglacials), 7, 17-22,

1.6, 33-38

glass (beads and bracelets), 239-240,

274-275, 284-303 passim

glottochronology, 104, 113-116

gold, 275-277, 288, 292, 294, 303

Golo, 173, 187-189, Plates 25-26

Go Mun, 269

Gondwanaland, 7

gourd, 208, 217, 231, 249, 287

Grenzbank, 1.8, 29-30, 46-48, 65

grindstones, $161,170,173,181,6.10$,

197-198, Plate 52

Gua Baik, 167-168

Gua Berhala, 260

Gua Bintong, 163, 260

Gua Bukit Ta'at, 163

Gua Cha, 83-86, 6.2, 163-167, 260-265, 287, Plates 17,44

Gua Chawas, 163, 168, 316

Gua Gunung Runtuh, 85-86, 163, 168

Gua Harimau, 168, 262

Gua Kajang, 262

Gua Kechil, 165-167, 262, Plate 42

Gua Kelawar, 163, 262

Gua Kerbau, 167-168

Gua Lawa, 37, 87, 196-198, Plate 29

Gua Madu, 167

Gua Musang, 262

Guangdong, 208, 210, 213-214, 227

Guanyindong Cave, 61

Gua Peraling, 163, 168, Plate 18

Guar Kepah, 88, 169, 264, Plates 19-20

Gua Sireh, 117, 158, 175, 236-239

Gua Siti Nafisah, 188, 301

Gunung Cantalan, 197

Gunung Kidul, 290-291

Gunungmegang, Plate 52

Gunung Mulu, 13

Gunung Padang, 290

Gunung Piring, 304, Plate 60 
Guri Cave, 37

Guthe Expedition, 297, 302

Hagop Bilo, 177-181, 189, 200, 302304, Plate 60

Hainan, 106, 111

Halmahera, 3, 5, 74, 105, 123-126, 133, $140,187-189,229,245,247,295$, 301,315

Han dynasty, 137, 275-278, 316, Plate 46

Hang Gon, 272-275

Harappan, 300

Hastinapura, 300

headhunting, 131, 148, 151, 153

Heap Cave, 258

hematite (red ochre), 161, 163, 169-170, $175,180-189$ passim, 197, 221, 240, 256

Hemudu, 206-213, 227, 317

Hinduism, Hindu civilizations. See India; Indianized States

hippopotamus, 23, 26-27

Hmong, 111

Hoabinhian, 58, 85-88, 158-173, 175 , $190,198,203,211,231,262,265$, 267, 306, Plates 17-21

Homo erectus, 23, 1.8, 29-55 passim, 93$94,308-309,315$; possible cultural evidence for, $55-68,160$

Homo ergaster, 41-42

Homo habilis, 41-45, 48

Homo sapiens, 41, 49-50, 54, 64, 66, 6995 passim, 308

horticulture. See agriculture

Huon Peninsula, 22, 1.6, 190

hunter and gatherer economy, 108, 111, 128-136, 155-200 passim, 201-203, $229,247-248,267,313$

Huxley's Line, 1.3, 7-8, 14, 16, 106

Iban, $122,129,145,148,151,248-249$, 313 , Plates 14, 38

Ifugao, 253
India, 33, 90, 93, 128, 136, 151, 190, $200,202,247,268,271-313$ passim Indianized states, 74, 124, 130, 136-144, 147-148, 252-253, 268-269, 278, 287, 296, Plate 12

Indo-Pacific Prehistory Association, 314, 318

Intertropical Front, 10-11, 34

Irian Jaya. See New Guinea

iron, 133, 150, 239, 249-252, 268-306 passim

irrigation. See agriculture

Islam, Islamic civilization, 74, 124, 128, $130,136,139-144,147-148,151$, 269,296

jade, 210, 217-218, 220, 303, Plate 32 Jakun, 47, 287

Jambi, 198

Japan, Japanese, 5, 79, 112, 155, 173, $179,190,200,252,306-307,309$

Java, 251-253, 269; biological anthropology, 86-87; civilizations of, 130, $137-142,146,152-153$, Plate 12; environmental features, $3-16$ passim, 38, 151, 244-248, 317; Homo erectus in, 39-95 passim, 308-309; languages, 102, 105, 115, 120-123; Neolithic and early Metal phases, 202, 219, 230-236, 244, 278-283, 287, 290-295, 304, 306, Plate 57; Pleistocene and Pliocene in, 17-18, 23-31 passim, 36, 52, Plates 1 and 2; stone industries, $55,57,2.9,190$ 191, 196-198

Javanese, 71, 316

Java Sea, 5, 8, 32

Jenderam Hilir, 260

Jetis fauna, 17, 25-26, 30, 61

Job's tears, 187, 245-246

Johor, 141, Plate 50

Jolo, 140

Jomon period and people, $72,79,83$, $200,211,306$ 
Kabuh formation, 28-31, 46, 48-49, 52 , 65

Kadai languages, 106, 111, 201

Kai, 278

Kajar, 290

Kalanay, 302, 304

Kalasan, 153, Plate 12

Kali Glagah, 17

Kalimantan. See Borneo

Kalumpang, 227-229, 239, 317, Plate 33

Kampong Pencu, 281, Plate 50

Kampong Sungei Lang, 278, 284

Kampung Dusun Raja, 260

Kandal, 281

Kantu, 250

Kapuas River, 32, 148

Kausambi, 300

Kayan, 129, 133, 149-151, 248

Kedah, 163, 179, 260, 262, 276, 287

Kedung Brubus fauna, 1.9, 28, 31

Keilor, 93

Kelabit, 253

Kelantan, 163, 167-168, 260, 262

Kendeng Lembu, 231

K'en-ting, 213-214

Kenyah, 149

Keqiutou, 213

Kerinci, 122, 189, 198, 281, Plate 49

Khao Talu Cave, 258

Khlong Thom, 277

Khok Phanom Di, 256-260, 262, 317

Kinabalu (Mt.), 9, 18, 38

Klang, 277-278, 281, 284, Plate 50

Kota Cina, 140

Kotaraya Lembak, 288-289

Kota Tampan, 68, 155, 160, 168, 316

Kota Tongkat, 165-166

Kota Waringin, 279

Kow Swamp, 54, 93-94

Ko-ying, 137

Kradenanrejo, 281, 292

Kra Isthmus, 276

Kuala Selinsing, 277, 286

Kuala Trengganu, 278
Kubu, 132

Kuningan, 288

Kushan, 278

Kutei, 138

Laetoli, 42

Lahanan, 147

Lake Diatas, 233

Lake Mungo, 93

Lake Padang, 233

Lal lo, 221

Lambanapu, 304

Lamongan, 279, 281, 292

Lampung, 281, 288

landbridges, $14-28$ passim

Land Dayaks, 237, 240

Lang Ca, 270

Lang Rongrien, 160-161, 179

Lang Vac, 270, 272

Lantian, 54, 61

Lan Yü, 245, 247

Lao, Laos, 161, 272, 277, 306

Lapita, 224, 227, 234-236, 244, 247, 313,317 , Plate 35

Laurente Cave, 220

lead, 286

Leang Bua, 304

Leang Buidane, 87, 297-304

Leang Burung, 181, 184-185, 190, 193, 195, 200, 229, Plates 28,60

Leang Cadang, 87

Leang Tuwo Mane'e, 191-193, 224-225, 229

Lebak Sibedug, 290

Lesser Sundas, 231, 251, 279; archaeology, 67, 295-296, 303-305; biological anthropology, 73-74; environmental features, 5-24, 157 passim; landbridges along, 24-25, 308; languages and peoples, 105, 124, 135, 138, 142$143,148-149,152-153,287$

Leuwiliang, 292

lexicostatistics, 104, 113-116

Liang Toge, 86 
Lingga Islands, 7, 136, 141

Lingling-o, 217, 273-274, 303

Liujiang, 83, 93-94

Lobang Jeragan, 238-240

Lombok, 8, 12, 24, 67, 105, 120, 304

Longgupo Cave, 45

lontar palm, 254

loom. See weaving

lost wax casting (cire perdue), 271, 278

Lubang Angin, 236-241, Plate 36

Lumajang, 284, 316

Lun Dayeh, 250, 253

Lungshanoid, 214-215

Lupar River, 32-33

Luzon, 8, 57, 67, 71, 105, 131-132, 140, $170,189,202,219,221,232,241$, $245,247,252-253$, Plate 41

Maanyan, 122, 145

Madagascar, 97, 105-106, 114, 122-123, $136,150,276,311$

Madai, 37-38, 175-181, 224-229, 301302 , Plate 24

Madura, 120-121, 281, 283, Plate 49

Magala, 240-241

Magapit, 221

Magsuhot, 303

Mai Da Nuoc, 88

Majapahit, 138

Majiabang, 208

Makassar (Makassarese, Strait of Makassar), 8, 32, 115, 140, 245, 281, 283

Malacca (Melaka, Strait of Malacca), 135-137, 140-141, 276, 287

malaria, 256-258

Malay (people and language), 71, 88, 97, $115,117,120-123,130-153$ passim, $211,287,316$

Malay Peninsula: biological anthropology, 71-73, 77, 85-88; civilizations of, $276,279,316$; environmental features, 1-14 passim, 38, 157-158, 247; languages of, 96-106 passim, 120, 271; Neolithic and early Metal phases, 219, 237, 244-245, 255-267, 270, 272, 277-279, 284-287, 290; peoples of, 71-73, 129-133, 137-148 passim; Pleistocene in, 23, 31, 35-36, 310 ; stone industries, $57,68,155-$ 171 passim, 179

Malayic languages, 119-122

Malayo Polynesian languages, 103-124 passim, 150, 152, 236, 241-242

Malesia, 13-14

Maloh, 149

Manobo, 135

Manuaba, 281, Plate 48

Manunggul, 239, 303

Manus. See Admiralty Islands

Mariana Islands, 234-236

Marjan Cave, 197

Maros points, 194-200, 229

Maros region, 181, 184-186, 193-196, 304

Masbate, 221-222

Mata Menge, 67-68

megaliths, megalithic traditions, 139, 146, 152-153, 287-290, 306, Plate 61. See also sarcophagi; slab graves

Meganthropus, 48-49

Melanau, 141, 150-151

Melanesia, Melanesians, 11, 149, 154, $173,187,189,234-236,247,251$, 309-313; biological anthropology, 71-81 passim, 90, 93; languages of, 97-99, 105-106, 119, 123-124. See also Lapita

Melolo, 87, 303-305

Mentawai Islands, 138, 144, 152, 247 , 250-251, Plate 6

Miao. See Hmong

microliths, 193-200

Micronesia, Micronesians, 79, 88, 90 , $92,95,105,146,235-236$

Mien, 111

millet, 109-110, 205, 208, 213, 215, 217, $231,242,245-246,250$ 
Minahasa, 12, 120, 151-152, 184-185, Plate 5

Minanga Sipakko, 227

Minangkabau, 115, 122, 143-144, 150, Plate 9

Minatogawa, 83

Mindanao, 25, 71, 133-135, 250, 302

mitochondrial DNA, 54-55, 72, 77, 315

Mnong Gar, 248

Moh Khiew, 85, 160-161

Mojokerto. See Perning

Moluccas (Maluku), 3, 5, 8, 16, 58, 67, 73-74, 105, 119-124 passim, 135141 passim, 172-173, 187-189, 219, 229-247 passim, 275-279, 295, 300304, Plates 25, 27

Mongoloids, 1, 54, 70-95 passim, 131, $202,215,258,297,311$

monkeys, $24,38,52,106,163,174,179$, $185,197,208,230-231$

Mon-Khmer (languages and people), $73,85,97,121,131,265,267$. 287

Morotai, 74, 86, 124-126, 187-189, 301, Plate 27

mortars. See grindstones

Motu, 115

Murrayians, 93-94

Muruts, 141, 151, Plate 8

Musang Cave (Luzon), 189

Musi River, 32

Natufians, 205

Negri Sembilan, 143-144

Negritos, 71-74, 76-77, 81, 85, 92-93, 99, 111, 117, 128-136 passim, 153, $158,205,247,265-267$, Plate 2

Negros, 71, 303

New Britain, 187, 224

New Caledonia, 116, 234

New Guinea, 187-188, 202-203, 234$235,251,278-279,313$; biological anthropology, 71, 73, 81, 89-95 passim; environmental features, 3,9 ,
$12,18,21,24,233,247,249,254$, 309-310; languages of, 97, 105, 115, 117, 123-127, 154; Pleistocene in, 18, $22,34,36,162,315$; stone industries, $56,155,171,173,190$

New Ireland, 187

Ngaju, 122

Ngandong, 25-26, 31, 1.8, 1.9, 2.3, 46, $50-53,57,65-67,94,315$

Ngawi, 46, 50, 66

Ngebung, 1.10, 57, 65, 68

Niah, 36-38, 83-85, 88, 93-94, 155, $172-175,180,236,238-241,272$, 306, Plates 10, 21, 22, 36, 37

Nias, $122,138,143,146,149-150,247$. 287

Nicobar Islands, 97

Nihewan, 61

Nikiniki, 317, Plate 35

Nil Kham Heng, 275, 279

Niu-ma-t'ou, 214

Nong Chae Sao, 259

Nong Nor, 203, 257-258, 275

Non Pa Wai, 257

Notopuro formation, 31,65

Nuaulu, 144, 250

Nusa Tenggara. See Lesser Sundas

Nyong, 264-265

obsidian, 171-172, 184-189, 198, 224, $7.12,227,229,235$

Oceanic (language subgroup), 103, 105, 114-115, 119, 123, 153, 234, 236, 313

Oc Eo, $275,277,286$

Olorgesailie, 56

O-luan-pi, 214

Ongbah Cave, 284, 286

Orang Asli, 85, 95, 97, 158, 165, 258. See also Aslian languages; Negritos; Senoi

Orang Laut, 135-136, 202

Orangutan, 25, 28, 40, 174, 181

Oryza. See rice 
Pa-chia-ts'un, 212

Pacitanian, 57, 59, 64, 67-68

Pacung, 281

Pageralam, Plate 52

Pahang, 71, 163, 165-168, 262, 264, 285

Paiwan, 217

Pakauman, 290

Palauan (Belauan), 105

Palawan, 7-8, 36, 85, 170, 173, 189, 191, 221-222, 239, 273, 9.13, 302303

Palembang, 137, 140, 288

Pallava, 137, 286

Pamwak, 189

Panay, 71

Pandanus, 29, 233, 235, 240, 247

Panganreang Tudea, 195

Pangkajene Kepaulauan, 193

Pangkungliplip, 294

Pangolin, 36-38, 106, 174

Pantar, 124-126

panther, 26-27

Papuan languages, 74, 79, 97-99, 116$117,123-127,234,236,295,301$, 310

Pasemah Plateau, 288-289

Paso, 185-186

peacocks, 278, Plate 47

Pea Sim Sim, 233

Peiligang, 208

Peinan, 215-223, 256, 318, Plates 31-32

Pejaten, 292

Pejeng, 281, 292, 294, Plate 48

Pematang, Plate 55

Penan. See Punan

Pengtoushan, 208, 317

Peninsular Malaysia. See Malay Peninsula

Perak, 68, 85, 160-168 passim, 262, 284286, Plate 51

Perlis, 163, 260, 262

Perning, 30, 43, 46

Pescadores Islands, 212-213

Petpuruh Cave, 197
Phalanger (Cuscus), 16, 24, 185, 188$189,193,230,235$

Philippines: biological anthropology, 71-73, 77, 81, 87-92; languages, 97, 102-105, 110-111, 117-124, 201;

Neolithic and early Metal phases, 215-249 passim, 273-274, 295-297, 302-303, 307, 311, 317; peoples of, 71, 131-153 passim, 247, 251-255, 265-267, 300; Pleistocene in, 1-16 passim, 24, 35, 157; stone industries, 57, 67, 170-173, 189-191

Phu Hoa, 272, 275

pigs, 24-27, 38, 52, 108, 111, 116, 146,

$149,164,169,173-174,179,185-$

$187,193,197,208,215-219,223$, 229-230, 235, 241, 256, 259, 287, 303 , Plate 46

Pinatubo, 131

Pintu, 170

Pithecanthropus. See Homo erectus

Plain of Jars (Laos), 272

Plawangan, 292, 304, 306

pollen analyses, 213, 233-234

Polynesia, Polynesians, 11, 72, 79, 88, 90, 92, 95, 105, 123-124, 129, 139,

$146,153,236,244,252,310,313$

Polynesian chestnut (Inocarpus), 231

porcupine, 174, 179, 231

Prajekan, 284

Prambanan, 137

projectile points (including arrowheads and spearheads): 178-179, 195-200, 212-221 passim, 227, 242, 258, 262, 279; of bronze and iron, 269, 271, 284-285, 288, 292, 302-303, Plates $29,32,33,42$. See also bows and arrows; Maros points

Proto-Malays, 74-75, 128

Pucangan formation, 28-30, 46-49

Puger, 87

Punan, 132-134, 147, 152, 202, 244 ,

Plates 7, 11

Punung, 1.9 
Purnavarman, 293

Pusu Samang Tas, 302

Quynh Van, 88

Rabel Cave, 220

Ramapithecus, 40

rats. See rodents

reaping knives, 206, 213-227 passim, $242,259,264-265,269,274,284$,

286, 317, Plate 38

red ochre. See hematite

Rejang (Sumatra), 143-144

Rejang River (Sarawak), 148

Rengasdengklok, 292

rhinoceros, 1.5, 25, 37-38, 67, 163, 170, $174-175,181,208$

Riau, 7, 135-136, 141

rice, $12,109-112,117,144,147-148$,

$152,162,164,203-223$ passim, 237-

254 passim, 256-259, 271, 287, 294,

Plates 38,40

rodents, $16,25,31,67,163,174,179$,

$185-186,188,193,235,249$, Plate 44

Rome, Roman, 275-277

Roti, 149, 254, 281, 283

Rouletted Ware, 292-295, Plate 59

Ryukyu Islands, 5, 200

Sabah, 3, 145, 160, 175-181, 224-227, 237, 239, 281, 296, 300-304, Plates 8, 39

sago, 12, 109-110, 133-134, 189, 242, $245,247,250-251$

Sahulland (Sahul Shelf), 1.3, 7-9, 16, 21, 34-36, 88

Sa Huynh Culture, 271-275, 284, 302303

Sailendras, 137, 153

Salayar, 278, Plate 47

Sama, Samal, 135-136

Sambungmacan, 46, 50, 66

Samoa, 102, 232-234

Sampung, 196-198
Sangeang Island, 278, Plates 45,46

Sangihe, 4, 120

Sangiran, 1.8, 1.10, 29-31, 2.3, 2.4, 46$48,57,64-65,67$, Plate 1

Sanskrit, 122, 137-138, 275, 293, 316

Santa Cruz, 235

Sarawak, 3, 13-14, 83-85, 117, 129, 133$135,140,145,148,150-152,236-$ 241, 248-249, 273, 302-303, 317, Plates 7, 11, 14, 36, 37. See also Niah sarcophagi (stone), 215, 290, 293-294, Plate 58

Satavahana, 278

Satus, 279-280

Savu, 149, 254, 281

sawah. See agriculture, irrigation

sea level changes, 21-22, 24, 28, 31-38 passim, 157-158, 161, 169, 177, 258, 310

Seberang Perak, 168

Selako, 122, 145

Selangor, 260, 284-285, 316, Plate 50

Semang. See Negritos

Sembiran, 281, 294-295, 300, 304, 306, 317, Plate 59

Semelai, 144

Senoi, 71, 85, 129-132, 147, 154, 165 , $245,255,265-267$, Plate 3

Seram, 7, 8, 143-144, 231, 250

shell middens, $163-170$ passim, 179 185 passim, 191, 213, 221, 317

shell tools, 108, 173, 187-189, 197, 201, 222-224, 235, 262, Plates 25, 34, 44.

See also fishhooks

shell ornaments, 108, 221-224, 227$230,235,256-258,262,286,297,304$

Shixia, 208

sickles. See reaping knives

Sidorejo, 66

Sinodonts, 90

Sino-Malayan fauna, 25

Sino-Tibetan, 97, 101, 112

Sisupalgarh, 295

Situ Gunung, 233 
Siva-Malayan fauna, 23-25

skin pigmentation, 71-76

slab graves, 215-217, 284-293, Plates 32,

$51,53,54$

snakes, $174,179,185-186,288$

Sodong Cave, 197

Solo River, 13, 29, 31, 39, 46, 49, 50-53

Solomon Islands, 19, 187, 234

Sonviian, 159-160

Song dynasty, 140, 206, 275

South China Sea, 5, 8, 11, 32

SPAFA, 314, 318

spearheads. See projectile points

spindle whorls, 7.2, 211-223 passim, 259,274

Spirit Cave, 162

Sri Lanka, 294-295, 307

Srivijaya, 122, 137, 141, 288, 316

Stegodon, 23-25, 31, 52, 61, 66, 187, 308, 310

subak (Bali), 139

sugarcane, 109-111, 217, 242, 250

Sukajadi, 87, 169, Plate 20

Sula, 7

Sulawesi: environmental features, 3-16 passim, 157; languages, 105, 119 124; Neolithic and early Metal phases, 219, 227-230, 242, 245-246, 296, 304, 306, Plates 33, 35, 61; peoples, 135, 138, 142-154 passim, 287. Plates 5, 13; Pleistocene, Pliocene: 23-25, 35, 308; stone industries: $57-$ $58,181,184-186,190-196,200$

Sulu (Archipelago and Sea), 8, 22, 135$136,140,189$

Sumatra: civilizations of (see also Srivijaya) and environmental features, 318 passim, 38, 233-234, 244-245. 251; languages, 105, 115, 120-124; Neolithic and early Metal phases, $219,231,236,251,275-279,281-$ 283, 287-289, 292, 295, 304, 316317; peoples, 131-132, 137-152 passim, Plates 9, 15, 52-56; Pleis- tocene in, 23, 31-36 passim, 315;

stone industries, $158,161-162,169-$ 171,189

Sumba, 3, 5, 8, 21, 25, 74, 87, 146, 149, 290, 296-297

Sumbawa, 67, 105, 290

Sunda Banda Arc, 3-7

Sundadonts, 90

Sundaland, Sunda Shelf: environmental features, 3-16 passim, 52, 309-310; Pleistocene and Pliocene, 21-38 passim, 57, 157-158, 171; populations and languages, 88, 90-92, 106, 120-124

Sundanese, 115

Sunget, 221

Sungkai, Plate 51

Sun Moon Lake (Taiwan), 213

Swartkrans, 51

Tabon Caves, 83-85, 88, 94, 189, 272, 302-306

Tagalog, 115

Taiwan, 3, 8, 34, 91-92 , 210-211, 311; archaeology, 158, 170, 200, 203, 208, 211-251 passim, 304, 317-318, Plates $30,31,32$; languages, 97-99, 102

119 passim, 201, 214

T'ai-yuan Culture, 215

Talasea, 224, 227

Talaud Islands, 87, 120, 191-192, 195, $219,236,281,295,297-304$

Tampanian, 68

Tana Ai, 143

Tanjungara, 288, Plate 54

Tanjung Pinang, 86, 188, 301, Plate 27

Tanshishan, 213

Tapadong, 302

Ta-p'en-k'eng, 203, 211-217, 245, 317, Plate 30

Tapir, 37, 174

taro, 109, 111, 144, 234-235, 242-251

passim, Plate 39

Tasaday, 133-135, 152 
Tasmania, Tasmanians, 84

Tattooing, 108, 135, 151, 220

Tausug, 135

Taxila, 300

Tebingtinggi, 290

Tegurwangi, 288, Plate 53

Tembeling, 264-265, 278

Temuan, 144, 147, 267, 287

Ternate, 140-141

Thailand, 2, 137, 158-162, 237, 244, $247,252,255-260,269,272-277$, $279,284,287,294,306,316,318$

Thai (Tai), Thais, 88, 97, 101, 106, 111$112,121,140,255$

Tianko Panjang Cave, 189, 198

Tidore, 140-141

tiger, 1.5, 26-27, 37-38, 174, 181, 278

Timor, 3, 5, 7-8, 16, 21, 24-25, 57, 67, $74,123-126,143-144,149-150,186-$ $187,219,229-231,235,242$, Plates 4 , 16,35

tin, $277,279,286$

Tingkayu, 160, 176-180, 190, 309, Plate 23

Toalian industry, 66, 181, 185, 191-197, 229,306

Toba eruption, 68, 160, 316

Tobelo, 74

Togutil, 133

Toraja, 145, 150-151, Plate 13

Trinil (and Trinil fauna), 25-26, 1.8, 1.9, 28-31, 39, 46, 49-50, 61, Plate 1

Tuban, 197

Tugu, 293

tulang mawas, 284-285

Uai Bobo, 7.14

Uattamdi, 229, 300-301, Plate 34

Ulu Leang, 181, 185, 193-196, 229, 304, Plate 35

Um Kapat Papo, 188

Vanuatu, 234, 247

Viet Khe, 270
Vietnam, 2, 88, 237, 241-252 passim, $255,294,306,318$; early Metal phase, 268-279, 283-284; languages, 97-99, 105-106, 120-122, 317; stone industries, 155-162, 165, 168, 170, 173. See also Cham; Dong Son

Villafranchian, 17, 23

Wahgi Valley, 251

Wajak, 86, 88, 93-94

Walanae Valley. See Cabenge

wallaby, 188-189, 235

Wallace Line, 7, 24. See also Huxley's Line

Wallacea, 1.3, 7-9, 14, 16, 21, 34, 88, 92, $309,311,315$

water buffalo, 26-27, 37, 52, 111-112, $197,202,208,252,271,288,294$. Plate 55

Watualang, 52, 66

weaving (and backstrap loom), 108, 131, $150,152,215,224,242,251$

Weber's Line, 8

Wetef, 187

Wonotunggal, Plate 56

Xiqiaoshan, 227

Xitou, 213

Yami, 247

yams, 109, 135, 162, 242-250 passim

Yangzi River, 206

Yao. See Mien

Yayoi Period, 306

Yellow River, 206, 208, 316

Younger Dryas, 33, 205-206

Yuanmou, 45, 61-63

Yüan-shan Culture, 215-223, Plate 30

Yunnan, 262, 271, 283-284, 318

Zhejiang, 206-213, 241, 317

Zhou dynasty, 316

Zhoukoudian, 39, 2.3, 2.4, 49, 54, 66, 94

Ziyang, 83 


\section{About the Author}

Peter Bellwood was born in Leicester, England, and studied archaeology at Cambridge University. In 1967 he was appointed Lecturer in Archaeology at Auckland University, then moved to the Australian National University in 1973, where he is currently a Reader in Archaeology. He has undertaken archaeological research in Polynesia and Island Southeast Asia, particularly in Malaysia and Indonesia. His previous books include Man's Conquest of the Pacific (1978), The Polynesians (1987), and Archaeological Research in South-Eastern Sabah (1988). His current research (1996) is in the Moluccas Islands of northeastern Indonesia. He is also Secretary-General of the Indo-Pacific Prehistory Association and editor of the IPPA Bulletin. 